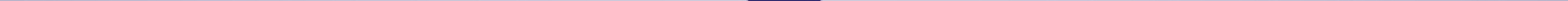




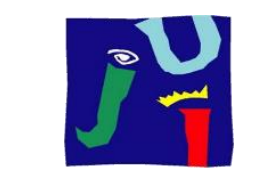

UNIVERSITAT

JAUME• |

DEPARTAMENT DE QUÍMICA FÍSICA I ANALÍTICA

ÀREA DE QUÍMICA FÍSICA

\title{
DESARROLLO DE SULFUROS CLÚSTER DE METALES DEL GRUPO SEIS Y SUS APLICACIONES CATALÍTICAS
}

\author{
Carmina Alfonso Padilla
}

TESIS DOCTORAL

Dirigida por:

Prof. Rosa $\mathrm{M}^{\mathrm{a}}$ Llusar Barelles

Dra. Marta Feliz Rodríguez 


\section{By \\ UNIVERSITAT \\ JAUME•I}

ROSA $M^{\mathrm{a}}$ LLUSAR BARELLES, CATEDRÁTICA DEL ÁREA DE QUÍMICA FÍSICA DE LA UNIVERSITAT JAUME I, Y MARTA FELIZ RODRÍGUEZ, CIENTÍFICA TITULAR DEL INSTITUTO DE TECNOLOGÍA QUÍMICA DE LA UNIVERSITAT POLITÈCNICA DE VALÈNCIA

CERTIFICAN: Que Carmina Alfonso Padilla, licenciada en Química por la Universitat Jaume I, ha realizado bajo su dirección, en el área de Química Física de esta universidad, el trabajo titulado: "Desarrollo de Sulfuros Clúster de Metales del Grupo Seis y sus Aplicaciones Catalíticas", que constituye su memoria de Tesis Doctoral para optar al grado de Doctora en Química, y que cumple los requisitos para la obtención de la Mención Internacional.

Y para que conste a los efectos oportunos, firmamos el presente certificado en Castellón a 24 de octubre de 2016. 


\section{AGRADECIMIENTOS}

Después de unos cuantos años, por fin llegó el momento de cerrar una etapa muy importante y enriquecedora, tanto personal como profesionalmente. Llegar hasta aquí no habría sido posible sin el aliento de muchas personas, algunas son "de toda la vida", y otras, no menos importantes, me las he ido encontrando por el camino. Espero no dejarme a nadie porque todos y cada uno de los que voy a nombrar tenéis algo de culpa de que hoy esté escribiendo estas líneas, y como nunca me ha costado dar las gracias, advierto que igual me queda un poco largo...jallá voy!

En primer lugar, me gustaría agradecer la labor de mis directoras, Rosa y Marta. A Rosa, por darme la oportunidad de desarrollar la tesis doctoral en el grupo de Materiales Moleculares y por ayudarme a crecer como persona y como investigadora. A Marta, por estar al pie del cañón, siempre animándome a comenzar cualquier proyecto por complicado que parezca. A las dos, jmuchas gracias! Vull agrair de manera especial a Sixte les hores que ha invertit en aquest treball i la seua paciència a l'hora d'ensenyar-me com funciona la Química Computacional, gràcies!

Me gustaría dar las gracias a todos los buenos maestros y profesores que he tenido a lo largo de mi vida académica, desde el colegio Madre $\mathrm{M}^{\mathrm{a}}$ Rosa Molas de Onda hasta la Universitat Jaume I, pasando por el IES Serra d'Espadà y el Cork Institute of Technology. ¡Sin vuestro buen trabajo yo no habría llegado hasta aquí!

Con mis compañeros de laboratorio he compartido risas, lloros, viajes...habéis sido mucho más que compañeros de trabajo. Elena y Eva, sabéis lo que significáis para mí. Gracias por estar ahí para cualquier cosa, científica o personal, por poder contar con vuestro apoyo y disfrutar de vuestra alegría permanente. A David, amb tu he compartit uns quants anys de carrera i uns quants més de despatx. Gràcies per tant! Por supuesto no me puedo olvidar de Tomás, tú me enseñaste con mucha paciencia algunas de las técnicas que hoy domino, además de los buenos ratos personales que compartimos, e Iván, encara que vam coincidir poc de temps a la 
universitat, vull donar-te les gràcies per la teua ajuda a Rostock. También me gustaría dar las gracias a Marta Blasco, Emma, Alba, Aitor y María, nuestros chicos de TFG, TFM o colaboración, le habéis dado mucha "vidilla" al laboratorio y ha sido muy fácil compartir espacio con vosotros. A los visitantes extranjeros como Pavel, Maxim S., Artem, Niels, Maxim R., Ruben y especialmente Carolina, gracias por vuestros consejos y por el tiempo compartido. A los técnicos de Serveis Centrals, gracias por vuestro trabajo, sin él el mío no habría salido adelante. También me gustaría agradecer al resto de compañeros del área de Química Física su interés en mi trabajo y los momentos compartidos en almuerzos, salidas en Magdalena y comidas de Navidad. Y con mucho cariño a Merche, nuestra secretaria, sense tu aquest departament no funcionaria ni la meitat de bé, gràcies! Finally, I would like to thank all the people I met during my months in Rostock (Germany), especially Professor Matthias Beller, it was a pleasure to work with you and your team, thanks for everything!

En el terreno personal soy muy afortunada, así que allá va mi lista interminable de personas a quien agradecer...A mis amigas, mis "deceneras", con las que he compartido "momentazos" desde la fundación de Radio Macuto en el conservatorio: Andrea, Anna, Cris, Isa, Lore, Maijo, Maribel, Merx y Silvia, tenemos muchas cosas que celebrar en muy poco tiempo, iy esta es la primera! Gracias por tantos años de amistad, aunque algunas estéis lejos es muy bonito que siempre hagamos por vernos en cuanto volvéis al "poble”. ¡Sois grandes! ¡Y vuestros maridos también!

A mi "Equipo A": Maria, David, Ana, Javi, Morena, Paloma y Lidia, gracias por tantos momentos bonitos vividos, por esta amistad desde tiempos inmemorables, he crecido con vosotros y esta tesis tiene mucho de vuestro cariño. Maria, W\&W forever! T'estime!

A mis “X. E.", desde 2005 dando guerra: Fus, Lara, María y Sandra. En los momentos duros, cuando me pregunto por qué estudié esta carrera, me viene a la 
mente vuestra amistad y se disipan todas las dudas, ¡sois las mejores compañeras que me pudieron tocar! Nuestros jueves universitarios siguen sin tener rival hoy en día y lo sabéis. ¡Espero con ansia nuestra próxima visita a las tascas, yo invito a la zurra! ¡Os quiero!

A los Pericos y Pericas, especialmente a Santi Solsona por el diseño desinteresado de la portada de la tesis (jartista! Gracias por tu generosidad y por estar dispuesto a ayudarme con tu mejor sonrisa). Y gracias a todos por acogerme como una más, por vuestra amistad y cariño, cada vez que me habéis preguntado por la tesis, en especial en estas últimas fiestas, sé que lo habéis hecho de corazón. ¡Una parte de lo que hay aquí escrito es vuestra!

A esos amigos que siempre están ahí cuando los necesitas...la responsable de mi pelo, pero mucho antes que eso AMIGA con mayúsculas. Gracias Ana por 12 años de amistad y por guardarme el turno a mediodía para que la tesis saliera adelante, ¡reinas 2004 forever! Delfín y Silvia, desde nuestros tiempos futboleros en Vilafamés pasando por las promociones a tercera del San Pedro, siempre habéis estado ahí en los buenos y malos momentos. ¡Gracias por vuestro apoyo y espero que compartamos en un futuro muchos momentos especiales como este! Inma, els teus ànims des de Puçol m’han arribat encara que estiguem a uns quants quilòmetres. La quedada la tenim pendent, ara amb Celia i Darío! \#Iloveyourubiameua

La vida te da sorpresas y una de ellas fue encontraros. Gracias "Diosas del Olimpo": Amparo, Ana, Guillermina, Laura y Rosa, gracias por tantas conversaciones "chorra" al día, pero también por estar ahí cuando hace falta y por animarme tanto estos últimos meses con la tesis. ¡Espero que sigamos compartiendo muchos “momentazos" juntas! Y que por fin quedemos para ver Dirty Dancing, ipor Dios!

Acabamos la carrera juntos e iniciamos este camino que hoy acaba también juntos. Vicente, has sido mi gran apoyo durante los años de doctorado, en especial durante mi estancia en Alemania y ayudándome con el proceso de escritura. Gracias 
por todos estos años, quién nos iba a decir que detrás de los bailes en Ettro venía esto...pasa el tiempo y cuando estoy contigo soy más "yo" que nunca, ipor muchas más risas y buenos momentos! ¡Te quiero! Y por supuesto, también me gustaría agradecer a la familia Cabrera-Vilar su apoyo en estos años, me habéis visto crecer profesionalmente y sé que estáis orgullosos de mí, igracias!

Llegó el momento de agradecer a mi familia y a la gente que siento como mi familia aunque no nos una la sangre. Gracias a todos mis tíos y primos, tanto "Padillones" como "Family Alfonso", he apreciado mucho vuestro interés y cariño durante el desarrollo de este trabajo. A Queta, Jose ( $\mathrm{Pa}$ ) y Cris y también a Charo, Pepe, Diana y Miguel: sois mis tíos y primos aunque la genética diga lo contrario, gracias por tanto desde que soy una enana. ¡Tenemos mucho que celebrar!

No sé si mi familia está más orgullosa de mí o si yo estoy más orgullosa de ellos en estos momentos, lo que sí que sé es que todo lo que he hecho en esta vida se lo debo a mis padres, a su esfuerzo, trabajo, sacrificio, educación y cariño. Sois el pilar de mi vida, nunca viviré los suficientes años para devolveros todo lo que me habéis dado. GRACIAS, jos quiero mucho!

Y si mis padres son mis pilares, mis hermanos son lo mejor que me ha pasado. Yoli, gracias por tu apoyo, por tus tonterías en la mesa, por todos los momentos compartidos, hemos crecido juntas y mi vida sin ti hubiera sido mucho más aburrida. Siempre tienes un "te quiero" preparado, y hoy soy yo la que te lo da a ti: jte quiero ginxi! Víctor, cuando tenías 10 años me dijiste que fuera a por el Nobel y casi me muero de un ataque de risa. Espero que un doctorado sea suficiente y que estés igual de orgulloso de mí, jte quiero enano!

Y por último, un recordatorio muy especial a mis abuelos que tan contentos estarían hoy de verme aquí. Sé que mi abuela Carmen siempre confió en que acabaría haciendo algo grande, jesto va por ti abuelita! 
A mis padres y hermanos

A Vicente 


\section{RESUMEN}

Esta tesis doctoral describe la síntesis, estructura y aplicaciones catalíticas de una nueva familia de calcogenuros clúster trinucleares de metales del grupo 6 funcionalizados con ligandos difosfina, aminofosfina y, por primera vez, aminodifosfina. El capítulo 1 recoge una introducción sobre el desarrollo de la química de clústeres metálicos y, más concretamente, de los calcogenuros de metales del grupo 6 desde los inicios de la química de clústeres hasta nuestros días. También se detallan algunas de las aplicaciones más interesantes de estos complejos en medicina y catálisis. A continuación en el capítulo 2 se enumeran los objetivos de este trabajo.

En el capítulo 3 se describen la síntesis y caracterización estructural de hidruros y fluoruros clúster de fórmula $\left[\mathrm{M}_{3} \mathrm{~S}_{4} \mathrm{X}_{3}(\mathrm{dppe})_{3}\right]^{+}(\mathrm{M}=\mathrm{Mo}, \mathrm{W} ; \mathrm{X}=\mathrm{H}, \mathrm{F})$ y se realiza un estudio comparativo de su actividad catalítica en la hidrodefluoración regioselectiva de la pentafluoropiridina en función de la basicidad del ligando.

En el capítulo 4 se recoge la síntesis de nuevos clústeres con unidad $\mathrm{M}_{3} \mathrm{~S}_{4}(\mathrm{M}$ $=\mathrm{Mo}, \mathrm{W})$ funcionalizados con la aminofosfina ediprp y se discute su estructura cristalina. El análisis de las estructuras cristalinas muestra la coordinación específica del ligando aminofosfina a la unidad trimetálica, con el átomo de fósforo en posición trans al azufre apuntado y el de nitrógeno en posición trans a los azufres puente. La actividad catalítica de estos clústeres funcionalizados con ligandos aminofosfina en la reducción catalítica de 2,2,2-trifluoroacetofenona se compara con la del clúster $\left[\mathrm{Mo}_{3} \mathrm{~S}_{4} \mathrm{Cl}_{4}\left(\mathrm{PPh}_{3}\right)_{3}\left(\mathrm{H}_{2} \mathrm{O}\right)_{2}\right]$ en presencia de varias aminofosfinas que difieren en la naturaleza primaria, secundaria o terciaria, del grupo amino.

El capítulo 5 trata sobre la funcionalización de clústeres $\mathrm{Mo}_{3} \mathrm{~S}_{4}$ con aminofosfinas quirales derivadas de la prolina, lo que resulta en clústeres ópticamente puros que presentan tres centros estereogénicos. La caracterización estructural de estos complejos permite asignar su configuración absoluta de forma inequívoca como 
P. Las estructuras cristalinas obtenidas experimentalmente se comparan con las estructuras optimizadas mediante la Teoría del Funcional de la Densidad (DFT). También se calculan y discuten los órdenes de enlace presentes en estos clústeres mediante cálculos de Orbitales Naturales de Enlace (NBO) y se presenta un análisis de las interacciones más relevantes basándonos en la Función de Localización Electrónica (ELF). Además, se discute la actividad catalítica de estos clústeres en la reducción de sustratos orgánicos.

El capítulo 6 muestra por primera vez la síntesis y estructura cristalina de un clúster con unidad $\mathrm{Mo}_{3} \mathrm{~S}_{4}$ funcionalizado con un ligando aminodifosfina. La característica más destacable de este clúster es la coordinación parcial del ligando a la unidad trimetálica, lo que resulta en la presencia de tres grupos fosfino no coordinados. Motivados por este hecho, se analiza la reactividad de este compuesto frente a diferentes complejos que contienen metales de transición con el fin de obtener clústeres heterodimetálicos. Por último, se discute la actividad catalítica de algunos de los clústeres obtenidos en reacciones de reducción de sustratos orgánicos así como en la hidrogenación de $\mathrm{CO}_{2}$.

En el capítulo 7 se detallan los procedimientos de síntesis y caracterización, tanto teórica como experimental. Finalmente, en el capítulo 8 se recogen las conclusiones extraídas tras la realización de este trabajo y en el capítulo 9 se detallan las publicaciones científicas derivadas de la presente tesis doctoral. 
This PhD Thesis describes the synthesis, crystal structures and catalytic applications of a new family of group 6 chalcogenide metal clusters bearing diphosphine, aminophosphine and, for the first time, aminodiphosphine ligands. Chapter 1 is concerned with a brief historical introduction of metal clusters and, more specifically, of group 6 chalcogenide metal clusters from the former cluster chemistry until today. Some interesting applications of these complexes in medicine and catalysis are also described. Next, the main objectives of this work are provided in Chapter 2.

Chapter 3 describes the synthesis, structural characterization and catalytic activity of hydride and fluoride clusters of formula $\left[\mathrm{M}_{3} \mathrm{~S}_{4} \mathrm{X}_{3}(\mathrm{dppe})_{3}\right]^{+}(\mathrm{M}=\mathrm{Mo}, \mathrm{W}$; $\mathrm{X}=\mathrm{H}, \mathrm{F})$. The influence of the diphosphine basicity in the catalytic hydrodefluorination of pentafluoropyridine is discussed.

Chapter 4 examines the synthesis and structural characterization of new $\mathrm{M}_{3} \mathrm{~S}_{4}$ $(\mathrm{M}=\mathrm{Mo}, \mathrm{W})$ clusters bearing aminophosphine ediprp ligands. The analysis of this crystal structures shows that the phosphorus atom of the aminophosphine ligand is placed trans to the capping sulphur, while the nitrogen one is coordinated trans to the bridging sulphurs. As a result, only one isomer is formed. The catalytic activity of these clusters in the reduction of 2,2,2-trifluoroacetophenone is compared to that observed for $\left[\mathrm{Mo}_{3} \mathrm{~S}_{4} \mathrm{Cl}_{4}\left(\mathrm{PPh}_{3}\right)_{3}\left(\mathrm{H}_{2} \mathrm{O}\right)_{2}\right]^{+}$in the presence of several aminophosphines containing primary, secondary and tertiary amines.

Functionalization of $\mathrm{Mo}_{3} \mathrm{~S}_{4}$ clusters with chiral aminophosphines derived from proline is explored in chapter 5. Optically pure complexes with three stereogenic centers are obtained, and their structural characterization leads us to the assignation of unequivocal absolute configurations as $P$. These experimental crystal structures are compared to theoretical ones, calculated by computational methods. Electronic distribution analyses by means of $\mathrm{NBO}$ and ELF calculations are also detailed. Finally, the catalytic activity of these clusters is assessed. 
Chapter 6 describes for the first time the structure of a molybdenum trinuclear cluster bearing an aminodiphosphine ligand. The most important feature of this cluster is the partial coordination of the aminodiphosphine ligand to the trinuclear unit, resulting in three dangling phosphino groups. Due to this peculiar structure, the reactivity of this cluster versus other transition metal complexes to form heterodimetallic clusters is assessed. The catalytic activity of some homo and heterodimetallic aminodiphosphino clusters in the reduction of organic substrates and $\mathrm{CO}_{2}$ hydrogenation is also described.

All the synthetic procedures employed in this work, together with the theoretical and experimental characterization of all compounds are presented in Chapter 7. Finally, the general conclusions of this work are detailed in Chapter 8. Chapter 9 enumerates the scientific articles derived from this $\mathrm{PhD}$ Thesis. 
ca.

(R)-PPro

(S)-PPro

CID

$\operatorname{cod}$

Cp

$\mathrm{dba}$

depe

DFT

DIPEA

dmen

DMF

dmpe

DMSO

dppe

DUPHOS

ediprp

edpp

edtbup

ELF

ESI

Et

et al.

GC

$\mathrm{H}_{3} \mathrm{nta}$

HDF
Circa (aproximadamente)

(R)-2-[(difenilfosfino)metil]pirrolidina

(S)-2-[(difenilfosfino)metil]pirrolidina

\section{Collision Induced Dissociation}

Ciclooctadieno

Ciclopentadienilo

Dibencilidenacetonato

1,2-bis(dietilfosfino)etano

Density Functional Theory

N,N-di-i-propiletilamina

$\mathrm{N}, \mathrm{N}$-dimetiletilendiamina

Dimetilformamida

1,2-bis(dimetilfosfino)etano

dimetilsulfóxido

1,2-bis(difenilfosfino)etano

1,2-bis(2,5-dimetilfosfolano)benceno

2-(aminoetil)di-i-propilfosfina

2-(aminoetil)difenilfosfina

2-(aminoetil)di-t-butilfosfina

Electron Localization Function

Electrospray Ionization

Etilo

Et alii (y otros autores)

Gas Chromatography

Ácido nitrilotriacético

Hidrodefluoración 
HMDS

HOESY

HPLC

i.e.

i $\operatorname{Pr}$

IR

$m / z$

$\mathrm{M}^{+}$

$\mathrm{Me}$

Me-BPE

MS

$\mathrm{NAO}$

NBO

$\mathrm{NHO}$

ORTEP

$\mathrm{Ph}$

$\mathrm{PiPr}_{2}-\mathrm{NMe}_{2}$-indeno

P-N

PNP

P-P

$\mathrm{RMN}$

TBA

tBu

THF

TON

TRISPHAT

T'THA

u.m.a.
Hexametildisilazano

Heteronuclear Overhauser Effect Spectroscopy

High Performance Liquid Chromatography

Id est (esto es)

i-propilo

Infrarrojo

Relación masa/carga

Pico molecular

Metilo

1,2-bis(2,5-dimetilfosfolano)etano

Mass spectrometry

Natural Atomic Orbitals

Natural Bond Orbitals

Natural Hybrid Orbitals

Oak Ridge Thermal-Ellipsoid Plot

Fenilo

1-di-i-propilfosfino-2-(N,N-dimetilamino)-1H-indeno

Aminofosfina

Bis[(2-di-i-propilfosfino)etil] amina

Difosfina

Resonancia Magnética Nuclear

Tetrabutilamonio

t-butilo

Tetrahidrofurano

Turnover Number

Tris(tetraclorobencenodiolato)fosfato (V)

Ácido etilendiaminotetraacético

Unidad de masa atómica 


\section{ÍNDICE}

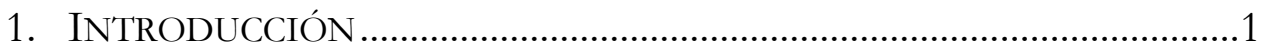

1.1. AspeCtos GENERALES SOBRE LOS CLÚSTERES METÁLICOS ................... 3

1.2. CALCogenuros Clúster trinuCleares de metales

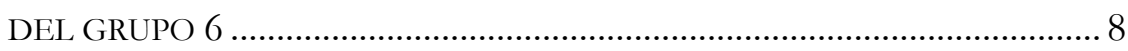

1.3. Aplicaciones DE CALCOGENUROS CLÚSTER TRINUCLEARES DE

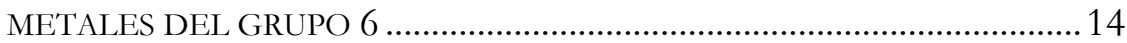

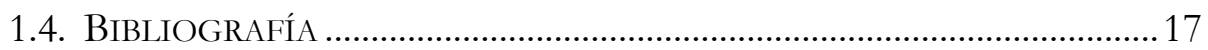

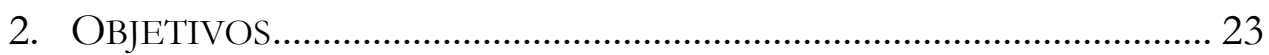

3. CLÚSTERES FUNCIONALIZADOS CON DIFOSFINAS:

HIDRODEFLUORACIÓN CATALÍTICA..................................................... 27

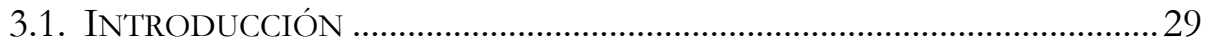

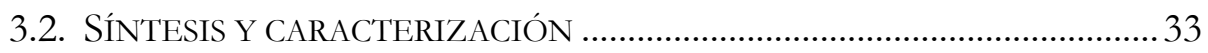

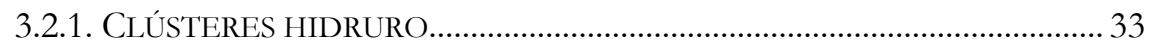

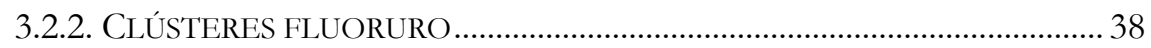

3.3. HidrodeFLUORACIÓN CATALÍTICA DE LA

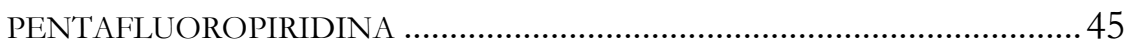

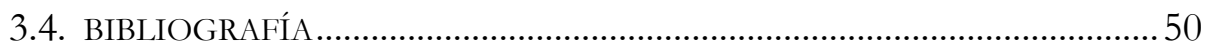

4. ClÚSTERES FUNCIONALIZADOS CON AMINOFOSFINAS Y SU

ACTIVIDAD EN LA REDUCCIÓN CATALÍTICA DE CETONAS .............. 53

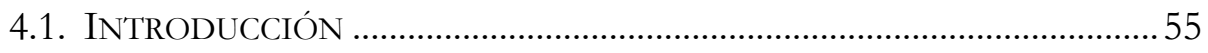

4.2. SíNTESIS, CARACTERIZACIÓN Y ESTUDIOS EN FASE GAS........................59

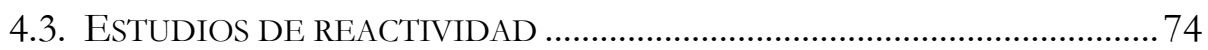


4.3.1. REACTIVIDAD FRENTE A BOROHIDRURO............................................... 75

4.3.2. REACTIVIDAD FRENTE A ALCOHOLES ................................................... 79

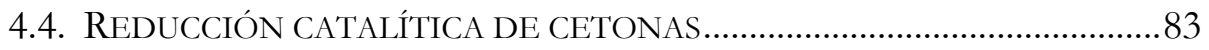

4.5. BIBLIOGRAFÍA …………………………………………………………....99

5. ClÚSTERES FUNCIONALIZADOS CON AMINOFOSFINAS QUIRALES: ESTUDIO TEÓRICO-EXPERIMENTAL ...................................................95

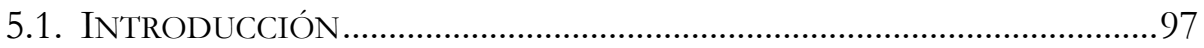

5.2. SÍNTESIS Y CARACTERIZACIÓN..........................................................102

5.3. ESTUDIO TEÓRICO .............................................................................111

5.3.1. OPTIMIZACIÓN DE GEOMETRÍAS.......................................................... 111

5.3.2. ANÁLISIS DE DISTRIBUCIÓN ELECTRÓNICA........................................... 114

5.3.2.1. ORbitALES NATURALES DE ENLACE (NBO) ........................................114

5.3.2.2. DENSIDAD ELECTRÓNICA (ELF) ……………………………………....117

5.4. Pruebas CATALÍTICAS …………………………………………….....122

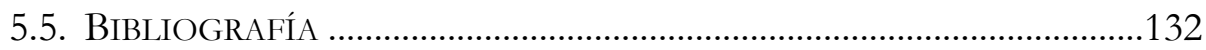

6. CLÚSTERES FUNCIONALIZADOS CON AMINODIFOSFINAS Y SUS

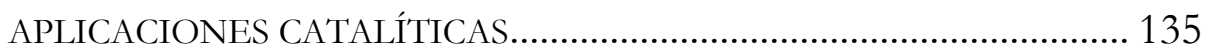

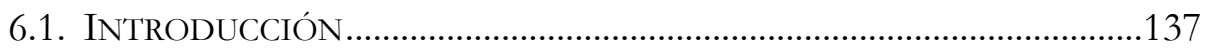

6.2. SÍNTESIS Y CARACTERIZACIÓN............................................................141

6.3. REACTIVIDAD FRENTE A OTROS METALES ...........................................149

6.3.1. REACTIVIDAD FRENTE A COBRE ...................................................... 150

6.3.2. REACTIVIDAD FRENTE A NÍQUEL .................................................... 152

6.3.3. REACTIVIDAD FRENTE A PALADIO................................................... 152

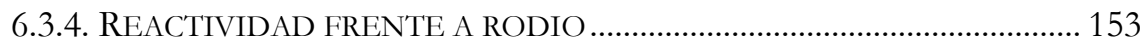

6.3.5. REACTIVIDAD FRENTE A IRIDIO ...................................................... 155

6.3.6. REACTIVIDAD FRENTE A ORO …………………………………....... 156 


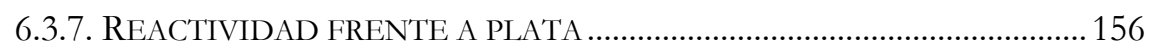

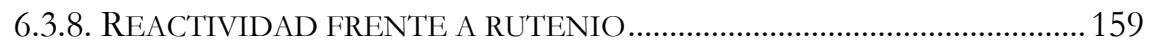

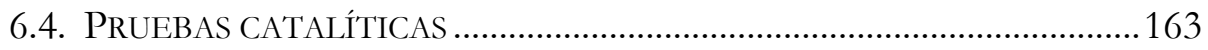

6.4.1. REDUCCIÓN CATALÍTICA DE ALDEHÍDOS Y CETONAS ...................... 165

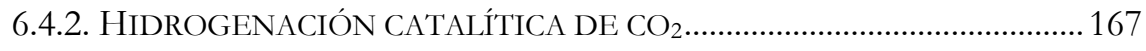

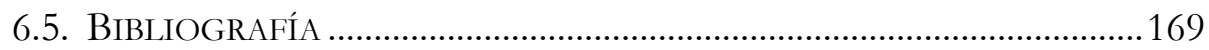

7. SECCIÓN EXPERIMENTAL.............................................................. 175

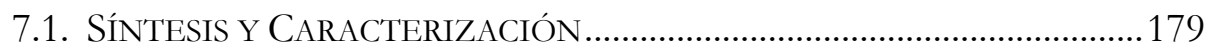

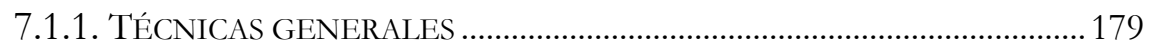

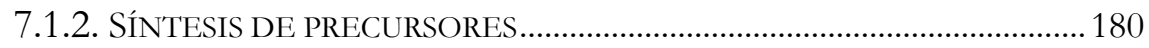

7.1.2.1. FASES SÓLIDAS POLIMÉRICAS.................................................................. 180

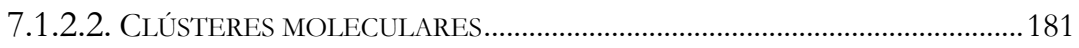

7.1.3. CLÚSTERES FUNCIONALIZADOS CON DIFOSFINAS ........................... 185

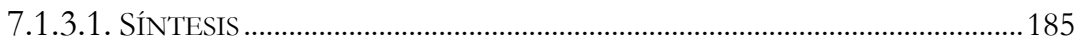

7.1.3.2. HidRODEFLUORACIÓN CATALÍTICA DE LA

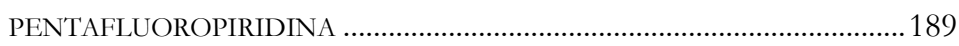

7.1.4. CLÚSTERES FUNCIONALIZADOS CON AMINOFOSFINAS................... 189

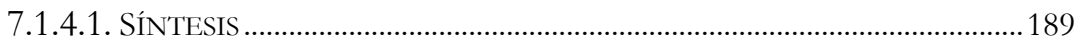

7.1.4.2. REDUCCIÓN CATALÍTICA DE SUSTRATOS ORGÁNICOS ...........................194

7.1.4.3. ESTUDIOS DE REACTIVIDAD FRENTE A ALCOHOLES............................... 194

7.1.5. ClÚSTERES FUNCIONALIZADOS CON AMINODIFOSFINAS ................ 195

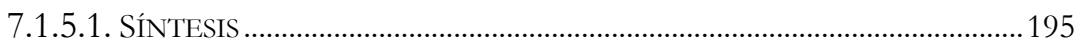

7.1.5.2. PRUEBAS DE REACTIVIDAD FRENTE A OTROS METALES........................197

7.1.5.3. REDUCCIÓN CATALÍTICA DE ALDEHÍDOS, CETONAS Y ÉSTERES.........200

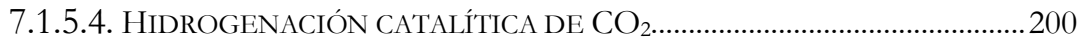

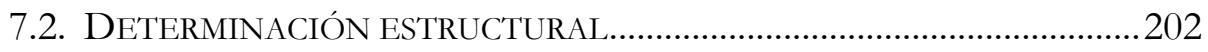

7.2.1. GENERALIDADES DE LA TOMA DE DATOS Y RESOLUCIÓN...............202 
7.2.2. CLÚSTERES FUNCIONALIZADOS CON DIFOSFINAS ............................ 204

7.2.2.1. ESTRUCTURA DE $\left.\left[\mathrm{Mo}_{3} \mathrm{~S}_{4} \mathrm{H}_{3} \text { (dppe }\right)_{3}\right] \mathrm{PF}_{6}(\mathbf{1}(\mathrm{PF}))$....................................204

7.2.2.2. EstruCtURA DE $\left[\mathrm{Mo}_{3} \mathrm{~S}_{4} \mathrm{~F}_{3}(\mathrm{dppe})_{3}\right] \mathrm{PF}_{6} \cdot \mathrm{H}_{2} \mathrm{O}\left(2(\mathrm{PF} 6) \cdot \mathrm{H}_{2} \mathrm{O}\right)$...............209

7.2.3. CLÚSTERES FUNCIONALIZADOS CON AMINOFOSFINAS ................... 214

7.2.3.1. EstruCtura DE $\left[\mathrm{Mo}_{3} \mathrm{~S}_{4} \mathrm{Cl}_{3}(\mathrm{edi} p \mathrm{prp})_{3}\right] \mathrm{Cl} \cdot \mathrm{CH}_{2} \mathrm{Cl}_{2}\left(\mathbf{5}(\mathrm{Cl}) \cdot \mathrm{CH}_{2} \mathrm{Cl}_{2}\right)$........214

7.2.3.2. ESTRUCTURA DE $\left[\mathrm{Mo}_{3} \mathrm{~S}_{4} \mathrm{Cl}_{3}(\text { edi prp })_{3}\right] \mathrm{Cl} \cdot \mathrm{CH}_{2} \mathrm{Cl}_{2}\left(\mathbf{5 a}(\mathrm{Cl}) \cdot \mathrm{CH}_{2} \mathrm{Cl}_{2}\right) \ldots . .221$

7.2.3.3. ESTRUCTURA DE $\left[\mathrm{W}_{3} \mathrm{~S}_{4} \mathrm{Br}_{3}(\text { ediprp })_{3}\right] \mathrm{BPh}_{4}\left(\mathbf{6}\left(\mathrm{BPh}_{4}\right)\right) \ldots \ldots \ldots \ldots \ldots \ldots \ldots \ldots \ldots \ldots . . .226$

7.2.3.4. EstruCtura De $(P)-\left[\mathrm{Mo}_{3} \mathrm{~S}_{4} \mathrm{Cl}_{3}((1 S, 2 \mathrm{R})-\mathrm{PPro})_{3}\right] \mathrm{BF}_{4}\left((P)-7\left(\mathrm{BF}_{4}\right)\right) \ldots . . .233$

7.2.3.5. Estructura DE $(P)-\left[\mathrm{Mo}_{3} \mathrm{~S}_{4} \mathrm{Cl}_{3}((1 S, 2 S)-\mathrm{PPro})_{3}\right] \mathrm{BF}_{4} \cdot 3\left(\mathrm{C}_{7} \mathrm{H}_{8}\right)$

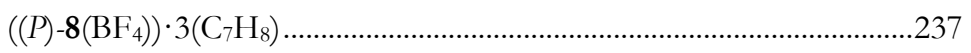

7.2.4. Clústeres FUnCIONALIZADOS CON AMINODIFOSFINAS ............... 241

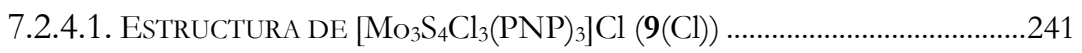

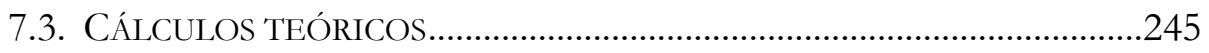

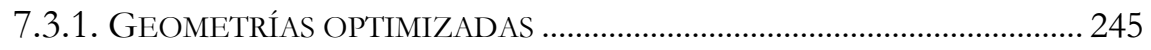

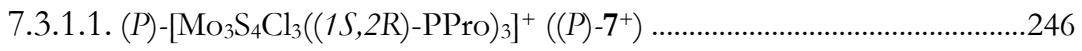

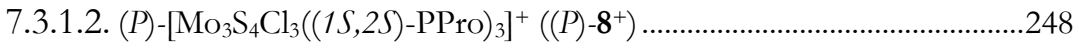

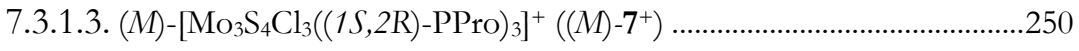

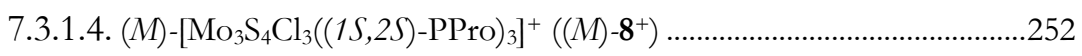

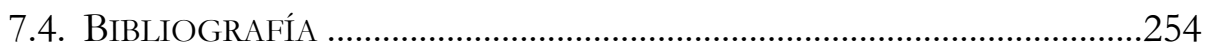

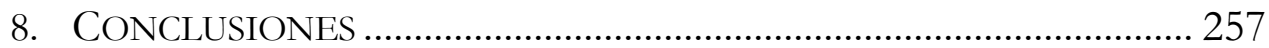

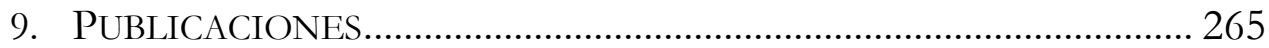


1. INTRODUCCIÓN 


\section{INTRODUCCIÓN}

1.1 Aspectos generales sobre los clústeres metálicos

1.2 Calcogenuros clúster trinucleares de metales del grupo 6

1.3 Aplicaciones de calcogenuros clúster trinucleares de metales del grupo 6

1.4 Bibliografía

"Ciencia es todo aquello sobre lo cual cabe siempre discusión." José Ortega y Gasset 


\subsection{ASPECTOS GENERALES SOBRE LOS CLÚSTERES METÁlICOS}

El término "clúster" fue introducido por F. A. Cotton hace más de 50 años para designar los compuestos formados por dos o más metales que presentan una interacción directa entre ellos. ${ }^{[1]}$ Cuando F. A. Cotton definió este término existían aproximadamente una docena de compuestos clúster, sin embargo el desarrollo de técnicas de caracterización como la difracción de rayos $\mathrm{X}$ y recientemente la microscopía óptica han contribuido al crecimiento exponencial de este campo de la química de coordinación. [2,3] Los clústeres metálicos se pueden funcionalizar con diferentes ligandos que les confieren propiedades muy diversas: quiralidad, ${ }^{[4]}$ solubilidad en agua, ${ }^{[5]}$ magnetismo ${ }^{[6]}$ o luminiscencia, ${ }^{[7]}$ entre otras. Esta característica los convierte en compuestos altamente atractivos en diferentes campos de investigación, desde la química de coordinación hasta la medicina.

Si buceamos en la historia de los clústeres metálicos encontramos que en la década de 1850 el químico sueco Christian Bloomstrand describió un compuesto de molibdeno que contenía dos tipos de halógenos, cloro y bromo.[8] Posteriormente, en los inicios del siglo XX, se describieron complejos de fórmula $\left[\mathrm{M}_{6} \mathrm{X}_{12}\right] \mathrm{X}_{2} \cdot 7 \mathrm{H}_{2} \mathrm{O}$ $(\mathrm{M}=\mathrm{Nb}, \mathrm{Ta} ; \mathrm{X}=\mathrm{Cl}, \mathrm{Br})^{[9-11]}$ cuya estructura, representada en la figura 1.1 , fue determinada décadas más tarde gracias a los estudios de rayos $\mathrm{X}$ de Pauling, Vaughan y Sturdivant.[12]

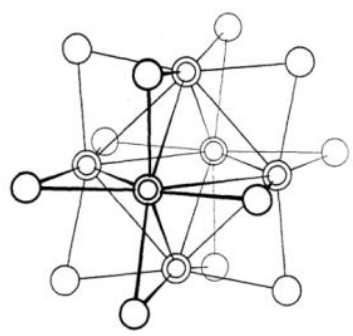

Figura 1.1. Estructura de los clústeres $\left[\mathrm{M}_{6} \mathrm{X}_{12}\right]^{2-}(\mathrm{M}=\mathrm{Nb}$, Ta; $\mathrm{X}=\mathrm{Cl}, \mathrm{Br})$. Los dos círculos representan los metales y los círculos los halógenos. Figura reproducida con permiso de $J$. Am. Chem. Soc. 1950, 72, 5477 (referencia [12]). Copyright 1950 American Chemical Society. 
El trabajo del cristalógrafo sueco C. Brosset en los años 40 resultó muy importante para el desarrollo de los clústeres metálicos, ya que determinó las estructuras de una serie de complejos basados en la unidad $\left[\mathrm{Mo}_{6} \mathrm{Cl}_{8}\right]^{4+}$, cuyos átomos de molibdeno forman un octaedro y se encuentran unidos a ocho átomos de cloro apuntados $\left(\mu_{3}-\mathrm{Cl}\right)$ situados en las caras triangulares del poliedro.[13,14]

En los años 60, al mismo tiempo que se establecía el término "clúster" para designar estos compuestos, tuvo lugar el descubrimiento del complejo $\left[\mathrm{Re}_{3} \mathrm{Cl}_{12}\right]^{3-}$, que se considera el primer compuesto que contiene enlaces múltiples metal-metal.[15] Poco tiempo después, los estudios cristalográficos de L. F. Dahl permitieron conocer las estructuras de los clústeres de hierro y cobalto con ligandos carbonilo, que se muestran en la figura 1.2.[16] Llegados a este punto, el número de clústeres sintetizados crece tan rápidamente que resulta muy difícil crear una lista de los compuestos clúster que se han descrito hasta nuestros días.
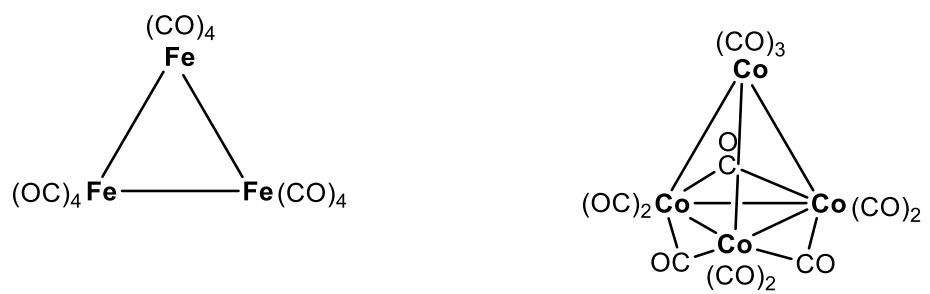

Figura 1.2. Estructura molecular de los clústeres $\mathrm{Fe}_{3}(\mathrm{CO})_{12}$ y $\mathrm{Co}_{4}(\mathrm{CO})_{12}$.

Las unidades clúster triangulares y octaédricas pueden originar redes tridimensionales mediante la formación de puentes halogenuro o mediante procesos de condensación basados en la compartición de vértices, aristas o caras. ${ }^{[17,18]}$ En la última década, se ha descrito el uso de sistemas multinucleares con posiciones lábiles para construir jaulas moleculares tridimensionales cuyas propiedades son fácilmente modificables. ${ }^{[19,20]}$ En este sentido, el grupo de Llusar publicó en 2009 la síntesis de un nanoclúster de molibdeno dodecanuclear con estructura de adamantano, formado 
a partir de entidades trinucleares $\mathrm{Mo}_{3} \mathrm{~S}_{7}$ que se encuentran enlazadas mediante puentes yoduro (ver figura 1.3).[21]

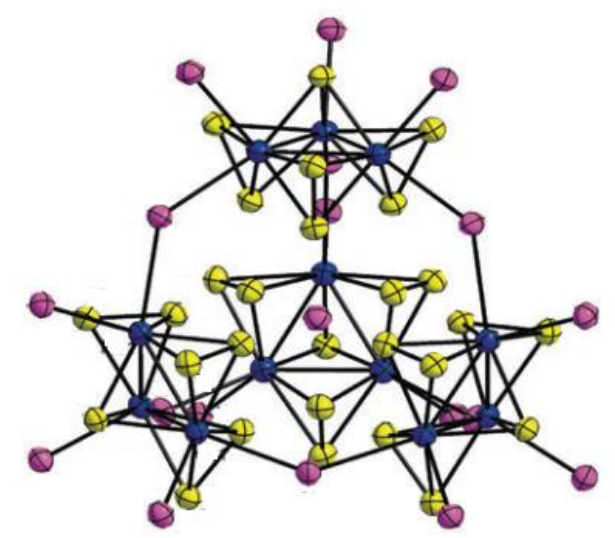

Figura 1.3. Estructura del nanoclúster $\left[\mathrm{Mo}_{3} \mathrm{~S}_{7} \mathrm{I}_{3}\right]_{4}(\mu-\mathrm{I})_{6} \mathrm{I}$ adaptada de la referencia [21] con permiso de la Royal Society of Chemistry. Los átomos de molibdeno están representados en azul, los de azufre en amarillo y los de yodo en morado.

En los años 70, Malatesta describió la síntesis de complejos clúster de oro que contenían 6, 9 u 11 átomos de este metal.[22] Posteriormente, los grupos de Mingos y Steggerda estudiaron la química de clústeres moleculares de oro funcionalizados con fosfinas o ligandos tiolato con el fin de obtener complejos clúster de mayor nuclearidad, cuyo diámetro en muchas ocasiones se aproxima a dimensiones nanométricas. ${ }^{[3]}$ Estos clústeres de oro han sido objeto de numerosos estudios tanto desde un punto de vista experimental como teórico. ${ }^{[23,24]}$ Con las técnicas actuales es posible caracterizar completamente clústeres nanométricos que contienen más de cien átomos de oro, como por ejemplo el complejo de fórmula $\mathrm{Au}_{102}(\mathrm{p}-\mathrm{MBA})_{44}(\mathrm{p}-\mathrm{MBA}=$ ácido $p$-mercaptobenzoico) (ver figura 1.4).[25] 


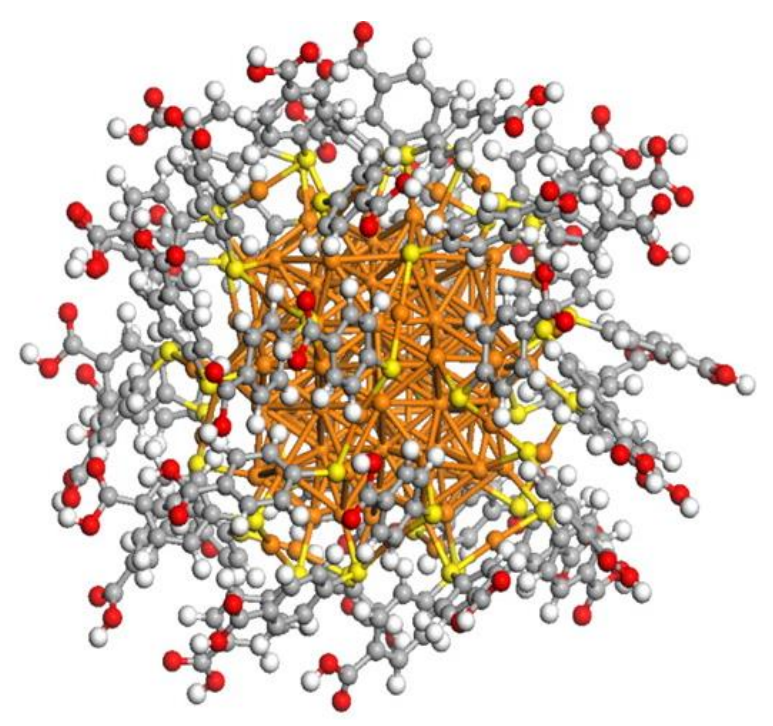

Figura 1.4. Estructura del nanoclúster $\mathrm{Au}_{102}(\mathrm{p}-\mathrm{MBA})_{44}$ de la referencia [23]. Los átomos de oro están representados en color naranja, los de azufre en amarillo, los de carbono en gris, los de oxígeno en rojo y los de hidrógeno en blanco. Copyright 2008 National Academy of Sciences.

Desde un punto de vista electrónico, los clústeres de metales de transición se pueden clasificar en dos grandes grupos, i.e. electrónicamente pobres y electrónicamente ricos. Los primeros presentan metales en estados de oxidación medios o altos y contienen ligandos con un carácter mayoritariamente $\sigma$-dador. Los últimos presentan metales en estados de oxidación muy bajos (ca. 0), con ligandos con un carácter $\pi$-aceptor.

Nuestro trabajo se ha centrado en clústeres trinucleares electrónicamente pobres, en los que el número de electrones de valencia es aproximadamente el necesario para formar enlaces sencillos entre metales adyacentes. Por lo tanto, un clúster trinuclear electrónicamente pobre deberá presentar entre 5 y 8 electrones de valencia para formar enlaces metal-metal. En concreto, esta tesis doctoral trata sobre la síntesis, caracterización y aplicaciones de clústeres con unidad central $\mathrm{M}_{3} \mathrm{~S}_{4}(\mathrm{M}=$ Mo, W), que se caracterizan por su gran robustez consecuencia de la presencia de puentes de sulfuro que mantienen unidos los átomos metálicos. Esta característica 
permite modificar el entorno de coordinación de esta unidad con la finalidad de conferir a los complejos una propiedad determinada. A continuación se muestra una descripción general de los clústeres $\mathrm{M}_{3} \mathrm{Q}_{4}(\mathrm{M}=\mathrm{Mo}, \mathrm{W} ; \mathrm{Q}=\mathrm{S}$, Se) que abarca aspectos sintéticos, estructurales y electrónicos, así como una descripción de sus aplicaciones más importantes desarrolladas en los últimos años. 


\subsection{CALCOGENUROS CLÚSTER TRINUCLEARES DE METALES DEL GRUPO 6}

La química de los calcogenuros de metales del grupo 6 está dominada por entidades trinucleares con unidades $\mathrm{M}_{3} \mathrm{Q}_{7}$ y $\mathrm{M}_{3} \mathrm{Q}_{4}(\mathrm{M}=\mathrm{Mo}, \mathrm{W} ; \mathrm{Q}=\mathrm{S}$, Se) tal como se muestra en la figura 1.5. Ambos sistemas presentan una unidad triangular formada por tres enlaces simples metal-metal y un calcógeno $\mu_{3}-\mathrm{Q}$ apuntado a los tres centros metálicos.[17] $\mathrm{La}$ diferencia entre las unidades $\mathrm{M}_{3} \mathrm{Q}_{7}$ y $\mathrm{M}_{3} \mathrm{Q}_{4}$ reside en la naturaleza de los calcógenos puente. Mientras que la primera unidad posee puentes dicalcogenuro $\mu-\mathrm{Q}_{2}$ con un calcógeno en posición ecuatorial y el otro en posición axial respecto al plano trimetálico, en la última estos calcógenos puente son monoatómicos ( $\mu$-Q).[26]
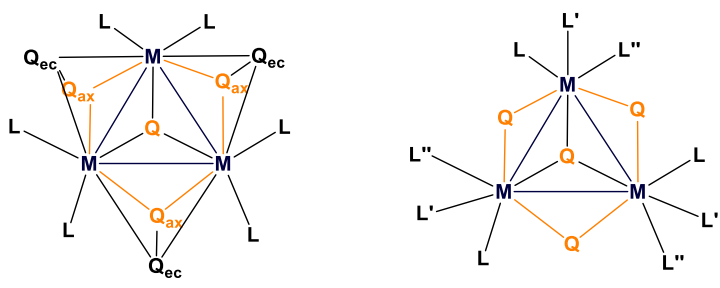

Figura 1.5. Estructuras de los clústeres con unidad $\mathrm{M}_{3} \mathrm{Q}_{7}$ (izquierda) y $\mathrm{M}_{3} \mathrm{Q}_{4}$ (derecha).

En función de la disposición de los ligandos L, L’ y L” periféricos estas unidades pueden presentar una simetría $C_{3 \mathrm{v}}$ o $C_{3}$. Cuando estos ligandos son equivalentes, la unidad clúster presenta una simetría $C_{3 v}$ con un eje de rotación $C_{3}$ que pasa a través del calcógeno apuntado $\mu_{3}-\mathrm{Q}$ y tres planos que contienen un átomo metálico cada uno. Sin embargo, cuando los ligandos periféricos no son equivalentes y están dispuestos de forma que no existen planos de simetría, el clúster tiene una simetría $C_{3}$. En este último caso, estas unidades clúster presentan quiralidad intrínseca.

Sin tener en cuenta los enlaces metal-metal, los centros metálicos de la unidad $\mathrm{M}_{3} \mathrm{Q}_{7}$ muestran un entorno heptacoordinado. La característica más interesante de 
estos clústeres reside en la disposición espacial de los puentes dicalcogenuro: mientras que el calcógeno situado en posición axial (trans al calcógeno apuntado) tiene un elevado carácter electrofílico que favorece la formación de agregados supramoleculares, el calcógeno situado en posición ecuatorial (coplanar al plano metálico) presenta una elevada labilidad, hecho que permite la conversión de los clústeres con unidad $\mathrm{M}_{3} \mathrm{Q}_{7}$ a $\mathrm{M}_{3} \mathrm{Q}_{4}$ mediante la reducción de los puentes dicalcogenuro utilizando agentes desulfurizantes como tiocianato o fosfinas.[27-29] En consecuencia, la reducción de los puentes disulfuro de las unidades $\mathrm{M}_{3} \mathrm{Q}_{7}$ resulta un buen método para la preparación de clústeres cuboidales $\mathrm{M}_{3} \mathrm{Q}_{4}$. Este cambio estructural provoca que el entorno de coordinación de los centros metálicos pase a ser octaédrico sin cambiar el estado de oxidación del metal.

El primer compuesto molecular con unidad $\mathrm{M}_{3} \mathrm{Q}_{4}$ fue descrito por L. F. Dahl y colaboradores en los inicios de la década de los 70.[30] Se trata del complejo $\left[\mathrm{Mo}_{3} \mathrm{~S}_{4} \mathrm{Cp}_{3}\right]^{+}(\mathrm{Cp}=$ ciclopentadienilo $)$, sintetizado mediante un proceso de autoorganización a partir de $\mathrm{MoCp}(\mathrm{CO})_{3} \mathrm{Cl}$ y $\left[\mathrm{Sn}\left(\mathrm{CH}_{3}\right)_{3}\right]_{2} \mathrm{~S}$. Utilizando este mismo procedimiento, posteriormente el grupo de A. Müller aisló el clúster $\left[\mathrm{Mo}_{3}\left(\mu_{3}-\mathrm{S}\right)(\mu-\right.$ $\left.\left.\mathrm{S}_{2}\right)_{3}\left(\mathrm{~S}_{2}\right)_{3}\right]\left(\mathrm{NH}_{4}\right)_{2}$ que constituye el primer clúster molecular con unidad central $\mathrm{Mo}_{3} \mathrm{~S}_{7 .}{ }^{[31]}$ Actualmente, este complejo es el precursor más utilizado para obtener clústeres moleculares de fórmula $\left[\mathrm{Mo}_{3} \mathrm{~S}_{7} \mathrm{X}_{6}\right]^{2-}(\mathrm{X}=\mathrm{Cl}, \mathrm{Br})$ tras su reacción con hidrácidos HX.[32]

El descubrimiento del método de escisión de las fases poliméricas de fórmula $\left\{\mathrm{M}_{3} \mathrm{Q}_{7} \mathrm{X}_{4}\right\}_{\mathrm{n}}(\mathrm{M}=\mathrm{Mo}, \mathrm{W})$ representadas en la figura 1.6 para dar lugar a clústeres moleculares con unidad $\mathrm{M}_{3} \mathrm{Q}_{4}$ permitió extender esta química al wolframio.[27,29,33-35] El grupo de investigación de Cotton describió este procedimiento por primera vez en el año 1989,[27] y a fecha de hoy sigue siendo muy utilizado para obtener clústeres de fórmula $\left[\mathrm{M}_{3} \mathrm{~S}_{4} \mathrm{X}_{3} \text { (quelato) }\right]^{+}(\mathrm{M}=\mathrm{Mo}, \mathrm{W} ; \mathrm{X}=\mathrm{Cl}, \mathrm{Br}$; quelato = difosfina, aminofosfina) en los que se basa la presente tesis doctoral.[36-38] En el caso del molibdeno, a pesar de que este procedimiento se utiliza ampliamente, la preparación 
de clústeres con unidad $\mathrm{Mo}_{3} \mathrm{~S}_{4}$ a partir de precursores moleculares de fórmula $\left[\mathrm{Mo}_{3} \mathrm{~S}_{7} \mathrm{X}_{6}\right]^{2-}(\mathrm{X}=\mathrm{Cl}, \mathrm{Br})$ proporciona por regla general rendimientos más altos.[39,40]

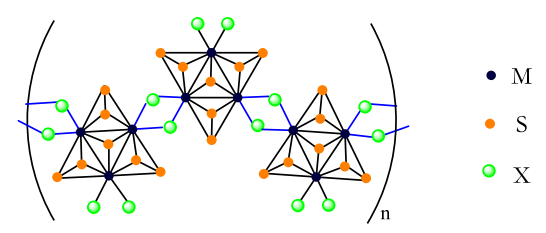

Figura 1.6. Representación esquemática de las fase poliméricas $\left\{\mathrm{M}_{3} \mathrm{~S}_{7} \mathrm{X}_{4}\right\}_{n}(\mathrm{M}=\mathrm{Mo}, \mathrm{W} ; \mathrm{X}$ $=\mathrm{Cl}, \mathrm{Br})$.

La coordinación específica de los ligandos externos en los clústeres $\left[\mathrm{M}_{3} \mathrm{~S}_{4} \mathrm{X}_{3} \text { (difosfina) }\right]^{+}$, con un átomo de fósforo trans al azufre apuntado y otro trans a los azufres puente, resulta en compuestos quirales con simetría $C_{3}$. Dependiendo del sentido de giro de los ligandos $\mathrm{X}$, los dos enantiómeros posibles se designan con las letras $P$ (sentido horario) y $M$ (sentido antihorario), tal como se representa en la figura 1.7 .

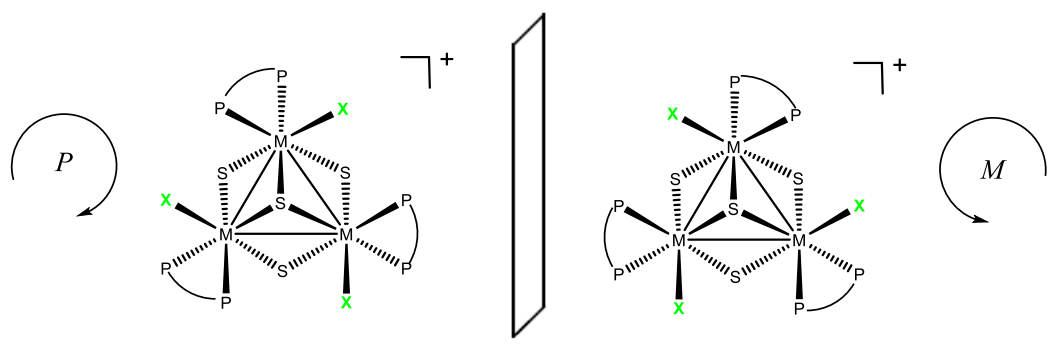

Figura 1.7. Representación de los enantiómeros $P$ (izquierda) y $M$ (derecha) que se forman en la síntesis de los clústeres de fórmula general $\left[\mathrm{M}_{3} \mathrm{~S}_{4} \mathrm{X}_{3}(\text { difosfina })\right]^{+}$.

Para la obtención de clústeres $\left[\mathrm{M}_{3} \mathrm{~S}_{4} \mathrm{X}_{3} \text { (difosfina) }\right]^{+}$ópticamente puros o enriquecidos en uno de los enantiómeros se han seguido varias estrategias. La primera de ellas consiste en la coordinación de difosfinas ópticamente puras, mientras que la segunda consiste en la resolución de la mezcla racémica utilizando aniones quirales. ${ }^{[4,37,41]}$ Concretamente, la escisión de fases poliméricas $\left\{\mathrm{M}_{3} \mathrm{Q}_{7} \mathrm{X}_{4}\right\}_{\mathrm{n}}(\mathrm{M}=\mathrm{Mo}$, $\mathrm{W} ; \mathrm{Q}=\mathrm{S}$, Se; $\mathrm{X}=\mathrm{Cl}, \mathrm{Br})$ con difosfinas quirales como (+)-1,2-bis $((2 \mathrm{R}, 5 \mathrm{R})-2,5-$ dimetilfosfolano)etano ((R,R)-Me-BPE), (-)-1,2-bis((2S,5S)-2,5-dimetilfosfolano)- 
etano $((S, S)-$ Me-BPE) y (-)-1,2-bis((2R,5R)-2,5-dimetilfosfolano)benceno $((R, R)$ DUPHOS) conduce a complejos clúster ópticamente puros. ${ }^{[4,37]}$ Mientras que el uso de difosfinas $(R, R)$ da lugar a enantiómeros clúster $P$, la utilización de difosfinas $(S, S)$ resulta en enantiómeros $M$. Por otra parte, la asociación del clúster $\left[\mathrm{Mo}_{3} \mathrm{~S}_{4} \mathrm{Cl}_{3}(\mathrm{dppe})\right]^{+}$ (dppe $=1,2$-bis(difenilfosfino)etano) con el anión quiral $\Delta$-TRISPHAT (tris(tetraclorobencenodiolato)fosfato $(\mathrm{V})$ ) da lugar a disoluciones enriquecidas en uno de los enantiómeros.[41]

Cotton y Haas construyeron en 1964 mediante cálculos semiempíricos un diagrama de orbitales moleculares para la unidad clúster $\mathrm{M}_{3} \mathrm{~S}_{4}$ a partir de la combinación de orbitales atómicos teniendo en cuenta la simetría de la molécula.[1,42] Según este diagrama, ilustrado en la figura 1.8, estos clústeres pueden situar seis electrones asociados al enlace intermetálico con la configuración $\left(1 \mathrm{a}_{1}\right)^{2}(1 \mathrm{e})^{4}$. Este estudio justifica que la mayoría de los clústeres con unidad $\mathrm{M}_{3} \mathrm{~S}_{4}$ descritos hasta la fecha contienen seis electrones asociados a la unidad trimetálica y presentan un estado de oxidación formal para cada metal de IV.

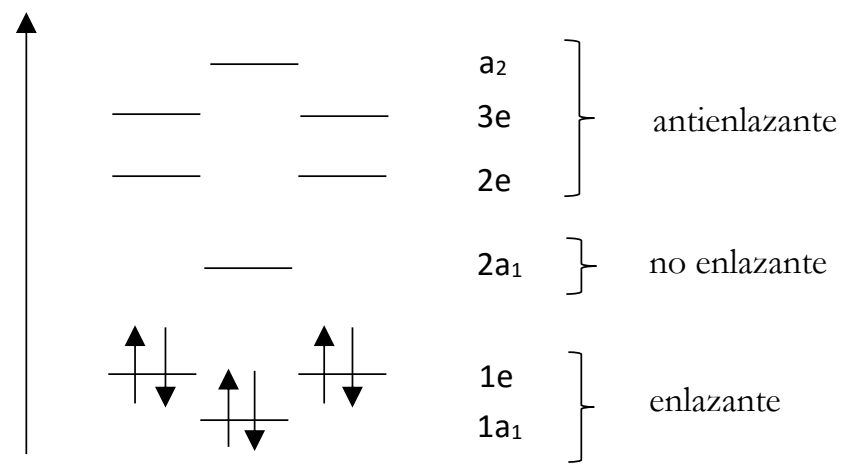

Figura 1.8. Diagrama energético simplificado propuesto por Cotton y Haas para la unidad $\mathrm{M}_{3} \mathrm{~S}_{4}$.

Los clústeres $\mathrm{M}_{3} \mathrm{~S}_{4}$ pueden, además, actuar como metaloligandos frente a un segundo metal M' de naturaleza variada (bloque p o de transición) para formar compuestos homo- o heterodimetálicos, lo que implica el paso de una estructura de cubo incompleto a una estructura de tipo cubano $\mathrm{M}_{3} \mathrm{M}^{\prime} \mathrm{S}_{4 .}{ }^{\left[{ }^{[43-45]} \text { Esta estrategia de }\right.}$ 
síntesis se conoce con el nombre de construcción por bloques [3+1] y fue descrita por Shibahara y colaboradores en el año 1986, donde hacen reaccionar hierro metal con el clúster $\left[\mathrm{Mo}_{3} \mathrm{~S}_{4}\left(\mathrm{H}_{2} \mathrm{O}\right)_{9}\right]^{+}$, con la consiguiente formación del complejo $\left[\mathrm{Mo}_{3} \mathrm{~S}_{4} \mathrm{Fe}\left(\mathrm{H}_{2} \mathrm{O}\right)_{10}\right]^{+} .{ }^{[46]}$ Se han identificado tres variantes estructurales de estos compuestos denominadas estructura de cubo sencillo, estructura de cubo doble y estructura tipo sándwich (ver figura 1.9).
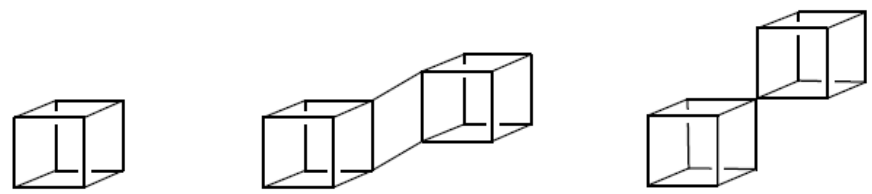

Figura 1.9. Variantes estructurales de los clústeres $\mathrm{M}_{3} \mathrm{M}^{\prime} \mathrm{S}_{4}$ : cubo sencillo (izquierda), cubo doble con interacciones directas entre aristas (centro) y estructura tipo sándwich (derecha).

A diferencia de los clústeres trinucleares, los complejos heterodimetálicos existen con una población electrónica de entre 13 y 17 electrones para la formación del enlace en la unidad clúster.[45,47] Por ejemplo, se han descrito clústeres de fórmula $\left[\mathrm{Mo}_{3} \mathrm{CoS}_{4} \mathrm{Cl}_{3} \mathrm{X}(\mathrm{dmpe})_{3}\right]^{\mathrm{n}+}(\mathrm{X}=\mathrm{Cl}, \mathrm{n}=1 ; \mathrm{X}=\mathrm{Cl}, \mathrm{n}=0 ; \mathrm{X}=\mathrm{CO}, \mathrm{n}=0)$ que presentan configuraciones electrónicas variables, en concreto de 14, 15 y 16 electrones, respectivamente. ${ }^{[48]}$ El diagrama de orbitales propuesto por Harris predice la existencia de tres orbitales moleculares enlazantes asociados a la formación del enlace metal-metal ( $1 \mathrm{e}, 1 \mathrm{a}_{1}$ y $\left.2 \mathrm{e}\right)$, dos orbitales no enlazantes $\left(2 \mathrm{a}_{1}\right.$ y $\left.3 \mathrm{e}\right)$ y dos antienlazantes $\left(4 \mathrm{e}\right.$ y $\left.3 \mathrm{a}_{1}\right) \cdot{ }^{[49]} \mathrm{La}$ ocupación de los orbitales tanto enlazantes como no enlazantes resulta en un clúster con 16 electrones y una configuración electrónica $(1 \mathrm{e})^{4}\left(1 \mathrm{a}_{1}\right)^{2}(2 \mathrm{e})^{4}\left(2 \mathrm{a}_{1}\right)^{2}(3 \mathrm{e})^{4}$, tal y como se representa en la figura 1.10 . 


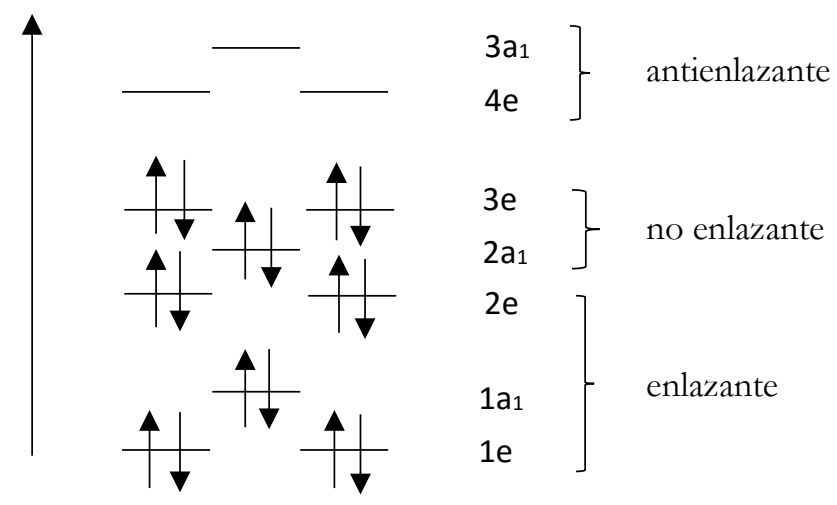

Figura 1.10. Diagrama energético simplificado propuesto por Harris para clústeres con unidad $\mathrm{M}_{3} \mathrm{M}^{\prime} \mathrm{S}_{4}$ que presentan 16 electrones.

La presente tesis doctoral se centra en complejos clúster con unidad $\mathrm{M}_{3} \mathrm{~S}_{4}$ funcionalizados con difosfinas, aminofosfinas y aminodifosfinas, así como en la reactividad de algunos de estos clústeres frente a otros complejos de metales de transición. A continuación describimos una visión general de las aplicaciones más relevantes hasta la fecha de los clústeres calcogenuro de metales del grupo 6. 


\subsection{APLICACIONES DE CALCOGENUROS CLÚSTER TRINUCLEARES DE METALES DEL GRUPO 6}

Los clústeres de metales de transición del grupo 6 encuentran aplicaciones en áreas tan diversas como la medicina, ${ }^{[5,51]}$ la catálisis homogénea ${ }^{[4,52-57]}$ y heterogénea ${ }^{[58-60]} \mathrm{O}$ la óptica no lineal.[61-63] En esta sección nos centraremos en las aplicaciones de clústeres con unidad central $\mathrm{M}_{3} \mathrm{~S}_{4}$ y $\mathrm{M}_{3} \mathrm{M}^{\prime} \mathrm{S}_{4}$, de mayor interés para esta tesis doctoral.

Durante la última década, se han estudiado las aplicaciones de algunos calcogenuros clúster de wolframio en medicina. En concreto, los clústeres con unidad central $\mathrm{W}_{3} \mathrm{~S}_{4}$ y $\mathrm{W}_{3} \mathrm{SO}_{3}$ funcionalizados con ligandos derivados del $\mathrm{H}_{3}$ nta (ácido nitrilotriacético) y TTHA (ácido etilendiaminotetraacético) son eficientes como agentes de contraste de rayos X.[50,51] Recientemente la empresa farmacéutica Bayer ha desarrollado una patente en la que han descrito la utilidad de un clúster de unidad $\mathrm{W}_{3} \mathrm{O}_{2}$ como sonda molecular de rayos X.[64]

Un campo de investigación en el cual los clústeres calcogenuro de metales de transición del grupo 6 han tenido un éxito notable ha sido la catálisis. En la naturaleza existen procesos catalíticos en los que los centros activos polinucleares tienen una estructura de tipo cubano. Por ejemplo, el cofactor de Fe-Mo en la enzima nitrogenasa, principal responsable de la fijación de nitrógeno en las plantas, presenta una subunidad $\mathrm{MoFe}_{3} \mathrm{~S}_{4}$ en su centro activo. Esta enzima convierte el nitrógeno atmosférico en amoníaco mediante transferencias de electrones y protones, lo que ha propiciado el estudio de estos sistemas clúster. ${ }^{[65]}$ En cuanto a la catálisis heterogénea, los clústeres con unidad central $\mathrm{Mo}_{3} \mathrm{M}^{\prime} \mathrm{S}_{4}\left(\mathrm{M}^{\prime}=\mathrm{Ru}, \mathrm{Rh}, \mathrm{Ir}, \mathrm{Pd}, \mathrm{Pt}\right)$ se han soportado sobre alúmina y se ha evaluado su capacidad para generar $\mathrm{MoS}_{2}$ tras su tratamiento térmico a alta temperatura. Posteriormente, se ha investigado su actividad en reacciones de hidrotratamiento. ${ }^{[59]}$ 
Una de las aplicaciones catalíticas más interesantes es la utilización del clúster $\left[\mathrm{Mo}_{3} \mathrm{~S}_{4}\left(\mathrm{Cp}^{\prime}\right)_{3}\right](\mathrm{pts})\left(\mathrm{Cp}^{\prime}=\right.$ metilciclopentadienil) impregnado sobre sílice dopada de tipo p como catalizador en reacciones de generación de hidrógeno (ver figura 1.11).[58] La utilización del hidrógeno como fuente de energía limpia en sustitución de los combustibles fósiles es actualmente una de las tendencias más investigadas con el fin de reducir el impacto medioambiental de las energías no renovables.
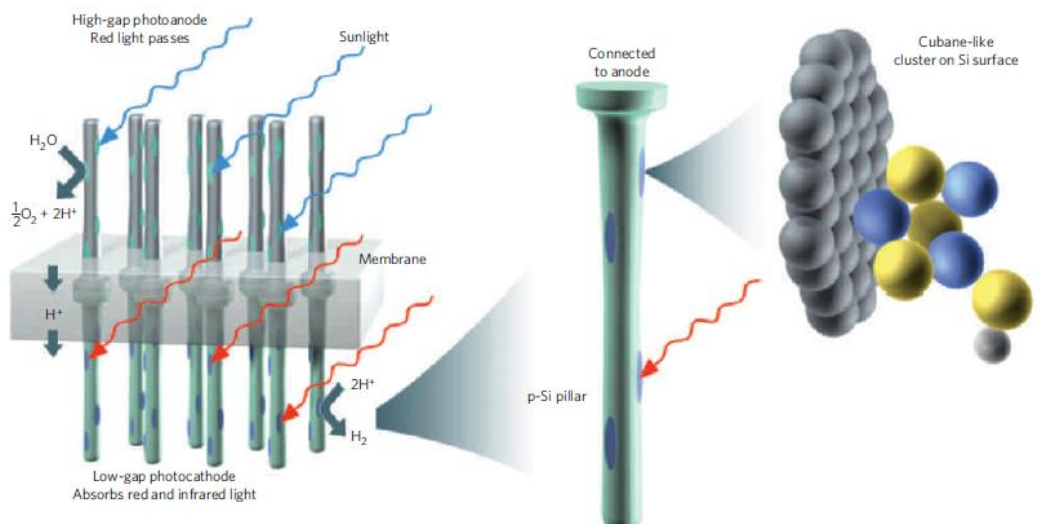

Figura 1.11. Esquema de la generación de hidrógeno catalizada por el clúster $\left[\mathrm{Mo}_{3} \mathrm{~S}_{4}\left(\eta_{5}-\right.\right.$ $\left.\left.\mathrm{Cp}^{\prime}\right)_{3}\right]$ (pts) soportado sobre sílice dopada de tipo p. Ilustración reproducida con permiso de Nature Publishing Group. Copyright 2011 (referencia [58]).

En el ámbito de la catálisis homogénea encontramos un gran número de aplicaciones de estos complejos, en especial de los que presentan unidades $\mathrm{M}_{3} \mathrm{M}^{\prime} \mathrm{S}_{4}$. Por ejemplo, los clústeres con unidad $\mathrm{Mo}_{3} \mathrm{PdS}_{4}$ son activos en reacciones de adición de alcoholes y ácidos carboxílicos a alquinos enlazados a grupos aceptores de densidad electrónica.[53,55] Por otra parte, los clústeres ópticamente puros con unidad central $\mathrm{Mo}_{3} \mathrm{CuS}_{4}$ catalizan la ciclopropanación de olefinas con un modesto exceso enantiomérico del $25 \%$, debido a la lejanía entre el centro activo $(\mathrm{Cu})$ y las difosfinas quirales coordinadas a los átomos de molibdeno. ${ }^{[4]}$ Los complejos de unidad $\mathrm{Mo}_{3} \mathrm{NiS}_{4}$ catalizan la ciclación intramolecular de ácidos alquinoicos terminales para dar enol lactonas.[56] Además, la reacción de polimerización de metilmetacrilato y la 
descomposición de hidracina para dar lugar a amoníaco y nitrógeno son procesos catalizados por clústeres de unidad $\mathrm{Mo}_{3} \mathrm{RuS}_{4}\left[{ }^{[65,66]}\right.$

En la gran mayoría de procesos catalizados por complejos heterodimetálicos con estructura de cubano, la actividad catalítica está centrada en el heterometal. Sin embargo, los clústeres homometálicos con unidades $\mathrm{Mo}_{3} \mathrm{~S}_{4}$ y $\mathrm{W}_{3} \mathrm{~S}_{4}$ también han mostrado ser activos en varios procesos catalíticos. Concretamente, los clústeres de fórmula $\left[\mathrm{M}_{3} \mathrm{~S}_{4} \mathrm{H}_{3}(\mathrm{dmpe})_{3}\right]^{+}(\mathrm{M}=\mathrm{Mo}, \mathrm{W} ;$ dmpe $=$ 1,2-bis(dimetilfosfino)etano) catalizan el proceso de hidrodefluoración de la pentafluoropiridina, que transcurre mediante un mecanismo de metátesis de enlace $\sigma$ que implica la descoordinación de uno de los átomos de fósforo de la difosfina. [57]

En los últimos años, Beller y Llusar han demostrado que los clústeres $\left[\mathrm{Mo}_{3} \mathrm{~S}_{4} \mathrm{H}_{3}(\text { dmpe })_{3}\right]^{+}, \quad\left[\mathrm{Mo}_{3} \mathrm{~S}_{4} \mathrm{Cl}_{3}(\mathrm{edpp})_{3}\right]^{+}$(edpp $=2$-(aminoetil)difenilfosfina $) \mathrm{y}$ $\left[\mathrm{Mo}_{3} \mathrm{~S}_{4} \mathrm{Cl}_{3}(\mathrm{dmen})_{3}\right]^{+}(\mathrm{dmen}=\mathrm{N}, \mathrm{N}$-dimetiletilendiamina $)$ son excelentes catalizadores o precatalizadores en la reducción selectiva de nitroderivados para obtener anilinas funcionalizadas. ${ }^{[52,54,67]} \mathrm{La}$ reducción puede transcurrir mediante un mecanismo de transferencia de hidrógeno cuando se emplea una mezcla de ácido fórmico y trietilamina como agente reductor, ${ }^{[52,67]}$ o mediante hidrosililación cuando se utilizan silanos en el proceso de reducción. ${ }^{[54]}$

La búsqueda de catalizadores eficientes que sustituyan a los metales nobles constituye un tema de gran actualidad. En el caso concreto de esta tesis doctoral, las aplicaciones de los nuevos clústeres sintetizados se han dirigido al ámbito de la catálisis homogénea. 


\subsection{BIBLIOGRAFÍA}

[1] Cotton, F. A. Inorg. Chem. 1964, 3, 1217.

[2] Braunstein, P.; Oro, L. A.; Raithby, P. Metal Clusters in Chemistry Vol. II, 1999.

[3] Mingos, M. P. Gold Clusters, Colloids and Nanoparticles I; Springer, 2014.

[4] Feliz, M.; Guillamon, E.; Llusar, R.; Vicent, C.; Stiriba, S. E.; Perez-Prieto, J.;

Barberis, M. Chem.-Eur. J. 2006, 12, 1486.

[5] Beltran, T. F.; Llusar, R.; Sokolov, M.; Basallote, M. G.; Fernandez-Trujillo,

M. J.; Pino-Chamorro, J. A. Inorg. Chem. 2013, 52, 8713.

[6] Tirfoin, R.; Chamoreau, L.-M.; Li, Y.; Fleury, B.; Lisnard, L.; Journaux, Y.

Dalton Trans. 2014, 43, 16805.

[7] Goswami, N.; Baksi, A.; Giri, A.; Xavier, P. L.; Basu, G.; Pradeep, T.; Pal, S.

K. Nanoscale 2014, 6, 1848.

[8] Bloomstrand, C. J. Prakt. Chemie 1857, 71, 449.

[9] Chabrié, M. C. Comptes Rend. 1907, 144, 804.

[10] Chapin, W. H. J. Am. Chem. Soc. 1910, 32, 323.

[11] Harned, H. S. J. Am. Chem. Soc. 1913, 35, 1078.

[12] Vaughan, P. A.; Sturdivant, J. H.; Pauling, L. J. Am. Chem. Soc. 1950, 72, 5477.

[13] Brosset, C. Arkiv för Kemi, Miner. Geol. 1945, Band 20A, No. 7.

[14] Brosset, C. Arkiv för Kemi, Miner. Geol. 1946, Band 22A.

[15] Cotton, F. A.; Murillo, C. A.; Walton, R. A. Multiple Bonds between Metal Atoms; Oxford University Press, 1993.

[16] Wei, C. H.; Dahl, L. F. J. Am. Chem. Soc. 1966, 88, 1821.

[17] Müller, A.; Jostes, R.; Cotton, F. A. Angew. Chem. Int. Ed. 1980, 19, 875.

[18] Mingos, M. P.; Wales, D. J. Introduction to Cluster Chemistry; Prentice-Hall International Editions, 1990.

[19] Cotton, F. A.; Lin, C.; Murillo, C. A. Acc. Chem. Res. 2001, 34, 759.

[20] Chisholm, M. H.; Macintosh, A. M. Chem. Rev. 2005, 105, 2949.

[21] Falvello, L. R.; Llusar, R.; Triguero, S.; Vicent, C. Chem. Commun. 2009, 3440. 
[22] Malatesta, L. Gold Bulletin 1975, 8, 48.

[23] Walter, M.; Akola, J.; Lopez-Acevedo, O.; Jadzinsky, P. D.; Calero, G.; Ackerson, C. J.; Whetten, R. L.; Grönbeck, H.; Häkkinen, H. Proc. Natl. Acad. Sci. 2008, 105, 9157.

[24] Häkkinen, H. In Metal Nanoparticles and Nanoalloys; Johnston, R. L., Wilcoxon, J. P., Eds.; Elsevier: Amsterdam, 2012; Vol. 3.

[25] Jadzinsky, P. D.; Calero, G.; Ackerson, C. J.; Bushnell, D. A.; Kornberg, R. D. Science 2007, 318, 430.

[26] Llusar R., Vicent C. in Inorganic Chemistry in Focus III, Chapter 7; Meyer, G.; Naumann, D.; Wesemann, L.; Wiley-VCH, 2006.

[27] Cotton, F. A.; Kibala, P. A.; Matusz, M.; McCaleb, C. S.; Sandor, R. B. W. Inorg. Chem. 1989, 28, 2623.

[28] Cotton, F. A.; Kibala, P. A.; Miertschin, C. S. Inorg. Chem. 1991, 30, 548.

[29] Fedin, V. P.; Sokolov, M. N.; Geras'ko, O. A.; Kolesov, B. A.; Fedorov, V. Y.; Mironov, A. V.; Yufit, D. S.; Slovohotov, Y. L.; Struchkov, Y. T. Inorg. Chim. Acta 1990, 175, 217.

[30] Vergamini, P. J.; Vahrenkamp, H.; Dahl, L. F. J. Am. Chem. Soc. 1971, 93, 6327.

[31] Müller, A.; Sarkar, S.; Bhattacharyya, R. G.; Pohl, S.; Dartmann, M. Angew. Chem. Int. Ed. 1978, 17, 535.

[32] Fedin, V. P.; Sokolov, M. N.; Mironov, Y. V.; Kolesov, B. A.; Tkachev, S. V.; Fedorov, V. Y. Inorg. Chim. Acta 1990, 167, 39.

[33] Fedin, V. P.; Sokolov, M. N.; Myakishev, K. G.; Geras'ko, O. A.; Fedorov, V. Y.; Maciček, J. Polyhedron 1991, 10, 1311.

[34] Saito, T.; Yoshikawa, A.; Yamagata, T.; Imoto, H.; Unoura, K. Inorg. Chem. 1989, 28,3588 .

[35] Fedin, V. P.; Sokolov, M. N.; Fedorov, V. Y.; Yufit, D. S.; Struchkov, Y. T. Inorg. Chim. Acta 1991, 179, 35. 
[36] Estevan, F.; Feliz, M.; Llusar, R.; Mata, J. A.; Uriel, S. Polyhedron 2001, 20, 527.

[37] Guillamon, E. M.; Blasco, M.; Llusar, R. Inorg. Chim. Acta 2015, 424, 248.

[38] Beltran, T. F.; Pino-Chamorro, J. A.; Fernandez-Trujillo, M. J.; Safont, V. S.; Basallote, M. G.; Llusar, R. Inorg. Chem. 2015, 54, 607.

[39] Basallote, M. G.; Fernández-Trujillo, M. J.; Pino-Chamorro, J. Á.; Beltrán, T. F.; Corao, C.; Llusar, R.; Sokolov, M.; Vicent, C. Inorg. Chem. 2012, 51, 6794.

[40] Alfonso, C.; Feliz, M.; Safont, V. S.; Llusar, R. Dalton Trans. 2016, 45, 7829.

[41] Frantz, R.; Guillamon, E.; Lacour, J.; Llusar, R.; Polo, V.; Vicent, C. Inorg. Chem. 2007, 46, 10717.

[42] Cotton, F. A.; Haas, T. E. Inorg. Chem. 1964, 3, 10.

[43] Hidai, M.; Kuwata, S.; Mizobe, Y. Acc. Chem. Res. 2000, 33, 46.

[44] Hernandez-Molina, R.; Sokolov, M. N.; Sykes, A. G. Acc. Chem. Res. 2001, 34, 223.

[45] Llusar, R.; Uriel, S. Eur. J. Inorg. Chem. 2003, 1271.

[46] Shibahara, T.; Akashi, H.; Kuroya, H. J. Am. Chem. Soc. 1986, 108, 1342.

[47] Sorribes, I.; Lloret, F.; Waerenborgh, J. C.; Polo, V.; Llusar, R.; Vicent, C. Inorg. Chem. 2012, 51, 10512.

[48] Feliz, M.; Llusar, R.; Uriel, S.; Vicent, C.; Coronado, E.; Gómez-García, C. J. Chem.-Eur. J. 2004, 10, 4308.

[49] Harris, S. Polyhedron 1989, 8, 2843.

[50] Yu, S.-B.; Droege, M.; Downey, S.; Segal, B.; Newcomb, W.; Sanderson, T.; Crofts, S.; Suravajjala, S.; Bacon, E.; Earley, W.; Delecki, D.; Watson, A. D. Inorg. Chem. 2001, 40, 1576.

[51] Yu, S.-B.; Droege, M.; Segal, B.; Kim, S.-H.; Sanderson, T.; Watson, A. D. Inorg. Chem. 2000, 39, 1325.

[52] Sorribes, I.; Wienhöfer, G.; Vicent, C.; Junge, K.; Llusar, R.; Beller, M. Angew. Chem. Int. Ed. 2012, 51, 7794. 
[53] Murata, T.; Mizobe, Y.; Gao, H.; Ishii, Y.; Wakabayashi, T.; Nakano, F.; Tanase, T.; Yano, S.; Hidai, M. J. Am. Chem. Soc. 1994, 116, 3389.

[54] Pedrajas, E.; Sorribes, I.; Junge, K.; Beller, M.; Llusar, R. ChemCatChem 2015, $7,2675$.

[55] Wakabayashi, T.; Ishii, Y.; Murata, T.; Mizobe, Y.; Hidai, M. Tetrahedron Lett. $1995,36,5585$.

[56] Takei, I.; Wakebe, Y.; Suzuki, K.; Enta, Y.; Suzuki, T.; Mizobe, Y.; Hidai, M. Organometallics 2003, 22, 4639.

[57] Beltran, T. F.; Feliz, M.; Llusar, R.; Mata, J. A.; Safont, V. S. Organometallics 2011, 30, 290.

[58] Hou, Y.; Abrams, B. L.; Vesborg, P. C. K.; Björketun, M. E.; Herbst, K.; Bech, L.; Setti, A. M.; Damsgaard, C. D.; Pedersen, T.; Hansen, O.; Rossmeisl, J.; Dahl, S.; Nørskov, J. K.; Chorkendorff, I. Nat. Mater. 2011, 10, 434.

[59] Herbst, K.; Brorson, M.; Carlsson, A. J. Mol. Catal. A: Chem. 2010, 325, 1.

[60] Fedin, V. P.; Czyzniewska, J.; Prins, R.; Weber, T. Appl. Catal. A. 2001, 213, 123.

[61] Feliz, M.; Garriga, J. M.; Llusar, R.; Uriel, S.; Humphrey, M. G.; Lucas, N. T.; Samoc, M.; Luther-Davies, B. Inorg. Chem. 2001, 40, 6132.

[62] Feliz, M.; Llusar, R.; Uriel, S.; Vicent, C.; Humphrey, M. G.; Lucas, N. T.; Samoc, M.; Luther-Davies, B. Inorg. Chim. Acta 2003, 349, 69.

[63] Recatala, D.; Llusar, R.; Barlow, A.; Wang, G.; Samoc, M.; Humphrey, M. G.; Guschin, A. L. Dalton Trans. 2015, 44, 13163.

[64] WO 2013/000970: Bayer Intellectual Property GmbH, Berlin (Germany), 2013.

[65] Seino, H.; Hidai, M. Chem. Sci. 2011, 2, 847.

[66] Takei, I.; Dohki, K.; Kobayashi, K.; Suzuki, T.; Hidai, M. Inorg. Chem. 2005, 44,3768 . 
[67] Beltrán, T. F., Tesis Doctoral, Universitat Jaume I, 2013. 
2. OBJETIVOS 
2. OBJETIVOS

"Cuando es obvio que las metas no se pueden conseguir, no ajustes las metas, ajusta los pasos para conseguirlas.”

Confucio 
El estudio de los clústeres de metales de transición es un campo de investigación multidisciplinar que ha crecido de forma exponencial durante los últimos años. Esto se debe a la versatilidad de estos compuestos, que presentan aplicaciones en campos tan diversos como la catálisis, las ciencias médicas o la óptica no lineal. El desarrollo de nuevas estrategias racionales de síntesis permite la funcionalización de la unidad clúster dirigida al uso de estos compuestos en aplicaciones específicas. Esta tesis doctoral trata principalmente sobre los clústeres de metales de transición del grupo 6 con unidad central $\mathrm{M}_{3} \mathrm{~S}_{4}$ y su funcionalización con ligandos difosfina, aminofosfina y aminodifosfina con la finalidad de obtener complejos que presenten actividad catalítica en reacciones de elevado interés industrial. Los objetivos concretos de este trabajo son los siguientes:

(i) Desarrollo y caracterización estructural de clústeres hidruro y fluoruro con unidad central $\mathrm{M}_{3} \mathrm{~S}_{4}(\mathrm{M}=\mathrm{Mo}, \mathrm{W})$ funcionalizados con difosfinas $\mathrm{y}$ estudio comparativo de su actividad catalítica en la hidrodefluoración de la pentafluoropiridina en función de la basicidad del ligando.

(ii) Funcionalización química de la unidad $\mathrm{M}_{3} \mathrm{~S}_{4}(\mathrm{M}=\mathrm{Mo}, \mathrm{W})$ con ligandos aminofosfina, optimización de las estrategias de síntesis de estos complejos y caracterización estructural.

(iii) Estudio combinado teórico-experimental sobre la obtención de clústeres con unidad central $\mathrm{Mo}_{3} \mathrm{~S}_{4}$ funcionalizados con aminofosfinas quirales, análisis de sus estructuras cristalinas y asignación de su configuración absoluta.

(iv) Desarrollo y análisis estructural de clústeres de molibdeno funcionalizados con ligandos aminodifosfina y estudio de su reactividad frente a otros complejos metálicos.

(v) Estudio de la actividad catalítica de clústeres de fórmula $\left[\mathrm{Mo}_{3} \mathrm{~S}_{4} \mathrm{Cl}_{3} \text { (quelato) }\right]^{+}$(quelato $=$aminofosfina, aminodifosfina) en reacciones de reducción de sustratos orgánicos e hidrogenación de $\mathrm{CO}_{2}$. 
3. CLÚSTERES FUNCIONALIZADOS

CON DIFOSFINAS:

HIDRODEFLUORACIÓN CATALÍTICA 
3. CLÚSTERES FUNCIONALIZADOS CON DIFOSFINAS: HIDRODEFLUORACIÓN CATALÍTICA.

3.1 Introducción

3.2 Síntesis y caracterización

3.2.1 Clústeres hidruro

3.2.2 Clústeres fluoruro

3.3 Hidrodefluoración catalítica de la pentafluoropiridina

3.4 Bibliografía

"La verdad en la ciencia puede ser definida como la hipótesis de trabajo que mejor se ajusta para abrir el camino a la siguiente mejor ajustada."

Konrad Lorenz 


\subsection{INTRODUCCIÓN}

Los compuestos organofluorados están presentes en medicamentos, compuestos agroquímicos, polímeros y otros productos industriales importantes, ${ }^{[1,2]}$ y su creciente demanda ha favorecido el desarrollo de rutas sintéticas donde no solo la formación del enlace C-F sino también su activación juegan un papel destacado. ${ }^{[3-5]} \mathrm{El}$ enlace carbono-flúor es el enlace $\sigma$ más fuerte que se conoce. Debido a la elevada electronegatividad del flúor nos encontramos ante un enlace muy polar, corto y con baja polarizabilidad, lo que provoca que su activación sea un reto perseguido durante las últimas décadas.[6] La eliminación selectiva de átomos de flúor en sustratos organofluorados proporciona precursores interesantes para la obtención de compuestos con alto interés industrial, $[7,8]$ y el procedimiento sintético más sencillo para conseguirlo consiste en la sustitución de enlaces C-F por enlaces C-H en presencia de un catalizador metálico y una fuente de hidrógeno. Este procedimiento, comúnmente conocido como hidrodefluoración (HDF), ha sido ampliamente estudiado ${ }^{[9-13]}$ y se representa en la ecuación 3.1:

$$
[\mathrm{M}]-\mathrm{H}+\mathrm{R}-\mathrm{F} \rightarrow[\mathrm{M}]-\mathrm{F}+\mathrm{R}-\mathrm{H}
$$

La hidrodefluoración es un proceso de gran interés a nivel industrial ya que permite obtener compuestos selectivamente fluorados y además proporciona una vía de destrucción de los clorofluorocarbonos (CFC) y perfluoroalcanos, gases que provocan el conocido "efecto invernadero".[14]

Muchos de los catalizadores utilizados los procesos de hidrodefluoración están basados en metales de transición situados en la parte derecha del sistema periódico. No obstante, los complejos de metales de transición de la parte izquierda han recibido un interés creciente en los últimos años.[1] Por ejemplo, el grupo de Lentz publicó en el 2010 la hidrodefluoración catalítica de hexafluoropropeno promovida por complejos fluorados de titanio como precatalizadores y silanos como 
fuente de hidrógeno, para dar lugar a diferentes isómeros monohidrogenados. ${ }^{[15]} \mathrm{El}$ mecanismo de esta reacción implica el ataque nucleofílico del ligando hidruro al sustrato orgánico electrofílico mediante la inserción del alqueno y la consecuente $\beta$ eliminación del fluoruro.

Por otra parte, Rosenthal y colaboradores demostraron en el año 2007 que complejos hidruro de zirconio catalizan la hidrodefluoración de la pentafluoropiridina a temperatura ambiente en presencia de un compuesto de aluminio con ligandos hidruro, obteniéndose como único producto de reacción la 2,3,5,6-tetrafluoropiridina. ${ }^{[16]}$ El ciclo catalítico propuesto por Rosenthal se muestra en la figura 3.1.

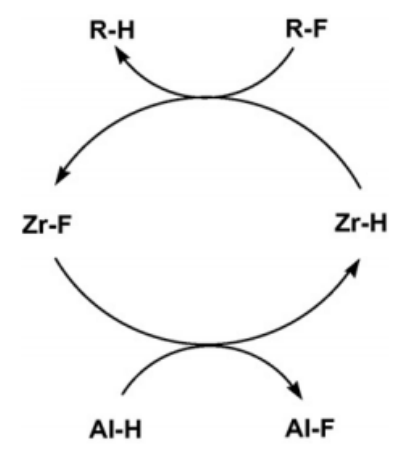

Figura 3.1. Ciclo catalítico propuesto por Rosenthal y colaboradores.

En la literatura existen muchos ejemplos de reacciones de hidrodefluoración catalizadas por complejos de metales de transición electrónicamente ricos. En el año 2005, Holland y colaboradores publicaron un artículo clave donde describieron la conversión catalítica de la pentafluoropiridina en 2,3,5,6-tetrafluoropiridina en presencia de un silano utilizando como catalizador un complejo de hierro y flúor. En este artículo proponen, además, un ciclo catalítico que se ha convertido en uno de los más aceptados por la comunidad científica, en el cual las especies hidruro se generan a partir de especies fluoradas gracias al uso de silanos como fuente de hidrógeno.[13] 
Por otra parte, Whittlesey y colaboradores utilizan un complejo hidruro de rutenio $\mathrm{N}$-heterocíclico y diferentes silanos en la hidrodefluoración catalítica de fluoroarenos, aunque en este caso la reacción es regioselectiva hacia la formación del isómero hidrogenado en la posición orto. ${ }^{[10]}$ Esta selectividad se atribuye a un mecanismo que implica la descoordinación de una de las fosfinas coordinadas al rutenio. ${ }^{[17]}$ Recientemente, el grupo de investigación de Peris ha publicado la actividad catalítica de un complejo de rutenio y paladio en la hidrodefluoración tándem de sustratos aromáticos y alifáticos fluorados. El paladio facilita la activación del enlace C-F, mientras que el rutenio permite la reducción del sustrato mediante transferencia de hidrógeno gracias a la adición de terbutóxido de sodio e isopropanol.[9]

En 2011 Llusar y colaboradores publicaron que los hidruros clúster de molibdeno y wolframio de fórmula general $\left[\mathrm{M}_{3} \mathrm{~S}_{4} \mathrm{H}_{3}(\mathrm{dmpe})_{3}\right]^{+}(\mathrm{M}=\mathrm{Mo}$, W; dmpe $=$ 1,2-bis(dimetilfosfino)etano) son activos en la hidrodefluoración de la pentafluoropiridina. ${ }^{[18]}$ Esta reacción resultó ser regioselectiva en la posición para del anillo aromático fluorado, formándose como producto 2,3,5,6-tetrafluoropiridina. La reacción estudiada transcurre mediante un mecanismo de metátesis de enlace $\sigma$ que implica la descoordinación de uno de los átomos de fósforo de la difosfina, tal como se muestra en la figura 3.2.

El paso clave del mecanismo de la hidrodefluoración propuesta por Llusar es la descoordinación parcial de la difosfina dmpe, energéticamente accesible mediante irradiación con microondas, por tanto la elección de la fosfina es clave en el éxito del proceso. Existen otras evidencias experimentales de la descoordinación de difosfinas en este tipo de compuestos, en concreto en el caso del clúster $\left[\mathrm{Mo}_{3} \mathrm{~S}_{4} \mathrm{Cl}_{3}(\mathrm{dppe})_{3}\right]^{+}$ (dppe $=1,2$-bis(difenilfosfino)etano) que genera una vacante de coordinación en el molibdeno a elevadas temperaturas.[19] 


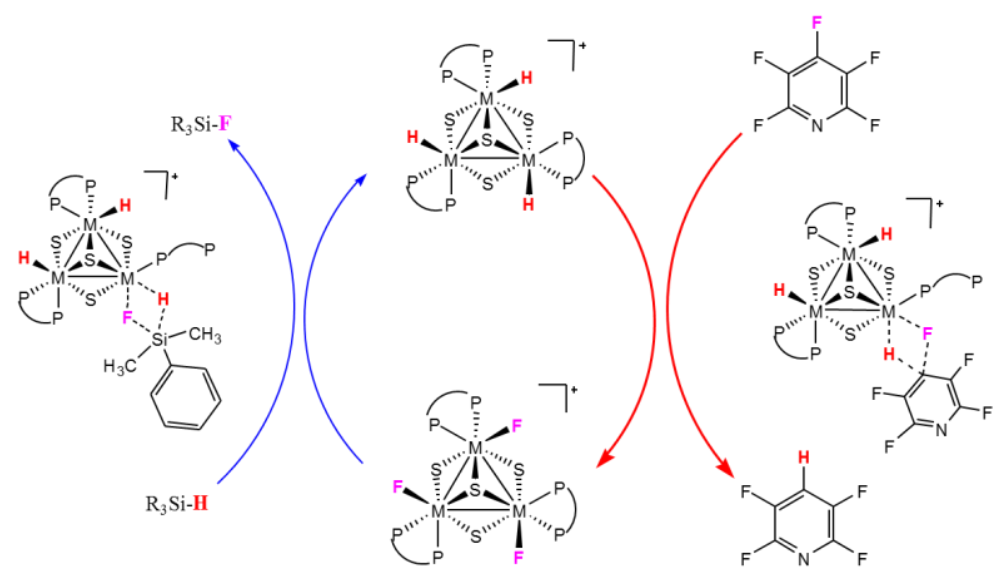

Figura 3.2. Ciclo catalítico de HDF propuesto por Llusar y colaboradores. Figura reproducida con permiso de Organometallics 2011, 30, 290 (referencia [18]). Copyright 2011 American Chemical Society.

El trabajo que se presenta en este capítulo parte de la hipótesis de que la coordinación de una fosfina menos básica a la unidad trimetálica, en concreto la dppe, podría favorecer esta descoordinación. A continuación se discuten los procesos de síntesis y caracterización de hidruros y fluoruros clúster funcionalizados con dppe, así como su papel en la hidrodefluoración de la pentafluoropiridina mediante un estudio comparativo. 


\subsection{SÍNTESIS Y CARACTERIZACIÓN}

\subsubsection{CLÚSTERES HIDRURO}

Los complejos de fórmula $\left[\mathrm{M}_{3} \mathrm{~S}_{4} \mathrm{H}_{3}(\text { dppe })_{3}\right]+(\mathrm{M}=\mathrm{Mo}, \mathrm{W})$ se han preparado mediante la adaptación de procesos descritos en la literatura para compuestos análogos. Los precursores halogenados se han hecho reaccionar con un exceso de borohidruro sódico en metanol, en el caso del compuesto de molibdeno, y en tetrahidrofurano en el caso del clúster de wolframio.

Cotton y colaboradores publicaron en 1989 la síntesis del primer clúster hidruro de fórmula $\left.\left[\mathrm{W}_{3} \mathrm{~S}_{4} \mathrm{H}_{3} \text { (difosfina) }\right)_{3}\right]^{+[20]}$ y una década después, el grupo de investigación de Llusar preparó el hidruro trinuclear de wolframio con ligandos dppe, descrito en esta tesis doctoral como $3^{+} .{ }^{[21]}$ A pesar de que es un complejo conocido desde hace años, la síntesis detallada en la ecuación 3.2 constituye una mejora de la estrategia de preparación puesto que se ha aumentado el rendimiento de un $46 \%$ a un $97 \%$ gracias a un cambio de disolvente.[22] Mientras que en la síntesis original se utiliza metanol, en la síntesis descrita en esta tesis doctoral se utiliza THF, que resulta ser crucial para la obtención de este hidruro de wolframio en rendimientos elevados. Un hecho similar ya se observó a principios de esta década cuando Llusar y colaboradores aislaron el complejo $\left[\mathrm{Mo}_{3} \mathrm{~S}_{4} \mathrm{H}_{3}(\mathrm{dmpe})_{3}\right]^{+}$, cuya síntesis se lleva a cabo también en THF.[23]

$$
\left[\mathrm{W}_{3} \mathrm{~S}_{4} \mathrm{Br}_{3}(\mathrm{dppe})_{3}\right]^{+}+3 \mathrm{NaBH}_{4} \stackrel{\mathrm{THF}}{\longrightarrow}\left[\mathrm{W}_{3} \mathrm{~S}_{4} \mathrm{H}_{3}(\mathrm{dppe})_{3}\right]^{+}+3 \mathrm{BH}_{3}+3 \mathrm{NaBr} \text { (3.2) }
$$

La síntesis del hidruro de molibdeno funcionalizado con dppe se ha abordado inicialmente siguiendo el mismo procedimiento utilizado para su análogo de wolframio $3^{+}$, aunque en esta ocasión el uso de THF ha dificultado enormemente su aislamiento. El complejo $\left[\mathrm{Mo}_{3} \mathrm{~S}_{4} \mathrm{H}_{3}(\mathrm{dppe})_{3}\right]\left(\mathrm{BPh}_{4}\right) \quad\left(\mathbf{1}\left(\mathrm{BPh}_{4}\right)\right)$ se ha preparado finalmente tras disolver el precursor clorado en metanol y añadir un exceso de borohidruro sódico. El producto final ha precipitado como un sólido puro en el seno 
de la reacción mediante la adición de $\mathrm{NaBPh}_{4}$. En la figura 3.3 se observa el esquema de la reacción de obtención de este hidruro.
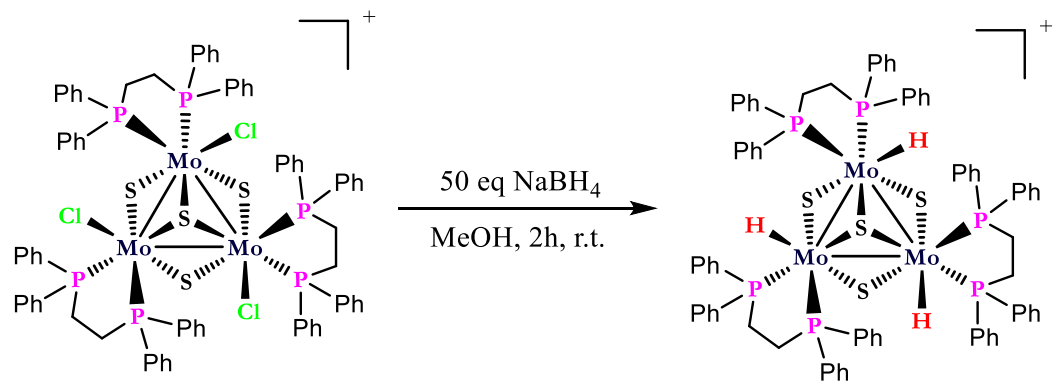

Figura 3.3. Procedimiento de síntesis para la obtención del clúster $\mathbf{1}^{+}$.

El clúster $\mathbf{1}^{+}$se puede manipular en estado sólido y en disolución con una exposición no excesivamente prolongada al aire, hecho que ha facilitado su caracterización mediante espectroscopia de RMN, espectrometría de masas y difracción de rayos X en monocristal. La cristalización de este compuesto se ha llevado a cabo mediante la difusión lenta de hexano sobre una disolución del clúster en diclorometano. El complejo $1\left(\mathrm{PF}_{6}\right)$ cristaliza en el grupo espacial no centrosimétrico Cc a partir de una mezcla racémica, lo que indica la cristalización preferencial de uno de los dos enantiómeros. Esta situación fue observada previamente por Llusar y colaboradores para un clúster con estructura similar de fórmula $\left[\mathrm{Mo}_{3} \mathrm{NiS}_{4} \mathrm{Cl}_{4}(\mathrm{dmpe})_{3}\right]$.[24]

Siguiendo la tendencia de los clústeres trinucleares funcionalizados con difosfinas hasta la fecha, el ligando se encuentra coordinado de una forma específica, con un átomo de fósforo situado trans al azufre apuntado y el otro trans a los azufres puente. El entorno de coordinación de cada molibdeno lo completa un ligando hidruro, que no se detecta mediante difracción de rayos $\mathrm{X}$ pero sí se confirma su presencia por ${ }^{1} \mathrm{H}-\mathrm{RMN}$ y espectrometría de masas, tal y como se verá más adelante. En la figura 3.4 se muestra una representación ORTEP de esta estructura. Asimismo, en la tabla 3.1 se listan las distancias de enlace más destacadas del compuesto $1\left(\mathrm{PF}_{6}\right)$, 
comparadas con las del precursor halogenado y el hidruro análogo funcionalizado con dmpe.

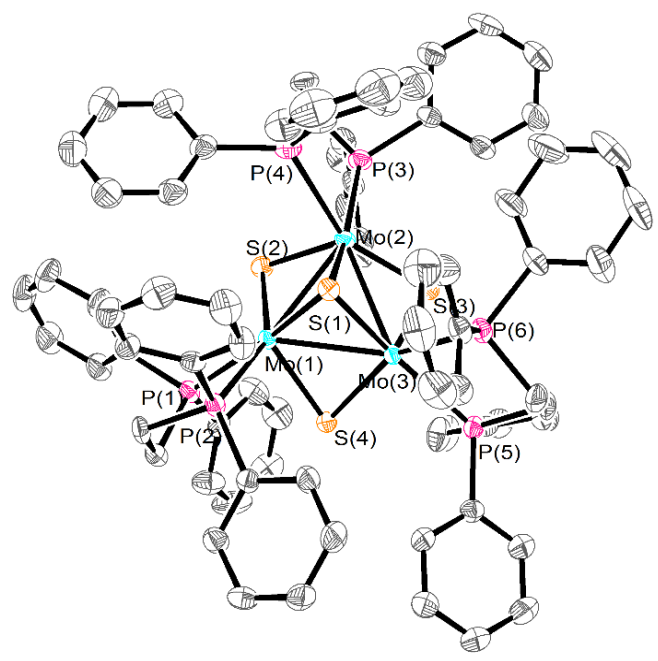

Figura 3.4. Representación ORTEP (elipsoides al 50\% de probabilidad) del complejo $\mathbf{1}^{+}$. El contraion y los átomos de hidrógeno se han omitido para una mayor claridad.

Tabla 3.1. Selección de distancias de enlace promedio para los compuestos $\mathbf{1}^{+}$, $\left[\mathrm{Mo}_{3} \mathrm{~S}_{4} \mathrm{Cl}_{3}(\mathrm{dppe})_{3}\right]^{+}$y $\left[\mathrm{Mo}_{3} \mathrm{~S}_{4} \mathrm{H}_{3}(\mathrm{dmpe})_{3}\right]^{+}$. La desviación estándar de los valores promedio se encuentra representada entre corchetes.

\begin{tabular}{|c|c|c|c|}
\hline Distancia (Å) & $1^{+}$ & {$\left[\mathrm{Mo}_{3} \mathrm{~S}_{4} \mathrm{Cl}_{3}(\mathrm{dppe})_{3}\right]^{+(\mathrm{a})}$} & {$\left[\mathrm{Mo}_{3} \mathrm{~S}_{4} \mathrm{H}_{3}(\mathrm{dmpe})_{3}\right]^{+(\mathrm{b})}$} \\
\hline Mo-Mo & $2,7663[5]$ & $2,777[6]$ & $2,744[5]$ \\
\hline $\operatorname{Mo}-\left(\mu_{3}-S\right)$ & $2,3642[13]$ & $2,359[5]$ & $2,346[4]$ \\
\hline Mo- $(\mu-S)^{(c)}$ & $2,3315[13]$ & $2,280[5]$ & $2,324[2]$ \\
\hline Mo- $(\mu-S)^{(d)}$ & $2,3242[13]$ & $2,320[4]$ & $2,329[4]$ \\
\hline Mo-P(1)(e) & $2,5133[13]$ & $2,576[6]$ & $2,467[12]$ \\
\hline $\mathrm{Mo}-\mathrm{P}(2)^{(\mathrm{f})}$ & $2,5557[13]$ & $2,658[12]$ & $2,524[9]$ \\
\hline
\end{tabular}

(a)Datos extraídos de la referencia [21]. (b)Datos extraídos de la referencia [23] (c)Distancia trans al enlace Mo-X (X = H, Cl). ${ }^{(\mathrm{d})}$ Distancia trans al enlace Mo-P(2). ${ }^{(\mathrm{e})}$ Distancia trans al enlace Mo- $\left(\mu_{3}-\right.$ S). ${ }^{(\mathrm{f} D}$ Distancia trans al enlace Mo- $(\mu-\mathrm{S})$. [] Desviación estándar del promedio. 
Las distancias Mo-Mo y Mo-S se encuentran dentro del rango observado para otros complejos $\mathrm{M}_{3} \mathrm{Q}_{4}(\mathrm{M}=\mathrm{Mo}, \mathrm{W} ; \mathrm{Q}=\mathrm{S}, \mathrm{Se})$ y son coherentes con un estado de oxidación IV para los átomos de molibdeno. ${ }^{[25]}$ En el caso de las distancias Mo-S, se observa que los enlaces molibdeno-azufre puente son ligeramente más cortos que los existentes entre el molibdeno y el azufre apuntado, tendencia que se repite en los clústeres de unidad $\mathrm{M}_{3} \mathrm{Q}_{4}$ publicados hasta la fecha. Además, en el caso de los hidruros, las distancias Mo- $(\mu-S)$ son muy similares entre ellas. Este hecho se atribuye a un alargamiento de la distancia Mo-S trans al enlace Mo-H debido a la mayor influencia trans del ligando hidruro con respecto al cloruro.

Las distancias Mo-P del clúster $\mathbf{1}^{+}$son más largas que las encontradas en su análogo funcionalizado con dmpe, lo que se puede atribuir a efectos estéricos y electrónicos. En cambio, estas distancias son más cortas si las comparamos con las del clúster precursor $\left[\mathrm{Mo}_{3} \mathrm{~S}_{4} \mathrm{Cl}_{3}(\mathrm{dppe})_{3}\right]^{+}$, circunstancia que se puede relacionar con el menor tamaño del ligando hidruro con respecto al cloruro.

La naturaleza del clúster $\mathbf{1}^{+}$se ha confirmado mediante $\mathrm{RMN}$ de ${ }^{31} \mathrm{P}\left\{{ }^{1} \mathrm{H}\right\}$, en cuyo espectro se observan dos señales de igual intensidad a 33,10 y 59,29 ppm que se corresponden con los dos grupos de tres átomos de fósforo no equivalentes, localizados por encima y por debajo del plano que conforman los tres metales (ver figura 3.5).

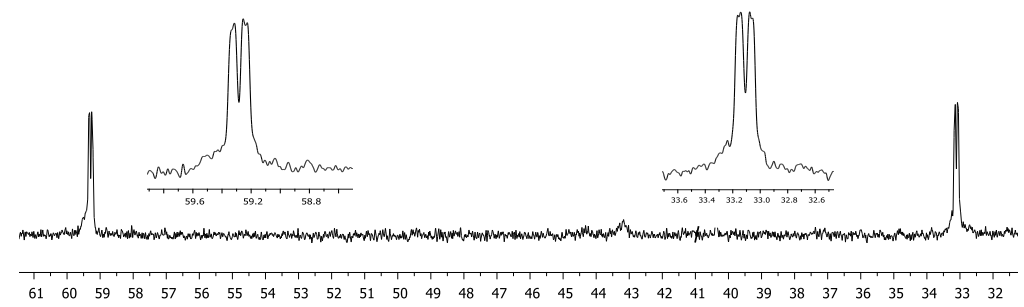

Figura 3.5. Espectro de $\mathrm{RMN}$ de ${ }^{31} \mathrm{P}\left\{{ }^{1} \mathrm{H}\right\}$ del compuesto $\left[\mathrm{Mo}_{3} \mathrm{~S}_{4} \mathrm{H}_{3}(\mathrm{dppe})_{3}\right] \mathrm{BPh}_{4}\left(\mathbf{1}\left(\mathrm{BPh}_{4}\right)\right)$. 
La multiplicidad de las señales es de doblete debido al acoplamiento existente entre los átomos de fósforo no equivalentes de la misma difosfina. La constante de acoplamiento ${ }^{2}$ Jp-p se ha calculado y ha resultado ser de 7,9 Hz. Un estudio de RMN de formación de pares iónicos de fórmula $\left(\left[\mathrm{W}_{3} \mathrm{Q}_{4} \mathrm{X}_{3}(\mathrm{dmpe})_{3}\right]^{+}, \mathrm{Y}^{-}\right)(\mathrm{Q}=\mathrm{S}, \mathrm{Se} ; \mathrm{X}=$ $\left.\mathrm{H}, \mathrm{OH}, \mathrm{Br} ; \mathrm{Y}^{-}=\mathrm{PF}_{6}^{-}, \mathrm{BF}_{4}^{-}\right)$publicado por los grupos de Llusar y Basallote, ha permitido asociar estas señales a cada uno de los grupos de átomos de fósforo no equivalentes presentes en el clúster. Mediante experimentos de desacoplamiento de protón ${ }^{1} \mathrm{H}$ y ${ }^{19} \mathrm{~F}$-HOESY, los investigadores relacionaron la señal que aparece a campos más altos con los átomos de fósforo que se encuentran en posición trans a los azufres puente y la señal que aparece a campos más bajos con los átomos de fósforo trans al azufre apuntado.[26] De esta forma y por analogía con los clústeres estudiados en ese trabajo, la señal que aparece a 33,10 ppm se corresponde con los primeros, mientras que la que aparece a 59,29 ppm se corresponde con los últimos.

Este compuesto también se ha caracterizado mediante ${ }^{1} \mathrm{H}-\mathrm{RMN}$, donde se observa una señal centrada en -1,05 ppm con multiplicidad de doble doblete en la zona hidrídica (ver figura 3.6), de forma análoga a lo observado para otros hidruros de fórmula general $\left.\left[\mathrm{M}_{3} \mathrm{~S}_{4} \mathrm{H}_{3} \text { (difosfina) }\right)_{3}\right]^{+}$.[21,23] Esta señal confirma la existencia de los tres ligandos hidruro coordinados a los tres átomos de molibdeno y su multiplicidad es consecuencia del acoplamiento entre los ligandos hidruro y los dos fósforos no equivalentes que se encuentran enlazados al mismo átomo metálico, con unas constantes de acoplamiento ${ }^{2} J_{\text {H-P de }} 37,0$ y 75,0 Hz. 


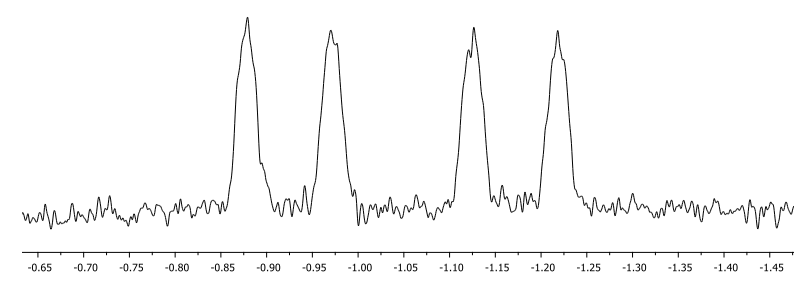

Figura 3.6. Ampliación de la zona hidrídica del espectro de $\mathrm{RMN}$ de ${ }^{1} \mathrm{H}$ del compuesto 1(BPh4).

Por último, este complejo también ha sido caracterizado mediante espectrometría de masas, utilizando la técnica de electrospray como fuente de ionización (ESI). En el espectro se observa un pico base centrado en 1614 u.m.a. que corresponde al ion molecular $\left[\mathrm{Mo}_{3} \mathrm{~S}_{4} \mathrm{H}_{3}(\mathrm{dppe})_{3}\right]^{+}$. La señal se ha asignado por comparación entre la distribución isotópica experimental y la simulada, calculada con el programa MassLynx, ${ }^{[27]}$ y se ha encontrado buena concordancia entre ellas tal y como se puede apreciar en la figura 3.7.

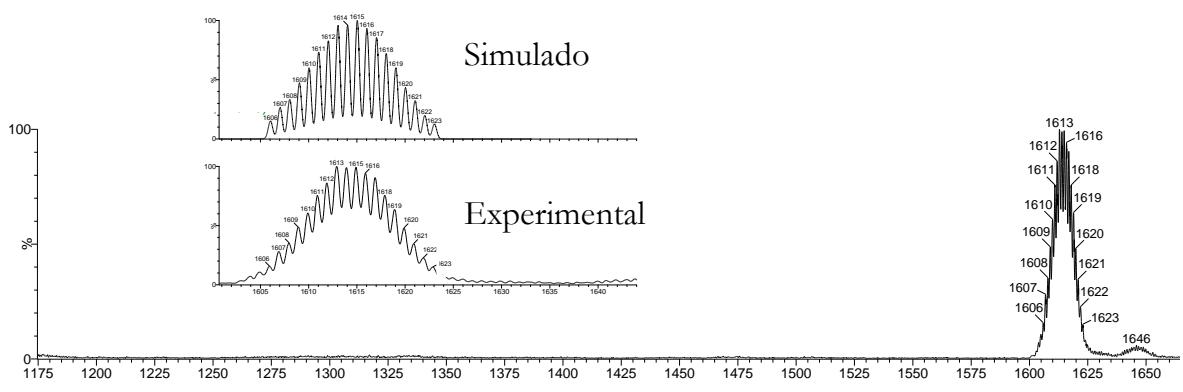

Figura 3.7. Espectros de masas simulado y experimental del clúster $\mathbf{1}^{+}$.

\subsubsection{CLÚSTERES FLUORURO}

La síntesis de los complejos clúster fluorados de fórmula $\left[\mathrm{M}_{3} \mathrm{~S}_{4} \mathrm{~F}_{3}(\mathrm{dppe})_{3}\right]^{+}(\mathrm{M}=\mathrm{Mo}$, W) se ha abordado inicialmente siguiendo la misma estrategia que en el caso de los complejos $\left[\mathrm{M}_{3} \mathrm{~S}_{4} \mathrm{~F}_{3}(\mathrm{dmpe})_{3}\right]^{+}$, que constituyen uno de los pocos precedentes existentes en la literatura para este tipo de clústeres trinucleares.[18] Esta ruta de síntesis se basa en la reacción del clúster hidruro precursor con un exceso de ácido 
hexafluorofosfórico $\left(\mathrm{HPF}_{6}\right)$ a temperatura ambiente en una mezcla de acetonitrilo:agua (2:1). En nuestro caso no se ha observado reacción más allá de la descomposición del hidruro, tanto en el complejo de molibdeno como en el de wolframio. Por esta razón, se ha decidido cambiar de estrategia y prescindir de los clústeres hidruro como precursores debido a su inestabilidad frente a este ácido.

Los compuestos trinucleares fluorados de fórmula $\left[\mathrm{M}_{3} \mathrm{~S}_{4} \mathrm{~F}_{3}(\mathrm{dppe})_{3}\right]^{+}(\mathrm{M}=$ Mo, W) se han obtenido en rendimientos moderados (65\% y $60 \%$, respectivamente) mediante el intercambio de los ligandos cloruro o bromuro por los ligandos fluoruro provenientes de una sal inorgánica como es el fluoruro de cesio (CsF), en presencia de acetonitrilo tal como se ilustra en la figura 3.8. Cabe destacar que la reacción tiene lugar tras 24 horas a reflujo en el caso del clúster de wolframio con un cambio de coloración de azul a morado, mientras que la reacción con el clúster de molibdeno tiene lugar a $60^{\circ} \mathrm{C}$ y resulta en un cambio de coloración de verde claro a verde oscuro. Si intentamos llevar a cabo esta reacción a temperatura ambiente, no se observa ningún cambio de color.

Los compuestos se han purificado suspendiéndolos en una mezcla de tolueno:acetona (98:2) para solubilizar los restos de complejos mononucleares resultantes de la degradación parcial de los precursores. Tras la filtración de esta suspensión, se han obtenido los complejos fluorados puros. Estos clústeres fluorados se han caracterizado mediante espectroscopia de RMN, espectrometría de masas y difracción de rayos $\mathrm{X}$ en monocristal.

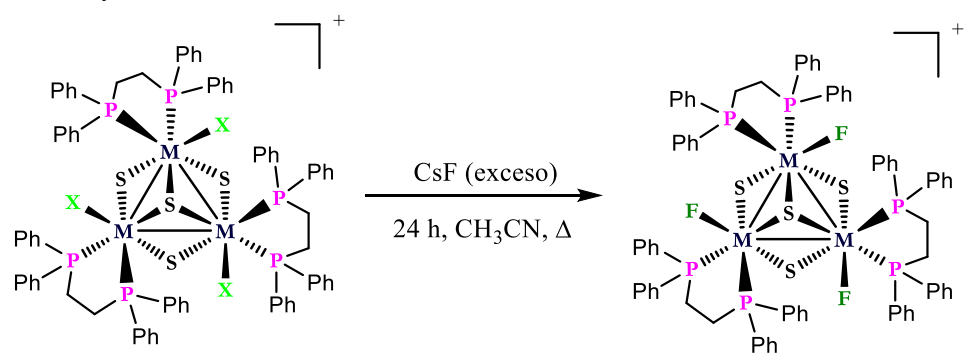

Figura 3.8. Procedimiento general para la obtención de clústeres $\left[\mathrm{M}_{3} \mathrm{~S}_{4} \mathrm{~F}_{3}(\mathrm{dppe})_{3}\right]^{+}$. 
Los espectros de RMN de fósforo muestran, tal como se observa en la figura 3.9, dos señales de igual intensidad y multiplicidad doblete a 22,2 ppm y 30,4 ppm en el caso del compuesto de molibdeno (arriba), y a 6,7 ppm y 7,7 ppm en el caso del compuesto de wolframio (abajo). Las señales corresponden a los dos núcleos de fósforo no equivalentes situados por encima y por debajo del plano trimetálico. Al igual que se ha descrito en la sección 3.2.1, se pueden asociar las señales que aparecen a campos más bajos a los fósforos localizados trans al azufre apuntado, mientras que las que aparecen a campos más altos se pueden asignar a los fósforos que se encuentran trans a los azufres puente. Además, la multiplicidad que muestran es debida al acoplamiento de cada átomo de fósforo de las difosfinas con el átomo de flúor coordinado al mismo metal.
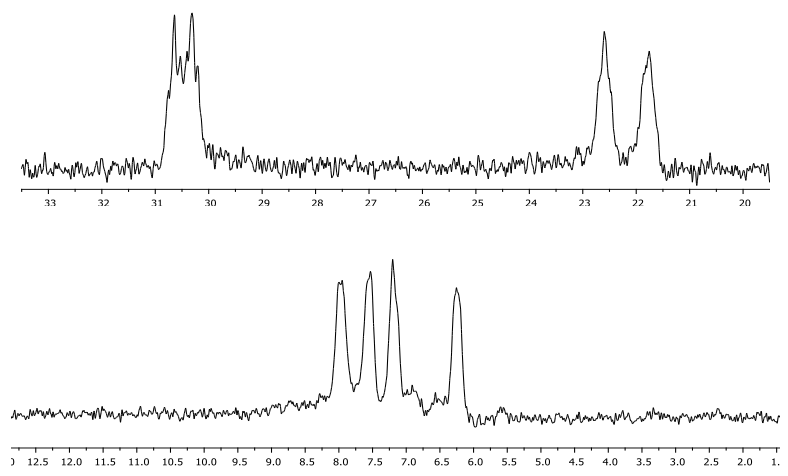

Figura 3.9. Espectros de $\mathrm{RMN}$ de ${ }^{31} \mathrm{P}\left\{{ }^{1} \mathrm{H}\right\} \mathrm{RMN}$ de $\left[\mathrm{Mo}_{3} \mathrm{~S}_{4} \mathrm{~F}_{3}(\mathrm{dppe})_{3}\right]^{+}\left(\mathbf{2}^{+}\right)$(arriba) y $\left[\mathrm{W}_{3} \mathrm{~S}_{4} \mathrm{~F}_{3}(\mathrm{dppe})_{3}\right]^{+}\left(4^{+}\right)$(abajo).

Las constantes de acoplamiento ${ }^{2} \mathrm{~J}$ P-F se han calculado para ambos clústeres, siendo de $103,3 \mathrm{~Hz}$ y 40,1 Hz para el complejo $2^{+}$y de $51,0 \mathrm{~Hz}$ y $114,2 \mathrm{~Hz}$ para el complejo $4^{+}$. Estas constantes de acoplamiento también se han calculado a partir de los espectros de RMN de ${ }^{19} \mathrm{~F}$, y el resultado obtenido nos permite caracterizar de forma inequívoca ambos compuestos. Al caracterizar los clústeres mediante RMN de ${ }^{19} \mathrm{~F}$ se observa una única señal asociada a los tres átomos de flúor equivalentes presentes en los dos compuestos, hecho que corrobora su simetría $C_{3}$. En el caso del complejo $\mathbf{2}^{+}$la señal aparece a $-177,50 \mathrm{ppm}$, mientras que en el compuesto $4^{+}$aparece 
a -182,40 ppm. La multiplicidad de esta señal es, en ambos casos, de doble doblete (ver figura 3.10), consecuencia del acoplamiento entre los átomos de fósforo de los ligandos difosfina y los ligandos fluoruro.
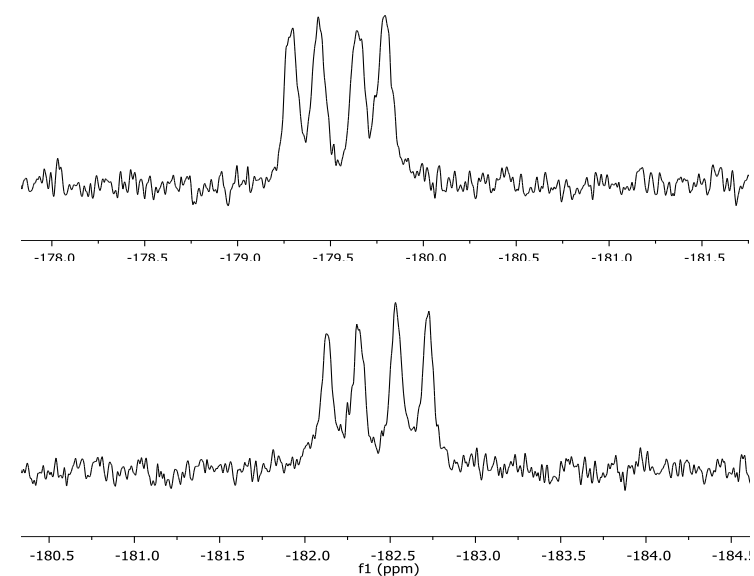

Figura 3.10. Espectros de $\mathrm{RMN}{ }^{19} \mathrm{~F}$ de $\left[\mathrm{Mo}_{3} \mathrm{~S}_{4} \mathrm{~F}_{3}(\mathrm{dppe})_{3}\right]^{+}\left(\mathbf{2}^{+}\right)$(arriba) y $\left[\mathrm{W}_{3} \mathrm{~S}_{4} \mathrm{~F}_{3}(\mathrm{dppe})_{3}\right]^{+}$ $\left(4^{+}\right)$(abajo).

Las constantes de acoplamiento ${ }^{2} \mathrm{~J} F-\mathrm{P}$ se han calculado para $\mathbf{2}^{+}$y $\mathbf{4}^{+}$, siendo de 40,9 Hz y 103,1 Hz para el complejo de molibdeno, y de 50,8 Hz y 112,9 Hz para el complejo de wolframio. Estos resultados están en excelente concordancia con los obtenidos en el caso de los acoplamientos ${ }^{2}$ JP-F para el RMN de ${ }^{31} \mathrm{P}\left\{{ }^{1} \mathrm{H}\right\}$. La tabla 3.2 muestra un resumen de los desplazamientos químicos y las constantes de acoplamiento para los RMN de fósforo y flúor, con el fin de obtener una visión conjunta del papel de esta técnica en la caracterización de nuestros compuestos. 
Tabla 3.2. Desplazamientos químicos de los átomos de flúor y fósforo de los complejos $\mathbf{2}^{+}$ y $4^{+}$y sus constantes de acoplamiento.

\begin{tabular}{ccccc}
\hline \hline Compuesto & $\begin{array}{c}\boldsymbol{\delta}(\mathbf{p p m}) \\
{ }^{31} \mathbf{P}\left\{{ }^{1} \mathbf{H}\right\}- \\
\mathbf{R M N}\end{array}$ & $\begin{array}{c}\boldsymbol{\delta}(\mathbf{p p m}) \\
{ }^{19} \mathbf{F}-\mathbf{R M N}\end{array}$ & ${ }^{2} \mathbf{J}_{\mathbf{P}-\mathbf{F}}(\mathbf{H z})$ & ${ }^{2} \mathbf{J}_{\mathrm{F}-\mathbf{P}}(\mathbf{H z})$ \\
\hline$\left[\mathrm{Mo}_{3} \mathrm{~S}_{4} \mathrm{~F}_{3}(\mathrm{dppe})_{3}\right]^{+}\left(\mathbf{2}^{+}\right)$ & 22,2 y 30,4 & $-177,5$ & 103,3 y 40,1 & 103,1 y 40,9 \\
{$\left[\mathrm{~W}_{3} \mathrm{~S}_{4} \mathrm{~F}_{3}(\mathrm{dppe})_{3}\right]^{+}\left(\mathbf{4}^{+}\right)$} & 6,7 y 7,7 & $-182,4$ & 51,0 y 114,2 & 50,8 y 112,9 \\
\hline \hline
\end{tabular}

Los clústeres $2^{+}$y $4^{+}$se han caracterizado mediante espectrometría de masas utilizando la técnica de electrospray como fuente de ionización (ESI). En ambos casos, se observa que el pico base se corresponde con el ion molecular de fórmula $\left.\left[\mathrm{M}_{3} \mathrm{~S}_{4} \mathrm{~F}_{3} \text { (dppe) }\right]_{3}\right]^{+}$, de forma que el pico de 1668 u.m.a. se asigna al complejo de molibdeno $2^{+}$y el pico de 1931 u.m.a. al complejo de wolframio $4^{+}$. Como se observa en la figura 3.11, en los dos clústeres existe correspondencia entre la distribución isotópica simulada y la distribución isotópica experimental.

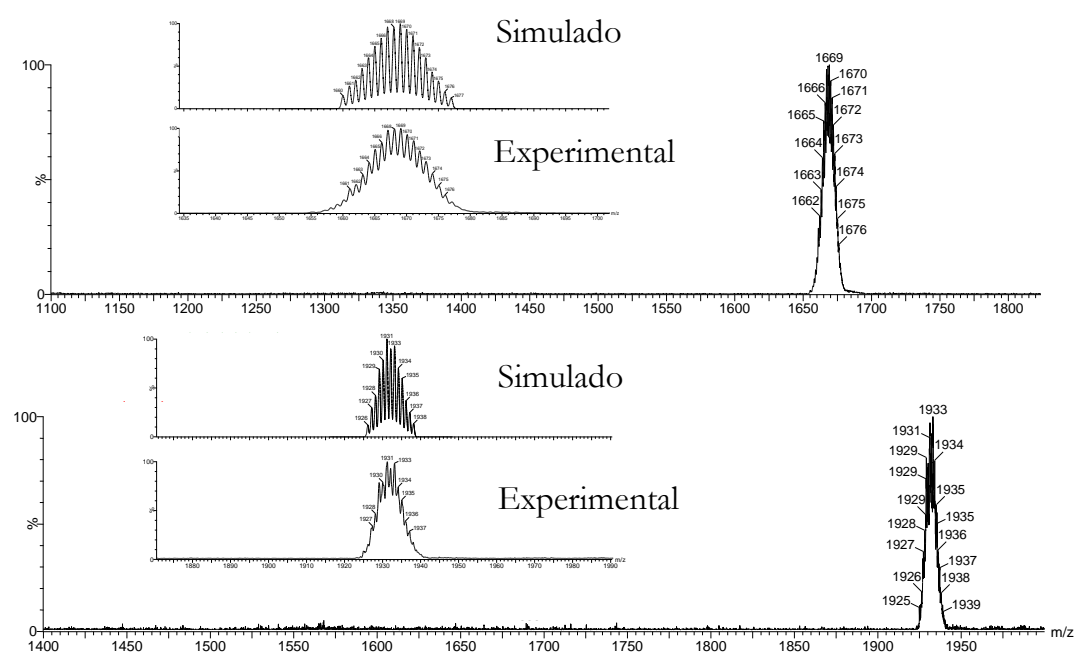

Figura 3.11. Espectros de masas simulados y experimentales de los clústeres $\left[\mathrm{Mo}_{3} \mathrm{~S}_{4} \mathrm{~F}_{3}(\text { dppe })_{3}\right]^{+}\left(2^{+}\right)$(arriba) y $\left[\mathrm{W}_{3} \mathrm{~S}_{4} \mathrm{~F}_{3}(\text { dppe })_{3}\right]^{+}\left(4^{+}\right)$(abajo). 
La caracterización del complejo $2^{+}$se ha llevado a cabo también mediante difracción de rayos $\mathrm{X}$ en monocristal. Este compuesto cristaliza en el grupo espacial centrosimétrico $\mathrm{P} 21 / \mathrm{c}$ como sal de hexafluorofosfato y además se observa la cristalización de una molécula de agua. El clúster $2^{+}$presenta la misma organización molecular que el clúster hidruro $\mathbf{1}^{+}$, con un átomo de fósforo trans al azufre apuntado y el otro trans al azufre puente. Los átomos metálicos y los átomos de azufre ocupan vértices adyacentes de un cubo con una posición vacante que resulta en una estructura de tipo cubano incompleta. En la figura 3.12 se muestra una representación ORTEP de la estructura cristalina del complejo $2^{+}$. Todos los intentos de obtención de cristales del complejo $4^{+}$adecuados para su caracterización por difracción de rayos X en monocristal han resultado infructuosos.

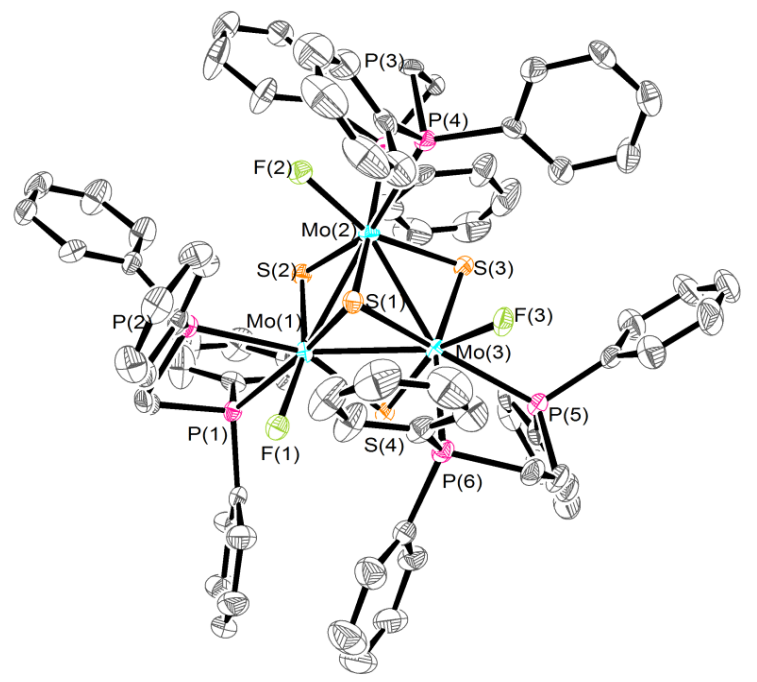

Figura 3.12. Representación ORTEP (elipsoides al 50\% de probabilidad) del complejo $\mathbf{2}^{+}$. El contraión y los átomos de hidrógeno se han omitido para una mayor claridad.

Las distancias de enlace más relevantes de esta estructura, las de su análogo funcionalizado con dmpe y las de la estructura del clúster hidruro $1^{+}$se recogen a continuación en la tabla 3.3. Las diferencias entre las distancias Mo- $(\mu-\mathrm{S})$ presentes 
los compuestos fluorados son mayores que las del complejo hidruro. Esto es debido a la menor influencia trans del ligando fluoruro en comparación con el hidruro, hecho que provoca que la distancia Mo-S trans al enlace Mo-F sea más pequeña que la que se encuentra trans al Mo-H en el complejo $\mathbf{1}^{+}$. Las distancias Mo-F son similares en ambos clústeres fluorados, y a su vez se asemejan a las Mo-F terminales publicadas para el clúster $\left[\mathrm{Mo}_{3} \mathrm{~S}_{4} \mathrm{~F}_{7}(\mathrm{FHF})_{2}\right] \mathrm{K}_{5} .{ }^{[28]}$ Los enlaces Mo-P sufren una elongación significativa en el compuesto $\mathbf{1}^{+}$frente a su análogo de dmpe que se puede atribuir a efectos electrónicos y estéricos debido al cambio de difosfina. Asimismo, estas distancias son más largas en el complejo fluorado $\mathbf{2}^{+}$que en el hidruro $\mathbf{1}^{+}$debido al mayor tamaño del ligando fluoruro.

Tabla 3.3. Selección de distancias de enlace promedio para los compuestos $2^{+}$y $\left[\mathrm{Mo}_{3} \mathrm{~S}_{4} \mathrm{~F}_{3}(\mathrm{dmpe})_{3}\right]^{+}$. La desviación estándar de los valores promedio se encuentra representada entre corchetes.

\begin{tabular}{cccc}
\hline \hline Distancia $(\AA)$ & $2^{+}$ & [Mos $\left._{3} \mathbf{S}_{4} \mathbf{F}_{3}(\mathbf{d m p e})_{3}\right]^{+(a)}$ & $1^{+}$ \\
\hline Mo-Mo & $2,7584[6]$ & $2,7497[17]$ & $2,7663[5]$ \\
Mo- $\left(\mu_{3}-\mathrm{S}\right)$ & $2,3550[14]$ & $2,351[4]$ & $2,3642[13]$ \\
Mo- $(\mu-S)^{(b)}$ & $2,2824[14]$ & $2,283[4]$ & $2,3315[13]$ \\
Mo- $(\mu-S)^{(c)}$ & $2,3173[14]$ & $2,343[4]$ & $2,3242[13]$ \\
Mo-P(1)(d) & $2,5555[15]$ & $2,504[4]$ & $2,5133[13]$ \\
Mo-P(2)(e) & $2,6232[15]$ & $2,567[4]$ & $2,5557[13]$ \\
Mo-X & $2,038[3]$ & $2,046[8]$ & - \\
\hline \hline
\end{tabular}

(a)Datos extraídos de la referencia [18]. ${ }^{(\mathrm{b})}$ Distancia trans al enlace Mo-X $(\mathrm{X}=\mathrm{F}, \mathrm{H}) .{ }^{(\mathrm{c})}$ Distancia trans al enlace Mo-P(2). (d)Distancia trans al enlace Mo- $\left(\mu_{3}-\mathrm{S}\right)$. (e) Distancia trans al enlace Mo- $(\mu-\mathrm{S})$. [] Desviación estándar del promedio. 


\subsection{HIDRODEFLUORACIÓN CATALÍTICA DE LA PENTAFLUOROPIRIDINA}

La actividad de los complejos hidruro $1\left(\mathrm{BPh}_{4}\right)$ y $3\left(\mathrm{BPh}_{4}\right)$ y fluoruro $2\left(\mathrm{BPh}_{4}\right)$ y $4\left(\mathrm{BPh}_{4}\right)$ en la hidrodefluoración catalítica de la pentafluoropiridina se ha estudiado de manera análoga al trabajo de Llusar y colaboradores. ${ }^{[18]}$ Se ha utilizado $\mathrm{Me}_{2} \mathrm{PhSiH}$ como fuente de hidrógeno y se ha monitorizado la evolución de la reacción mediante RMN de ${ }^{19} \mathrm{~F} .{ }^{[28]}$ La comparación con un patrón comercial de elevada pureza ha permitido la identificación del producto de reacción, 2,3,5,6-tetrafluoropiridina, lo que confirma que la reacción ocurre selectivamente hacia la obtención del producto monohidrogenado sustituido en posición para. En la figura 3.13 se muestra un esquema de la reacción:
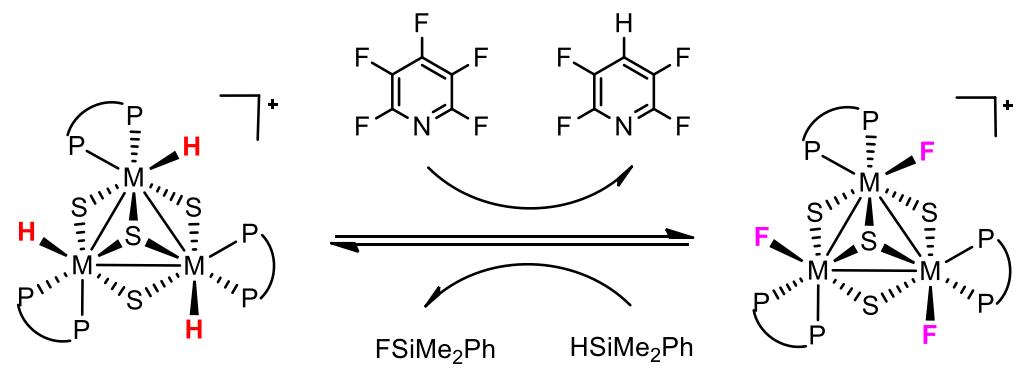

Figura 3.13. Esquema general de la hidrodefluoración catalítica de la pentafluoropiridina.

Antes de realizar los estudios catalíticos, hemos estudiado la reactividad de los complejos $\mathbf{1}^{+}$y $\mathbf{3}^{+}$frente a la pentafluoropiridina con el objetivo de observar la formación deseable de complejos fluorados. Esta reacción se ha realizado bajo irradiación microondas en acetonitrilo, en presencia de un exceso de 50 equivalentes de pentafluoropiridina y se ha monitorizado por espectrometría de masas durante un tiempo de 4 horas, tal como se ilustra en la figura 3.14. El resultado ha sido una sustitución secuencial de ligandos hidruro por ligandos fluoruro que queda demostrada por la aparición de los picos correspondientes a las especies monosustituidas, disustituidas y trisustituidas a una relación $\mathrm{m} / \mathrm{z}$ de 1895, 1913 y 
1931, respectivamente. En la siguiente figura se muestra la evolución de la reacción para el complejo $\mathbf{1}^{+}$:

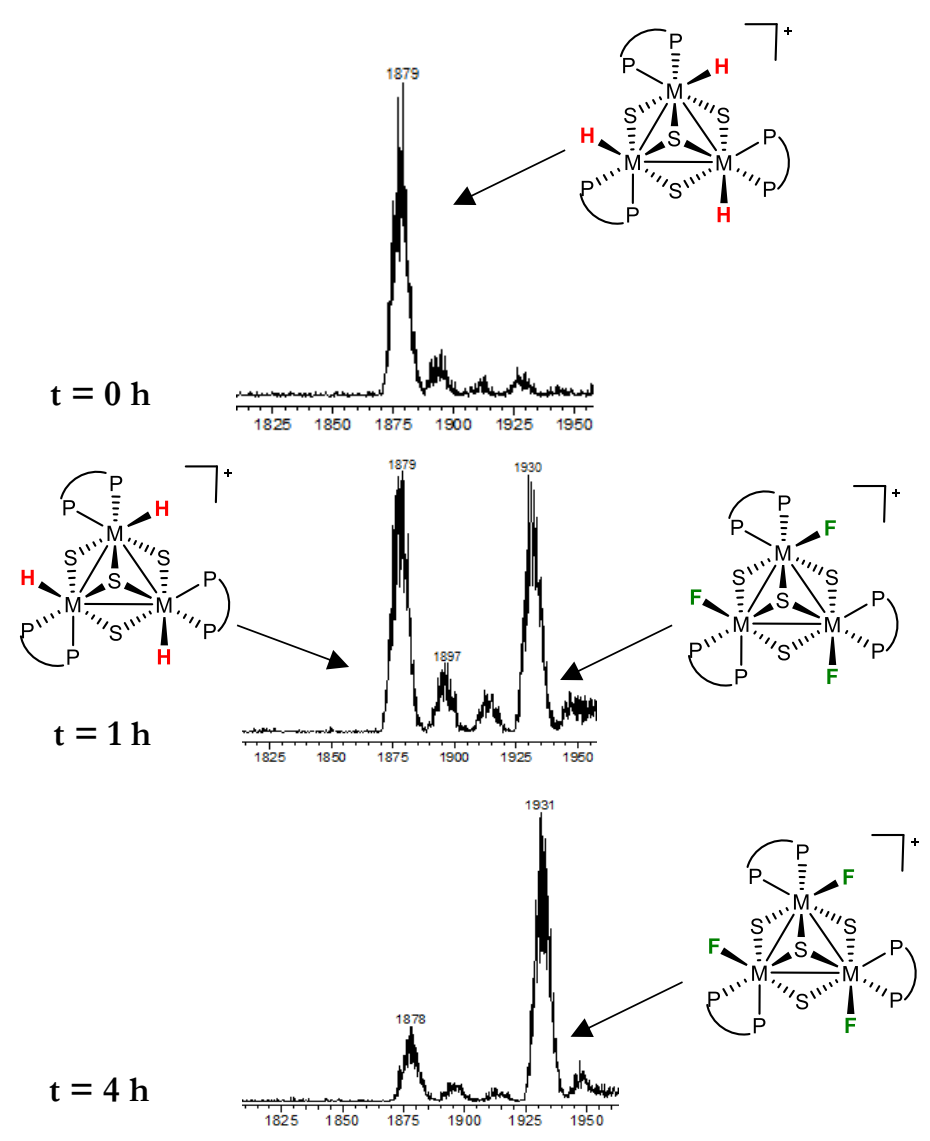

Figura 3.14. Seguimiento por espectrometría de masas de la reacción entre $\left[\mathrm{W}_{3} \mathrm{~S}_{4} \mathrm{H}_{3}(\mathrm{dppe})_{3}\right]^{+}$ $\left(3^{+}\right)$y pentafluoropiridina a tiempo inicial, tras 1 hora de reacción y tras 4 horas de reacción.

El producto orgánico de hidrodefluoración se ha identificado como 2,3,5,6tetrafluoropiridina mediante $\mathrm{RMN}$ de ${ }^{19} \mathrm{~F}$, debido a la aparición de dos señales a un desplazamiento químico de -94 ppm y -142 ppm. Por tanto, una vez demostrada la capacidad que presentan estos clústeres para activar el enlace C-F de la pentafluoropiridina, la reacción catalítica se ha llevado a cabo añadiendo un silano con el fin de regenerar la especie activa, es decir, el clúster hidruro. 
Las condiciones de reacción del estudio catalítico (carga de hidruro, tiempo de reacción y temperatura) se han optimizado hasta obtener una conversión máxima. El mejor resultado se ha obtenido con $0,7 \mathrm{~mol} \%$ de catalizador a $115{ }^{\circ} \mathrm{C}$ bajo radiación microondas, con una potencia máxima de $70 \mathrm{~W}$. Una vez el equipo alcanza la temperatura indicada, esta permanece constante durante todo el proceso, de forma que la presión y la potencia se ajustan dinámicamente hasta alcanzar el equilibrio necesario para mantener la reacción a $115{ }^{\circ} \mathrm{C}$. Si se sobrepasan la potencia y temperatura indicadas, se ha comprobado mediante espectrometría de masas que tiene lugar la descomposición del clúster. Estas condiciones energéticas son más suaves que las requeridas para la misma reacción utilizando catalizadores análogos de dmpe, donde los mejores rendimientos se observaron a $180{ }^{\circ} \mathrm{C}$ con una carga de catalizador de $1 \mathrm{~mol} \%$.

Los fluoruros $2^{+}$y $4^{+}$se han utilizado como precatalizadores en esta reacción para estudiar la generación de especies hidruro en presencia de $\mathrm{Me}_{2} \mathrm{PhSiH}$, y la consiguiente hidrodefluoración catalítica de la pentafluoropiridina. La reacción se ha llevado a cabo en las mismas condiciones que con los clústeres hidruro.

La naturaleza del producto de reacción se ha confirmado mediante RMN de ${ }^{19} \mathrm{~F}$, donde se observan dos señales a $-94 \mathrm{ppm}$ y $-142 \mathrm{ppm}$ correspondientes a la 2,3,5,6-tetrafluoropiridina y también se observa una señal a -162 ppm que se corresponde con el coproducto de la reacción, $\mathrm{Me} 2 \mathrm{PhSiF}$. En ningún experimento se han encontrado señales correspondientes a isómeros de sustitución en posición meta, orto ni de doble hidrodefluoración, por tanto se confirma que la reacción es altamente selectiva. Esta transformación también se ha monitorizado por espectrometría de masas para comprobar que la unidad clúster está presente a lo largo de todo el ciclo catalítico. Esto se confirma por la aparición durante el transcurso de la reacción de un pico a 1877 u.m.a. que corresponde al ion molecular $\left[\mathrm{W}_{3} \mathrm{~S}_{4} \mathrm{H}_{3}(\mathrm{dppe})_{3}\right]^{+}$en el caso del compuesto $3^{+}$, y un pico a 1614 u.m.a. que corresponde a $\left[\mathrm{Mo}_{3} \mathrm{~S}_{4} \mathrm{H}_{3}(\mathrm{dppe})_{3}\right]^{+}$en el caso del compuesto $\mathbf{1}^{+}$. 
Los resultados de la hidrodefluoración catalítica de la pentafluoropiridina con hidruros clúster de fórmula $\left[\mathrm{M}_{3} \mathrm{~S}_{4} \mathrm{H}_{3}(\mathrm{dppe})_{3}\right]^{+}\left(\mathbf{1}^{+}\right.$y $\left.\mathbf{3}^{+}\right)$como catalizadores y fluoruros clúster de fórmula $\left[\mathrm{Mo}_{3} \mathrm{~S}_{4} \mathrm{~F}_{3}(\mathrm{dppe})_{3}\right]^{+}\left(\mathbf{2}^{+}\right.$y $\left.\mathbf{4}^{+}\right)$como precatalizadores se muestran a continuación en la tabla 3.4.

Tabla 3.4. Hidrodefluoración catalítica de la pentafluoropiridina.

\begin{tabular}{|c|c|c|c|c|c|}
\hline Entrada & Catalizador & $\begin{array}{l}\text { Tiempo } \\
\text { de } \\
\text { reacción } \\
\text { (h) }\end{array}$ & $\begin{array}{l}\text { Condiciones } \\
\text { de } \\
\text { reacción }^{(a)}\end{array}$ & $\begin{array}{c}\text { Rendimiento } \\
(\%)^{(\mathbf{b})}\end{array}$ & TON(c) \\
\hline 1 & {$\left[\mathrm{Mo}_{3} \mathrm{~S}_{4} \mathrm{H}_{3}(\mathrm{dppe})_{3}\right]^{+}$} & 1 & Microondas & 21 & 30 \\
\hline 2 & {$\left[\mathrm{Mo}_{3} \mathrm{~S}_{4} \mathrm{~F}_{3}(\mathrm{dppe})_{3}\right]^{+}$} & 2 & Microondas & 19 & 27 \\
\hline 3 & {$\left[\mathrm{~W}_{3} \mathrm{~S}_{4} \mathrm{H}_{3}(\mathrm{dppe})_{3}\right]^{+}$} & 30 & Reflujo & 55 & 79 \\
\hline 4 & {$\left[\mathrm{~W}_{3} \mathrm{~S}_{4} \mathrm{H}_{3}(\mathrm{dppe})_{3}\right]^{+}$} & 2,5 & Microondas & 87 & 124 \\
\hline 5 & {$\left[\mathrm{~W}_{3} \mathrm{~S}_{4} \mathrm{~F}_{3}(\mathrm{dppe})_{3}\right]^{+}$} & 0,75 & Microondas & 65 & 94 \\
\hline
\end{tabular}

(a) Cantidades: 0,7 mol \% de catalizador/precatalizador, 0,38 mmol de pentafluoropiridina, 1,9 mmol de $\mathrm{HSiMe}_{2} \mathrm{Ph}, 1 \mathrm{~mL}$ de acetonitrilo.

(b) Rendimiento basado en ${ }^{19} \mathrm{~F}-\mathrm{RMN}$ utilizando fluorobenceno como patrón interno

(c) TON (turnover number): moles de producto de $\mathrm{HDF} /$ moles de catalizador

El clúster $3^{+}$resulta ser el catalizador más eficiente, presentando un rendimiento de $87 \%$ y un TON de 124 (entrada 4), mientras que el clúster hidruro de molibdeno, $\mathbf{1}^{+}$, reacciona rápidamente aunque solo presenta un $21 \%$ de rendimiento y un TON de 30 (entrada 1). En general, los clústeres de wolframio presentan mayor actividad catalítica que los de molibdeno. Destaca el hecho de que la reacción funciona de manera notable a reflujo utilizando como catalizador el complejo $3^{+}$, situación que no se observa con los complejos análogos de dmpe.[18]

Cuando la reacción se lleva a cabo con los fluoruros clúster $2^{+}$y $4^{+}$, se obtienen rendimientos y TON menores que en el caso de los hidruros (ver tabla 3.4). 
Estos resultados se atribuyen a la poca eficiencia del $\mathrm{Me}_{2} \mathrm{PhSiH}$ para generar especies hidruro.

En conclusión, los métodos sintéticos descritos para el desarrollo de clústeres de fórmula $\left[\mathrm{M}_{3} \mathrm{~S}_{4} \mathrm{X}_{3}(\mathrm{dmpe})_{3}\right]^{+}(\mathrm{M}=\mathrm{Mo}, \mathrm{W} ; \mathrm{X}=\mathrm{H}, \mathrm{F})$ se pueden adaptar fácilmente para sintetizar los complejos análogos de dppe. Los clústeres $\left[\mathrm{M}_{3} \mathrm{~S}_{4} \mathrm{H}_{3}(\mathrm{dppe})_{3}\right]^{+}$son activos en la hidrodefluoración catalítica de la pentafluoropiridina y se pueden regenerar a partir de los complejos $\left[\mathrm{M}_{3} \mathrm{~S}_{4} \mathrm{~F}_{3}(\mathrm{dppe})_{3}\right]^{+}$en presencia de $\mathrm{Me}_{2} \mathrm{PhSiH}$. Esta reacción ocurre de forma selectiva dando lugar a un único producto de hidrodefluoración sustituido en posición para. Además, la sustitución de la difosfina dmpe por la dppe, que presenta una menor basicidad, se traduce en un aumento de la actividad catalítica, ya que la reacción tiene lugar en condiciones más suaves y a tiempos menores. Se observa además actividad catalítica cuando la reacción tiene lugar a reflujo, situación que con los complejos de dmpe no ocurre. 


\subsection{BIBLIOGRAFÍA}

[1] Purser, S.; Moore, P. R.; Swallow, S.; Gouverneur, V. Chem. Soc. Rev. 2008, 37,320 .

[2] O'Hagan, D. J. Fluor. Chem. 2010, 131, 1071.

[3] Amii, H.; Uneyama, K. Chem. Rev. 2009, 109, 2119.

[4] Nova, A.; Mas-Ballesté, R.; Lledós, A. Organometallics 2011, 31, 1245.

[5] Clot, E.; Eisenstein, O.; Jasim, N.; Macgregor, S. A.; McGrady, J. E.; Perutz, R. N. Acc. Chem. Res. 2011, 44, 333.

[6] Hiyama, T. Organofluorine Compounds. Chemistry and Applications:; Springer, 2000.

[7] C-H and C-X Bond Functionalization: Transition Metal Mediation; Ribas, X., Ed.; RSC, Cambridge, UK, 2013.

[8] Kuehnel, M. F.; Lentz, D.; Braun, T. Angew. Chem. 2013, 52, 3328.

[9] Sabater, S.; Mata, J. A.; Peris, E. Nat. Commun. 2013, 4.

[10] Reade, S. P.; Mahon, M. F.; Whittlesey, M. K. J. Am. Chem. Soc. 2009, 131, 1847.

[11] Kraft, B. M.; Lachicotte, R. J.; Jones, W. D. J. Am. Chem. Soc. 2001, 123, 10973.

[12] Yow, S.; Gates, S. J.; White, A. J. P.; Crimmin, M. R. Angew. Chem. Int. Ed. 2012, 51, 12559 .

[13] Vela, J.; Smith, J. M.; Yu, Y.; Ketterer, N. A.; Flaschenriem, C. J.; Lachicotte, R. J.; Holland, P. L. J. Am. Chem. Soc. 2005, 127, 7857.

[14] Alonso, F.; Beletskaya, I. P.; Yus, M. Chem. Rev. 2002, 102, 4009.

[15] Kuhnel, M. F.; Lentz, D. Angew. Chem. Int. Ed. 2010, 49, 2933.

[16] Jäger-Fiedler, U.; Klahn, M.; Arndt, P.; Baumann, W.; Spannenberg, A.; Burlakov, V. V.; Rosenthal, U. J. Mol. Catal. A: Chem. 2007, 261, 184.

[17] Panetier, J. A.; Macgregor, S. A.; Whittlesey, M. K. Angew. Chem. Int. Ed. 2011, 50, 2783. 
[18] Beltran, T. F.; Feliz, M.; Llusar, R.; Mata, J. A.; Safont, V. S. Organometallics 2011, 30, 290.

[19] Frantz, R.; Guillamón, E.; Lacour, J.; Llusar, R.; Polo, V.; Vicent, C. Inorg. Chem. 2007, 46, 10717.

[20] Cotton, F. A.; Llusar, R.; Eagle, C. T. J. Am. Chem. Soc. 1989, 111, 4332.

[21] Estevan, F.; Feliz, M.; Llusar, R.; Mata, J. A.; Uriel, S. Polyhedron 2001, 20, 527.

[22] Alfonso, C.; Beltran, T. F.; Feliz, M.; Llusar, R. J. Clust. Sci. 2015, 26, 199.

[23] Algarra, A. G.; Basallote, M. G.; Fernandez-Trujillo, M. J.; Feliz, M.; Guillamon, E.; Llusar, R.; Sorribes, I.; Vicent, C. Inorg. Chem. 2010, 49, 5935.

[24] Andrés, J.; Feliz, M.; Fraxedas, J.; Hernández, V.; López-Navarrete, J. T.; Llusar, R.; Sauthier, G.; Sensato, F. R.; Silvi, B.; Bo, C.; Campanera, J. M. Inorg. Chem. 2007, 46, 2159.

[25] Llusar, R.; Uriel, S. Eur. J. Inorg. Chem. 2003, 2003, 1271.

[26] Algarra, A. S. G.; Basallote, M. G.; Fernandez-Trujillo, M. J.; Llusar, R.; Safont, V. S.; Vicent, C. Inorg. Chem. 2006, 45, 5774.

[27] MassLynx 4.1 ed, Waters Ltd., Milford, MA, 2006.

[28] Mironov, Y. V.; Yarovoi, S. S.; Solodovnikov, S. F.; Fedorov, V. E. J. Mol. Struct. 2003, 656, 195. 


\section{CLÚSTERES FUNCIONALIZADOS CON AMINOFOSFINAS Y SU ACTIVIDAD EN LA REDUCCIÓN CATALÍTICA DE CETONAS}


4. CLÚSTERES FUNCIONALIZADOS CON AMINOFOSFINAS Y SU ACTIVIDAD EN LA REDUCCIÓN CATALÍTICA DE CETONAS

4.1 Introducción

4.2 Síntesis, caracterización y estudios en fase gas

4.3 Estudios de reactividad

4.3.1 Reactividad frente a borohidruro

4.3.2 Reactividad frente a alcoholes

4.4 Reducción catalítica de cetonas

4.5 Bibliografía

"La verdadera ciencia enseña, sobre todo, a dudar y a ser ignorante." Miguel de Unamuno 


\subsection{INTRODUCCIÓN}

Los complejos de metales de transición funcionalizados con aminofosfinas presentan una actividad elevada en procesos catalíticos de hidrogenación, de gran importancia tanto a escala de laboratorio como a nivel industrial.[1-3] A pesar de que el hidrógeno molecular es la fuente de hidrogenación más limpia en términos medioambientales, su manipulación presenta una serie de riesgos, especialmente cuando se requiere trabajar a presiones elevadas. Por ello resulta interesante la búsqueda de alternativas tales como las reacciones de transferencia de hidrógeno, ámbito en el que los complejos de metales de transición funcionalizados con aminofosfinas juegan un papel relevante. ${ }^{[4-7]}$

La reducción de sustratos carbonílicos en los que el grupo $\mathrm{C}=\mathrm{O}$ se transforma en un grupo alcohol tiene un marcado interés industrial, ${ }^{[8]}$ ya que los alcoholes son intermedios en la síntesis de medicamentos, aromatizantes y fertilizantes, entre otros.[9,10] Por otra parte, la hidrogenación de $\mathrm{CO}_{2}$ constituye hoy en día un reto ya que permite obtener derivados con valor comercial a la vez que reduce el contenido de este gas en la atmósfera.[11,12]

La naturaleza química de un sustrato orgánico determina su tendencia a reaccionar con hidrógeno. En el caso de los sustratos que contienen un grupo carbonilo, las cetonas y los aldehídos son los más sencillos de hidrogenar, mientras que las amidas y ésteres presentan una mayor dificultad.[13] Por otra parte, la reducción de derivados de ácido carbónico (carbonatos, carbamatos y urea) constituye uno de los retos en la hidrogenación de sustratos carbonílicos, tal como muestra la figura 4.1. 
Dificultad de hidrogenación

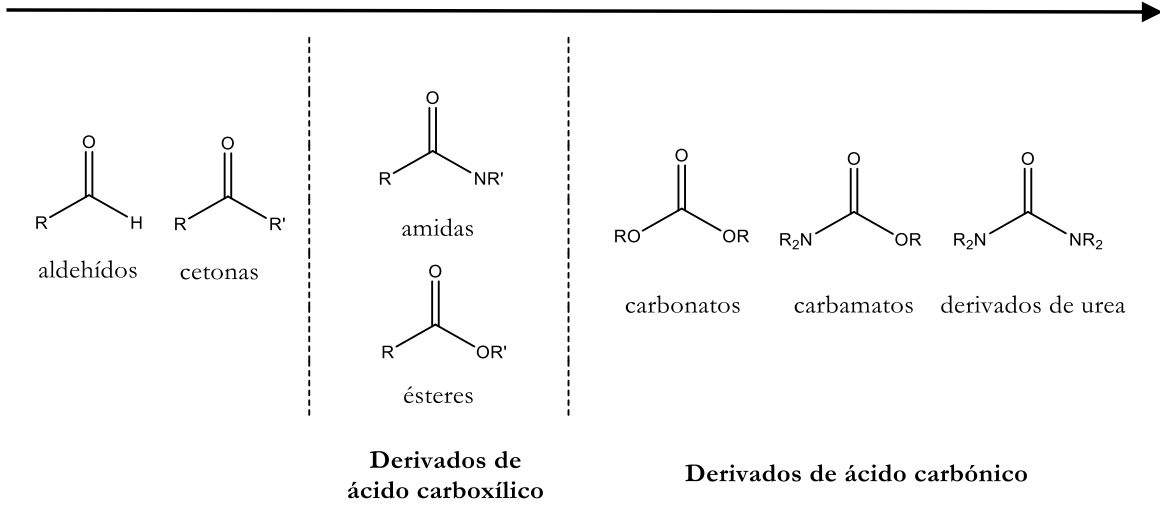

Figura 4.1. Orden creciente de la dificultad de hidrogenación de sustratos carbonílicos. Figura adaptada de la referencia [13].

El grupo amino juega un papel crucial en diversos tipos de reacciones catalíticas, como hidrogenaciones, reacciones de transferencia de hidrógeno, deshidrogenaciones y epoxidación de olefinas, entre otras, ya que puede comportarse como un grupo de coordinación, fuente de protones o dador y aceptor de hidrógeno. ${ }^{[8]}$ En este contexto, las aminofosfinas constituyen una familia de ligandos muy versátil, ya que combinan el carácter $\pi$-aceptor del átomo de fósforo con el $\sigma$ dador del átomo de nitrógeno. Esta combinación puede ayudar a estabilizar los intermedios de reacción que se forman durante un ciclo catalítico. Además, estos ligandos pueden ejercer un grado de regiocontrol mediante el fenómeno conocido como efecto trans, que tiene lugar debido a que la posición trans al átomo $\pi$-aceptor (P) es más electrofílica que la posición trans al átomo $\sigma$-dador $(\mathrm{N})$ y por tanto la primera posición se encuentra más expuesta a un ataque nucleofílico.[14]

La naturaleza hemilábil de los ligandos aminofosfina es debida a la mayor labilidad del enlace metal-N frente a la del enlace metal-P, que facilita la creación de vacantes de coordinación y en consecuencia la reacción con agentes externos. Sin embargo esto no se cumple en los clústeres trinucleares de molibdeno, en los que los ligandos difosfina requieren un menor aporte energético para descoordinar uno de 
los átomos de fósforo que la necesaria para romper el enlace molibdeno-fósforo o molibdeno-nitrógeno en las aminofosfinas. ${ }^{[15]}$

El elevado número de ejemplos de complejos mononucleares derivados con aminofosfinas contrasta con el limitado número de clústeres publicados funcionalizados con estos ligandos. En 1987, Süss-Fink y colaboradores publicaron la síntesis de clústeres trinucleares de osmio derivados con la amida de N,P,Ptrifenilfosfinoso.[16] Posteriormente, el grupo de Komiya ha descrito la síntesis y reactividad de clústeres heterodimetálicos de platino-molibdeno y paladio-cobalto funcionalizados con 2-(difenilfosfino)-N,N-dietiletanamina que facilitan la inserción de $\mathrm{CO}_{2} \cdot[17-19]$

En cuanto a los clústeres con unidad $\mathrm{M}_{3} \mathrm{~S}_{4}(\mathrm{M}=\mathrm{Mo}, \mathrm{W})$, el grupo de Llusar ha descrito recientemente la síntesis de complejos de fórmula $\left[\mathrm{M}_{3} \mathrm{~S}_{4} \mathrm{X}_{3}(\mathrm{edpp})_{3}\right]^{+}(\mathrm{M}$

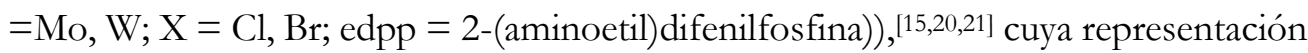
esquemática se muestra en la figura 4.2. Estos clústeres de molibdeno catalizan selectivamente la reducción de nitroderivados, con buenos resultados en términos de conversión y rendimiento.[20]

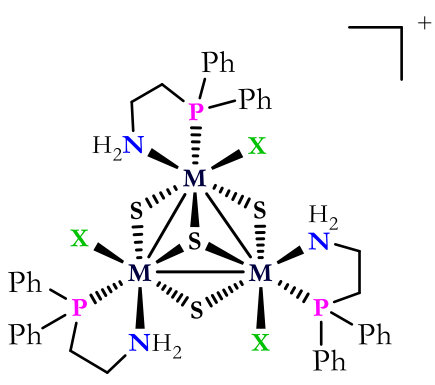

Figura 4.2. Representación esquemática de los clústeres $\left[\mathrm{M}_{3} \mathrm{~S}_{4} \mathrm{X}_{3}(\mathrm{edpp})_{3}\right]^{+}(\mathrm{M}=\mathrm{Mo}, \mathrm{W} ; \mathrm{X}$ $=\mathrm{Cl}, \mathrm{Br})$.

En esta tesis doctoral hemos extendido la química de los clústeres $\mathrm{M}_{3} \mathrm{~S}_{4}(\mathrm{M}$ $=\mathrm{Mo}, \mathrm{W})$ al ligando aminofosfina ediprp (2-(aminoetil)di-i-propilfosfina)), con el fin de investigar su actividad catalítica, un campo poco explorado hasta la fecha. A 
continuación se describen de forma detallada la síntesis y caracterización de nuevos complejos clúster, su reactividad y su papel en la reducción catalítica de 2,2,2trifluoroacetofenona. 


\subsection{SÍNTESIS, CARACTERIZACIÓN Y ESTUDIOS EN FASE GAS}

La escisión de fases sólidas poliméricas de fórmula $\left\{\mathrm{M}_{3} \mathrm{~S}_{7} \mathrm{X}_{4}\right\}_{\mathrm{n}}(\mathrm{M}=\mathrm{Mo}, \mathrm{W} ; \mathrm{X}=\mathrm{Cl}$, $\mathrm{Br})$ resulta una excelente vía de síntesis de clústeres cuboidales $\left[\mathrm{M}_{3} \mathrm{~S}_{4} \mathrm{X}_{3} \text { (difosfina) }\right]^{+}$ y $\left.\left[\mathrm{M}_{3} \mathrm{~S}_{4} \mathrm{X}_{3} \text { (aminofosfina) }\right)_{3}\right]^{+}$en rendimientos elevados. ${ }^{[21,22]}$ Las unidades triangulares $\mathrm{M}_{3}\left(\mu_{3}-\mathrm{S}\right)\left(\mu-\mathrm{S}_{2}\right)_{3}$ de estas fases sólidas se encuentran conectadas entre ellas por cuatro puentes halogenuro formando una estructura unidimensional en zigzag, tal como se muestra en la figura 4.3. Durante el proceso de escisión de las fases poliméricas $\left\{\mathrm{M}_{3} \mathrm{~S}_{7} \mathrm{X}_{4}\right\}_{\mathrm{n}}$, tiene lugar la ruptura de los puentes halogenuro y la reducción de los puentes disulfuro $\mu-S_{2}$ a puentes sulfuro $\mu-S$ en un solo paso, con la consiguiente modificación estructural de la unidad $\mathrm{M}_{3}\left(\mu_{3}-\mathrm{S}\right)\left(\mu-\mathrm{S}_{2}\right)_{3}$ para dar lugar a la unidad $\mathrm{M}_{3}\left(\mu_{3}-\right.$ $S)(\mu-S)_{3}$. La fuerza motriz de esta reacción reside en la capacidad de los ligandos fosfina para reducir los puentes disulfuro y coordinarse a la unidad clúster molecular $\mathrm{M}_{3} \mathrm{~S}_{4}$. Esta ruta sintética, publicada por Cotton y colaboradores en el año 1989 y denominada también reducción dimensional,[23] sigue vigente hoy en día como una ruta eficiente para la obtención de clústeres de fórmula $\left.\left[\mathrm{M}_{3} \mathrm{~S}_{4} \mathrm{X}_{3} \text { (quelato) }\right]_{3}\right]^{+}(\mathrm{M}=\mathrm{Mo}$, $\mathrm{W} ; \mathrm{X}=\mathrm{Cl}, \mathrm{Br}$; quelato = difosfina, aminofosfina) en un solo paso.

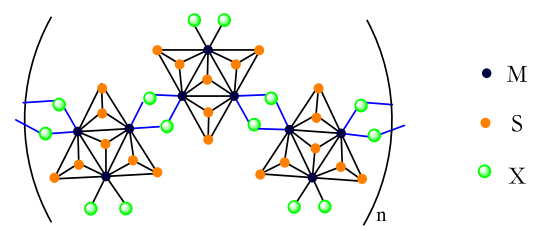

Figura 4.3. Representación esquemática de la fase polimérica $\left\{\mathrm{M}_{3} \mathrm{~S}_{7} \mathrm{X}_{4}\right\}_{\mathrm{n}}(\mathrm{M}=\mathrm{Mo}, \mathrm{W} ; \mathrm{X}=$ $\mathrm{Cl}, \mathrm{Br})$.

Además de las fases sólidas poliméricas, el complejo clúster molecular de fórmula $\left[\mathrm{Mo}_{3} \mathrm{~S}_{7} \mathrm{X}_{6}\right]^{2-}(\mathrm{X}=\mathrm{Cl}, \mathrm{Br})$ es un excelente precursor para la obtención de clústeres $\left.\left[\mathrm{Mo}_{3} \mathrm{~S}_{4} \mathrm{X}_{3} \text { (quelato) }\right]_{3}\right]^{+}$(quelato $=$difosfina, aminofosfina). ${ }^{[15,24]} \mathrm{Al}$ igual que en la reacción de escisión, la fuerza motriz de este proceso se atribuye a la reducción 
de los puentes disulfuro a la vez que el ligando quelato ocupa las posiciones de coordinación externas del metal. Por otra parte, la síntesis de clústeres de fórmula $\left.\left[\mathrm{Mo}_{3} \mathrm{~S}_{4} \mathrm{X}_{3} \text { (quelato) }\right]_{3}\right]^{+}$puede llevarse también a cabo en dos etapas con el fin de disminuir el aporte de ligando difosfina o aminofosfina. El primer paso consiste en la obtención del clúster $\left[\mathrm{Mo}_{3} \mathrm{~S}_{4} \mathrm{Cl}_{4}\left(\mathrm{PPh}_{3}\right)_{3}\left(\mathrm{H}_{2} \mathrm{O}\right)_{2}\right]$, que se utiliza como precursor de otros complejos derivados de la unidad $\mathrm{Mo}_{3} \mathrm{~S}_{4}$ (ver figura 4.4).[25] Esta ruta está limitada a los clústeres de molibdeno, ya que el precursor molecular de wolframio de fórmula $\left[\mathrm{W}_{3} \mathrm{~S}_{7} \mathrm{Br}_{6}\right]^{2-}$ no se ha podido sintetizar hasta la fecha.

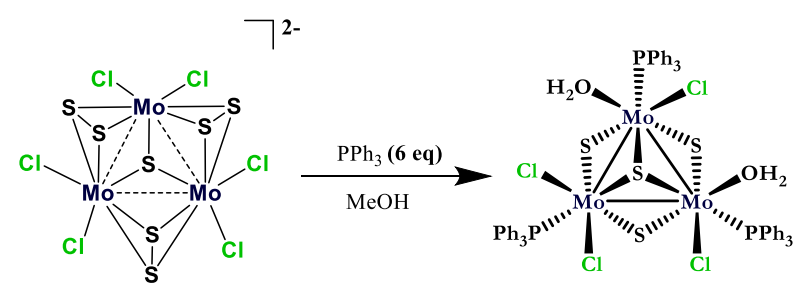

Figura 4.4. Esquema de la síntesis de $\left[\mathrm{Mo}_{3} \mathrm{~S}_{4} \mathrm{Cl}_{4}\left(\mathrm{PPh}_{3}\right)_{3}\left(\mathrm{H}_{2} \mathrm{O}\right)_{2}\right]$.

El complejo $\left[\mathrm{Mo}_{3} \mathrm{~S}_{4} \mathrm{Cl}_{3}(\text { ediprp) })_{3}\right]^{+}$se ha preparado a partir de una disolución del clúster neutro $\left[\mathrm{Mo}_{3} \mathrm{~S}_{4} \mathrm{Cl}_{4}\left(\mathrm{PPh}_{3}\right)_{3}\left(\mathrm{H}_{2} \mathrm{O}\right)_{2}\right]$ en etanol que se hace reaccionar con 3 equivalentes de ediprp según el esquema de reacción que se muestra en la figura 4.5. La reacción transcurre con un cambio de coloración de verde oscuro a verde claro y tras 3 horas a temperatura ambiente, la mezcla se ha concentrado hasta $1 / 4$ de su volumen inicial y el producto se ha precipitado con pentano, se ha centrifugado y se ha separado por decantación. Para completar el proceso de purificación, el sólido verde obtenido se ha disuelto en metanol y se ha precipitado añadiendo un exceso de $\mathrm{NaBPh}_{4}$, para dar $\left[\mathrm{Mo}_{3} \mathrm{~S}_{4} \mathrm{Cl}_{3}(\text { ediprp })_{3}\right]\left(\mathrm{BPh}_{4}\right)\left(\mathbf{5}\left(\mathrm{BPh}_{4}\right)\right)$ con un rendimiento del $85 \%$.

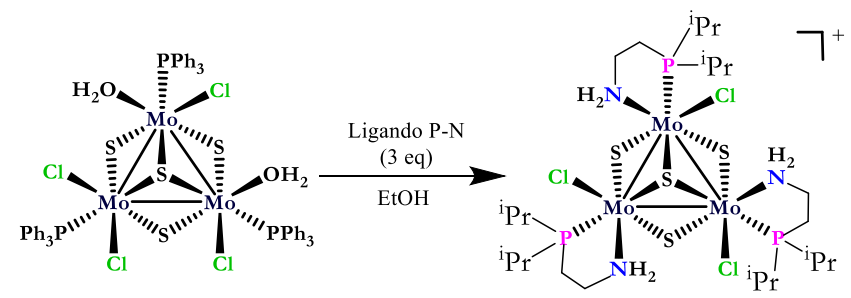

Figura 4.5. Esquema de la síntesis del clúster $\left[\mathrm{Mo}_{3} \mathrm{~S}_{4} \mathrm{Cl}_{3}(\text { ediprp })_{3}\right]\left(5^{+}\right)$. 
El clúster análogo de wolframio de fórmula $\left[\mathrm{W}_{3} \mathrm{~S}_{4} \mathrm{Br}_{3}(\text { ediprp })_{3}\right]^{+}\left(\mathbf{6}^{+}\right)$se ha preparado mediante el procedimiento de escisión que se muestra en la figura 4.6, i.e. a partir de fases sólidas poliméricas. La fase sólida $\left\{\mathrm{W}_{3} \mathrm{~S}_{7} \mathrm{Br}_{4}\right\}_{n}$ se ha suspendido en acetonitrilo y se ha añadido un exceso de aminofosfina ediprp. La mezcla de reacción se lleva a reflujo durante 24 horas con el consiguiente cambio de color de naranja a azul oscuro, en presencia de 3,5 equivalentes de ácido bromhídrico 0,5 $\mathrm{M}$ en acetonitrilo. La adición de ácidos hidrácidos asegura la formación de la especie halogenada, según se ha descrito en la bibliografía para clústeres trinucleares de molibdeno y wolframio funcionalizados con aminofosfinas.[15,21,22,26]

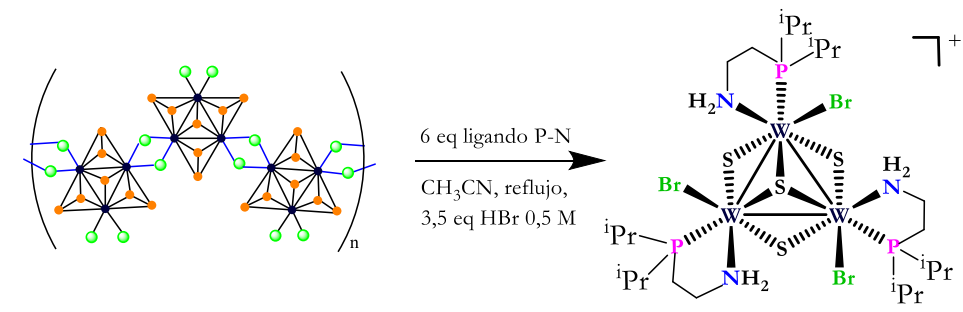

Figura 4.6. Esquema de la síntesis del complejo $\left[\mathrm{W}_{3} \mathrm{~S}_{4} \mathrm{Br}_{3}\left(\text { ediprp }_{3}\right]^{+}\left(\mathbf{6}^{+}\right)\right.$.

El compuesto $\mathbf{6}\left(\mathrm{BPh}_{4}\right)$ se ha obtenido de forma analíticamente pura con un rendimiento del 60,1 \% tras filtrar la mezcla de reacción, concentrar a vacío, redisolver en metanol, precipitar con $\mathrm{NaBPh}_{4}$ y lavar el producto con metanol y éter dietílico. La precipitación con $\mathrm{NaBPh}_{4}$ y posterior lavado han permitido eliminar los restos de aminofosfina protonada detectada por RMN de fósforo en el crudo de reacción, cuya formación se atribuye a la adición de $\mathrm{HBr}$.

Por otra parte, el clúster neutro $\left[\mathrm{W}_{3} \mathrm{~S}_{4} \mathrm{Br}_{4}\left(\mathrm{PPh}_{3}\right)_{3}\left(\mathrm{H}_{2} \mathrm{O}\right)_{2}\right]$ constituye un buen precursor para la obtención de clústeres $\left.\left[\mathrm{W}_{3} \mathrm{~S}_{4} \mathrm{Br}_{3} \text { (difosfina) }\right)_{3}\right]^{+}$.[24] La preparación del complejo $\left[\mathrm{W}_{3} \mathrm{~S}_{4} \mathrm{Br}_{4}\left(\mathrm{PPh}_{3}\right)_{3}\left(\mathrm{H}_{2} \mathrm{O}\right)_{2}\right]$ tiene lugar mediante la escisión de la fase sólida $\left\{\mathrm{W}_{3} \mathrm{~S}_{7} \mathrm{Br}_{4}\right\}_{\mathrm{n}}$ con trifenilfosfina, ya que no existe el clúster molecular de fórmula $\left[\mathrm{W}_{3} \mathrm{~S}_{7} \mathrm{Br}_{6}\right]^{2-}$. El clúster $\mathbf{6}^{+}$también se ha preparado a partir del precursor $\left[\mathrm{W}_{3} \mathrm{~S}_{4} \mathrm{Br}_{4}\left(\mathrm{PPh}_{3}\right)_{3}\left(\mathrm{H}_{2} \mathrm{O}\right)_{2}\right]$ disuelto en etanol en presencia de 3,2 equivalentes de ediprp 
y 3,5 equivalentes de una disolución de $\mathrm{HBr}$ 0,5 M en etanol. Tras 3 horas de reacción, la mezcla se ha concentrado hasta $1 / 4$ de su volumen inicial, el producto se ha precipitado con pentano y se ha identificado como $\mathbf{6}^{+}$con un rendimiento del $76 \%$.

Los clústeres $5^{+}$y $\mathbf{6}^{+}$se han caracterizado mediante RMN de fósforo, espectrometría de masas y difracción de rayos $\mathrm{X}$ en monocristal. Los espectros de RMN de ${ }^{31} \mathrm{P}\left\{{ }^{1} \mathrm{H}\right\}$ de los clústeres $\mathbf{5}\left(\mathrm{BPh}_{4}\right)$ y $\mathbf{6}\left(\mathrm{BPh}_{4}\right)$ (ver figura 4.7$)$ muestran una única señal de multiplicidad singulete a 53,45 ppm y 24,33 ppm, respectivamente. En el caso del complejo $\mathbf{6}\left(\mathrm{BPh}_{4}\right)$ se observan además dos señales satélite a 23,60 y 25,06 ppm originadas por el acoplamiento entre los núcleos de ${ }^{31} \mathrm{P}$ y $183 \mathrm{~W}$, con ${ }^{1} \mathrm{JP}$-W de 88,2 Hz. La relación de intensidades entre estas señales satélite y la señal mayoritaria es de 1:15:1 aproximadamente, debido a la abundancia natural del isótopo ${ }^{183} \mathrm{~W}(14,3 \%)$. Estos resultados son coherentes con la presencia de tres átomos de fósforo químicamente equivalentes para cada complejo clúster y también con los espectros de RMN de clústeres de fórmula $\left[\mathrm{M}_{3} \mathrm{~S}_{4} \mathrm{X}_{3}(\mathrm{edpp})_{3}\right](\mathrm{M}=\mathrm{Mo}$, W; $\mathrm{X}=\mathrm{Cl}, \mathrm{Br} ; \mathrm{H}) .[20,21]$ Esto sugiere que los tres ligandos aminofosfina se coordinan de forma equivalente a los tres metales de la unidad $\mathrm{M}_{3} \mathrm{~S}_{4}$, lo que resulta en un único isómero de todos los posibles.
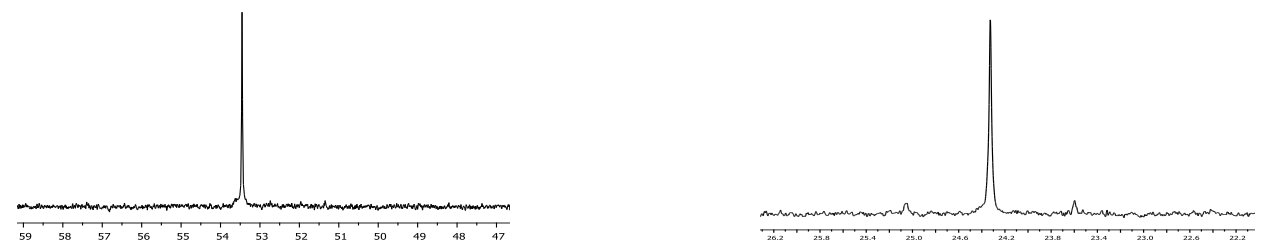

Figura 4.7. Espectros de $\mathrm{RMN}$ de ${ }^{31} \mathrm{P}\left\{{ }^{1} \mathrm{H}\right\}$ de los complejos $\mathbf{5}\left(\mathrm{BPh}_{4}\right)$ (izquierda) y $\mathbf{6}\left(\mathrm{BPh}_{4}\right)$ (derecha).

La caracterización de $5^{+}$y $\mathbf{6}^{+}$se ha completado mediante espectrometría de masas utilizando como fuente de ionización electrospray (ESI-MS). En el espectro del complejo $\mathbf{5}^{+}$bajo condiciones suaves $\left(\mathrm{U}_{\mathrm{c}}=20 \mathrm{~V}\right)$ se observa un único pico a 1006 u.m.a. que se corresponde con el ion molecular $\left[\mathrm{Mo}_{3} \mathrm{~S}_{4} \mathrm{Cl}_{3}(\text { ediprp) }]_{3}\right]^{+}$. Por otra parte, 
en las mismas condiciones se observa un único pico a 1403 u.m.a. en el espectro del clúster $\mathbf{6}^{+}$que se asigna al ion molecular $\left[\mathrm{W}_{3} \mathrm{~S}_{4} \mathrm{Br}_{3}(\text { ediprp) }]_{3}\right]^{+}$. Ambos picos han sido asignados por comparación de su distribución isotópica experimental con la distribución isotópica simulada tal como se detalla en la figura 4.8.
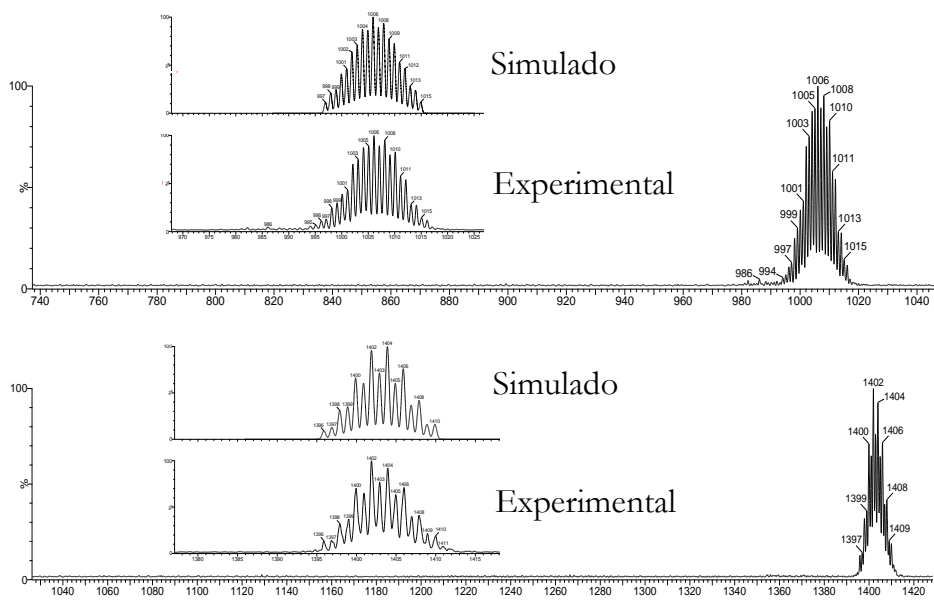

Figura 4.8. Espectros de masas simulados y experimentales a $\mathrm{U}_{\mathrm{c}}=20 \mathrm{~V}$ de los clústeres $\mathbf{5}^{+}$ (arriba) y $\mathbf{6}^{+}$(abajo).

Con el fin de profundizar el comportamiento de estos clústeres en fase gas, hemos registrado los espectros de masas de $\mathbf{5}^{+}$y $\mathbf{6}^{+}$a mayores voltajes de cono y también hemos llevado a cabo estudios de disociación inducida por colisión (CID). La fragmentación del clúster $5^{+}$a diferentes voltajes de cono $\left(\mathrm{U}_{\mathrm{c}}=20-70 \mathrm{~V}\right)$ a partir de muestras disueltas acetonitrilo se muestra en la figura 4.9. Observamos pérdidas sucesivas de moléculas de $\mathrm{HCl}$ formadas por el ligando cloruro y uno de los átomos de hidrógeno enlazados a una de las aminas, con la consecuente formación de grupos $\mathrm{Mo}=\mathrm{NHR}$ en función del voltaje de cono aplicado. A partir de $50 \mathrm{~V}$ aparece un pico minoritario centrado en 970 u.m.a. que se asigna a la especie [M-HCl]+..15] Las especies $\left[\mathrm{M}-(\mathrm{HCl})_{2}\right]^{+}(\mathrm{m} / \mathrm{z}, 934)$ y $\left[\mathrm{M}-(\mathrm{HCl})_{3}\right]^{+}(\mathrm{m} / \mathrm{z}, 896)$ aparecen a partir de $60 \mathrm{~V}$. La pérdida secuencial de moléculas de $\mathrm{HX}$ es el resultado de interacciones intramoleculares entre el ligando haluro externo y uno de los átomos de hidrógeno 
del grupo amino.[15] En ningún momento se observan pérdidas de ligandos aminofosfina.

$70 \mathrm{~V}$

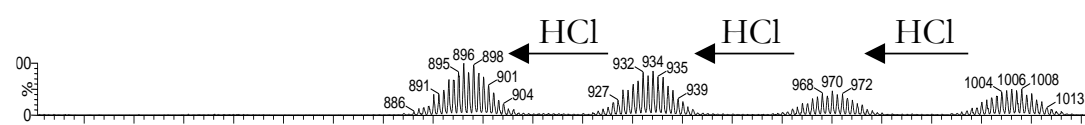

$60 \mathrm{~V}$

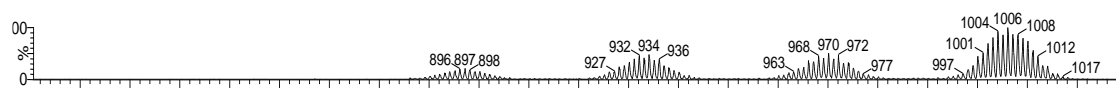

$50 \mathrm{~V}$

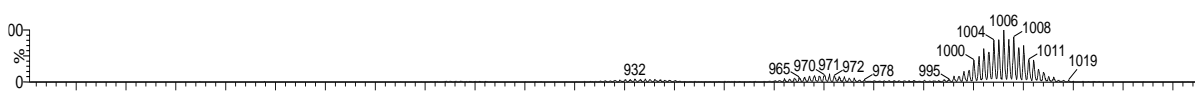

$40 \mathrm{~V}$

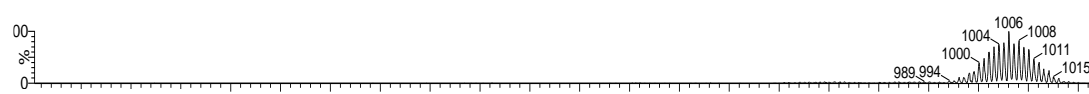

$30 \mathrm{~V}$

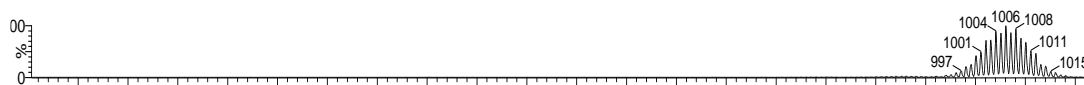

$20 \mathrm{~V}$

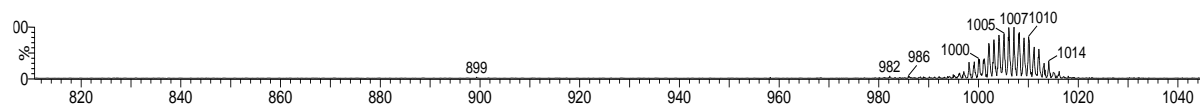

Figura 4.9. Espectros de masas del complejo $\mathbf{5}^{+}$y asignación de picos a voltajes de cono de 20 a $70 \mathrm{~V}$.

Los resultados de fragmentación del complejo $\mathbf{5}^{+}$siguen la tendencia observada para los clústeres análogos funcionalizados con aminofosfinas y contrastan con los descritos para los clústeres que contienen difosfinas, donde se observan pérdidas de hasta dos ligandos difosfina. ${ }^{[15,27]}$ En el caso del cluster $\mathbf{6}^{+}$se reproducen los resultados del clúster $5^{+}$, con pérdidas sucesivas de $\mathrm{HBr}$ a partir de $40 \mathrm{~V}$ que dan lugar a especies $[\mathrm{M}-\mathrm{HBr}]^{+}(m /$ z 1321$),[\mathrm{M}-(\mathrm{HBr}) 2]^{+}(m / z, 1239)$ y $[\mathrm{M}-(\mathrm{HBr}) 3]^{+}(m /$ z 1160).

Los experimentos de disociación inducida por colisión (CID) se han llevado a cabo a energías de colisión ( $\mathrm{E}_{\text {lab }}$ ) de 40 y $60 \mathrm{~V}$ y un voltaje de cono de $20 \mathrm{~V}$, mediante la selección y posterior fragmentación de los picos moleculares $\mathbf{5}^{+}$y $\mathbf{6}^{+}$. Estos experimentos requieren de tres analizadores en el espectrómetro de masas. En el primer analizador se selecciona el pico del catión correspondiente, a continuación se 
hace interaccionar el catión con argón en la celda de colisión para inducir su fragmentación y finalmente en el tercer analizador se detectan los productos de fragmentación.

En el caso del clúster $\mathbf{5}^{+}$, los estudios mediante CID a Elab $=40 \mathrm{~V}$ muestran pérdidas sucesivas de $\mathrm{HCl}$, así como un pico minoritario a 773 u.m.a que se asocia a pérdidas simultáneas de un ligando aminofosfina desprotonado (ediprp-H) y dos moléculas de $\mathrm{HCl}$ (ver figura 4.10). A $E_{\text {lab }}=60 \mathrm{~V}$ la especie insaturada $\left[\mathrm{M}-(\mathrm{HCl})_{3}\right]^{+}$ domina el espectro. En el clúster $\mathbf{6}^{+}$, la selección y posterior fragmentación del pico molecular a $\mathrm{E}_{\mathrm{lab}}=40 \mathrm{~V}$ proporciona una pérdida sucesiva de moléculas de $\mathrm{HBr}$ dando lugar a las especies $[\mathrm{M}-\mathrm{HBr}]^{+}(\mathrm{m} / \mathrm{z}, 1321)$, $\left[\mathrm{M}-(\mathrm{HBr})_{2}\right]^{+}(\mathrm{m} / \mathrm{z}, 1239)$ y $[\mathrm{M}-$ $\left.(\mathrm{HBr})_{3}\right]^{+}(m / z, 1160)$. A E $E_{\text {lab }}=60 \mathrm{~V}$ la especie insaturada $\left[\mathrm{M}-(\mathrm{HBr})_{3}\right]^{+}$domina el espectro. En este caso no se observan pérdidas de ligando aminofosfina.

Los resultados de los experimentos CID sugieren que la aminofosfina ediprp se comporta de manera similar a la edpp estudiada previamente por Llusar y colaboradores, y de manera diferente a las difosfinas coordinadas a unidades $\mathrm{M}_{3} \mathrm{~S}_{4}$. Los mayores voltajes de cono y energías de colisión necesarios para la fragmentación de $\mathbf{5}^{+}$y $\mathbf{6}^{+}$sugieren una menor labilidad del ligando ediprp frente a edpp. Los patrones de fragmentación observados para $5^{+}$y $\mathbf{6}^{+}$contrastan con la idea del carácter hemilábil de los ligandos aminofosfina y apoyan la formación de especies insaturadas $\mathrm{M}=\mathrm{NHR}$. 

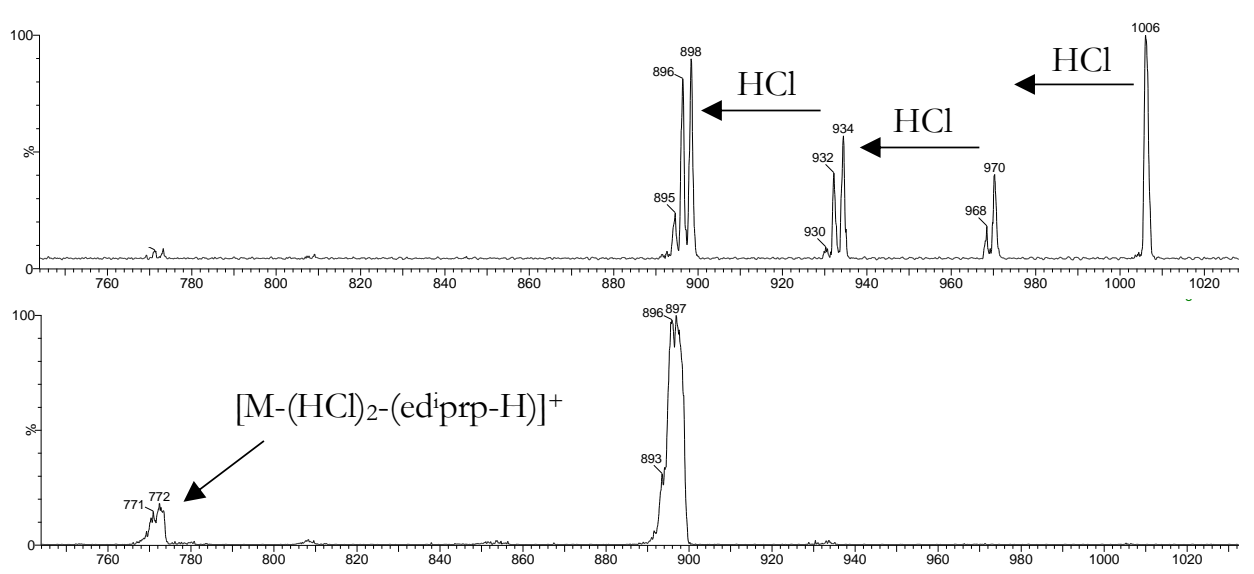

Figura 4.10. Espectro de masas del experimento de fragmentación CID a $\mathrm{E}_{\mathrm{lab}}=40 \mathrm{~V}$ (arriba) y $\mathrm{E}_{\mathrm{lab}}=60 \mathrm{~V}$ (abajo) para el complejo $\mathbf{5}^{+}$.

Las estructuras cristalinas de las sales de los cationes $\mathbf{5}^{+}$y $\mathbf{6}^{+}$se han determinado por difracción de rayos $\mathrm{X}$ en monocristal. Cristales aptos para dicho estudio se han obtenido mediante la difusión lenta de pentano sobre disoluciones de $\mathbf{5}(\mathrm{Cl})$ y $\mathbf{6}\left(\mathrm{BPh}_{4}\right)$ en diclorometano. El complejo catiónico $\mathbf{5}^{+}$cristaliza como una sal de cloruro en el grupo espacial P-1, el cual no impone ninguna restricción por simetría a excepción del centro de inversión. La estructura presenta dos unidades clúster cristalográficamente independientes y dos contraiones, así como una molécula de diclorometano de cristalización. Uno de los contraiones se encuentra desordenado en dos posiciones con ocupaciones relativas de 0,58 y 0,42 , de forma que la carga del complejo se encuentra balanceada. Además, uno de los carbonos pertenecientes al ligando isopropilo se encuentra desordenado en dos posiciones $(\mathrm{C}(44)$ y $\mathrm{C}(45))$ con ocupaciones relativas del 50 \%. Los átomos de hidrógeno se incluyen en posiciones idealizadas y se refinan como átomos "jinete", con un parámetro térmico de desplazamiento proporcional al átomo al que se encuentra enlazado (1,2 cuando están unidos a un carbono secundario o aromático; 1,5 cuando están unidos a un carbono primario o un oxígeno), excepto los enlazados a los carbonos $C(44)$ y $C(45)$, que no se han incluido en el modelo. 
Por otra parte, el complejo $\mathbf{6}^{+}$cristaliza como una sal de tetrafenilborato en el grupo espacial monoclínico $\mathrm{P} 2{ }_{1} / \mathrm{c}$. En este caso también se observa la presencia de dos unidades clúster cristalográficamente independientes, así como de dos contraiones. El último mapa de diferencia de Fourier muestra una serie de picos cuyas intensidades oscilan entre 3,05 y 1,60 y que se encuentran demasiado cerca de los átomos de wolframio para ser incluidos en el modelo cristalográfico. La figura 4.11 muestra la representación ORTEP de los clústeres $\mathbf{5}^{+}$y $\mathbf{6}^{+}$. En la figura se incluye únicamente una de las unidades cristalográficamente independientes.

La unidad clúster en estos complejos trinucleares presenta una disposición análoga a la observada en otros complejos con unidad $\mathrm{M}_{3} \mathrm{~S}_{4}(\mathrm{M}=\mathrm{Mo}$, W).[12,22] Los metales forman un triángulo casi equilátero, con un azufre apuntado $\mu_{3}-\mathrm{S}$ que queda por encima del plano generado por los metales y tres azufres puente $\mu$-S que se sitúan por debajo de este plano. Esta disposición resulta en una estructura de tipo cubano incompleto. Cada metal presenta un entorno de coordinación pseudooctaédrico, sin considerar los enlaces metal-metal, definido por tres átomos de azufre, un átomo de fósforo, otro de nitrógeno y el halógeno correspondiente. 

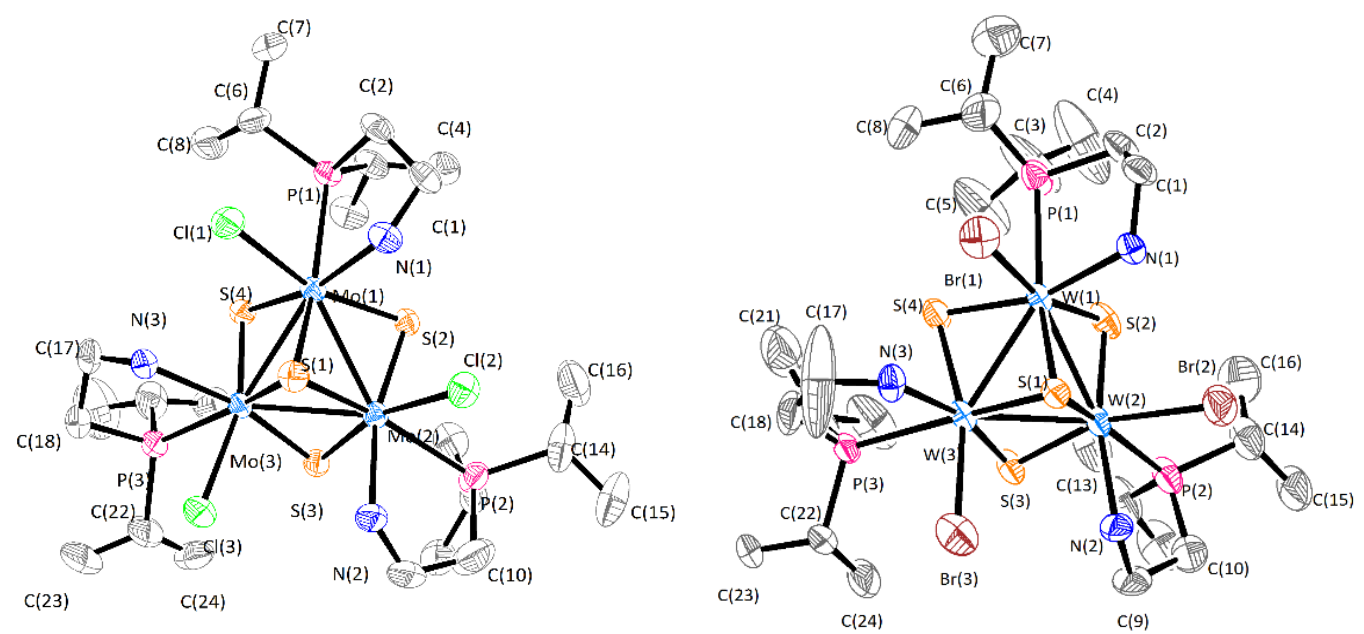

Figura 4.11. Representación ORTEP de uno de los clústeres cristalográficamente independientes de las estructuras de $\left[\mathrm{Mo}_{3} \mathrm{~S}_{4} \mathrm{Cl}_{3}(\text { ediprp) }]_{3}\right]^{+}\left(\mathbf{5}^{+}\right)$y $\left[\mathrm{W}_{3} \mathrm{~S}_{4} \mathrm{Br}_{3}(\text { ediprp })_{3}\right]^{+}\left(\mathbf{6}^{+}\right)$ (elipsoides al 50\% de probabilidad). Los átomos de hidrógeno, el disolvente y el contraión han sido omitidos para mayor claridad.

Al igual que ocurre en otros clústeres análogos derivados con aminofosfinas, el átomo de fósforo se encuentra en posición trans al azufre apuntado $\mu_{3}-\mathrm{S}$, mientras que el de nitrógeno se encuentra en posición trans a los azufres puente $\mu-S .{ }^{[15,21]}$ Esta disposición se observa con mayor claridad en la figura 4.12, donde se aprecia que el átomo de cloro terminal del complejo $\mathbf{5}^{+}$y el nitrógeno de la aminofosfina se encuentran en el mismo lado respecto al plano trimetálico.

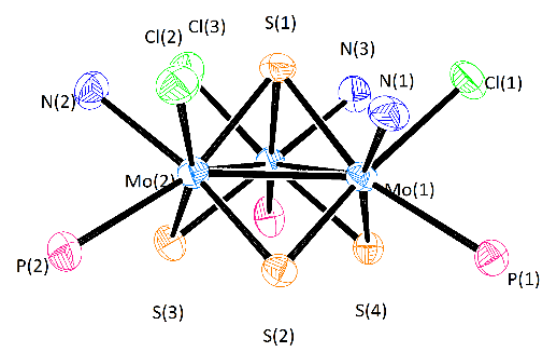

Figura 4.12. Representación lateral del clúster $\mathbf{5}^{+}$. Los átomos de carbono se han omitido para una mayor claridad. 
En la tabla 4.1 se listan, con fines comparativos, las distancias de enlace promedio más relevantes para los compuestos $\mathbf{5}(\mathrm{Cl})$ y $\mathbf{6}\left(\mathrm{BPh}_{4}\right)$ junto a las de los clústeres

$$
\left[\mathrm{Mo}_{3} \mathrm{~S}_{4} \mathrm{Cl}_{3}(\mathrm{edpp})_{3}\right]\left(\mathrm{BPh}_{4}\right),{ }^{[15]}
$$

$\left[\mathrm{Mo}_{3} \mathrm{~S}_{4} \mathrm{Cl}_{3}(\mathrm{dmpe})_{3}\right]\left(\mathrm{PF}_{6}\right),{ }^{[28]}$ $\left[\mathrm{W}_{3} \mathrm{~S}_{4} \mathrm{Br}_{3}(\mathrm{edpp})_{3}\right]\left(\mathrm{BPh}_{4}\right),{ }^{[21]}$ y $\left[\mathrm{W}_{3} \mathrm{~S}_{4} \mathrm{Br}_{3}(\mathrm{dmpe})_{3}\right]\left(\mathrm{PF}_{6}\right){ }^{[29]}$ Las distancias metal-metal son similares a las observadas en complejos análogos funcionalizados tanto con difosfinas como con aminofosfinas y concuerdan con un estado de oxidación IV del metal. Las distancias $\mathrm{M}-\left(\mu_{3}-\mathrm{S}\right)$ son significativamente mayores que las $\mathrm{M}-(\mu-\mathrm{S})$, siguiendo la tendencia observada en el resto de clústeres estructuralmente similares. Las distancias M- $(\mu-S)$ son semejantes entre ellas, tal como ocurre en los complejos $\left[\mathrm{Mo}_{3} \mathrm{~S}_{4} \mathrm{Cl}_{3}(\mathrm{edpp})_{3}\right]\left(\mathrm{BPh}_{4}\right)$ y $\left[\mathrm{W}_{3} \mathrm{~S}_{4} \mathrm{Br}_{3}(\mathrm{edpp})_{3}\right]\left(\mathrm{BPh}_{4}\right) \cdot\left[{ }^{[15,21]}\right.$ Este hecho difiere del observado en los clústeres funcionalizados con difosfinas. En estos últimos, la mayor influencia trans de las fosfinas frente a los ligandos cloruro y bromuro provoca que la distancia $\mathrm{M}-(\mu-\mathrm{S})$ en posición trans al enlace metal-fósforo sea significativamente mayor que la distancia $\mathrm{M}-(\mu-\mathrm{S})$ trans al enlace metal-halógeno. En cambio, en los clústeres funcionalizados con aminofosfinas estas distancias se acercan hasta casi igualarse debido a que los ligandos amina, cloruro y bromuro presentan una influencia trans muy similar. Las distancias Mo-P de los clústeres $\mathbf{5}(\mathrm{Cl})$ y $\mathbf{6}\left(\mathrm{BPh}_{4}\right)$ son aproximadamente $0,035 \AA$ más largas que en los análogos de edpp y esta diferencia asciende hasta casi $0,050 \AA$ si los comparamos con los clústeres funcionalizados con dmpe de la tabla 4.1. Este hecho se puede atribuir al mayor impedimento estérico de los grupos isopropilo de la aminofosfina ediprp frente a los grupos fenilo de la edpp y los metilos de la dmpe. Las distancias M-N de 2,255[7] en $\mathbf{5}^{+}$y 2,263[13] en $\mathbf{6}^{+}$son coherentes con la presencia de un enlace sencillo metal-nitrógeno.[15] 
Tabla 4.1. Selección de distancias de enlace promedio para los compuestos 5(Cl), $\mathbf{6}\left(\mathrm{BPh}_{4}\right), \quad\left[\mathrm{Mo}_{3} \mathrm{~S}_{4} \mathrm{Cl}_{3}\left(\mathrm{edpp}_{3}\right]\left(\mathrm{BPh}_{4}\right)\right.$, $\left[\mathrm{Mo}_{3} \mathrm{~S}_{4} \mathrm{Cl}_{3}(\mathrm{dmpe})_{3}\right]\left(\mathrm{PF}_{6}\right), \quad\left[\mathrm{W}_{3} \mathrm{~S}_{4} \mathrm{Br}_{3}(\mathrm{edpp})_{3}\right]\left(\mathrm{BPh}_{4}\right)$, y $\left[\mathrm{W}_{3} \mathrm{~S}_{4} \mathrm{Br}_{3}(\mathrm{dmpe})_{3}\right]\left(\mathrm{PF}_{6}\right)$. La desviación estándar de los valores promedio se encuentra representada entre corchetes.

\begin{tabular}{ccccccc}
\hline \hline $\begin{array}{c}\text { Distancia } \\
(\AA)\end{array}$ & $\mathbf{5}^{+}$ & {$\left[\mathbf{M o}_{3} \mathbf{S}_{4} \mathbf{C l}_{3}(\mathbf{e d p p})_{3}\right]^{+(\mathbf{a})}$} & {$\left[\mathbf{M o}_{3} \mathbf{S}_{4} \mathbf{C l}_{3}(\mathbf{d m p e})_{3}\right]^{+(\mathbf{b})}$} & $\mathbf{6}^{+}$ & {$\left[\mathbf{W}_{3} \mathbf{S}_{4} \mathbf{B r}_{3}(\mathbf{e d p p})_{3}\right]^{+(\mathbf{c})}$} & {$\left[\mathbf{W}_{3} \mathbf{S}_{4} \mathbf{B r}_{3}(\mathbf{d m p e})_{3}\right]^{+(\mathbf{d})}$} \\
\hline $\mathrm{M}-\mathrm{M}$ & $2,760[6]$ & $2,7463[7]$ & $2,766(4)$ & $2,755[7]$ & $2,7520[3]$ & $2,759(2)$ \\
$\mathrm{M}-\left(\mu \mu_{3}-\mathrm{S}\right)$ & $2,347[4]$ & $2,3611[13]$ & $2,360(9)$ & $2,357[7]$ & $2,3450[16]$ & $2,37(1)$ \\
$\mathrm{M}-(\mu-\mathrm{S})^{(\mathrm{e})}$ & $2,296[6]$ & $2,2960[12]$ & $2,290(7)$ & $2,300[6]$ & $2,3117[15]$ & $2,30(1)$ \\
$\mathrm{M}-(\mu-\mathrm{S})^{(+)}$ & $2,300[5]$ & $2,2863[12]$ & $2,336(7)$ & $2,306[5]$ & $2,3091[15]$ & $2,33(1)$ \\
$\mathrm{M}-\mathrm{P}$ & $2,576[10]$ & $2,5414[14]$ & $2,534(8)$ & $2,564[7]$ & $2,5306[16]$ & $2,51(1)$ \\
$\mathrm{M}-\mathrm{N}$ & $2,255[7]$ & $2,2733[4]$ & - & $2,263[13]$ & $2,286[5]$ & - \\
$\mathrm{M}-\mathrm{X}$ & $2,510[12]$ & $2,4625[15]$ & $2,473(7)$ & $2,613[19]$ & $2,4947[16]$ & $2,641(4)$ \\
\hline \hline
\end{tabular}

(a)Datos extraídos de la referencia [15]. (b)Datos extraídos de la referencia [22]. (c)Datos extraídos de la referencia [21]. (d)Datos extraídos de la referencia [22]. ${ }^{(\mathrm{e})}$ Distancia trans al enlace $\mathrm{M}-\mathrm{X}(\mathrm{M}=\mathrm{Mo}, \mathrm{W}, \mathrm{X}=\mathrm{Cl}, \mathrm{Br}) .{ }^{\left({ }^{\mathrm{f}}\right.}$ Distancia trans al enlace $\mathrm{M}-\mathrm{N}$ (M-P para los clústeres funcionalizados con difosfinas).

() Desviación estándar de la medida.

[] Desviación estándar del promedio. 
Los halógenos y los grupos amino se encuentran coordinados hacia el mismo lado del plano que forman los tres metales, lo que permite múltiples interacciones $\mathrm{X} \cdots \mathrm{HN}(\mathrm{X}=\mathrm{Cl}, \mathrm{Br})$. Estas interacciones pueden ser vecinales, i.e. entre el halógeno coordinado a un metal y uno de los hidrógenos del grupo amino coordinado al metal adyacente, o geminales, que se dan cuando el halógeno y el grupo amino se encuentran coordinados al mismo átomo metálico.

En el caso del compuesto $5^{+}$se observan distancias vecinales $\mathrm{Cl} \cdots \mathrm{HN}$ que oscilan entre 2,386 $\AA$ y 2,480 $\AA$. Las distancias $\mathrm{Cl} \cdots \mathrm{HN}$ geminales son mayores que las vecinales y toman valores en el rango de 2,645-2,705 A. Ambas distancias son más cortas que las observadas en el clúster análogo $\left[\mathrm{Mo}_{3} \mathrm{~S}_{4} \mathrm{Cl}_{3}(\mathrm{edpp})_{3}\right]^{+} .{ }^{[15]}$ Las dos unidades cristalográficamente independientes del clúster $\mathbf{6}^{+}$presentan distancias vecinales $\mathrm{Br} \cdots \mathrm{HN}$ de entre 2,344 y 2,449 $\AA$.[21] Una de estas unidades muestra una distancia vecinal más larga, de 2,794 A. Análogamente, las distancias geminales toman valores en el rango de 2,677-2,778 $\AA$, excepto una de ellas que es de $2,924 \AA$.

Durante el estudio cristalográfico del compuesto $\mathbf{5}^{+}$, se ha identificado una nueva fase, etiquetada como $\mathbf{5} \mathbf{a}^{+}$, con una celda unidad monoclínica que presenta valores de $\mathrm{a}=13,7649(3) \AA, \mathrm{b}=20,8591$ (4) $\AA, \mathrm{c}=16,3323(4) \AA$ y difiere de la triclínica reportada anteriormente para el clúster $5(\mathrm{Cl})$, que presenta valores de a $=$ $12,9349(6) \AA, \mathrm{b}=15,9802(4) \AA \mathrm{y} \mathrm{c}=22,0560(7) \AA$. La estructura de esta fase monoclínica se ha resuelto con éxito en el grupo espacial $\mathrm{P} 2{ }_{1} / \mathrm{n}$ y aunque la unidad $\mathrm{Mo}_{3} \mathrm{~S}_{4}$ presenta una estructura cuboidal análoga a la familia de clústeres de fórmula $\left[\mathrm{M}_{3} \mathrm{~S}_{4} \mathrm{X}_{3} \text { (quelato) }\right]^{+}(\mathrm{M}=\mathrm{Mo}, \mathrm{W} ; \mathrm{X}=\mathrm{Cl}, \mathrm{Br}$; quelato $=$ difosfina, aminofosfina, diamina) descritos hasta la fecha, ${ }^{[15,22,26,30,31]}$ la disposición de los ligandos externos es diferente. Esta nueva fase estructural carece de la simetría $C_{3}$ característica de estos compuestos, ya que los tres átomos metálicos no poseen el mismo entorno de coordinación. La figura 4.13 muestra un diagrama ORTEP de la estructura $5 \mathbf{a}(\mathrm{Cl})$. Los carbonos enlazados al $\mathrm{P}(1)$ presentan una elevada agitación térmica y por ello se han refinado de forma isotrópica. 


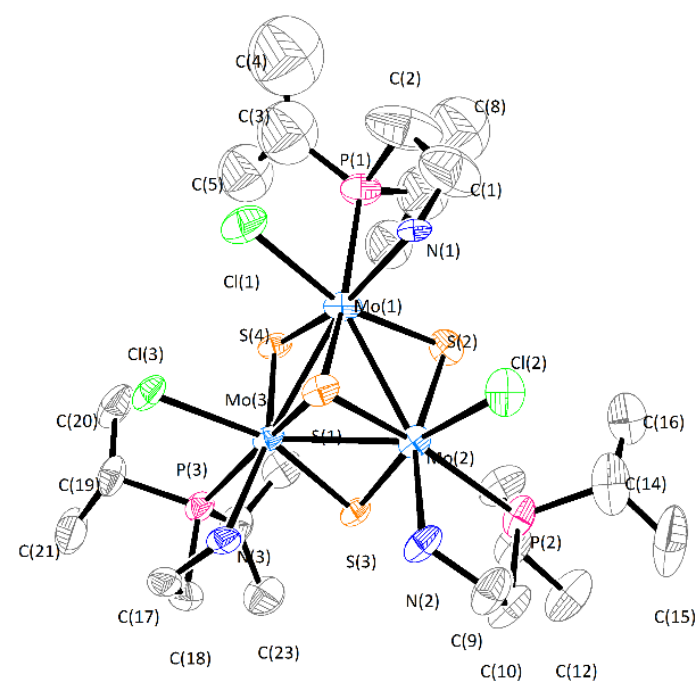

Figura 4.13. Diagrama ORTEP de la estructura del complejo $\mathbf{5} \mathbf{a}^{+}$. Los hidrógenos, el contraión y el disolvente se han omitido por claridad.

En la estructura $\mathbf{5 a ^ { + }}$, el átomo de nitrógeno $\mathrm{N}(3)$ y el de cloro $\mathrm{Cl}(3)$ intercambian sus posiciones respecto a la $\mathbf{5}^{+}$, de manera que los halógenos no están dispuestos alrededor de la unidad $\mathrm{Mo}_{3} \mathrm{~S}_{4}$ formando una hélice y en consecuencia se pierde la simetría $C_{3}$ del clúster. La tabla 4.2 lista con fines comparativos las distancias interatómicas más relevantes de esta estructura singular junto con las de $\mathbf{5}^{+}$. Las diferencias más significativas las encontramos en la longitud de enlace Mo-N, que en el caso de $\mathbf{5} \mathbf{a}^{+}$presenta valores de 2,288, 2,290 y 2,382 Å. El enlace más largo corresponde a la distancia entre $\mathrm{Mo}(1)$ y $\mathrm{N}(1)$.

Respecto a las distancias vecinales $\mathrm{Cl} \cdots \mathrm{HN}$, a diferencia de las tres interacciones observadas para la estructura $5^{+}$, existe un único contacto vecinal a destacar entre el $\mathrm{Cl}(2)$ y el $\mathrm{HN}(1)$, que presenta un valor de 2,419 ̊. Las distancias geminales $\mathrm{Cl} \cdots \mathrm{HN}$ oscilan entre 2,796 y $2,814 \AA$ y son mayores que la vecinal y que las observadas en $\mathbf{5}^{+}$. 
Tabla 4.2. Selección de distancias de enlace promedio para $\mathbf{5} \mathbf{a}^{+}$y $\mathbf{5}^{+}$. La desviación estándar de los valores promedio se encuentra representada entre corchetes.

\begin{tabular}{|c|c|c|}
\hline Distancia (§̊) & $5 a^{+}$ & $5^{+}$ \\
\hline Мo-Mo & $2,781[18]$ & $2,760[6]$ \\
\hline Mo- $\left(\mu_{3}-S\right)$ & $2,354[6]$ & $2,347[4]$ \\
\hline Mo- $(\mu-S)^{(a)}$ & $2,299[12]$ & $2,296[6]$ \\
\hline Mo- $(\mu-S)(b)$ & $2,296[3]$ & $2,300[5]$ \\
\hline Mo-P & $2,564[7]$ & $2,576[10]$ \\
\hline $\mathrm{Mo}-\mathrm{N}$ & $2,320[44]$ & $2,255[7]$ \\
\hline $\mathrm{Mo}-\mathrm{Cl}$ & $2,495[19]$ & $2,510[12]$ \\
\hline
\end{tabular}

(a) Distancia trans al enlace Mo-Cl. (b) Distancia trans al enlace Mo-N. [] Desviación estándar del promedio.

La cristalización de esta fase $\mathbf{5} \mathbf{a}^{+}$minoritaria, cuya presencia no evidencia la espectroscopia de $\mathrm{RMN}$ de ${ }^{31} \mathrm{P}\left\{{ }^{1} \mathrm{H}\right\}$, es un hecho singular cuyo hallazgo ha sido puramente fortuito. Cabe destacar que no hemos sido capaces de identificar esta fase en otras mezclas de reacción ni dirigir el procedimiento de síntesis hacia su formación. 


\subsection{ESTUDIOS DE REACTIVIDAD}

Los complejos de metales de transición funcionalizados con ligandos difosfina y aminofosfina juegan un papel importante en catálisis homogénea. ${ }^{[3,14,32]}$ En este contexto, los complejos hidruro juegan un papel protagonista, ya que son especies activas en diversos procesos de interés industrial como la hidrogenación de $\mathrm{CO}_{2}$ y la reducción de sustratos carbonílicos.[1,8,12] Por otra parte, los complejos hidroxo y alcoxo se postulan como intermedios en estas transformaciones catalíticas. ${ }^{33,34]}$

Los complejos metálicos funcionalizados con ligandos alcoxo pueden activar el $\mathrm{CO}_{2}$ mediante el mecanismo propuesto por Darensbourg y colaboradores, donde el dióxido de carbono se inserta en el enlace metal-oxígeno del complejo. Esto se debe a que el átomo de carbono interacciona con el par electrónico solitario del oxígeno para dar el estado de transición que se ilustra en la figura 4.14.[35]

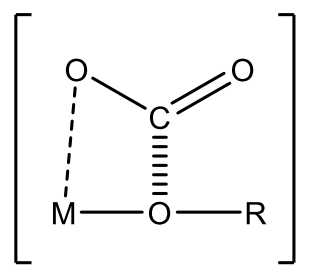

Figura 4.14. Estado de transición propuesto para la inserción del $\mathrm{CO}_{2}$ en el enlace M-OR por Darensbourg y colaboradores.

La existencia de complejos clúster hidruro, hidroxo y alcoxo es limitada en contraste con los numerosos complejos monometálicos descritos que contienen estos ligandos, por ello la obtención de clústeres de fórmula $\left.\left[\mathrm{M}_{3} \mathrm{~S}_{4} \mathrm{X}_{3} \text { (quelato) }\right)_{3}\right]^{+}(\mathrm{M}=\mathrm{Mo}$, $\mathrm{W} ; \mathrm{X}=\mathrm{H}, \mathrm{OH}, \mathrm{OR}$; quelato = difosfina, aminofosfina) resulta un reto en la química de los clústeres metálicos. A continuación se discuten los resultados derivados de la reactividad de los clústeres $\left[\mathrm{Mo}_{3} \mathrm{~S}_{4} \mathrm{Cl}_{3}(\text { ediprp) })_{3}\right]^{+}\left(5^{+}\right)$y $\left[\mathrm{W}_{3} \mathrm{~S}_{4} \mathrm{Br}_{3}(\text { ediprp })_{3}\right]^{+}\left(\mathbf{6}^{+}\right)$frente a sales de borohidruro y alcoholes dirigida a la formación de clústeres hidruro y alcoxo, respectivamente. 


\subsubsection{REACTIVIDAD FRENTE A BOROHIDRURO}

En la actualidad se conocen diversos ejemplos de clústeres hidruro de metales del grupo seis funcionalizados con ligandos difosfina. ${ }^{[22,36-38]}$ Los hidruros con unidad central $\mathrm{W}_{3} \mathrm{~S}_{4}$ son más estables que sus análogos de molibdeno. Esto se pone de manifiesto en la pronta publicación de la estructura cristalina del primer hidruro de wolframio de fórmula $\left[\mathrm{W}_{3} \mathrm{~S}_{4} \mathrm{H}_{3}(\mathrm{dmpe})_{3}\right]^{+}$, que tuvo lugar en $1989,{ }^{[36]}$ mientras que la del primer hidruro de molibdeno análogo no se publicó hasta el año 2010.[37] Estos clústeres se preparan en atmósfera inerte mediante la adición de una sal de borohidruro en exceso a una disolución del precursor halogenado en THF o metanol. Mediante esta vía de síntesis, el primer hidruro clúster de fórmula $\left[\mathrm{W}_{3} \mathrm{~S}_{4} \mathrm{H}_{3}(\text { aminofosfina })_{3}\right]^{+}$(aminofosfina $=$edpp) ha sido aislado recientemente por el grupo de Llusar. ${ }^{20]}$ En este trabajo hemos seguido el mismo procedimiento con el fin de sustituir los halógenos por hidruros para obtener complejos de fórmula $\left[\mathrm{M}_{3} \mathrm{~S}_{4} \mathrm{H}_{3}(\text { ediprp) }]_{3}\right]^{+}(\mathrm{M}=\mathrm{Mo}, \mathrm{W})$, según el esquema de reacción de la figura 4.15.
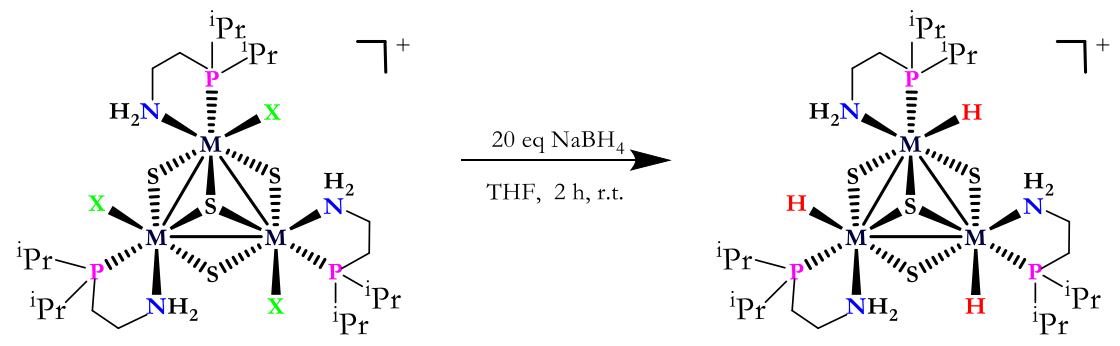

Figura 4.15. Esquema de la síntesis de hidruros de fórmula $\left[\mathrm{M}_{3} \mathrm{~S}_{4} \mathrm{H}_{3}(\text { ediprp) }]^{+}\right.$.

En el caso del wolframio, la reacción entre $\left[\mathrm{W}_{3} \mathrm{~S}_{4} \mathrm{Br}_{3}(\text { ediprp) })_{3}\right]^{+}$y $\mathrm{BH}_{4}$ transcurre mediante un cambio de coloración de azul intenso a rosa fucsia, al igual que se observa en el clúster análogo funcionalizado con edpp. ${ }^{[20]}$ Tras filtrar la mezcla de reacción, la disolución resultante se lleva a sequedad y se caracteriza mediante espectrometría de masas. La figura 4.16 muestra el espectro de masas del producto de reacción en acetonitrilo, en el cual se observa un pico mayoritario a 1166 u.m.a. asociado al compuesto $\left[\mathrm{W}_{3} \mathrm{~S}_{4} \mathrm{H}_{3}\left(\text { ediprp }_{3}\right]^{+}\right.$, que resulta de la sustitución de los 
ligandos bromuro por hidruro. La asignación se ha realizado por comparación entre el espectro de masas simulado y experimental, que presentan una buena concordancia. Adicionalmente, se observan picos a relaciones $\mathrm{m} / \mathrm{z}$ de 1182, 1196, 1210, 1226, 1242 y 1254 que resultan de la sustitución parcial de ligandos hidruro por hidroxo, metoxo y etoxo, fenómeno que con gran probabilidad ocurre durante el registro del espectro.

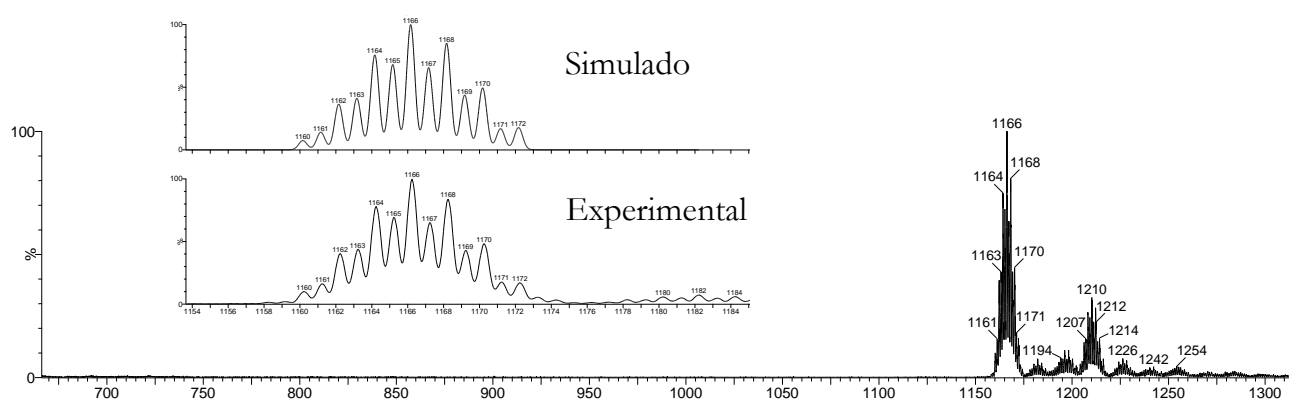

Figura 4.16. Espectro de masas simulado y experimental del producto de la reacción entre $\left[\mathrm{W}_{3} \mathrm{~S}_{4} \mathrm{Br}_{3}(\text { ediprp) }]_{3}\right]^{+}$y $\mathrm{NaBH}_{4}$.

Con el fin de analizar la pureza del producto de reacción, este se ha caracterizado mediante RMN de fósforo en acetona deuterada. El espectro, representado en la figura 4.17, muestra un pico mayoritario a 40,74 ppm que apoya la formulación del producto como $\left[\mathrm{W}_{3} \mathrm{~S}_{4} \mathrm{H}_{3} \text { (ediprp) }_{3}\right]^{+}$y es coherente con la sustitución de tres ligandos bromuro por hidruros, sin que se produzcan cambios en la disposición del resto de ligandos. Esta señal concuerda con la presencia de tres átomos de fósforo equivalentes que apuntan hacia el mismo lado del plano definido por los tres metales. También se observan dos señales satélite correspondientes al acoplamiento del núcleo de fósforo y del de wolframio, con un valor ${ }^{1} \mathrm{~J}$ P-W de 90,5 Hz. La presencia de otros picos, marcados en la figura 4.17 con un asterisco, apunta a que el sólido aislado contiene impurezas y por lo tanto que el proceso de purificación requiere trabajo adicional. 


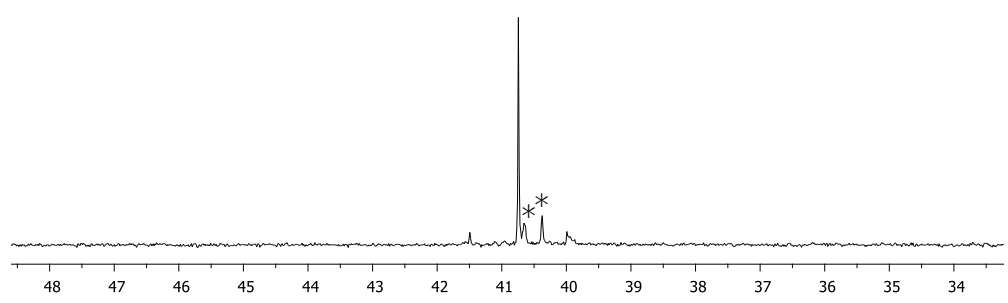

Figura 4.17. $\mathrm{RMN}$ de ${ }^{31} \mathrm{P}\left\{{ }^{1} \mathrm{H}\right\}$ de la reacción entre $\left[\mathrm{W}_{3} \mathrm{~S}_{4} \mathrm{Br}_{3}(\text { ediprp })_{3}\right]^{+}$y $\mathrm{NaBH}_{4}$.

La tabla 4.3 muestra un resumen comparativo de las señales de RMN de fósforo de los precursores de wolframio de fórmula $\left[\mathrm{W}_{3} \mathrm{~S}_{4} \mathrm{Br}_{3}(\text { edpp })_{3}\right]^{+}$y $\left[\mathrm{W}_{3} \mathrm{~S}_{4} \mathrm{Br}_{3} \text { (ediprp) }_{3}\right]^{+}$, junto con la de los complejos hidruro de fórmula $\left[\mathrm{W}_{3} \mathrm{~S}_{4} \mathrm{H}_{3}(\text { edpp })_{3}\right]^{+}$y $\left[\mathrm{W}_{3} \mathrm{~S}_{4} \mathrm{H}_{3}(\text { ediprp) }]_{3}\right]^{+}$. La diferencia entre las señales del precursor halogenado y el hidruro es de 16,41 ppm para los compuestos de ediprp y de 15,04 ppm para los de edpp. Este resultado apoya la formulación del producto de reacción entre $\left[\mathrm{W}_{3} \mathrm{~S}_{4} \mathrm{Br}_{3}\left(\text { ediprp }_{3}\right]^{+}\right.$y $\mathrm{BH}_{4^{-}}$como $\left[\mathrm{W}_{3} \mathrm{~S}_{4} \mathrm{H}_{3}(\text { ediprp) })_{3}\right]^{+}$.

Tabla 4.3. Desplazamiento químico en el $\mathrm{RMN}$ de ${ }^{31} \mathrm{P}\left\{{ }^{1} \mathrm{H}\right\}$ para los clústeres $\left[\mathrm{W}_{3} \mathrm{~S}_{4} \mathrm{Br}_{3}(\text { ediprp) })_{3}\right]^{+}\left(\mathbf{6}^{+}\right),\left[\mathrm{W}_{3} \mathrm{~S}_{4} \mathrm{Br}_{3}(\text { edpp })_{3}\right]^{+},\left[\mathrm{W}_{3} \mathrm{~S}_{4} \mathrm{H}_{3}(\text { edpp })_{3}\right]^{+}$y $\left[\mathrm{W}_{3} \mathrm{~S}_{4} \mathrm{H}_{3}(\text { ediprp })_{3}\right]^{+}$.

\begin{tabular}{cc}
\hline \hline Compuesto & $\boldsymbol{\delta}$ (ppm) \\
\hline$\left[\mathrm{W}_{3} \mathrm{~S}_{4} \mathrm{Br}_{3}\left(\text { edi }^{\mathrm{i} p r p}\right)_{3}\right]^{+}$ & 24,33 \\
{$\left[\mathrm{~W}_{3} \mathrm{~S}_{4} \mathrm{Br}_{3}(\text { edpp })_{3}\right]^{+(\mathrm{a})}$} & 15,43 \\
{$\left[\mathrm{~W}_{3} \mathrm{~S}_{4} \mathrm{H}_{3}(\text { edi prp) }]_{3}^{+}\right.$} & 40,74 \\
{$\left[\mathrm{~W}_{3} \mathrm{~S}_{4} \mathrm{H}_{3}(\text { edpp })_{3}\right]^{+(\mathrm{b})}$} & 30,47 \\
\hline \hline
\end{tabular}

(a) Datos extraídos de la referencia [21]. (b) Datos extraídos de la referencia [20].

La presencia de tres ligandos hidruro en el producto de reacción entre $\left[\mathrm{W}_{3} \mathrm{~S}_{4} \mathrm{Br}_{3}(\text { ediprp) }]^{+}\right.$y $\mathrm{BH}_{4}{ }^{-}$queda confirmada por su espectro de $\mathrm{RMN}$ de ${ }^{1} \mathrm{H}$ (ver figura 4.18). El espectro muestra una señal con multiplicidad doblete en la región hidrídica, con un desplazamiento de 0,74 ppm. Esta señal aparece como un doblete debido al acoplamiento entre los ligandos hidruro y los núcleos de fósforo, con una 
constante ${ }^{2} \mathrm{~J}$-H de 4,8 Hz, lo que corrobora la formulación del producto como $\left[\mathrm{W}_{3} \mathrm{~S}_{4} \mathrm{H}_{3}\left(\text { ediprp}_{3}\right]^{+}\right.$.

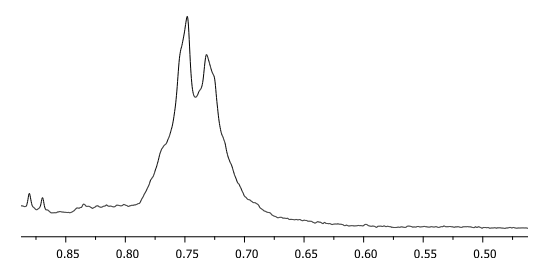

Figura 4.18. Zona hidrídica ampliada del espectro de $\mathrm{RMN}$ de ${ }^{1} \mathrm{H}$ del producto de reacción entre $\left[\mathrm{W}_{3} \mathrm{~S}_{4} \mathrm{Br}_{3}\left(\text { ediprp}_{3}\right]^{+}\right.$y $\mathrm{BH}_{4}$.

Se han realizado numerosos intentos de purificación del producto $\left[\mathrm{W}_{3} \mathrm{~S}_{4} \mathrm{H}_{3}(\text { ediprp) }]_{3}\right]^{+}$. El primero de ellos consiste en llevar el producto a sequedad y redisolver posteriormente en diclorometano, tal como se describe para complejos análogos.[20,37] De este modo se eliminan las sales formadas durante la reacción, insolubles en este disolvente. En nuestro caso, este procedimiento va acompañado de un cambio de color de rosa fucsia a morado que apunta a la sustitución de los ligandos hidruro por cloruros. Esta sustitución se confirma por la aparición de un pico a 1270 u.m.a. en el espectro de masas, que se asocia a la especie $\left[\mathrm{W}_{3} \mathrm{~S}_{4} \mathrm{Cl}_{3}(\text { ediprp) }]^{+}\right.$.

Adicionalmente, hemos tratado de obtener muestras puras del clúster $\left[\mathrm{W}_{3} \mathrm{~S}_{4} \mathrm{H}_{3} \text { (ediprp) }\right]_{3}^{+}$mediante cristalización en mezclas THF:éter y THF:pentano. Los mejores resultados se obtienen en THF:pentano a baja temperatura, pero los cristales no resultan aptos para su caracterización mediante difracción de rayos $\mathrm{X}$ en monocristal.

Por otra parte, la reacción entre el clúster $\left[\mathrm{Mo}_{3} \mathrm{~S}_{4} \mathrm{Cl}_{3}(\text { ediprp) }]^{+}\left(\mathbf{5}^{+}\right)\right.$y $\mathrm{NaBH}_{4}$ no conduce a la formación del clúster hidruro de fórmula $\left[\mathrm{Mo}_{3} \mathrm{~S}_{4} \mathrm{H}_{3} \text { (ediprp) }\right]^{+}$. A pesar de observar repetidamente cambios de coloración de verde a marrón característicos de los hidruros de molibdeno, ${ }^{[37,38]}$ no hemos podido identificar el producto de reacción mediante las técnicas habituales. 
En conclusión, la reacción entre $\left[\mathrm{W}_{3} \mathrm{~S}_{4} \mathrm{Br}_{3}(\text { ediprp) }]_{3}\right]^{+}$y $\mathrm{BH}_{4}^{-}$conduce a la formación del hidruro de fórmula $\left.\left[\mathrm{W}_{3} \mathrm{~S}_{4} \mathrm{H}_{3} \text { (ediprp) }\right]_{3}\right]^{+}$, pero su elevada reactividad nos impide aislarlo de forma analíticamente pura. Este procedimiento de síntesis no ha podido extenderse a los clústeres análogos de molibdeno.

\subsubsection{REACTIVIDAD FRENTE A ALCOHOLES}

Recientemente, el grupo de Llusar ha descrito que los clústeres de fórmula $\left[\mathrm{M}_{3} \mathrm{~S}_{4} \mathrm{X}_{3}(\mathrm{edpp})_{3}\right]^{+}(\mathrm{M}=\mathrm{Mo}, \mathrm{W} ; \mathrm{X}=\mathrm{Cl}, \mathrm{Br})$ reaccionan con metanol o etanol en presencia de un exceso de trietilamina a $45^{\circ} \mathrm{C}$ para dar clústeres de fórmula general $\left[\mathrm{M}_{3} \mathrm{~S}_{4}(\mathrm{OR})_{3}(\mathrm{edpp})_{3}\right]^{+}\left(\mathrm{M}=\mathrm{Mo}, \mathrm{W} ; \mathrm{R}=\mathrm{CH}_{3}, \mathrm{CH}_{2} \mathrm{CH}_{3}\right)$ con altos rendimientos (86$93 \%$ ) (ver figura 4.19).[20] Con el fin de obtener una nueva familia de clústeres con ligandos terminales alcoxo funcionalizados con ediprp, se ha evaluado la reactividad de $5^{+}$y $\mathbf{6}^{+}$frente a alcoholes en presencia de trietilamina siguiendo el procedimiento descrito en la figura 4.19.
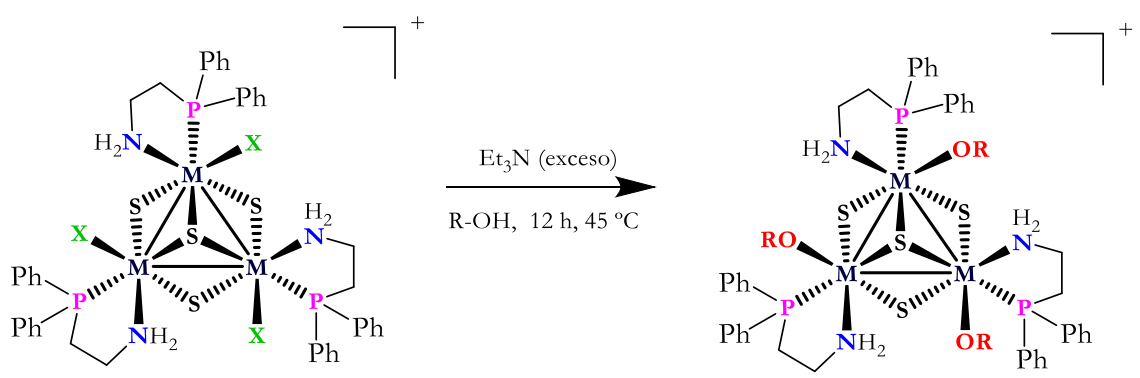

Figura 4.19. Esquema general de la obtención de clústeres de fórmula $\left[\mathrm{M}_{3} \mathrm{~S}_{4}(\mathrm{OR})_{3}(\mathrm{edpp})_{3}\right]^{+}$ $\left(\mathrm{M}=\mathrm{Mo}, \mathrm{W} ; \mathrm{R}=\mathrm{CH}_{3}, \mathrm{CH}_{2} \mathrm{CH}_{3}\right)$.

El clúster $\left[\mathrm{Mo}_{3} \mathrm{~S}_{4} \mathrm{Cl}_{3}(\text { ediprp) }]_{3}\right]^{+}\left(\mathbf{5}^{+}\right)$se ha disuelto en metanol y tras alcanzar esta disolución una temperatura de $45^{\circ} \mathrm{C}$ hemos añadido un exceso de trietilamina. La reacción transcurre con un cambio de coloración inmediato de verde claro a verde oscuro y tras 4 horas, se analizó una muestra del crudo de reacción mediante RMN de ${ }^{31} \mathrm{P}\left\{{ }^{1} \mathrm{H}\right\}$ y espectrometría de masas. En el espectro de RMN aparecen varias 
señales entre 50 y 55 ppm que sugieren una mezcla de especies, hipótesis que se confirma mediante espectrometría de masas (ver figura 4.20).

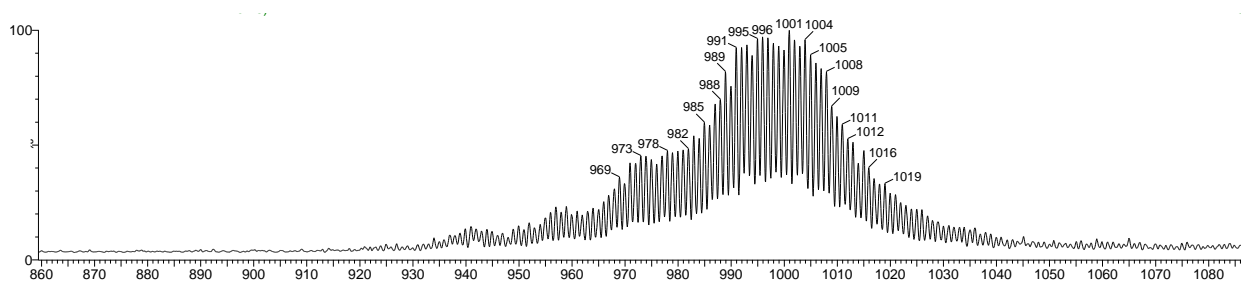

Figura 4.20. Espectro de masas del crudo tras 4 horas de reacción entre $\mathbf{5}^{+}$y metanol en presencia de trietilamina.

En el espectro de masas del producto de reacción entre $\mathbf{5}^{+}$y metanol en presencia de trietilamina (figura 4.20) aparecen solapadas tres señales: una perteneciente a la especie $\left[\mathrm{Mo}_{3} \mathrm{~S}_{4}\left(\mathrm{OCH}_{3}\right)_{3}(\text { ediprp) }]_{3}\right]^{+}$centrada en 994 u.m.a., otra a 971 u.m.a., que se asigna a la especie $\left[\mathrm{Mo}_{3} \mathrm{~S}_{4} \mathrm{Cl}_{2}(\text { ediprp-H})_{3}\right]^{+}$con un grupo $\mathrm{Mo}=\mathrm{NHR}$ cuya formación tiene lugar en el equipo de medida y por último, la señal correspondiente al precursor $\mathbf{5}^{+}$a 1006 u.m.a. En este contexto, podemos afirmar que los ligandos cloruro se han sustituido por ligandos metoxo, pero que dicha sustitución no es completa, ya que todavía se detecta la presencia de producto de partida. Tras 12 horas, la mezcla de reacción se ha concentrado a vacío en atmósfera inerte y el producto se ha precipitado con éter. Cabe destacar que un aumento en el tiempo de reacción no provoca un mayor alcance de la misma hacia el producto trisustituido. Durante el proceso de concentración hemos observado un cambio de color de verde oscuro a verde claro. La caracterización mediante RMN de fósforo y espectrometría de masas del sólido aislado tras la precipitación ha revelado que el producto de reacción es de nuevo el complejo $5^{+}$, es decir, el clúster precursor halogenado. Este resultado se ha confirmado mediante la aparición de una única señal a 53,45 ppm en el espectro de $\mathrm{RMN}$ de ${ }^{31} \mathrm{P}\left\{{ }^{1} \mathrm{H}\right\}$ y un único pico a 1006 u.m.a. en el espectro de masas. Tras esta sorprendente conclusión se ha llevado a cabo la misma 
reacción a temperatura ambiente, obteniendo en todos los casos el clúster de partida como producto de reacción.

Adicionalmente, hemos investigado la reacción del clúster $\left[\mathrm{Mo}_{3} \mathrm{~S}_{4} \mathrm{Cl}_{3}(\text { ediprp) }]_{3}\right]^{+}\left(\mathbf{5}^{+}\right)$y etanol en presencia de un exceso de trietilamina. Tras 12 horas de reacción, se ha aislado un sólido verde que ha resultado ser una mezcla de clústeres con diferentes sustituciones. El espectro de RMN de fósforo muestra varias señales difíciles de interpretar y el espectro de masas indica que el producto final es una mezcla de especies entre las que se encuentran el clúster de partida y una especie mixta con un ligando etoxo y dos ligandos cloruro. Al contrario de lo que ocurría con el metanol, en ningún caso se ha observado la coordinación de más de un ligando etoxo a la unidad trimetálica. Asimismo, esta reacción es completamente irreversible.

Por otra parte, el clúster $\left[\mathrm{W}_{3} \mathrm{~S}_{4} \mathrm{Br}_{3}(\text { ediprp })_{3}\right]^{+}\left(\mathbf{6}^{+}\right)$se ha hecho reaccionar con metanol en presencia de un exceso de trietilamina y se han obtenido resultados diferentes a los de su análogo de molibdeno. Tras 12 horas de reacción, hemos podido aislar un sólido violeta que, según los antecedentes descritos en la bibliografía, podría tratarse del complejo con tres ligandos $\mathrm{OCH}_{3} .{ }^{20]}$ Sin embargo, los análisis mediante RMN de fósforo y espectrometría de masas han mostrado que nos encontramos de nuevo ante una mezcla de productos entre los que se identifican diversas especies mixtas que contienen ligandos metoxo, hidroxo y bromuro. Una situación similar ha ocurrido al hacer reaccionar el clúster $\mathbf{6}^{+}$con etanol en presencia de un exceso de trietilamina, en cuyo espectro hemos identificado especies mixtas que contienen ligandos etoxo, hidroxo y bromuro.

En conclusión, la reactividad de los clústeres $5^{+}$y $\mathbf{6}^{+}$frente a alcoholes en presencia de trietilamina es significativamente distinta a la de sus análogos funcionalizados con edpp. En nuestro caso, los clústeres de fórmula $\left[\mathrm{M}_{3} \mathrm{~S}_{4}\left(\mathrm{OR}_{3}\right)_{3}(\text { ediprp })_{3}\right]^{+}\left(\mathrm{M}=\mathrm{Mo}, \mathrm{W} ; \mathrm{R}=\mathrm{OCH}_{3}, \mathrm{OCH}_{2} \mathrm{CH}_{3}\right)$ no son estables y por tanto no se han podido aislar. 


\subsection{REDUCCIÓN CATALÍTICA DE CETONAS}

Las transformaciones de sustratos orgánicos mediante hidrogenación catalítica son procesos fundamentales en química orgánica, tanto a nivel de laboratorio como a escala industrial.[1] En concreto, los hidruros de metales de transición destacan por su actividad catalítica en este tipo de procesos.[3,39,40] Los complejos de metales de transición funcionalizados con ligandos aminofosfina son activos tanto en reacciones de hidrogenación como de transferencia de hidrógeno, tal como indicábamos en la sección 4.1.[2-7] Recientemente se han descrito complejos hidruro monometálicos de rutenio e iridio funcionalizados con aminofosfinas que presentan actividad en reacciones de reducción de cetonas, iminas y amidas, entre otros sustratos.[3,39,41]

La mayoría de complejos clúster activos en la hidrogenación de sustratos orgánicos son complejos derivados con fosfinas. En el año 2001 Duckett, Dyson y colaboradores describen la hidrogenación directa de alquinos catalizada por un complejo trinuclear de rutenio funcionalizado con trifenilfosfina. ${ }^{[42]}$ Más recientemente, Nordlander y colaboradores han descrito una serie de clústeres trinucleares y tetranucleares de rutenio funcionalizados con fosfinas derivadas con grupos ferroceno capaces de hidrogenar una serie de ácidos carboxílicos $\alpha$ insaturados en presencia de hidrógeno molecular.[43-45]

Beller, Llusar y colaboradores han demostrado que el hidruro $\left[\mathrm{Mo}_{3} \mathrm{~S}_{4} \mathrm{H}_{3}(\text { dmpe })_{3}\right]^{+}$cataliza la reducción de nitroarenos funcionalizados a sus correspondientes anilinas mediante un proceso de transferencia de hidrógeno, en presencia de una mezcla de ácido fórmico y trietilamina como agente reductor. ${ }^{[40]} \mathrm{El}$ ciclo catalítico propuesto para esta transformación se ilustra en la figura 4.21. 


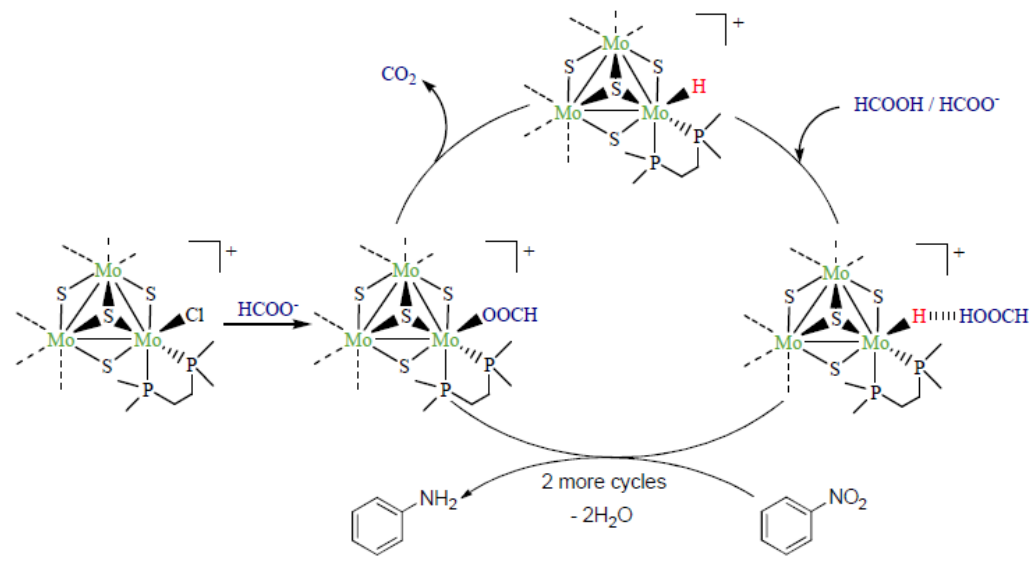

Figura 4.21. Ciclo catalítico propuesto por Beller y Llusar para la reducción de nitrobenceno catalizada por el clúster $\left[\mathrm{Mo}_{3} \mathrm{~S}_{4} \mathrm{H}_{3}(\mathrm{dmpe})_{3}\right]^{+}$. Figura adaptada de la referencia [40] con permiso de Wiley-VCH. Copyright 2012 Wiley-VCH Verlag GmbH \& Co.

Además, estos autores identifican las especie activa como el clúster hidruro $\left[\mathrm{Mo}_{3} \mathrm{~S}_{4} \mathrm{H}_{3}(\mathrm{dmpe})_{3}\right]^{+}$, que también se puede generar in situ a partir del clúster precursor $\left[\mathrm{Mo}_{3} \mathrm{~S}_{4} \mathrm{Cl}_{3}(\mathrm{dmpe})_{3}\right]^{+}$mediante la reacción con ácido fórmico en medio básico. De esta manera, la especie hidruro se genera tras la sustitución de los ligandos cloruro por los ligandos formiato y la posterior liberación de $\mathrm{CO}_{2}$, tal como muestra la figura 4.21. La reducción de nitroderivados también puede ser catalizada por complejos diamino con unidad $\mathrm{Mo}_{3} \mathrm{~S}_{4}$, aunque en este caso la mezcla de ácido fórmico y trietilamina no es un agente reductor efectivo y es necesario utilizar silanos como dadores de hidrógeno.[31]

La sustitución de ligandos difosfina o diamina por aminofosfinas, concretamente la edpp, resulta en especies precatalizadoras de fórmula $\left[\mathrm{Mo}_{3} \mathrm{~S}_{4} \mathrm{X}_{3}(\mathrm{edpp})_{3}\right]^{+}(\mathrm{X}=\mathrm{Cl}, \mathrm{Br})$ activas en procesos de transferencia de hidrógeno de nitroarenos cuando se utiliza la mezcla de ácido fórmico y trietilamina como agente reductor.[20] En este caso, varias especies clúster coexisten durante el proceso catalítico según la monitorización por PSI (Pressuriz̨ed Sample Infusion) en combinación con espectrometría de masas, lo que imposibilita la identificación de la especie activa. 
Dada la dificultad de aislar los complejos hidruro de fórmula $\left[\mathrm{M}_{3} \mathrm{~S}_{4} \mathrm{H}_{3} \text { (ediprp) }\right]_{3}^{+}(\mathrm{M}=\mathrm{Mo}, \mathrm{W})$ descrita en la sección 4.3.1, las pruebas catalíticas se han realizado con el clúster halogenado de molibdeno de fórmula $\left[\mathrm{Mo}_{3} \mathrm{~S}_{4} \mathrm{Cl}_{3}(\text { ediprp })_{3}\right]^{+}\left(\mathbf{5}^{+}\right)$. En esta tesis hemos decidido ampliar el campo de aplicación en catálisis de los complejos clúster funcionalizados con aminofosfinas a otros sustratos, concretamente cetonas. La elección de esta reacción catalítica se debe a que la obtención de alcoholes a partir de procedimientos de reducción de sustratos carbonílicos presenta un elevado interés industrial. Debido a la importancia de los derivados organofluorados en la química industrial, descrita previamente en el capítulo 3,[46,47] hemos elegido la cetona fluorada 2,2,2-trifluoroacetofenona como sustrato modelo, cuya reducción conduce previsiblemente a la formación de $\alpha$ (trifluorometil)bencil alcohol.

La introducción de átomos de flúor en moléculas orgánicas pequeñas es una estrategia muy utilizada en la química farmacéutica, ya que el flúor modifica notablemente sus propiedades y aumenta su afinidad a los receptores moleculares. ${ }^{[48,49]}$ En este contexto, las moléculas que contienen grupos trifluorometilo $\left(\mathrm{CF}_{3}\right)$ son especialmente relevantes, ya que presentan una elevada actividad biológica. Concretamente, los alcoholes $\alpha$-trifluorometilados como el $\alpha$ (trifluorometil)bencil alcohol se utilizan ampliamente como intermedios en el diseño de medicamentos como los mostrados a modo de ejemplo en la figura 4.22.[50] 

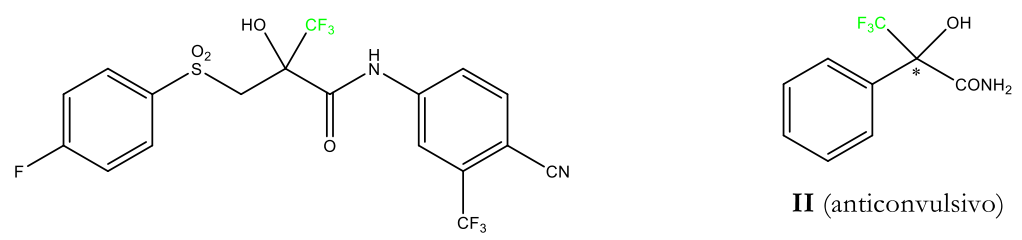

II (anticonvulsivo)

I (agente anticancerígeno)

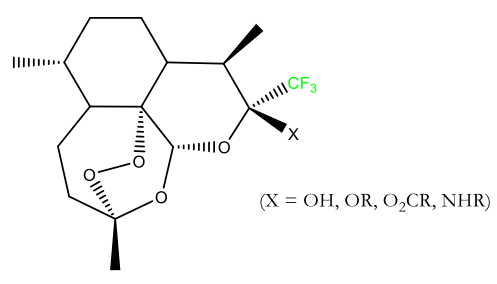

III (agente antimalaria)

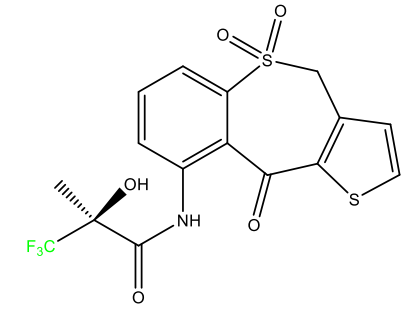

IV (medicamento para desórdenes urinarios)

Figura 4.22. Compuestos biológicamente activos que contienen un grupo trifluorometilo.

Los ensayos catalíticos de la reducción de 2,2,2-trifluoroacetofenona con el fin de obtener el alcohol correspondiente, según el esquema de la tabla 4.4, se han llevado a cabo en THF a $70{ }^{\circ} \mathrm{C}$, utilizando $0,1 \mathrm{mmol}$ de sustrato, $5 \mathrm{~mol} \%$ de clúster $\mathbf{5}^{+}$y 5 equivalentes de agente reductor durante 18 horas. Las pruebas realizadas con una menor cantidad de catalizador (1-3 mol \%) y a un menor tiempo (12-15 horas) resultan en conversiones y rendimientos más pobres. Como agentes reductores hemos utilizado hidrógeno molecular, una mezcla HCOOH:Et $3 \mathrm{~N}(5: 2)$ y una serie de silanos $\left(\mathrm{PhSiH}_{3}\right.$ y $\left.\mathrm{Ph}_{2} \mathrm{SiH}_{2}\right)$. El producto de reacción se ha identificado mediante comparación con un estándar comercial de elevada pureza. Para su cuantificación, se ha utilizado n-hexadecano como patrón interno.

La tabla 4.4 lista las conversiones y rendimientos obtenidos al variar el agente reductor para la reducción catalítica de 2,2,2-trifluoroacetofenona al alcohol correspondiente. La ausencia de reacción al utilizar hidrógeno molecular (entrada 1), nos lleva a afirmar que la reacción no tiene lugar mediante un mecanismo de hidrogenación directa. El uso de la mezcla $\mathrm{HCOOH}_{\mathrm{E}} \mathrm{H}_{3} \mathrm{~N}$ (5:2) resulta en conversiones moderadas y rendimientos de alcohol muy bajos, lo que apunta a una 
baja selectividad hacia el alcohol del mecanismo de transferencia de hidrógeno (entrada 2). Los silanos son los agentes reductores que mejores resultados presentan en términos de conversión y rendimiento (entradas 3 y 4), siendo el $\mathrm{PhSiH}_{3}$ el mejor de ellos (entrada 4).

Tabla 4.4. Reducción catalítica de 2,2,2-trifluoroacetofenona con el clúster $\mathbf{5}^{+(a)}$.

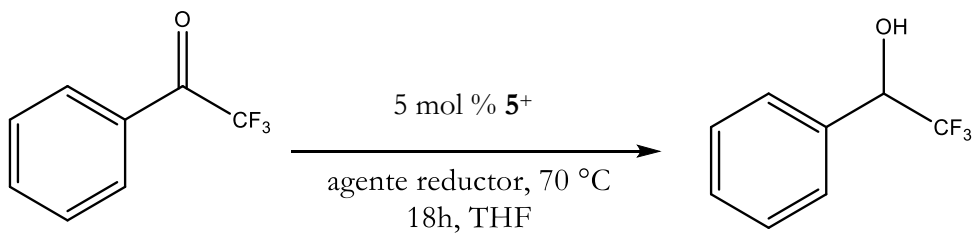

\begin{tabular}{cccc}
\hline \hline Entrada & Agente reductor & Conversión (\%) $^{(\mathrm{b})}$ & Rendimiento (\%) $^{(\mathrm{b})}$ \\
\hline 1 & $\mathrm{H}_{2}$ & 0 & 0 \\
2 & $\mathrm{HCOOH}: \mathrm{Et}_{3} \mathrm{~N}(5: 2)$ & 84 & 24 \\
3 & $\mathrm{Ph}_{2} \mathrm{SiH}_{2}$ & 76 & 61 \\
4 & $\mathrm{PhSiH}_{3}$ & 95 & 83 \\
\hline \hline
\end{tabular}

(a)Condiciones de reacción: 0,1 mmol sustrato, $5 \mathrm{~mol} \%$ catalizador, 5 equivalentes agente reductor. (b)Determinados por GC utilizando n-hexadecano como estándar interno.

La integridad de la unidad $\mathrm{Mo}_{3} \mathrm{~S}_{4}$ durante el transcurso de la reacción en presencia de $\mathrm{PhSiH}_{3}$ ha sido confirmada mediante espectrometría de masas, donde se observa que la entidad trinuclear no se ha fragmentado en especies de menor nuclearidad. Se ha registrado un espectro de masas en el transcurso de uno de los ensayos catalíticos en presencia de $\mathrm{PhSiH}_{3}$ y se ha observado un pico mayoritario a 1006 u.m.a. que corresponde al clúster $\mathbf{5}^{+}$y un pico minoritario a 1080 u.m.a. cuya relación masa/carga es consistente con la formulación $\left[\mathrm{Mo}_{3} \mathrm{~S}_{4} \mathrm{Cl}_{2}(\mathrm{edi} p r p){ }_{3}\left(\mathrm{PhSiH}_{3}\right)\right]^{+}$.

La actividad de los complejos derivados con ligandos aminofosfina está intimamente relacionada con la naturaleza del grupo amino. ${ }^{[8]}$ Ikariya y colaboradores han demostrado que los complejos de rutenio funcionalizados con ligandos 
aminofosfina que contienen un grupo amino primario presentan una mayor actividad en la hidrogenación catalítica de ésteres que los que contienen un grupo amino secundario, mientras que las aminofosfinas que contienen un grupo amino terciario apenas presentan actividad.[13] Este estudio apunta claramente a que los hidrógenos del grupo amino juegan un papel destacado en el proceso de hidrogenación. Sin embargo, Stradiotto y colaboradores han demostrado que un complejo de rutenio funcionalizado con la aminofosfina 1-di-i-propilfosfino-2-(N.N-dimetilamino)-1Hindeno ( $\mathrm{PiPr}_{2}-\mathrm{NMe}_{2}$-indeno), que contiene un grupo amino terciario, es eficaz en la reducción de cetonas.[51] Con estos antecedentes y nuestros resultados positivos en la reducción catalítica de 2,2,2-trifluoroacetofenona en presencia del clúster precatalizador $\mathbf{5}^{+}$, hemos decidido llevar a cabo un estudio exploratorio in situ combinando la especie clúster $\left[\mathrm{Mo}_{3} \mathrm{~S}_{4} \mathrm{Cl}_{4}\left(\mathrm{PPh}_{3}\right)_{3}\left(\mathrm{H}_{2} \mathrm{O}\right)_{2}\right]$, inactiva en este tipo de procesos, y una serie de ligandos aminofosfina de distinta naturaleza. Para ello, se han seleccionado una familia de aminofosfinas comerciales, representadas en la figura 4.23, que contienen aminas primarias (edpp, ediprp, 2-(aminoetil)di-t-butilfosfina (edtbup)), secundarias ((R)- y (S)-2-[(difenilfosfino)metil]pirrolidina ((R)- y (S)-PPro)) y terciarias ( $\mathrm{PiPr}_{2}-\mathrm{NMe}_{2}$-indeno).

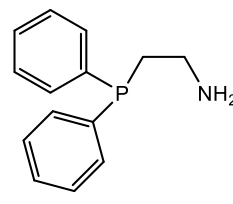

edpp

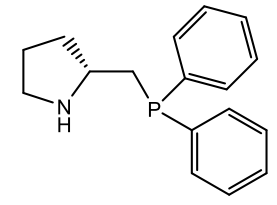

(R)-PPro

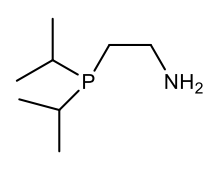

ediprp

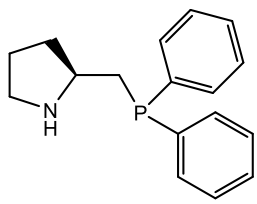

(S)-PPro

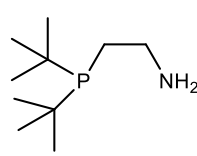

ed ${ }^{\mathrm{t} b u p}$

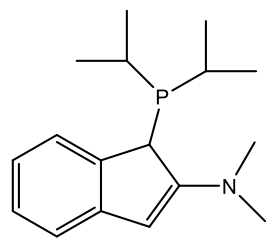

$\mathrm{P}^{\mathrm{i}} \mathrm{Pr}_{2}-2-\mathrm{NMe}_{2}$-indeno

Figura 4.23. Ligandos aminofosfina elegidos para la evaluación del papel de los hidrógenos del grupo amino en la reducción catalítica de 2,2,2-trifluoroacetofenona. 
Las pruebas de reducción catalítica se realizan con el sustrato modelo 2,2,2trifluoroacetofenona bajo condiciones análogas a las optimizadas al utilizar el clúster $5^{+}$como precatalizador. Concretamente, los ensayos se han llevado a cabo en THF a $70{ }^{\circ} \mathrm{C}$ durante 18 horas, utilizando un $5 \mathrm{~mol} \%$ de clúster precursor $\left[\mathrm{Mo}_{3} \mathrm{~S}_{4} \mathrm{Cl}_{4}\left(\mathrm{PPh}_{3}\right)_{3}\left(\mathrm{H}_{2} \mathrm{O}\right)_{2}\right], 3$ equivalentes de ligando aminofosfina y 5 equivalentes de $\mathrm{PhSiH}_{3}$ como agente reductor. Bajo estas condiciones, el clúster $\left[\mathrm{Mo}_{3} \mathrm{~S}_{4} \mathrm{Cl}_{4}\left(\mathrm{PPh}_{3}\right)_{3}\left(\mathrm{H}_{2} \mathrm{O}\right)_{2}\right]$ en ausencia de aminofosfina no presenta actividad catalítica. Los resultados obtenidos se resumen en la tabla 4.5.

Tabla 4.5. Reducción catalítica in situ de 2,2,2-trifluoroacetofenona con el clúster precursor $\left[\mathrm{Mo}_{3} \mathrm{~S}_{4} \mathrm{Cl}_{4}\left(\mathrm{PPh}_{3}\right)_{3}\left(\mathrm{H}_{2} \mathrm{O}\right)_{2}\right]$ y 3 equivalentes de ligando aminofosfina ${ }^{(\mathrm{a})}$.<smiles>O=C(c1ccccc1)C(F)(F)F</smiles>
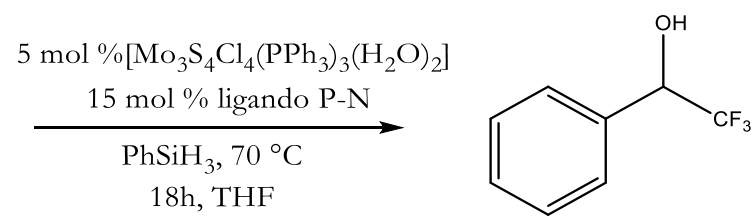

\begin{tabular}{cccc}
\hline \hline Entrada & Ligando & Conversión (\%) $)^{(\mathrm{b})}$ & Rendimiento (\%) $\mathbf{b}^{(\mathrm{b})}$ \\
\hline 1 & ediprp & 94 & 85 \\
2 & edtbup & $>99$ & 88 \\
3 & edpp & 91 & 43 \\
4 & (R)-PPro & 80 & 45 \\
5 & $(S)$-PPro & 62 & 33 \\
6 & PiPr $_{2}-\mathrm{NMe}_{2}$-indeno & $>99$ & 52 \\
\hline \hline
\end{tabular}

(a) Condiciones de reacción: 0,1 $\mathrm{mmol}$ sustrato, $5 \mathrm{~mol} \%$ catalizador, 5 equivalentes agente reductor. (b)Determinados por GC utilizando n-hexadecano como estándar interno.

Las aminofosfinas que contienen grupos amina primarios y cadenas alquílicas enlazadas al fósforo, como la ediprp y la edtbup, muestran los mejores resultados en términos de conversión y rendimiento (entradas 1 y 2 , tabla 4.5). Cabe destacar que la actividad catalítica descrita en la entrada 1 de la tabla 4.5 es comparable a la 
observada al utilizar el clúster aislado $5^{+}$, que se ha descrito anteriormente en la entrada 4 de la tabla 4.4. La sustitución de las cadenas alquílicas de las aminofosfinas por sustituyentes aromáticos, concretamente fenilos, no afecta a la conversión pero disminuye el rendimiento aproximadamente en un $50 \%$ (entrada 3, tabla 4.5). Las aminofosfinas con grupos amina secundarios como la $(R)$ - y la $(S)$-PPro presentan conversiones moderadas y rendimientos pobres (entradas 4 y 5 , tabla 4.5). También en este caso, los resultados obtenidos son comparables con los que se describen posteriormente en el capítulo 5 para los clústeres aislados funcionalizados con estas aminofosfinas (entrada 3, tabla 5.6; entrada 3, tabla 5.7). Por último, el ligando $\mathrm{PiPr}_{2^{-}}$ $\mathrm{NMe}_{2}$-indeno presenta resultados excelentes en términos de conversión y moderados en cuanto a rendimiento (entrada 6, tabla 4.5).

En conclusión, las aminofosfinas que contienen un grupo amino primario y cadenas alquílicas enlazadas al átomo de fósforo (ediprp y edtbup) favorecen la reducción de la 2,2,2-trifluoroacetofenona a $\alpha$-(trifluorometil)bencil alcohol catalizada por clústeres trimetálicos con unidad central $\mathrm{Mo}_{3} \mathrm{~S}_{4}$ en presencia de $\mathrm{PhSiH}_{3}$. Además, las conversiones y los rendimientos obtenidos son comparables a los observados para los clústeres aislados. 


\subsection{BIBLIOGRAFÍA}

[1] Blaser, H.-U.; Malan, C.; Pugin, B.; Spindler, F.; Steiner, H.; Studer, M. Adv. Synth. Catal. 2003, 345, 103.

[2] Guo, R.; Morris, R. H.; Song, D. J. Am. Chem. Soc. 2005, 127, 516.

[3] John, J. M.; Bergens, S. H. Angew. Chem. Int. Edit. 2011, 50, 10377.

[4] Braunstein, P.; Graiff, C.; Naud, F.; Pfaltz, A.; Tiripicchio, A. Inorg. Chem. 2000, 39, 4468.

[5] Clarke, Z. E.; Maragh, P. T.; Dasgupta, T. P.; Gusev, D. G.; Lough, A. J.; Abdur-Rashid, K. Organometallics 2006, 25, 4113.

[6] Hounjet, L. J.; Bierenstiel, M.; Ferguson, M. J.; McDonald, R.; Cowie, M. Inorg. Chem. 2010, 49, 4288.

[7] Aydemir, M.; Baysal, A.; Turgut, Y. Appl. Organomet. Chem. 2011, 25, 270.

[8] Zhao, B. G.; Han, Z. B.; Ding, K. L. Angew. Chem. Int. Edit. 2013, 52, 4744.

[9] Andersson, P. G.; Munslow, I. J. Modern Reduction Methods, 2008, Wiley-VCH Verlag GmbH \& Co, Weinheim.

[10] Carey, J. S.; Laffan, D.; Thomson, C.; Williams, M. T. Org. Biomol. Chem. 2006, 4, 2337.

[11] Schmeier, T. J.; Dobereiner, G. E.; Crabtree, R. H.; Hazari, N. J. Am. Chem. Soc. 2011, 133, 9274.

[12] Klankermayer, J.; Wesselbaum, S.; Beydoun, K.; Leitner, W. Angew. Chem. Int. Edit. 2016, 55, 7296.

[13] Ito, M.; Ootsuka, T.; Watari, R.; Shiibashi, A.; Himizu, A.; Ikariya, T. J. Am. Chem. Soc. 2011, 133, 4240.

[14] Carroll, M. P.; Guiry, P. J. Chem. Soc. Rev. 2014, 43, 819.

[15] Beltran, T. F.; Safont, V. S.; Llusar, R. Eur. J. Inorg. Chem. 2016.

[16] Sussfink, G.; Pellinghelli, M. A.; Tiripicchio, A. J. Organomet. Chem. 1987, 320, 101. 
[17] Komine, N.; Tsutsuminai, S.; Hirano, M.; Komiya, S. J. Organomet. Chem. 2007, 692, 4486.

[18] Tsutsuminai, S.; Komine, N.; Hirano, M.; Komiya, S. Organometallics 2003, 22, 4238.

[19] Tsutsuminai, S.; Komine, N.; Hirano, M.; Komiya, S. Organometallics 2004, 23, 44.

[20] Beltrán, T. F., Tesis Doctoral, Universitat Jaume I, 2013.

[21] Beltran, T. F.; Pino-Chamorro, J. A.; Fernandez-Trujillo, M. J.; Safont, V. S.;

Basallote, M. G.; Llusar, R. Inorg. Chem. 2015, 54, 607.

[22] Estevan, F.; Feliz, M.; Llusar, R.; Mata, J. A.; Uriel, S. Polyhedron 2001, $20,527$.

[23] Cotton, F. A.; Kibala, P. A.; Matusz, M.; McCaleb, C. S.; Sandor, R. B. W. Inorg. Chem. 1989, 28, 2623.

[24] Basallote, M. G.; Fernández-Trujillo, M. J.; Pino-Chamorro, J. Á.; Beltrán, T. F.; Corao, C.; Llusar, R.; Sokolov, M.; Vicent, C. Inorg. Chem. 2012, 51, 6794.

[25] Sasaki, M.; Sakane, G.; Ouchi, T.; Shibahara, T. J. Clust. Sci. 1998, 9, 25.

[26] Feliz, M.; Guillamon, E.; Llusar, R.; Vicent, C.; Stiriba, S. E.; Perez-Prieto, J.; Barberis, M. Chem.-Eur. J. 2006, 12, 1486.

[27] Feliz, M., Tesis Doctoral, Universitat Jaume I, 2003.

[28] Cotton, F. A.; Llusar, R. Polybedron 1987, 6, 1741.

[29] Cotton, F. A.; Mandal, S. K. Inorg. Chim. Acta 1992, 192, 71.

[30] Alfonso, C.; Feliz, M.; Safont, V. S.; Llusar, R. Dalton Trans. 2016, 45, 7829.

[31] Pedrajas, E.; Sorribes, I.; Junge, K.; Beller, M.; Llusar, R. ChemCatChem 2015, 7, 2675.

[32] Sorribes, I.; Wienhöfer, G.; Vicent, C.; Junge, K.; Llusar, R.; Beller, M. Angew. Chem. Int. Edit. 2012, 51, 7794.

[33] Khusnutdinova, J. R.; Milstein, D. Angew. Chem. Int. Edit. 2015, 54, 12236.

[34] Hamilton, R. J.; Bergens, S. H. J. Am. Chem. Soc. 2008, 130, 11979. 
[35] Darensbourg, D. J.; Lee, W.-Z.; Phelps, A. L.; Guidry, E. Organometallics 2003, 22, 5585 .

[36] Cotton, F. A.; Llusar, R.; Eagle, C. T. J. Am. Chem. Soc. 1989, 111, 4332.

[37] Algarra, A. G.; Basallote, M. G.; Fernandez-Trujillo, M. J.; Feliz, M.; Guillamon, E.; Llusar, R.; Sorribes, I.; Vicent, C. Inorg. Chem. 2010, 49, 5935.

[38] Alfonso, C.; Beltran, T. F.; Feliz, M.; Llusar, R. J. Clust. Sci. 2015, 26, 199.

[39] Abdur-Rashid, K.; Guo, R. W.; Lough, A. J.; Morris, R. H.; Song, D. T. Adv. Synth. Catal. 2005, 347, 571.

[40] Sorribes, I.; Wienhofer, G.; Vicent, C.; Junge, K.; Llusar, R.; Beller, M. Angew. Chem. Int. Edit. 2012, 51, 7794.

[41] Xie, J. B.; Xie, J. H.; Liu, X. Y.; Zhang, Q. Q.; Zhou, Q. L. Chem.-Asian J. 2011, 6, 899.

[42] Blazina, D.; Duckett, S. B.; Dyson, P. J.; Lohman, J. A. B. Angew. Chem. Int. Ed. 2001, 40, 3874.

[43] Moberg, V.; Duquesne, R.; Contaldi, S.; Rohrs, O.; Nachtigall, J.; Damoense, L.; Hutton, A. T.; Green, M.; Monari, M.; Santelia, D.; Haukka, M.; Nordlander, E. Chem.-Eur. J. 2012, 18, 12458.

[44] Moberg, V.; Haukka, M.; Koshevoy, I. O.; Ortiz, R.; Nordlander, E. Organometallics 2007, 26, 4090.

[45] Abdel-Magied, A. F.; Singh, A. K.; Haukka, M.; Richmond, M. G.; Nordlander, E. Chem. Commun. 2014, 50, 7705.

[46] O'Hagan, D. J. Fluor. Chem. 2010, 131, 1071.

[47] Purser, S.; Moore, P. R.; Swallow, S.; Gouverneur, V. Chem. Soc. Rev. 2008, 37,320 .

[48] Ojima, I. Fluorine in Medicinal Chemistry and Chemical Biology: Blackwell Publishing, Chichester, UK, 2009.

[49] Kirsch, P. Modern Fluoroorganic Chemistry: Synthesis, Reactivity, Applications. Wiley-VCH Verlag GmbH \& Co, Weinheim, 2013. 
[50] Nie, J.; Guo, H.-C.; Cahard, D.; Ma, J.-A. Chem. Rev. 2011, 111, 455.

[51] Lundgren, R. J.; Rankin, M. A.; McDonald, R.; Schatte, G.; Stradiotto, M. Angew. Chem. Int. Ed. 2007, 46, 4732. 
5. CLÚSTERES FUNCIONALIZADOS CON AMINOFOSFINAS QUIRALES: ESTUDIO TEÓRICO-EXPERIMENTAL 


\section{CLÚSTERES FUNCIONALIZADOS CON AMINOFOSFINAS QUIRALES: ESTUDIO TEÓRICO-EXPERIMENTAL}

\subsection{Introducción}

\subsection{Síntesis y caracterización}

5.3 Estudio teórico

5.3.1 Optimización de geometrías

5.3.2 Análisis de distribución electrónica

5.3.2.1 Orbitales naturales de enlace (NBO)

5.3.2.2 Densidad electrónica (ELF)

5.4 Pruebas catalíticas

5.5 Bibliografía

“[...] Ser tú no es una experiencia gratificante a nivel atómico. Pese a toda su devota atención, tus átomos no se preocupan en realidad por ti, de hecho, ni siquiera saben que estás ahí.[...] Sin embargo, por la razón que sea, durante el período de tu experiencia, tus átomos responderán a un único impulso riguroso: que tú sigas siendo tú.” Bill Bryson, Una breve historia de casi todo 


\subsection{INTRODUCCIÓN}

Los compuestos quirales tienen una gran importancia tanto en química como en biología. Durante las últimas décadas, la demanda de este tipo de compuestos en industrias como la farmacéutica, agroquímica o perfumera ha aumentado considerablemente, por lo que el control de la estereoselectividad de una reacción resulta crucial a la hora de sintetizar fármacos o moléculas aromatizantes. ${ }^{[1,2] ~} \mathrm{La}$ importancia de controlar esta esta selectividad se puede ilustrar con diversos ejemplos de suma importancia en nuestra vida diaria. El ibuprofeno, uno de los medicamentos antiinflamatorios más consumido a nivel mundial, presenta dos formas enantioméricas de las cuales solo la denominada $S$ tiene actividad farmacológica (ver figura 5.1).[3] El enantiómero $R$ de este compuesto no provoca ningún daño en el ser humano, de hecho generalmente el ibuprofeno comercializado es una mezcla racémica de ambos.
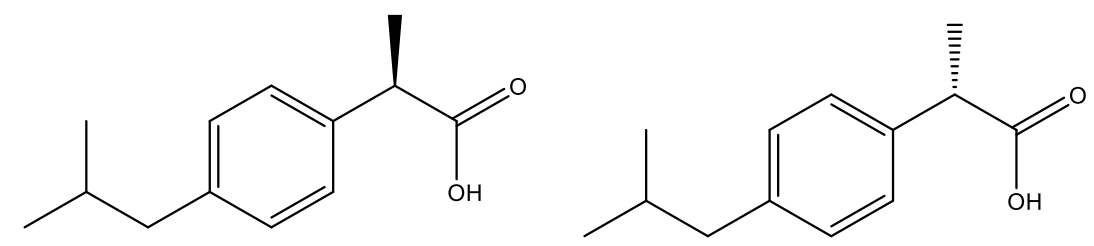

Figura 5.1. (S)-ibuprofeno (izquierda) y (R)-ibuprofeno (derecha).

Existen otros casos en los que el enantiómero inactivo en términos farmacológicos es tóxico y la administración de una mezcla racémica puede provocar daños irreversibles. Un claro ejemplo se encuentra en el uso de la talidomida en mujeres embarazadas durante los años 60 . El enantiómero que lograba frenar las náuseas era la $(\mathrm{R})$-talidomida, mientras que la $(S)$-talidomida, con una actividad biológica completamente diferente, provocó graves defectos físicos y malformaciones en los niños nacidos posteriormente.[4,5] Por tanto, el control de la estereoselectividad de una reacción resulta de gran importancia para obtener 
fármacos enantiopuros y así conseguir una mayor eficacia de los tratamientos administrados.

En este contexto cobra importancia la catálisis asimétrica, que supone una vía de síntesis para obtener compuestos enantiopuros a partir de sustratos no quirales con la ayuda de catalizadores que presentan una fuente de quiralidad, i.e. enzimas, organocatalizadores o catalizadores metálicos. ${ }^{[6]} \mathrm{El}$ creciente interés en este campo durante las pasadas décadas, propició que en el año 2001 los profesores William S. Knowles, Ryoji Noyori y Karl B. Sharpless fueran galardonados con el premio Nobel de Química por el desarrollo de catalizadores de metales de transición con aplicación en oxidaciones e hidrogenaciones estereoselectivas. Debido al interés industrial de estas reacciones y de la catálisis asimétrica en general, en este capítulo de la tesis doctoral se presentan una serie clústeres de unidad $\mathrm{Mo}_{3} \mathrm{~S}_{4}$ funcionalizados con ligandos aminofosfina quirales y se discuten sus posibles aplicaciones en reacciones de reducción catalítica de sustratos orgánicos.

En la literatura se han descrito ejemplos de complejos funcionalizados con ligandos P-N quirales que presentan actividad en este tipo de reacciones, la mayoría de ellos monometálicos. Zhou y colaboradores desarrollaron en el año 2010 una nueva clase de ligandos P-N conocidos como SpiroAP (ver figura 5.2), que utilizados in situ en presencia de un complejo de iridio monometálico eran capaces de hidrogenar cetonas $\alpha, \beta$-insaturadas para convertirlas en alcoholes alílicos quirales.[7]

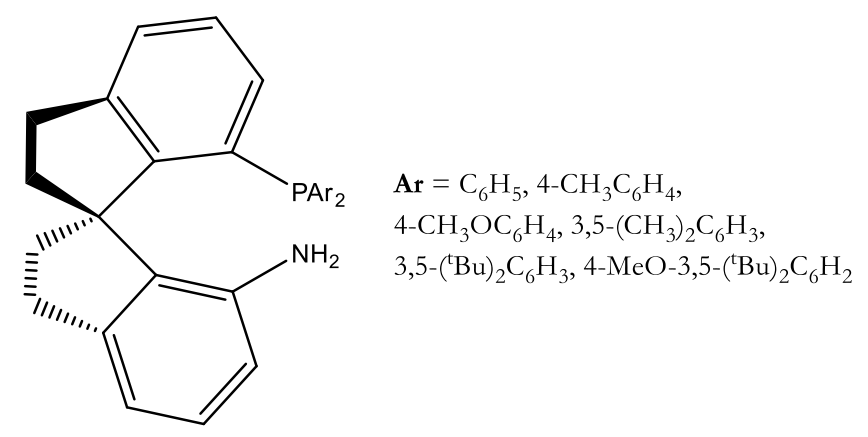

Figura 5.2. Familia de ligandos SpiroAP de Zhou y colaboradores. 
Por otra parte, el grupo de Matthew L. Clarke ha publicado recientemente la actividad catalítica de un complejo de iridio funcionalizado con ligandos diaminofosfina en la hidrogenación asimétrica de cetonas, ${ }^{[8]}$ mientras que Li y colaboradores llevan a cabo la misma transformación utilizando como catalizador un complejo monometálico de paladio funcionalizado con diaminofosfinas quirales.[9]

Los ejemplos de catálisis mediada por complejos clúster son menos frecuentes.[10] Destacan los trabajos publicados por Nordlander y colaboradores, en los que clústeres tetranucleares de rutenio funcionalizados con difosfinas quirales presentan actividad en la hidrogenación de ácidos carboxílicos $\alpha$-insaturados con buenos resultados de conversión, rendimiento y exceso enantiomérico.[11-13]

Durante la última década, Llusar y colaboradores han descrito una ruta sintética estereoselectiva para la preparación de clústeres de fórmula general $\left[\mathrm{M}_{3} \mathrm{~S}_{4} \mathrm{Cl}_{3}\left(\text { difosfina }^{*}\right)_{3}\right]^{+}$(difosfina* $=$difosfina quiral), que consiste en la reacción de fases poliméricas de fórmula $\left\{\mathrm{M}_{3} \mathrm{~S}_{7} \mathrm{X}_{4}\right\}_{\mathrm{n}}(\mathrm{M}=\mathrm{Mo}, \mathrm{W})$ con difosfinas quirales como $(R, R)$ - y $(S, S)$-Me-BPE, ${ }^{14,15]}$ donde los ligandos tienen simetría $\mathrm{C}_{2}$ y los centros estereogénicos se encuentran en los átomos de carbono. Por regla general, el uso de difosfinas $(R, R)$ conduce a clústeres $P$ ópticamente puros, mientras que la obtención de clústeres $M$ es posible al coordinar difosfinas $(S, S)$ a la unidad $\mathrm{M}_{3} \mathrm{~S}_{4}$. Con la letra $P$ se designa al enantiómero que sitúa los haluros en sentido horario, y con la letra $M$ al que los sitúa en sentido antihorario, siempre con el azufre apuntado $\mu_{3}-\mathrm{S}$ mirando hacia el lector, tal como se ilustra en la figura 5.3. 


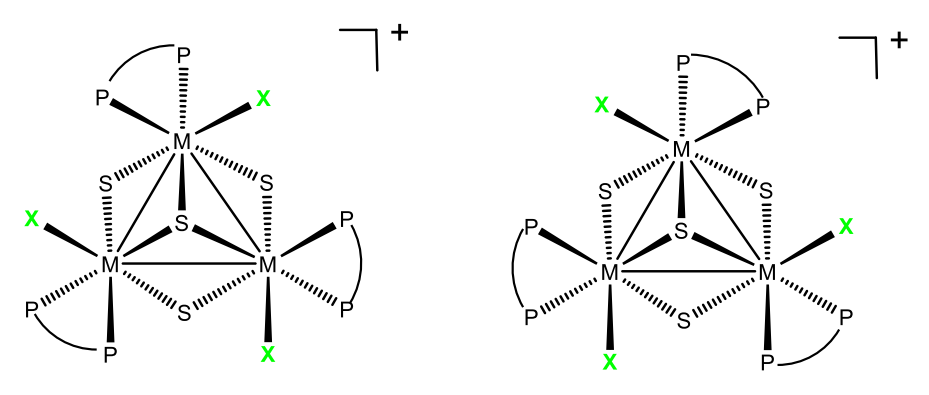

Figura 5.3. Representación simplificada del par enantiomérico $(P)-\left[\mathrm{M}_{3} \mathrm{~S}_{4} \mathrm{Cl}_{3}(\text { difosfina* })_{3}\right]^{+}$ (izquierda) y $(M)-\left[\mathrm{M}_{3} \mathrm{~S}_{4} \mathrm{Cl}_{3}(\text { difosfina* })_{3}\right]^{+}$(derecha).

La incorporación de cobre a las anteriores especies $\mathrm{Mo}_{3} \mathrm{~S}_{4}$ resulta en compuestos heterodimetálicos $\mathrm{Mo}_{3} \mathrm{CuS}_{4}$ que conservan la configuración absoluta de los clústeres de partida y presentan actividad catalítica en reacciones de ciclopropanación, con buenos rendimientos y enantioselectividades moderadas.[14] Asimismo, el grupo de Llusar ha descrito recientemente la actividad catalítica de clústeres con unidad central $\mathrm{M}_{3} \mathrm{~S}_{4}$ funcionalizados con ligandos difosfina, aminofosfina y diamina en reacciones como la hidrodefluoración de la pentafluoropiridina y la reducción de nitrocompuestos.[16-20]

Motivados por estos resultados, unidos al papel destacado del grupo NH en catálisis ${ }^{[21]}$ y al interés industrial de las reacciones enantioselectivas, hemos decidido explorar nuevas rutas sintéticas para la obtención de clústeres funcionalizados con aminofosfinas quirales, y estudiar su papel en la reducción asimétrica de diversos sustratos orgánicos. Para ello, se han elegido dos aminofosfinas enantiómeras derivadas de la prolina: $(R)-$ y $(S)-2-[($ difenilfosfino)metil]pirrolidina $((R)-P P r o$ y $(S)$ PPro)), que se encuentran disponibles comercialmente. Su estructura se muestra en la figura 5.4. 

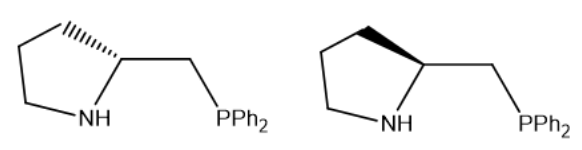

Figura 5.4. Estructuras de las aminofosfinas quirales (R)-PPro (izquierda) y (S)-PPro (derecha).

La funcionalización de compuestos monometálicos de rutenio con aminofosfinas o diaminas quirales derivadas de la prolina ha dado lugar a numerosas especies catalíticamente activas, concretamente en reacciones de hidrogenación de imidas, carboxamidas y cetonas, con buenos rendimientos y enantioselectividades. ${ }^{22-}$ 24] Esta circunstancia hace atractiva la combinación de estos ligandos con la unidad clúster $\mathrm{Mo}_{3} \mathrm{~S}_{4}$ con vistas a su potencial papel en catálisis. En este capítulo se detallan el método de síntesis, los estudios teóricos y las pruebas catalíticas realizadas con estos nuevos compuestos. 


\subsection{SÍNTESIS Y CARACTERIZACIÓN}

Las fases poliméricas $\left\{\mathrm{M}_{3} \mathrm{~S}_{7} \mathrm{X}_{4}\right\}_{\mathrm{n}}(\mathrm{M}=\mathrm{Mo}, \mathrm{W} ; \mathrm{X}=\mathrm{Cl} ; \mathrm{Br})$ junto con los precursores moleculares de fórmula $\left[\mathrm{M}_{3} \mathrm{~S}_{7} \mathrm{X}_{6}\right]^{2-}(\mathrm{M}=\mathrm{Mo}, \mathrm{W} ; \mathrm{X}=\mathrm{Cl}, \mathrm{Br})$ constituyen un buen punto de partida para la obtención de clústeres cuboidales $\mathrm{M}_{3} \mathrm{~S}_{4}$ con ligandos difosfina y aminofosfina en rendimientos elevados. ${ }^{[25,26]}$ La fuerza motriz de ambas reacciones se atribuye a la reducción de los puentes disulfuro de la unidad central $\mathrm{M}_{3} \mathrm{~S}_{7}$, con la consecuente coordinación del ligando quelato al metal dando lugar a un cambio estructural que conduce a la unidad $\mathrm{M}_{3} \mathrm{~S}_{4}$.

Otra estrategia consiste en reducir los puentes disulfuro del clúster $\left[\mathrm{Mo}_{3} \mathrm{~S}_{7} \mathrm{X}_{6}\right]^{2-}(\mathrm{X}=\mathrm{Cl}, \mathrm{Br})$ con un exceso de trifenilfosfina en una primera etapa para generar el complejo $\left[\mathrm{Mo}_{3} \mathrm{~S}_{4} \mathrm{Cl}_{4}\left(\mathrm{PPh}_{3}\right)_{3}\left(\mathrm{H}_{2} \mathrm{O}\right)_{2}\right]$.[27] Seguidamente, en una segunda etapa de reacción, se añaden cantidades estequiométricas del ligando difosfina o aminofosfina. ${ }^{[28]}$ Resulta evidente que una de las ventajas de este procedimiento de síntesis es la económica, ya que se utiliza una menor cantidad de ligando al no tener que reducir los puentes disulfuro, trabajo que realiza la trifenilfosfina en el paso previo. En este caso, este procedimiento ha sido el elegido para preparar los clústeres de fórmula $\left[\mathrm{Mo}_{3} \mathrm{~S}_{4} \mathrm{Cl}_{3}(\mathrm{PPro})_{3}\right]^{+}$debido al elevado coste de los ligandos enantiopuros (R)-PPro y (S)-PPro. El esquema de reacción se muestra en la ecuación 5.1.

$$
\left[\mathrm{Mo}_{3} \mathrm{~S}_{4} \mathrm{Cl}_{4}\left(\mathrm{PPh}_{3}\right)\left(\mathrm{H}_{2} \mathrm{O}\right)_{2}\right]+3 \mathrm{PPro} \rightarrow\left[\mathrm{Mo}_{3} \mathrm{~S}_{4} \mathrm{Cl}_{3}(\mathrm{PPro})_{3}\right] \mathrm{Cl}+3 \mathrm{PPh}_{3}+2 \mathrm{H}_{2} \mathrm{O}
$$

La reacción del clúster precursor $\left[\mathrm{Mo}_{3} \mathrm{~S}_{4} \mathrm{Cl}_{4}\left(\mathrm{PPh}_{3}\right)\left(\mathrm{H}_{2} \mathrm{O}\right)_{2}\right]$ con cantidades estequiométricas de la aminofosfina (R)-PPro o su enantiómera ( $S$ )-PPro, nos conduce a la formación de los clústeres ópticamente activos $\left[\mathrm{Mo}_{3} \mathrm{~S}_{4} \mathrm{Cl}_{3}((1 S, 2 \mathrm{R})\right.$ PPro $\left.)_{3}\right] \mathrm{Cl}(\mathbf{7}(\mathrm{Cl}))$ y $\left[\mathrm{Mo}_{3} \mathrm{~S}_{4} \mathrm{Cl}_{3}((1 S, 2 S)-\mathrm{PPro})_{3}\right] \mathrm{Cl}(\mathbf{8}(\mathrm{Cl}))$. La coordinación específica de la aminofosfina quiral a la unidad trimetálica conlleva la formación de dos centros estereogénicos adicionales: los átomos de nitrógeno del ligando y la unidad clúster $\mathrm{Mo}_{3} \mathrm{~S}_{4}$. En cuanto al nitrógeno del ligando, los estudios estructurales que se discutirán 
más adelante muestran que, al coordinarse al metal, se convierte en un centro estereogénico que adopta de forma inequívoca la configuración $S$ en ambos compuestos. En cuanto a los átomos metálicos, los estudios previos de Llusar y colaboradores demuestran que la coordinación de difosfinas quirales $(R, R)$ conducen de forma inequívoca a clústeres de configuración absoluta $P$, mientras que difosfinas $(S, S)$ a clústeres $M \cdot[14,15,29,30]$ En este caso, la caracterización mediante ${ }^{31} \mathrm{P}\left\{{ }^{1} \mathrm{H}\right\}-\mathrm{RMN}$ nos dio un indicio de que nuestros compuestos no seguían esta tendencia: en ambos espectros se observa una única señal que corresponde a los tres átomos de fósforo equivalentes a desplazamientos ligeramente diferentes: $32,3 \mathrm{ppm}$ para el complejo $7^{+}$ y $32,1 \mathrm{ppm}$ en el caso del complejo $\mathbf{8}^{+}$, tal y como se puede observar en la figura 5.5.

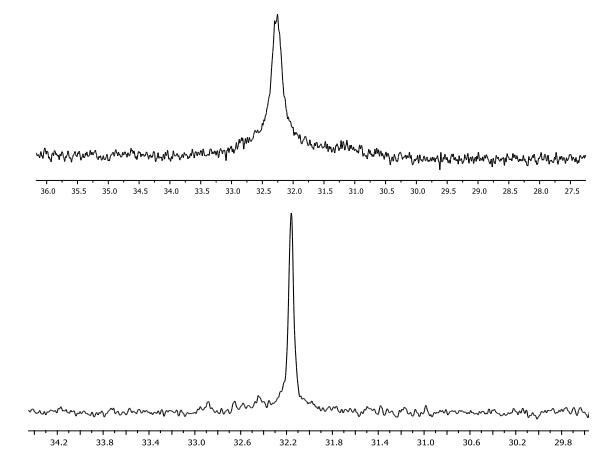

Figura 5.5. Espectros de ${ }^{31} \mathrm{P}\left\{{ }^{1} \mathrm{H}\right\}-\mathrm{RMN}$ de los complejos $\mathbf{7}^{+}$(arriba) y $\mathbf{8}^{+}$(abajo).

El hecho de que los complejos $\mathbf{7}^{+}$y $\mathbf{8}^{+}$no sean enantiómeros entre sí se ha confirmado mediante la caracterización de estos compuestos por dicroísmo circular, donde no se observan señales estrictamente antípodas, hecho que sí ocurría en el caso de clústeres funcionalizados con difosfinas quirales enantiómeras. ${ }^{[14]} \mathrm{El}$ espectro del complejo $7(\mathrm{Cl})$ muestra dos señales a $\lambda_{\text {máx }}$ de $260 \mathrm{~nm}$ y $301 \mathrm{~nm}$ a $+51,7$ mdeg y $+14,7$ mdeg respectivamente, mientras que el espectro de $\mathbf{8}(\mathrm{Cl})$ presenta dos señales de signo opuesto a las anteriores, a $\lambda_{\text {máx }}$ de $254 \mathrm{~nm}$ y $313 \mathrm{~nm}$, a -46 mdeg y -3,2 mdeg, como ilustra la figura 5.6. Los signos opuestos de estas dos bandas sugieren una importante contribución del ligando a los orbitales involucrados en estas transiciones. 


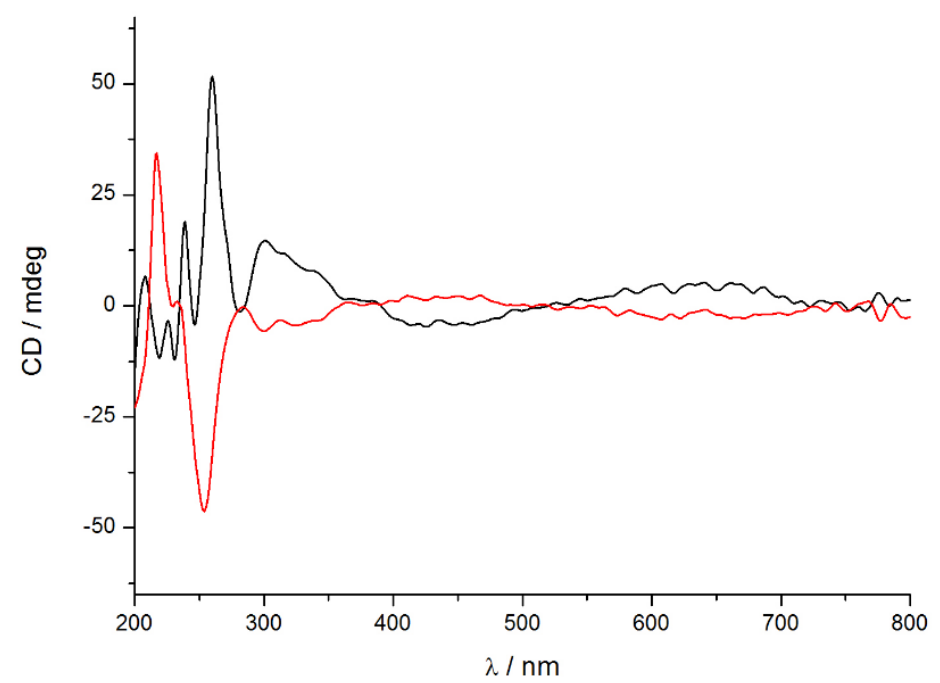

Figura 5.6. Espectros de dicroísmo circular de los complejos $7(\mathrm{Cl})$ (negro) y $8(\mathrm{Cl})$ (rojo).

Las bandas registradas por debajo de $250 \mathrm{~nm}$ son del mismo signo en los compuestos $7^{+}$y $\mathbf{8}^{+}$, y pueden asignarse de manera tentativa a transiciones entre orbitales relacionados con el nitrógeno estereogénico. ${ }^{[31,32]}$ Los valores de rotación específica son, aproximadamente, un orden de magnitud más pequeños que los referenciados para complejos análogos funcionalizados con difosfinas quirales, que exhiben valores desde -400 mdeg hasta +450 mdeg. ${ }^{[14]}$

Una vez demostrado que los complejos $7^{+}$y $\mathbf{8}^{+}$no son enantiómeros entre sí, se ha procedido a determinar su configuración absoluta mediante difracción de rayos X en monocristal. Ambas estructuras se han resuelto en el grupo espacial cúbico no centrosimétrico P213 con parámetros de Flack cercanos a cero donde las unidades $\mathrm{Mo}_{3} \mathrm{~S}_{4}$ se sitúan sobre un eje $\mathrm{C}_{3}$. Los tres átomos de molibdeno definen un triángulo equilátero con la presencia de un azufre apuntado $\mu_{3}-\mathrm{S}$ y tres azufres puente $\mu$-S situados en planos opuestos con respecto al plano definido por los tres metales. La configuración absoluta se ha determinado como $P$ en ambos complejos, ya que los ligandos cloruro giran alrededor de la unidad $\mathrm{Mo}_{3} \mathrm{~S}_{4}$ en el sentido de las agujas del reloj, siempre con el azufre $\mu_{3}-\mathrm{S}$ apuntando hacia el lector. La representación ORTEP de ambas estructuras se muestra en la figura 5.7. 

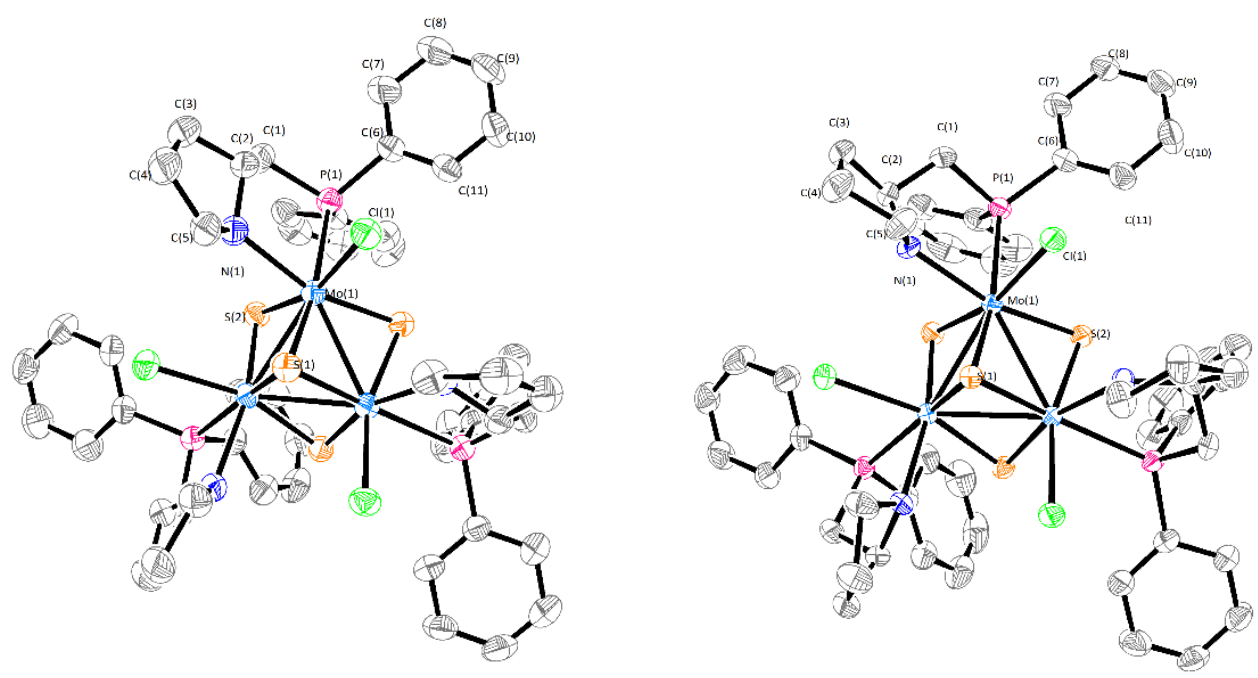

Figura 5.7. Representaciones ORTEP de $(P)-\left[\mathrm{Mo}_{3} \mathrm{~S}_{4} \mathrm{Cl}_{3}((1 S, 2 \mathrm{R})-\mathrm{PPro})_{3}\right]^{+}\left((P)-\mathbf{7}^{+}\right)$ (izquierda) y $(P)-\left[\mathrm{Mo}_{3} \mathrm{~S}_{4} \mathrm{Cl}_{3}((1 S, 2 S)-\mathrm{PPro})_{3}\right]^{+}\left((P)-8^{+}\right)$(derecha). Los átomos de hidrógeno, contraiones y disolvente se han omitido por claridad.

La difracción de rayos $\mathrm{X}$ en monocristal confirma que la quiralidad de las aminofosfinas se preserva tras su coordinación al átomo metálico y que el nitrógeno del anillo pirrolidina se convierte en un centro estereogénico al coordinarse de forma inequívoca con una conformación $S$ en ambos complejos (ver figura 5.8 más adelante). En la tabla 5.1 se muestran las distancias de enlace más relevantes de ambas estructuras, así como las de un clúster trinuclear de molibdeno funcionalizado con ligandos aminofosfina de elevada semejanza estructural. 
Tabla 5.1. Selección de distancias de enlace promedio para los compuestos $(P)-7^{+},(P)-\mathbf{8}^{+}$y $\left[\mathrm{Mo}_{3} \mathrm{~S}_{4} \mathrm{Cl}_{3}(\mathrm{edpp})_{3}\right]^{+}$. La desviación estándar de los valores promedio se encuentra representada entre corchetes.

\begin{tabular}{cccc}
\hline \hline Distancia $(\AA)$ & $(P)-7^{+}$ & $(P)-8^{+}$ & {$\left[\mathbf{M o}_{3} \mathbf{S}_{4} \mathbf{C l}_{3}(\mathbf{e d p p})_{3}\right]^{+(a)[33]}$} \\
\hline Mo-Mo & $2,752(1)$ & $2,757(1)$ & $2,7463[7]$ \\
Mo- $\left(\mu_{3}-\mathrm{S}\right)$ & $2,364(3)$ & $2,353(3)$ & $2,3611[13]$ \\
Mo- $(\mu-S)^{(b)}$ & $2,297(2)$ & $2,294(2)$ & $2,2960[12]$ \\
Mo- $(\mu-S)^{(c)}$ & $2,277(2)$ & $2,293(2)$ & $2,2863[12]$ \\
Mo-P & $2,547(2)$ & $2,550(2)$ & $2,5414[14]$ \\
Mo-N & $2,300(7)$ & $2,289(7)$ & $2,2733[4]$ \\
Mo-Cl & $2,461(2)$ & $2,484(2)$ & $2,4625[15]$ \\
\hline \hline
\end{tabular}

(a)Datos extraídos de la referencia [33]. ${ }^{(\mathrm{b})}$ Distancia trans al enlace Mo-Cl. (c) Distancia trans al enlace Mo-N.

() Desviación estándar de la medida.

[] Desviación estándar del promedio.

Las distancias Mo-Mo y Mo-S siguen las tendencias observadas en otros compuestos similares.[17,30,34] Cada átomo de molibdeno presenta un entorno pseudooctaédrico definido por tres átomos de azufre, uno de cloro y los átomos de nitrógeno y fósforo del ligando aminofosfina. La coordinación específica del ligando sigue la tendencia observada en clústeres análogos hasta la fecha, en los que el átomo de fósforo se encuentra en posición trans al azufre apuntado, mientras que el átomo de nitrógeno se encuentra trans a los azufres puente. ${ }^{26,33]}$ Las distancias de enlace en los clústeres $(P)-7^{+}$y $(P)-8^{+}$son semejantes a las descritas para el clúster análogo $\left[\mathrm{Mo}_{3} \mathrm{~S}_{4} \mathrm{Cl}_{3}(\mathrm{edpp})_{3}\right]^{+}$. Las dos distancias $\mathrm{Mo}-(\mu-\mathrm{S})$ tienden a igualarse si las comparamos con las observadas para clústeres funcionalizados con difosfinas, debido a la similar influencia trans de los ligandos amino y cloruro.[18] Además, tal y como se ha detallado anteriormente, la coordinación del ligando quiral conlleva la formación 
de un nuevo centro estereogénico en el nitrógeno pirrolidínico, que se incorpora a un sistema bicíclico [3.3.0] tal y como muestra la figura 5.8.
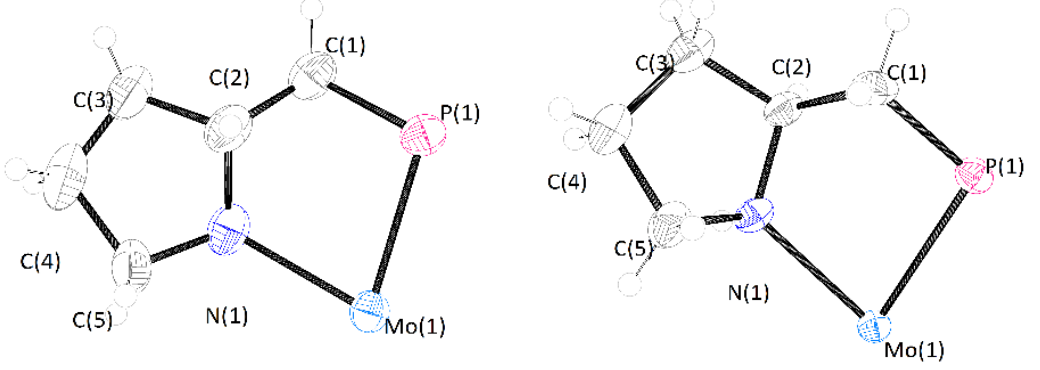

Figura 5.8. Representaciones ORTEP de los sistemas bicíclicos [3.3.0] formados al coordinarse los ligandos aminofosfina al molibdeno en los complejos $(P)-7^{+}$(izquierda) y $(P)-8^{+}($derecha $)$.

Este sistema bicíclico adopta una conformación cis en el complejo $(P)-\mathbf{8}^{+}$, que presenta una mayor estabilidad que la conformación trans en compuestos de similar disposición estructural como son los biciclooctanos.[35] Los ángulos diedros $\mathrm{C}(5)-\mathrm{N}$ $\mathrm{C}(2)-\mathrm{C}(1)$ y $\mathrm{C}(3)-\mathrm{C}(2)-\mathrm{N}-\mathrm{Mo}$ calculados para $(P)-\mathbf{8}^{+}$confirman la conformación cis de este sistema bicíclico y constatan las diferencias estructurales de este complejo con $(P)-7^{+}$, en el cual ambos anillos se encuentran aproximadamente en el mismo plano (ver tabla 5.2).

Tabla 5.2. Ángulos diedros de los sistemas bicíclicos [3.3.0] formados al coordinarse los ligandos (R)- y (S)-PPro a la unidad clúster trimetálica.

\begin{tabular}{ccc}
\hline \hline Ángulos diedros $\left(^{(}\right)$ & $(P)-7^{+}$ & $(P)-8^{+}$ \\
\hline $\mathrm{C}(5)-\mathrm{N}-\mathrm{C}(2)-\mathrm{C}(1)$ & 11,269 & 96,860 \\
$\mathrm{C}(3)-\mathrm{C}(2)-\mathrm{N}-\mathrm{Mo}$ & 179,416 & 14,402 \\
\hline \hline
\end{tabular}


En los complejos con unidad $\mathrm{Mo}_{3} \mathrm{~S}_{4}$ funcionalizados con aminofosfinas, los átomos de cloro y los grupos amino se encuentran en el mismo lado del plano que forman los tres metales, lo que permite múltiples interacciones entre el átomo de hidrógeno de esta amina secundaria y el cloro coordinado al metal adyacente, identificadas como interacciones vecinales.[26] En el complejo $(P)-8^{+}$se observa que esta distancia $\mathrm{Cl} \cdots \mathrm{HN}$ es de $2,379 \AA$, produciendo una interacción que se postula como uno de los factores estabilizantes de este compuesto (ver figura 5.9). La misma distancia también se ha medido para el compuesto $(P)-7^{+}$y resulta ser más notablemente más larga $(2,941 \AA)$.

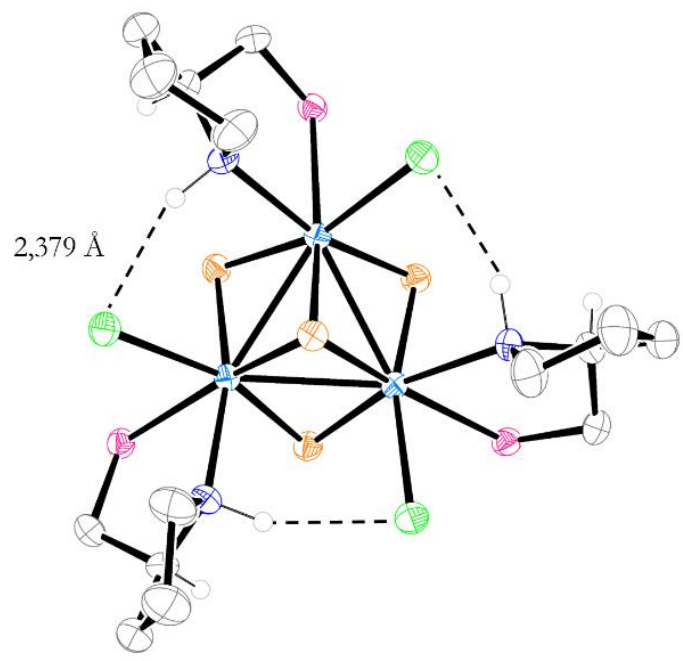

Figura 5.9. Representación ORTEP del complejo $(P)-8^{+}$con sus interacciones $\mathrm{Cl} \cdots \mathrm{HN}$ vecinales. Los grupos fenilo se han omitido por claridad.

Las interacciones entre el grupo NH y el átomo de cloro coordinados al mismo metal, identificadas como geminales, también han sido estudiadas. Las distancias $\mathrm{Cl} \cdots \mathrm{HN}$ geminales son de $3,055 \AA$ para el compuesto $(P)-7^{+}$y $3,170 \AA$ para el $(P)-\mathbf{8}^{+}$, ligeramente más largas que en el complejo funcionalizado con edpp, que presentaba un valor medio de 3,021 Å.[18]

Además de los estudios de RMN, dicroísmo circular y difracción de rayos $\mathrm{X}$ en monocristal, la caracterización de estos clústeres se ha completado mediante 
espectrometría de masas, utilizando como fuente de ionización electrospray (ESI-MS). En los espectros de la figura 5.10 se identifican claramente los picos correspondientes a los iones moleculares $\left[\mathrm{Mo}_{3} \mathrm{~S}_{4} \mathrm{Cl}_{3}(\mathrm{PPro})_{3}\right]^{+}$que se pueden asignar a los complejos $(P)-7^{+}$y $(P)-8^{+}$. Ambos picos aparecen a una relación $m / z$ de 1330 en concordancia con los experimentos anteriores, puesto que ambos complejos presentan la misma masa molecular.
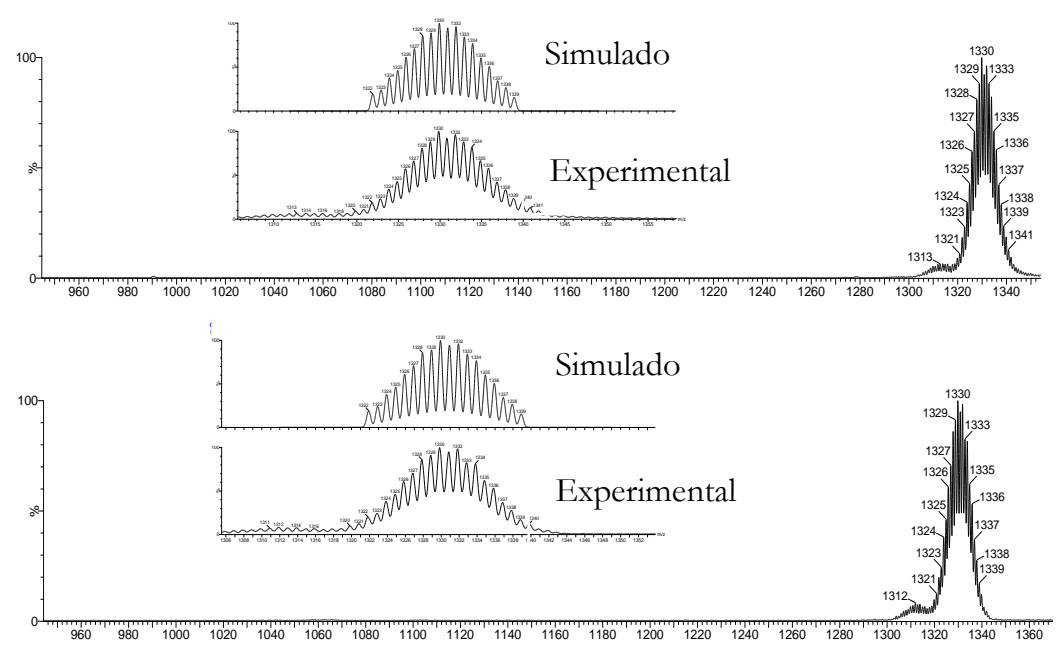

Figura 5.10. Espectros de masas experimentales y simulados de los complejos $(P)-7^{+}$(arriba) y $(P)-8^{+}$(abajo).

En conclusión, esta sección demuestra que la reacción entre el precursor $\left[\mathrm{Mo}_{3} \mathrm{~S}_{4} \mathrm{Cl}_{4}\left(\mathrm{PPh}_{3}\right)\left(\mathrm{H}_{2} \mathrm{O}\right)_{2}\right]$ y las aminofosfinas enantiómeras (R)- o (S)-PPro es diastereoselectiva hacia la formación de clústeres ópticamente puros con configuración $P$. Además, se ha comprobado que la conformación de la aminofosfina se mantiene tras su coordinación a la unidad trinuclear y que esta coordinación específica provoca la transformación del nitrógeno en un nuevo centro estereogénico de configuración $S$. El hecho de que estos clústeres no hayan seguido la tendencia observada para los clústeres funcionalizados con difosfinas quirales, en los cuales los ligandos $(R, R)$ daban lugar a clústeres con configuración $P$ y los ligandos $(S, S)$ a 
clústeres $M,[14,15,29]$ ha propiciado la realización de cálculos teóricos DFT, con el fin de analizar las diferencias de energía entre los cuatro posibles isómeros: $(P)-7^{+},(P)$ $\mathbf{8}^{+},(M)-\mathbf{7}^{+}$y $(M)-\mathbf{8}^{+}$e identificar las causas que dirigen la diastereoselectividad de este proceso de síntesis. 


\subsection{ESTUDIO TEÓRICO}

\subsubsection{OPTIMIZACIÓN DE GEOMETRÍAS}

Las geometrías de los cuatro isómeros posibles, los aislados experimentalmente $(P)$ $7^{+}$y $(P)-\mathbf{8}^{+}$, y los clústeres hipotéticos $(M)-7^{+}$y $(M)-\mathbf{8}^{+}$se han optimizado con el paquete de programas Gaussian 09[36] mediante cálculos DFT con el funcional híbrido B3LYP. Como punto de partida hemos tomado las estructuras cristalinas de $(P)-7^{+}$y $(P)-\mathbf{8}^{+}$, mientras que las estructuras iniciales de los complejos $(M)-7^{+}$y $(M)$ $8^{+}$se han construido intercambiando las posiciones del ligando cloruro y el nitrógeno de la aminofosfina, sin alterar la configuración de los centros estereogénicos presentes en el ligando. La tabla 5.3 lista las distancias de enlace de las geometrías optimizadas, así como las energías relativas de los cuatro diastereoisómeros.

Tabla 5.3. Selección de distancias de enlace promedio calculadas al nivel B3LYP/lanl2dz para las geometrías optimizadas de los compuestos $(P)-\mathbf{7}^{+},(P)-\mathbf{8}^{+},(M)-\mathbf{7}^{+}$y $(M)-\mathbf{8}^{+}$y sus energías.

\begin{tabular}{ccccc}
\hline \hline Distancia $(\AA)$ & $(P)-7^{+}$ & $(P)-8^{+}$ & $(M)-7^{+}$ & $(M)-8^{+}$ \\
\hline Mo-Mo & $2,8074[8]$ & $2,8054[3]$ & $2,8329[12]$ & $2,8446[18]$ \\
Mo- $\left(\mu 3_{-}-\mathrm{S}\right)$ & $2,4377[5]$ & $2,4431[9]$ & $2,4604[19]$ & $2,4491[11]$ \\
Mo- $(\mu-S)^{(a)}$ & $2,3788[1]$ & $2,3673[6]$ & $2,3558[5]$ & $2,3712[7]$ \\
Mo- $(\mu-S))^{(b)}$ & $2,3800[1]$ & $2,3869[2]$ & $2,3763[7]$ & $2,3761[8]$ \\
Mo-P & $2,6605[3]$ & $2,6309[14]$ & $2,6720[41]$ & $2,6334[19]$ \\
Mo-N & $2,2885[14]$ & $2,2912[16]$ & $2,2957[5]$ & $2,3615[9]$ \\
Mo-Cl & $2,5500[9]$ & $2,5699[9]$ & $2,6009[17]$ & $2,5438[25]$ \\
\hline \hline Energía libre & 4,8 & 0,0 & 20,2 & 20,0 \\
(kcal/mol) $\mathbf{c}$ & & & & \\
\hline \hline
\end{tabular}

(a) Distancia trans al enlace Mo-Cl. (b)Distancia trans al enlace Mo-N. (c)Relativas al complejo $(P)-8^{+}$

[] Desviación estándar del promedio. 
Los menores valores de energía libre corresponden a los clústeres $(P)-7^{+}$y $(P)-\mathbf{8}^{+}$, que son los obtenidos experimentalmente. La figura 5.11 muestra la geometría optimizada del clúster catiónico $(P)-\mathbf{8}^{+}$, tomado como referencia por ser el más estable. Esta mayor estabilidad energética concuerda con la estabilización esperada atribuida a la conformación cis del sistema bicíclico y a las cortas distancias observadas entre los átomos de cloro y los hidrógenos del grupo amino unidos a metales vecinos (ver figura 5.9). Mientras que el clúster $(P)-7^{+}$resulta ser $15,4 \mathrm{kcal} / \mathrm{mol}$ más estable que el $(M)-7^{+}$, el complejo $(P)-8^{+}$es $20,0 \mathrm{kcal} / \mathrm{mol}$ más estable que el $(M)-\mathbf{8}^{+}$, lo que confirma la preferencia termodinámica hacia la formación de especies $P$, tal como se ha comprobado de manera experimental.

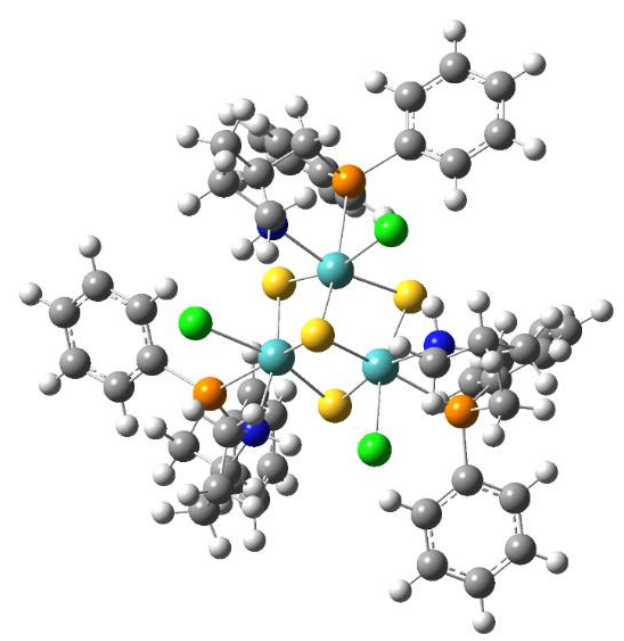

Figura 5.11. Geometría optimizada del complejo $(P)-\left[\mathrm{Mo}_{3} \mathrm{~S}_{4} \mathrm{Cl}_{3}((1 S, 2 S)-\mathrm{PPro})_{3}\right]^{+}\left((P)-\mathbf{8}^{+}\right)$

Las distancias de enlace optimizadas para los complejos $(P)-7^{+}$y $(P)-\mathbf{8}^{+}$ concuerdan con las determinadas experimentalmente a partir de difracción de rayos $\mathrm{X}$ (ver tabla 5.1), aunque por regla general las distancias teóricas son un poco más largas que las experimentales. Las distancias optimizadas para los isómeros $(M)-7^{+} \mathrm{y}$ (M) $-\mathbf{8}^{+}$, no obtenidos experimentalmente, siguen tendencias similares a las observadas en otros clústeres estructuralmente análogos.[26,33] En todos los casos las longitudes de enlace entre molibdeno y azufre apuntado son más largas que las distancias Mo-( $\mu$-S). A su vez las distancias Mo- $(\mu-\mathrm{S})$ trans al enlace Mo-Cl y trans al 
enlace Mo-N presentan valores similares a diferencia de lo observado en los clústeres análogos funcionalizados con difosfinas. ${ }^{[3,37]}$ Los valores optimizados reproducen, por tanto, la influencia trans similar de los ligandos cloruro y amino.

La tabla 5.4 lista los ángulos diedros experimentales y optimizados, relacionados con las conformaciones del sistema bicíclico formado tras la coordinación del ligando al metal (ver figura 5.8). Los valores optimizados para (P)$7^{+}$y $(P)-8^{+}$concuerdan con los valores experimentales, de los cuales difieren como máximo en $3^{\circ}$. La conformación cis de este sistema bicíclico, observada en la estructura cristalina de $(P)-\mathbf{8}^{+}$y también en la geometría optimizada del mismo nombre, se postula como uno de los factores que provocan la mayor estabilidad del compuesto $(P)-8^{+}$.

Tabla 5.4. Ángulos diedros de los sistemas bicíclicos [3.3.0] de los complejos $(P)-7^{+}$y $(P)-\mathbf{8}^{+}$ y las geometrías optimizadas $(P)-\mathbf{7}^{+},(P)-\mathbf{8}^{+},(M)-\mathbf{7}^{+}$y $(M)-\mathbf{8}^{+}$.

\begin{tabular}{ccc|cccc}
\hline \hline & \multicolumn{3}{c|}{ Experimentales } & \multicolumn{4}{|c}{ Optimizados } \\
\hline Ángulos diedros ( $\left.\mathbf{(}^{\mathbf{}}\right)$ & $(P)-\mathbf{7}^{+}$ & $(P)-\mathbf{8}^{+}$ & $(P)-\mathbf{7}^{+}$ & $(P)-\mathbf{8}^{+}$ & $(M)-\mathbf{7}^{+}$ & $(M)-\mathbf{8}^{+}$ \\
\hline $\mathrm{C}(5)-\mathrm{N}-\mathrm{C}(2)-\mathrm{C}(1)^{(\mathrm{a})}$ & 11,269 & 96,860 & 8,770 & 95,723 & 24,866 & 81,800 \\
$\mathrm{C}(3)-\mathrm{C}(2)-\mathrm{N}-\mathrm{Mo}$ & 179,416 & 14,402 & 179,573 & 14,052 & 6,480 & 4,885 \\
\hline \hline
\end{tabular}

(a)La numeración de los átomos hace referencia a la figura 5.8.

La distancia $\mathrm{Cl} \cdots \mathrm{HN}$ vecinal ha resultado ser de $2,320 \AA$ para la estructura optimizada de $(P)-\mathbf{8}^{+}$, valor muy similar al experimental, lo que refuerza la idea de su carácter estabilizante. Sin embargo, en el complejo $(M)-7^{+}$se ha observado una distancia de tan solo 2,255 $\AA$ entre el ligando cloruro y el hidrógeno enlazado al nitrógeno en posición geminal. Con objeto de profundizar en la naturaleza de estas interacciones se han llevado a cabo estudios de distribución electrónica mediante cálculos NBO (Natural Bond Orbital) y ELF (Electron Localization Function). 


\subsubsection{ANÁLISIS DE DISTRIBUCIÓN ELECTRÓNICA}

\subsubsection{ORBITALES NATURALES DE ENLACE (NBO)}

$\mathrm{El}$ análisis NBO permite obtener orbitales localizados a partir de funciones de onda ab-initio. Estos orbitales se pueden relacionar con enlaces, pares libres de electrones y antienlaces, proporcionando información sobre la naturaleza electrónica de una interacción molecular en un complejo. El orbital NBO localizado de un enlace $\sigma_{\mathrm{AB}}$ entre los átomos A y B se forma a partir de los orbitales naturales híbridos (NHO) $h_{A}$ y h $h_{B}$ según la ecuación 5.2,[38,39] y a su vez, estos orbitales naturales híbridos están compuestos por una serie de orbitales atómicos naturales (NAO) optimizados para la función de onda elegida.

$$
\sigma_{\mathrm{AB}}=\mathrm{c}_{\mathrm{A}} \mathrm{h}_{\mathrm{A}}+\mathrm{c}_{\mathrm{B}} \mathrm{h}_{\mathrm{B}}
$$

Los orbitales naturales de enlace requieren simultáneamente ortonormalidad y máxima ocupación, de esta forma se pueden obtener expresiones matemáticas de propiedades atómicas y de enlace. Las funciones de onda ab-initio transformadas a la forma NBO concuerdan con los conceptos de la teoría de Lewis, lo que permite describir las contribuciones covalentes en moléculas. Esta transformación también puede dar lugar a orbitales antienlazantes $\sigma^{*}{ }_{\mathrm{AB}}$ tal como muestra la ecuación 5.3.

$$
\sigma^{*}{ }_{\mathrm{AB}}=\mathrm{c}_{\mathrm{A}} \mathrm{h}_{\mathrm{A}}-\mathrm{c}_{\mathrm{B}} \mathrm{h}_{\mathrm{B}}
$$

La energía asociada a estos antienlaces se puede evaluar de forma numérica eliminando estos orbitales del conjunto de bases y recalculando la energía total, que es la suma del componente enlazante (contribución covalente) y antienlazante (no covalente).

$$
\mathrm{E}=\mathrm{E}_{\sigma \sigma}+\mathrm{E}_{\sigma \sigma^{*}}
$$

Las contribuciones antienlazantes $\mathrm{E}_{\sigma \sigma^{*}}$ usualmente tienen un valor menor del $1 \% \mathrm{si}$ las comparamos con las contribuciones enlazantes $\mathrm{E}_{\sigma \sigma}$, por lo que el dominio de las contribuciones de tipo Lewis parece evidente. ${ }^{[40]}$ 
A partir del análisis mediante $\mathrm{NBO}$ se pueden estudiar una serie de propiedades de un compuesto, entre las que se encuentran la carga natural de sus átomos y los órdenes de enlace, por lo que resulta una herramienta útil para conocer de forma más amplia la naturaleza de los enlaces que tienen lugar en nuestros clústeres. Para la realización de este estudio se ha utilizado el paquete de software Gaussian $09^{[36]}$ con el objetivo de conocer los órdenes de enlace más importantes de los cuatro isómeros $(P)-\mathbf{7}^{+},(P)-\mathbf{8}^{+},(M)-\mathbf{7}^{+}$y $(M)-\mathbf{8}^{+}$. Los cálculos se han realizado al nivel B3LYP utilizando la base 3-21G. En la tabla 5.5 se muestran los órdenes de enlace obtenidos mediante estos cálculos.

Tabla 5.5. Órdenes de enlace obtenidos con los cálculos NBO en el nivel B3LYP con la base $3-21 G$.

\begin{tabular}{ccccc}
\hline \hline & \multicolumn{4}{c}{ Orden de enlace (B promedio) $^{(\mathrm{a})}$} \\
\hline Enlace & $(P)-7^{+}$ & $(P)-\mathbf{8}^{+}$ & $(M)-7^{+}$ & $(M)-\mathbf{8}^{+}$ \\
\hline Mo-Mo & 0,7505 & 0,7506 & 0,7428 & 0,7361 \\
Mo-P & 0,6261 & 0,6397 & 0,6182 & 0,6345 \\
Mo-N & 0,4755 & 0,4784 & 0,4889 & 0,4468 \\
Mo-Cl & 0,7263 & 0,6864 & 0,6552 & 0,7400 \\
N-H & 0,7667 & 0,7294 & 0,7472 & 0,7818 \\
$\mathrm{Cl} \cdots \mathrm{HN}$ & 0,0131 & 0,0519 & - & 0,0019 \\
$\mathrm{Cl} \cdots \mathrm{HN}_{\text {gem }}$ & - & - & 0,0425 & 0,0054 \\
\hline \hline
\end{tabular}

(a) Índices de enlace de Wiberg.

Los enlaces Mo-Mo son similares entre los cuatro isómeros y tienen un carácter mayoritariamente covalente, ya que se acercan al orden de enlace formal de la unidad, de acuerdo con la visión del enlace de Lewis. Una situación similar se observa para los enlaces $\mathrm{N}-\mathrm{H}$, aunque existen diferencias destacables entre los isómeros. Los órdenes de enlace más pequeños corresponden a los complejos $(P)-\mathbf{8}^{+}$ 
y $(M)-7^{+}$, lo que está en concordancia con la participación de los átomos de hidrógeno en sendas interacciones vecinal y geminal. Esta situación se repite para los enlaces Mo-Cl, con un carácter mayoritariamente covalente, donde los órdenes de enlace más pequeños son los asociados a $(P)-\mathbf{8}^{+}$y $(M)-7^{+}$debido a la participación del cloro en estas interacciones.

Los enlaces Mo-P presentan un menor carácter covalente que los $\mathrm{Mo}-\mathrm{Cl}$, ya que el orden de enlace es ligeramente menor. Sin embargo, llaman la atención los órdenes de enlace Mo-N, todos menores de 0,5, lo que sugiere una pérdida importante del carácter covalente asociado a este enlace en comparación con la visión de enlace de Lewis.

Con excepción del isómero $(M)-7^{+}$, el resto presentan interacciones $\mathrm{Cl} \cdots \mathrm{HN}$ vecinales, donde el mayor orden de enlace corresponde al clúster $(P)-\mathbf{8}^{+}$. En el caso de las interacciones geminales, únicamente detectadas en los isómeros $M$, el mayor orden de enlace se observa para el clúster $(M)-7^{+}$. Estos resultados concuerdan con los obtenidos durante la optimización de geometrías, donde se encontraban dos distancias $\mathrm{Cl} \cdots \mathrm{HN}$ cortas: una vecinal de 2,320 $\AA$ para $(P)-\mathbf{8}^{+}$, y una geminal de 2,255 $\AA$ para $(M)-7^{+}$. El orden de enlace de la interacción geminal presente en $(M)-7^{+}$es, aproximadamente, un $17 \%$ más pequeño que el de la interacción vecinal encontrada en el compuesto $(P)-\mathbf{8}^{+}$, lo que está en concordancia con las energías relativas calculadas: el isómero $(P)-\mathbf{8}^{+}$es $20,2 \mathrm{kcal} / \mathrm{mol}$ más estable que el $(M)-7^{+}$. Cabe destacar que la interacción vecinal $\mathrm{Cl} \cdots \mathrm{HN}$ con mayor orden de enlace corresponde a una distancia más larga que la geminal, una tendencia contraria a lo habitual.

Por otra parte, el compuesto $(M)-\mathbf{8}^{+}$presenta una interacción vecinal lejana que provoca una ligera estabilización energética frente al compuesto $(M)-7^{+}$, que no presenta este tipo de interacción. Los órdenes de enlaces $\mathrm{Mo}-\mathrm{Cl}$ también siguen esta tendencia: en los isómeros $(P)-\mathbf{8}^{+}$y $(M)-7^{+}$son más pequeños debido a la 
participación del átomo de cloro en las interacciones $\mathrm{Cl} \cdots \mathrm{HN}$ vecinal y geminal, respectivamente.

\subsubsection{DENSIDAD ELECTRÓNICA (ELF)}

La función de localización electrónica (ELF) fue propuesta por Becke y Edgecombe en el año 1990 a partir de la densidad de probabilidad condicional expresada en la ecuación 5.5.[41]

$$
P_{\text {cond }}^{\alpha \alpha}\left(r_{1}, r_{2}\right)=\frac{\pi_{\alpha \alpha}\left(r_{1}, r_{2}\right)}{\rho_{\alpha}}
$$

$P_{c o n d}^{\alpha \alpha}\left(r_{1}, r_{2}\right)$ representa la densidad de probabilidad para que dos electrones del mismo espín se sitúen en $\mathrm{r}_{1}$ y $\mathrm{r}_{2}$. Cuando $\mathrm{r}_{1}=\mathrm{r}_{2}$ esta función se anula y su curvatura es una medida de la probabilidad de encontrar un electrón del mismo espín alrededor de la posición $\mathrm{r}_{1}$. La laplaciana de la densidad de probabilidad condicional cuando $\mathrm{r}_{1}=\mathrm{r}_{2}$ se representa como $D^{\sigma}(r)$ tal como se describe en la ecuación 5.6, donde $\phi_{i}(r)$ son orbitales de Hartree-Fock u orbitales de Kohn-Sham.

$$
D^{\sigma}(r)=\frac{1}{2} \sum_{i}\left|\nabla \phi_{i}(r)\right|^{2}-\frac{1}{8} \frac{\left|\nabla \rho^{\sigma}(r)\right|^{2}}{\rho^{\sigma}(r)}
$$

Cuando $D^{\sigma}(r)$ toma valores altos, los electrones con espines paralelos pueden aproximarse unos a otros, en cambio para valores de $D^{\sigma}(r)$ pequeños los electrones permanecen separados. De esta manera, los electrones con espines antiparalelos se localizarán en regiones donde $D^{\sigma}(r)$ tome valores pequeños.

Otra interpretación de la función de localización electrónica es la propuesta por Silvi y Savin en 1996 al considerar la laplaciana de la densidad de probabilidad condicional como un exceso de la energía cinética debido a la repulsión de Pauli, que se atribuye al término $\mathrm{T}_{\mathrm{s}}$ (ver ecuación 5.7).[42]

$$
D^{\sigma}(r)=T_{s}-\frac{1}{8} \frac{\left|\nabla \rho^{\sigma}(r)\right|^{2}}{\rho^{\sigma}(r)}
$$


Con el objetivo de obtener una función de localización electrónica independiente de la densidad, se define la energía cinética del gas homogéneo de electrones, $D_{0}^{\sigma}$, con la misma densidad electrónica según la ecuación 5.8,

$$
D_{0}^{\sigma}=2^{(5 / 3)} \cdot C_{F} \cdot\left(\rho^{\sigma}(r)\right)^{(5 / 3)}
$$

Donde $\mathrm{C}_{\mathrm{F}}$ es la constante de Fermi. La función ELF se define tal como se esquematiza en la ecuación 5.9.

$$
\eta(r)=\frac{1}{1+\left(\frac{D^{\sigma}(r)}{D_{0}^{\sigma}(r)}\right)^{2}}
$$

Los valores que toma esta función se encuentran entre 0 y 1 , de forma que los valores cercanos a 1 implican regiones de localización de pares electrónicos y baja repulsión de Pauli, donde los electrones poseen baja energía cinética. Por el contrario, valores de la función ELF muy pequeños indican que la probabilidad de apareamiento de electrones es baja ya que existe una elevada repulsión de Pauli y los electrones poseen una gran energía cinética.

De acuerdo con la interpretación de Silvi y Savin, la función ELF divide el espacio molecular en dominios de localización, es decir, en atractores y sus correspondientes cuencas de localización electrónica de una manera coherente con la Teoría de Lewis. ${ }^{[42]}$ Los atractores se sitúan en zonas donde el exceso de energía cinética es mínimo, es decir, donde los electrones se encuentran apareados. Las cuencas se clasifican en función de si contienen o no un núcleo en su interior. De esta forma, las que contienen un núcleo se dividen en cuencas de core $(\mathrm{C}(\mathrm{A}), \mathrm{A}=$ símbolo atómico) y cuencas hidrogenadas $(\mathrm{V}(\mathrm{H}))$. El resto de cuencas son cuencas de valencia $(\mathrm{V}(\mathrm{A}, \ldots))$, que representan la densidad electrónica distribuida entre las cuencas de core (cuencas de enlace) o a su alrededor (cuencas asociadas a pares libres).

La clasificación de las cuencas de valencia está basada en su conectividad a las cuencas de core. De esta forma, una cuenca de valencia está conectada a una cuenca de core si ambos atractores están limitados por una misma separatriz. Si 
existen varios cores conectados a una misma cuenca de valencia se determina el orden sináptico del atractor perteneciente a esta cuenca. Las cuencas monosinápticas se atribuyen a los pares de electrones solitarios, mientras que las cuencas disinápticas $\mathrm{V}(\mathrm{A}, \mathrm{B})$ están relacionadas con enlaces covalentes entre los átomos A y B. Por otra parte, las cuencas de orden polisináptico $\mathrm{V}(\mathrm{A}, \mathrm{B}, \ldots)$ se asocian a enlaces multicéntricos. La presencia o ausencia de atractores di- o polisinápticos es una indicación de interacción con electrones compartidos o de capas cerradas, respectivamente.

La función ELF permite dividir el espacio molecular en dominios de localización, que se definen como unidades con significado químico. Un dominio es reducible si contiene más de un atractor, mientras que si únicamente contiene uno se conoce como irreducible. Si se colocan en un diagrama los valores de la función ELF en los cuales tienen lugar las divisiones de los dominios, se obtiene un diagrama de bifurcación como el del clúster modelo $\left[\mathrm{Mo}_{3} \mathrm{~S}_{4} \mathrm{Cl}_{3}\left(\mathrm{PH}_{3}\right)_{3}\right]^{+}$que se muestra en la figura 5.12, estudiado por Llusar y colaboradores en el año 2005.[43]

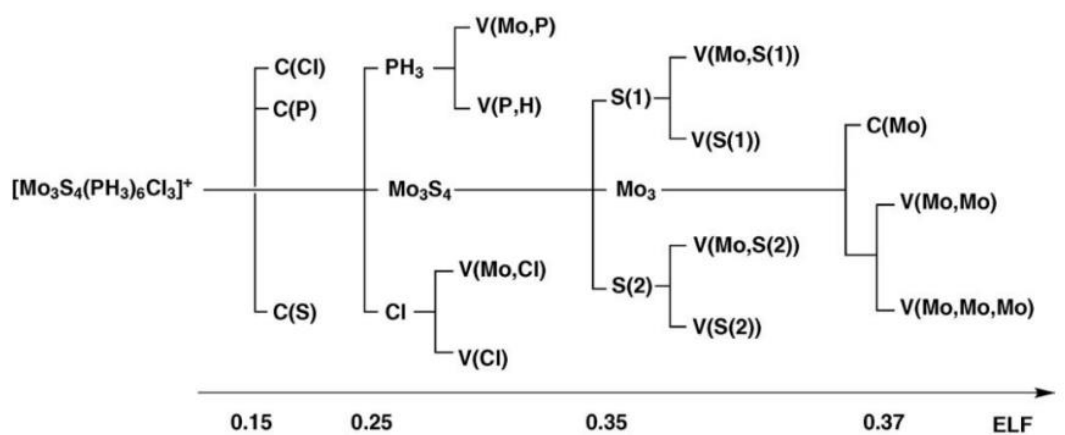

Figura 5.12. Diagrama de bifurcación de $\left[\mathrm{Mo}_{3} \mathrm{~S}_{4} \mathrm{Cl}_{3}\left(\mathrm{PH}_{3}\right)_{6}\right]^{+}$adaptado de la referencia [43] con permiso de la Royal Society of Chemistry.

En esta sección se describen los análisis ELF realizados en el fragmento $\mathrm{Cl} \cdots \mathrm{HN}$ de los cuatro isómeros con el programa TopMod[44] utilizando las geometrías optimizadas al nivel B3LYP/3-21G. En todos los isómeros se observan cuencas disinápticas $\mathrm{V}(\mathrm{N}-\mathrm{H})$ y $\mathrm{V}(\mathrm{Mo}-\mathrm{P})$, lo que indica la presencia de enlaces 
covalentes entre estos átomos, así como dos cuencas monosinápticas $\mathrm{V}(\mathrm{Cl})$, una orientada hacia el átomo de hidrógeno y otra hacia el de molibdeno. En el caso de la cuenca que apunta hacia el átomo de hidrógeno se observa la población más alta en el isómero $(P)-\mathbf{8}^{+}\left(3,91 \mathrm{e}^{-}\right)$, la cual disminuye hasta $3,77 \mathrm{e}^{-}$en el isómero $(M)-\mathbf{7}^{+}$. Estos resultados indican una mayor contribución electrónica de $\mathrm{V}(\mathrm{Cl})$ a la interacción vecinal presente en el complejo $(P)-\mathbf{8}^{+}$, y a su vez justifican desde un punto de vista topológico la menor energía del clúster $(P)-\mathbf{8}^{+}$.

No se encuentran cuencas disinápticas $\mathrm{V}(\mathrm{Cl}-\mathrm{H})$ en los clústeres $(P)-8^{+}$y $(M)$ $7^{+}$debido al carácter mayoritariamente iónico de estas interacciones, en concordancia con los análisis NBO. En ninguno de los isómeros se observan cuencas disinápticas $\mathrm{V}(\mathrm{Mo}-\mathrm{Cl})$ ni tampoco $\mathrm{V}(\mathrm{Mo}-\mathrm{N})$, lo que indica que estos enlaces son mayoritariamente iónicos. El carácter iónico del enlace $\mathrm{Mo}-\mathrm{Cl}$ se ha estudiado previamente en el modelo $\left[\mathrm{Mo}_{3} \mathrm{~S}_{4} \mathrm{Cl}_{3}\left(\mathrm{PH}_{3}\right)_{6}\right]^{+}$con el conjunto de funciones de base $3-21 \mathrm{G}^{* *} \cdot[43]$

La isosuperficie de ELF a $\eta=0,75$ del complejo $(P)-\mathbf{8}^{+}$se muestra en la figura 5.13. Las interacciones vecinales $\mathrm{Cl} \cdots \mathrm{HN}$ están representadas con una línea discontinua de color amarillo. 


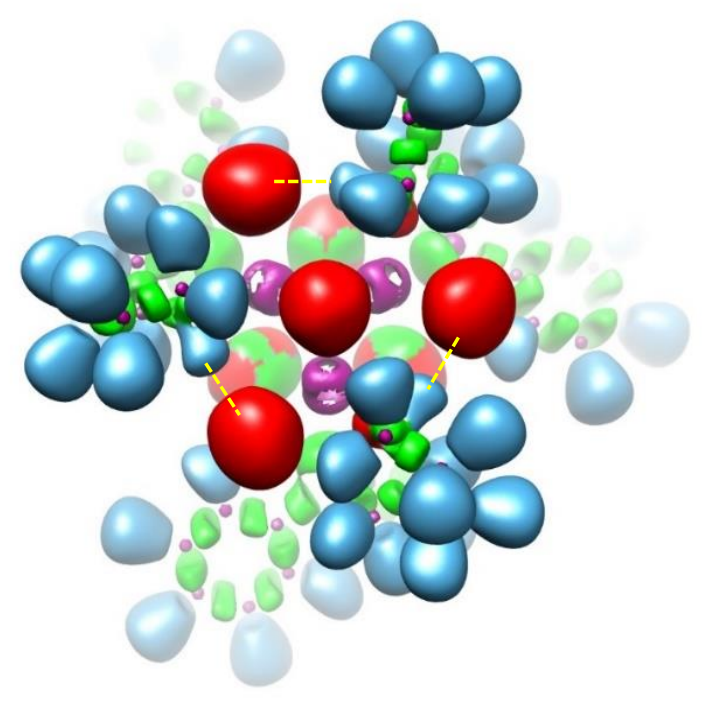

Figura 5.13. Isosuperficie de ELF a $\eta=0,75$ del clúster $(P)-8^{+}$. Las cuencas de core se representan en color morado, las monosinápticas en rojo, las disinápticas en verde y las hidrogenadas en azul. Se ha difuminado parte de esta representación en función de su profundidad para una mejor visualización de las cuencas.

Tras el cálculo de las geometrías optimizadas y los análisis NBO y ELF podemos concluir que, a pesar de que la distancia $\mathrm{Cl} \cdots \mathrm{HN}$ geminal en $(M)-7^{+}$es ligeramente más corta que la vecinal en $(P)-\mathbf{8}^{+}$, la interacción vecinal que se produce en $(P)-\mathbf{8}^{+}$es más fuerte. Esto es debido a que esta interacción vecinal presenta un mayor orden de enlace y a que la cuenca $\mathrm{V}(\mathrm{Cl})$ que interacciona con la $\mathrm{V}(\mathrm{H})$ está más poblada en $(P)-\mathbf{8}^{+}$que en $(M)-7^{+}$. Estos factores, junto con la conformación cis del anillo bicíclico, contribuyen a una mayor estabilización del complejo $(P)-\mathbf{8}^{+}$y están en concordancia con los valores de las energías relativas calculadas. Además, los valores de energía apoyan la obtención experimental de dos únicos isómeros: $(P)-7^{+}$ y $(P)-8^{+}$. 


\subsection{PRUEBAS CATALÍTICAS}

La actividad catalítica de los clústeres $\mathrm{Mo}_{3} \mathrm{~S}_{4}$ en la reducción de sustratos orgánicos a la que hacíamos referencia en la introducción de este capítulo, puede modularse mediante la elección adecuada de los ligandos externos. En el caso de algunos complejos monometálicos de rutenio, la funcionalización con ligandos aminofosfina y diamina derivados de la prolina resulta en catalizadores altamente selectivos en procesos de hidrogenación asimétrica. ${ }^{[22,24]}$ Motivados por estos estudios, hemos decidido investigar el potencial de los clústeres $(P)-\mathbf{7}^{+}$y $(P)-\mathbf{8}^{+}$como catalizadores para la reducción asimétrica de sustratos orgánicos con vistas a la obtención de aminas secundarias ópticamente puras. Los ensayos catalíticos se han llevado a cabo en el Leibniz Institut für Katalyse (Rostock, Alemania) en colaboración con el profesor Matthias Beller.

Inicialmente, hemos elegido como sustrato modelo el compuesto fenil(1feniletiliden)amina y hemos investigado la actividad catalítica de los clústeres $(P)-7^{+}$y $(P)-8^{+}$bajo diferentes condiciones de reacción, con el fin de obtener la amina secundaria correspondiente (ver figura 5.14).
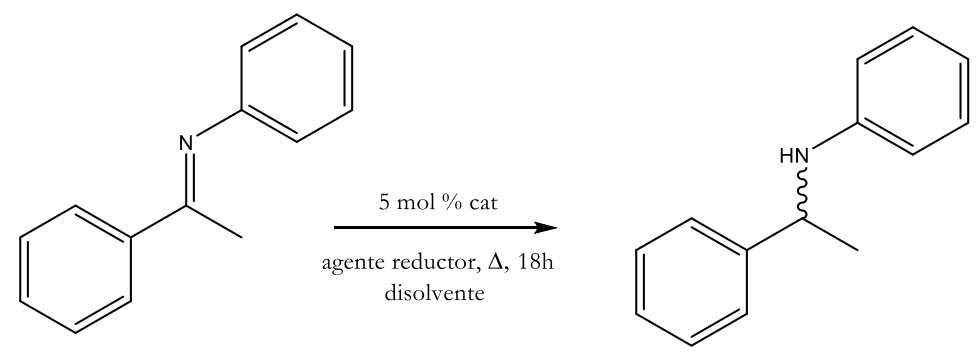

Figura 5.14. Esquema de reacción de la reducción asimétrica de fenil(1-feniletiliden)amina.

Las reacciones se han llevado a cabo en presencia de un 5 mol \% de catalizador, utilizando metanol, etanol o THF como disolventes y en presencia de hidrógeno molecular (20-40 bar), 5 equivalentes de una mezcla 5:2 de $\mathrm{HCOOH}: \mathrm{Et}_{3} \mathrm{~N}$ 
o la misma cantidad de $\mathrm{PhSiH}_{3}$ como agentes reductores. El seguimiento de las reacciones se ha realizado mediante cromatografía de gases con detector FID y los productos obtenidos se han identificado mediante comparación con un estándar comercial de elevada pureza. Para su cuantificación, se ha utilizado n-hexadecano como patrón interno. Los resultados obtenidos han sido negativos en todos los casos, y solo se han podido identificar como productos la acetofenona y la anilina, consecuencia de la reacción del sustrato con agua.

Alternativamente, se ha partido de acetofenona y anilina con vistas a obtener la amina secundaria correspondiente (ver figura 5.15). Las reacciones se han llevado a cabo en presencia de un $5 \mathrm{~mol} \%$ de $(P)-7^{+} \mathrm{o}(P)-\mathbf{8}^{+}$, variando el disolvente (metanol, etanol o THF), a diferentes temperaturas $\left(70-120^{\circ} \mathrm{C}\right)$ y utilizando como agentes reductores hidrógeno molecular (30 bar), 5 equivalentes de una mezcla 5:2 de HCOOH:Et ${ }_{3} \mathrm{~N}$ o la misma cantidad de $\mathrm{PhSiH}_{3}$. De nuevo no se han obtenido resultados positivos.<smiles>CC(=O)c1ccccc1</smiles><smiles>Nc1ccccc1</smiles>

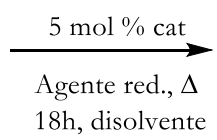<smiles>CC(Nc1ccccc1)c1ccccc1</smiles>

Figura 5.15. Esquema de reacción de la aminación reductiva de acetofenona y anilina.

A continuación, hemos decidido investigar la reducción catalítica de una selección de cetonas aromáticas fluoradas con el fin de obtener alcoholes quirales. Los alcoholes aromáticos fluorados ópticamente puros son intermedios muy importantes en la industria farmacéutica, tal como se ha explicado con detalle en el capítulo 4 de la presente tesis doctoral. 
Inicialmente se ha realizado una prueba utilizando como sustrato modelo la acetofenona con el objetivo de establecer las condiciones óptimas de reacción. Hemos estudiado los efectos de la fuente de hidrógeno, del disolvente y de la temperatura. En cuanto a la fuente de hidrógeno, se han probado una gran variedad de agentes reductores entre los que se encuentran el hidrógeno molecular, $\mathrm{PhSiH}_{3}$, $\mathrm{Ph}_{2} \mathrm{SiH}_{2}$, trietoxisilano, $\mathrm{PhMe}_{2} \mathrm{SiH}$, HMDS (hexametildisilazano) y la mezcla HCOOH:Et 3 N (5:2). Se ha observado actividad para dos silanos, $\mathrm{PhSiH}_{3}$ y $\mathrm{Ph}_{2} \mathrm{SiH}_{2}$, y para la mezcla HCOOH:Et $3 \mathrm{~N}$ (5:2). Los mejores resultados en términos de conversión y rendimiento se obtienen a $70{ }^{\circ} \mathrm{C}$ en presencia de THF y de 5 equivalentes de agente reductor, por lo que se establecen como condiciones óptimas de reacción. En la siguiente página se muestran los resultados obtenidos para los diferentes sustratos (tabla 5.6).

El estudio se ha limitado a cetonas fluoradas debido al interés de los alcoholes resultantes en la industria farmacéutica, y elevado coste de los ligandos quirales nos ha llevado a restringir el campo de aplicación de los sustratos. Las pruebas iniciales de hidrogenación de cetonas fluoradas se han realizado con el clúster $(P)-7^{+}$. 
Tabla 5.6. Reducción catalítica de cetonas aromáticas fluoradas con el clúster $(P)-7^{+}$(a).
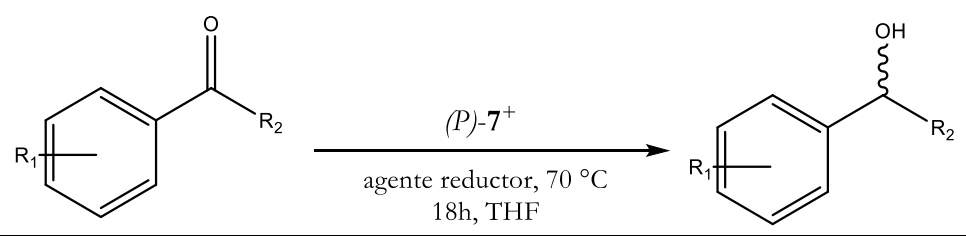

\begin{tabular}{|c|c|c|c|c|}
\hline Entrada & Sustrato & Agente reductor & Conversión $(\%)^{(b)}$ & Rendimiento $(\%)^{(b)}$ \\
\hline 1 & \multirow{4}{*}{$\mathrm{R}_{1}=\mathrm{H}, \mathrm{R}_{2}=\mathrm{CF}_{3}$} & $\mathrm{H}_{2}$ & 0 & 0 \\
\hline 2 & & $\mathrm{Ph}_{2} \mathrm{SiH}_{2}$ & 74 & 21 \\
\hline 3 & & $\mathrm{PhSiH}_{3}$ & 82 & 43 \\
\hline 4 & & $\mathrm{HCOOH}: \mathrm{Et}_{3} \mathrm{~N}(5: 2)$ & 80 & 29 \\
\hline 5 & \multirow{4}{*}{$\mathrm{R}_{1}=4-\mathrm{F}, \mathrm{R}_{2}=\mathrm{CH}_{3}$} & $\mathrm{H}_{2}$ & 0 & 0 \\
\hline 6 & & $\mathrm{Ph}_{2} \mathrm{SiH}_{2}$ & $\mathrm{nd}(\mathrm{c})$ & nd \\
\hline 7 & & $\mathrm{PhSiH}_{3}$ & nd & nd \\
\hline 8 & & $\mathrm{HCOOH}: \mathrm{Et}_{3} \mathrm{~N}(5: 2)$ & 0 & 0 \\
\hline 9 & \multirow{2}{*}{$\mathrm{R}_{1}=4-\mathrm{CF}_{3}, \mathrm{R}_{2}=\mathrm{CH}_{3}$} & $\mathrm{H}_{2}$ & 0 & 0 \\
\hline 10 & & $\mathrm{Ph}_{2} \mathrm{SiH}_{2}$ & nd & nd \\
\hline
\end{tabular}

(a) Condiciones de reacción: 0,1 mmol sustrato, $5 \mathrm{~mol} \%$ catalizador, 5 equivalentes agente reductor. (b)Determinados por GC utilizando n-hexadecano como estándar interno. (c)No detectado en la suficiente cantidad para poder cuantificarlo 
Tabla 5.6. (Continuación). Reducción catalítica de cetonas aromáticas fluoradas con el clúster (P)-7+(a).

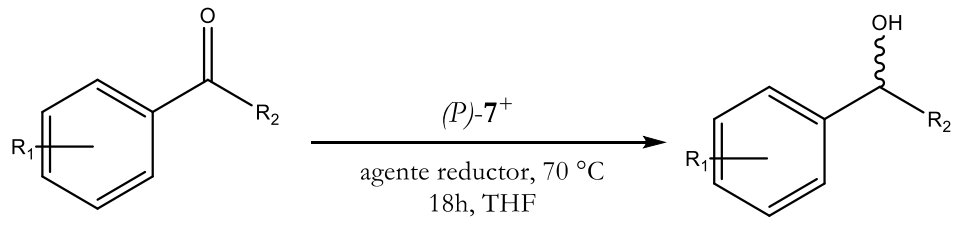

\begin{tabular}{ccccc}
\hline \hline Entrada & Sustrato & Agente reductor & Conversión (\%) $^{(\mathrm{b})}$ & Rendimiento (\%) $^{(\mathrm{b})}$ \\
\hline 11 & $\mathrm{R}_{1}=4-\mathrm{CF}_{3}, \mathrm{R}_{2}=\mathrm{CH}_{3}$ & $\mathrm{PhSiH}$ & 50 & 27 \\
12 & & $\mathrm{HCOOH}: \mathrm{Et}{ }_{3} \mathrm{~N}(5: 2)$ & 0 & 0 \\
\hline 13 & $\mathrm{H}_{2}$ & 0 & $\mathrm{nd}$ \\
14 & $\mathrm{R}_{1}=2-\mathrm{F}, \mathrm{R}_{2}=\mathrm{CH}_{3}$ & $\mathrm{Ph}_{2} \mathrm{SiH}_{2}$ & $\mathrm{nd}(\mathrm{c})$ & 32 \\
15 & & $\mathrm{PhSiH}_{3}$ & 51 & 0 \\
\hline \hline
\end{tabular}

(a)Condiciones de reacción: 0,1 mmol sustrato, $5 \mathrm{~mol} \%$ catalizador, 5 equivalentes agente reductor. (b)Determinados por GC

utilizando n-hexadecano como estándar interno. (c)No detectado en la suficiente cantidad para poder cuantificarlo. 
Los mejores resultados en cuanto a conversión y rendimiento se han obtenido al reducir el sustrato 2,2,2-trifluoroacetofenona en presencia de $\mathrm{PhSiH}_{3}$ (entrada 3, tabla 5.6), al igual que ocurría en el capítulo 4 con los clústeres funcionalizados con aminofosfinas, aunque en este caso la conversión y el rendimiento son mucho menores. La reacción no ha tenido lugar en presencia de hidrógeno molecular para ninguno de los sustratos (entradas 1, 5, 9 y 13, tabla 5.6), por lo que la hidrogenación directa no es posible. La utilización de la mezcla reductora $\mathrm{HCOOH}: \mathrm{Et}_{3} \mathrm{~N}(5: 2)$ ha funcionado en buenos niveles de conversión pero con bajos rendimientos únicamente para la 2,2,2-trifluoroacetofenona (entrada 4, tabla 5.6), al contrario de lo que ocurría con la reducción de nitroderivados en presencia del clúster $\left[\mathrm{Mo}_{3} \mathrm{~S}_{4} \mathrm{H}_{3}(\mathrm{dmpe})_{3}\right]^{+} .{ }^{[19]}$.

La obtención de alcoholes fluorados en posición para no ha tenido lugar (entradas 5-8, tabla 5.6), mientras que en posición orto se han obtenido conversiones moderadas y rendimientos bajos al utilizar $\mathrm{PhSiH}_{3}$ como agente reductor (entrada 5, tabla 5.6). Hemos observado conversión moderada y rendimientos bajos en la obtención de alcoholes trifluorometilados en posición para (entrada 11, tabla 5.6).

A pesar de los bajos rendimientos obtenidos, la importancia de este tipo de reacciones radica en la capacidad del catalizador para obtener un producto final ópticamente puro. Desafortunadamente, los análisis mediante HPLC han demostrado que en todos los casos se obtiene una mezcla racémica del alcohol fluorado resultante.

Adicionalmente, hemos estudiado la reducción de 2,2,2-trifluoroacetofenona, el sustrato que mejores resultados ha mostrado en términos de conversión y rendimiento, en presencia del complejo $(P)-\mathbf{8}^{+}$para comparar la actividad de ambos diastereoisómeros en esta reacción. La tabla 5.7 lista los resultados obtenidos. 
Tabla 5.7. Reducción catalítica de 2,2,2-trifluoroacetofenona con el clúster $(P)-\mathbf{8}^{+(a)}$.

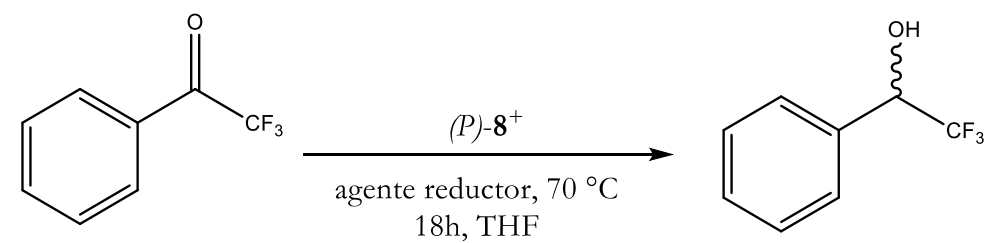

\begin{tabular}{|c|c|c|c|}
\hline Entrada & Agente reductor & Conversión (\%) & Rendimiento $(\%)^{(b)}$ \\
\hline 1 & $\mathrm{H}_{2}$ & 0 & 0 \\
\hline 2 & $\mathrm{Ph}_{2} \mathrm{SiH}_{2}$ & 58 & 15 \\
\hline 3 & $\mathrm{PhSiH}_{3}$ & 66 & 35 \\
\hline 4 & $\mathrm{HCOOH}: \mathrm{Et}_{3} \mathrm{~N}$ & 49 & 16 \\
\hline
\end{tabular}

(a) Condiciones de reacción: 0,1 mmol sustrato, $5 \mathrm{~mol} \%$ catalizador, 5 equivalentes agente reductor. (b)Determinados por GC utilizando n-hexadecano como estándar interno.

Los resultados en cuanto a conversión y rendimiento son peores con el clúster $(P)-\mathbf{8}^{+}$(tabla 5.7) que con el $(P)-7^{+}$(tabla 5.6). Este comportamiento se puede atribuir de manera tentativa a la mayor estabilidad energética del complejo $(P)-\mathbf{8}^{+}$ estudiada detalladamente en las secciones anteriores. De nuevo, el mejor resultado se ha obtenido al utilizar $\mathrm{PhSiH}_{3}$ como agente reductor (entrada 3, tabla 5.7), aunque en este caso las conversiones y rendimientos son inferiores a las obtenidas utilizando el clúster $(P)-7^{+}$como catalizador. Al igual que en el caso anterior, no se ha encontrado ninguna evidencia de la obtención del alcohol ópticamente puro correspondiente.

Con el fin de mejorar los resultados de conversión y rendimiento, hemos llevado a cabo la reacción a $100{ }^{\circ} \mathrm{C}$ en un tubo apto para soportar elevadas presiones. Las conversiones y rendimientos se han elevado hasta $90 \%$ y $74 \%$, respectivamente, en el caso del clúster $(P)-7^{+}$y hasta $78 \%$ y $73 \%$ en el caso del complejo $(P)-\mathbf{8}^{+}$. Sin embargo, tal como se esperaba al aumentar la temperatura, en los análisis mediante HPLC del producto de reacción no se ha detectado exceso enantiomérico. 
Tras los estudios anteriores, hemos decidido disminuir la temperatura hasta $50^{\circ} \mathrm{C}$ para intentar favorecer la estereoselectividad del proceso catalítico en detrimento de los valores de conversión y rendimiento, pero el análisis del producto final ha revelado que nos encontramos de nuevo ante una mezcla racémica.

Llegados a este punto hemos decidido cambiar la estrategia y probar nuevos ligandos P-N quirales en la reducción asimétrica de 2,2,2-trifluoroacetofenona in situ en las condiciones de reacción optimizadas anteriormente. Para ello se ha utilizado el clúster $\left[\mathrm{Mo}_{3} \mathrm{~S}_{4} \mathrm{Cl}_{4}\left(\mathrm{PPh}_{3}\right)_{3}\left(\mathrm{H}_{2} \mathrm{O}\right)_{2}\right]$, que ha demostrado previamente ser un buen precursor al reaccionar con cantidades estequiométricas de ligandos aminofosfina [28] (ver sección 5.2). Las aminofosfinas quirales elegidas son (1S,2S)-2-(difenilfosfino)1,2-difeniletilamina (ligando 1) y (1S,2S)-1-amino-2-(difenilfosfino)indano (ligando 2), ilustradas en la figura 5.16. La tabla 5.8 muestra los resultados obtenidos en la reducción catalítica in situ de 2,2,2-trifluoroacetofenona en presencia de estos ligandos.

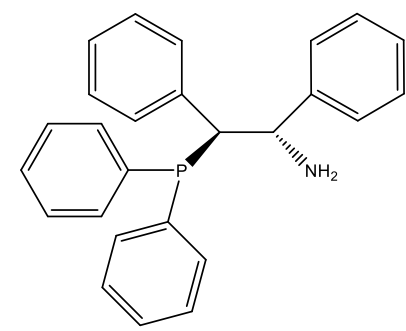

Ligando 1

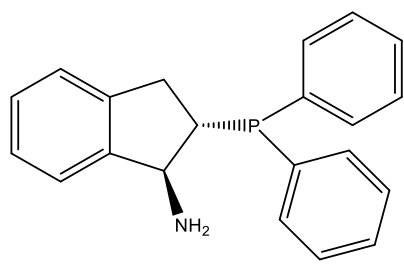

Ligando 2

Figura 5.16. Aminofosfinas quirales utilizadas para la reducción catalítica de 2,2,2trifluoroacetofenona. 
Tabla 5.8. Reducción catalítica in situ de 2,2,2-trifluoroacetofenona con los nuevos ligandos P-N quirales (a).

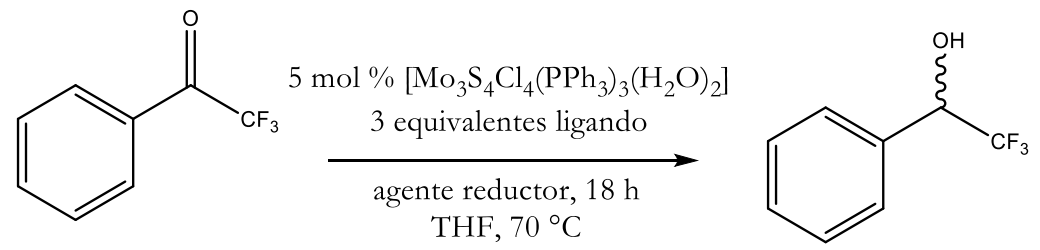

\begin{tabular}{ccccc}
\hline \hline Entrada & Ligando & Agente reductor & $\begin{array}{c}\text { Conversión } \\
\mathbf{( \% )}(\mathrm{b})\end{array}$ & $\begin{array}{c}\text { Rendimiento } \\
(\mathbf{\%} / \mathbf{( b )}\end{array}$ \\
\hline 1 & 1 & $\mathrm{PhSiH}_{3}$ & $\mathrm{nd}^{(\mathrm{c})}$ & $\mathrm{nd}$ \\
2 & & $\mathrm{HCOOH} / \mathrm{Et}_{3} \mathrm{~N}(5: 2)$ & 0 & 0 \\
3 & 2 & $\mathrm{PhSiH}_{3}$ & $>99$ & $>99$ \\
4 & & $\mathrm{HCOOH} / \mathrm{Et}_{3} \mathrm{~N}(5: 2)$ & 0 & 0 \\
\hline \hline
\end{tabular}

(a)Condiciones de reacción: 0,1 mmol sustrato, $5 \mathrm{~mol}$ \% clúster, $15 \mathrm{~mol} \%$ ligando, 5 equivalentes agente reductor. (b)Determinados por GC utilizando n-hexadecano como estándar interno. ${ }^{(c)} \mathrm{No}$ detectado en la suficiente cantidad para poder cuantificarlo.

Los mejores resultados de conversión y rendimiento se han obtenido con el ligando 2, (1S,2S)-1-amino-2-(difenilfosfino)indano, utilizando como reductor $\mathrm{PhSiH}_{3}$ (entrada 3, tabla 5.8), aunque el producto de reacción ha resultado ser de nuevo una mezcla racémica. La utilización del ligando 1 no muestra actividad catalítica (entradas 1 y 2, tabla 5.8). En ambos casos, la utilización de la mezcla $\mathrm{HCOOH}: \mathrm{Et}_{3} \mathrm{~N}$ no es efectiva, por lo que no tiene lugar la reacción mediante transferencia de hidrógeno (entradas 2 y 4, tabla 5.8). La reacción se ha llevado a cabo a $50{ }^{\circ} \mathrm{C}$ pero tampoco hemos detectado estereoselectividad en el proceso.

En conclusión, los clústeres $\mathrm{Mo}_{3} \mathrm{~S}_{4}$ en presencia de aminofosfinas ópticamente puras conducen a la obtención de alcoholes, pero el proceso de reducción no es enantioselectivo. Además, se obtienen mejores resultados en la 
reducción de 2,2,2-trifluoroacetofenona cuando utilizamos el ligando 2 in situ en lugar de los clústeres aislados $(P)-\mathbf{7}^{+}$y $(P)-\mathbf{8}^{+}$funcionalizados con (R)-PPro y $(S)$-PPro. 


\subsection{BIBLIOGRAFÍA}

[1] I. K. Reddy, R. M. Chirality in Drug Design and Development 2004.

[2] A. N. Collins, G. N. S., J. Crosby Chirality in Industry II: Developments in the Commercial Manufacture and Applications of Optically Active Compounds, 1997.

[3] S. Sarker, L. N. Chemistry for Pharmacy Students: General, Organic and Natural Product Chemistry, 2007.

[4] McBride, W. G. The Lancet (British edition) 1961, 2, 1358.

[5] McBride, W. G. The Lancet (British edition) 1965, 2, 855.

[6] Carroll, M. P.; Guiry, P. J. Chem. Soc. Rev. 2014, 43, 819.

[7] Xie, J. B.; Xie, J. H.; Liu, X. Y.; Kong, W. L.; Li, S.; Zhou, Q. L. J. Am. Chem. Soc. 2010, 132, 4538.

[8] Fuentes, J. A.; Carpenter, I.; Kann, N.; Clarke, M. L. Chem. Commun. 2013, 49, 10245.

[9] Zeng, L.; Wu, F.; Li, Y. Y.; Dong, Z. R.; Gao, J. X. J. Organomet. Chem. 2014, 762, 34 .

[10] Buchwalter, P.; Rosé, J.; Braunstein, P. Chem. Rev. 2015, 115, 28.

[11] Moberg, V.; Duquesne, R.; Contaldi, S.; Rohrs, O.; Nachtigall, J.; Damoense, L.; Hutton, A. T.; Green, M.; Monari, M.; Santelia, D.; Haukka, M.; Nordlander, E. Chem.-Eur. J. 2012, 18, 12458.

[12] Moberg, V.; Haukka, M.; Koshevoy, I. O.; Ortiz, R.; Nordlander, E. Organometallics 2007, 26, 4090.

[13] Abdel-Magied, A. F.; Singh, A. K.; Haukka, M.; Richmond, M. G.; Nordlander, E. Chem. Commun. 2014, 50, 7705.

[14] Feliz, M.; Guillamon, E.; Llusar, R.; Vicent, C.; Stiriba, S. E.; Perez-Prieto, J.; Barberis, M. Chem.-Eur. J. 2006, 12, 1486.

[15] Guillamon, E. M.; Blasco, M.; Llusar, R. Inorg. Chim. Acta 2015, 424, 248.

[16] Beltran, T. F.; Feliz, M.; Llusar, R.; Mata, J. A.; Safont, V. S. Organometallics 2011, 30, 290. 
[17] Alfonso, C.; Beltran, T. F.; Feliz, M.; Llusar, R. J. Clust. Sci. 2015, 26, 199.

[18] Beltrán, T. F., Tesis Doctoral, Universitat Jaume I, 2013.

[19] Sorribes, I.; Wienhofer, G.; Vicent, C.; Junge, K.; Llusar, R.; Beller, M. Angew. Chem. Int. Edit. 2012, 51, 7794.

[20] Pedrajas, E.; Sorribes, I.; Junge, K.; Beller, M.; Llusar, R. ChemCatChem 2015, $7,2675$.

[21] Zhao, B. G.; Han, Z. B.; Ding, K. L. Angew. Chem. Int. Edit. 2013, 52, 4744.

[22] Ito, M.; Kobayashi, C.; Himizu, A.; Ikariya, T. J. Am. Chem. Soc. 2010, 132, 11414.

[23] Ito, M.; Ootsuka, T.; Watari, R.; Shiibashi, A.; Himizu, A.; Ikariya, T. J. Am. Chem. Soc. 2011, 133, 4240.

[24] Denizalti, S.; Mercan, D.; Sen, B.; Gokce, A. G.; Cetinkaya, B. J. Organomet. Chem. 2015, 779, 62.

[25] Basallote, M. G.; Fernández-Trujillo, M. J.; Pino-Chamorro, J. Á.; Beltrán, T. F.; Corao, C.; Llusar, R.; Sokolov, M.; Vicent, C. Inorg. Chem. 2012, 51, 6794.

[26] Beltran, T. F.; Pino-Chamorro, J. A.; Fernandez-Trujillo, M. J.; Safont, V. S.; Basallote, M. G.; Llusar, R. Inorg. Chem. 2015, 54, 607.

[27] M. Sasaki, G. S., T. Ouchi, T. Shibahara J. Clust. Sci. 1998, 9, 25.

[28] Alfonso, C.; Feliz, M.; Safont, V. S.; Llusar, R. Dalton Trans. 2016, 45, 7829.

[29] Guillamón, E.; Llusar, R.; Pérez-Prieto, J.; Stiriba, S.-E. J. Organomet. Chem. 2008, 693, 1723.

[30] Algarra, A. G.; Basallote, M. G.; Fernandez-Trujillo, M. J.; Feliz, M.; Guillamon, E.; Llusar, R.; Sorribes, I.; Vicent, C. Inorg. Chem. 2010, 49, 5935.

[31] F. Egidi; R. Russo; I. Carnimeo; A. D'Urso; G. Mancini; Cappelli, C. J. Phys. Chem. A 2015, 119, 5396.

[32] Ringdahl, B. C., C.J. Acta Chem. Scand. 1980, 34B, 731.

[33] Beltran, T. F.; Safont, V. S.; Llusar, R. Eur. J. Inorg. Chem. 2016. DOI: 10.1002/ejic.201600586 
[34] Llusar, R.; Uriel, S. Eur. J. Inorg. Chem. 2003, 1271.

[35] Kuznetsov, V. F.; Yap, G. P. A.; Alper, H. Organometallics 2001, 20, 1300.

[36] Gaussian 09, M. J. Frisch; H. B. Schlegel; G. E. Scuseria; J. R. C. M. A. Robb, G. S., V. Barone, B. Mennucci, , H. N. G. A. Petersson, M. Caricato, X. Li, H. P. Hratchian, , J. B. A. F. Izmaylov, G. Zheng, J. L. Sonnenberg, M. Hada, , K. T. M. Ehara, R. Fukuda, J. Hasegawa, M. Ishida, T. Nakajima, , O. K. Y. Honda, H. Nakai, T. Vreven, J. A. Montgomery, Jr., , F. O. J. E. Peralta, M. Bearpark, J. J. Heyd, E. Brothers, V. N. Staroverov, K. N. Kudin, T. Keith, R. Kobayashi, J. Normand, A. Rendell, K. Raghavachari, J. C. Burant, S. S. Iyengar, J. Tomasi, , N. R. M. Cossi, J. M. Millam, M. Klene, J. E. Knox, J. B. Cross, C. Adamo, V. Bakken, J. Jaramillo, R. Gomperts, R. E. Stratmann, A. J. Austin, O. Yazyev, R. Cammi, C. Pomelli, J. W. Ochterski, , K. Morokuma, R. L. Martin, V. G. Zakrzewski, G. A. Voth, , J. J. Dannenberg P. Salvador, S. Dapprich, A. D. Daniels, J. B. Foresman, O. Farkas, J. V. Ortiz, J. Cioslowski, and G. and D. J. Fox; Gaussian, Inc., Wallingford CT, 2010.

[37] Estevan, F.; Feliz, M.; Llusar, R.; Mata, J. A.; Uriel, S. Polyhedron 2001, 20, 527.

[38] Reed, A. E.; Weinhold, F. J. Chem. Phys. 1983, 78, 4066.

[39] Reed, A. E.; Weinstock, R. B.; Weinhold, F. J. Chem. Phys. 1985, 83, 735.

[40] Reed, A. E.; Curtiss, L. A.; Weinhold, F. Chemical Reviews 1988, 88, 899.

[41] Becke, A. D.; Edgecombe, K. E. J. Chem. Phys. 1990, 92, 5397.

[42] Savin, A.; Silvi, B.; Coionna, F. Can. J. Chem. 1996, 74, 1088.

[43] Feliz, M.; Llusar, R.; Andres, J.; Berski, S.; Silvi, B. New J. Chem. 2002, $26,844$.

[44] S. Noury, X. K., F. Fuster and B. Silvi Comput. Chem 1999, 597. 
6. CLÚSTERES FUNCIONALIZADOS CON AMINODIFOSFINAS Y SUS APLICACIONES CATALÍTICAS 


\section{CLÚSTERES FUNCIONALIZADOS CON AMINODIFOSFINAS Y SUS APLICACIONES CATALÍTICAS}

6.1 Introducción

6.2 Síntesis y caracterización

6.3 Reactividad frente a otros metales

6.3.1 Reactividad frente a cobre

6.3.2 Reactividad frente a níquel

6.3.3 Reactividad frente a paladio

6.3.4 Reactividad frente a rodio

6.3.5 Reactividad frente a iridio

6.3.6 Reactividad frente a oro

6.3.7 Reactividad frente a plata

6.3.8 Reactividad frente a rutenio

6.4 Pruebas catalíticas

6.4.1 Reducción catalítica de aldehídos y cetonas

6.4.2 Hidrogenación catalítica de $\mathrm{CO}_{2}$

6.5 Bibliografía

[...] Que la nobleza del hombre, adquirida tras cien siglos de tentativas y errores, consistía en hacerse dueño de la materia, y que yo me había matriculado en Química porque me quería mantener fiel a esta nobleza. Que dominar la materia es comprenderla, y comprender la materia es preciso para conocer el universo y conocernos a nosotros mismos $[\ldots]$ ” Primo Levi, El Sistema Periódico 


\subsection{INTRODUCCIÓN}

Los ligandos tridentados aminodifosfina se utilizan ampliamente en química de coordinación para diseñar catalizadores activos en reacciones de deshidrogenación[1,2] e hidrogenación, ${ }^{[3,4]}$ entre otras. Históricamente, los catalizadores basados en metales nobles como el iridio o el rutenio han sido los más utilizados en este tipo de reacciones debido a su buena actividad y selectividad.[4,5] En los últimos años, la tendencia seguida en este campo de investigación responde a la búsqueda de catalizadores más económicos y menos contaminantes que los basados en estos metales preciosos, demasiado caros y perjudiciales para el medio ambiente. ${ }^{[6]}$ Hoy en día, muchos catalizadores y precatalizadores utilizados tanto en reacciones hidrogenación como de deshidrogenación están basados en complejos monometálicos de hierro, ya que es el metal más abundante de la corteza terrestre, no es tóxico y es económico.[2,7-10] Entre estos catalizadores y precatalizadores, se encuentran algunos funcionalizados con aminodifosfinas, como por ejemplo el publicado por el grupo de Milstein en el año 2011[11] o el preparado por Morris y colaboradores en el 2014.[3] Ambos se ilustran en la figura 6.1.
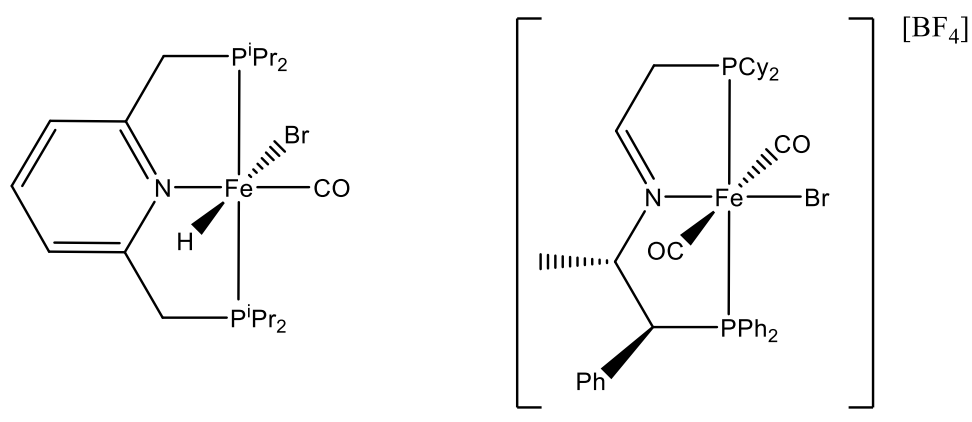

Figura 6.1. Catalizador desarrollado por Milstein (izquierda) para la hidrogenación de cetonas y precatalizador de Morris (derecha) para la hidrogenación de cetonas e iminas.

El molibdeno se encuentra dentro del grupo de metales económicos y durante las últimas décadas se ha demostrado su actividad catalítica en reacciones de hidrogenación, hidrosililación, hidrólisis y metátesis de olefinas, entre otras.[12-14] Por 
el diseño de catalizadores de molibdeno para esta última reacción, Richard R. Schrock obtuvo en 2005 el Premio Nobel de Química junto a Robert H. Grubbs e Yves Chauvin.

A pesar de la importancia del molibdeno en catálisis y del papel fundamental de los ligandos aminodifosfina en las reacciones de hidrogenación y deshidrogenación, no existen muchos ejemplos de complejos de molibdeno funcionalizados con estos ligandos. El grupo de investigación de Heinz Berke ha sintetizado recientemente compuestos de Mo y W con bajos estados de oxidación que contienen ligandos tridentados aminodifosfina y que además son activos en reacciones de hidrogenación de iminas y dióxido de carbono.[15,16] La figura 6.2 muestra el ciclo catalítico de la hidrogenación de $\mathrm{CO}_{2}$ publicado por Berke el año pasado. En este contexto, la síntesis de compuestos de molibdeno funcionalizados con aminodifosfinas y la búsqueda de sus aplicaciones catalíticas resulta un campo de investigación muy atractivo y poco explorado.

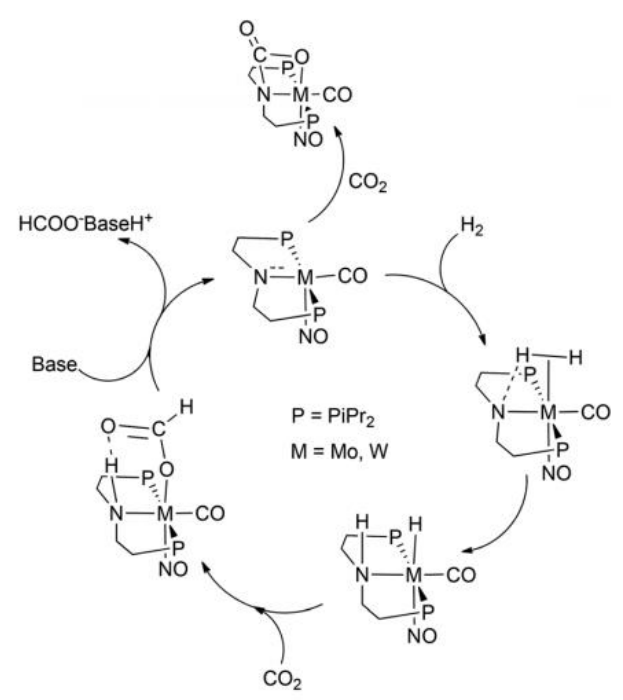

Figura 6.2. Ciclo catalítico de la hidrogenación de $\mathrm{CO}_{2}$ en presencia de complejos de Mo y W funcionalizados con aminodifosfinas publicado por Berke en 2015 adaptado de la referencia [16] con permiso de la Royal Society of Chemistry. 
Recientemente el grupo de Llusar ha demostrado que compuestos clúster con unidad trimetálica $\mathrm{Mo}_{3} \mathrm{~S}_{4}$ funcionalizados con ligandos difosfina, aminofosfina y diamina son eficientes en procesos de hidrogenación de cetonas (ver capítulos 4 y 5 ) y reducción de nitroderivados, ${ }^{[17,18]}$ por lo que se ha decidido extender esta química a los ligandos aminodifosfina y estudiar su actividad catalítica en procesos similares. La aminodifosfina bis[(2-di-i-propilfosfino)etil]amina (a partir de ahora PNP) ha sido el ligando seleccionado para realizar estas pruebas, debido a su éxito en reacciones de hidrogenación. ${ }^{[4,15]}$

Hasta la fecha, no se han encontrado en la literatura clústeres con unidad $\mathrm{M}_{3} \mathrm{Q}_{4}(\mathrm{M}=\mathrm{Mo}, \mathrm{W} ; \mathrm{Q}=\mathrm{O}, \mathrm{S})$ funcionalizados con ligandos aminodifosfina, aunque sí con otros ligandos tridentados, como por ejemplo 1,4,7-triazaciclononano,[19,20] bases conjugadas de los ácidos nitrilotriacético[21,22] e iminodiacético[23] y sus derivados, ${ }^{[24]}$ entre otros. La estructura de uno de ellos sintetizado por Cotton y colaboradores se muestra en la figura 6.3 .

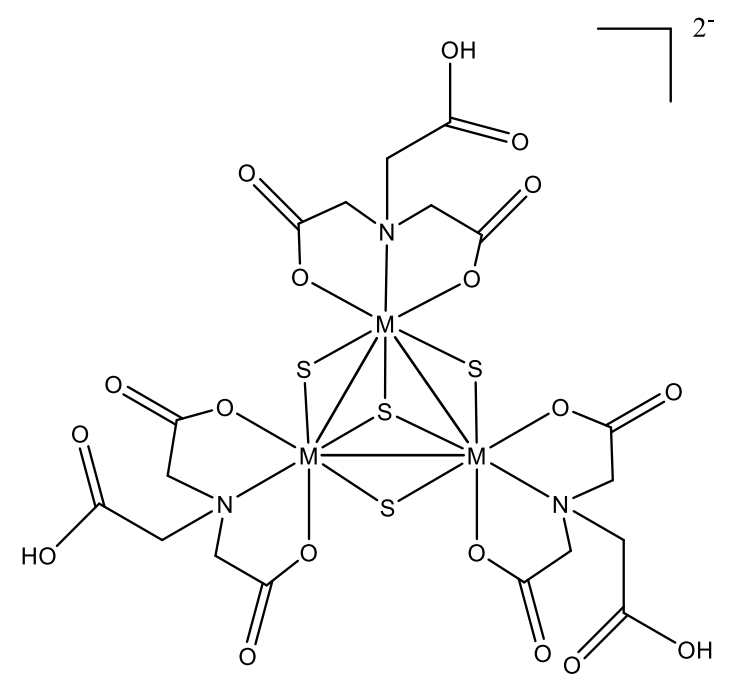

Figura 6.3. Estructura esquemática del clúster $\left[\mathrm{Mo}_{3} \mathrm{~S}_{4}(\mathrm{Hnta})_{3}\right]^{2-}\left(\mathrm{H}_{3}\right.$ nta $=$ ácido nitriloacético). 
La actividad catalítica de clústeres de unidad $\mathrm{Mo}_{3} \mathrm{~S}_{4}$ en reacciones tan importantes a nivel industrial como la hidrogenación de sustratos orgánicos, la escasez de complejos de molibdeno funcionalizados con aminodifosfinas y el papel crucial de estas en algunas reacciones catalíticas, nos han llevado a sintetizar el primer clúster $\mathrm{Mo}_{3} \mathrm{~S}_{4}$ con ligandos aminodifosfina y a estudiar su papel en reacciones catalíticas de hidrogenación. Además, la coordinación parcial del ligando PNP a la unidad clúster nos ha motivado a investigar la reactividad de nuestro compuesto frente a diferentes complejos metálicos. En este capítulo se describe la síntesis, caracterización, reactividad y actividad catalítica del primer clúster de unidad $\mathrm{Mo}_{3} \mathrm{~S}_{4}$ funcionalizado con la aminodifosfina PNP. 


\subsection{SÍNTESIS Y CARACTERIZACIÓN}

La síntesis en dos etapas de complejos $\left[\mathrm{Mo}_{3} \mathrm{~S}_{4} \mathrm{Cl}_{3} \text { (difosfina) }\right]^{+}$y $\left.\left[\mathrm{Mo}_{3} \mathrm{~S}_{4} \mathrm{Cl}_{3} \text { (aminofosfina) }\right]_{3}\right]^{+}$a partir del clúster molecular $\left[\mathrm{Mo}_{3} \mathrm{~S}_{7} \mathrm{Cl}_{6}\right]^{2-}$ es una estrategia alternativa a la reacción de escisión de fases poliméricas $\left\{\mathrm{Mo}_{3} \mathrm{~S}_{7} \mathrm{Cl}_{4}\right\}_{\mathrm{n}}$, tal como se ha descrito en los capítulos 4 y 5 de esta tesis doctoral.[25,26] Esta vía de síntesis resulta ser más económica ya que se evita el gasto del ligando difosfina o aminofosfina en la reducción de los puentes disulfuro de la unidad $\mathrm{Mo}_{3} \mathrm{~S}_{7}$ (ver figura 6.4), y permite obtener el producto deseado en altos rendimientos. Por estas razones ha sido la elegida mayoritariamente en nuestra investigación.

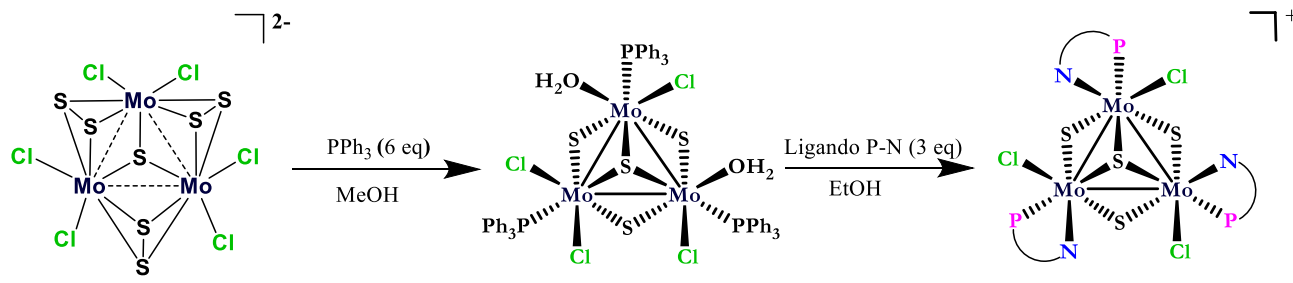

Figura 6.4. Esquema de la síntesis en dos etapas de clústeres $\mathrm{Mo}_{3} \mathrm{~S}_{4}$ funcionalizados con aminofosfinas a partir del precursor molecular $\left[\mathrm{Mo}_{3} \mathrm{~S}_{7} \mathrm{Cl}_{6}\right]^{2-}$.

Debido a los buenos resultados obtenidos con ligandos aminofosfina al llevar a cabo esta reacción, se ha decidido extender esta estrategia al ligando aminodifosfina abreviado como PNP. En una primera etapa de reacción, a una suspensión del precursor $\left[\mathrm{Mo}_{3} \mathrm{~S}_{7} \mathrm{Cl}_{6}\right]^{2-}$ en metanol se le han añadido 6 equivalentes de trifenilfosfina con la consiguiente formación de un precipitado verde en el seno de reacción que corresponde al clúster neutro $\left[\mathrm{Mo}_{3} \mathrm{~S}_{4} \mathrm{Cl}_{4}\left(\mathrm{PPh}_{3}\right)_{3}\left(\mathrm{H}_{2} \mathrm{O}\right)_{2}\right]$. El sólido obtenido se filtra y se lava con una mezcla fría de hexano y tolueno, así como con hexano a ebullición. En la siguiente etapa, este compuesto se ha suspendido en etanol y a la mezcla resultante se le han añadido tres equivalentes de ligando aminodifosfina. Al cabo de 3 horas, la disolución verde obtenida se ha concentrado en atmósfera inerte hasta aproximadamente $1 / 4$ de su volumen, para precipitar seguidamente el producto con 
hexano. El producto deseado de fórmula $\left[\mathrm{Mo}_{3} \mathrm{~S}_{4} \mathrm{Cl}_{3}(\mathrm{PNP})_{3}\right] \mathrm{Cl}(\mathbf{9}(\mathrm{Cl}))$ se ha aislado como un sólido verde en un rendimiento del $89 \%$ y se ha caracterizado mediante RMN de ${ }^{31} \mathrm{P}\left\{{ }^{1} \mathrm{H}\right\}$, espectrometría de masas y difracción de rayos $\mathrm{X}$ en monocristal.

La naturaleza de este clúster se ha confirmado mediante RMN de ${ }^{31} \mathrm{P}\left\{{ }^{1} \mathrm{H}\right\}$, en cuyo espectro se observan dos singuletes de igual intensidad a 47,63 ppm y -2,04 ppm, tal como se ilustra en la figura 6.5. Se descarta la presencia de aminodifosfina libre, cuya única señal en el espectro de RMN de fósforo aparece a 22,91 ppm. Las dos señales observadas en el espectro representado en la figura 6.5 indican la presencia de dos tipos de átomos de fósforo no equivalentes en nuestro compuesto, aunque no proporcionan información sobre la ordenación espacial de estos.[27,28]

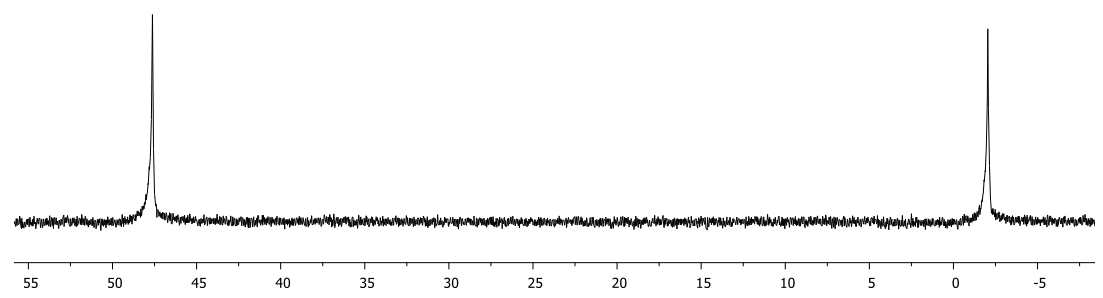

Figura 6.5. Espectro de $\mathrm{RMN}$ de ${ }^{31} \mathrm{P}\left\{{ }^{1} \mathrm{H}\right\}$ del compuesto de molibdeno $\mathrm{Mo}_{3} \mathrm{~S}_{4}$ funcionalizado con la aminodifosfina PNP.

El espectro de masas nos permite conocer más detalladamente la formulación de este complejo. En él se observa un único pico base centrado en 1438 u.m.a. que se relaciona con un compuesto monocargado de unidad trimetálica $\mathrm{Mo}_{3} \mathrm{~S}_{4}$, funcionalizado con tres ligandos PNP y con tres ligandos cloruro. Este pico se asocia al ion molecular $\left[\mathrm{Mo}_{3} \mathrm{~S}_{4} \mathrm{Cl}_{3}(\mathrm{PNP})_{3}\right]^{+}\left(\mathbf{9}^{+}\right)$. La figura 6.6 muestra la buena concordancia entre el espectro de masas simulado y el experimental. 


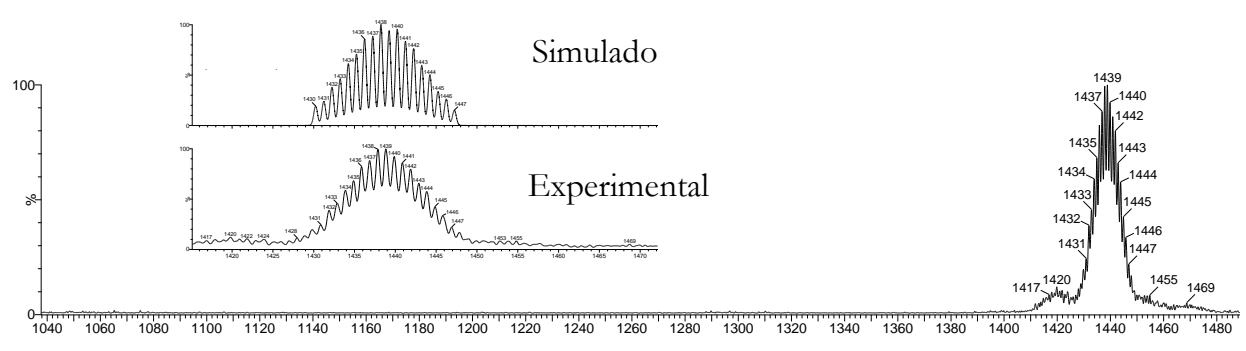

Figura 6.6. Espectros de masas simulado y experimental del clúster $\mathbf{9}^{+}$.

El resultado obtenido mediante espectrometría de masas es sorprendente, ya que la naturaleza del ligando PNP y los antecedentes encontrados en la literatura, tanto en complejos mononucleares funcionalizados con este ligando como en clústeres $\mathrm{Mo}_{3} \mathrm{~S}_{4}$ funcionalizados con otros ligandos tridentados, conducen a pensar que el ligando PNP se coordinará al molibdeno mediante sus dos átomos de fósforo y el de nitrógeno. Sin embargo, la presencia de ligandos cloruro y de una señal que claramente representa un complejo monocargado revela que no ha sido así. A pesar de toda la información que se ha obtenido gracias a la espectroscopia de RMN y la espectrometría de masas, sin la difracción de rayos $\mathrm{X}$ en monocristal no se puede conocer la disposición espacial de este ligando ni analizar su coordinación al molibdeno.

Afortunadamente, se han obtenido cristales adecuados para su caracterización mediante difusión lenta de hexano sobre una disolución concentrada del clúster como sal de cloruro en diclorometano y la resolución de su estructura cristalina nos ha permitido conocer la naturaleza del clúster de fórmula $\left[\mathrm{Mo}_{3} \mathrm{~S}_{4} \mathrm{Cl}_{3}(\mathrm{PNP})_{3}\right]^{+}\left(\mathbf{9}^{+}\right)$. Este compuesto ha cristalizado en el grupo espacial cúbico $\mathrm{Pa}-3$ de elevada simetría, con la unidad $\mathrm{Mos}_{4}$ situada sobre un eje terciario, en la que el ligando tridentado PNP se coordina al molibdeno a través de un átomo de fósforo (representado en la figura 6.7 como P(1)) y el átomo de nitrógeno, de manera análoga a los ligandos bidentados aminofosfina. El entorno de coordinación del metal lo completa el ligando cloruro, cuya presencia se ha detectado previamente mediante 
espectrometría de masas. En la estructura, uno de los átomos de fósforo de la aminodifosfina, identificado en la figura 6.7 como $\mathrm{P}(2)$, permanece sin coordinar.

La tabla 6.1, muestra las principales distancias de enlace para el compuesto $\mathbf{9}^{+}$junto con las distancias del compuesto $\left.\left[\mathrm{Mo}_{3} \mathrm{~S}_{4} \mathrm{Cl}_{3} \text { (ediprp) }\right]_{3}\right]^{+}\left(\mathbf{5}^{+}\right)$y las de un compuesto monometálico de molibdeno funcionalizado con el mismo ligando PNP.[16]

Tabla 6.1. Selección de distancias de enlace promedio para los compuestos $\mathbf{9}^{+}$, $\left[\mathrm{Mo}_{3} \mathrm{~S}_{4} \mathrm{Cl}_{3}(\text { ediprp) }]_{3}\right]^{+}\left(\mathbf{5}^{+}\right)$y $\mathrm{Mo}(\mathrm{NO})\left(\mathrm{Cl}_{2}\right)(\mathrm{PNP})$. La desviación estándar de los valores promedio se encuentra representada entre corchetes.

\begin{tabular}{cccc}
\hline \hline Distancia $(\AA)$ & $\mathbf{9}^{+}$ & {$\left[\mathbf{M o s}_{3} \mathbf{S}_{4} \mathbf{C l}_{3}\left(\mathbf{e d} \mathbf{e d p}_{3}\right]^{+}\left(\mathbf{5}^{+}\right)\right.$} & $\mathbf{M o}(\mathbf{N O})\left(\mathbf{C l}_{2}\right)(\mathbf{P N P})^{(\mathrm{a})}$ \\
\hline Mo-Mo & $2,7772(4)$ & 2,7600 & - \\
Mo- $\left(\mu_{3}-\mathrm{S}\right)$ & $2,3539(10)$ & 2,3468 & - \\
Mo- $(\mu-S)^{(c)}$ & $2,2936(8)$ & 2,2959 & - \\
Mo- $(\mu-S)^{(\mathrm{d})}$ & $2,2923(8)$ & 2,3000 & $2,5371[1]$ \\
Mo-P(1) & $2,5746(8)$ & 2,5760 & $2,255(2)$ \\
Mo-N(e) & $2,346(3)$ & 2,2552 & $2,464[38]$ \\
Mo-Cl & $2,4910(8)$ & 2,5097 & - \\
\hline \hline
\end{tabular}

(a)Datos extraídos de la referencia [16] (c)Distancia trans al enlace Mo-Cl. (d)Distancia trans al enlace Mo-N. (e)El valor de Mo-N es el propio de la aminodifosfina.

() Desviación estándar de la medida. [] Desviación estándar del promedio. 

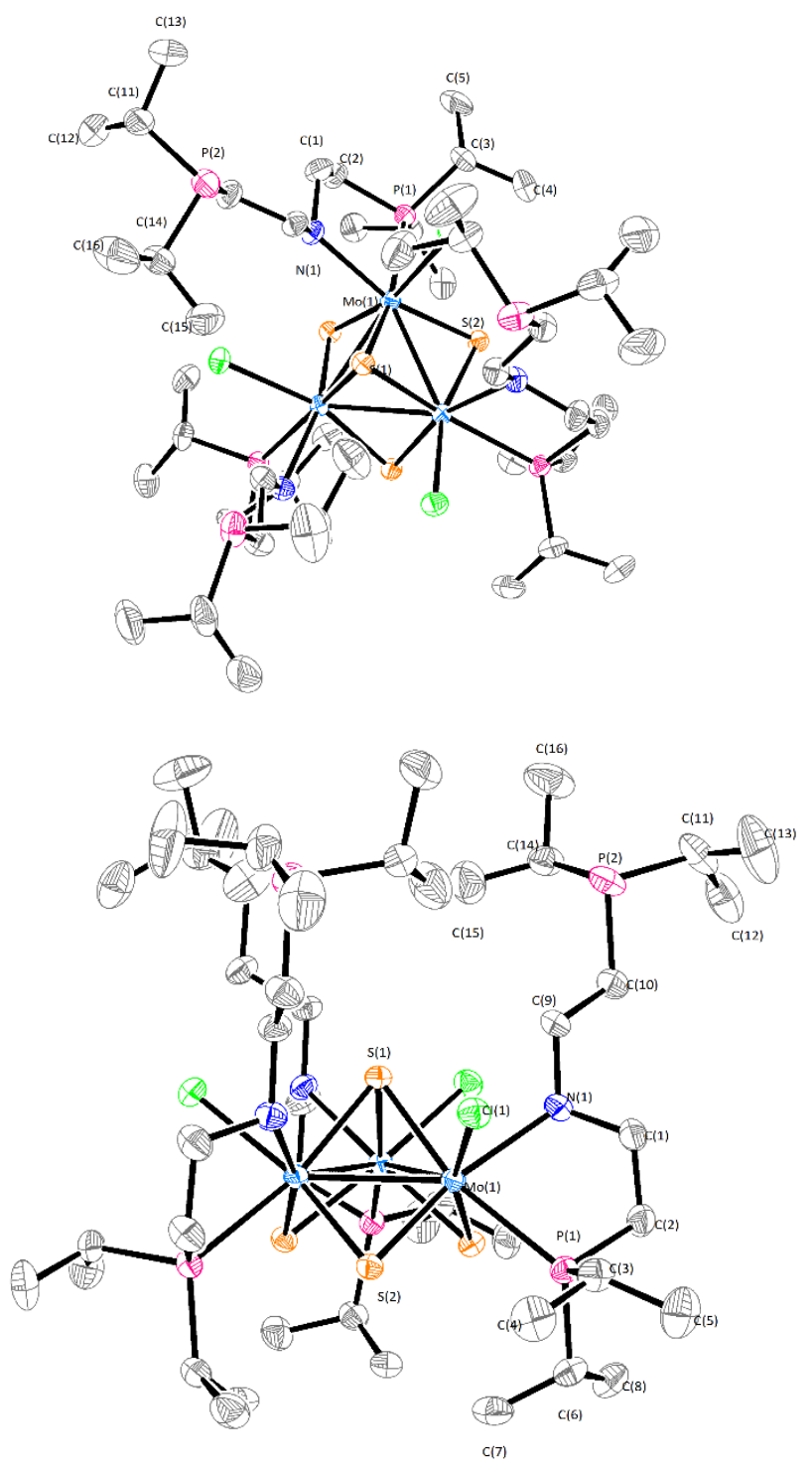

Figura 6.7. Representaciones ORTEP (elipsoides al $50 \%$ de probabilidad) frontal (arriba) y lateral (abajo) del compuesto $\mathbf{9}^{+}$. El contraión $\mathrm{Cl}^{-}$y los hidrógenos se han omitido por claridad. 
Las distancias de enlace para el compuesto $\mathbf{9}^{+}$son similares a las encontradas en el clúster $5^{+}$y también en otros clústeres de estructura análoga funcionalizados con aminofosfinas. ${ }^{[25,26,29]}$ Las distancias Mo-Mo son coherentes con un estado de oxidación IV para el átomo de molibdeno y las longitudes de enlace Mo-S siguen la misma tendencia que aquellas observadas para otros clústeres similares: la distancia de enlace entre molibdeno y azufre apuntado (Mo- $\left.\left(\mu_{3}-\mathrm{S}\right)\right)$ es mayor que las distancias entre molibdeno y azufres puente (Mo- $(\mu-\mathrm{S}))$. Las distancias Mo-( $\mu-\mathrm{S})$ son aproximadamente iguales entre ellas, hecho que se observa repetidamente en los clústeres trinucleares funcionalizados con aminofosfinas descritos en la literatura y en esta tesis doctoral (capítulos 4 y 5).[26,29] Este hecho se debe a la similar influencia trans que presentan los ligandos amina y cloruro, lo que contrasta con lo ocurrido en los clústeres halogenados funcionalizados con difosfinas.[27,28] Se observa una diferencia significativa en la distancia Mo-N del complejo $\mathbf{9}^{+}$en relación con las observadas hasta el momento en clústeres de fórmula $\left.\left[\mathrm{M}_{3} \mathrm{~S}_{4} \mathrm{X}_{3} \text { (aminofosfina) }\right)_{3}\right]^{+}(\mathrm{M}=$ Mo, W; $\mathrm{X}=\mathrm{Cl}, \mathrm{Br}$ ). En el caso del clúster $\mathbf{9}^{+}$esta distancia Mo-N es más larga que las observadas en el resto de clústeres, y la diferencia, que oscila entre $0,04 \AA$ y 0,10 Å en función de la aminofosfina coordinada, se asocia de manera tentativa a la mayor congestión estérica del ligando PNP.

Tal como se observa en otros clústeres funcionalizados con aminofosfinas, el átomo de fósforo se coordina en posición trans al azufre apuntado, mientras que el átomo de nitrógeno lo hace en posición trans a los azufres puente. En este caso, al coordinar un ligando aminodifosfina, se observa que el segundo átomo de fósforo del ligando permanece sin coordinar al metal, de manera que en el compuesto $\mathbf{9}^{+}$ encontramos tres grupos fosfino situados en el mismo lado del plano trimetálico en posición cis al azufre apuntado (ver figura 6.7). El comportamiento inesperado de este ligando nos ha abierto una puerta a explorar la reactividad de los grupos fosfina no coordinados presentes en $\mathbf{9}^{+}$. 
La tabla 6.1 también incluye una comparativa con las distancias de enlace encontradas para el único complejo de molibdeno halogenado funcionalizado con la aminodifosfina PNP hasta la fecha. En esta situación nos encontramos ante el complejo mononuclear $\mathrm{Mo}(\mathrm{NO})\left(\mathrm{Cl}_{2}\right)(\mathrm{PNP})$, en el cual el ligando PNP se encuentra coordinado de manera tridentada al metal, ${ }^{[16]}$ tal como se ilustra en la figura 6.8 .

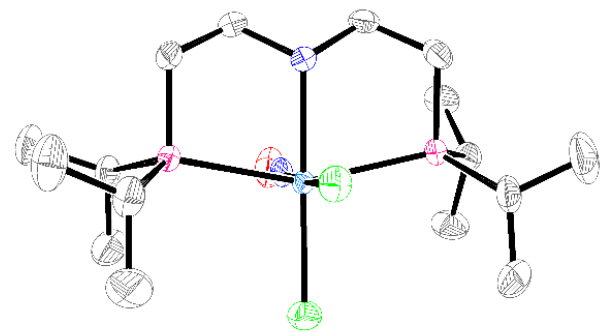

Figura 6.8. Representación ORTEP de la estructura cristalina del complejo $\mathrm{Mo}(\mathrm{NO})\left(\mathrm{Cl}_{2}\right)(\mathrm{PNP})$ donde se observa la coordinación tridentada del ligando. Datos extraídos de la referencia [16].

Las distancias Mo-P, Mo-N (átomo de nitrógeno perteneciente al ligando aminodifosfina) y $\mathrm{Mo}-\mathrm{Cl}$ siguen la misma tendencia que las observadas en el complejo $\mathbf{9}^{+}$, teniendo en cuenta que nos encontramos ante un complejo mononuclear donde el molibdeno presenta un estado de oxidación II, significativamente más bajo que el que presenta en el clúster trimetálico $\mathbf{9}^{+}$. La distancia Mo-N, considerablemente más corta que en el complejo $\mathbf{9}^{+}$y muy similar a la encontrada en el clúster $\mathbf{5}^{+}$, refuerza la idea expuesta anteriormente: la no coordinación del átomo de fósforo $\mathrm{P}(2)$ y la congestión estérica que esta situación produce, provoca un alargamiento del enlace Mo-N en el clúster $\mathbf{9}^{+}$en comparación con otros complejos similares.

En conclusión, hemos sintetizado el primer clúster trinuclear de molibdeno con un ligando aminodifosfina y, tras su caracterización, hemos detectado una coordinación parcial del ligando PNP. El hecho de que este ligando tridentado se coordine como un ligando bidentado provoca que un átomo de fósforo de cada 
aminodifosfina, identificado como $\mathrm{P}(2)$, permanezca sin coordinar. De esta forma el clúster $\left[\mathrm{Mo}_{3} \mathrm{~S}_{4} \mathrm{Cl}_{3}(\mathrm{PNP})_{3}\right]^{+}$presenta tres grupos fosfino no coordinados al metal situados en posición cis al azufre apuntado. La presencia de estos grupos resulta interesante, especialmente con vistas a la coordinación de un segundo metal de transición y de ese modo, al desarrollo de complejos heterodimetálicos con propiedades catalíticas. Esquemáticamente, el clúster $\mathbf{9}^{+}$se puede representar tal y como aparece en la figura 6.9, a pesar de que este dibujo no representa la posición exacta de los tres átomos de fósforo $\mathrm{P}(2)$ por ser una representación en 2 dimensiones. Es por esto que la resolución de la estructura cristalina ha resultado crucial para descifrar la naturaleza de este compuesto y la orientación espacial del ligando PNP.

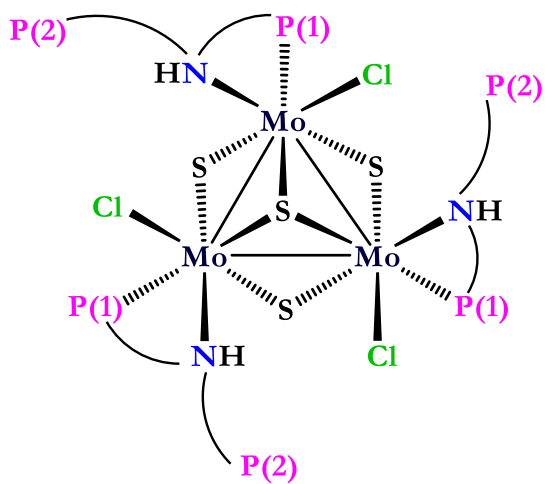

Figura 6.9. Representación esquemática del clúster $9^{+}$.

En los siguientes apartados se detalla la reactividad de este clúster frente a otros metales y la búsqueda de aplicaciones catalíticas con interés industrial que se ha realizado en esta tesis doctoral para los complejos funcionalizados con esta aminodifosfina. 


\subsection{REACTIVIDAD FRENTE A OTROS METALES}

En la literatura se han descrito numerosos ejemplos de clústeres $\mathrm{M}_{3} \mathrm{~S}_{4}(\mathrm{M}=\mathrm{Mo}, \mathrm{W})$ que reaccionan frente a otros metales de transición y se han discutido las propiedades catalíticas de los compuestos heterodimetálicos resultantes.[28,30-33] Los clústeres $\mathrm{M}_{3} \mathrm{~S}_{4}$ se pueden considerar como unidades cuboidales incompletas, donde los metales y los calcógenos ocupan los vértices alternos de un cubo, con una posición metálica vacante. Estos clústeres son capaces de actuar como metaloligandos frente a un segundo metal M' para formar compuestos heterodimetálicos $\mathrm{M}_{3} \mathrm{M}^{\prime} \mathrm{S}_{4}$, en los que todas las posiciones del cubo se encuentran ocupadas. Esta estrategia de síntesis se conoce por el nombre de "construcción por bloques [3+1]" y se ilustra en la figura 6.10 .

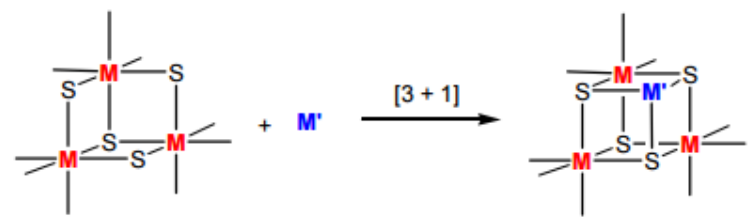

Figura 6.10. Formación de clústeres heterodimetálicos $\mathrm{M}_{3} \mathrm{M}^{\prime} \mathrm{S}_{4}$ mediante la estrategia de construcción por bloques [3+1].

Sin embargo, en ninguno de los ejemplos estudiados existe un ligando que, como el PNP, se coordine a la unidad tri- o tetrametálica solo parcialmente. Es por esto que la reactividad del compuesto $\mathbf{9}^{+}$frente a otros metales resulta una incógnita, ya que pueden ocurrir dos situaciones por separado o conjuntamente: que se forme un complejo con estructura de tipo cubano $\mathrm{Mo}_{3} \mathrm{M}^{\prime} \mathrm{S}_{4}$, o que los átomos de fósforo $\mathrm{P}(2)$ sin coordinar reaccionen con el complejo metálico añadido, para dar lugar a un nuevo tipo de clústeres heterodimetálicos.

A continuación se detallan las reacciones que se han llevado a cabo entre el complejo $\mathbf{9}^{+}$y diferentes compuestos metálicos, con el objetivo de estudiar la 
reactividad de los átomos de fósforo no coordinados en combinación con la de la unidad trimetálica $\mathrm{Mo}_{3} \mathrm{~S}_{4}$.

\subsubsection{REACTIVIDAD FRENTE A COBRE}

En este apartado hemos investigado la reactividad del clúster $\mathbf{9}^{+}$frente a $\mathrm{CuCl}$. La elección de este metal viene motivada, en primer lugar, por la capacidad de los clústeres $\mathrm{M}_{3} \mathrm{~S}_{4}$ de incorporar cobre (I) para dar complejos $\mathrm{M}_{3} \mathrm{CuS}_{4} \mathrm{y}$, en segundo lugar, por la actividad de estos complejos heterodimetálicos en reacciones catalíticas como la ciclopropanación de alquenos y diazocompuestos, descritas por Llusar y colaboradores en diferentes publicaciones. ${ }^{28,34]}$ Además, los complejos de cobre (I) funcionalizados con monofosfinas catalizan reacciones de alquilación reductiva de enonas.[35] Por tanto, la presencia de átomos de fósforo sin coordinar de la aminodifosfina y la posibilidad de que los mismos reaccionen con el cobre, amplía el ámbito de las aplicaciones catalíticas de estos sistemas.

En nuestro caso la reacción entre el clúster $\mathbf{9}^{+}$y $\mathrm{CuCl}$ se ha llevado a cabo a temperatura ambiente, mediante la adición de 3,5 equivalentes de la sal de cobre a una disolución del clúster en diclorometano. La adición de esta sal comporta un cambio de color inmediato, de verde a rojo, lo que a priori apunta a que nuestro compuesto reacciona con el cobre. Cabe destacar que la formación de clústeres de tipo cubano con unidad $\mathrm{Mo}_{3} \mathrm{CuS}_{4}$ funcionalizados con difosfinas transcurre también con un cambio de coloración de verde a rojo.[28] Tras 24 horas de reacción, la disolución roja se filtra para eliminar el $\mathrm{CuCl}$ sin reaccionar y se lleva a sequedad. Para la caracterización del sólido rojo obtenido se han utilizado las técnicas habituales, i.e. espectroscopia RMN, espectrometría de masas y difracción de rayos $\mathrm{X}$ en monocristal.

El espectro de $\mathrm{RMN}$ de ${ }^{31} \mathrm{P}\left\{{ }^{1} \mathrm{H}\right\}$ presenta una señal mayoritaria de multiplicidad singulete a un desplazamiento de 44,63 ppm. Esta situación sería coherente con la presencia de un único tipo de átomos de fósforo, resultado de la 
sustitución de los ligandos cloruro por los átomos de fósforo $\mathrm{P}(2)$. A pesar del resultado obtenido, resulta bastante improbable que ambos átomos de fósforo se hayan coordinado al metal hacia el mismo lado del plano que forman los tres molibdenos, por lo que este espectro de RMN resulta muy complicado de interpretar. El espectro de masas del compuesto anterior no muestra ningún pico que se pueda asociar con la presencia de unidades $\mathrm{Mo}_{3} \mathrm{~S}_{4} \mathrm{O} \mathrm{Mo}_{3} \mathrm{CuS}_{4}$, de forma que estas técnicas no nos han resultado útiles para la caracterización del sólido obtenido.

La difracción de rayos $\mathrm{X}$ en monocristal nos ha proporcionado más información, ya que se han obtenido cristales adecuados para su medición mediante difusión lenta de tolueno sobre una disolución concentrada del sólido en diclorometano. Desafortunadamente, no se ha podido resolver esta estructura en su totalidad, pero sí que se han observado dos datos importantes que nos ayudan a entender la naturaleza de este compuesto. En primer lugar, la estructura de cubo incompleto que presentaba la unidad $\mathrm{Mo}_{3} \mathrm{~S}_{4}$ en el compuesto $\mathbf{9}^{+}$se ha transformado en una estructura tipo cubano debido a la incorporación de un átomo de cobre a la unidad trimetálica, tal como se muestra en la figura 6.11 .

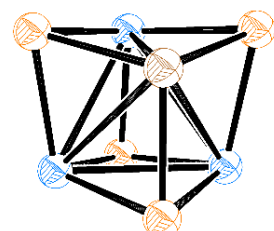

Figura 6.11. Representación ORTEP de la unidad central $\mathrm{Mo}_{3} \mathrm{CuS}_{4}$. Los átomos de molibdeno están representados en azul, los de azufre en naranja y el de cobre en marrón.

En segundo lugar, los tres átomos de fósforo $\mathrm{P}(2)$ no coordinados al molibdeno han reaccionado con la sal de cobre, pero el desorden observado en la estructura cristalina y la dificultad de su resolución no nos han permitido descifrar qué tipo de reacción ha ocurrido. Ningún intento posterior de síntesis y caracterización de este compuesto heterodimetálico nos ha permitido obtener más 
información en cuanto a su composición y estructura más allá de lo detallado en este apartado.

\subsubsection{REACTIVIDAD FRENTE A NÍQUEL}

Se ha investigado la reactividad del complejo $\mathbf{9}^{+}$frente al compuesto $\mathrm{Ni}(\mathrm{cod})_{2}$ en presencia de $\mathrm{TBACl}$, siguiendo los procedimientos descritos en la literatura para la obtención de compuestos análogos de fórmula $\left[\mathrm{Mo}_{3} \mathrm{NiS}_{4} \mathrm{Cl}_{4}(\text { difosfina) })_{3}\right]$ (difosfina $=$ dmpe, (R,R)-Me-BPE).[36,37] El interés de este tipo de compuestos con unidad tetrametálica $\mathrm{Mo}_{3} \mathrm{NiS}_{4}$ viene motivado por su actividad en la ciclación intramolecular de ácidos alquinoicos terminales para dar enol lactonas [38]. Además, los sistemas níquel-monofosfina juegan un papel importante en reacciones de acoplamiento cruzado.[39,40]

La reacción entre el clúster $\mathbf{9}^{+}$y $\mathrm{Ni}(\operatorname{cod})_{2}(\operatorname{cod}=$ ciclooctadieno $)$ se ha llevado a cabo en diferentes condiciones, a temperatura ambiente o a reflujo, en presencia de disolventes como acetonitrilo o diclorometano y con un exceso más o menos elevado de $\mathrm{Ni}(\operatorname{cod})_{2}$ y TBACl. El sólido verde-marrón aislado no se ha podido caracterizar mediante espectrometría de masas, donde no se observan picos asociados a la presencia de unidades tri- o tetrametálicas, ni mediante espectroscopia de RMN de fósforo, en el cual no se observa ninguna señal, quizás debido a la aparición de especies paramagnéticas en el transcurso de la reacción.

\subsubsection{REACTIVIDAD FRENTE A PALADIO}

Los clústeres con unidad $\mathrm{Mo}_{3} \mathrm{PdS}_{4}$ presentan actividad catalítica en reacciones de adición de alcoholes y ácidos carboxílicos a alquinos unidos a grupos aceptores de densidad electrónica.[41,42] Además, los sistemas Pd-monofosfina son de gran relevancia en química orgánica y organometálica por su papel como catalizadores en reacciones de acoplamiento carbono-carbono. ${ }^{[43-45]}$ El reconocimiento internacional de su trascendencia hizo merecedores del premio Nobel de Química en el año 2010 a Richard F. Heck, Ei-ichi Negishi y Akira Suzuki. 
En nuestro caso el clúster $\mathbf{9}^{+}$se ha hecho reaccionar con un exceso de $\mathrm{Pd}_{2}(\mathrm{dba})_{3}$ en presencia de TBACl. Como disolvente se ha utilizado diclorometano y tras 24 horas de reacción se ha obtenido una disolución marrón a partir de la cual es posible aislar un sólido marrón-grisáceo, cuya caracterización mediante las técnicas habituales no ha sido posible.

\subsubsection{REACTIVIDAD FRENTE A RODIO}

Brorson y colaboradores publicaron en el año 2001 una serie de clústeres con unidad $\mathrm{Mo}_{3} \mathrm{M}^{\prime} \mathrm{S}_{4}$, donde M' representa rodio, iridio, osmio o rutenio.[46] Además, existen ejemplos de clústeres heterodimetálicos con unidades $\mathrm{MoRh}_{2}{ }^{[47]}$ y $\mathrm{MoRh},{ }^{[48]}$ aunque hasta la fecha no se han descrito aplicaciones catalíticas. Sin embargo, los complejos monometálicos de rodio (I) funcionalizados con ligandos monofosfina son catalizadores activos en hidrogenaciones asimétricas. ${ }^{[49]}$ En este contexto, se ha explorado la reactividad de este metal de transición frente al clúster $\mathbf{9}^{+}$con el fin de obtener un complejo heterodimetálico dirigido a hidrogenaciones catalíticas. Un procedimiento bien establecido para la obtención de compuestos de tipo [RhCl(cod)(monofosfina)] consiste en la adición de 2 equivalentes de monofosfina al dímero $\left[\{\mathrm{RhCl}(\operatorname{cod})\}_{2}\right] .{ }^{[50,51]}$ En esta sección se ha seguido esta estrategia de síntesis con el fin de obtener sistemas rodio-monofosfina-clúster que imiten los descritos anteriormente.

El clúster $\mathbf{9}^{+}$se ha disuelto en diclorometano y a esta disolución se le ha añadido aproximadamente un equivalente del dímero $\left[\{\mathrm{RhCl}(\operatorname{cod})\}_{2}\right]$ en atmósfera inerte. Una vez transcurridas 24 horas y tras concentrar la disolución hasta aproximadamente $1 / 4$ de su volumen, se ha precipitado el sólido verde obtenido con éter. Este sólido se ha caracterizado mediante RMN de ${ }^{31} \mathrm{P}\left\{{ }^{1} \mathrm{H}\right\}$, tal como se muestra en la figura 6.12. 


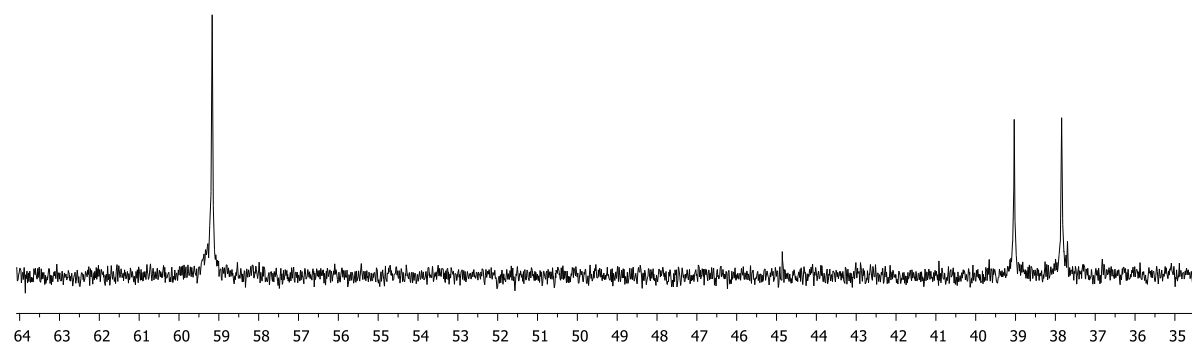

Figura 6.12. Espectro de $\mathrm{RMN}$ de ${ }^{31} \mathrm{P}\left\{{ }^{1} \mathrm{H}\right\}$ del compuesto que resulta de la reacción entre $\mathbf{9}^{+} \mathrm{y}\left[\{\mathrm{RhCl}(\operatorname{cod})\}_{2}\right]$.

Por primera vez en esta serie de pruebas de reactividad con metales de transición, nos encontramos ante un espectro que muestra varias señales de fósforo bien definidas. Se observan dos grupos de señales: un singulete de mayor intensidad a 59,17 ppm y dos singuletes menos intensos a 39,03 ppm y 37,84 ppm que parecen estar relacionados entre sí. El área integrada por estas dos últimas señales es la misma que la del singulete a 59,17 ppm, lo que indica que hay dos grupos de tres fósforos químicamente no equivalentes asociados a la unidad clúster. Por tanto, los dos singuletes menos intensos a los que hacemos referencia anteriormente son en realidad una única señal que presenta multiplicidad doblete con una constante de acoplamiento ${ }^{1} \mathrm{~J}_{\mathrm{Rh}} \mathrm{P}$ de $143 \mathrm{~Hz}$, que se asocia a una interacción entre los núcleos de ${ }^{31} \mathrm{P}$ y ${ }^{103} \mathrm{Rh}$. Este resultado sugiere la coordinación de cada uno de los átomos de fósforo $\mathrm{P}(2)$ a un átomo de rodio, formando tres sistemas equivalentes rodiomonofosfina. El desplazamiento químico de esta señal y su constante de acoplamiento se aproximan a los descritos para compuestos de tipo $\left[\mathrm{RhCl}(\mathrm{cod})\right.$ (monofosfina)].[50,51] Por ejemplo el complejo $\left[\mathrm{RhCl}(\mathrm{cod})\left(\mathrm{PPh}_{3}\right)\right]$ muestra una única señal de resonancia a 31,3 ppm en el espectro de RMN de fósforo, con una constante de acoplamiento ${ }^{1} \mathrm{~J}$ Rh-P de 150,1 Hz. ${ }^{[50]} \mathrm{El}$ singulete observado a 59,17 ppm se asigna al átomo de fósforo coordinado al molibdeno, identificado en el clúster precursor $9^{+}$como $\mathrm{P}(1)$, pero que muestra un desplazamiento de 9 ppm hacia campos más bajos. Basándonos en la caracterización por RMN de fósforo descrita anteriormente, una formulación plausible para el producto de reacción sería 
$\left[\mathrm{Mo}_{3} \mathrm{~S}_{4} \mathrm{Cl}_{3}\left(\kappa^{2}(\mathrm{P}, \mathrm{N})-\left(\mathrm{RhCl}(\mathrm{cod})\left(\kappa^{1}(\mathrm{P})-\mathrm{PNP}\right)\right)_{3}\right]^{+}\right.$, cuya representación esquemática se muestra en la figura 6.13. No obstante, la espectroscopia de RMN ha sido la única técnica que ha aportado información para la caracterización del producto de reacción.

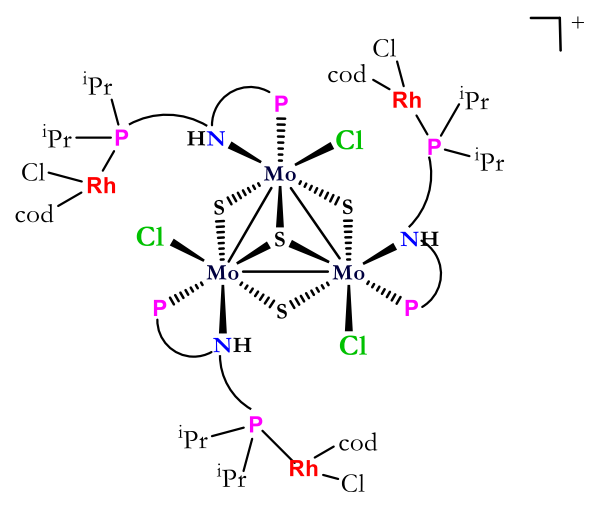

Figura 6.13. Representación esquemática del complejo $\left[\mathrm{Mo}_{3} \mathrm{~S}_{4} \mathrm{Cl}_{3}\left(\kappa^{2}(\mathrm{P}, \mathrm{N})-\left(\mathrm{RhCl}(\operatorname{cod})\left(\kappa^{1}(\mathrm{P})-\right.\right.\right.\right.$ PNP) $\left.)_{3}\right]^{+}$.

\subsubsection{REACTIVIDAD FRENTE A IRIDIO}

El grupo de investigación de Mark Humphrey ha descrito recientemente varios ejemplos de clústeres mixtos de Mo-Ir.[52-54] Por otra parte, Brorson y colaboradores publicaron hace más de una década la síntesis de clústeres con unidad $\mathrm{Mo}_{3} \mathrm{IrS}_{4}$. Sin embargo, no se han encontrado referencias que valoren la actividad catalítica de estos complejos. En cuanto a la actividad catalítica de los sistemas Ir-monofosfina, recientemente el grupo de Timothy J. Donohoe ha demostrado que estos complejos catalizan eficientemente reacciones de acoplamiento entre cetonas y metanol.[55]

El precursor utilizado en estos experimentos es análogo al de rodio descrito en la sección anterior y es uno de los utilizados por el grupo de Brorson en la síntesis de los compuestos anteriormente mencionados. Se ha añadido un exceso de $\left[\{\operatorname{IrCl}(\mathrm{cod})\}_{2}\right]$ sobre una disolución del clúster $\mathbf{9}^{+}$en diclorometano y unas gotas de etanol para solubilizar el compuesto de iridio. Tras 24 horas de reacción, se ha aislado el producto mediante su precipitación con hexano y se ha procedido a su 
caracterización mediante las técnicas habituales. Ninguna de ellas aporta información sobre el producto de reacción.

\subsubsection{REACTIVIDAD FRENTE A ORO}

Hidai et al. publicaron en 2010 la síntesis y estudio de las propiedades electrónicas de los primeros complejos clúster de unidad trimetálica $\mathrm{Mo}_{3} \mathrm{AuS}_{4}$, utilizando complejos de $\mathrm{Au}(\mathrm{I})$ funcionalizados con monofosfinas como precursores, [56] por lo que el estudio de este tipo de compuestos se encuentra en su fase inicial. Además, existen estudios computacionales que demuestran la posibilidad de mejorar las propiedades magnéticas de clústeres de oro mediante el dopaje de estos con átomos de molibdeno.[57] Adicionalmente, los complejos de Au (I) funcionalizados con monofosfinas destacan por sus propiedades luminiscentes.[58,59]

Siguiendo los procedimientos explicados previamente para otros metales, se ha añadido un exceso de $\mathrm{AuCl}\left(\mathrm{PPh}_{3}\right)$ a una disolución del clúster $\mathbf{9}^{+}$en diclorometano, y se ha observado un cambio de color progresivo de verde a marrón. Tras 24 horas de reacción se ha aislado el producto obtenido mediante precipitación. Ninguna de las técnicas habituales ha aportado información sobre el producto obtenido.

\subsubsection{REACTIVIDAD FRENTE A PLATA}

La publicación del grupo de Hidai a la que se hace referencia en la sección 6.3.6 también incluye por primera vez la síntesis de clústeres con unidad tetrametálica $\mathrm{Mos}_{3} \mathrm{AgS}_{4 .}{ }^{[56]}$ Para ello, utilizan cantidades estequiométricas de un complejo de plata (I) y trifenilfosfina que reacciona a temperatura ambiente con el clúster precursor. En nuestro caso, más allá de la coordinación de la plata a la unidad trimetálica, pretendemos estudiar la posibilidad de que una sal de plata sea capaz de declorar nuestro clúster, lo que permitiría la coordinación de los tres átomos de fósforo $\mathrm{P}(2)$ a los respectivos átomos de molibdeno. 
Se ha investigado la reacción del clúster $\mathbf{9}^{+}$previamente disuelto en diclorometano con un exceso de una sal de plata (I), concretamente $\mathrm{AgBF}_{4}$. En este caso se han tenido que añadir unas gotas de éter para favorecer la disolución de esta sal, insoluble en diclorometano. A temperatura ambiente se ha observado un cambio de color inmediato de verde a marrón, acompañado de la aparición de un precipitado mayoritario de color marrón y uno minoritario de color blanco, presumiblemente $\mathrm{AgCl}$. Al cabo de 4 horas de reacción se ha filtrado el sólido obtenido y se ha redisuelto en metanol. Se ha filtrado de nuevo para eliminar el precipitado blanco y, tras la evaporación del disolvente, se ha aislado un sólido marrón microcristalino que se ha podido caracterizar mediante espectroscopia de RMN.

En el espectro de RMN de ${ }^{31} \mathrm{P}\left\{{ }^{1} \mathrm{H}\right\}$ (ver figura 6.14) se observan dos señales de multiplicidad doblete centradas en 27,09 ppm y 22,91 ppm con una constante de acoplamiento 2Jp-p de 36,4 Hz. Esta situación indica la existencia de dos grupos de átomos de fósforo no equivalentes. Además, la multiplicidad de estas señales es análoga a la descrita para un elevado número de clústeres $\mathrm{Mo}_{3} \mathrm{~S}_{4}$ funcionalizados con ligandos difosfina, ${ }^{[27]}$ lo que sugiere la coordinación de los átomos de fósforo $\mathrm{P}(2)$ a los metales, con la consiguiente descoordinación del ligando cloruro. La aparición durante el transcurso de la reacción de un precipitado blanco asociado a la formación de $\mathrm{AgCl}$ confirma esta reacción de sustitución.

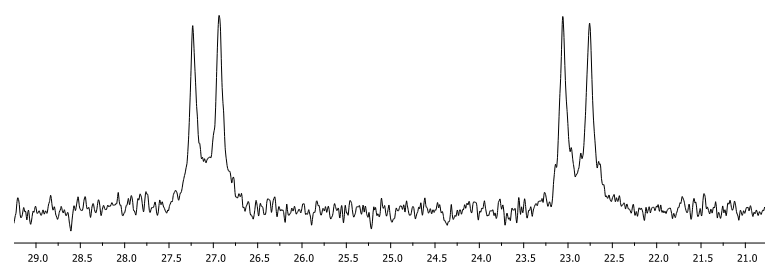

Figura 6.14. Espectro de RMN de ${ }^{31} \mathrm{P}\left\{{ }^{1} \mathrm{H}\right\}$ del compuesto que resulta de la reacción entre $9^{+} \mathrm{y} \mathrm{AgBF} 4$.

El espectro de RMN sugiere que el ligando aminodifosfina se encuentra coordinado de forma tridentada, lo que resulta en un complejo de fórmula 
$\left[\mathrm{Mo}_{3} \mathrm{~S}_{4}(\mathrm{PNP})_{3}\right]^{4+}\left(\mathbf{1 0} \mathbf{4}^{4+}\right)$, la cual concuerda con el resultado obtenido mediante análisis elemental. A pesar de no conocer la disposición espacial de los átomos de fósforo, el espectro de RMN indica que no son químicamente equivalentes, por lo que no pueden apuntar hacia el mismo lado del plano formado por la unidad trimetálica. La figura 6.15 representa de forma esquemática una estructura compatible con los datos espectroscópicos obtenidos.

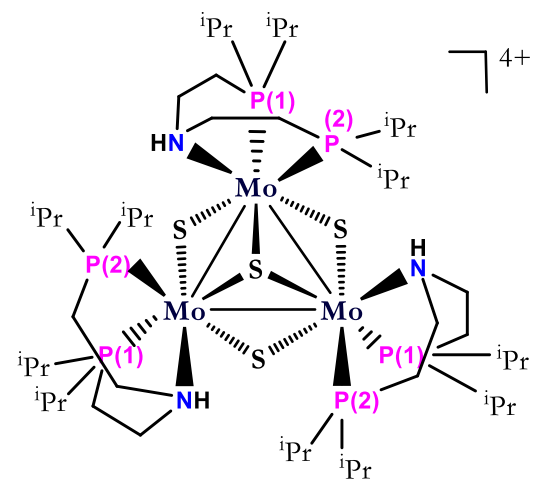

Figura 6.15. Representación esquemática de la estructura del clúster 10 $\mathbf{1 0}^{4+}$.

La coordinación del átomo de fósforo $\mathrm{P}(2)$ al molibdeno resulta en la formación de un anillo de cinco miembros Mo-N-C-C-P(2) de elevada estabilidad termodinámica, equivalente al que se observa para el mismo compuesto en el caso del $\mathrm{P}(1)$ y en otros clústeres funcionalizados con aminofosfinas.[26,29,60] El átomo de fósforo $\mathrm{P}(2)$ también se puede coordinar al molibdeno adyacente dando lugar a un anillo de seis miembros Mo-N-C-C-P(2)-Mo. Sin embargo, la ausencia de una estructura cristalina nos impide descartar la formación de este último anillo y por lo tanto la coordinación del fósforo $\mathrm{P}(2)$ al átomo de molibdeno adyacente.

Este compuesto también se ha intentado caracterizar mediante espectrometría de masas, pero no se ha identificado ningún pico asociado a la especie multicargada $\mathbf{1 0}^{4+}$. Hasta la fecha no se han podido obtener cristales adecuados para su caracterización mediante difracción de rayos X en monocristal. 


\subsubsection{REACTIVIDAD FRENTE A RUTENIO}

El rutenio es un metal de transición ampliamente utilizado en catálisis. Se han descrito numerosos ejemplos de reacciones de hidrogenación, ${ }^{[5,61]}$ de alquilaciónn[62] y de metátesis de olefinas catalizadas por complejos mononucleares de este metal. El profesor Robert Grubbs obtuvo el premio Nobel de Química en 2005, conjuntamente con los profesores Yves Chauvin y Richard R. Schrock, por su contribución a este último campo.[63-65]

En gran parte de los catalizadores monometálicos el rutenio está coordinado a una fosfina monodentada, ${ }^{[6-68]}$ situación que podría ocurrir en nuestro clúster si cada uno de los átomos de fósforo $\mathrm{P}(2)$ se coordinaran a un átomo de rutenio. También existe la posibilidad de que un átomo de rutenio complete la estructura cuboidal que forma la unidad $\mathrm{Mo}_{3} \mathrm{~S}_{4}$ para dar un compuesto con unidad tetrametálica $\mathrm{Mo}_{3} \mathrm{RuS}_{4}$, cuyo precedente ha sido descrito en la literatura por Brorson y colaboradores. ${ }^{[46]}$

En primer lugar, el compuesto $\mathbf{9}^{+}$se ha hecho reaccionar con el clúster $\mathrm{Ru}_{3}(\mathrm{CO})_{12}$ tanto en diclorometano como en acetonitrilo. A pesar de que se observa un cambio de color de verde a rojo, el producto obtenido no se ha podido identificar mediante espectroscopia de RMN ni mediante espectrometría de masas. El hecho de que no se hayan observado señales características de un clúster con unidad $\mathrm{Mo}_{3} \mathrm{~S}_{4} \mathrm{O}$ $\mathrm{Mo}_{3} \mathrm{RuS}_{4}$ nos ha llevado a cambiar el precursor de rutenio. En segundo lugar, se ha probado la reacción del clúster $\mathbf{9}^{+}$con los dímeros $\left[\left\{\mathrm{RuCl}_{2} \mathrm{Ar}_{2}\right](\mathrm{Ar}=p\right.$-cimeno, benceno), que resultan ser buenos precursores para la formación de sistemas Rufosfina. ${ }^{[69,70]}$ El clúster $\mathbf{9}^{+}$se ha disuelto en diclorometano y a esta disolución se le ha añadido un exceso del dímero $\left[\left\{\mathrm{RuCl}_{2} \mathrm{Ar}\right\}_{2}\right]$. Cuando se utiliza el precursor de rutenio que contiene benceno, la reacción se lleva a cabo en una mezcla diclorometano:metanol (1:1) para favorecer su solubilización. En ambos casos se ha observado un cambio inmediato de coloración de verde a marrón y tras tres horas, se 
ha conseguido aislar un sólido marrón. El procedimiento de purificación de este sólido ha sido sencillo: tras una filtración para eliminar restos de precursor de rutenio, se ha evaporado el disolvente para obtener el sólido deseado. Estos sólidos se han caracterizado mediante RMN de ${ }^{31} \mathrm{P}\left\{{ }^{1} \mathrm{H}\right\}$ tal como se muestra en la figura 6.16 .

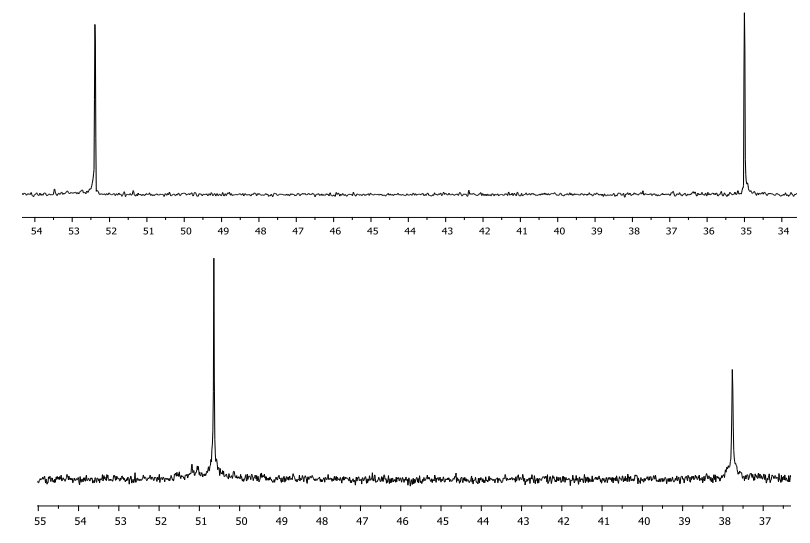

Figura 6.16. Espectros de RMN de ${ }^{31} \mathrm{P}\left\{{ }^{1} \mathrm{H}\right\}$ de los compuestos resultantes de la reacción entre $\mathbf{9}^{+}$y $\left[\left\{\mathrm{RuCl}_{2}(p \text {-cimeno) }\}_{2}\right]\right.$ (arriba) y $\mathbf{9}^{+}$y $\left[\left\{\mathrm{RuCl}_{2} \text { (benceno) }\right\}_{2}\right]$ (abajo).

Los espectros de RMN de los productos de reacción muestran dos singuletes a 52,39 ppm y 35,00 ppm en el primer caso y a 50,64 ppm y 37,76 ppm en el segundo que se corresponden con los dos grupos de átomos de fósforo no equivalentes presentes en la aminodifosfina. Al realizar la integración de las dos señales de cada espectro obtenemos el mismo valor para ambas. Los desplazamientos no coinciden con los observados en el precursor $\mathbf{9}^{+}$, por lo que podemos afirmar que ha tenido lugar una reacción entre el complejo de rutenio y nuestro clúster. Tal como se deduce de los espectros de RMN mostrados en la figura 6.17, los dos precursores de rutenio reaccionan de forma análoga con el clúster $\mathbf{9}^{+}$y probablemente tenga lugar la coordinación de tres átomos de rutenio a los tres átomos de aminodifosfina que permanecían sin coordinar $(\mathrm{P}(2))$, aunque no se descarta todavía la formación de la unidad tetrametálica $\mathrm{Mo}_{3} \mathrm{RuS}_{4}$. 
La caracterización mediante espectrometría de masas nos ha ayudado a conocer más profundamente la naturaleza de estos compuestos. En el caso de la reacción con el precursor de rutenio que contiene $p$-cimeno, se observa un pico a 2356 u.m.a. que se puede asociar con el ion molecular $\left[\mathrm{Mo}_{3} \mathrm{~S}_{4} \mathrm{Cl}_{3}(\kappa)(\mathrm{P}, \mathrm{N})-\left(\mathrm{RuCl}_{2}(p-\right.\right.$ cimeno) $\left.\left.\left(\kappa^{1}(\mathrm{P})-\mathrm{PNP}\right)\right)_{3}\right]^{+}$(de ahora en adelante $\mathbf{1 1}^{+}$) cuyo perfil isotópico concuerda con el simulado (ver figura 6.17). De igual forma, en el caso del precursor que contiene benceno se observa un pico a 2189 u.m.a. que corresponde al ion molecular $\left[\mathrm{Mo}_{3} \mathrm{~S}_{4} \mathrm{Cl}_{3}\left(\kappa^{2}(\mathrm{P}, \mathrm{N})-\left(\mathrm{RuCl}_{2} \text { (benceno) }\left(\kappa^{1}(\mathrm{P})-\mathrm{PNP}\right)\right)_{3}\right]^{+}\left(\mathbf{1 2}^{+}\right)\right.$.

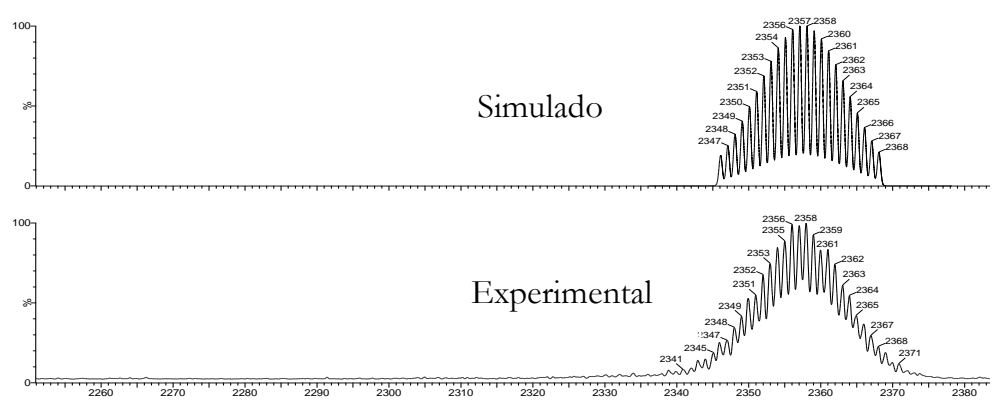

Figura 6.17. Espectro de masas simulado y experimental del compuestos $\mathbf{1 1}^{+}$.

Por otra parte, en el espectro de masas del compuesto $11^{+}$, se observan picos a 312 u.m.a. y 353 u.m.a. que son coherentes con la presencia de complejos mononucleares de rutenio derivados de la ruptura del dímero precursor. El primer pico se asocia la especie $\left[\mathrm{RuCl}(p \text {-cimeno })\left(\mathrm{CH}_{3} \mathrm{CN}\right)\right]^{+}$y el segundo a la especie $\left[\mathrm{RuCl}(p \text {-cimeno })\left(\mathrm{CH}_{3} \mathrm{CN}\right)_{2}\right]^{+}$, ambas generadas en el proceso de ionización por electrospray al reaccionar las impurezas de rutenio presentes en las muestras con el acetonitrilo elegido como disolvente durante el proceso de caracterización. En el caso del complejo $12^{+}$, estas señales aparecen a 256 u.m.a. y 297 u.m.a. para las especies $\left[\mathrm{RuCl}(\text { benceno }) \mathrm{CH}_{3} \mathrm{CN}\right]^{+}$y $\left[\mathrm{RuCl}(\text { benceno })\left(\mathrm{CH}_{3} \mathrm{CN}\right)_{2}\right]^{+}$, respectivamente. Para eliminar estas impurezas ha sido necesaria la adsorción de los complejos $11^{+}$y $\mathbf{1 2}^{+}$, previamente disueltos en diclorometano, en sendas columnas cromatográficas de gel de sílice y su consiguiente elución con una disolución de $\mathrm{KPF}_{6}$ en acetona para 
obtenerlos puros como sales de hexafluorofosfato. Se han obtenido cristales de ambos compuestos pero su patrón de difracción no tenía la calidad necesaria para su caracterización estructural.

La combinación de la espectroscopia de RMN y la espectrometría de masas concuerdan con las estructuras esquemáticas de estos compuestos que se muestran en la figura 6.18. El análisis elemental de los clústeres $11^{+}$y $12^{+}$ha permitido establecer de manera inequívoca que nos encontramos ante dos complejos heterodimetálicos novedosos.

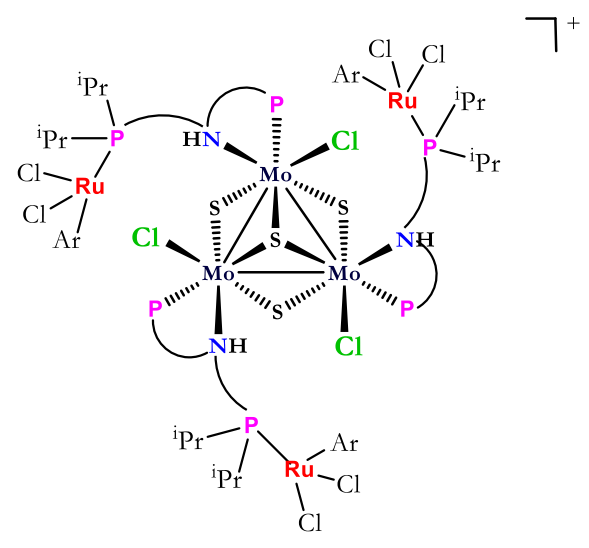

Figura 6.18. Estructura esquematizada de los compuestos $11^{+}(\mathrm{Ar}=p$-cimeno $)$ y $12^{+}(\mathrm{Ar}=$ benceno). 


\subsection{PRUEBAS CATALÍTICAS}

Los clústeres descritos en este capítulo son catalizadores potenciales en una gran variedad de transformaciones químicas. Existen numerosos ejemplos de sistemas $\mathrm{Mo}_{3} \mathrm{~S}_{4}$ y $\mathrm{Mo}_{3} \mathrm{M}^{\prime} \mathrm{S}_{4}$ que son activos como catalizadores en reacciones de hidrodefluoración catalítica, ${ }^{[71,72]}$ ciclopropanación de diazocompuestos, [28]

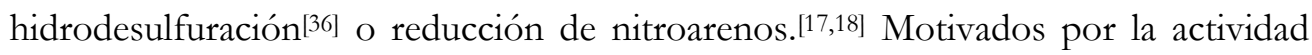
catalítica de los clústeres $\mathrm{Mo}_{3} \mathrm{~S}_{4}$ funcionalizados con aminofosfinas en la reducción de cetonas descrita en los capítulos 4 y 5 y por la importancia de esta transformación en la industria química, hemos decidido investigar la actividad catalítica del complejo $\left[\mathrm{Mo}_{3} \mathrm{~S}_{4} \mathrm{Cl}_{3}(\mathrm{PNP})_{3}\right]^{+}\left(\boldsymbol{9}^{+}\right)$en la reducción de aldehídos y cetonas.

Por otra parte, la característica singular del clúster $\left[\mathrm{Mo}_{3} \mathrm{~S}_{4} \mathrm{Cl}_{3}\left(\kappa^{2}(\mathrm{P}, \mathrm{N})\right.\right.$ $\left.\left(\mathrm{RuCl}_{2}(p \text {-cimeno })\left(\kappa^{1}(\mathrm{P})-\mathrm{PNP}\right)\right)_{3}\right]^{+}\left(\mathbf{1 1}^{+}\right)$, que combina la unidad trinuclear con la funcionalidad del ligando aminodifosfina y la presencia de tres grupos fosfina coordinados a tres átomos de rutenio, nos ha llevado a investigar la hidrogenación catalítica de $\mathrm{CO}_{2}$. El interés de esta reacción radica en su potencial para eliminar el $\mathrm{CO}_{2}$ atmosférico, así como en su capacidad para almacenar hidrógeno.[73,74] Además, la actividad catalítica de los sistemas rutenio-fosfina en la hidrogenación de $\mathrm{CO}_{2}$ ha sido descrita previamente por numerosos autores. ${ }^{67,75,76]}$

La hidrogenación de $\mathrm{CO}_{2}$ presenta una gran relevancia desde el punto de vista medioambiental y constituye un objetivo prioritario en los programas de investigación de muchas agencias, tanto nacionales como internacionales. Esta reacción nos permite obtener compuestos de elevado interés industrial como hidrocarburos saturados, y también moléculas funcionales como derivados de ácidos carboxílicos, alcoholes o aldehídos. Klankermayer, Leitner y colaboradores han acuñado recientemente el término "ajedrez catalítico", en el que exponen que la hidrogenación catalítica de $\mathrm{CO}_{2}$ debe controlarse de manera estratégica tal como sucede en una partida de ajedrez (ver figura 6.19).[74] De esta forma, las diferentes 
etapas de esta reacción se muestran en el eje $\mathrm{Y}$ en función de los equivalentes de $\mathrm{H}_{2}$ necesarios para conseguirlas. Por otra parte, el eje X representa la complejidad molecular en sentido creciente según los enlaces carbono-carbono o carbonoheteroátomo que se forman.

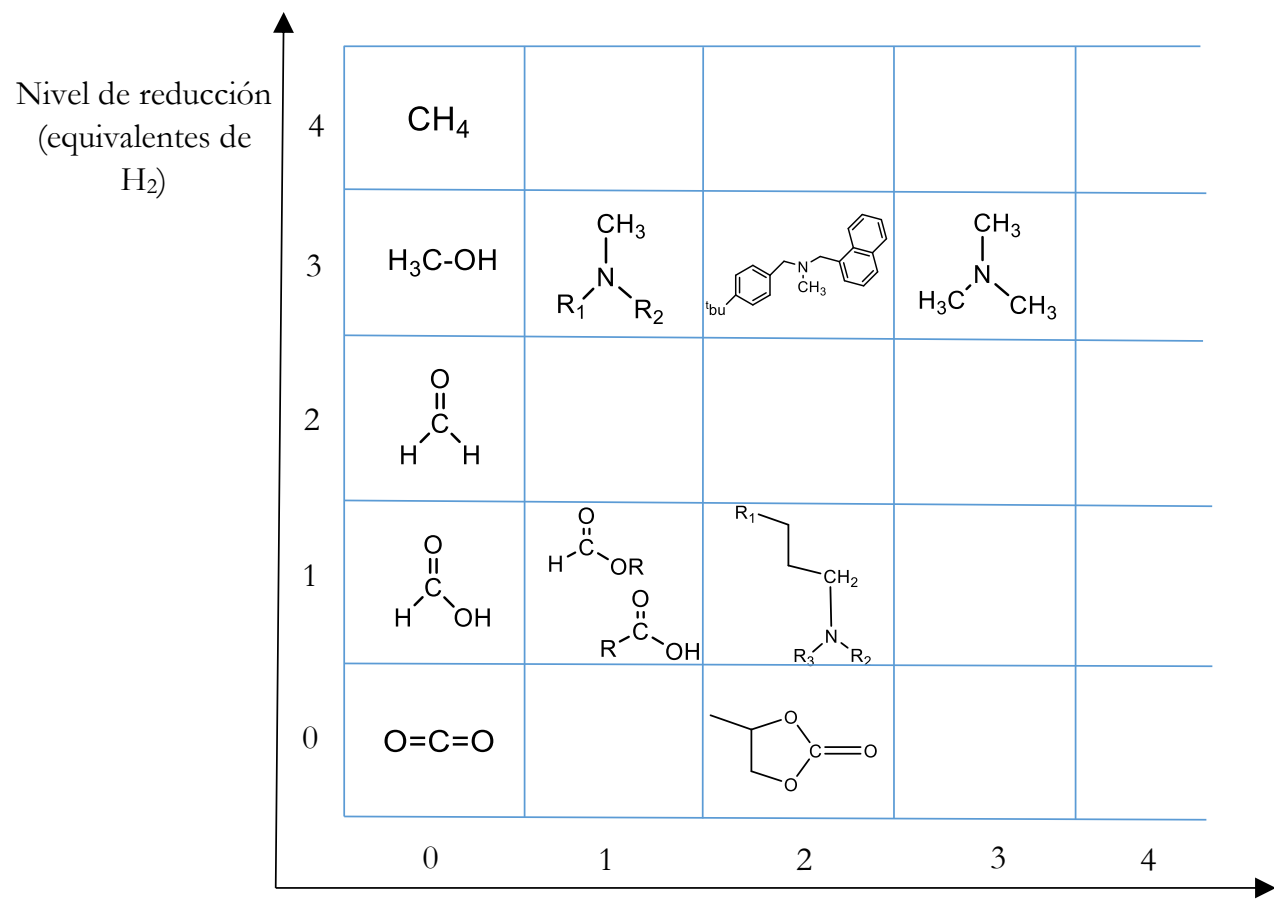

Complejidad molecular (nuevos enlaces C-C y C-heteroátomo)

Figura 6.19. Ajedrez catalítico de Klankermayer, Leitner y colaboradores. Figura adaptada de la referencia [74].

Tal como se observa en la figura 6.19, la adición de un equivalente de $\mathrm{H}_{2}$ proporcionaría ácido fórmico en una reacción 100 \% eficiente. Sin embargo, en muchas ocasiones es necesario aumentar la cantidad de hidrógeno molecular para realizar esta transformación. Las pruebas preliminares de hidrogenación catalítica de 
$\mathrm{CO}_{2}$ realizadas en esta sección se han dirigido hacia la formación de ácido fórmico catalizada por el complejo heterodimetálico $11^{+}$.

\subsubsection{REDUCCIÓN CATALÍTICA DE ALDEHÍDOS Y CETONAS}

La reducción catalítica de aldehídos y cetonas se ha llevado a cabo en el Leibniz Institut für Katalyse (Rostock, Alemania), en colaboración con el profesor Matthias Beller y en el Instituto de Tecnología Química (Valencia). Los sustratos utilizados son similares a los descritos en los capítulos 4 y 5, así como las condiciones de reacción.

El mayor número de ensayos se ha llevado a cabo en presencia de $\mathrm{Ph}_{2} \mathrm{SiH}_{2}$ como agente reductor, ya que proporciona los mejores resultados en presencia de 0,1 mmol de sustrato, $5 \mathrm{~mol} \%$ de catalizador $\left[\mathrm{Mo}_{3} \mathrm{~S}_{4} \mathrm{Cl}_{3}(\mathrm{PNP})_{3}\right]^{+}\left(\mathbf{9}^{+}\right)$y 5 equivalentes de agente reductor. Como medio de reacción se ha elegido metanol debido a la inestabilidad del clúster $\mathbf{9}^{+}$en THF. La tabla 6.2 muestra los resultados de la reducción catalítica de cetonas y aldehídos catalizada por este clúster.

Los mejores resultados se obtienen, al igual que en los capítulos 4 y 5 , para la 2,2,2-trifluoroacetofenona (entrada 1), a pesar de que la conversión y el rendimiento son notablemente menores que para los clústeres de fórmula $\left.\left[\mathrm{Mo}_{3} \mathrm{~S}_{4} \mathrm{Cl}_{3} \text { (aminofosfina }\right)_{3}\right]^{+}$. Hemos observado conversión moderada y rendimientos bajos muy similares entre ellos para la metil-3-piridilcetona (entrada 2) y para la 4fenil-2-butanona (entrada 3).

Por otra parte, la reducción de benzaldehído presenta resultados similares a los de la reducción de cetonas en términos de conversión y rendimiento cuando se utiliza $\mathrm{Ph}_{2} \mathrm{SiH}_{2}$ como agente reductor (entrada 4). Sin embargo, esta reducción es la única que tiene lugar tanto en presencia de $\mathrm{Ph}_{2} \mathrm{SiH}_{2}$ como de la mezcla reductora HCOOH:Et 3 N (5:2) (entrada 5). En este último caso, la conversión es notablemente menor que la observada en presencia de silanos (entrada 5). La reducción de nitrilos en presencia de $\mathrm{HCOOH}: \mathrm{Et}_{3} \mathrm{~N}$ (5:2) y $\mathrm{Ph}_{2} \mathrm{SiH}_{2}$ también se ha probado sin éxito manteniendo las condiciones de reacción anteriores. 
Tabla 6.2. Reducción catalítica de cetonas y aldehídos con el clúster 9+(a).

Entrada

(a)Condiciones de reacción: 0,1 mmol sustrato, $5 \mathrm{~mol} \%$ catalizador, 5 equivalentes agente reductor. (b)Determinados por GC utilizando $\mathrm{n}$ hexadecano como estándar interno. 
Adicionalmente, la hidrogenación directa de ésteres en presencia de bases se ha llevado a cabo sin éxito en un reactor a presión a $100{ }^{\circ} \mathrm{C}$ de temperatura, con una presión de hidrógeno de entre 30 y 50 bar y se han añadido cantidades variables de una base (10-20 equivalentes). Previamente, se han realizado pruebas de reactividad entre las bases ${ }^{\mathrm{BuOK}}, \mathrm{NaOMe},\left[\left(\mathrm{CH}_{3}\right)_{3} \mathrm{Si}_{2} \mathrm{NK}, \mathrm{K}_{2} \mathrm{CO}_{3}\right.$, DIPEA y Et ${ }_{3} \mathrm{~N}$ y el clúster $9^{+}$y en todos los casos se ha observado degradación del complejo clúster, excepto en presencia de DIPEA y Et $t_{3} \mathrm{~N}$, con las que se observa la oxidación de los fósforos no coordinados al metal, resultando en una mezcla de especies $\left[\mathrm{Mo}_{3} \mathrm{~S}_{4} \mathrm{Cl}_{3}\left(\mathrm{PNP}(\mathrm{O})_{\mathrm{n}}\right)\right]^{+}$ $(\mathrm{n}=0-3)$.

En conclusión, tras el análisis global de los resultados de reducción podemos confirmar que el clúster $\mathbf{9}^{+}$es un catalizador menos activo en la reducción de cetonas que los complejos $\left[\mathrm{Mo}_{3} \mathrm{~S}_{4} \mathrm{Cl}_{3}(\text { aminofosfina })_{3}\right]^{+}$. Este hecho se puede atribuir principalmente a la congestión estérica que provoca la presencia de los átomos de fósforo no coordinados.

\subsubsection{HIDROGENACIÓN CATALÍTICA DE $\mathrm{CO}_{2}$}

Los estudios catalíticos se han llevado a cabo en el Instituto de Tecnología Química (Valencia) en un reactor de acero que contiene un recipiente de teflón. Las pruebas se han llevado a cabo en condiciones análogas a las descritas por Byers y colaboradores, $[67]$ i.e. en presencia de $1 \mu \mathrm{mol}$ de clúster $\left[\mathrm{Mo}_{3} \mathrm{~S}_{4} \mathrm{Cl}_{3}\left(\kappa^{2}(\mathrm{P}, \mathrm{N})-\left(\mathrm{RuCl}_{2}(p-\right.\right.\right.$

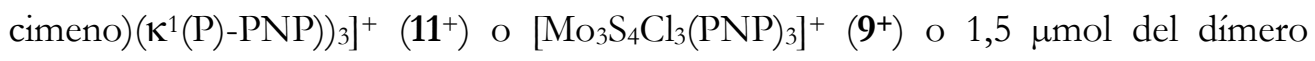
$\left.\left[\left\{\mathrm{RuCl}_{2}(p \text {-cimeno })\right\}_{2}\right]\right)$ y 600 equivalentes de tributilamina en DMSO anhidro a $50{ }^{\circ} \mathrm{C}$ durante 21 horas. Según la prueba, también se han añadido 5 equivalentes de $\mathrm{KHCO}_{3}$ como aditivo. La relación de presión de $\mathrm{CO}_{2}: \mathrm{H}_{2}$ es de 1:2 aproximadamente. El esquema de la reacción catalítica y los resultados preliminares obtenidos se muestran en la tabla 6.3. 
Tabla 6.3. Hidrogenación catalítica de $\mathrm{CO}_{2}{ }^{(\mathrm{a})}$.

$$
\begin{aligned}
& \text { Complejo metálico } \\
& \mathrm{CO}_{2}+\mathrm{H}_{2} \underset{50{ }^{\circ} \mathrm{C}}{600 \mathrm{eq} \mathrm{Bu}_{3} \mathrm{~N}} \mathrm{HCOOH}-\mathrm{NBu}_{3}
\end{aligned}
$$

\begin{tabular}{ccccc}
\hline \hline Entrada & Complejo & Aditivo & $\begin{array}{c}\text { Presión } \mathbf{C O}_{2}: \mathbf{H}_{2} \\
\text { (bar) }\end{array}$ & TON(b) \\
\hline 1 & $\mathbf{1 1}^{+}$ & - & $22: 47$ & 207 \\
2 & $\mathbf{1 1}^{+}$ & $5 \mathrm{eq} \mathrm{KHCO}_{3}$ & $22: 47$ & 221 \\
3 & $\mathbf{1 1}^{+}$ & $5 \mathrm{eq} \mathrm{KHCO}_{3}$ & $6: 13$ & 13 \\
4 & $\mathbf{9}^{+}$ & $5 \mathrm{eq} \mathrm{KHCO}_{3}$ & $22: 47$ & 0 \\
5 & $\left.\left[\left\{\mathrm{RuCl}_{2} p \text {-cimeno }\right)\right\}_{2}\right]$ & $5 \mathrm{eq} \mathrm{KHCO}_{3}$ & $22: 47$ & 103 \\
\hline \hline
\end{tabular}

(a) Condiciones de reacción: $1 \mu \mathrm{mol}$ de $\mathbf{9}^{+}$u 11 $1^{+} ; 1,5 \mu \mathrm{mol}$ de $\left[\left\{\mathrm{RuCl}_{2}(p \text {-cimeno })\right\}_{2}\right]$. (b) TON (turnover number): moles de producto/moles de catalizador. Determinado por $\mathrm{RMN}$ de ${ }^{1} \mathrm{H}$ utilizando DMF como patrón interno.

La hidrogenación de $\mathrm{CO}_{2}$ tiene lugar en presencia del complejo heterodimetálico $11^{+}$(entradas 1, 2 y 3), tanto en ausencia como en presencia de $\mathrm{KHCO}_{3}$. Si se utilizan 5 equivalentes de $\mathrm{KHCO}_{3}$ como aditivo el catalizador presenta mayor actividad (entrada 2). Al aumentar la presión se obtienen mejores resultados en términos de TON. La reacción también tiene lugar en presencia del dímero $\left[\left\{\mathrm{RuCl}_{2}(p \text {-cimeno })\right\}_{2}\right]$ (entrada 5), aunque presenta un TON menor que con el complejo heterodimetálico $\mathbf{1 1}^{+}$. En las mismas condiciones, la actividad catalítica del complejo $\mathbf{1 1}^{+}$es mayor que la del dímero $\left[\left\{\mathrm{RuCl}_{2}(\not \text {-cimeno })\right\}_{2}\right]$. El complejo de molibdeno $\mathbf{9}^{+}$es inactivo bajo las condiciones investigadas (entrada 4).

En conclusión, el complejo $\mathbf{1 1}^{+}$con enlaces rutenio-fosfina cataliza la reducción de $\mathrm{CO}_{2}$ a $\mathrm{HCOOH}$ siendo el $\mathrm{KHCO}_{3}$ un buen aditivo. Este estudio preliminar abre las puertas a futuras aplicaciones del complejo $\mathbf{1 1}^{+}$en catálisis. 


\subsection{BIBLIOGRAFÍA}

[1] Bielinski, E. A.; Lagaditis, P. O.; Zhang, Y.; Mercado, B. Q.; Würtele, C.; Bernskoetter, W. H.; Hazari, N.; Schneider, S. J. Am. Chem. Soc. 2014, 136, 10234.

[2] Sharninghausen, L. S.; Mercado, B. Q.; Crabtree, R. H.; Hazari, N. Chem. Commun. 2015, 51, 16201.

[3] Lagaditis, P. O.; Sues, P. E.; Sonnenberg, J. F.; Wan, K. Y.; Lough, A. J.; Morris, R. H. J. Am. Chem. Soc. 2014, 136, 1367.

[4] Schmeier, T. J.; Dobereiner, G. E.; Crabtree, R. H.; Hazari, N. J. Am. Chem. Soc. 2011, 133, 9274.

[5] Tang, W.; Zhang, M.; Wang, C.; Xiao, J. Synlett 2016, 27, 1748.

[6] Bullock, R. M. Catalysis without precious metals; Wiley-VCH Verlag GmbH \& Co. KGaA, 2010.

[7] Morris, R. H. Chem. Soc. Rev. 2009, 38, 2282.

[8] Junge, K.; Schroder, K.; Beller, M. Chem. Commun. 2011, 47, 4849.

[9] Zhou, S. L.; Fleischer, S.; Junge, K.; Das, S.; Addis, D.; Beller, M. Angen. Chem. Int. Edit. 2010, 49, 8121.

[10] Boddien, A.; Mellmann, D.; Gartner, F.; Jackstell, R.; Junge, H.; Dyson, P. J.; Laurenczy, G.; Ludwig, R.; Beller, M. Science 2011, 333, 1733.

[11] Langer, R.; Leitus, G.; Ben-David, Y.; Milstein, D. Angew. Chem. Int. Edit. 2011, 50, 2120.

[12] Schrock, R. R. In Catalysis without Precious Metals; Wiley-VCH Verlag GmbH \& Co. KGaA, 2010.

[13] Bullock, R. M. In Catalysis without Precious Metals; Wiley-VCH Verlag GmbH \& Co. KGaA, 2010.

[14] Bazan, G. C.; Oskam, J. H.; Cho, H. N.; Park, L. Y.; Schrock, R. R. J. Am. Chem. Soc. 1991, 113, 6899.

[15] Chakraborty, S.; Blacque, O.; Fox, T.; Berke, H. Chem. Asian J. 2014, 9, 328. 
[16] Chakraborty, S.; Blacque, O.; Berke, H. Dalton Trans. 2015, 44, 6560.

[17] Sorribes, I.; Wienhofer, G.; Vicent, C.; Junge, K.; Llusar, R.; Beller, M. Angew. Chem. Int. Edit. 2012, 51, 7794.

[18] Pedrajas, E.; Sorribes, I.; Junge, K.; Beller, M.; Llusar, R. ChemCatChem 2015, $7,2675$.

[19] Cotton, F. A.; Dori, Z.; Llusar, R.; Schwotzer, W. Inorg. Chem. 1986, 25, 3654.

[20] Murata, T.; Mizobe, Y.; Gao, H.; Ishii, Y.; Wakabayashi, T.; Nakano, F.; Tanase, T.; Yano, S.; Hidai, M. J. Am. Chem. Soc. 1994, 116, 3389.

[21] Shibahara, T.; Hattori, H.; Kuroya, H. J. Am. Chem. Soc. 1984, 106, 2710.

[22] Shibahara, T.; Yamasaki, M.; Akashi, H.; Katayama, T. Inorg. Chem. 1991, 30, 2693.

[23] Takashi, S.; Shun, Y.; Masataka, M.; Masaaki, K. Bull. Chem. Soc. Jpn. 1999, 72, 2271.

[24] Duval, S.; Dumur, F.; Guénée, L.; Marrot, J.; Simonnet-Jégat, C.; Cadot, E. Eur. J. Inorg. Chem. 2013, 1149.

[25] Beltrán, T. F., Tesis Doctoral, Universitat Jaume I, 2013.

[26] Alfonso, C.; Feliz, M.; Safont, V. S.; Llusar, R. Dalton Trans. 2016, 45, 7829.

[27] Estevan, F.; Feliz, M.; Llusar, R.; Mata, J. A.; Uriel, S. Polyhedron 2001, 20, 527.

[28] Feliz, M.; Guillamon, E.; Llusar, R.; Vicent, C.; Stiriba, S. E.; Perez-Prieto, J.; Barberis, M. Chem.-Eur.J. 2006, 12, 1486.

[29] Beltran, T. F.; Pino-Chamorro, J. A.; Fernandez-Trujillo, M. J.; Safont, V. S.; Basallote, M. G.; Llusar, R. Inorg. Chem. 2015, 54, 607.

[30] Llusar, R.; Uriel, S. Eur. J. Inorg. Chem. 2003, 1271.

[31] Hidai, M.; Kuwata, S.; Mizobe, Y. Acc. Chem. Res. 2000, 33, 46.

[32] Hernández-Molina, R.; Kalinina, I. V.; Abramov, P. A.; Sokolov, M. N.; Virovets, A. V.; Platas, J. G.; Llusar, R.; Polo, V.; Vicent, C.; Fedin, V. P. Inorg. Chem. 2008, 47, 306.

[33] Seino, H.; Hidai, M. Chem. Sci. 2011, 2, 847. 
[34] Feliz, M.; Garriga, J. M.; Llusar, R.; Uriel, S.; Humphrey, M. G.; Lucas, N. T.; Samoc, M.; Luther-Davies, B. Inorg. Chem. 2001, 40, 6132.

[35] Lipshutz, B. H.; Papa, P. Angew. Chem. Int. Edit. 2002, 41, 4580.

[36] Feliz, M.; Llusar, R.; Uriel, S.; Vicent, C.; Brorson, M.; Herbst, K. Polyhedron 2005, 24, 1212.

[37] Guillamón, E., Tesis Doctoral, Universitat Jaume I, 2009.

[38] Takei, I.; Wakebe, Y.; Suzuki, K.; Enta, Y.; Suzuki, T.; Mizobe, Y.; Hidai, M. Organometallics 2003, 22, 4639.

[39] Quasdorf, K. W.; Antoft-Finch, A.; Liu, P.; Silberstein, A. L.; Komaromi, A.; Blackburn, T.; Ramgren, S. D.; Houk, K. N.; Snieckus, V.; Garg, N. K. J. Am. Chem. Soc. 2011, 133, 6352.

[40] Guan, B. T.; Wang, Y.; Li, B. J.; Yu, D. G.; Shi, Z. J. J. Am. Chem. Soc. 2008, $130,14468$.

[41] Murata, T.; Mizobe, Y.; Gao, H.; Ishii, Y.; Wakabayashi, T.; Nakano, F.; Tanase, T.; Yano, S.; Hidai, M.; Echizen, I.; Nanikawa, H.; Motomura, S. J. Am. Chem. Soc. 1994, 116, 3389.

[42] Wakabayashi, T.; Ishii, Y.; Murata, T.; Mizobe, Y.; Hidai, M. Tetrahedron Lett. 1995, 36, 5585.

[43] Miyaura, N.; Suzuki, A. Chem. Rev. 1995, 95, 2457.

[44] Heck, R. F.; Nolley, J. P. J. Org. Chem. 1972, 37, 2320.

[45] Negishi, E.; King, A. O.; Okukado, N. J. Org. Chem. 1977, 42, 1821.

[46] Herbst, K.; Monari, M.; Brorson, M. Inorg. Chem. 2001, 40, 2979.

[47] Zhu, B.-H.; Shibata, Y.; Muratsugu, S.; Yamanoi, Y.; Nishihara, H. Angen. Chem. Int. Edit. 2009, 48, 3858.

[48] Coutinho, K. J.; Dickson, R. S.; Koutsantonis, G. A.; Skelton, B. W.; White, A. H. Z. Anorg. Allg. Chem. 2008, 634, 669.

[49] Jerphagnon, T.; Renaud, J.-L.; Bruneau, C. Tetrahedron: Asymm. 2004, 15, 2101. 
[50] Tiburcio, J.; Bernès, S.; Torrens, H. Polyhedron 2006, 25, 1549.

[51] García, J. M.; Coll, D. S.; Ocando-Mavárez, E.; Ascanio, J.; Pekerar, S.; Atencio, R.; Gonzalez, T.; Briceño, A.; Avila, E.; Rosales, M. Inorg. Chim. Acta 2014, 414, 250.

[52] Fu, J.; Moxey, G. J.; Cifuentes, M. P.; Humphrey, M. G. J. Organomet. Chem. 2015, 792, 46.

[53] Simpson, P. V.; Randles, M. D.; Gupta, V.; Fu, J.; Moxey, G. J.; Schwich, T.; Morshedi, M.; Cifuentes, M. P.; Humphrey, M. G. Dalton Trans. 2015, 44, 7292.

[54] Fu, J.; Moxey, G. J.; Morshedi, M.; Barlow, A.; Randles, M. D.; Simpson, P. V.; Schwich, T.; Cifuentes, M. P.; Humphrey, M. G. J. Organomet. Chem. 2016, $812,135$.

[55] Shen, D.; Poole, D. L.; Shotton, C. C.; Kornahrens, A. F.; Healy, M. P.; Donohoe, T. J. Angew. Chem. Int. Edit. 2015, 54, 1642.

[56] Chen, P.; Chen, Y.; Zhou, Y.; Peng, Y.; Qu, J.; Hidai, M. Dalton Trans. 2010, $39,5658$.

[57] Cao, J.; Li, Q.; Wang, Z. X.; Gao, L. J.; Fu, F.; Fan, B.; Wang, Y. J. Clust. Sci. 2016, 27, 993.

[58] Jenkins, D. E.; Sykora, R. E.; Assefa, Z. Inorg. Chim. Acta 2013, 406, 293.

[59] Bardají, M.; Miguel-Coello, A. B.; Espinet, P. Inorg. Chim. Acta 2012, 392, 91.

[60] Beltran, T. F.; Safont, V. S.; Llusar, R. Eur. J. Inorg. Chem. 2016. DOI: 10.1002/ejic.201600586

[61] Rohmann, K.; Kothe, J.; Haenel, M. W.; Englert, U.; Hölscher, M.; Leitner, W. Angew. Chem. Int. Edit. 2016, 55, 8966.

[62] Schlepphorst, C.; Maji, B.; Glorius, F. ACS Catal. 2016, 6, 4184.

[63] Nguyen, S. T.; Grubbs, R. H.; Ziller, J. W. J. Am. Chem. Soc. 1993, 115, 9858.

[64] Schwab, P.; France, M. B.; Ziller, J. W.; Grubbs, R. H. Angew. Chem. Int. Edit. $1995,34,2039$. 
[65] Schwab, P.; Grubbs, R. H.; Ziller, J. W. J. Am. Chem. Soc. 1996, 118, 100.

[66] Arockiam, P. B.; Fischmeister, C.; Bruneau, C.; Dixneuf, P. H. Green Chem. 2013, 15, 67.

[67] Drake, J. L.; Manna, C. M.; Byers, J. A. Organometallics 2013, 32, 6891.

[68] Lastra-Barreira, B.; Diaz-Alvarez, A. E.; Menendez-Rodriguez, L.; Crochet, P. RSC Advances 2013, 3, 19985.

[69] Jensen, S. B.; Rodger, S. J.; Spicer, M. D. J. Organomet. Chem. 1998, 556, 151.

[70] Chen, Y.; Valentini, M.; Pregosin, P. S.; Albinati, A. Inorg. Chim. Acta 2002, 327,4 .

[71] Beltran, T. F.; Feliz, M.; Llusar, R.; Mata, J. A.; Safont, V. S. Organometallics 2011, 30, 290.

[72] Alfonso, C.; Beltran, T. F.; Feliz, M.; Llusar, R. J. Clust. Sci. 2015, 26, 199.

[73] Wang, W.; Wang, S.; Ma, X.; Gong, J. Chem. Soc. Rev. 2011, 40, 3703.

[74] Klankermayer, J.; Wesselbaum, S.; Beydoun, K.; Leitner, W. Angew. Chem. Int. Edit. 2016, 55, 7296.

[75] Filonenko, G. A.; van Putten, R.; Schulpen, E. N.; Hensen, E. J. M.; Pidko, E. A. ChemCatChem 2014, 6, 1526.

[76] Elek, J.; Nádasdi, L.; Papp, G.; Laurenczy, G.; Joó, F. Appl. Catal. A 2003, 255,59 . 
7. SECCIÓN EXPERIMENTAL 


\section{SECCIÓN EXPERIMENTAL}

7.1 Síntesis y caracterización

7.1.1. Técnicas generales

7.1.2. Síntesis de precursores

1. Fases sólidas poliméricas

2. Clústeres moleculares

7.1.3. Clústeres funcionalizados con difosfinas

7.1.3.1 Síntesis

7.1.3.2 Hidrodefluoración catalítica de la pentafluoropiridina

7.1.4. Clústeres funcionalizados con aminofosfinas

7.1.4.1 Síntesis

7.1.4.2 Reducción catalítica de sustratos orgánicos

7.1.4.3 Estudios de reactividad frente a alcoholes

7.1.5. Síntesis de clústeres funcionalizados con aminodifosfinas

7.1.5.1 Síntesis

7.1.5.2 Pruebas de reactividad frente a otros metales

7.1.5.3 Reducción catalítica de aldehídos, cetonas y ésteres

7.1.5.4 Hidrogenación catalítica de $\mathrm{CO}_{2}$

7.2 Determinación estructural

7.2.1. Generalidades de la toma de datos y resolución

7.2.2. Clústeres funcionalizados con difosfinas

7.2.2.1 Estructura de $\left[\mathrm{Mo}_{3} \mathrm{~S}_{4} \mathrm{H}_{3}(\mathrm{dppe})_{3}\right] \mathrm{PF}_{6}\left(\mathbf{1}\left(\mathrm{PF}_{6}\right)\right)$

7.2.2.2 Estructura de $\left[\mathrm{Mo}_{3} \mathrm{~S}_{4} \mathrm{~F}_{3}(\mathrm{dppe})_{3}\right] \mathrm{PF}_{6} \cdot \mathrm{H}_{2} \mathrm{O}$ $\left(2\left(\mathrm{PF}_{6}\right) \cdot \mathrm{H}_{2} \mathrm{O}\right)$

7.2.3. Clústeres funcionalizados con aminofosfinas 
7.2.3.1 Estructura de $\quad\left[\mathrm{Mo}_{3} \mathrm{~S}_{4} \mathrm{Cl}_{3}(\text { ediprp })_{3}\right] \mathrm{Cl}$ $\cdot \mathrm{CH}_{2} \mathrm{Cl}_{2}\left(\mathbf{5}(\mathrm{Cl}) \cdot \mathrm{CH}_{2} \mathrm{Cl}_{2}\right)$

7.2.3.2 Estructura de $\left.\left[\mathrm{Mo}_{3} \mathrm{~S}_{4} \mathrm{Cl}_{3} \text { (ediprp) }\right)_{3}\right] \mathrm{Cl}$ $\mathrm{CH}_{2} \mathrm{Cl}_{2}\left(\mathbf{5} \mathbf{a}(\mathrm{Cl}) \cdot \mathrm{CH}_{2} \mathrm{Cl}_{2}\right)$

7.2.3.3 Estructura de $\left[\mathrm{W}_{3} \mathrm{~S}_{4} \mathrm{Br}_{3}(\right.$ ediprp) 3$] \mathrm{BPh}_{4}$ $\left(\mathbf{6}\left(\mathrm{BPh}_{4}\right)\right)$

7.2.3.4 Estructura de $(P)-\left[\mathrm{Mo}_{3} \mathrm{~S}_{4} \mathrm{Cl}_{3}((1 S, 2 \mathrm{R})-\right.$ $\left.\mathrm{PPro}_{3}\right] \mathrm{BF}_{4}\left((\mathrm{P})-\mathbf{7}\left(\mathrm{BF}_{4}\right)\right)$

7.2.3.5 Estructura de $(P)-\left[\mathrm{Mo}_{3} \mathrm{~S}_{4} \mathrm{Cl}_{3}((1 S, 2 S)\right.$ PPro $\left.)_{3}\right] \mathrm{BF}_{4} \cdot 3 \mathrm{C}_{7} \mathrm{H}_{8}\left((P)-8\left(\mathrm{BF}_{4}\right) \cdot 3 \mathrm{C}_{7} \mathrm{H}_{8}\right)$

7.2.4. Clústeres funcionalizados con aminodifosfinas

7.2.4.1 Estructura de $\left[\mathrm{Mo}_{3} \mathrm{~S}_{4} \mathrm{Cl}_{3}(\mathrm{PNP})_{3}\right] \mathrm{Cl}(\mathbf{9}(\mathrm{Cl}))$

7.3 Cálculos teóricos

7.3.1 Geometrías optimizadas

7.3.1.1 $(P)-\left[\mathrm{Mo}_{3} \mathrm{~S}_{4} \mathrm{Cl}_{3}((1 S, 2 \mathrm{R})-\mathrm{PPro})_{3}\right]^{+}\left((P)-7^{+}\right)$

7.3.1.2 $(P)-\left[\mathrm{Mo}_{3} \mathrm{~S}_{4} \mathrm{Cl}_{3}((1 S, 2 S)-\mathrm{PPro})_{3}\right]^{+}\left((P)-\mathbf{8}^{+}\right)$

7.3.1.3 $(M)-\left[\mathrm{Mo}_{3} \mathrm{~S}_{4} \mathrm{Cl}_{3}((1 S, 2 \mathrm{R})-\mathrm{PPro})_{3}\right]^{+}\left((M)-7^{+}\right)$

7.3.1.4 $(M)-\left[\mathrm{Mo}_{3} \mathrm{~S}_{4} \mathrm{Cl}_{3}((1 S, 2 S)-\mathrm{PPro})_{3}\right]^{+}\left((M)-\mathbf{8}^{+}\right)$

7.4 Bibliografía 
"La ciencia y la vida cotidiana no pueden ni deben separarse. La ciencia me proporciona una explicación parcial de la vida en la medida en que se basa en los hechos, la experiencia y la experimentación."

Rosalind Franklin 


\subsection{SÍNTESIS Y CARACTERIZACIÓN}

\subsubsection{TÉCNICAS GENERALES}

Las reacciones en estado sólido se han llevado a cabo en tubos de pyrex de $18 \mathrm{~mm}$ de diámetro externo, $14 \mathrm{~mm}$ de diámetro interno y aproximadamente $20 \mathrm{~cm}$ de largo. Se introducen los reactivos en el tubo, se realizan ciclos de vacío y llenado de nitrógeno a la temperatura del nitrógeno líquido y, seguidamente, con la ayuda de un soplete se sellan a vacío y se introducen en el horno tubular donde tiene lugar la reacción, a la temperatura deseada y durante el tiempo establecido.

Las reacciones en disolución se han realizado, a menos que se indique lo contrario, en atmósfera inerte de nitrógeno utilizando las técnicas estándar de Schlenk. El acetonitrilo, diclorometano y tetrahidrofurano utilizados en las síntesis se han purificado utilizando el equipo MBRAUN SPS-800. El etanol se ha desgasificado burbujeando una corriente de nitrógeno durante 30 minutos antes de su utilización.

Los ensayos de catálisis bajo radiación microondas se realizaron en un equipo MW Discover Labmate (CEM Corp.). Los espectros de masas (ESI-MS) se han registrado en un equipo de triple cuadrupolo (Micromass Quattro LC). La composición de los picos se asigna por comparación de la distribución isotópica experimental con la teórica, obtenida utilizando el programa MassLynx 4.1.[1] Las medidas de análisis elemental se han realizado en un analizador Euro EA 3000 CHN.

Los espectros de resonancia magnética nuclear $(\mathrm{RMN})$ de ${ }^{31} \mathrm{P},{ }^{19} \mathrm{~F}$ y ${ }^{1} \mathrm{H}$ se han registrado en los equipos Varian Mercury Vx $300 \mathrm{MHz}$ y recientemente en Bruker 400 y 300 Avance III HD utilizando disolventes deuterados. Los espectros de dicroísmo circular se han registrado a temperatura ambiente en un espectropolarímetro JASCO J-180 utilizando una cubeta de cuarzo de $1 \mathrm{~cm}$ de recorrido. Los análisis por cromatografía de gases se llevaron a cabo en un cromatógrafo de gases Agilent, modelo 7820A, equipado con una columna capilar HP-5, con dimensiones $30 \mathrm{~m}$ x 0,320 $\mathrm{mm} \times 0,25 \mu \mathrm{m}$. 


\subsubsection{SÍNTESIS DE PRECURSORES}

Las síntesis de los complejos clúster descritas en esta tesis doctoral requieren la preparación previa de precursores que se utilizan como punto de partida para su obtención. Estos precursores se han preparado siguiendo los procedimientos experimentales publicados en la literatura, que se describen a continuación.

\subsubsection{FASES SÓLIDAS POLIMÉRICAS}

\section{$\left\{\mathrm{Mo}_{3} \mathrm{~S}_{7} \mathrm{Cl}_{4}\right\}_{\mathrm{n}}{ }^{[2]}$}

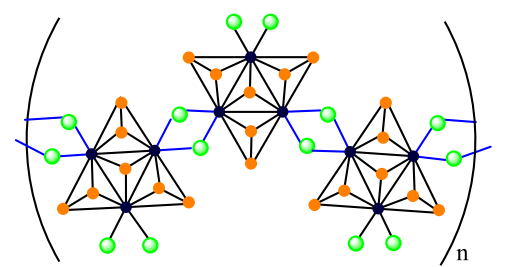

En un tubo de pyrex se introducen molibdeno en polvo $(1,000 \mathrm{~g}, 10,40 \mathrm{mmol})$, azufre $(0,34,10,40$ $\mathrm{mmol})$ y $\mathrm{S}_{2} \mathrm{Cl}_{2}(0,56 \mathrm{~mL}, 6,90 \mathrm{mmol})$. Después de desoxigenar los reactivos, se sella el tubo a vacío.

La reacción en estado sólido se lleva a cabo en un horno tubular a $425^{\circ} \mathrm{C}$ durante 48 horas. A continuación, se deja que el fundido alcance la temperatura ambiente, se lava con diclorometano y se seca aplicando vacío, obteniéndose 1,900 g (rendimiento 84\%) de un sólido rojo teja.

IR (polietileno, cm-1): 561 (f, $\mathrm{S}_{\text {ax }}-\mathrm{S}_{\mathrm{ec}}$ ), 540 (f, $\mathrm{S}_{\mathrm{ax}}-\mathrm{S}_{\mathrm{ec}}$ ), 460 (m, Mo- $\left.\mu_{3}-\mathrm{S}\right), 395$ (d, Mo$\left.\mu_{2-} \mathrm{S}_{\mathrm{ax}}\right), 365\left(\mathrm{~d}, \mathrm{Mo}-\mu_{2-} \mathrm{S}_{\mathrm{ec}}\right.$ ), 342 (f, Mo- $\mu_{2-} \mathrm{S}_{\mathrm{ec}}$ ). 


\section{$\left\{\mathbf{W}_{3} \mathrm{~S}_{7} \mathrm{Br}_{4}\right\}_{\mathrm{n}}{ }^{[3]}$}

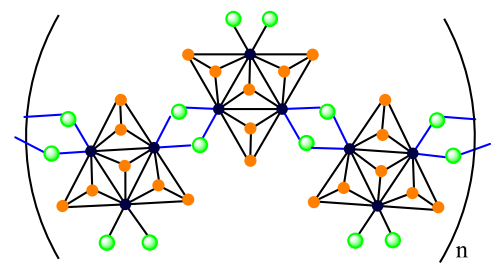

En un tubo de pyrex se introducen wolframio en polvo $(1,250 \mathrm{~g}, 6,80 \mathrm{mmol})$, azufre $(0,508 \mathrm{~g}, 0,16$ $\mathrm{mmol})$ y $\mathrm{Br}_{2}(0,24 \mathrm{~mL}, 4,67 \mathrm{mmol})$. Estos reactivos se desgasifican y se sella el tubo a vacío. La reacción se lleva a cabo en un horno tubular, a $300{ }^{\circ} \mathrm{C}$ durante 120 horas. Seguidamente, se deja enfriar el producto, se lava con diclorometano y se seca a vacío, obteniéndose 2,400 g (rendimiento 97\%) de un sólido marrón.

IR (polietileno, $\left.\mathrm{cm}^{-1}\right)$ : $554\left(\mathrm{~m}, \mathrm{~S}_{\mathrm{ax}}-\mathrm{S}_{\mathrm{ec}}\right), 543\left(\mathrm{f}, \mathrm{S}_{\mathrm{ax}}-\mathrm{S}_{\mathrm{ec}}\right), 445\left(\mathrm{~d}, \mathrm{~W}-\mu_{3}-\mathrm{S}\right), 378\left(\mathrm{~W}-\mu_{2-}\right.$ $\mathrm{S}_{\mathrm{ec}}$ ), $337\left(\mathrm{~d}, \mathrm{~W}-\mu_{2-} \mathrm{S}_{\mathrm{ec}}\right), 329$ (f, W- $\left.\mu_{2-} \mathrm{S}_{\mathrm{ax}}\right), 318\left(\mathrm{~d}, \mathrm{~W}-\mu_{2-} \mathrm{S}_{\mathrm{ax}}\right)$.

\subsubsection{CLÚSTERES MOLECULARES}

\section{$\left(\mathrm{NH}_{4}\right)_{2}\left[\mathrm{Mo}_{3} \mathrm{~S}_{13}\right] \cdot \mathrm{nH}_{2} \mathrm{O}(\mathrm{n}=0-2)^{[4]}$}

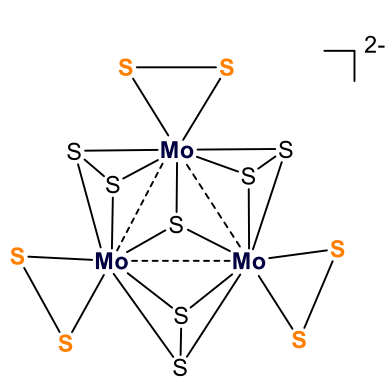

Se disuelven $27 \mathrm{~g}$ de azufre elemental $(0,84 \mathrm{mmol})$ en 120 $\mathrm{mL}$ de $\left(\mathrm{NH}_{4}\right)_{2} \mathrm{~S}$ al $20 \%$, y se observa que la disolución toma paulatinamente una coloración rojiza. Por otra parte, en un Erlenmeyer se prepara una disolución de $\left(\mathrm{NH}_{4}\right)_{6}\left[\mathrm{Mo}_{7} \mathrm{O}_{24}\right] \cdot 4 \mathrm{H}_{2} \mathrm{O}(4 \mathrm{~g}, 3,24 \mathrm{mmol})$ en $20 \mathrm{~mL}$ de agua y se calienta. A continuación, se añade lentamente la

primera disolución al Erlenmeyer que contiene la disolución de molibdato, se cubre el matraz con un vidrio de reloj y se deja reaccionar durante 5 días a $85{ }^{\circ} \mathrm{C} \sin$ agitación. Una vez transcurrido este tiempo, se deja que la mezcla alcance la temperatura ambiente, se filtra y se lava sucesivamente con agua, etanol, disulfuro de carbono y éter dietílico. Se obtienen 5,05 g (rendimiento 88\%) de un sólido rojo microcristalino.

Análisis Elemental calc. para $\mathrm{H}_{8} \mathrm{Mo}_{3} \mathrm{~N}_{2} \mathrm{~S}_{13} \cdot \mathrm{H}_{2} \mathrm{O}(\%): \mathrm{H}, 1,33 ; \mathrm{N}, 3,69 ; \mathrm{S}, 54,93$; Exp (\%): H, 1,10; N, 3,35; S, 55,80. 


\section{$\left(\mathrm{Bu}_{4} \mathrm{~N}\right)_{2}\left[\mathrm{Mo}_{3} \mathrm{~S}_{7} \mathrm{Cl}_{6}\right]$}

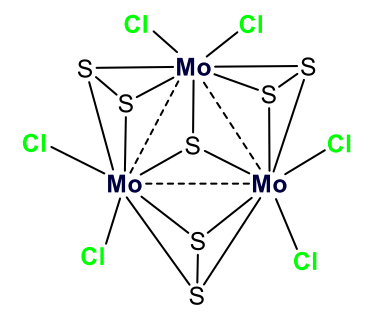

$7^{2-}$ Se disuelven $0,200 \quad \mathrm{~g} \quad(0,26 \quad \mathrm{mmol}) \quad \mathrm{de}$ $\left(\mathrm{NH}_{4}\right)_{2}\left[\mathrm{Mo}_{3} \mathrm{~S}_{13}\right] \cdot \mathrm{H}_{2} \mathrm{O}$ en $30 \mathrm{~mL}$ de $\mathrm{HCl}(37 \%$ de pureza) y se calienta la mezcla a reflujo durante 4 horas. Se filtra la disolución resultante para eliminar el azufre elemental generado durante el transcurso de la reacción, y al filtrado se le añade un exceso de $\mathrm{Bu}_{4} \mathrm{NCl}$ hasta observar la precipitación de un sólido naranja. Se lava el precipitado con agua, metanol y éter dietílico, obteniéndose 0,249 g (rendimiento 80\%) de un producto caracterizado como $\left(\mathrm{Bu}_{4} \mathrm{~N}\right)_{2}\left[\mathrm{Mo}_{3} \mathrm{~S}_{7} \mathrm{Cl}_{6}\right]$.

ESI(-)-MS $\left(\mathrm{CH}_{3} \mathrm{CN}, 20 \mathrm{~V}\right): m / z: 361[\mathrm{M}]^{2-}$.

$\left[\mathrm{Mo}_{3} \mathrm{~S}_{4} \mathrm{Cl}_{4}\left(\mathrm{PPh}_{3}\right)_{3}\left(\mathrm{H}_{2} \mathrm{O}\right)_{2}\right]^{[2],[6]}$

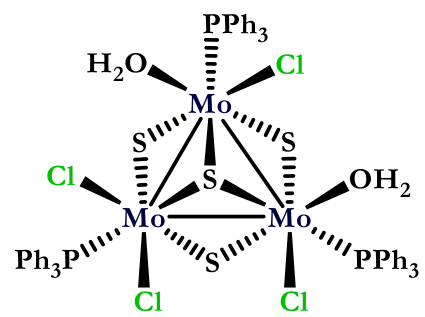

Se prepara una disolución de 0,200 g $(0,165 \mathrm{mmol}) \mathrm{de}$ $\left(\mathrm{Bu}_{4} \mathrm{~N}\right)_{2}\left[\mathrm{Mo}_{3} \mathrm{~S}_{7} \mathrm{Cl}_{6}\right]$ en $5 \mathrm{~mL}$ de metanol y, a continuación, se le añade un exceso de trifenilfosfina $(0,290 \mathrm{~g}, 1,224 \mathrm{mmol})$. Se deja reaccionar durante 25 minutos y se observa la formación de una suspensión de color verde. La mezcla de reacción se filtra, y se lava el sólido con metanol, una mezcla fría de hexano-tolueno (1:1) y con hexano a ebullición para eliminar los subproductos de reacción. Se obtienen 0,205 g del compuesto deseado (rendimiento $90 \%)$.

${ }^{31} \mathrm{P}\left\{{ }^{1} \mathrm{H}\right\} \mathrm{RMN}\left(\mathrm{CD}_{3} \mathrm{CN}, 121 \mathrm{MHz}\right) \delta(\mathrm{ppm})=28,17 ; 24,41 ;-4,4\left(\mathrm{PPh}_{3}\right.$ libre $)$. 


\section{$\left[\mathrm{Mo}_{3} \mathrm{~S}_{4} \mathrm{Cl}_{3}(\mathrm{dppe})_{3}\right] \mathrm{X}\left(\mathrm{X}=\mathrm{PF}_{6}, \mathrm{BPh}_{4}\right)^{[7]}$}

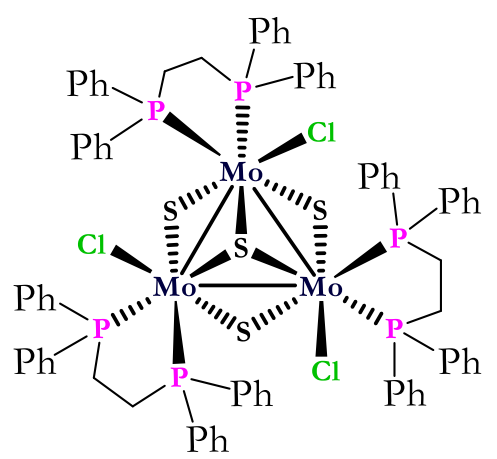

A una suspensión de $\left\{\mathrm{Mo}_{3} \mathrm{~S}_{7} \mathrm{Cl}_{4}\right\}_{\mathrm{n}}(0,15 \mathrm{~g}, 0,23$ mmol) en acetonitrilo se le añade un exceso de dppe $(0,600 \mathrm{~g}, 1,51 \mathrm{mmol})$ bajo atmósfera de nitrógeno. A los cinco minutos se observa que la disolución toma un color verde. Se deja reaccionar durante 48 horas a reflujo. Después de enfriar la disolución a temperatura ambiente, se separa el precipitado por filtración. Se recoge el filtrado y se lleva a sequedad, se redisuelve en diclorometano y se carga en una columna de gel de sílice. Se lava con acetona y se eluye el producto con una disolución de $\mathrm{KPF}_{6}$ en acetona. Se obtiene una disolución concentrada de color verde que se lleva a sequedad; el precipitado obtenido se redisuelve en diclorometano y se filtra para eliminar las sales inorgánicas. El filtrado se lleva a sequedad y se obtienen $385 \mathrm{mg}$ (rendimiento 90\%) de un sólido verde estable al aire caracterizado como $\left[\mathrm{Mo}_{3} \mathrm{~S}_{4} \mathrm{Cl}_{3}(\mathrm{dppe})_{3}\right] \mathrm{PF}_{6}$. Para cambiar el contraión se disuelve el sólido anterior en metanol y se añade un exceso de $\mathrm{NaBPh}_{4}$. Se observa la precipitación de un sólido verde en el seno de la disolución, que corresponde al compuesto $\left[\mathrm{Mo}_{3} \mathrm{~S}_{4} \mathrm{Cl}_{3}(\mathrm{dppe})_{3}\right] \mathrm{BPh}_{4}$. A continuación se filtra, se lava con metanol y se seca al aire.

${ }^{31} \mathrm{P}\left\{{ }^{1} \mathrm{H}\right\} \mathrm{NMR}\left(\mathrm{CD}_{3} \mathrm{CN}, 121 \mathrm{MHz}\right) \delta(\mathrm{ppm}): 26,92\left(\mathrm{t},{ }^{3} J\left({ }^{31} \mathrm{P},{ }^{31} \mathrm{P}\right)=10 \mathrm{~Hz}\right), 36,32$ $\left(\mathrm{t},{ }^{3} \mathrm{~J}\left({ }^{31} \mathrm{P},{ }^{31} \mathrm{P}\right)=10 \mathrm{~Hz}\right)$.

ESI-MS $\left(\mathrm{CH}_{3} \mathrm{CN}, 80 \mathrm{~V}\right) \mathrm{m} / \approx: 1717\left(\mathrm{M}^{+}\right), 1319\left(\mathrm{M}^{+}-\right.$dppe $)$

UV-Vis $\left(\mathrm{CH}_{3} \mathrm{CN}\right) \mathrm{nm}: 628$ (b), 408 (b), 350 (sh), 302 (sh). 
$\left[\mathrm{W}_{3} \mathrm{~S}_{4} \mathrm{Br}_{3}(\mathrm{dppe})_{3}\right] \mathrm{X}\left(\mathrm{X}=\mathrm{PF}_{6}, \mathrm{BPh}_{4}\right)^{[7]}$

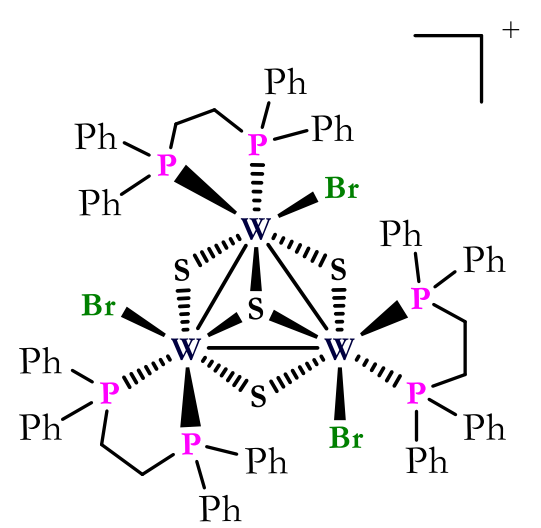

A una suspensión de $\left\{\mathrm{W}_{3} \mathrm{~S}_{7} \mathrm{Br}_{4}\right\}_{n}(0,1 \mathrm{~g}, 0,091$
mmol) en acetonitrilo, se le añade un exceso de dppe (200 mg, 0,5 mmol) bajo atmósfera de nitrógeno. Se deja reaccionar durante 48 horas a reflujo, y a continuación se filtra la mezcla de reacción y el filtrado de color azul se lleva a sequedad. Se redisuelve en la mínima cantidad de diclorometano y se carga en una columna cromatográfica de sílice. Se eluye con una disolución de $\mathrm{KPF}_{6}$ en acetona y se obtiene una disolución de color azul que se lleva a sequedad. El precipitado obtenido se redisuelve en diclorometano, se filtra para eliminar las impurezas y se lleva de nuevo a sequedad, para obtener un sólido azul (160 mg, rendimiento 77\%) caracterizado como $\left[\mathrm{W}_{3} \mathrm{~S}_{4} \mathrm{Br}_{3}(\text { dppe })_{3}\right] \mathrm{PF}_{6}$. El cambio de contraión se realiza de forma análoga al complejo de molibdeno descrito anteriormente.

${ }^{31} \mathrm{P}\left\{{ }^{1} \mathrm{H}\right\} \operatorname{NMR}\left(\mathrm{CD}_{3} \mathrm{CN}, 121 \mathrm{MHz}\right) \delta(\mathrm{ppm}): 2,94\left(\mathrm{~d},{ }^{2} J\left({ }^{31} \mathrm{P},{ }^{31} \mathrm{P}\right)=5,5 \mathrm{~Hz},{ }^{1} J\left({ }^{31} \mathrm{P}\right.\right.$, $\left.\left.{ }^{183} \mathrm{~W}\right)=151,46 \mathrm{~Hz}\right), 7,90\left(\mathrm{~d},{ }^{2} J\left({ }^{31} \mathrm{P},{ }^{31} \mathrm{P}\right)=5,5 \mathrm{~Hz},{ }^{1} J\left({ }^{31} \mathrm{P},{ }^{183} \mathrm{~W}\right)=159,36 \mathrm{~Hz}\right)$.

ESI-MS $\left(\mathrm{CH}_{3} \mathrm{CN}, 80 \mathrm{~V}\right) \mathrm{m} / \approx: 2115\left(\mathrm{M}^{+}\right), 1717\left(\mathrm{M}^{+}\right.$-dppe)

UV-Vis $\left(\mathrm{CH}_{3} \mathrm{CN}\right) \mathrm{nm}: 570$ (b), 365 (sh), 327 (b), 288 (b). 


\subsubsection{CLÚSTERES FUNCIONALIZADOS CON DIFOSFINAS}

\subsubsection{SÍNTESIS}

\section{$\left[\mathrm{Mo}_{3} \mathrm{~S}_{4} \mathrm{H}_{3}(\text { dppe })_{3}\right] \mathrm{BPh}_{4}\left(1\left(\mathrm{BPh}_{4}\right)\right)$}

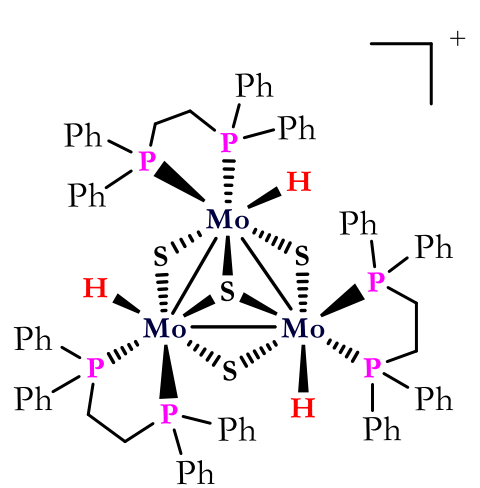

A una disolución de $\left[\mathrm{Mo}_{3} \mathrm{~S}_{4} \mathrm{Cl}_{3}(\mathrm{dppe})_{3}\right] \mathrm{BPh}_{4}(90,4$
$\mathrm{mg}, 0,0485 \mathrm{mmol})$ en $15 \mathrm{~mL}$ de metanol se le añade un exceso de $\mathrm{NaBH}_{4}(91,78 \mathrm{mg}, 2,4261 \mathrm{mmol})$ bajo atmósfera de nitrógeno. Se deja reaccionar la mezcla durante 2 horas, y a continuación se le añade un exceso de $\mathrm{NaBPh}_{4}$ para provocar la precipitación del compuesto deseado en el seno de la reacción. El sólido marrón obtenido se filtra y se lava sucesivamente con metanol y éter dietílico $(70,4 \mathrm{mg}$, rendimiento 75\%).

${ }^{31} \mathrm{P}\left\{{ }^{1} \mathrm{H}\right\} \mathrm{RMN}\left(\mathrm{CD}_{3} \mathrm{CN}, 121 \mathrm{MHz}\right) \delta(\mathrm{ppm})=33,10(\mathrm{~d}, 3 \mathrm{P}), 59,29(\mathrm{~d}, 3 \mathrm{P}) \cdot{ }^{2} \mathrm{~J}\left({ }^{31} \mathrm{P},{ }^{31} \mathrm{P}\right)$ $=7,9 \mathrm{~Hz})$.

${ }^{1} \mathrm{H} \mathrm{RMN}\left(\mathrm{CD}_{3} \mathrm{CN}, 282 \mathrm{MHz}\right) \delta(\mathrm{ppm})=-1,05\left(\mathrm{dd}, 3 \mathrm{H},{ }^{2} \mathrm{~J}\left({ }^{1} \mathrm{H},{ }^{31} \mathrm{P}\right)=37,0 \mathrm{~Hz}\right.$, $\left.{ }^{2} \mathrm{~J}\left({ }^{1} \mathrm{H},{ }^{31} \mathrm{P}\right)=75,0 \mathrm{~Hz}\right)$.

ESI-MS $\left(\mathrm{CH}_{3} \mathrm{CN}, 20 \mathrm{~V}\right) \mathrm{m} /$ ₹: $1614[\mathrm{M}+]$.

Análisis Elemental calc. para $\mathrm{Mo}_{3} \mathrm{~S}_{4} \mathrm{P}_{6} \mathrm{C}_{102} \mathrm{H}_{95} \mathrm{~B}$ (\%): C: 63,36; H: 4,95; S: 6,63; Exp (\%): C: 63,95; H: 5,21; S: 6,97. 
$\left[\mathrm{Mo}_{3} \mathrm{~S}_{4} \mathrm{~F}_{3}(\mathrm{dppe})_{3}\right] \mathrm{X}\left(\mathrm{X}=\mathrm{PF}_{6}, \mathrm{BPh}_{4}\right)(2(\mathrm{X}))$

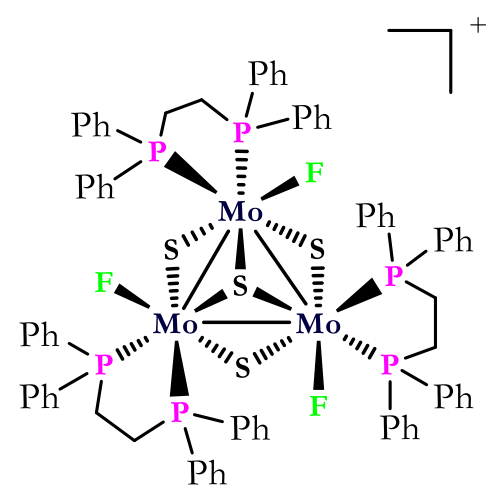

A una disolución de $\left[\mathrm{Mo}_{3} \mathrm{~S}_{4} \mathrm{Cl}_{3}(\mathrm{dppe})_{3}\right] \mathrm{PF}_{6}(97,4$ $\mathrm{mg}, 0,0523 \mathrm{mmol}$ ) en acetonitrilo seco se le añade un exceso de 6,5 equivalentes de CsF. La mezcla se calienta a $60{ }^{\circ} \mathrm{C}$ durante 24 horas, y una vez transcurrido este tiempo se filtra. El filtrado se lleva a sequedad, se redisuelve en diclorometano y se filtra de nuevo para eliminar las sales inorgánicas. Después de evaporar el disolvente, obtenemos 61,6 mg de un sólido verde caracterizado como $\left[\mathrm{Mo}_{3} \mathrm{~S}_{4} \mathrm{~F}_{3}(\mathrm{dppe})_{3}\right] \mathrm{PF}_{6}$ (rendimiento $65 \%$ ). El cambio de contraión de $\mathrm{PF}_{6}^{-}$a $\mathrm{BPh}_{4}$ - se realiza disolviendo el sólido obtenido en metanol y añadiendo un exceso de $\mathrm{NaBPh}_{4}$. Se observa la precipitación de un sólido verde en el seno de la disolución, que corresponde al complejo $\left[\mathrm{Mo}_{3} \mathrm{~S}_{4} \mathrm{~F}_{3}(\mathrm{dppe})_{3}\right] \mathrm{BPh}_{4}$. A continuación, se filtra, se lava con metanol y se seca al aire. ${ }^{31} \mathrm{P}\left\{{ }^{1} \mathrm{H}\right\} \mathrm{RMN}\left(\mathrm{CD}_{3} \mathrm{CN}, 121 \mathrm{MHz}\right) \delta(\mathrm{ppm})=-143,3$ (multiplete, $1 \mathrm{P},{ }^{1} \mathrm{~J}\left({ }^{31} \mathrm{P},{ }^{19} \mathrm{~F}\right)=$ 706,4 Hz), 22,2 (d, 3P, ${ }^{2} \mathrm{~J}\left({ }^{31} \mathrm{P},{ }^{19} \mathrm{~F}\right)=103,3 \mathrm{~Hz}, 30,4\left(\mathrm{~d}, 3 \mathrm{P},{ }^{2} \mathrm{~J}\left({ }^{31} \mathrm{P},{ }^{19} \mathrm{~F}\right)=40,1 \mathrm{~Hz}\right)$. ${ }^{19} \mathrm{~F} \operatorname{RMN}\left(\mathrm{CD}_{3} \mathrm{CN}, 282 \mathrm{MHz}\right) \delta(\mathrm{ppm})=-177,5\left(\mathrm{dd}, 3 \mathrm{~F},{ }^{2} \mathrm{~J}\left({ }^{19} \mathrm{~F},{ }^{31} \mathrm{P}\right)=40,9 \mathrm{~Hz}\right.$, $\left.{ }^{2} \mathrm{~J}\left({ }^{19} \mathrm{~F},{ }^{31} \mathrm{P}\right)=103,1 \mathrm{~Hz}\right),-72,9\left(\mathrm{~d}, 6 \mathrm{~F},{ }^{1} \mathrm{~J}\left({ }^{19} \mathrm{~F},{ }^{31} \mathrm{P}\right)=700,3 \mathrm{~Hz}\right)$.

ESI-MS $\left(\mathrm{CH}_{3} \mathrm{CN}, 20 \mathrm{~V}\right) \mathrm{m} / \approx_{:} 1668[\mathrm{M}+]$.

Análisis Elemental calc. para $\mathrm{Mo}_{3} \mathrm{~S}_{4} \mathrm{P}_{7} \mathrm{~F}_{9} \mathrm{C}_{78} \mathrm{H}_{72}$ (\%): C: 51,66; H: 4,00; S: 7,07; Exp (\%): C: 52,03; H: 4,20; S: 7,31. 


\section{$\left[\mathrm{W}_{3} \mathrm{~S}_{4} \mathrm{H}_{3}(\mathrm{dppe})_{3}\right] \mathrm{BPh}_{4}\left(3\left(\mathrm{BPh}_{4}\right)\right)$}

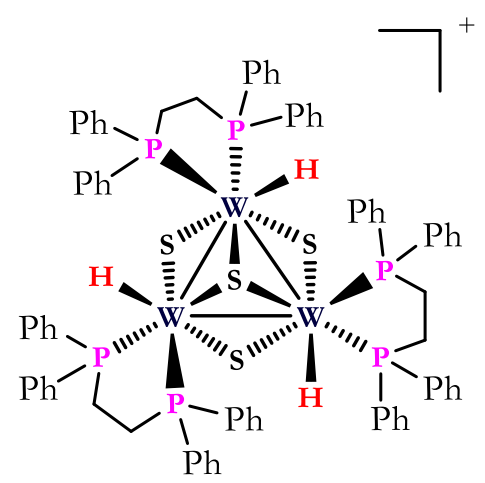

Este compuesto se sintetiza realizando una modificación del procedimiento experimental publicado en la literatura[7], lo que constituye una mejora del rendimiento. A una disolución de $\left[\mathrm{W}_{3} \mathrm{~S}_{4} \mathrm{Br}_{3}(\mathrm{dppe})_{3}\right] \mathrm{BPh}_{4}(100,8 \mathrm{mg}, 0,041 \mathrm{mmol})$ en THF se le añade lentamente un exceso de $\mathrm{NaBH}_{4}$ (14,8 mg, 0,40 mmol) bajo atmósfera inerte. El color de la disolución cambia de azul a rosa de forma gradual en un tiempo aproximado de 15 minutos. Se deja reaccionar durante una hora y media, tras la cual la mezcla de reacción se filtra y el filtrado se evapora a sequedad. A continuación, se redisuelve en diclorometano y se lava repetidamente con agua (3x20 mL). Se seca la fase orgánica con $\mathrm{MgSO}_{4}$ anhidro, se filtra y se evapora a sequedad, para obtener el compuesto deseado como un sólido rosa (87,9 mg, 97 \% rendimiento).

${ }^{31} \mathrm{P}\left\{{ }^{1} \mathrm{H}\right\} \mathrm{NMR}\left(\mathrm{CD}_{3} \mathrm{CN}, 121 \mathrm{MHz}\right) \delta(\mathrm{ppm}): 38,56\left(\mathrm{~s},{ }^{1} \mathrm{~J}\left({ }^{1} \mathrm{P},{ }^{183} \mathrm{~W}\right)=181,3 \mathrm{~Hz}\right), 7,05$ $\left(\mathrm{s},{ }^{1} J\left(31 \mathrm{P},{ }^{183} \mathrm{~W}\right)=97,6 \mathrm{~Hz}\right)$.

${ }^{1} \mathrm{H} \mathrm{NMR}\left(\mathrm{CD}_{3} \mathrm{CN}, 282 \mathrm{MHz}\right): \delta(\mathrm{ppm}): 0,97\left(\mathrm{dd},{ }^{2} J\left({ }^{1} \mathrm{H},{ }^{31} \mathrm{P}\right)=58 \mathrm{~Hz},{ }^{2} \mathrm{~J}\left({ }^{1} \mathrm{H},{ }^{31} \mathrm{P}\right)=\right.$ $23 \mathrm{~Hz})$.

ESI-MS $\left(\mathrm{CH}_{3} \mathrm{CN}, 80 \mathrm{~V}\right) \mathrm{m} /$ z: $1877\left(\mathrm{M}^{+}\right)$.

$\mathrm{UV}-\mathrm{V}$ is $\left(\mathrm{CH}_{3} \mathrm{CN}\right) \mathrm{nm}: 502$ (b), 327 (b), 274 (b).

Análisis Elemental calc. para $\mathrm{W}_{3} \mathrm{~S}_{4} \mathrm{P}_{7} \mathrm{~F}_{6} \mathrm{C}_{78} \mathrm{H}_{75}$ (\%): C: 45,33; H: 3,80; S: 6,45; Exp (\%): C: 45,43; H: 3,92; S: 6,38. 
$\left[\mathrm{W}_{3} \mathrm{~S}_{4} \mathrm{~F}_{3}(\mathrm{dppe})_{3}\right] \mathrm{X}\left(\mathrm{X}=\mathrm{PF}_{6}, \mathrm{BPh}_{4}\right)(4(\mathrm{X}))$

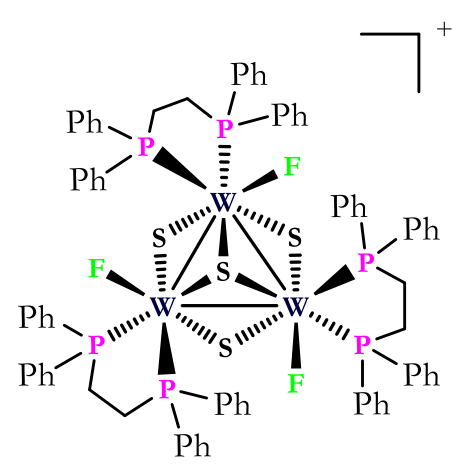

A una disolución de $\left[\mathrm{W}_{3} \mathrm{~S}_{4} \mathrm{Br}_{3}(\mathrm{dppe})_{3}\right] \mathrm{PF}_{6}(95,7 \mathrm{mg}$, $0,0423 \mathrm{mmol}$ ) en $20 \mathrm{~mL}$ de acetonitrilo seco se le añade un exceso de 4 equivalentes de CsF $(28,9 \mathrm{mg}$, 0,191 mmol) bajo atmósfera de nitrógeno. La mezcla se agita a reflujo durante 24 horas, tras las que se observa un cambio de color de azul a morado. A continuación se filtra, y después de llevar el filtrado a sequedad se redisuelve en diclorometano. Se filtra de nuevo para eliminar las sales inorgánicas formadas y se evapora el disolvente. El sólido morado obtenido se lava abundantemente con una mezcla de tolueno-acetona (98:2), obteniéndose 52,7 mg de $\left[\mathrm{W}_{3} \mathrm{~S}_{4} \mathrm{~F}_{3}(\mathrm{dppe})_{3}\right] \mathrm{PF}_{6}$ (rendimiento $60 \%$ ). El cambio de contraión se realiza mediante el procedimiento habitual, es decir, disolviendo el compuesto en metanol y añadiendo un exceso de $\mathrm{NaBPh}_{4}$, para posteriormente filtrar el precipitado obtenido.

${ }^{31} \mathrm{P}\left\{{ }^{1} \mathrm{H}\right\} \mathrm{NMR}\left(\mathrm{CD}_{3} \mathrm{CN}, 121 \mathrm{MHz}\right) \delta(\mathrm{ppm})=-143,1$ (multiplete, $1 \mathrm{P},{ }^{1} \mathrm{~J}\left({ }^{31} \mathrm{P},{ }^{19} \mathrm{~F}\right)=$ 706,5 Hz), 7,7 (d, 3P, $\left.{ }^{2} \mathrm{~J}\left({ }^{31} \mathrm{P},{ }^{19} \mathrm{~F}\right)=114,2 \mathrm{~Hz}\right), 6,7\left(\mathrm{~d}, 3 \mathrm{P},{ }^{2} \mathrm{~J}\left({ }^{31} \mathrm{P},{ }^{19} \mathrm{~F}\right)=51,0 \mathrm{~Hz}\right)$.

${ }^{19} \mathrm{~F}$ NMR $\left(\mathrm{CD}_{3} \mathrm{CN}, 282 \mathrm{MHz}\right) \delta(\mathrm{ppm})=-182,4\left(\mathrm{dd}, 3 \mathrm{~F},{ }^{2} \mathrm{~J}\left({ }^{19} \mathrm{~F},{ }^{31} \mathrm{P}\right)=50,8 \mathrm{~Hz}\right.$, $\left.{ }^{2} \mathrm{~J}\left({ }^{19} \mathrm{~F},{ }^{31} \mathrm{P}\right)=112,9 \mathrm{~Hz}\right),-75.5\left(\mathrm{~d}, 6 \mathrm{~F},{ }^{1} \mathrm{~J}\left({ }^{19} \mathrm{~F},{ }^{31} \mathrm{P}\right)=711,6 \mathrm{~Hz}\right)$.

ESI-MS $\left(\mathrm{CH}_{3} \mathrm{CN}, 20 \mathrm{~V}\right) \mathrm{m} / \mathrm{z}_{\mathrm{i}} 1931[\mathrm{M}+]$.

Análisis Elemental calc. para $\mathrm{W}_{3} \mathrm{~S}_{4} \mathrm{P}_{7} \mathrm{~F}_{9} \mathrm{C}_{78} \mathrm{H}_{72}$ (\%): C: 45,10; H: 3,49; S: 6,18; Exp (\%): C: 45,26; H: 3,62; S: 6,25. 


\subsubsection{HIDRODEFLUORACIÓN CATALÍTICA DE LA PENTA- FLUOROPIRIDINA}

Los estudios catalíticos se han llevado a cabo a reflujo utilizando un matraz de fondo redondo, y también bajo radiación microondas, usando un tubo de vidrio compatible con el equipo. Los recipientes de reacción se cargan con pentafluoropiridina $(0,38$ mmol, $40 \mu \mathrm{L})$, fluorobenceno como referencia interna $(0,38 \mathrm{mmol}, 36 \mu \mathrm{L})$, dimetilfenilsilano $(1,9 \mathrm{mmol}, 296 \mu \mathrm{L})$, el catalizador $(0,7 \%)$ y $1 \mathrm{~mL}$ de acetonitrilo seco. En el caso de la reacción llevada a cabo en el microondas, la potencia del equipo se ajusta dinámicamente para alcanzar la temperatura necesaria, que resulta ser de 115 ${ }^{\circ} \mathrm{C}$, sin sobrepasar un máximo de $70 \mathrm{~W}$ para evitar la descomposición del clúster. La reacción tiene lugar durante el tiempo apropiado según el catalizador utilizado, y los rendimientos y conversiones se calculan monitorizando la reacción mediante ${ }^{19} \mathrm{~F}$ RMN. La confirmación de la naturaleza de la 2,3,5,6-tetrafluoropiridina se deduce por comparación con un estándar comercial y con datos extraídos de la literatura.[8]

\subsubsection{CLÚSTERES FUNCIONALIZADOS CON AMINOFOSFINAS}

\subsubsection{SÍNTESIS}

\section{$\left[\mathrm{Mo}_{3} \mathrm{~S}_{4} \mathrm{Cl}_{3}(\text { ediprp) })_{3}\right] \mathrm{BPh}_{4}\left(5\left(\mathrm{BPh}_{4}\right)\right)$}

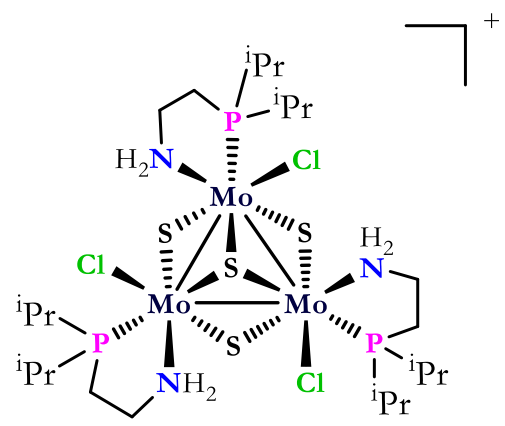

y se añade pentano hasta observar la precipitación de un sólido verde. Se separa el sólido por centrifugación, se redisuelve en metanol y se precipita con un exceso de 
$\mathrm{NaBPh}_{4}$, obteniendo 43,3 mg del compuesto caracterizado como $\left.\left[\mathrm{Mo}_{3} \mathrm{~S}_{4} \mathrm{Cl}_{3} \text { (ediprp) }\right)_{3}\right] \mathrm{BPh}_{4}$ (rendimiento $85 \%$ ).

${ }^{31} \mathrm{P}\left\{{ }^{1} \mathrm{H}\right\} \mathrm{NMR}\left(121 \mathrm{MHz}, \mathrm{CD}_{2} \mathrm{Cl}_{2}\right) \delta(\mathrm{ppm})=53,45(\mathrm{~s}, 3 \mathrm{P})$.

ESI-MS $\left(\mathrm{CH}_{3} \mathrm{CN}, 20 \mathrm{~V}\right) \mathrm{m} /$ z: $1006[\mathrm{M}+]$.

Análisis Elemental calc. para $\mathrm{Mo}_{3} \mathrm{~S}_{4} \mathrm{P}_{3} \mathrm{~N}_{3} \mathrm{Cl}_{3} \mathrm{C}_{48} \mathrm{H}_{80} \mathrm{~B}$ (\%): C: 43,50; H: 6,08; N: 3,17 S: 9,68; $\operatorname{Exp}(\%):$ C: 43,95; H: 6,18; N: 3,32 S: 9,79.

\section{Reacción entre $\left[\mathrm{Mo}_{3} \mathrm{~S}_{4} \mathrm{Cl}_{3}(\right.$ ediprp) 3$] \mathrm{BPh}_{4}$ y $\mathrm{NaBH}_{4}$}

A una disolución de 50,1 $\mathrm{mg}$ de $\left[\mathrm{Mo}_{3} \mathrm{~S}_{4} \mathrm{Cl}_{3}(\text { ediprp) })_{3}\right] \mathrm{BPh}_{4}\left(\mathbf{5}\left(\mathrm{BPh}_{4}\right)\right)$ en $10 \mathrm{~mL}$ de $\mathrm{THF}$, se le añaden 20 equivalentes de $\mathrm{NaBH}_{4}$. La reacción transcurre mediante un cambio de coloración de verde a marrón. Al cabo de 2 horas, la disolución se filtra para eliminar el $\mathrm{NaBH}_{4}$ que no ha reaccionado y se evapora el disolvente a vacío. El sólido marrón obtenido se ha intentado caracterizar mediante las técnicas habituales pero no se ha observado ninguna señal correspondiente a la formación del hidruro.

\section{$\left[\mathrm{W}_{3} \mathrm{~S}_{4} \mathrm{Br}_{3}\left(\text { ed }^{\mathrm{i}} \mathrm{prp}\right)_{3}\right] \mathrm{BPh}_{4}\left(6\left(\mathrm{BPh}_{4}\right)\right)$}

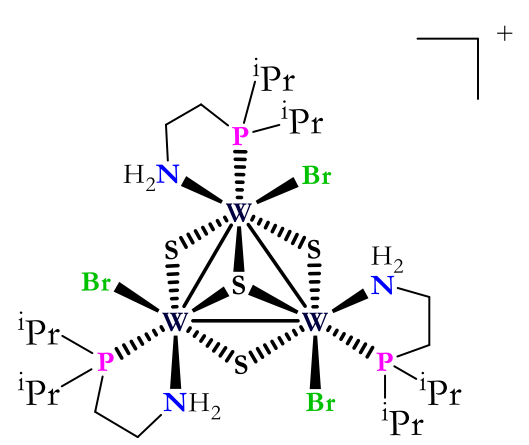

A una suspensión de $\left\{\mathrm{W}_{3} \mathrm{~S}_{7} \mathrm{Br}_{4}\right\}_{\mathrm{n}}(0,3030 \mathrm{~g}, 0,276$ $\mathrm{mmol}$ ) en $30 \mathrm{~mL}$ de acetonitrilo se le añaden 3,5 equivalentes $(1,682 \mathrm{~mL}$ ) de $\mathrm{HBr} 0,5 \mathrm{M}$ (en $\left.\mathrm{CH}_{3} \mathrm{CN}\right)$. A continuación se añaden 6,2 equivalentes de aminofosfina $(0,2763 \mathrm{~g}, 1,714$ mmol) bajo atmósfera de nitrógeno y se deja reaccionar durante 24 horas, tras las cuales se observa la formación de una disolución azul. Después de que la mezcla alcance la temperatura ambiente, se filtra para eliminar impurezas y se evapora el disolvente en el rotavapor. A continuación, se redisuelve el sólido en metanol, y se le añade un exceso de $\mathrm{NaBPh}_{4}$ para precipitar el clúster deseado $(0,289 \mathrm{~g}$, rendimiento $60,1 \%)$. 
${ }^{31} \mathrm{P}\left\{{ }^{1} \mathrm{H}\right\} \operatorname{NMR}\left(162 \mathrm{MHz}, \mathrm{CD}_{2} \mathrm{Cl}_{2}\right) \delta(\mathrm{ppm})=24,33\left(\mathrm{~s}, 3 \mathrm{P},{ }^{1} \mathrm{~J}\left({ }^{11} \mathrm{P},{ }^{183} \mathrm{~W}\right)=88,2 \mathrm{~Hz}\right)$. ESI-MS $\left(\mathrm{CH}_{3} \mathrm{CN}, 20 \mathrm{~V}\right) \mathrm{m} / \approx: 1403[\mathrm{M}+]$.

Análisis Elemental calc. para $\mathrm{W}_{3} \mathrm{~S}_{4} \mathrm{P}_{3} \mathrm{~N}_{3} \mathrm{Br}_{3} \mathrm{C}_{48} \mathrm{H}_{80} \mathrm{~B}$ (\%): C: 33,47; H: 4,68; N: $2,45 \mathrm{~S}$ : 7,44; $\operatorname{Exp}(\%)$ : C: 34,12; H: 4,87; N: 3,02 S: 7,95.

\section{Reacción entre $\left[\mathrm{W}_{3} \mathrm{~S}_{4} \mathrm{Br}_{3}(\text { ediprp) })_{3}\right] \mathrm{BPh}_{4}$ y $\mathrm{NaBH}_{4}$}

A una disolución de $\left.\left[\mathrm{W}_{3} \mathrm{~S}_{4} \mathrm{Br}_{3} \text { (ediprp) }\right)_{3}\right] \mathrm{BPh}_{4}\left(\mathbf{6}\left(\mathrm{BPh}_{4}\right)\right.$ en $10 \mathrm{~mL}$ de THF se le añaden 20 equivalentes de $\mathrm{NaBH}_{4}$. Se observa un cambio de color gradual de azul a rosa. $\mathrm{Al}$ cabo de 2 horas, se filtra la disolución obtenida para eliminar el $\mathrm{NaBH}_{4}$ que no ha reaccionado y a continuación se evapora el disolvente a vacío, para obtener un sólido rosa. Debido a los problemas de purificación encontrados descritos en el capítulo 4, no se ha podido calcular el rendimiento ni confirmar de forma inequívoca que nos encontramos ante el complejo $\left[\mathrm{W}_{3} \mathrm{~S}_{4} \mathrm{H}_{3}\left(\right.\right.$ ediprp$\left._{3}\right] \mathrm{BPh}_{4}$.

${ }^{31} \mathrm{P}\left\{{ }^{1} \mathrm{H}\right\} \operatorname{NMR}\left(162 \mathrm{MHz}\right.$, acetona- $\left.\mathrm{d}_{6}\right) \delta(\mathrm{ppm})=40,74\left(\mathrm{~s}, 3 \mathrm{P},{ }^{1} \mathrm{~J}\left({ }^{31} \mathrm{P},{ }^{183} \mathrm{~W}\right)=90,5\right.$ $\mathrm{Hz})$.

${ }^{1} \mathrm{H}$ NMR $\left(162 \mathrm{MHz}\right.$, acetona- $\left.\mathrm{d}_{6}\right) \delta(\mathrm{ppm})=0,74\left(\mathrm{~d},{ }^{2} J\left({ }^{1} \mathrm{H},{ }^{31} \mathrm{P}\right)=4,8 \mathrm{~Hz}\right)$.

ESI-MS $\left(\mathrm{CH}_{3} \mathrm{CN}, 20 \mathrm{~V}\right) \mathrm{m} / \approx: 1166[\mathrm{M}+]$.

\section{$(P)-\left[\mathrm{Mo}_{3} \mathrm{~S}_{4} \mathrm{Cl}_{3}((1 S, 2 R)-\mathrm{PPro})_{3}\right] \mathrm{Cl}(7(\mathrm{Cl}),(P)-7(\mathrm{Cl}))$}

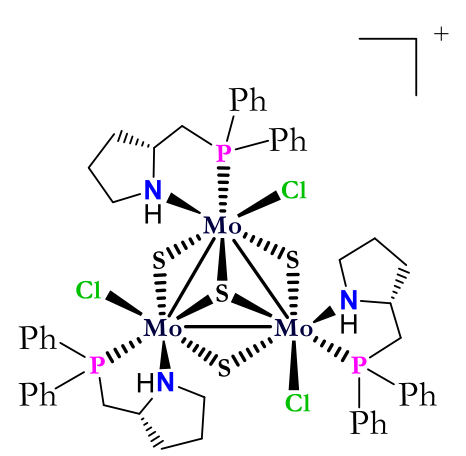

A una suspensión verde de $\left[\mathrm{Mo}_{3} \mathrm{~S}_{4} \mathrm{Cl}_{4}(\mathrm{PPh})_{3}\left(\mathrm{H}_{2} \mathrm{O}\right)_{2}\right]$ (57,0 $\mathrm{mg}, 0,0413 \mathrm{mmol})$ en $5 \mathrm{~mL}$ de etanol se le añaden 3,2 equivalentes del ligando enantioméricamente puro (R)-2[(difenilfosfino)metil]pirrolidina (36,5 $\mathrm{mg}, \quad 0,1355$ mmol) bajo nitrógeno. Transcurridas 3 horas, la disolución verde obtenida se concentra a vacío en atmósfera inerte y se añade hexano hasta observar la precipitación del producto 
deseado. Finalmente, el sólido verde obtenido se separa por centrifugación, obteniéndose 46,7 mg (rendimiento 81\%).

${ }^{31} \mathrm{P}\left\{{ }^{1} \mathrm{H}\right\} \mathrm{NMR}\left(121 \mathrm{MHz}, \mathrm{CD}_{2} \mathrm{Cl}_{2}\right) \delta(\mathrm{ppm})=32,30(\mathrm{~s}, 3 \mathrm{P})$.

ESI-MS $\left(\mathrm{CH}_{3} \mathrm{CN}, 20 \mathrm{~V}\right) \mathrm{m} / \mathrm{z}_{1} 1330[\mathrm{M}+]$.

Análisis Elemental calc. para $\mathrm{Mo}_{3} \mathrm{~S}_{4} \mathrm{P}_{3} \mathrm{~N}_{3} \mathrm{Cl}_{4} \mathrm{C}_{51} \mathrm{H}_{60}$ (\%): C: 44,85; H: 4,43; N: 3,08 S: 9,39; $\operatorname{Exp}(\%):$ C: 45,31; H: 5,00; N: 3,59 S: 9,57.

\section{$(P)-\left[\mathrm{Mo}_{3} \mathrm{~S}_{4} \mathrm{Cl}_{3}((1 S, 2 S)-\mathrm{PPro})_{3}\right] \mathrm{Cl}(8(\mathrm{Cl}),(P)-8(\mathrm{Cl}))$}

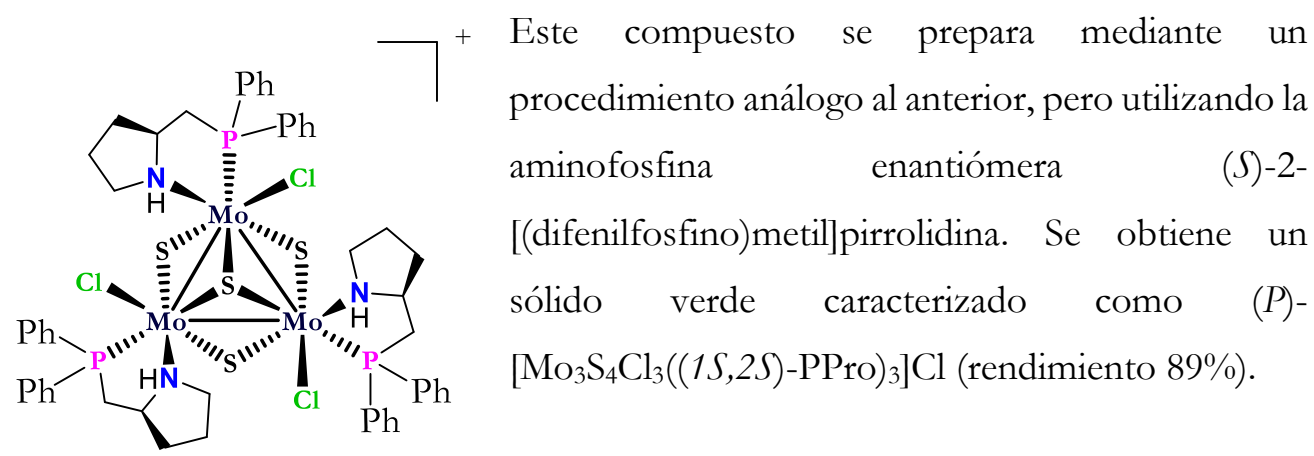

${ }^{31} \mathrm{P}\left\{{ }^{1} \mathrm{H}\right\}$ NMR $\left(121 \mathrm{MHz}, \mathrm{CD}_{2} \mathrm{Cl}_{2}\right) \delta(\mathrm{ppm})=32,10(\mathrm{~s}, 3 \mathrm{P})$.

ESI-MS $\left(20 \mathrm{~V}, \mathrm{CH}_{3} \mathrm{CN}\right) \mathrm{m} / \mathrm{z}_{1} 1330[\mathrm{M}+]$.

Análisis Elemental calc. para $\mathrm{Mo}_{3} \mathrm{~S}_{4} \mathrm{P}_{3} \mathrm{~N}_{3} \mathrm{Cl}_{4} \mathrm{C}_{51} \mathrm{H}_{60}$ (\%): C: 44,85; H: 4,43; N: $3,08 \mathrm{~S}$ : 9,39; $\operatorname{Exp}(\%):$ C: 45,42; H: 4,93; N: 3,26 S: 9,47.

\section{Reacción entre $\left[\mathrm{Mo}_{3} \mathrm{~S}_{4} \mathrm{Cl}_{4}(\mathrm{PPh})_{3}\left(\mathrm{H}_{2} \mathrm{O}\right)_{2}\right]$ y edtbup}

A una disolución de 50,4 mg del precursor $\left[\mathrm{Mo}_{3} \mathrm{~S}_{4} \mathrm{Cl}_{4}(\mathrm{PPh})_{3}\left(\mathrm{H}_{2} \mathrm{O}\right)_{2}\right]$ en $10 \mathrm{~mL}$ de etanol, se le añaden 3,2 equivalentes de edtbup (2-(di-t-butilfosfino)etilamina). La mezcla se deja reaccionar durante 3 horas a temperatura ambiente y la disolución marrón obtenida se concentra en atmósfera inerte hasta $1 / 4$ de su volumen inicial. A continuación, se precipita el producto con pentano, obteniéndose un sólido marrón. La caracterización de este sólido se ha realizado mediante RMN de fósforo y 
espectrometría de masas, sin observarse en ningún momento la coordinación del ligando a la unidad clúster. Esta reacción se ha repetido en presencia de 3,5 equivalentes de una disolución $0,5 \mathrm{M}$ de $\mathrm{HCl}$ en acetonitrilo, obteniéndose los mismos resultados.

\section{Reacción entre $\left\{\mathrm{Mo}_{3} \mathrm{~S}_{7} \mathrm{Cl}_{4}\right\}_{n}$ y edtbup}

A una suspensión de 101,2 $\mathrm{mg}$ de polímero $\left\{\mathrm{Mo}_{3} \mathrm{~S}_{7} \mathrm{Cl}_{4}\right\}_{\mathrm{x}}$ en $20 \mathrm{~mL}$ de acetonitrilo, se le añaden 6,2 equivalentes de edtbup en un matraz Schlenk. Se calienta a reflujo en presencia de 3,5 equivalentes de una disolución $0,5 \mathrm{M}$ de $\mathrm{HCl}$ en acetonitrilo y tras 24 horas de reacción, se deja enfriar a temperatura ambiente, se filtra y se concentra el disolvente hasta $1 / 4$ del volumen inicial aproximadamente. A continuación se precipita el producto con éter, obteniéndose un sólido marrón que se caracteriza mediante las técnicas habituales sin observar la coordinación del ligando a la unidad clúster.

\section{Reacción entre $\left[\mathrm{Mo}_{3} \mathrm{~S}_{4} \mathrm{Cl}_{4}(\mathrm{PPh})_{3}\left(\mathrm{H}_{2} \mathrm{O}\right)_{2}\right]$ y $\mathrm{PiPr}_{2}-\mathrm{NMe}_{2}$-indeno}

Se disuelven 51,3 mg de precursor $\left[\mathrm{Mo}_{3} \mathrm{~S}_{4} \mathrm{Cl}_{4}(\mathrm{PPh})_{3}\left(\mathrm{H}_{2} \mathrm{O}\right)_{2}\right]$ en $10 \mathrm{~mL}$ de etanol y a continuación se añaden 3,2 equivalentes del ligando $\mathrm{PiPr}_{2}-\mathrm{NMe}_{2}$-indeno (1-di-ipropilfosfino-2-(N.N-dimetilamino)-1H-indeno). Tras 3 horas de reacción a temperatura ambiente se obtiene una disolución marrón, que se filtra y se concentra hasta $1 / 4$ del volumen inicial. A continuación se precipita el producto mediante la adición de pentano. El sólido marrón obtenido se caracteriza por RMN de fósforo y espectrometría de masas sin observarse evidencias de la coordinación del ligando a la unidad clúster. Esta reacción se ha repetido en presencia de 3,5 equivalentes de una disolución 0,5 M de $\mathrm{HCl}$ en acetonitrilo, obteniéndose idénticos resultados.

\section{Reacción entre $\left\{\mathrm{Mo}_{3} \mathrm{~S}_{7} \mathrm{Cl}_{4}\right\}_{\mathrm{n}}$ y $\mathrm{PiPr}_{2}-\mathrm{NMe}_{2}$-indeno}

Se suspenden $100,3 \mathrm{mg}$ de polímero $\left\{\mathrm{Mo}_{3} \mathrm{~S}_{7} \mathrm{Cl}_{4}\right\}_{\mathrm{x}}$ en $20 \mathrm{~mL}$ de acetonitrilo y se añaden 6,2 equivalentes de la aminofosfina $\mathrm{PiPr}_{2}-\mathrm{NMe}_{2}$-indeno. A continuación se 
calienta la mezcla a reflujo en presencia de 3,5 equivalentes de una disolución 0,5 M de $\mathrm{HCl}$ en acetonitrilo durante 24 horas. La reacción transcurre mediante un cambio de coloración de naranja a marrón oscuro. Una vez finalizada la reacción y alcanzada la temperatura ambiente, se filtra la mezcla y se concentra hasta $1 / 4$ de su volumen inicial. El producto, un sólido marrón, se obtiene mediante precipitación con éter. Este sólido se caracteriza por RMN de fósforo y espectrometría de masas, sin observarse evidencias de la coordinación del ligando a la unidad clúster.

\subsubsection{REDUCCIÓN CATALÍTICA DE SUSTRATOS ORGÁNICOS}

Los estudios catalíticos con estos clústeres se han llevado a cabo a diferentes temperaturas utilizando matraces Schlenk, que se cargan con 2,5 mL de THF seco, 0,1 mmol de sustrato orgánico, $20 \mu \mathrm{L}$ de n-hexadecano como referencia interna, $5 \%$ mol de catalizador y 5 equivalentes de agente reductor. La mezcla de reacción se agita durante 18 horas. Transcurrido este tiempo, se deja enfriar a temperatura ambiente, se diluye con $15 \mathrm{~mL}$ de acetato de etilo y se toma una muestra para ser inyectada en el cromatógrafo de gases. Si el agente reductor elegido es un silano, antes de tomar muestra se añaden además $5 \mathrm{~mL}$ de una disolución $3 \mathrm{M}$ de $\mathrm{NaOH}$, se agita la mezcla durante 3 horas y, a continuación, se toma una muestra de la fase orgánica y se inyecta en el cromatógrafo de gases. La naturaleza del producto final se determina por comparación con un estándar comercial.

\subsubsection{ESTUDIOS DE REACTIVIDAD FRENTE A ALCOHOLES}

En un matraz Schlenk se introducen $20 \mathrm{mg}$ de $\left.\left[\mathrm{Mo}_{3} \mathrm{~S}_{4} \mathrm{Cl}_{3} \text { (ediprp) }\right)_{3}\right] \mathrm{BPh}_{4}$ o de $\left[\mathrm{W}_{3} \mathrm{~S}_{4} \mathrm{Br}_{3}\right.$ (ediprp) $\left.{ }_{3}\right] \mathrm{BPh}_{4}$ y se disuelven en $5 \mathrm{~mL}$ de metanol o etanol según la prueba. A continuación se añaden 37 equivalentes de trietilamina seca y desgasificada, se calienta la mezcla a $45{ }^{\circ} \mathrm{C}$. Al cabo de 12 horas, la disolución se concentra en atmósfera inerte hasta aproximadamente $1 / 4$ de su volumen y se precipita el producto obtenido con éter dietílico. 


\subsubsection{CLÚSTERES FUNCIONALIZADOS CON AMINODIFOSFINAS}

\subsubsection{SÍNTESIS}

\section{$\left[\mathrm{Mo}_{3} \mathrm{~S}_{4} \mathrm{Cl}_{3}(\mathrm{PNP})_{3}\right] \mathrm{Cl}(9(\mathrm{Cl}))$}

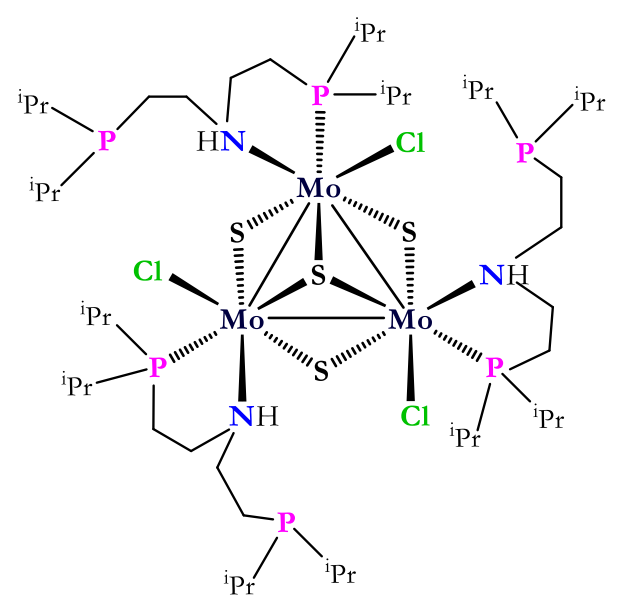

$\longrightarrow \begin{array}{ll}\mathrm{A} \quad \text { una disolución de } \\ {\left[\mathrm{Mo}_{3} \mathrm{~S}_{4} \mathrm{Cl}_{4}\left(\mathrm{PPh}_{3}\right)_{3}\left(\mathrm{H}_{2} \mathrm{O}\right)_{2}\right]} & \text { en }\end{array}$ etanol (86,7 $\mathrm{mg}, 0,063 \mathrm{mmol})$, se le añade un exceso de aminodifosfina $(63,0 \mathrm{mg}, 0,206$ mmol) y se deja reaccionar durante 3 horas. Una vez ha finalizado la reacción, se concentra en atmósfera inerte hasta aproximadamente un cuarto

del volumen inicial y se precipita el producto final con hexano. Se separa el sólido verde por centrifugación, obteniéndose 76,9 mg del compuesto deseado (rendimiento $83 \%)$.

31P $\left\{{ }^{1} \mathrm{H}\right\}$ NMR $\left(121 \mathrm{MHz}, \mathrm{CD}_{2} \mathrm{Cl}_{2}\right) \delta(\mathrm{ppm})=47,63(\mathrm{~s}, 3 \mathrm{P}),-2,04(\mathrm{~s}, 3 \mathrm{P})$

ESI-MS $\left(\mathrm{CH}_{3} \mathrm{CN}, 20 \mathrm{~V}\right) \mathrm{m} / \tau_{:} 1438[\mathrm{M}+]$.

Análisis Elemental calc. para $\mathrm{Mo}_{3} \mathrm{~S}_{4} \mathrm{Cl}_{4} \mathrm{P}_{6} \mathrm{~N}_{3} \mathrm{C}_{48} \mathrm{H}_{111}$ (\%): C: 39,10; H: 7,58; N: 2,85, S: 8,70; $\operatorname{Exp}(\%): C: 37,92 ; H: 7,65$; N: 2,60; S: 8,64. 


\section{$\left[\mathrm{Mo}_{3} \mathrm{~S}_{4}(\mathrm{PNP})_{3}\right]\left(\mathrm{BF}_{4}\right)_{4}\left(10\left(\mathrm{BF}_{4}\right)_{4}\right)$}

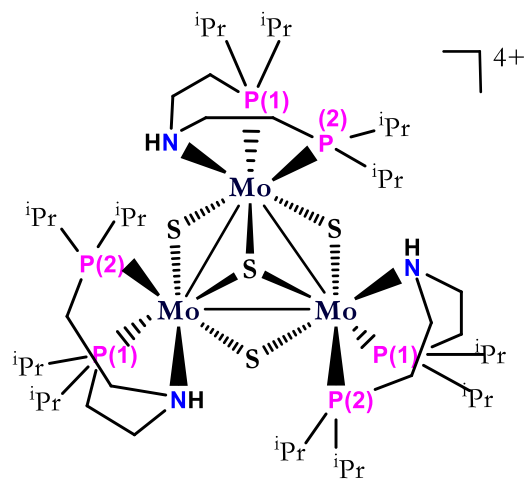

A una disolución del precursor $\left[\mathrm{Mo}_{3} \mathrm{~S}_{4} \mathrm{Cl}_{3}(\mathrm{PNP})_{3}\right] \mathrm{Cl}(19,5 \mathrm{mg}, 0,013 \mathrm{mmol})$ en $10 \mathrm{~mL}$ de diclorometano, se le añaden 6 equivalentes $(15,4 \mathrm{mg})$ de $\mathrm{AgBF}_{4}$ y $1 \mathrm{~mL}$ de éter gota a gota para solubilizar la sal de plata. Al cabo de 30 minutos aproximadamente se observa un cambio de coloración de verde a marrón. Una vez transcurridas 4 horas de reacción, se filtra el precipitado obtenido y se recoge con diclorometano. A continuación, se redisuelve en metanol, se filtra de nuevo y se evapora el disolvente para obtener el compuesto deseado como un sólido marrón microcristalino $(18,3 \mathrm{mg}$, rendimiento $83 \%)$.

${ }^{31} \mathrm{P}\left\{{ }^{1} \mathrm{H}\right\} \operatorname{NMR}(121 \mathrm{MHz}, \mathrm{DMSO}) \delta(\mathrm{ppm})=27,09\left(\mathrm{~d}, 3 \mathrm{P},{ }^{2} \mathrm{~J}\left({ }^{31} \mathrm{P}, 31 \mathrm{P}\right)=36,1 \mathrm{~Hz}\right)$, 22,91 (d, 3P, $\left.{ }^{2} \mathrm{~J}\left({ }^{31} \mathrm{P}, 31 \mathrm{P}\right)=36,7 \mathrm{~Hz}\right)$.

Análisis Elemental calc. para $\mathrm{Mo}_{3} \mathrm{~S}_{4} \mathrm{P}_{6} \mathrm{~N}_{3} \mathrm{C}_{48} \mathrm{H}_{111} \mathrm{BF}_{4}(\%)$ : C: 40,62; H: 7,88; N: 2,96; S: 9,03; $\operatorname{Exp}(\%)$ C: 41,53; H: 8,11; N: 3,05; S: 9,32.

\section{$\left[\mathrm{Mo}_{3} \mathrm{~S}_{4} \mathrm{Cl}_{3}\left(\boldsymbol{\kappa}^{2}(\mathbf{P}, \mathbf{N})-\left(\mathrm{RuCl}_{2} \operatorname{Ar}\left(\boldsymbol{\kappa}^{1}(\mathbf{P})-\mathbf{P N P}\right)\right)_{3}\right]\left(\mathbf{P F}_{6}\right)(\mathrm{Ar}=p\right.$-cimeno, benceno $)$}

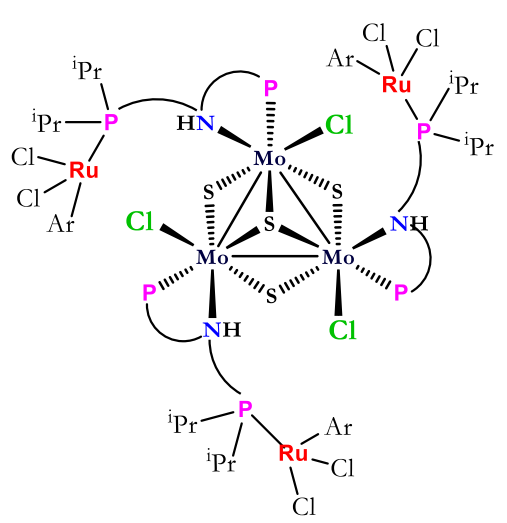
(11 $\left.\left(\mathrm{PF}_{6}\right), 12\left(\mathrm{PF}_{6}\right)\right)$

A una disolución del complejo $\left[\mathrm{Mo}_{3} \mathrm{~S}_{4} \mathrm{Cl}_{3}(\mathrm{PNP})_{3}\right] \mathrm{Cl}(50,3 \mathrm{mg}, 0,034 \mathrm{mmol})$ en diclorometano seco, se le añaden 3,2 equivalentes del dímero $\left(\mathrm{RuCl}_{2} \mathrm{Ar}\right)_{2}(\mathrm{Ar}=p$ cimeno, benceno). En el caso de utilizar el precursor de rutenio que contiene benceno, se utiliza una mezcla diclorometano:metanol

(1:1) como disolvente de la reacción. Inmediatamente se observa un cambio de coloración de verde a marrón. Una vez transcurridas 3 horas de reacción, se evapora 
el disolvente y se redisuelve el sólido marrón resultante en etanol. La mezcla de reacción se filtra y se evapora a sequedad. A continuación, se redisuelve el producto en diclorometano y se introduce en una columna cromatográfica. Tras lavar con diclorometano y acetona, se eluye el producto deseado con una disolución de $\mathrm{KPF}_{6}$ (10 mg/L) en acetona. La fracción de color marrón se recoge, se evapora a sequedad, se redisuelve en diclorometano, se filtra y se evapora a sequedad de nuevo, obteniéndose un sólido marrón caracterizado como $\left[\mathrm{Mo}_{3} \mathrm{~S}_{4} \mathrm{Cl}_{3}\left(\kappa^{2}(\mathrm{P}, \mathrm{N})\right.\right.$ $\left.\left(\mathrm{RuCl}_{2} \mathrm{Ar}\left(\kappa^{1}(\mathrm{P})-\mathrm{PNP}\right)\right)_{3}\right]^{+}$.

\section{Caracterización de 11( $\left.\mathrm{PF}_{6}\right)$}

Rendimiento: $85 \%$

${ }^{31} \mathrm{P}\left\{{ }^{1} \mathrm{H}\right\} \operatorname{NMR}\left(162 \mathrm{MHz}, \mathrm{CD}_{2} \mathrm{Cl}_{2}\right) \delta(\mathrm{ppm})=52,39(\mathrm{~s}, 3 \mathrm{P}), 35,00(\mathrm{~d}, 3 \mathrm{P})$.

ESI-MS $\left(\mathrm{CH}_{3} \mathrm{CN}, 20 \mathrm{~V}\right) \mathrm{m} / \gtrless_{2} 2356[\mathrm{M}+]$.

Análisis Elemental calc. para $\mathrm{Mo}_{3} \mathrm{Ru}_{3} \mathrm{~S}_{4} \mathrm{P}_{7} \mathrm{~N}_{3} \mathrm{~F}_{6} \mathrm{Cl}_{9} \mathrm{C}_{78} \mathrm{H}_{153}$ (\%): C: 37,44; H: 6,16; N: 1,68; S: 5,13; $\operatorname{Exp}(\%):$ C: 38,02; H: 6,95; N: 1,86; S: 6,01.

\section{Caracterización de 12( $\left(\mathrm{PF}_{6}\right)$}

Rendimiento: $82 \%$

${ }^{31} \mathrm{P}\left\{{ }^{1} \mathrm{H}\right\} \operatorname{NMR}\left(162 \mathrm{MHz}, \mathrm{CD}_{2} \mathrm{Cl}_{2}\right) \delta(\mathrm{ppm})=50,64(\mathrm{~s}, 3 \mathrm{P}), 37,76(\mathrm{~d}, 3 \mathrm{P})$.

ESI-MS $\left(\mathrm{CH}_{3} \mathrm{CN}, 20 \mathrm{~V}\right) \mathrm{m} / \gtrless^{2} 2189[\mathrm{M}+]$.

Análisis Elemental calc. para $\mathrm{Mo}_{3} \mathrm{Ru}_{3} \mathrm{~S}_{4} \mathrm{P}_{7} \mathrm{~N}_{3} \mathrm{~F}_{6} \mathrm{Cl}_{9} \mathrm{C}_{66} \mathrm{H}_{129}$ (\%): C: 36,21; H: 5,94; N: 1,92; S: 5,86; $\operatorname{Exp}(\%)$ : C: 37,05; H: 6,25; N: 2,12; S: 6,21.

\subsubsection{PRUEBAS DE REACTIVIDAD FRENTE A OTROS METALES}

\section{Reacción de $\left[\mathrm{Mo}_{3} \mathrm{~S}_{4} \mathrm{Cl}_{3}(\mathrm{PNP})_{3}\right] \mathrm{Cl}$ con $\mathrm{CuCl}$}

A una disolución de $\left[\mathrm{Mo}_{3} \mathrm{~S}_{4} \mathrm{Cl}_{3}(\mathrm{PNP})_{3}\right] \mathrm{Cl}$ en diclorometano $(18,5 \mathrm{mg}$ en $5 \mathrm{~mL})$ se le añaden 3,5 equivalentes de $\mathrm{CuCl}(4,35 \mathrm{mg})$. La reacción transcurre a temperatura ambiente mediante un cambio de coloración de verde a rojo y al cabo de 24 horas, se filtra la disolución y se lleva a sequedad. Las pruebas de caracterización mediante 
RMN de fósforo y espectrometría de masas no han permitido conocer la naturaleza del producto de reacción, en cambio, la resolución parcial de la estructura obtenida mediante la difracción de rayos $\mathrm{X}$ en monocristal muestra que tenemos un clúster con unidad central $\mathrm{Mo}_{3} \mathrm{CuS}_{4}$.

\section{Reacción de $\left[\mathrm{Mo}_{3} \mathrm{~S}_{4} \mathrm{Cl}_{3}(\mathrm{PNP})_{3}\right] \mathrm{Cl}$ con $\mathrm{Ni}(\operatorname{cod})_{2}$ y TBACl}

A una disolución de $\left[\mathrm{Mo}_{3} \mathrm{~S}_{4} \mathrm{Cl}_{3}(\mathrm{PNP})_{3}\right] \mathrm{Cl}$ en diclorometano $(21,5 \mathrm{mg}$ en $5 \mathrm{~mL})$ se le añaden 3,2 equivalentes de $\mathrm{Ni}(\operatorname{cod})_{2}$ (12,8 mg) y 3,2 equivalentes de $\mathrm{TBACl}(12,9 \mathrm{mg})$. Tras 24 horas de reacción a temperatura ambiente se observa una disolución marrón que contiene un precipitado verde de textura aceitosa. Todos los intentos de caracterización de este precipitado han resultado infructuosos. La reacción también se ha llevado a cabo con 5 y 10 equivalentes de $\mathrm{Ni}(\operatorname{cod})_{2}$ y a reflujo obteniéndose los mismos resultados.

\section{Reacción de $\left[\mathrm{Mo}_{3} \mathrm{~S}_{4} \mathrm{Cl}_{3}(\mathrm{PNP})_{3}\right] \mathrm{Cl}$ con $\mathrm{Pd}_{2}(\mathrm{dba})_{3}$ y TBACl}

Se disuelven 21,2 $\mathrm{mg}$ de $\left[\mathrm{Mo}_{3} \mathrm{~S}_{4} \mathrm{Cl}_{3}(\mathrm{PNP})_{3}\right] \mathrm{Cl}$ en $5 \mathrm{~mL}$ de diclorometano y se añaden 3,2 equivalentes de $\mathrm{Pd}_{2}(\mathrm{dba})_{3}$ (42 mg) y el mismo exceso de TBACl (12,9 mg). Tras 24 horas de reacción, se aísla un sólido marrón-grisáceo cuya estructura no se ha podido descifrar.

\section{Reacción de $\left[\mathrm{Mo}_{3} \mathrm{~S}_{4} \mathrm{Cl}_{3}(\mathrm{PNP})_{3}\right] \mathrm{Cl}$ con $\left[\{\mathrm{RhCl}(\operatorname{cod})\}_{2}\right]$}

A una disolución de $\left[\mathrm{Mo}_{3} \mathrm{~S}_{4} \mathrm{Cl}_{3}(\mathrm{PNP})_{3}\right] \mathrm{Cl}$ en diclorometano $(18,4 \mathrm{mg}$ en $5 \mathrm{~mL})$ se le añaden 1,3 equivalentes de $\left[\{\mathrm{RhCl}(\mathrm{cod})\}_{2}\right](8,0 \mathrm{mg})$. Tras 24 horas de reacción, se concentra la disolución y se precipita el sólido con éter dietílico. La caracterización mediante RMN de fósforo adjunta a continuación sugiere la formación del compuesto $\left[\mathrm{Mo}_{3} \mathrm{~S}_{4} \mathrm{Cl}_{3}\left(\kappa^{2}(\mathrm{P}, \mathrm{N})-\left(\mathrm{RhCl}(\operatorname{cod})\left(\kappa^{1}(\mathrm{P})-\mathrm{PNP}\right)\right)_{3}\right]^{+}\right.$, aunque la presencia de este compuesto no se ha podido confirmar mediante otras técnicas.

${ }^{31} \mathrm{P}\left\{{ }^{1} \mathrm{H}\right\} \operatorname{NMR}\left(121 \mathrm{MHz}, \mathrm{CD}_{2} \mathrm{Cl}_{2}\right) \delta(\mathrm{ppm})=59,17$ (s, 3P), 38,44 (d, 3P, $\left.{ }^{1} \mathrm{~J}\left({ }^{31} \mathrm{P},{ }^{103 \mathrm{Rh}}\right)=143 \mathrm{~Hz}\right)$. 


\section{Reacción de $\left[\mathrm{Mo}_{3} \mathrm{~S}_{4} \mathrm{Cl}_{3}(\mathrm{PNP})_{3}\right] \mathrm{Cl}$ con $\left[\{\operatorname{IrCl}(\operatorname{cod})\}_{2}\right]$}

Se disuelven 9,5 $\mathrm{mg}$ de clúster precursor en $5 \mathrm{~mL}$ de dicolorometano y se añaden 1,2 equivalentes de $\left[\{\mathrm{IrCl}(\operatorname{cod})\}_{2}\right](5,2 \mathrm{mg})$. A continuación se añaden unas gotas de etanol para solubilizar el complejo de iridio. Tras 24 horas de reacción, se concentra la disolución obtenida y se precipita el producto con hexano, que resulta ser un sólido verde. No se ha podido obtener información sobre este sólido mediante las técnicas de caracterización habituales.

\section{Reacción de $\left[\mathrm{Mo}_{3} \mathrm{~S}_{4} \mathrm{Cl}_{3}(\mathrm{PNP})_{3}\right] \mathrm{Cl}$ con $\left[\mathrm{AuCl}\left(\mathrm{PPh}_{3}\right)\right]$}

A una disolución de 19,5 mg del clúster $\left[\mathrm{Mo}_{3} \mathrm{~S}_{4} \mathrm{Cl}_{3}(\mathrm{PNP})_{3}\right] \mathrm{Cl}$ en $5 \mathrm{~mL}$ de diclorometano se le añaden 3,2 equivalentes de [ $\left.\mathrm{AuCl}\left(\mathrm{PPh}_{3}\right)\right](20,9 \mathrm{mg})$. Tras 24 horas de reacción, se concentra la disolución obtenida y se precipita el producto con hexano. Las técnicas de caracterización habituales no nos proporcionan ninguna información sobre la naturaleza del producto obtenido.

\section{Reacción de $\left[\mathrm{Mo}_{3} \mathrm{~S}_{4} \mathrm{Cl}_{3}(\mathrm{PNP})_{3}\right] \mathrm{Cl}$ con $\mathrm{Ru}_{3}(\mathrm{CO})_{12}$}

Se disuelven 11,1 $\mathrm{mg}$ del clúster precursor en $5 \mathrm{~mL}$ de diclorometano y se le añade 1 equivalente del clúster $\mathrm{Ru}_{3}(\mathrm{CO})_{12}(5,1 \mathrm{mg})$. Tras 24 horas de reacción, se observa un cambio de coloración de verde a rojo, se concentra la disolución y se precipita el producto con éter. El precipitado rojo obtenido no se ha podido caracterizar mediante las técnicas habituales. 


\subsubsection{REDUCCIÓN CATALÍTICA DE ALDEHÍDOS, CETONAS Y ÉSTERES}

Los estudios catalíticos de reducción de aldehídos y cetonas se han llevado a cabo de forma análoga a los descritos en la sección 7.1.4.2. Los matraces Schlenk se cargan con 2,5 mL de metanol, $0,1 \mathrm{mmol}$ de sustrato orgánico, $20 \mu \mathrm{L}$ de n-hexadecano como referencia interna, $5 \mathrm{~mol} \%$ de catalizador y 5 equivalentes de agente reductor. La mezcla de reacción se agita a reflujo durante 18 horas. Transcurrido este tiempo, se deja enfriar a temperatura ambiente, se diluye con $15 \mathrm{~mL}$ de acetato de etilo y se toma una muestra para ser inyectada en el cromatógrafo de gases. Si el agente reductor elegido es un silano, antes de tomar muestra se añaden además $5 \mathrm{~mL}$ de una disolución $3 \mathrm{M}$ de $\mathrm{NaOH}$, se agita la mezcla durante 3 horas y, a continuación, se toma una muestra de la fase orgánica y se inyecta en el cromatógrafo de gases. La naturaleza del producto final se determina por comparación con un estándar comercial.

Adicionalmente, la hidrogenación directa de ésteres se ha llevado a cabo en un reactor de vidrio de $2 \mathrm{~mL}$ de capacidad equipado con control de presión. Se ha añadido una cantidad mayor de sustrato $(1 \mathrm{mmol})$, manteniendo siempre la relación sustrato-catalizador mencionada anteriormente y adaptando la cantidad de disolvente a la capacidad del reactor. La temperatura se ha ajustado a $100{ }^{\circ} \mathrm{C}$ en todas las reacciones. La presión de hidrógeno, de entre 30 y 50 bar y las cantidades variables de una base, de entre 10 y 20 equivalentes, se han ajustado según la prueba.

\subsubsection{HIDROGENACIÓN CATALÍTICA DE $\mathrm{CO}_{2}$}

Los estudios catalíticos se han llevado a cabo en un reactor de acero equipado con control de presión y válvula de ruptura que contiene un recipiente de teflón de $15 \mathrm{~mL}$ de capacidad. El recipiente se ha cargado con $1 \mu \mathrm{mol}$ de clúster $\left[\mathrm{Mo}_{3} \mathrm{~S}_{4} \mathrm{Cl}_{3}\left(\kappa^{2}(\mathrm{P}, \mathrm{N})\right.\right.$ $\left.\left(\mathrm{RuCl}_{2}(\not \text {-cimeno })\left(\kappa^{1}(\mathrm{P})-\mathrm{PNP}\right)\right)_{3}\right]^{+}\left(\mathbf{1 1}^{+}\right)$o $\left[\mathrm{Mo}_{3} \mathrm{~S}_{4} \mathrm{Cl}_{3}(\mathrm{PNP})_{3}\right]^{+}\left(\boldsymbol{9}^{+}\right)$o $1,5 \mu \mathrm{mol} \mathrm{de}$ complejo $\left[\left\{\mathrm{RuCl}_{2}(p \text {-cimeno })\right\}_{2}\right]$ en $1 \mathrm{~mL}$ de DMSO anhidro y 600 equivalentes de 
tributilamina. Se han añadido también 5 equivalentes de $\mathrm{KHCO}_{3}$ cuando se han realizado pruebas con este aditivo. El reactor se ha presurizado en primer lugar con $\mathrm{CO}_{2}$ y tras alcanzar la temperatura de $50{ }^{\circ} \mathrm{C}$, se ha presurizado con $\mathrm{H}_{2}$ hasta conseguir una relación $\mathrm{CO}_{2}: \mathrm{H}_{2}$ de 1:2. La mezcla se ha dejado reaccionar durante 21 horas. La presencia de ácido fórmico se ha determinado mediante $\mathrm{RMN}$ de ${ }^{1} \mathrm{H}$ y la cuantificación se ha realizado utilizando DMF de elevada pureza como patrón interno. 


\subsection{DETERMINACIÓN ESTRUCTURAL}

\subsubsection{GENERALIDADES DE LA TOMA DE DATOS Y RESOLUCIÓN}

La toma de datos y la resolución de las estructuras cristalinas que aparecen en esta tesis doctoral se han realizado utilizando el procedimiento general descrito a continuación. La recogida de datos se ha llevado a cabo en un difractómetro Agilent Supernova equipado con un detector Atlas CCD, empleando una radiación monocromática Mo-K $\alpha(\lambda=0.71073 \AA)$. La integración de los datos se ha realizado mediante el programa CrysAlis ${ }^{\text {Pro }}{ }^{[9]}$ y se aplican correcciones basadas en el método multi-scan. ${ }^{[10]}$ Las estructuras se han resuelto mediante métodos directos o de Patterson utilizando SHELXS-2013 y SHELXS-97,[11] y se han refinado con SHELXL[12] utilizando el método de mínimos cuadrados. La estructura correspondiente al apartado 7.2.3.3 se ha resuelto mediante el método charge-flipping utilizando Superflip. ${ }^{[13]} \mathrm{El}$ paquete $\mathrm{Olex}^{2} 1.2^{[14]}$ se ha utilizado como interfaz en todas las ocasiones. A menos que se indique lo contrario, todos los átomos se han refinado anisotrópicamente excepto los hidrógenos. Estos se incluyen en posiciones idealizadas y se refinan como átomos "jinete", con un parámetro térmico de desplazamiento proporcional al átomo al que se encuentra enlazado (1,2 cuando están unidos a un carbono secundario o aromático; 1,5 cuando están unidos a un carbono primario o un oxígeno).

Los datos cristalográficos, parámetros de refino y condiciones de registro se especifican en una tabla completada para cada estructura cristalina. Adicionalmente, se detalla una selección de distancias de enlace y ángulos interatómicos asociados a la primera esfera de coordinación de los metales. Las estructuras se han representado utilizando el programa ORTEP3 v2.02,[15] aunque los átomos de hidrógeno se han omitido para mayor claridad. En estas representaciones se ha seguido un código de colores específico. Los átomos de molibdeno y wolframio se han representado en azul claro, los de azufre en naranja, los fósforos en rosa, los nitrógenos en azul, los 
átomos de oxígeno en rojo, el boro en amarillo, y los átomos de carbono en gris. Respecto a los halógenos, el flúor y el cloro se han representado en color verde, mientras que para el bromo se ha utilizado el color granate. 


\subsubsection{CLÚSTERES FUNCIONALIZADOS CON DIFOSFINAS}

\subsubsection{ESTRUCTURA DE $\left[\mathrm{Mo}_{3} \mathrm{~S}_{4} \mathrm{H}_{3}(\mathrm{dppe})_{3}\right] \mathrm{PF}_{6}\left(1\left(\mathrm{PF}_{6}\right)\right)$}

La cristalización del compuesto $\mathbf{1}\left(\mathrm{PF}_{6}\right)$ se ha llevado a cabo mediante difusión lenta de hexano sobre una disolución del clúster en diclorometano. El refino de esta estructura se ha realizado con éxito en el grupo espacial no centrosimétrico Cc, lo que indica la cristalización preferencial de uno de los enantiómeros del clúster a partir de una mezcla racémica. La existencia de tres ligandos hidruro coordinados a los tres metales se ha confirmado mediante experimentos de ${ }^{1} \mathrm{H}-\mathrm{RMN}$. Todos los átomos del clúster han sido refinados anisotrópicamente, excepto los átomos de hidrógeno que se han generado en posiciones geométricas.

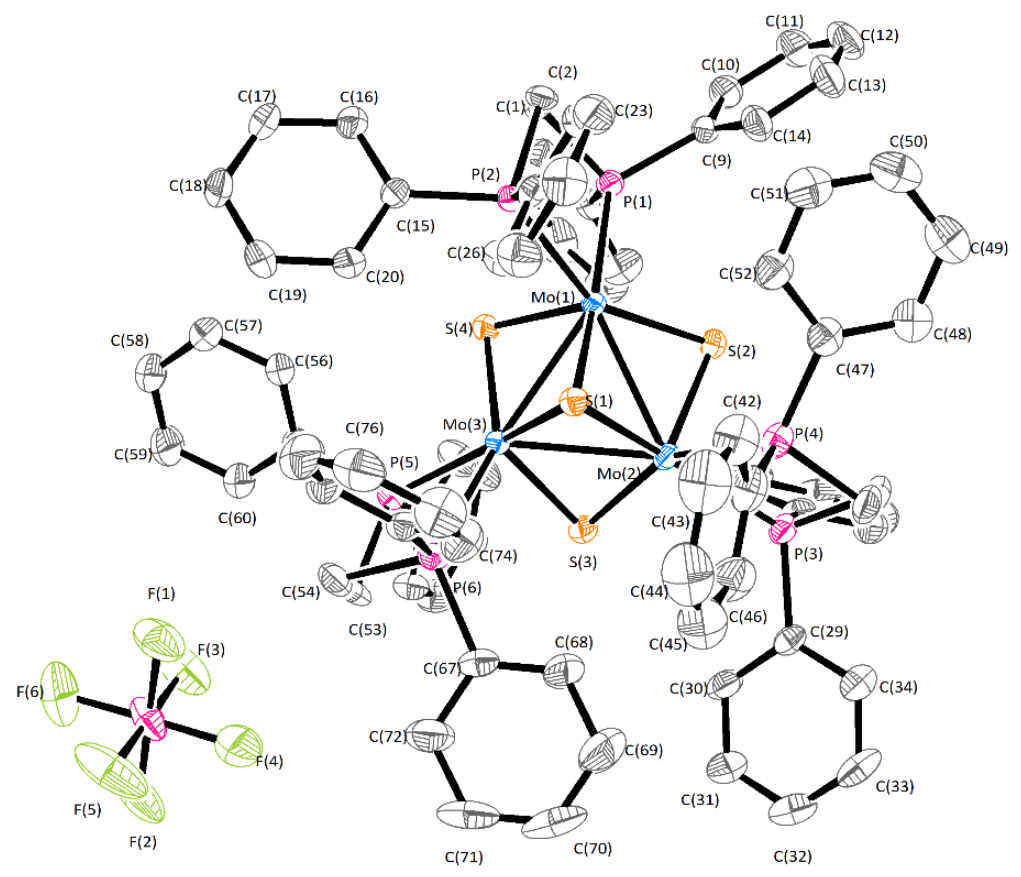

Figura 7.1. Representación ORTEP del clúster $\left[\mathrm{Mo}_{3} \mathrm{~S}_{4} \mathrm{H}_{3}(\mathrm{dppe})_{3}\right] \mathrm{PF}_{6}$ (elipsoides al $50 \%$ de probabilidad). 
Tabla 7.1. Parámetros cristalográficos, condiciones de registro y refino.

Datos cristalográficos

\begin{tabular}{|c|c|c|c|}
\hline Especie & {$\left[\mathrm{Mo}_{3} \mathrm{~S}_{4} \mathrm{H}_{3}(\mathrm{dppe})_{3}\right] \mathrm{PF}_{6}$} & & \\
\hline Fórmula & $\mathrm{C}_{78} \mathrm{H}_{72} \mathrm{~F}_{6} \mathrm{Mo}_{3} \mathrm{P}_{7} \mathrm{~S}_{4}$ & $\begin{array}{l}\text { Sistema } \\
\text { cristalino }\end{array}$ & Monoclínico \\
\hline Grupo espacial & $\mathrm{Cc}$ & Peso molecular & 1756,20 \\
\hline $\mathbf{a}(\AA)$ & $29,4364(12)$ & $\boldsymbol{\alpha}\left({ }^{\circ}\right)$ & 90,00 \\
\hline $\mathbf{b}(\AA)$ & $13,9514(3)$ & $\beta\left({ }^{\circ}\right)$ & $127,208(6)$ \\
\hline $\mathbf{c}(\AA)$ & $22,7696(9)$ & $\gamma\left({ }^{\circ}\right)$ & 90,00 \\
\hline $\mathbf{Z}$ & 4 & Vol $\left(\AA^{3}\right)$ & $7447,5(7)$ \\
\hline Color & Marrón & $\begin{array}{l}\text { Tamaño del } \\
\text { cristal }(\mathrm{mm})\end{array}$ & $\begin{array}{l}0,173 \times 0,107 \times \\
0,056\end{array}$ \\
\hline $\mathbf{d}_{\text {calc }}\left(\mathrm{mg} / \mathrm{mm}^{3}\right)$ & 1,566 & $\lambda(\AA)$ & 0,71073 \\
\hline $\begin{array}{l}\text { Coeficiente de } \\
\text { absorción }\left(\mathrm{mm}^{-1}\right)\end{array}$ & 0,819 & $F(000)$ & 3556 \\
\hline
\end{tabular}

Parámetros experimentales

\begin{tabular}{|c|c|c|c|}
\hline Temperatura $(\mathrm{K})$ & $150,05(10)$ & $\mathbf{R}($ int $)$ & 0,0353 \\
\hline Tiempo por marco & 190 & $\mathbf{R}(\sigma)$ & 0,0464 \\
\hline Límites $2 \theta\left(^{\circ}\right)$ & 5,84 y 58,85 & $\begin{array}{l}\text { Límites h, k, } 1 \\
\text { (mín/máx) }\end{array}$ & $\begin{array}{l}-40 \leq \mathrm{h} \leq 38 \\
-19-\leq \mathrm{k} \leq 17 \\
-31-\leq 1 \leq 30\end{array}$ \\
\hline $\begin{array}{l}\mathrm{N}^{\mathrm{o}} \text { de reflexiones } \\
\text { registradas }\end{array}$ & 43623 & $\begin{array}{l}\mathrm{N}^{\circ} \text { de reflexiones } \\
\text { independientes }\end{array}$ & 17371 \\
\hline
\end{tabular}

Resolución y refino

$\begin{array}{llll}\mathbf{N}^{\mathbf{o}} \text { de variables } & 883 & \text { GooF en } \mathbf{F}^{2} & 1,073 \\ \mathbf{N}^{\mathbf{o}} \text { de restricciones } & 2 & & \\ \mathbf{R} 1 \text { final }([\mathbf{I}>\text { 2sigma(I)]) } & 0,0345 & \text { R1 (todos los datos) } & 0,0449 \\ \text { wR2 final }([\mathbf{I}>\text { 2sigma(I)] }) & 0,0705 & \text { wR2 (todos los datos) } & 0,0771 \\ \begin{array}{l}\text { Densidad electrónica } \\ \text { residual }\left(\mathrm{e} \cdot \AA^{-3}\right)\end{array} & 0,65 /-0,45 & \text { Max. desplazam./ } \boldsymbol{0} & 0,001 \\ \text { Parámetro de Flack } & -0,055(12) & & \end{array}$

$$
R 1=\sum|| F_{0}|-| F_{1}|| / \sum F_{0} \quad w R 2=\left[\sum\left[w\left(F_{0}^{2}-F_{c}^{2}\right)^{2}\right] / \sum\left[w\left(F_{0}^{2}\right)^{2}\right]\right]^{1 / 2}
$$


Tabla 7.2. Distancias de enlace representativas $(\AA)$.

\begin{tabular}{cccccc}
\hline Átomo Átomo & Distancia & Átomo Átomo & Distancia \\
\hline $\operatorname{Mo}(1)$ & $\mathrm{Mo}(2)$ & $2,7599(6)$ & $\mathrm{Mo}(2)$ & $\mathrm{S}(2)$ & $2,3276(13)$ \\
$\mathrm{Mo}(1)$ & $\mathrm{Mo}(3)$ & $2,7605(6)$ & $\mathrm{Mo}(2)$ & $\mathrm{S}(3)$ & $2,3335(14)$ \\
$\mathrm{Mo}(2)$ & $\mathrm{Mo}(3)$ & $2,7785(6)$ & $\mathrm{Mo}(2)$ & $\mathrm{P}(3)$ & $2,5204(14)$ \\
$\mathrm{Mo}(1)$ & $\mathrm{S}(1)$ & $2,3757(13)$ & $\mathrm{Mo}(2)$ & $\mathrm{P}(4)$ & $2,5564(14)$ \\
$\mathrm{Mo}(1)$ & $\mathrm{S}(2)$ & $2,3203(13)$ & $\mathrm{Mo}(3)$ & $\mathrm{S}(1)$ & $2,3570(13)$ \\
$\mathrm{Mo}(1)$ & $\mathrm{S}(4)$ & $2,3322(13)$ & $\mathrm{Mo}(3)$ & $\mathrm{S}(3)$ & $2,3347(13)$ \\
$\mathrm{Mo}(1)$ & $\mathrm{P}(1)$ & $2,5051(13)$ & $\mathrm{Mo}(3)$ & $\mathrm{S}(4)$ & $2,3172(12)$ \\
$\mathrm{Mo}(1)$ & $\mathrm{P}(2)$ & $2,5438(13)$ & $\mathrm{Mo}(3)$ & $\mathrm{P}(5)$ & $2,5146(14)$ \\
$\mathrm{Mo}(2)$ & $\mathrm{S}(1)$ & $2,3604(13)$ & $\mathrm{Mo}(3)$ & $\mathrm{P}(6)$ & $2,5664(13)$ \\
\hline
\end{tabular}


Tabla 7.3. Ángulos de enlace representativos $\left({ }^{\circ}\right)$.

\begin{tabular}{|c|c|c|c|c|c|c|c|}
\hline \multicolumn{3}{|c|}{ Átomo Átomo Átomo } & \multirow{2}{*}{$\begin{array}{c}\text { Ángulo } \\
59,792(14)\end{array}$} & \multicolumn{3}{|c|}{ Átomo Átomo Átomo } & \multirow{2}{*}{$\begin{array}{l}\text { Ángulo } \\
54,10(3)\end{array}$} \\
\hline $\operatorname{Mo}(1)$ & $\mathrm{Mo}(2)$ & $\operatorname{Mo}(3)$ & & $S(1)$ & $\mathrm{Mo}(1)$ & $\operatorname{Mo}(2)$ & \\
\hline $\mathrm{S}(2)$ & $\operatorname{Mo}(2)$ & $\operatorname{Mo}(1)$ & $53,45(3)$ & $\mathrm{S}(1)$ & $\operatorname{Mo}(1)$ & $\operatorname{Mo}(3)$ & $54,00(3)$ \\
\hline$S(2)$ & $\operatorname{Mo}(2)$ & $\operatorname{Mo}(3)$ & $103,27(4)$ & $S(1)$ & $\operatorname{Mo}(1)$ & $\mathrm{P}(2)$ & $87,63(4)$ \\
\hline$S(2)$ & $\operatorname{Mo}(2)$ & $S(1)$ & $104,52(5)$ & $S(1)$ & $\operatorname{Mo}(1)$ & $\mathrm{P}(1)$ & $166,53(5)$ \\
\hline$S(2)$ & $\operatorname{Mo}(2)$ & $\mathrm{P}(3)$ & $85,35(5)$ & $\mathrm{P}(1)$ & $\operatorname{Mo}(1)$ & $\operatorname{Mo}(2)$ & $136,43(4)$ \\
\hline$S(2)$ & $\operatorname{Mo}(2)$ & $\mathrm{P}(4)$ & $92,57(5)$ & $\mathrm{P}(1)$ & $\operatorname{Mo}(1)$ & $\operatorname{Mo}(3)$ & $135,41(4)$ \\
\hline $\mathrm{S}(3)$ & $\operatorname{Mo}(2)$ & $\operatorname{Mo}(1)$ & $101,50(4)$ & $\mathrm{P}(1)$ & $\mathrm{Mo}(1)$ & $\mathrm{P}(2)$ & $80,44(4)$ \\
\hline$S(3)$ & $\operatorname{Mo}(2)$ & $\operatorname{Mo}(3)$ & $53,49(3)$ & $\mathrm{Mo}(2)$ & $\mathrm{Mo}(3)$ & $\operatorname{Mo}(1)$ & $59,769(15)$ \\
\hline$S(3)$ & $\mathrm{Mo}(2)$ & $\mathrm{S}(2)$ & $108,67(5)$ & $S(4)$ & $\mathrm{Mo}(3)$ & $\operatorname{Mo}(2)$ & $102,89(3)$ \\
\hline$S(3)$ & $\operatorname{Mo}(2)$ & $S(1)$ & $104,53(5)$ & $S(4)$ & $\operatorname{Mo}(3)$ & $\operatorname{Mo}(1)$ & $53,83(3)$ \\
\hline$S(3)$ & $\operatorname{Mo}(2)$ & $\mathrm{P}(3)$ & $87,34(5)$ & $S(4)$ & $\mathrm{Mo}(3)$ & $S(3)$ & $107,52(5)$ \\
\hline$S(3)$ & $\operatorname{Mo}(2)$ & $\mathrm{P}(4)$ & $154,19(5)$ & $S(4)$ & $\mathrm{Mo}(3)$ & $S(1)$ & $105,24(5)$ \\
\hline$S(1)$ & $\operatorname{Mo}(2)$ & $\mathrm{Mo}(1)$ & $54,62(3)$ & $S(4)$ & $\mathrm{Mo}(3)$ & $\mathrm{P}(5)$ & $84,69(5)$ \\
\hline$S(1)$ & $\operatorname{Mo}(2)$ & $\operatorname{Mo}(3)$ & $53,86(3)$ & $S(4)$ & $\operatorname{Mo}(3)$ & $\mathrm{P}(6)$ & $150,84(5)$ \\
\hline$S(1)$ & $\operatorname{Mo}(2)$ & $\mathrm{P}(3)$ & $160,75(5)$ & $S(3)$ & $\mathrm{Mo}(3)$ & $\operatorname{Mo}(2)$ & $53,45(3)$ \\
\hline$S(1)$ & $\operatorname{Mo}(2)$ & $\mathrm{P}(4)$ & $83,29(5)$ & $\mathrm{S}(3)$ & $\mathrm{Mo}(3)$ & $\operatorname{Mo}(1)$ & $101,45(4)$ \\
\hline $\mathrm{P}(3)$ & $\operatorname{Mo}(2)$ & $\operatorname{Mo}(1)$ & $138,66(4)$ & $\mathrm{S}(3)$ & $\mathrm{Mo}(3)$ & $\mathrm{S}(1)$ & $104,60(5)$ \\
\hline $\mathrm{P}(3)$ & $\mathrm{Mo}(2)$ & $\mathrm{Mo}(3)$ & $140,75(4)$ & $S(3)$ & $\mathrm{Mo}(3)$ & $\mathrm{P}(5)$ & $85,58(5)$ \\
\hline $\mathrm{P}(3)$ & $\operatorname{Mo}(2)$ & $\mathrm{P}(4)$ & $79,75(5)$ & $S(3)$ & $\mathrm{Mo}(3)$ & $\mathrm{P}(6)$ & $95,04(5)$ \\
\hline $\mathrm{P}(4)$ & $\mathrm{Mo}(2)$ & $\mathrm{Mo}(1)$ & $102,96(4)$ & $\mathrm{S}(1)$ & $\mathrm{Mo}(3)$ & $\mathrm{Mo}(2)$ & $53,97(3)$ \\
\hline $\mathrm{P}(4)$ & $\operatorname{Mo}(2)$ & $\mathrm{Mo}(3)$ & $136,66(4)$ & $\mathrm{S}(1)$ & $\operatorname{Mo}(3)$ & $\operatorname{Mo}(1)$ & $54,63(3)$ \\
\hline $\operatorname{Mo}(2)$ & $\mathrm{Mo}(1)$ & $\operatorname{Mo}(3)$ & $60,439(15)$ & $\mathrm{S}(1)$ & $\mathrm{Mo}(3)$ & $\mathrm{P}(5)$ & $162,43(5)$ \\
\hline $\mathrm{S}(2)$ & $\operatorname{Mo}(1)$ & $\operatorname{Mo}(2)$ & $53,70(3)$ & $\mathrm{S}(1)$ & $\mathrm{Mo}(3)$ & $\mathrm{P}(6)$ & $86,01(5)$ \\
\hline$S(2)$ & $\operatorname{Mo}(1)$ & $\mathrm{Mo}(3)$ & $104,01(4)$ & $\mathrm{P}(5)$ & $\mathrm{Mo}(3)$ & $\operatorname{Mo}(2)$ & $138,89(3)$ \\
\hline $\mathrm{S}(2)$ & $\operatorname{Mo}(1)$ & $S(4)$ & $110,81(5)$ & $\mathrm{P}(5)$ & $\operatorname{Mo}(3)$ & $\operatorname{Mo}(1)$ & $138,27(3)$ \\
\hline $\mathrm{S}(2)$ & $\operatorname{Mo}(1)$ & $\mathrm{P}(2)$ & $150,07(5)$ & $\mathrm{P}(5)$ & $\operatorname{Mo}(3)$ & $\mathrm{P}(6)$ & $78,75(4)$ \\
\hline$S(2)$ & $\operatorname{Mo}(1)$ & $S(1)$ & $104,26(5)$ & $\mathrm{P}(6)$ & $\operatorname{Mo}(3)$ & $\operatorname{Mo}(2)$ & $105,38(4)$ \\
\hline$S(2)$ & $\operatorname{Mo}(1)$ & $\mathrm{P}(1)$ & $83,61(5)$ & $\mathrm{P}(6)$ & $\mathrm{Mo}(3)$ & $\operatorname{Mo}(1)$ & $139,94(4)$ \\
\hline$S(4)$ & $\mathrm{Mo}(1)$ & $\mathrm{Mo}(2)$ & $103,04(3)$ & $\mathrm{Mo}(1)$ & $S(2)$ & $\mathrm{Mo}(2)$ & $72,85(4)$ \\
\hline$S(4)$ & $\operatorname{Mo}(1)$ & $\operatorname{Mo}(3)$ & $53,33(3)$ & $\operatorname{Mo}(3)$ & $S(4)$ & $\operatorname{Mo}(1)$ & $72,84(4)$ \\
\hline$S(4)$ & $\mathrm{Mo}(1)$ & $\mathrm{P}(2)$ & $92,10(5)$ & $C(15)$ & $\mathrm{P}(2)$ & $\mathrm{Mo}(1)$ & $124,16(17)$ \\
\hline$S(4)$ & $\operatorname{Mo}(1)$ & $S(1)$ & $104,17(5)$ & $C(15)$ & $\mathrm{P}(2)$ & $C(2)$ & $103,5(2)$ \\
\hline$S(4)$ & $\mathrm{Mo}(1)$ & $\mathrm{P}(1)$ & $82,63(4)$ & $\mathrm{C}(2)$ & $\mathrm{P}(2)$ & $\mathrm{Mo}(1)$ & $104,70(17)$ \\
\hline $\mathrm{P}(2)$ & $\operatorname{Mo}(1)$ & $\mathrm{Mo}(2)$ & $141,08(3)$ & $C(21)$ & $\mathrm{P}(2)$ & $\operatorname{Mo}(1)$ & $116,02(17)$ \\
\hline $\mathrm{P}(2)$ & $\mathrm{Mo}(1)$ & $\operatorname{Mo}(3)$ & $105,14(3)$ & $C(21)$ & $\mathrm{P}(2)$ & $C(15)$ & $100,5(2)$ \\
\hline
\end{tabular}


Tabla 7.3. Ángulos de enlace representativos $\left(^{\circ}\right)$ (continuación).

\begin{tabular}{cccccccc}
\hline ÁtomoÁtomo Átomo & Ángulo & \multicolumn{4}{c}{ ÁtomoÁtomoÁtomo } & Ángulo \\
\hline $\mathrm{C}(21)$ & $\mathrm{P}(2)$ & $\mathrm{C}(2)$ & $106,4(2)$ & $\mathrm{C}(3)$ & $\mathrm{P}(1)$ & $\mathrm{C}(1)$ & $106,9(3)$ \\
$\mathrm{Mo}(2)$ & $\mathrm{S}(3)$ & $\mathrm{Mo}(3)$ & $73,05(4)$ & $\mathrm{C}(9)$ & $\mathrm{P}(1)$ & $\mathrm{Mo}(1)$ & $120,74(19)$ \\
$\mathrm{Mo}(1)$ & $\mathrm{S}(1)$ & $\mathrm{Mo}(2)$ & $71,29(4)$ & $\mathrm{C}(9)$ & $\mathrm{P}(1)$ & $\mathrm{C}(1)$ & $103,7(2)$ \\
$\mathrm{Mo}(1)$ & $\mathrm{S}(1)$ & $\mathrm{Mo}(3)$ & $71,36(4)$ & $\mathrm{C}(1)$ & $\mathrm{P}(1)$ & $\mathrm{Mo}(1)$ & $107,31(17)$ \\
$\mathrm{Mo}(3)$ & $\mathrm{S}(1)$ & $\mathrm{Mo}(2)$ & $72,17(4)$ & $\mathrm{C}(67)$ & $\mathrm{P}(6)$ & $\mathrm{Mo}(3)$ & $124,90(19)$ \\
$\mathrm{C}(27)$ & $\mathrm{P}(3)$ & $\mathrm{Mo}(2)$ & $105,83(18)$ & $\mathrm{C}(67)$ & $\mathrm{P}(6)$ & $\mathrm{C}(73)$ & $102,1(2)$ \\
$\mathrm{C}(29)$ & $\mathrm{P}(3)$ & $\mathrm{Mo}(2)$ & $118,77(18)$ & $\mathrm{C}(67)$ & $\mathrm{P}(6)$ & $\mathrm{C}(54)$ & $102,0(3)$ \\
$\mathrm{C}(29)$ & $\mathrm{P}(3)$ & $\mathrm{C}(27)$ & $103,5(3)$ & $\mathrm{C}(73)$ & $\mathrm{P}(6)$ & $\mathrm{Mo}(3)$ & $115,33(17)$ \\
$\mathrm{C}(29)$ & $\mathrm{P}(3)$ & $\mathrm{C}(35)$ & $102,4(2)$ & $\mathrm{C}(73)$ & $\mathrm{P}(6)$ & $\mathrm{C}(54)$ & $105,3(3)$ \\
$\mathrm{C}(35)$ & $\mathrm{P}(3)$ & $\mathrm{Mo}(2)$ & $118,42(18)$ & $\mathrm{C}(54)$ & $\mathrm{P}(6)$ & $\mathrm{Mo}(3)$ & $105,11(18)$ \\
$\mathrm{C}(35)$ & $\mathrm{P}(3)$ & $\mathrm{C}(27)$ & $106,5(3)$ & $\mathrm{C}(42)$ & $\mathrm{C}(41)$ & $\mathrm{P}(4)$ & $123,4(5)$ \\
$\mathrm{C}(41)$ & $\mathrm{P}(4)$ & $\mathrm{Mo}(2)$ & $114,3(2)$ & $\mathrm{C}(46)$ & $\mathrm{C}(41)$ & $\mathrm{P}(4)$ & $117,8(5)$ \\
$\mathrm{C}(41)$ & $\mathrm{P}(4)$ & $\mathrm{C}(28)$ & $103,3(3)$ & $\mathrm{C}(68)$ & $\mathrm{C}(67)$ & $\mathrm{P}(6)$ & $119,1(5)$ \\
$\mathrm{C}(28)$ & $\mathrm{P}(4)$ & $\mathrm{Mo}(2)$ & $106,11(19)$ & $\mathrm{C}(72)$ & $\mathrm{C}(67)$ & $\mathrm{P}(6)$ & $123,0(5)$ \\
$\mathrm{C}(47)$ & $\mathrm{P}(4)$ & $\mathrm{Mo}(2)$ & $122,10(18)$ & $\mathrm{C}(74)$ & $\mathrm{C}(73)$ & $\mathrm{P}(6)$ & $120,7(5)$ \\
$\mathrm{C}(47)$ & $\mathrm{P}(4)$ & $\mathrm{C}(41)$ & $105,1(3)$ & $\mathrm{C}(78)$ & $\mathrm{C}(73)$ & $\mathrm{P}(6)$ & $120,4(5)$ \\
$\mathrm{C}(47)$ & $\mathrm{P}(4)$ & $\mathrm{C}(28)$ & $103,9(3)$ & $\mathrm{C}(52)$ & $\mathrm{C}(47)$ & $\mathrm{P}(4)$ & $119,7(5)$ \\
$\mathrm{C}(55)$ & $\mathrm{P}(5)$ & $\mathrm{Mo}(3)$ & $120,97(18)$ & $\mathrm{C}(30)$ & $\mathrm{C}(29)$ & $\mathrm{P}(3)$ & $118,7(4)$ \\
$\mathrm{C}(53)$ & $\mathrm{P}(5)$ & $\mathrm{Mo}(3)$ & $107,51(19)$ & $\mathrm{C}(48)$ & $\mathrm{C}(47)$ & $\mathrm{P}(4)$ & $122,0(5)$ \\
$\mathrm{C}(53)$ & $\mathrm{P}(5)$ & $\mathrm{C}(55)$ & $100,1(3)$ & $\mathrm{C}(27)$ & $\mathrm{C}(28)$ & $\mathrm{P}(4)$ & $110,7(4)$ \\
$\mathrm{C}(53)$ & $\mathrm{P}(5)$ & $\mathrm{C}(62)$ & $106,6(3)$ & $\mathrm{C}(20)$ & $\mathrm{C}(15)$ & $\mathrm{P}(2)$ & $118,7(4)$ \\
$\mathrm{C}(62)$ & $\mathrm{P}(5)$ & $\mathrm{Mo}(3)$ & $116,49(18)$ & $\mathrm{C}(16)$ & $\mathrm{C}(15)$ & $\mathrm{P}(2)$ & $122,9(4)$ \\
$\mathrm{C}(62)$ & $\mathrm{P}(5)$ & $\mathrm{C}(55)$ & $103,3(2)$ & $\mathrm{C}(56)$ & $\mathrm{C}(55)$ & $\mathrm{P}(5)$ & $122,5(4)$ \\
$\mathrm{C}(3)$ & $\mathrm{P}(1)$ & $\mathrm{Mo}(1)$ & $114,21(17)$ & $\mathrm{C}(60)$ & $\mathrm{C}(55)$ & $\mathrm{P}(5)$ & $118,6(4)$ \\
$\mathrm{C}(3)$ & $\mathrm{P}(1)$ & $\mathrm{C}(9)$ & $102,8(2)$ & $\mathrm{C}(54)$ & $\mathrm{C}(53)$ & $\mathrm{P}(5)$ & $106,7(4)$ \\
\hline
\end{tabular}




\subsubsection{ESTRUCTURA DE $\left[\mathrm{Mo}_{3} \mathrm{~S}_{4} \mathrm{~F}_{3}(\mathrm{dppe})_{3}\right] \mathrm{PF}_{6} \cdot \mathrm{H}_{2} \mathrm{O}\left(2\left(\mathrm{PF}_{6}\right) \cdot \mathrm{H}_{2} \mathrm{O}\right)$}

La obtención de cristales adecuados para la determinación estructural del compuesto $2\left(\mathrm{PF}_{6}\right)$ se ha llevado a cabo mediante una difusión líquido-líquido de hexano sobre una disolución concentrada del clúster en diclorometano. El clúster ha cristalizado como sal de hexafluorofosfato junto con una molécula de agua en el grupo espacial $\mathrm{P} 2{ }_{1} / \mathrm{c}$. En el último mapa de diferencia de Fourier se han localizado tres picos de intensidades 3,04, 3,00 y 2,43 respectivamente, pero se encuentran demasiado cerca de los tres átomos de molibdeno ( $c a .0,92 \AA$ ) para ser incluidos en nuestro modelo. Todos los átomos del clúster han sido refinados anisotrópicamente, con excepción de los hidrógenos, que se han generado en posiciones geométricas.

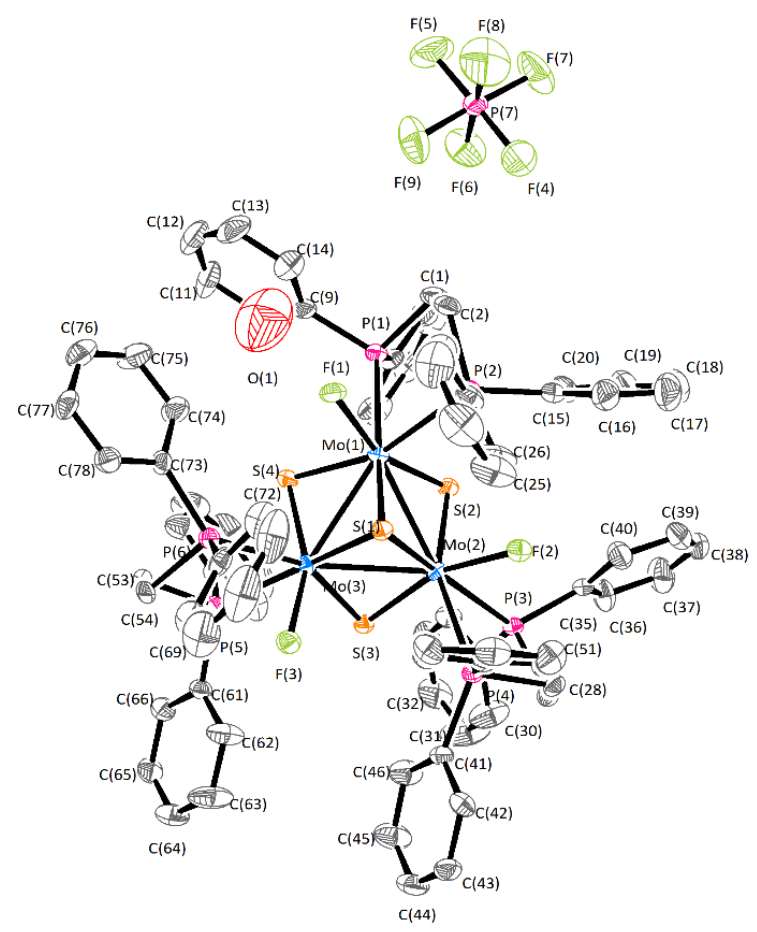

Figura 7.2. Representación ORTEP del clúster $\left[\mathrm{Mo}_{3} \mathrm{~S}_{4} \mathrm{~F}_{3}(\mathrm{dppe})_{3}\right] \mathrm{PF}_{6} \cdot \mathrm{H}_{2} \mathrm{O}$ (elipsoides al $50 \%$ de probabilidad). 
Tabla 7.4. Parámetros cristalográficos, condiciones de registro y refino.

Datos cristalográficos

Especie

$\left[\mathrm{Mo}_{3} \mathrm{~S}_{4} \mathrm{~F}_{3}(\mathrm{dppe})_{3}\right] \mathrm{PF}_{6} \cdot \mathrm{H}_{2} \mathrm{O}$

Fórmula

$\mathrm{C}_{78} \mathrm{H}_{74} \mathrm{~F}_{9} \mathrm{Mo}_{3} \mathrm{OP}_{7} \mathrm{~S}_{4}$

Sistema

Monoclínico

Grupo espacial

$$
\mathrm{P} 21 / \mathrm{c}
$$

a $(\AA)$

13,6191 (4)

cristalino

b $(\AA)$

$21,2898(6)$

c $(\AA)$

26,7358 (6)

Z

Color

4

Morado

Peso molecular 1831,22

$\alpha\left(^{\circ}\right)$

90,00

$\boldsymbol{\beta}\left(^{\circ}\right)$

$93,352(3)$

$\gamma\left({ }^{\circ}\right)$

90,00

Vol $\left(\AA^{3}\right)$

7738,7 (4)

Tamaño del 0,282 x $0,214 \times$

cristal (mm) $\quad 0,11$

$\mathbf{d}_{\text {calc }}\left(\mathrm{mg} / \mathrm{mm}^{3}\right)$

1,572

Coeficiente de $\quad 0,798$

$\lambda(\AA)$

F (000)

0,71073

3704

absorción $\left(\mathrm{mm}^{-1}\right)$

Parámetros experimentales

Temperatura (K) 149,95 (10)

Tiempo por marco 56

(s)

Límites $\mathbf{2 \theta}\left(^{\circ}\right) \quad 5,84$ y 58,848

$\mathbf{N}^{\mathbf{o}}$ de reflexiones 39617

registradas

$\begin{array}{ll}\mathbf{R}(\text { int) } & 0,0310 \\ \mathbf{R}(\boldsymbol{\sigma}) & 0,0319\end{array}$

Límites h, k, $1 \quad-15 \leq \mathrm{h} \leq 15$

(mín/máx) $\quad-23-\leq \mathrm{k} \leq 23$

$-24-\leq 1 \leq 29$

$\mathbf{N}^{\mathbf{o}}$ de reflexiones 11417

independientes

Resolución y refino

$\begin{array}{llll}\mathbf{N}^{\mathbf{o}} \text { de variables } & 1138 & \text { GooF en } \mathbf{F}^{2} & 1,062 \\ \mathbf{N}^{\mathbf{o}} \text { de restricciones } & 0 & & \\ \mathbf{R} 1 \text { final }([\mathbf{I}>\text { 2sigma(I) }]) & 0,0492 & \text { R1 (todos los datos) } & 0,0629 \\ \text { wR2 final }([\mathbf{I}>\text { 2sigma(I)]) } & 0,1038 & \text { wR2 (todos los datos) } & 0,1159 \\ \begin{array}{l}\text { Densidad electrónica } \\ \text { residual }\left(\mathrm{e} \cdot \AA^{-3}\right)\end{array} & 3,04 /-1,32 & \text { Max. desplazam./ } \boldsymbol{\sigma} & 0.001\end{array}$

$$
R 1=\sum|| F_{0}|-| F_{1}|| / \sum F_{0} \quad w R 2=\left[\sum\left[w\left(F_{0}^{2}-F_{c}^{2}\right)^{2}\right] / \sum\left[w\left(F_{0}^{2}\right)^{2}\right]\right]^{1 / 2}
$$


Tabla 7.5. Distancias de enlace representativas $(\AA)$.

\begin{tabular}{cccccc}
\hline Átomo Átomo & Distancia & Átomo Átomo & Distancia \\
\hline $\operatorname{Mo}(1)$ & $\operatorname{Mo}(2)$ & $2,7557(6)$ & $\operatorname{Mo}(2)$ & $\mathrm{S}(3)$ & $2,2842(14)$ \\
$\operatorname{Mo}(1)$ & $\operatorname{Mo}(3)$ & $2,7635(6)$ & $\operatorname{Mo}(2)$ & $\mathrm{P}(3)$ & $2,5692(15)$ \\
$\operatorname{Mo}(2)$ & $\operatorname{Mo}(3)$ & $2,7560(7)$ & $\operatorname{Mo}(2)$ & $\mathrm{P}(4)$ & $2,6318(14)$ \\
$\operatorname{Mo}(1)$ & $\mathrm{S}(1)$ & $2,3495(14)$ & $\operatorname{Mo}(2)$ & $\mathrm{F}(2)$ & $2,027(3)$ \\
$\operatorname{Mo}(1)$ & $\mathrm{S}(2)$ & $2,2808(15)$ & $\mathrm{Mo}(3)$ & $\mathrm{S}(1)$ & $2,3571(14)$ \\
$\operatorname{Mo}(1)$ & $\mathrm{S}(4)$ & $2,3207(15)$ & $\mathrm{Mo}(3)$ & $\mathrm{S}(3)$ & $2,3105(14)$ \\
$\operatorname{Mo}(1)$ & $\mathrm{P}(1)$ & $2,5505(15)$ & $\mathrm{Mo}(3)$ & $\mathrm{S}(4)$ & $2,2823(14)$ \\
$\operatorname{Mo}(1)$ & $\mathrm{P}(2)$ & $2,6102(15)$ & $\mathrm{Mo}(3)$ & $\mathrm{P}(5)$ & $2,5469(15)$ \\
$\operatorname{Mo}(1)$ & $\mathrm{F}(1)$ & $2,055(3)$ & $\mathrm{Mo}(3)$ & $\mathrm{P}(6)$ & $2,6276(15)$ \\
$\operatorname{Mo}(2)$ & $\mathrm{S}(1)$ & $2,3583(14)$ & $\mathrm{Mo}(3)$ & $\mathrm{F}(3)$ & $2,032(3)$ \\
$\operatorname{Mo}(2)$ & $\mathrm{S}(2)$ & $2,3206(14)$ & & & \\
\hline
\end{tabular}


Tabla 7.6. Ángulos de enlace representativos $\left(^{\circ}\right)$.

\begin{tabular}{|c|c|c|c|c|c|c|c|}
\hline \multicolumn{3}{|c|}{ ÁtomoÁtomoÁtomo } & \multirow{2}{*}{$\begin{array}{c}\text { Ángulo } \\
60,185(17)\end{array}$} & \multicolumn{3}{|c|}{ ÁtomoÁtomo Átomo } & \multirow{2}{*}{$\begin{array}{l}\text { Ángulo } \\
99,27(4)\end{array}$} \\
\hline $\operatorname{Mo}(1)$ & $\mathrm{Mo}(2)$ & $\operatorname{Mo}(3)$ & & $\mathrm{S}(4)$ & $\operatorname{Mo}(1)$ & $\operatorname{Mo}(2)$ & \\
\hline$S(2)$ & $\operatorname{Mo}(2)$ & $\operatorname{Mo}(1)$ & $52,55(4)$ & $S(4)$ & $\operatorname{Mo}(1)$ & $\operatorname{Mo}(3)$ & $52,47(4)$ \\
\hline $\mathrm{S}(2)$ & $\operatorname{Mo}(2)$ & $\operatorname{Mo}(3)$ & $97,75(4)$ & $\mathrm{S}(4)$ & $\operatorname{Mo}(1)$ & $\mathrm{P}(2)$ & $161,83(5)$ \\
\hline $\mathrm{S}(2)$ & $\mathrm{Mo}(2)$ & $S(1)$ & $105,31(5)$ & $\mathrm{S}(4)$ & $\operatorname{Mo}(1)$ & $\mathrm{S}(1)$ & $104,6(5)$ \\
\hline $\mathrm{S}(2)$ & $\mathrm{Mo}(2)$ & $\mathrm{P}(3)$ & $84,80(5)$ & $S(4)$ & $\operatorname{Mo}(1)$ & $\mathrm{P}(1)$ & $85,32(5)$ \\
\hline$S(2)$ & $\mathrm{Mo}(2)$ & $\mathrm{P}(4)$ & $157,23(5)$ & $\mathrm{P}(2)$ & $\mathrm{Mo}(1)$ & $\operatorname{Mo}(2)$ & $98,64(4)$ \\
\hline$S(3)$ & $\operatorname{Mo}(2)$ & $\mathrm{Mo}(1)$ & $99,57(4)$ & $\mathrm{P}(2)$ & $\mathrm{Mo}(1)$ & $\operatorname{Mo}(3)$ & $142,45(4)$ \\
\hline$S(3)$ & $\operatorname{Mo}(2)$ & $\operatorname{Mo}(3)$ & $53,58(4)$ & $S(1)$ & $\mathrm{Mo}(1)$ & $\operatorname{Mo}(2)$ & $54,32(4)$ \\
\hline$S(3)$ & $\operatorname{Mo}(2)$ & $\mathrm{S}(2)$ & $97,10(5)$ & $\mathrm{S}(1)$ & $\operatorname{Mo}(1)$ & $\operatorname{Mo}(3)$ & $54,17(4)$ \\
\hline$S(3)$ & $\operatorname{Mo}(2)$ & $S(1)$ & $106,20(5)$ & $\mathrm{S}(1)$ & $\operatorname{Mo}(1)$ & $\mathrm{P}(2)$ & $88,34(5)$ \\
\hline $\mathrm{S}(3)$ & $\mathrm{Mo}(2)$ & $\mathrm{P}(3)$ & $83,37(5)$ & $\mathrm{S}(1)$ & $\operatorname{Mo}(1)$ & $\mathrm{P}(1)$ & $162,52(5)$ \\
\hline$S(3)$ & $\operatorname{Mo}(2)$ & $\mathrm{P}(4)$ & $95,49(5)$ & $\mathrm{P}(1)$ & $\operatorname{Mo}(1)$ & $\operatorname{Mo}(2)$ & $139,26(4)$ \\
\hline$S(1)$ & $\mathrm{Mo}(2)$ & $\operatorname{Mo}(1)$ & $54,02(3)$ & $\mathrm{P}(1)$ & $\operatorname{Mo}(1)$ & $\operatorname{Mo}(3)$ & $137,78(4)$ \\
\hline $\mathrm{S}(1)$ & $\mathrm{Mo}(2)$ & $\operatorname{Mo}(3)$ & $54,21(4)$ & $\mathrm{P}(1)$ & $\operatorname{Mo}(1)$ & $\mathrm{P}(2)$ & $79,09(5)$ \\
\hline$S(1)$ & $\mathrm{Mo}(2)$ & $\mathrm{P}(3)$ & $164,74(5)$ & $\mathrm{F}(1)$ & $\mathrm{Mo}(1)$ & $\operatorname{Mo}(2)$ & $141,03(9)$ \\
\hline $\mathrm{S}(1)$ & $\operatorname{Mo}(2)$ & $\mathrm{P}(4)$ & $89,20(5)$ & $\mathrm{F}(1)$ & $\mathrm{Mo}(1)$ & $\operatorname{Mo}(3)$ & $103,15(9)$ \\
\hline $\mathrm{P}(3)$ & $\operatorname{Mo}(2)$ & $\mathrm{Mo}(1)$ & $137,34(4)$ & $\mathrm{F}(1)$ & $\mathrm{Mo}(1)$ & $\mathrm{S}(2)$ & $158,30(10)$ \\
\hline $\mathrm{P}(3)$ & $\operatorname{Mo}(2)$ & $\operatorname{Mo}(3)$ & $136,92(4)$ & $\mathrm{F}(1)$ & $\operatorname{Mo}(1)$ & $S(4)$ & $94,26(10)$ \\
\hline $\mathrm{P}(3)$ & $\operatorname{Mo}(2)$ & $\mathrm{P}(4)$ & $77,89(5)$ & $\mathrm{F}(1)$ & $\operatorname{Mo}(1)$ & $\mathrm{P}(2)$ & $73,48(10)$ \\
\hline $\mathrm{P}(4)$ & $\mathrm{Mo}(2)$ & $\operatorname{Mo}(1)$ & $142,85(4)$ & $\mathrm{F}(1)$ & $\operatorname{Mo}(1)$ & $S(1)$ & $86,95(9)$ \\
\hline $\mathrm{P}(4)$ & $\operatorname{Mo}(2)$ & $\operatorname{Mo}(3)$ & $104,99(4)$ & $\mathrm{F}(1)$ & $\mathrm{Mo}(1)$ & $\mathrm{P}(1)$ & $77,91(9)$ \\
\hline $\mathrm{F}(2)$ & $\operatorname{Mo}(2)$ & $\mathrm{Mo}(1)$ & $98,56(9)$ & $\operatorname{Mo}(2)$ & $\operatorname{Mo}(3)$ & $\operatorname{Mo}(1)$ & $59,903(16)$ \\
\hline $\mathrm{F}(2)$ & $\operatorname{Mo}(2)$ & $\operatorname{Mo}(3)$ & $140,05(9)$ & $S(4)$ & $\operatorname{Mo}(3)$ & $\operatorname{Mo}(2)$ & $100,24(4)$ \\
\hline $\mathrm{F}(2)$ & $\operatorname{Mo}(2)$ & $S(2)$ & $92,65(9)$ & $\mathrm{S}(4)$ & $\operatorname{Mo}(3)$ & $\operatorname{Mo}(1)$ & $53,74(4)$ \\
\hline $\mathrm{F}(2)$ & $\operatorname{Mo}(2)$ & $S(3)$ & $161,80(10)$ & $\mathrm{S}(4)$ & $\operatorname{Mo}(3)$ & $S(3)$ & $99,69(5)$ \\
\hline $\mathrm{F}(2)$ & $\operatorname{Mo}(2)$ & $S(1)$ & $85,83(10)$ & $S(4)$ & $\operatorname{Mo}(3)$ & $S(1)$ & $105,66(5)$ \\
\hline$F(2)$ & $\mathrm{Mo}(2)$ & $\mathrm{P}(3)$ & $82,25(10)$ & $\mathrm{S}(4)$ & $\operatorname{Mo}(3)$ & $\mathrm{P}(5)$ & $80,35(5)$ \\
\hline $\mathrm{F}(2)$ & $\mathrm{Mo}(2)$ & $\mathrm{P}(4)$ & $70,60(9)$ & $S(4)$ & $\mathrm{Mo}(3)$ & $\mathrm{P}(6)$ & $92,74(5)$ \\
\hline $\operatorname{Mo}(2)$ & $\mathrm{Mo}(1)$ & $\operatorname{Mo}(3)$ & $59,912(17)$ & $S(3)$ & $\operatorname{Mo}(3)$ & $\operatorname{Mo}(2)$ & $52,71(4)$ \\
\hline $\mathrm{S}(2)$ & $\operatorname{Mo}(1)$ & $\operatorname{Mo}(2)$ & $53,88(4)$ & $S(3)$ & $\operatorname{Mo}(3)$ & $\operatorname{Mo}(1)$ & $98,69(4)$ \\
\hline $\mathrm{S}(2)$ & $\operatorname{Mo}(1)$ & $\operatorname{Mo}(3)$ & $98,52(4)$ & $S(3)$ & $\operatorname{Mo}(3)$ & $\mathrm{S}(1)$ & $105,39(5)$ \\
\hline$S(2)$ & $\mathrm{Mo}(1)$ & $S(4)$ & $98,10(5)$ & $\mathrm{S}(3)$ & $\operatorname{Mo}(3)$ & $\mathrm{P}(5)$ & $82,44(5)$ \\
\hline$S(2)$ & $\mathrm{Mo}(1)$ & $\mathrm{P}(2)$ & $89,95(5)$ & $\mathrm{S}(3)$ & $\operatorname{Mo}(3)$ & $\mathrm{P}(6)$ & $156,12(5)$ \\
\hline$S(2)$ & $\mathrm{Mo}(1)$ & $S(1)$ & $106,85(5)$ & $S(1)$ & $\operatorname{Mo}(3)$ & $\operatorname{Mo}(2)$ & $54,25(4)$ \\
\hline $\mathrm{S}(2)$ & $\operatorname{Mo}(1)$ & $\mathrm{P}(1)$ & $85,39(5)$ & $S(1)$ & $\operatorname{Mo}(3)$ & $\mathrm{Mo}(1)$ & $53,92(4)$ \\
\hline
\end{tabular}


Tabla 7.6. Ángulos de enlace representativos ( ${ }^{\circ}$ (continuación).

\begin{tabular}{|c|c|c|c|c|c|c|c|}
\hline \multicolumn{3}{|c|}{ Átomo Átomo Átomo } & \multirow{2}{*}{\begin{tabular}{|c|} 
Ángulo \\
$168,83(5)$
\end{tabular}} & \multicolumn{3}{|c|}{ Átomo Átomo Átomo } & \multirow{2}{*}{$\begin{array}{c}\text { Ángulo } \\
116,35(18)\end{array}$} \\
\hline$S(1)$ & $\operatorname{Mo}(3)$ & $\mathrm{P}(5)$ & & $\mathrm{C}(47)$ & $\mathrm{P}(4)$ & $\mathrm{Mo}(2)$ & \\
\hline$S(1)$ & $\operatorname{Mo}(3)$ & $\mathrm{P}(6)$ & $90,56(5)$ & $C(47)$ & $\mathrm{P}(4)$ & $\mathrm{C}(41)$ & $103,4(2)$ \\
\hline $\mathrm{P}(5)$ & $\operatorname{Mo}(3)$ & $\operatorname{Mo}(2)$ & $134,84(4)$ & $\mathrm{C}(47)$ & $\mathrm{P}(4)$ & $\mathrm{C}(28)$ & $106,0(3)$ \\
\hline $\mathrm{P}(5)$ & $\operatorname{Mo}(3)$ & $\operatorname{Mo}(1)$ & $133,78(4)$ & $\mathrm{C}(55)$ & $\mathrm{P}(5)$ & $\operatorname{Mo}(3)$ & $114,49(19)$ \\
\hline $\mathrm{P}(5)$ & $\operatorname{Mo}(3)$ & $\mathrm{P}(6)$ & $79,64(5)$ & $\mathrm{C}(53)$ & $\mathrm{P}(5)$ & $\operatorname{Mo}(3)$ & $106,6(2)$ \\
\hline $\mathrm{P}(6)$ & $\operatorname{Mo}(3)$ & $\operatorname{Mo}(2)$ & $144,57(4)$ & $C(53)$ & $\mathrm{P}(5)$ & $C(55)$ & $107,4(3)$ \\
\hline $\mathrm{P}(6)$ & $\operatorname{Mo}(3)$ & $\operatorname{Mo}(1)$ & $105,09(4)$ & $C(53)$ & $\mathrm{P}(5)$ & $C(61)$ & $102,2(3)$ \\
\hline $\mathrm{F}(3)$ & $\operatorname{Mo}(3)$ & $\operatorname{Mo}(2)$ & $100,86(9)$ & $C(61)$ & $\mathrm{P}(5)$ & $\mathrm{Mo}(3)$ & $121,8(2)$ \\
\hline $\mathrm{F}(3)$ & $\operatorname{Mo}(3)$ & $\operatorname{Mo}(1)$ & $140,74(9)$ & $C(61)$ & $\mathrm{P}(5)$ & $C(55)$ & $103,1(3)$ \\
\hline $\mathrm{F}(3)$ & $\operatorname{Mo}(3)$ & $S(4)$ & $158,90(10)$ & $C(3)$ & $\mathrm{P}(1)$ & $\operatorname{Mo}(1)$ & $117,96(18)$ \\
\hline $\mathrm{F}(3)$ & $\operatorname{Mo}(3)$ & $\mathrm{S}(3)$ & $93,02(10)$ & $C(3)$ & $\mathrm{P}(1)$ & $C(9)$ & $103,1(3)$ \\
\hline $\mathrm{F}(3)$ & $\operatorname{Mo}(3)$ & $S(1)$ & $86,86(10)$ & $C(3)$ & $\mathrm{P}(1)$ & $\mathrm{C}(1)$ & $106,1(3)$ \\
\hline $\mathrm{F}(3)$ & $\operatorname{Mo}(3)$ & $\mathrm{P}(5)$ & $84,75(10)$ & $\mathrm{C}(9)$ & $\mathrm{P}(1)$ & $\operatorname{Mo}(1)$ & $116,14(19)$ \\
\hline $\mathrm{F}(3)$ & $\operatorname{Mo}(3)$ & $\mathrm{P}(6)$ & $69,81(9)$ & $\mathrm{C}(9)$ & $\mathrm{P}(1)$ & $C(1)$ & $102,9(3)$ \\
\hline $\operatorname{Mo}(1)$ & $S(2)$ & $\operatorname{Mo}(2)$ & $73,58(4)$ & $C(1)$ & $\mathrm{P}(1)$ & $\operatorname{Mo}(1)$ & $109,2(2)$ \\
\hline $\operatorname{Mo}(3)$ & $S(4)$ & $\operatorname{Mo}(1)$ & $73,79(4)$ & $C(67)$ & $\mathrm{P}(6)$ & $\operatorname{Mo}(3)$ & $114,59(19)$ \\
\hline$C(15)$ & $\mathrm{P}(2)$ & $\operatorname{Mo}(1)$ & $123,4(2)$ & $\mathrm{C}(67)$ & $\mathrm{P}(6)$ & $\mathrm{C}(73)$ & $101,9(3)$ \\
\hline$C(15)$ & $\mathrm{P}(2)$ & $C(2)$ & $102,6(3)$ & $\mathrm{C}(67)$ & $\mathrm{P}(6)$ & $C(54)$ & $107,0(3)$ \\
\hline$C(2)$ & $\mathrm{P}(2)$ & $\mathrm{Mo}(1)$ & $103,5(2)$ & $C(73)$ & $\mathrm{P}(6)$ & $\operatorname{Mo}(3)$ & $129,52(19)$ \\
\hline$C(21)$ & $\mathrm{P}(2)$ & $\operatorname{Mo}(1)$ & $112,3(2)$ & $C(73)$ & $\mathrm{P}(6)$ & $C(54)$ & $99,3(3)$ \\
\hline$C(21)$ & $\mathrm{P}(2)$ & $C(15)$ & $107,1(3)$ & $C(54)$ & $\mathrm{P}(6)$ & $\operatorname{Mo}(3)$ & $101,7(2)$ \\
\hline$C(21)$ & $\mathrm{P}(2)$ & $C(2)$ & $106,1(3)$ & $C(42)$ & $C(41)$ & $\mathrm{P}(4)$ & $119,1(4)$ \\
\hline $\mathrm{Mo}(2)$ & $S(3)$ & $\mathrm{Mo}(3)$ & $73,71(4)$ & $C(46)$ & $C(41)$ & $\mathrm{P}(4)$ & $121,6(4)$ \\
\hline $\mathrm{Mo}(1)$ & $S(1)$ & $\operatorname{Mo}(2)$ & $71,66(4)$ & $C(68)$ & $C(67)$ & $\mathrm{P}(6)$ & $123,3(5)$ \\
\hline $\operatorname{Mo}(1)$ & $\mathrm{S}(1)$ & $\operatorname{Mo}(3)$ & $71,91(4)$ & $C(72)$ & $C(67)$ & $\mathrm{P}(6)$ & $117,4(5)$ \\
\hline $\mathrm{Mo}(3)$ & $S(1)$ & $\mathrm{Mo}(2)$ & $71,53(4)$ & $C(74)$ & $C(73)$ & $\mathrm{P}(6)$ & $122,4(5)$ \\
\hline$C(27)$ & $\mathrm{P}(3)$ & $\operatorname{Mo}(2)$ & $108,5(2)$ & $\mathrm{C}(78)$ & $C(73)$ & $\mathrm{P}(6)$ & $118,8(5)$ \\
\hline$C(29)$ & $\mathrm{P}(3)$ & $\operatorname{Mo}(2)$ & $116,91(19)$ & $C(27)$ & $C(28)$ & $\mathrm{P}(4)$ & $108,5(4)$ \\
\hline C(29) & $\mathrm{P}(3)$ & $C(27)$ & $104,8(3)$ & $C(28)$ & $C(27)$ & $\mathrm{P}(3)$ & $110,6(4)$ \\
\hline C(29) & $\mathrm{P}(3)$ & $C(35)$ & $104,2(3)$ & $C(48)$ & $C(47)$ & $\mathrm{P}(4)$ & $118,4(4)$ \\
\hline$C(35)$ & $\mathrm{P}(3)$ & $\operatorname{Mo}(2)$ & $120,18(18)$ & $C(52)$ & $C(47)$ & $\mathrm{P}(4)$ & $122,6(5)$ \\
\hline$C(35)$ & $\mathrm{P}(3)$ & $C(27)$ & $100,0(3)$ & $C(20)$ & $C(15)$ & $\mathrm{P}(2)$ & $119,0(5)$ \\
\hline $\mathrm{C}(41)$ & $\mathrm{P}(4)$ & $\operatorname{Mo}(2)$ & $127,35(19)$ & $C(16)$ & $C(15)$ & $\mathrm{P}(2)$ & $123,2(5)$ \\
\hline$C(41)$ & $\mathrm{P}(4)$ & $C(28)$ & $99,1(3)$ & $C(56)$ & $C(55)$ & $\mathrm{P}(5)$ & $118,5(5)$ \\
\hline C(28) & $\mathrm{P}(4)$ & $\mathrm{Mo}(2)$ & $101,43(19)$ & $C(60)$ & $C(55)$ & $\mathrm{P}(5)$ & $121,9(6)$ \\
\hline
\end{tabular}




\subsubsection{CLÚSTERES FUNCIONALIZADOS CON AMINOFOSFINAS}

\subsubsection{ESTRUCTURA DE $\left[\mathrm{Mo}_{3} \mathrm{~S}_{4} \mathrm{Cl}_{3}(\text { ediprp) })_{3}\right] \mathrm{Cl} \cdot \mathrm{CH}_{2} \mathrm{Cl}_{2}\left(5(\mathrm{Cl}) \cdot \mathrm{CH}_{2} \mathrm{Cl}_{2}\right)$}

El clúster $\mathbf{5}(\mathrm{Cl})$ ha cristalizado tras la difusión lenta de pentano sobre diclorometano en el grupo espacial $\mathrm{P} 21 / \mathrm{c}$. Se observa la presencia de dos unidades clúster cristalográficamente independientes, así como de dos contraiones y una molécula de diclorometano. En una de las unidades, uno de los contraiones se encuentra desordenado en dos posiciones con ocupaciones relativas de 0,58 y 0,42 , por lo que la carga del complejo se encuentra balanceada. Un átomo de carbono perteneciente al ligando isopropilo se encuentra desordenado en dos posiciones $(\mathrm{C}(44)$ y $\mathrm{C}(45))$, con ocupación relativa del $50 \%$. Todos los átomos del complejo se han refinado anisotrópicamente, excepto los de hidrógeno que se han generado en posiciones geométricas. Los átomos de hidrógeno enlazados a $\mathrm{C}(44)$ y $\mathrm{C}(45)$ no se incluyen en el modelo.

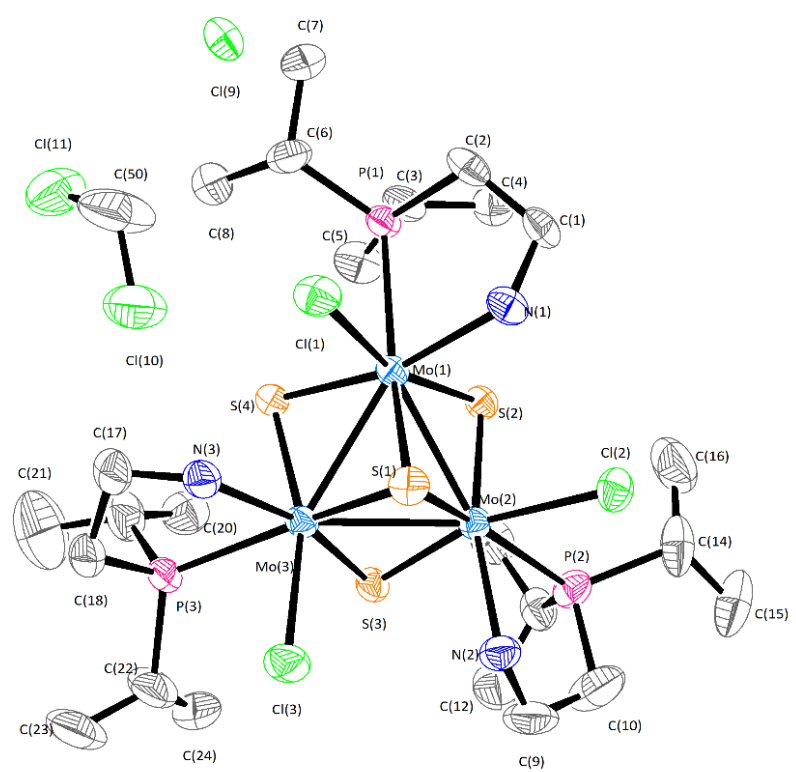

Figura 7.3. Representación ORTEP de uno de los clústeres $\left[\mathrm{Mo}_{3} \mathrm{~S}_{4} \mathrm{Cl}_{3}\right.$ (ediprp) 3 ] $\mathrm{Cl}$ (elipsoides al $50 \%$ de probabilidad). 
Tabla 7.7. Parámetros cristalográficos, condiciones de registro y refino.

Datos cristalográficos

\begin{tabular}{|c|c|c|c|}
\hline Especie & $2\left(\left[\mathrm{Mo}_{3} \mathrm{~S}_{4} \mathrm{Cl}_{3}\left(\text { ediprp }_{3}\right)_{3} \mathrm{Cl}\right.\right.$ & $\cdot \mathrm{CH}_{2} \mathrm{Cl}_{2}$ & \\
\hline Fórmula & $\mathrm{C}_{49} \mathrm{H}_{119} \mathrm{Cl}_{10} \mathrm{Mo}_{6} \mathrm{~N}_{6} \mathrm{P}_{6} \mathrm{~S}_{8}$ & $\begin{array}{l}\text { Sistema } \\
\text { cristalino }\end{array}$ & Triclínico \\
\hline Grupo espacial & P-1 & Peso molecular & 2164,93 \\
\hline $\mathbf{a}(\AA)$ & $12,9349(6)$ & $\boldsymbol{\alpha}\left({ }^{\circ}\right)$ & $77,986(3)$ \\
\hline $\mathbf{b}(\AA)$ & $15,9802(4)$ & $\boldsymbol{\beta}\left({ }^{\circ}\right)$ & $87,372(3)$ \\
\hline $\mathbf{c}(\AA)$ & $22,0560(7)$ & $\gamma\left({ }^{\circ}\right)$ & $78,457(3)$ \\
\hline $\mathbf{Z}$ & 2 & Vol $\left(\AA^{3}\right)$ & $4368,9(3)$ \\
\hline Color & Verde & $\begin{array}{l}\text { Tamaño del } \\
\text { cristal (mm) }\end{array}$ & $\begin{array}{l}0,156 \times 0,067 \times \\
0,049\end{array}$ \\
\hline $\mathbf{d}_{\text {calc }}\left(\mathrm{mg} / \mathrm{mm}^{3}\right)$ & 1,646 & $\lambda(\AA)$ & 0,71073 \\
\hline $\begin{array}{l}\text { Coeficiente de } \\
\text { absorción }\left(\mathrm{mm}^{-1}\right)\end{array}$ & 1,476 & F (000) & 2190,0 \\
\hline
\end{tabular}

Parámetros experimentales

\begin{tabular}{|c|c|c|c|}
\hline Temperatura (K) & $200,00(14)$ & $\mathbf{R}($ int $)$ & 0,0638 \\
\hline $\begin{array}{l}\text { Tiempo por marco } \\
\text { (s) }\end{array}$ & 300 & $\mathbf{R}(\sigma)$ & 0,0761 \\
\hline Límites $2 \theta\left(^{\circ}\right)$ & 5,702 a 51,996 & $\begin{array}{l}\text { Límites h, k, } 1 \\
\text { (mín/máx) }\end{array}$ & $\begin{array}{l}-15 \leq \mathrm{h} \leq 15 \\
-19-\leq \mathrm{k} \leq 19 \\
-27-\leq 1 \leq 27\end{array}$ \\
\hline $\begin{array}{l}N^{\circ} \text { de reflexiones } \\
\text { registradas }\end{array}$ & 48677 & $\begin{array}{l}\mathbf{N}^{\circ} \text { de reflexiones } \\
\text { independientes }\end{array}$ & 17122 \\
\hline
\end{tabular}

Resolución y refino

\begin{tabular}{|c|c|c|c|}
\hline $\mathbf{N}^{\mathbf{o}}$ de variables & 808 & GooF en $\mathrm{F}^{2}$ & 1,046 \\
\hline $\mathbf{N}^{o}$ de restricciones & 0 & & \\
\hline R1 final ([I>2sigma(I)]) & 0,0551 & R1 (todos los datos) & 0,0884 \\
\hline wR2 final ([I>2sigma(I)] $)$ & 0,1292 & wR2 (todos los datos) & 0,1538 \\
\hline $\begin{array}{l}\text { Densidad electrónica } \\
\text { residual }\left(\mathrm{e} \cdot \AA^{-3}\right)\end{array}$ & $2,10 /-1,18$ & Max. desplazam./ $\sigma$ & 0,001 \\
\hline
\end{tabular}

$$
R 1=\sum|| F_{0}|-| F_{1}|| / \sum F_{0} \quad w R 2=\left[\sum\left[w\left(F_{0}^{2}-F_{c}^{2}\right)^{2}\right] / \sum\left[w\left(F_{0}^{2}\right)^{2}\right]\right]^{1 / 2}
$$


Tabla 7.8. Distancias de enlace representativas $(\AA)$.

\begin{tabular}{|c|c|c|c|c|c|}
\hline Átomo & Átomo & Distancia & Átomo & Átomo & Distancia \\
\hline $\mathrm{Mo}(1)$ & $\operatorname{Mo}(2)$ & $2,7678(7)$ & $\operatorname{Mo}(4)$ & $\operatorname{Mo}(5)$ & $2,7661(9)$ \\
\hline $\operatorname{Mo}(1)$ & $\operatorname{Mo}(3)$ & $2,7563(7)$ & $\mathrm{Mo}(4)$ & $\operatorname{Mo}(6)$ & $2,7527(8)$ \\
\hline $\mathrm{Mo}(1)$ & $\mathrm{S}(1)$ & $2,3494(16)$ & $\operatorname{Mo}(4)$ & $\mathrm{S}(5)$ & $2,3457(17)$ \\
\hline $\operatorname{Mo}(1)$ & $\mathrm{S}(2)$ & $2,3041(16)$ & $\mathrm{Mo}(4)$ & $\mathrm{S}(6)$ & $2,2978(19)$ \\
\hline $\operatorname{Mo}(1)$ & $\mathrm{S}(4)$ & $2,2938(17)$ & $\mathrm{Mo}(4)$ & $\mathrm{S}(8)$ & $2,2950(18)$ \\
\hline $\operatorname{Mo}(1)$ & $\mathrm{P}(1)$ & $2,5766(16)$ & $\operatorname{Mo}(4)$ & $\mathrm{P}(4)$ & $2,5741(17)$ \\
\hline $\operatorname{Mo}(1)$ & $\mathrm{N}(1)$ & $2,251(5)$ & $\operatorname{Mo}(4)$ & $\mathrm{N}(4)$ & $2,259(5)$ \\
\hline $\operatorname{Mo}(1)$ & $\mathrm{Cl}(1)$ & $2,4990(16)$ & $\operatorname{Mo}(4)$ & $\mathrm{Cl}(4)$ & $2,5139(19)$ \\
\hline $\operatorname{Mo}(2)$ & $\mathrm{S}(1)$ & $2,3397(16)$ & $\operatorname{Mo}(5)$ & $\mathrm{S}(5)$ & $2,351(2)$ \\
\hline $\operatorname{Mo}(2)$ & $\mathrm{S}(2)$ & $2,3059(15)$ & $\operatorname{Mo}(5)$ & $\mathrm{S}(6)$ & $2,3053(17)$ \\
\hline $\operatorname{Mo}(2)$ & $\mathrm{S}(3)$ & $2,3036(17)$ & $\operatorname{Mo}(5)$ & $\mathrm{S}(7)$ & $2,2897(18)$ \\
\hline $\operatorname{Mo}(2)$ & $\mathrm{P}(2)$ & $2,5704(18)$ & $\operatorname{Mo}(5)$ & $\mathrm{P}(5)$ & $2,593(2)$ \\
\hline $\operatorname{Mo}(2)$ & $\mathrm{N}(2)$ & $2,261(5)$ & $\operatorname{Mo}(5)$ & $\mathrm{N}(5)$ & $2,253(5)$ \\
\hline $\operatorname{Mo}(2)$ & $\mathrm{Cl}(2)$ & $2,5275(17)$ & $\operatorname{Mo}(5)$ & $\mathrm{Cl}(5)$ & $2,4929(18)$ \\
\hline $\operatorname{Mo}(3)$ & $\mathrm{S}(1)$ & $2,3498(17)$ & $\operatorname{Mo}(6)$ & $\mathrm{S}(5)$ & $2,3454(19)$ \\
\hline $\operatorname{Mo}(3)$ & $\mathrm{S}(3)$ & $2,2952(17)$ & $\operatorname{Mo}(6)$ & $\mathrm{S}(7)$ & $2,305(2)$ \\
\hline $\operatorname{Mo}(3)$ & $\mathrm{S}(4)$ & $2,2917(15)$ & $\operatorname{Mo}(6)$ & $\mathrm{S}(8)$ & $2,2882(17)$ \\
\hline $\operatorname{Mo}(3)$ & $\mathrm{P}(3)$ & $2,582(2)$ & $\operatorname{Mo}(6)$ & $\mathrm{P}(6)$ & $2,560(2)$ \\
\hline $\operatorname{Mo}(3)$ & $\mathrm{N}(3)$ & $2,243(5)$ & $\operatorname{Mo}(6)$ & $\mathrm{N}(6)$ & $2,264(17)$ \\
\hline $\operatorname{Mo}(3)$ & $\mathrm{Cl}(3)$ & $2,5203(17)$ & $\operatorname{Mo}(6)$ & $\mathrm{Cl}(6)$ & $2,5046(19)$ \\
\hline $\operatorname{Mo}(3)$ & $\mathrm{Mo}(2)$ & $2,7606(8)$ & $\operatorname{Mo}(6)$ & $\operatorname{Mo}(5)$ & $2,7559(10)$ \\
\hline
\end{tabular}


Tabla 7.9. Ángulos de enlace representativos $\left({ }^{\circ}\right)$.

\begin{tabular}{|c|c|c|c|c|c|c|c|}
\hline \multicolumn{3}{|c|}{ Átomo Átomo Átomo } & \multirow{2}{*}{$\begin{array}{c}\text { Ángulo } \\
59,964(19)\end{array}$} & \multicolumn{3}{|c|}{ Átomo Átomo Átomo } & \multirow{2}{*}{$\begin{array}{l}\text { Ángulo } \\
88,87(6)\end{array}$} \\
\hline $\operatorname{Mo}(3)$ & $\mathrm{Mo}(1)$ & $\operatorname{Mo}(2)$ & & $\mathrm{S}(3)$ & $\operatorname{Mo}(3)$ & $\mathrm{P}(3)$ & \\
\hline $\mathrm{Cl}(1)$ & $\mathrm{Mo}(1)$ & $\operatorname{Mo}(3)$ & $98,41(4)$ & $\mathrm{S}(1)$ & $\operatorname{Mo}(3)$ & $\operatorname{Mo}(1)$ & $54,08(4)$ \\
\hline $\mathrm{Cl}(1)$ & $\operatorname{Mo}(1)$ & $\operatorname{Mo}(2)$ & $136,23(5)$ & $\mathrm{S}(1)$ & $\operatorname{Mo}(3)$ & $\operatorname{Mo}(2)$ & $53,77(4)$ \\
\hline $\mathrm{S}(1)$ & $\mathrm{Mo}(1)$ & $\operatorname{Mo}(3)$ & $54,10(4)$ & $\mathrm{S}(1)$ & $\operatorname{Mo}(3)$ & $\mathrm{Cl}(3)$ & $85,02(6)$ \\
\hline $\mathrm{S}(1)$ & $\mathrm{Mo}(1)$ & $\operatorname{Mo}(2)$ & $53,66(4)$ & $\mathrm{S}(1)$ & $\operatorname{Mo}(3)$ & $\mathrm{P}(3)$ & $160,38(6)$ \\
\hline $\mathrm{S}(1)$ & $\mathrm{Mo}(1)$ & $\mathrm{Cl}(1)$ & $82,63(6)$ & $\mathrm{P}(3)$ & $\operatorname{Mo}(3)$ & $\operatorname{Mo}(1)$ & $137,89(5)$ \\
\hline $\mathrm{S}(1)$ & $\operatorname{Mo}(1)$ & $\mathrm{P}(1)$ & $159,22(6)$ & $\mathrm{P}(3)$ & $\operatorname{Mo}(3)$ & $\operatorname{Mo}(2)$ & $142,12(5)$ \\
\hline $\mathrm{S}(4)$ & $\operatorname{Mo}(1)$ & $\operatorname{Mo}(3)$ & $53,02(4)$ & $\mathrm{P}(3)$ & $\operatorname{Mo}(3)$ & $\mathrm{Cl}(3)$ & $81,03(7)$ \\
\hline $\mathrm{S}(4)$ & $\operatorname{Mo}(1)$ & $\operatorname{Mo}(2)$ & $98,48(4)$ & $\mathrm{S}(4)$ & $\operatorname{Mo}(3)$ & $\operatorname{Mo}(1)$ & $53,09(4)$ \\
\hline $\mathrm{S}(4)$ & $\mathrm{Mo}(1)$ & $\mathrm{Cl}(1)$ & $95,28(6)$ & $\mathrm{S}(4)$ & $\operatorname{Mo}(3)$ & $\operatorname{Mo}(2)$ & $98,74(5)$ \\
\hline $\mathrm{S}(4)$ & $\mathrm{Mo}(1)$ & $\mathrm{S}(1)$ & $105,76(6)$ & $\mathrm{S}(4)$ & $\operatorname{Mo}(3)$ & $\mathrm{Cl}(3)$ & $162,65(7)$ \\
\hline $\mathrm{S}(4)$ & $\mathrm{Mo}(1)$ & $\mathrm{P}(1)$ & $87,61(5)$ & $C(42)$ & $\mathrm{P}(6)$ & $C(43)$ & $106,0(6)$ \\
\hline $\mathrm{S}(4)$ & $\mathrm{Mo}(1)$ & $\mathrm{S}(2)$ & $96,64(6)$ & $\operatorname{Mo}(6)$ & $\mathrm{S}(8)$ & $\mathrm{Mo}(4)$ & $73,83(5)$ \\
\hline $\mathrm{P}(1)$ & $\operatorname{Mo}(1)$ & $\operatorname{Mo}(3)$ & $140,50(4)$ & $\operatorname{Mo}(2)$ & $\mathrm{S}(3)$ & $\operatorname{Mo}(3)$ & $73,78(5)$ \\
\hline $\mathrm{P}(1)$ & $\mathrm{Mo}(1)$ & $\operatorname{Mo}(2)$ & $141,34(4)$ & $\operatorname{Mo}(5)$ & $\mathrm{S}(7)$ & $\operatorname{Mo}(6)$ & $73,72(6)$ \\
\hline $\mathrm{P}(1)$ & $\operatorname{Mo}(1)$ & $\mathrm{Cl}(1)$ & $80,32(6)$ & $\mathrm{Mo}(4)$ & $\mathrm{S}(6)$ & $\operatorname{Mo}(5)$ & $73,87(6)$ \\
\hline $\mathrm{S}(2)$ & $\mathrm{Mo}(1)$ & $\operatorname{Mo}(3)$ & $98,27(4)$ & C(38) & $\mathrm{P}(5)$ & $\operatorname{Mo}(5)$ & $117,9(3)$ \\
\hline $\mathrm{S}(2)$ & $\mathrm{Mo}(1)$ & $\operatorname{Mo}(2)$ & $53,13(4)$ & $C(38)$ & $\mathrm{P}(5)$ & $C(34)$ & $105,3(5)$ \\
\hline $\mathrm{S}(2)$ & $\mathrm{Mo}(1)$ & $\mathrm{Cl}(1)$ & $163,15(6)$ & $C(38)$ & $\mathrm{P}(5)$ & $C(35)$ & $110,2(5)$ \\
\hline $\mathrm{S}(2)$ & $\mathrm{Mo}(1)$ & $\mathrm{S}(1)$ & $105,42(6)$ & $C(34)$ & $\mathrm{P}(5)$ & $\operatorname{Mo}(5)$ & $99,0(4)$ \\
\hline $\mathrm{S}(2)$ & $\mathrm{Mo}(1)$ & $\mathrm{P}(1)$ & $88,31(5)$ & $C(34)$ & $\mathrm{P}(5)$ & $C(35)$ & $104,8(4)$ \\
\hline $\mathrm{N}(1)$ & $\mathrm{Mo}(1)$ & $\operatorname{Mo}(3)$ & $141,38(13)$ & $\mathrm{C}(35)$ & $\mathrm{P}(5)$ & $\operatorname{Mo}(5)$ & $118,1(6)$ \\
\hline $\mathrm{N}(1)$ & $\operatorname{Mo}(1)$ & $\operatorname{Mo}(2)$ & $94,80(14)$ & $\operatorname{Mo}(3)$ & $\mathrm{S}(1)$ & $\operatorname{Mo}(1)$ & $71,82(5)$ \\
\hline $\mathrm{N}(1)$ & $\operatorname{Mo}(1)$ & $\mathrm{Cl}(1)$ & 79,66(15) & $\operatorname{Mo}(2)$ & $\mathrm{S}(1)$ & $\operatorname{Mo}(1)$ & $71,64(9)$ \\
\hline $\mathrm{N}(1)$ & $\operatorname{Mo}(1)$ & $\mathrm{S}(1)$ & $87,68(14)$ & $\operatorname{Mo}(2)$ & $\mathrm{S}(1)$ & $\operatorname{Mo}(3)$ & $72,35(4)$ \\
\hline $\mathrm{N}(1)$ & $\mathrm{Mo}(1)$ & $\mathrm{S}(4)$ & $165,03(14)$ & $\mathrm{C}(22)$ & $\mathrm{P}(3)$ & $\operatorname{Mo}(3)$ & $119,5(4)$ \\
\hline $\mathrm{N}(1)$ & $\operatorname{Mo}(1)$ & $\mathrm{P}(1)$ & $77,68(14)$ & $\mathrm{C}(18)$ & $\mathrm{P}(3)$ & $\operatorname{Mo}(3)$ & $99,2(3)$ \\
\hline $\mathrm{N}(1)$ & $\operatorname{Mo}(1)$ & $\mathrm{S}(2)$ & $85,81(14)$ & $\mathrm{C}(18)$ & $\mathrm{P}(3)$ & $C(22)$ & $104,5(4)$ \\
\hline $\operatorname{Mo}(1)$ & $\mathrm{Mo}(3)$ & $\operatorname{Mo}(2)$ & $59,811(19)$ & C(19) & $\mathrm{P}(3)$ & $\operatorname{Mo}(3)$ & $118,0(3)$ \\
\hline $\mathrm{Cl}(3)$ & $\mathrm{Mo}(3)$ & $\operatorname{Mo}(1)$ & $139,08(5)$ & C(19) & $\mathrm{P}(3)$ & $C(22)$ & $108,1(5)$ \\
\hline $\mathrm{Cl}(3)$ & $\mathrm{Mo}(3)$ & $\operatorname{Mo}(2)$ & $98,60(5)$ & C(19) & $\mathrm{P}(3)$ & $C(18)$ & $105,1(4)$ \\
\hline $\mathrm{S}(3)$ & $\mathrm{Mo}(3)$ & $\operatorname{Mo}(1)$ & $99,24(4)$ & $\operatorname{Mo}(5)$ & $\mathrm{S}(5)$ & $\operatorname{Mo}(6)$ & $71,87(6)$ \\
\hline $\mathrm{S}(3)$ & $\mathrm{Mo}(3)$ & $\operatorname{Mo}(2)$ & $53,25(4)$ & $\operatorname{Mo}(5)$ & $\mathrm{S}(5)$ & $\operatorname{Mo}(4)$ & $72,17(5)$ \\
\hline $\mathrm{S}(3)$ & $\mathrm{Mo}(3)$ & $\mathrm{Cl}(3)$ & $92,21(6)$ & $\mathrm{Mo}(4)$ & $\mathrm{S}(5)$ & $\operatorname{Mo}(6)$ & $71,86(5)$ \\
\hline $\mathrm{S}(3)$ & $\mathrm{Mo}(3)$ & $\mathrm{S}(1)$ & $105,45(6)$ & $\operatorname{Mo}(3)$ & $\mathrm{S}(4)$ & $\operatorname{Mo}(1)$ & $73,90(5)$ \\
\hline
\end{tabular}


Tabla 7.9. Ángulos de enlace representativos ( $\left.{ }^{\circ}\right)$ (continuación).

\begin{tabular}{|c|c|c|c|c|c|c|c|}
\hline \multicolumn{3}{|c|}{ ÁtomoÁtomoÁtomo } & \multirow{2}{*}{$\begin{array}{c}\text { Ángulo } \\
99,6(2)\end{array}$} & \multicolumn{3}{|c|}{ ÁtomoÁtomo Átomo } & \multirow{2}{*}{$\begin{array}{l}\text { Ángulo } \\
52,89(5)\end{array}$} \\
\hline $\mathrm{C}(2)$ & $\mathrm{P}(1)$ & $\operatorname{Mo}(1)$ & & $S(7)$ & $\mathrm{Mo}(6)$ & $\operatorname{Mo}(5)$ & \\
\hline$C(2)$ & $\mathrm{P}(1)$ & $C(6)$ & $102,6(3)$ & $S(7)$ & $\operatorname{Mo}(6)$ & $\mathrm{Mo}(4)$ & $99,07(5)$ \\
\hline$C(6)$ & $\mathrm{P}(1)$ & $\operatorname{Mo}(1)$ & $117,5(2)$ & $S(7)$ & $\operatorname{Mo}(6)$ & $\mathrm{Cl}(6)$ & $90,67(8)$ \\
\hline$C(3)$ & $\mathrm{P}(1)$ & $\operatorname{Mo}(1)$ & $122,2(2)$ & $S(7)$ & $\mathrm{Mo}(6)$ & $\mathrm{P}(6)$ & $88,70(8)$ \\
\hline$C(3)$ & $\mathrm{P}(1)$ & $C(2)$ & $106,9(3)$ & $S(7)$ & $\mathrm{Mo}(6)$ & $\mathrm{S}(8)$ & $98,43(7)$ \\
\hline$C(3)$ & $\mathrm{P}(1)$ & $C(6)$ & $105,6(3)$ & $S(7)$ & $\mathrm{Mo}(6)$ & $S(5)$ & $105,49(7)$ \\
\hline $\operatorname{Mo}(1)$ & $S(2)$ & $\operatorname{Mo}(2)$ & $73,80(5)$ & $\mathrm{S}(5)$ & $\mathrm{Mo}(6)$ & $\operatorname{Mo}(5)$ & $54,16(6)$ \\
\hline C(41) & $\mathrm{N}(6)$ & $\mathrm{Mo}(6)$ & $118,6(6)$ & $\mathrm{S}(5)$ & $\mathrm{Mo}(6)$ & $\mathrm{Mo}(4)$ & $54,08(4)$ \\
\hline $\mathrm{C}(48)$ & $\mathrm{C}(47)$ & $\mathrm{P}(6)$ & $116,5(11)$ & $S(5)$ & $\operatorname{Mo}(6)$ & $\mathrm{Cl}(6)$ & $84,95(7)$ \\
\hline $\mathrm{C}(49)$ & $\mathrm{C}(47)$ & $\mathrm{P}(6)$ & $112,5(8)$ & $S(5)$ & $\operatorname{Mo}(6)$ & $\mathrm{P}(6)$ & $160,12(8)$ \\
\hline C(45) & $C(43)$ & $\mathrm{P}(6)$ & 116,6(11) & $\mathrm{N}(6)$ & $\operatorname{Mo}(6)$ & $\operatorname{Mo}(5)$ & $139,46(17)$ \\
\hline C(44) & $C(43)$ & $\mathrm{P}(6)$ & $125,5(15)$ & $\mathrm{N}(6)$ & $\mathrm{Mo}(6)$ & $\mathrm{Mo}(4)$ & $95,98(17)$ \\
\hline $\mathrm{C}(23)$ & $\mathrm{C}(22)$ & $\mathrm{P}(3)$ & $117,2(7)$ & $\mathrm{N}(6)$ & $\mathrm{Mo}(6)$ & $\mathrm{Cl}(6)$ & $79,48(18)$ \\
\hline C(24) & $\mathrm{C}(22)$ & $\mathrm{P}(3)$ & $114,4(6)$ & $\mathrm{N}(6)$ & $\operatorname{Mo}(6)$ & $\mathrm{P}(6)$ & $78,33(18)$ \\
\hline$S(4)$ & $\operatorname{Mo}(3)$ & $S(3)$ & $97,71(6)$ & $\mathrm{N}(6)$ & $\mathrm{Mo}(6)$ & $S(8)$ & $88,60(18)$ \\
\hline$S(4)$ & $\operatorname{Mo}(3)$ & $\mathrm{S}(1)$ & $105,81(6)$ & $\mathrm{N}(6)$ & $\mathrm{Mo}(6)$ & $\mathrm{S}(7)$ & $164,74(18)$ \\
\hline$S(4)$ & $\mathrm{Mo}(3)$ & $\mathrm{P}(3)$ & $84,96(6)$ & $\mathrm{N}(6)$ & $\mathrm{Mo}(6)$ & $S(5)$ & $85,38(18)$ \\
\hline $\mathrm{N}(3)$ & $\operatorname{Mo}(3)$ & $\operatorname{Mo}(1)$ & $94,10(14)$ & $\mathrm{Mo}(4)$ & $\mathrm{Mo}(6)$ & $\operatorname{Mo}(5)$ & $60,28(2)$ \\
\hline $\mathrm{N}(3)$ & $\operatorname{Mo}(3)$ & $\operatorname{Mo}(2)$ & $139,89(15)$ & $\mathrm{Cl}(5)$ & $\operatorname{Mo}(5)$ & $\operatorname{Mo}(6)$ & $138,64(6)$ \\
\hline $\mathrm{N}(3)$ & $\operatorname{Mo}(3)$ & $\mathrm{Cl}(3)$ & $80,86(14)$ & $\mathrm{Cl}(5)$ & $\operatorname{Mo}(5)$ & $\mathrm{Mo}(4)$ & $96,87(5)$ \\
\hline $\mathrm{N}(3)$ & $\operatorname{Mo}(3)$ & $S(3)$ & $165,74(15)$ & $S(7)$ & $\operatorname{Mo}(5)$ & $\mathrm{Mo}(6)$ & $53,39(6)$ \\
\hline $\mathrm{N}(3)$ & $\operatorname{Mo}(3)$ & $S(1)$ & $86,46(16)$ & $S(7)$ & $\operatorname{Mo}(5)$ & $\mathrm{Mo}(4)$ & $99,06(5)$ \\
\hline $\mathrm{N}(3)$ & $\operatorname{Mo}(3)$ & $\mathrm{P}(3)$ & $77,79(16)$ & $S(7)$ & $\operatorname{Mo}(5)$ & $\mathrm{Cl}(5)$ & $164,02(7)$ \\
\hline $\mathrm{N}(3)$ & $\operatorname{Mo}(3)$ & $S(4)$ & $86,23(14)$ & $S(7)$ & $\operatorname{Mo}(5)$ & $S(6)$ & $98,03(6)$ \\
\hline $\operatorname{Mo}(6)$ & $\mathrm{Mo}(4)$ & $\operatorname{Mo}(5)$ & $59,92(2)$ & $S(7)$ & $\operatorname{Mo}(5)$ & $\mathrm{P}(5)$ & $85,89(7)$ \\
\hline $\mathrm{Cl}(6)$ & $\mathrm{Mo}(6)$ & $\operatorname{Mo}(5)$ & $97,29(6)$ & $\mathrm{S}(7)$ & $\operatorname{Mo}(5)$ & $S(5)$ & $105,79(8)$ \\
\hline $\mathrm{Cl}(6)$ & $\operatorname{Mo}(6)$ & $\operatorname{Mo}(4)$ & $139,02(5)$ & $S(6)$ & $\operatorname{Mo}(5)$ & $\operatorname{Mo}(6)$ & $98,55(5)$ \\
\hline $\mathrm{P}(6)$ & $\mathrm{Mo}(6)$ & $\operatorname{Mo}(5)$ & $141,59(7)$ & $C(40)$ & $\mathrm{C}(38)$ & $\mathrm{P}(5)$ & $114,1(7)$ \\
\hline $\mathrm{P}(6)$ & $\operatorname{Mo}(6)$ & $\mathrm{Mo}(4)$ & $138,49(5)$ & $C(39)$ & $C(38)$ & $\mathrm{P}(5)$ & $116,4(8)$ \\
\hline $\mathrm{P}(6)$ & $\mathrm{Mo}(6)$ & $\mathrm{Cl}(6)$ & $80,97(7)$ & $C(10)$ & $\mathrm{P}(2)$ & $\operatorname{Mo}(2)$ & $99,4(3)$ \\
\hline $\mathrm{S}(8)$ & $\mathrm{Mo}(6)$ & $\operatorname{Mo}(5)$ & $99,25(6)$ & $C(10)$ & $\mathrm{P}(2)$ & $C(11)$ & 109,1(4) \\
\hline$S(8)$ & $\operatorname{Mo}(6)$ & $\mathrm{Mo}(4)$ & $53,20(5)$ & $C(10)$ & $\mathrm{P}(2)$ & $C(14)$ & $101,0(5)$ \\
\hline$S(8)$ & $\operatorname{Mo}(6)$ & $\mathrm{Cl}(6)$ & $163,42(8)$ & $C(11)$ & $\mathrm{P}(2)$ & $\operatorname{Mo}(2)$ & $122,3(3)$ \\
\hline$S(8)$ & $\operatorname{Mo}(6)$ & $\mathrm{P}(6)$ & $85,39(7)$ & $C(11)$ & $\mathrm{P}(2)$ & $C(14)$ & $103,6(3)$ \\
\hline $\mathrm{S}(8)$ & $\operatorname{Mo}(6)$ & $\mathrm{S}(5)$ & $105,70(7)$ & $C(14)$ & $\mathrm{P}(2)$ & $\operatorname{Mo}(2)$ & $119,0(3)$ \\
\hline
\end{tabular}


Tabla 7.9. Ángulos de enlace representativos $\left(^{\circ}\right)$ (continuación).

\begin{tabular}{|c|c|c|c|c|c|c|c|}
\hline \multicolumn{3}{|c|}{ Átomo Átomo Átomo } & \multirow{2}{*}{$\begin{array}{c}\text { Ángulo } \\
98,7(2)\end{array}$} & \multicolumn{3}{|c|}{ Átomo Átomo Átomo } & \multirow{2}{*}{$\begin{array}{l}\text { Ángulo } \\
98,79(5)\end{array}$} \\
\hline $\mathrm{C}(26)$ & $\mathrm{P}(4)$ & $\mathrm{Mo}(4)$ & & $\mathrm{S}(8)$ & $\mathrm{Mo}(4)$ & $\operatorname{Mo}(5)$ & \\
\hline$C(26)$ & $\mathrm{P}(4)$ & $\mathrm{C}(27)$ & $103,5(3)$ & $\mathrm{S}(8)$ & $\operatorname{Mo}(4)$ & $\mathrm{Cl}(4)$ & $90,31(7)$ \\
\hline $\mathrm{C}(30)$ & $\mathrm{P}(4)$ & $\operatorname{Mo}(4)$ & $119,3(3)$ & $\mathrm{S}(8)$ & $\operatorname{Mo}(4)$ & $\mathrm{S}(5)$ & $105,47(7)$ \\
\hline $\mathrm{C}(30)$ & $\mathrm{P}(4)$ & $C(26)$ & $104,8(4)$ & $\mathrm{S}(8)$ & $\operatorname{Mo}(4)$ & $\mathrm{P}(4)$ & $88,29(6)$ \\
\hline $\mathrm{C}(30)$ & $\mathrm{P}(4)$ & $\mathrm{C}(27)$ & $110,1(3)$ & $\mathrm{S}(6)$ & $\operatorname{Mo}(4)$ & $\operatorname{Mo}(6)$ & $98,83(5)$ \\
\hline $\mathrm{C}(27)$ & $\mathrm{P}(4)$ & $\operatorname{Mo}(4)$ & $117,3(2)$ & $\mathrm{S}(6)$ & $\operatorname{Mo}(4)$ & $\operatorname{Mo}(5)$ & $53,19(4)$ \\
\hline $\mathrm{C}(1)$ & $\mathrm{N}(1)$ & $\operatorname{Mo}(1)$ & $118,5(4)$ & $\mathrm{S}(6)$ & $\operatorname{Mo}(4)$ & $\mathrm{Cl}(4)$ & $163,36(7)$ \\
\hline $\mathrm{C}(9)$ & $\mathrm{N}(2)$ & $\operatorname{Mo}(2)$ & $116,3(4)$ & $\mathrm{S}(6)$ & $\operatorname{Mo}(4)$ & $\mathrm{S}(8)$ & $98,02(7)$ \\
\hline$C(25)$ & $\mathrm{N}(4)$ & $\operatorname{Mo}(4)$ & $116,6(4)$ & $\mathrm{S}(6)$ & $\operatorname{Mo}(4)$ & $\mathrm{S}(5)$ & $105,64(7)$ \\
\hline$C(26)$ & $C(25)$ & $\mathrm{N}(4)$ & $110,8(6)$ & $\mathrm{S}(6)$ & $\operatorname{Mo}(4)$ & $\mathrm{P}(4)$ & $86,33(6)$ \\
\hline $\mathrm{C}(2)$ & $C(1)$ & $\mathrm{N}(1)$ & $109,5(6)$ & $\mathrm{S}(5)$ & $\operatorname{Mo}(4)$ & $\operatorname{Mo}(6)$ & $54,06(5)$ \\
\hline$C(17)$ & $\mathrm{N}(3)$ & $\operatorname{Mo}(3)$ & $116,9(4)$ & $\mathrm{S}(5)$ & $\operatorname{Mo}(4)$ & $\operatorname{Mo}(5)$ & $54,00(5)$ \\
\hline C(33) & $\mathrm{N}(5)$ & $\operatorname{Mo}(5)$ & $117,3(5)$ & $S(5)$ & $\mathrm{Mo}(4)$ & $\mathrm{Cl}(4)$ & $85,77(7)$ \\
\hline$S(6)$ & $\operatorname{Mo}(5)$ & $\operatorname{Mo}(4)$ & $52,94(5)$ & $\mathrm{S}(5)$ & $\mathrm{Mo}(4)$ & $\mathrm{P}(4)$ & $159,93(6)$ \\
\hline$S(6)$ & $\operatorname{Mo}(5)$ & $\mathrm{Cl}(5)$ & $90,57(6)$ & $\mathrm{P}(4)$ & $\mathrm{Mo}(4)$ & $\operatorname{Mo}(6)$ & $141,25(5)$ \\
\hline$S(6)$ & $\operatorname{Mo}(5)$ & $\mathrm{P}(5)$ & $89,89(7)$ & $\mathrm{P}(4)$ & $\mathrm{Mo}(4)$ & $\operatorname{Mo}(5)$ & $139,44(5)$ \\
\hline $\mathrm{S}(6)$ & $\operatorname{Mo}(5)$ & $\mathrm{S}(5)$ & $105,24(7)$ & $\mathrm{P}(4)$ & $\operatorname{Mo}(4)$ & $\mathrm{Cl}(4)$ & $79,51(6)$ \\
\hline $\mathrm{P}(5)$ & $\operatorname{Mo}(5)$ & $\operatorname{Mo}(6)$ & $139,10(5)$ & $\mathrm{N}(4)$ & $\mathrm{Mo}(4)$ & $\operatorname{Mo}(6)$ & $139,63(13)$ \\
\hline $\mathrm{P}(5)$ & $\operatorname{Mo}(5)$ & $\operatorname{Mo}(4)$ & $142,81(5)$ & $\mathrm{N}(4)$ & $\operatorname{Mo}(4)$ & $\operatorname{Mo}(5)$ & $95,93(14)$ \\
\hline $\mathrm{P}(5)$ & $\operatorname{Mo}(5)$ & $\mathrm{Cl}(5)$ & $80,67(7)$ & $\mathrm{N}(4)$ & $\operatorname{Mo}(4)$ & $\mathrm{Cl}(4)$ & $80,33(16)$ \\
\hline $\mathrm{S}(5)$ & $\operatorname{Mo}(5)$ & $\operatorname{Mo}(6)$ & $53,98(5)$ & $\mathrm{N}(4)$ & $\operatorname{Mo}(4)$ & $\mathrm{S}(8)$ & $164,96(15)$ \\
\hline $\mathrm{S}(5)$ & $\operatorname{Mo}(5)$ & $\operatorname{Mo}(4)$ & $53,83(5)$ & $\mathrm{N}(4)$ & $\operatorname{Mo}(4)$ & $S(6)$ & $88,36(16)$ \\
\hline $\mathrm{S}(5)$ & $\operatorname{Mo}(5)$ & $\mathrm{Cl}(5)$ & $84,68(7)$ & $\mathrm{N}(4)$ & $\operatorname{Mo}(4)$ & $\mathrm{S}(5)$ & $85,69(13)$ \\
\hline $\mathrm{S}(5)$ & $\operatorname{Mo}(5)$ & $\mathrm{P}(5)$ & $159,03(7)$ & $\mathrm{N}(4)$ & $\operatorname{Mo}(4)$ & $\mathrm{P}(4)$ & $78,50(13)$ \\
\hline $\mathrm{N}(5)$ & $\operatorname{Mo}(5)$ & $\operatorname{Mo}(6)$ & $95,8(2)$ & $\operatorname{Mo}(1)$ & $\operatorname{Mo}(3)$ & $\operatorname{Mo}(2)$ & $60,226(19)$ \\
\hline $\mathrm{N}(5)$ & $\operatorname{Mo}(5)$ & $\mathrm{Mo}(4)$ & $138,9(2)$ & $\mathrm{Cl}(2)$ & $\operatorname{Mo}(2)$ & $\operatorname{Mo}(1)$ & $97,09(4)$ \\
\hline $\mathrm{N}(5)$ & $\operatorname{Mo}(5)$ & $\mathrm{Cl}(5)$ & $80,15(17)$ & $C(34)$ & $C(33)$ & $\mathrm{N}(5)$ & $109,8(7)$ \\
\hline $\mathrm{N}(5)$ & $\operatorname{Mo}(5)$ & $\mathrm{S}(7)$ & $88,61(17)$ & $\mathrm{N}(2)$ & $\mathrm{C}(9)$ & $C(10)$ & $113,9(7)$ \\
\hline $\mathrm{N}(5)$ & $\operatorname{Mo}(5)$ & $S(6)$ & $165,5(2)$ & $C(9)$ & $C(10)$ & $\mathrm{P}(2)$ & $113,1(6)$ \\
\hline $\mathrm{N}(5)$ & $\operatorname{Mo}(5)$ & $\mathrm{P}(5)$ & $77,6(2)$ & $C(13)$ & $\mathrm{C}(11)$ & $\mathrm{P}(2)$ & $112,3(5)$ \\
\hline $\mathrm{N}(5)$ & $\operatorname{Mo}(5)$ & $\mathrm{S}(5)$ & $85,2(2)$ & $\mathrm{C}(12)$ & $\mathrm{C}(11)$ & $\mathrm{P}(2)$ & $113,6(6)$ \\
\hline $\operatorname{Mo}(6)$ & $\operatorname{Mo}(5)$ & $\mathrm{Mo}(4)$ & $59,80(2)$ & $C(33)$ & $C(34)$ & $\mathrm{P}(5)$ & $109,9(6)$ \\
\hline $\mathrm{Cl}(4)$ & $\mathrm{Mo}(4)$ & $\operatorname{Mo}(6)$ & $97,68(5)$ & $\mathrm{N}(6)$ & $\mathrm{C}(41)$ & $C(42)$ & $109,8(9)$ \\
\hline $\mathrm{Cl}(4)$ & $\mathrm{Mo}(4)$ & $\operatorname{Mo}(5)$ & $139,76(5)$ & $C(41)$ & $\mathrm{C}(42)$ & $\mathrm{P}(6)$ & $113,0(7)$ \\
\hline $\mathrm{S}(8)$ & $\mathrm{Mo}(4)$ & $\operatorname{Mo}(6)$ & $52,97(5)$ & $\mathrm{C}(1)$ & $\mathrm{C}(2)$ & $\mathrm{P}(1)$ & $111,4(4)$ \\
\hline
\end{tabular}


Tabla 7.9. Ángulos de enlace representativos $\left(^{\circ}\right)$ (continuación).

\begin{tabular}{|c|c|c|c|c|c|c|c|}
\hline \multicolumn{3}{|c|}{ ÁtomoÁtomoÁtomo } & \multirow{2}{*}{$\begin{array}{l}\text { Ángulo } \\
113,6(5)\end{array}$} & \multicolumn{3}{|c|}{ ÁtomoÁtomo Átomo } & \multirow{2}{*}{$\begin{array}{c}\text { Ángulo } \\
80,44(16)\end{array}$} \\
\hline $\mathrm{C}(7)$ & $C(6)$ & $\mathrm{P}(1)$ & & $\mathrm{N}(2)$ & $\operatorname{Mo}(2)$ & $\mathrm{Cl}(2)$ & \\
\hline$C(8)$ & $C(6)$ & $\mathrm{P}(1)$ & $114,2(5)$ & $\mathrm{N}(2)$ & $\operatorname{Mo}(2)$ & $S(3)$ & $87,88(6)$ \\
\hline$C(15)$ & $\mathrm{C}(14)$ & $\mathrm{P}(2)$ & $113,4(7)$ & $\mathrm{N}(2)$ & $\operatorname{Mo}(2)$ & $\mathrm{S}(1)$ & $85,69(14)$ \\
\hline $\mathrm{Cl}(2)$ & $\operatorname{Mo}(2)$ & $\operatorname{Mo}(3)$ & $138,41(4)$ & $\mathrm{N}(2)$ & $\operatorname{Mo}(2)$ & $S(2)$ & $165,69(15)$ \\
\hline$S(3)$ & $\operatorname{Mo}(2)$ & $\operatorname{Mo}(1)$ & $98,69(5)$ & $\mathrm{N}(2)$ & $\operatorname{Mo}(2)$ & $\mathrm{P}(2)$ & $78,20(14)$ \\
\hline$S(3)$ & $\operatorname{Mo}(2)$ & $\operatorname{Mo}(3)$ & $52,97(4)$ & $C(47)$ & $\mathrm{P}(6)$ & $\operatorname{Mo}(6)$ & $117,5(4)$ \\
\hline$S(3)$ & $\operatorname{Mo}(2)$ & $\mathrm{Cl}(2)$ & $164,21(6)$ & $C(43)$ & $\mathrm{P}(6)$ & $\operatorname{Mo}(6)$ & $121,0(4)$ \\
\hline$S(3)$ & $\mathrm{Mo}(2)$ & $\mathrm{S}(1)$ & $105,51(6)$ & $C(43)$ & $\mathrm{P}(6)$ & $C(47)$ & $106,4(5)$ \\
\hline$S(3)$ & $\operatorname{Mo}(2)$ & $S(2)$ & $97,14(6)$ & $\mathrm{C}(42)$ & $\mathrm{P}(6)$ & $\operatorname{Mo}(6)$ & $98,2(3)$ \\
\hline$S(3)$ & $\operatorname{Mo}(2)$ & $\mathrm{P}(2)$ & $84,86(6)$ & $\mathrm{C}(42)$ & $\mathrm{P}(6)$ & $C(47)$ & $105,8(6)$ \\
\hline$S(1)$ & $\operatorname{Mo}(2)$ & $\operatorname{Mo}(1)$ & $53,99(4)$ & $C(16)$ & $C(14)$ & $\mathrm{P}(2)$ & $112,2(6)$ \\
\hline$S(1)$ & $\operatorname{Mo}(2)$ & $\operatorname{Mo}(3)$ & $54,11(5)$ & $C(25)$ & $\mathrm{C}(26)$ & $\mathrm{P}(4)$ & $110,7(5)$ \\
\hline$S(1)$ & $\operatorname{Mo}(2)$ & $\mathrm{Cl}(2)$ & $84,31(6)$ & $\mathrm{C}(5)$ & $\mathrm{C}(3)$ & $\mathrm{P}(1)$ & $111,4(5)$ \\
\hline$S(1)$ & $\operatorname{Mo}(2)$ & $\mathrm{P}(2)$ & $160,57(6)$ & $C(4)$ & $C(3)$ & $\mathrm{P}(1)$ & $113,3(5)$ \\
\hline$S(2)$ & $\operatorname{Mo}(2)$ & $\mathrm{Mo}(1)$ & $53,07(4)$ & $C(17)$ & $C(18)$ & $\mathrm{P}(3)$ & $108,5(5)$ \\
\hline $\mathrm{S}(2)$ & $\mathrm{Mo}(2)$ & $\operatorname{Mo}(3)$ & $98,11(5)$ & $C(37)$ & $\mathrm{C}(35)$ & $\mathrm{P}(5)$ & $114,1(6)$ \\
\hline $\mathrm{S}(2)$ & $\operatorname{Mo}(2)$ & $\mathrm{Cl}(2)$ & $91,88(6)$ & $C(36)$ & $C(35)$ & $\mathrm{P}(5)$ & $115,0(7)$ \\
\hline$S(2)$ & $\operatorname{Mo}(2)$ & $S(1)$ & $105,68(6)$ & $C(20)$ & $\mathrm{C}(19)$ & $\mathrm{P}(3)$ & $112,8(6)$ \\
\hline $\mathrm{S}(2)$ & $\operatorname{Mo}(2)$ & $\mathrm{P}(2)$ & $88,87(6)$ & $C(21)$ & $C(19)$ & $\mathrm{P}(3)$ & $118,6(8)$ \\
\hline $\mathrm{P}(2)$ & $\operatorname{Mo}(2)$ & $\mathrm{Mo}(1)$ & $141,94(5)$ & $C(18)$ & $\mathrm{C}(17)$ & $\mathrm{N}(3)$ & $109,9(6)$ \\
\hline $\mathrm{P}(2)$ & $\mathrm{Mo}(2)$ & $\operatorname{Mo}(3)$ & $137,74(5)$ & C(32) & $C(30)$ & $\mathrm{P}(4)$ & $113,6(6)$ \\
\hline $\mathrm{P}(2)$ & $\operatorname{Mo}(2)$ & $\mathrm{Cl}(2)$ & $82,38(6)$ & $C(31)$ & $C(30)$ & $\mathrm{P}(4)$ & $114,9(6)$ \\
\hline $\mathrm{N}(2)$ & $\operatorname{Mo}(2)$ & $\operatorname{Mo}(1)$ & $139,52(14)$ & $C(28)$ & $\mathrm{C}(27)$ & $\mathrm{P}(4)$ & $115,1(5)$ \\
\hline $\mathrm{N}(2)$ & $\operatorname{Mo}(2)$ & $\operatorname{Mo}(3)$ & $95,61(15)$ & $\mathrm{C}(29)$ & $C(27)$ & $\mathrm{P}(4)$ & $112,6(5)$ \\
\hline
\end{tabular}




\subsubsection{ESTRUCTURA DE $\left[\mathrm{Mo}_{3} \mathrm{~S}_{4} \mathrm{Cl}_{3}(\text { ediprp })_{3}\right] \mathrm{Cl} \cdot \mathrm{CH}_{2} \mathrm{Cl}_{2}\left(5 \mathrm{a}(\mathrm{Cl}) \cdot \mathrm{CH}_{2} \mathrm{Cl}_{2}\right)$}

La obtención de cristales del clúster $\mathbf{5 a}(\mathrm{Cl})$ adecuados para su caracterización ha tenido lugar tras la difusión lenta de pentano sobre una disolución del clúster $\mathbf{5}(\mathrm{Cl})$ en diclorometano. Esta estructura se ha obtenido únicamente en uno de los intentos de cristalización del clúster $\mathbf{5}^{+}$y se ha obtenido la misma celda unidad para todos los cristales extraídos de esta muestra. El clúster $\mathbf{5 a}(\mathrm{Cl})$ ha cristalizado en el grupo espacial $\mathrm{P} 2 / \mathrm{n}$ junto con una molécula de diclorometano. Los grupos isopropilo enlazados al fósforo $\mathrm{P}(1)$ se encuentran desordenados, por lo que a diferencia del resto de átomos de la estructura, se han refinado isotrópicamente debido a que presentan elevados parámetros de agitación térmica. En el último mapa de diferencia de Fourier aparecen una serie de picos cuyas intensidades oscilan entre 2,85 y 1,74, aunque se encuentran demasiado cerca de los átomos de molibdeno para ser incluidos en el modelo.

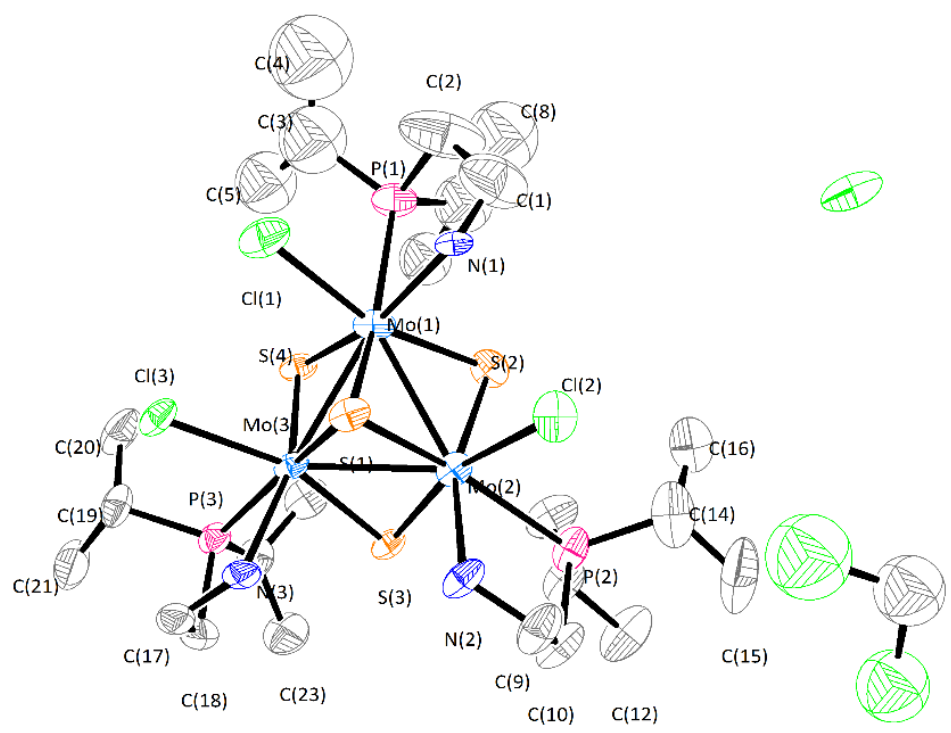

Figura 7.4. Representación ORTEP del clúster $\left[\mathrm{Mo}_{3} \mathrm{~S}_{4} \mathrm{Cl}_{3}(\text { ediprp })_{3}\right] \mathrm{Cl} \cdot \mathrm{CH}_{2} \mathrm{Cl}_{2}$ $\left(\mathbf{5 a}(\mathrm{Cl}) \cdot \mathrm{CH}_{2} \mathrm{Cl}_{2}\right)$ (elipsoides al $50 \%$ de probabilidad). 
Tabla 7.10. Parámetros cristalográficos, condiciones de registro y refino.

Datos cristalográficos

Especie

$\left.\left[\mathrm{Mo}_{3} \mathrm{~S}_{4} \mathrm{Cl}_{3} \text { (ediprp) }\right)_{3}\right] \mathrm{Cl} \cdot \mathrm{CH}_{2} \mathrm{Cl}_{2}$

Fórmula

$\mathrm{C}_{25} \mathrm{H}_{60} \mathrm{Cl}_{6} \mathrm{Mo}_{3} \mathrm{~N}_{3} \mathrm{P}_{3} \mathrm{~S}_{4}$

Sistema

Monoclínico

Grupo espacial

$$
\mathrm{P} 21 / \mathrm{n}
$$

cristalino

a $(\AA)$

$13,7649(3)$

Peso molecular 1124,43

b $(\AA)$

20,8591(4)

c $(\AA)$

$16,3323(3)$

Z

Color

4

Verde

$\alpha\left(^{\circ}\right)$

90,00

$\boldsymbol{\beta}\left(^{\circ}\right)$

109,274(2)

$\gamma\left({ }^{\circ}\right)$

90,00

Vol $\left(\AA^{3}\right)$

4426,56(17)

Tamaño del

$0,097 \times 0,080 \times$

cristal (mm)

0,018

$\mathbf{d}_{\text {calc }}\left(\mathrm{mg} / \mathrm{mm}^{3}\right)$

1,687

Coeficiente de

13,154

$\lambda(\AA)$

1,541814

absorción $\left(\mathrm{mm}^{-1}\right)$

F (000)

2272,0

\section{Parámetros experimentales}

Temperatura (K) 293(2)

Tiempo por marco 130

(s)

Límites $2 \theta\left(^{\circ}\right)$

7,13 y 133,202

25765

$\mathrm{N}^{\mathrm{o}}$ de reflexiones

registradas
R(int)

$\mathbf{R}(\sigma)$

0,0837

0,0595

Límites h, k, $1 \quad-16 \leq \mathrm{h} \leq 16$

(mín/máx)

$-23 \leq \mathrm{k} \leq 19$

$-19 \leq 1 \leq 19$

$\mathbf{N}^{\circ}$ de reflexiones 7709

independientes

Resolución y refino

\begin{tabular}{|c|c|c|c|}
\hline $\mathbf{N}^{\mathrm{o}}$ de variables & 364 & GooF en $F^{2}$ & 1,113 \\
\hline $\mathbf{N}^{o}$ de restricciones & 0 & & \\
\hline R1 final ([I>2sigma(I)]) & 0,0930 & R1 (todos los datos) & 0,1097 \\
\hline wR2 final ([I>2sigma(I)]) & 0,2377 & wR2 (todos los datos) & 0,2466 \\
\hline $\begin{array}{l}\text { Densidad electrónica } \\
\text { residual }\left(e \cdot \AA^{-3}\right)\end{array}$ & $2,85 /-1,32$ & Max. desplazam./ $\sigma$ & 0,000 \\
\hline
\end{tabular}

$$
R 1=\sum|| F_{0}|-| F_{1}|| / \sum F_{0} \quad w R 2=\left[\sum\left[w\left(F_{0}^{2}-F_{c}^{2}\right)^{2}\right] / \sum\left[w\left(F_{0}^{2}\right)^{2}\right]\right]^{1 / 2}
$$


Tabla 7.11. Distancias de enlace representativas $(\AA)$.

\begin{tabular}{cccccc}
\hline Átomo Átomo & Distancia & Átomo Átomo & Distancia \\
\hline $\operatorname{Mo}(1)$ & $\operatorname{Mo}(2)$ & $2,7898(14)$ & $\operatorname{Mo}(2)$ & $\mathrm{S}(3)$ & $2,284(3)$ \\
$\operatorname{Mo}(1)$ & $\operatorname{Mo}(3)$ & $2,7564(3)$ & $\operatorname{Mo}(2)$ & $\mathrm{P}(2)$ & $2,572(3)$ \\
$\operatorname{Mo}(2)$ & $\operatorname{Mo}(3)$ & $2,7976(13)$ & $\operatorname{Mo}(2)$ & $\mathrm{N}(2)$ & $2,288(9)$ \\
$\operatorname{Mo}(1)$ & $\mathrm{S}(1)$ & $2,347(3)$ & $\operatorname{Mo}(2)$ & $\mathrm{Cl}(2)$ & $2,521(3)$ \\
$\operatorname{Mo}(1)$ & $\mathrm{S}(2)$ & $2,313(3)$ & $\operatorname{Mo}(3)$ & $\mathrm{S}(1)$ & $2,361(3)$ \\
$\operatorname{Mo}(1)$ & $\mathrm{S}(4)$ & $2,296(3)$ & $\operatorname{Mo}(3)$ & $\mathrm{S}(3)$ & $2,301(3)$ \\
$\operatorname{Mo}(1)$ & $\mathrm{P}(1)$ & $2,564(3)$ & $\operatorname{Mo}(3)$ & $\mathrm{S}(4)$ & $2,293(3)$ \\
$\operatorname{Mo}(1)$ & $\mathrm{N}(1)$ & $2,382(8)$ & $\operatorname{Mo}(3)$ & $\mathrm{P}(3)$ & $2,556(3)$ \\
$\operatorname{Mo}(1)$ & $\mathrm{Cl}(1)$ & $2,484(4)$ & $\operatorname{Mo}(3)$ & $\mathrm{N}(3)$ & $2,290(9)$ \\
$\operatorname{Mo}(2)$ & $\mathrm{S}(1)$ & $2,353(3)$ & $\operatorname{Mo}(3)$ & $\mathrm{Cl}(3)$ & $2,479(3)$ \\
$\operatorname{Mo}(2)$ & $\mathrm{S}(2)$ & $2,300(3)$ & & & \\
\hline
\end{tabular}


Tabla 7.12. Ángulos de enlace representativos $\left(^{\circ}\right)$.

\begin{tabular}{|c|c|c|c|c|c|c|c|}
\hline \multicolumn{3}{|c|}{ ÁtomoÁtomoÁtomo } & \multirow{2}{*}{$\begin{array}{l}\text { Ángulo } \\
59,12(3)\end{array}$} & \multicolumn{3}{|c|}{ ÁtomoÁtomo Átomo } & \multirow{2}{*}{$\begin{array}{l}\text { Ángulo } \\
97,96(8)\end{array}$} \\
\hline $\operatorname{Mo}(1)$ & $\mathrm{Mo}(2)$ & $\operatorname{Mo}(3)$ & & $S(4)$ & $\mathrm{Mo}(1)$ & $\operatorname{Mo}(2)$ & \\
\hline$S(2)$ & $\operatorname{Mo}(2)$ & $\operatorname{Mo}(1)$ & $53,00(9)$ & $S(4)$ & $\operatorname{Mo}(1)$ & $\operatorname{Mo}(3)$ & $53,04(7)$ \\
\hline $\mathrm{S}(2)$ & $\operatorname{Mo}(2)$ & $\operatorname{Mo}(3)$ & $97,09(9)$ & $\mathrm{S}(4)$ & $\operatorname{Mo}(1)$ & $\mathrm{N}(1)$ & $165,0(2)$ \\
\hline $\mathrm{S}(2)$ & $\mathrm{Mo}(2)$ & $\mathrm{S}(1)$ & $105,24(12)$ & $S(4)$ & $\mathrm{Mo}(1)$ & $\mathrm{S}(1)$ & $106,44(15)$ \\
\hline $\mathrm{S}(2)$ & $\mathrm{Mo}(2)$ & $\mathrm{P}(2)$ & $88,53(12)$ & $S(4)$ & $\operatorname{Mo}(1)$ & $\mathrm{P}(1)$ & $87,30(11)$ \\
\hline$S(2)$ & $\mathrm{Mo}(2)$ & $\mathrm{N}(2)$ & $160,9(3)$ & $\mathrm{N}(1)$ & $\mathrm{Mo}(1)$ & $\mathrm{Mo}(2)$ & $95,6(2)$ \\
\hline$S(3)$ & $\operatorname{Mo}(2)$ & $\mathrm{Mo}(1)$ & $99,66(8)$ & $\mathrm{N}(1)$ & $\operatorname{Mo}(1)$ & $\operatorname{Mo}(3)$ & $141,0(2)$ \\
\hline$S(3)$ & $\operatorname{Mo}(2)$ & $\operatorname{Mo}(3)$ & $52,68(7)$ & $\mathrm{S}(1)$ & $\operatorname{Mo}(1)$ & $\operatorname{Mo}(2)$ & $53,70(8)$ \\
\hline$S(3)$ & $\operatorname{Mo}(2)$ & $\mathrm{S}(2)$ & $98,99(11)$ & $\mathrm{S}(1)$ & $\operatorname{Mo}(1)$ & $\operatorname{Mo}(3)$ & $54,40(8)$ \\
\hline$S(3)$ & $\operatorname{Mo}(2)$ & $S(1)$ & $104,19(11)$ & $\mathrm{S}(1)$ & $\operatorname{Mo}(1)$ & $\mathrm{N}(1)$ & $86,8(2)$ \\
\hline $\mathrm{S}(3)$ & $\mathrm{Mo}(2)$ & $\mathrm{P}(2)$ & $81,15(11)$ & $\mathrm{S}(1)$ & $\operatorname{Mo}(1)$ & $\mathrm{P}(1)$ & $160,84(12)$ \\
\hline$S(3)$ & $\operatorname{Mo}(2)$ & $\mathrm{N}(2)$ & $91,2(3)$ & $\mathrm{P}(1)$ & $\operatorname{Mo}(1)$ & $\operatorname{Mo}(2)$ & $139,02(10)$ \\
\hline$S(1)$ & $\mathrm{Mo}(2)$ & $\operatorname{Mo}(1)$ & $53,48(7)$ & $\mathrm{P}(1)$ & $\operatorname{Mo}(1)$ & $\operatorname{Mo}(3)$ & $140,26(9)$ \\
\hline$S(1)$ & $\mathrm{Mo}(2)$ & $\operatorname{Mo}(3)$ & $53,72(8)$ & $\mathrm{P}(1)$ & $\mathrm{Mo}(1)$ & $\mathrm{N}(1)$ & $78,2(2)$ \\
\hline$S(1)$ & $\mathrm{Mo}(2)$ & $\mathrm{P}(2)$ & $163,99(11)$ & $\mathrm{Cl}(1)$ & $\operatorname{Mo}(1)$ & $\mathrm{Mo}(2)$ & $140,22(10)$ \\
\hline $\mathrm{S}(1)$ & $\operatorname{Mo}(2)$ & $\mathrm{Cl}(2)$ & $85,79(11)$ & $\mathrm{Cl}(1)$ & $\mathrm{Mo}(1)$ & $\operatorname{Mo}(3)$ & 98,30(11) \\
\hline $\mathrm{P}(2)$ & $\operatorname{Mo}(2)$ & $\mathrm{Mo}(1)$ & $141,37(9)$ & $\mathrm{Cl}(1)$ & $\mathrm{Mo}(1)$ & $\mathrm{S}(2)$ & $163,59(13)$ \\
\hline $\mathrm{P}(2)$ & $\operatorname{Mo}(2)$ & $\operatorname{Mo}(3)$ & $133,83(9)$ & $\mathrm{Cl}(1)$ & $\operatorname{Mo}(1)$ & $S(4)$ & $92,93(13)$ \\
\hline $\mathrm{P}(2)$ & $\operatorname{Mo}(2)$ & $\mathrm{N}(2)$ & $77,0(3)$ & $\mathrm{Cl}(1)$ & $\operatorname{Mo}(1)$ & $\mathrm{N}(1)$ & $80,6(2)$ \\
\hline $\mathrm{N}(2)$ & $\mathrm{Mo}(2)$ & $\operatorname{Mo}(1)$ & $141,2(3)$ & $\mathrm{Cl}(1)$ & $\operatorname{Mo}(1)$ & $S(1)$ & $86,52(12)$ \\
\hline $\mathrm{N}(2)$ & $\operatorname{Mo}(2)$ & $\operatorname{Mo}(3)$ & $102,0(3)$ & $\mathrm{Cl}(1)$ & $\operatorname{Mo}(1)$ & $\mathrm{P}(1)$ & $79,37(13)$ \\
\hline $\mathrm{Cl}(2)$ & $\operatorname{Mo}(2)$ & $\mathrm{Mo}(1)$ & $96,10(10)$ & $\operatorname{Mo}(3)$ & $\mathrm{Mo}(1)$ & $\operatorname{Mo}(2)$ & $60,58(3)$ \\
\hline $\mathrm{Cl}(2)$ & $\operatorname{Mo}(2)$ & $\operatorname{Mo}(3)$ & $139,36(9)$ & $S(4)$ & $\operatorname{Mo}(3)$ & $\operatorname{Mo}(2)$ & $97,82(8)$ \\
\hline $\mathrm{Cl}(2)$ & $\operatorname{Mo}(2)$ & $S(2)$ & $89,90(12)$ & $S(4)$ & $\operatorname{Mo}(3)$ & $\operatorname{Mo}(1)$ & $53,14(8)$ \\
\hline $\mathrm{Cl}(2)$ & $\operatorname{Mo}(2)$ & $S(3)$ & $164,23(12)$ & $S(4)$ & $\mathrm{Mo}(3)$ & $S(3)$ & $99,03(11)$ \\
\hline $\mathrm{Cl}(2)$ & $\operatorname{Mo}(2)$ & $S(1)$ & $85,83(10)$ & $S(4)$ & $\operatorname{Mo}(3)$ & $S(1)$ & $106,08(10)$ \\
\hline $\mathrm{Cl}(2)$ & $\mathrm{Mo}(2)$ & $\mathrm{P}(2)$ & $86,12(12)$ & $\mathrm{S}(4)$ & $\operatorname{Mo}(3)$ & $\mathrm{P}(3)$ & $86,64(10)$ \\
\hline $\mathrm{Cl}(2)$ & $\mathrm{Mo}(2)$ & $\mathrm{N}(2)$ & $76,9(3)$ & $S(4)$ & $\mathrm{Mo}(3)$ & $\mathrm{N}(3)$ & $160,5(3)$ \\
\hline $\operatorname{Mo}(1)$ & $\mathrm{Mo}(3)$ & $\operatorname{Mo}(2)$ & $60,30(4)$ & $S(3)$ & $\mathrm{Mo}(3)$ & $\mathrm{Mo}(2)$ & $52,13(8)$ \\
\hline $\mathrm{S}(2)$ & $\operatorname{Mo}(1)$ & $\operatorname{Mo}(2)$ & $52,57(8)$ & $S(3)$ & $\operatorname{Mo}(3)$ & $\operatorname{Mo}(1)$ & 100,19(8) \\
\hline $\mathrm{S}(2)$ & $\operatorname{Mo}(1)$ & $\operatorname{Mo}(3)$ & $97,92(9)$ & $S(3)$ & $\operatorname{Mo}(3)$ & $\mathrm{S}(1)$ & $103,42(11)$ \\
\hline$S(2)$ & $\mathrm{Mo}(1)$ & $S(4)$ & $94,83(11)$ & $\mathrm{S}(3)$ & $\operatorname{Mo}(3)$ & $\mathrm{P}(3)$ & $80,43(10)$ \\
\hline $\mathrm{S}(2)$ & $\mathrm{Mo}(1)$ & $\mathrm{N}(1)$ & $88,3(2)$ & $\mathrm{S}(3)$ & $\operatorname{Mo}(3)$ & $\mathrm{N}(3)$ & $89,6(3)$ \\
\hline$S(2)$ & $\mathrm{Mo}(1)$ & $S(1)$ & $105,4(11)$ & $S(1)$ & $\operatorname{Mo}(3)$ & $\operatorname{Mo}(2)$ & $53,48(8)$ \\
\hline $\mathrm{S}(2)$ & $\operatorname{Mo}(1)$ & $\mathrm{P}(1)$ & $86,56(12)$ & $S(1)$ & $\operatorname{Mo}(3)$ & $\operatorname{Mo}(1)$ & $53,92(7)$ \\
\hline
\end{tabular}


Tabla 7.12. Ángulos de enlace representativos $\left(^{\circ}\right)$ (continuación).

\begin{tabular}{|c|c|c|c|c|c|c|c|}
\hline \multicolumn{3}{|c|}{ Átomo Átomo Átomo } & \multirow{2}{*}{$\begin{array}{c}\text { Ángulo } \\
165,71(10)\end{array}$} & \multicolumn{3}{|c|}{ Átomo Átomo Átomo } & \multirow{2}{*}{$\begin{array}{c}\text { Ángulo } \\
99,2(5)\end{array}$} \\
\hline $\mathrm{S}(1)$ & $\mathrm{Mo}(3)$ & $\mathrm{P}(3)$ & & $\mathrm{C}(18)$ & $\mathrm{P}(3)$ & $\mathrm{Mo}(3)$ & \\
\hline$S(1)$ & $\operatorname{Mo}(3)$ & $\mathrm{N}(3)$ & $88,6(3)$ & $C(18)$ & $\mathrm{P}(3)$ & $\mathrm{C}(22)$ & $107,7(8)$ \\
\hline $\mathrm{P}(3)$ & $\operatorname{Mo}(3)$ & $\operatorname{Mo}(2)$ & $132,51(8)$ & $\mathrm{C}(18)$ & $\mathrm{P}(3)$ & $\mathrm{C}(19)$ & $103,9(7)$ \\
\hline $\mathrm{P}(3)$ & $\operatorname{Mo}(3)$ & $\operatorname{Mo}(1)$ & $139,59(8)$ & $\mathrm{C}(19)$ & $\mathrm{P}(3)$ & $\operatorname{Mo}(3)$ & $118,5(5)$ \\
\hline $\mathrm{P}(3)$ & $\operatorname{Mo}(3)$ & $\mathrm{N}(3)$ & $77,6(3)$ & $\mathrm{C}(19)$ & $\mathrm{P}(3)$ & $\mathrm{C}(22)$ & $103,0(6)$ \\
\hline $\mathrm{N}(3)$ & $\operatorname{Mo}(3)$ & $\operatorname{Mo}(2)$ & $101,3(3)$ & $C(17)$ & $\mathrm{N}(3)$ & $\operatorname{Mo}(3)$ & $117,4(7)$ \\
\hline $\mathrm{N}(3)$ & $\operatorname{Mo}(3)$ & $\operatorname{Mo}(1)$ & $142,5(3)$ & $C(14)$ & $\mathrm{P}(2)$ & $\operatorname{Mo}(2)$ & $116,7(7)$ \\
\hline $\mathrm{Cl}(3)$ & $\operatorname{Mo}(3)$ & $\mathrm{Mo}(2)$ & $141,20(9)$ & $C(10)$ & $\mathrm{P}(2)$ & $\mathrm{Mo}(2)$ & $99,6(5)$ \\
\hline $\mathrm{Cl}(3)$ & $\operatorname{Mo}(3)$ & $\mathrm{Mo}(1)$ & $97,72(8)$ & $C(10)$ & $\mathrm{P}(2)$ & $\mathrm{C}(11)$ & $102,6(8)$ \\
\hline $\mathrm{Cl}(3)$ & $\operatorname{Mo}(3)$ & $S(4)$ & $91,09(10)$ & $C(14)$ & $\mathrm{P}(2)$ & $\mathrm{C}(11)$ & $110,6(8)$ \\
\hline $\mathrm{Cl}(3)$ & $\operatorname{Mo}(3)$ & $S(3)$ & $162,08(11)$ & $C(11)$ & $\mathrm{P}(2)$ & $\operatorname{Mo}(2)$ & $119,5(5)$ \\
\hline $\mathrm{Cl}(3)$ & $\operatorname{Mo}(3)$ & $S(1)$ & $87,75(11)$ & $C(14)$ & $\mathrm{P}(2)$ & $\mathrm{C}(41)$ & $104,7(9)$ \\
\hline $\mathrm{Cl}(3)$ & $\operatorname{Mo}(3)$ & $\mathrm{P}(3)$ & $85,48(11)$ & $C(2)$ & $\mathrm{P}(1)$ & $\operatorname{Mo}(1)$ & $99,9(7)$ \\
\hline $\mathrm{Cl}(3)$ & $\operatorname{Mo}(3)$ & $\mathrm{N}(3)$ & $76,5(3)$ & $C(2)$ & $\mathrm{P}(1)$ & $C(3)$ & $101,1(15)$ \\
\hline $\operatorname{Mo}(1)$ & $\mathrm{S}(2)$ & $\operatorname{Mo}(2)$ & $74,43(9)$ & $C(2)$ & $\mathrm{P}(1)$ & $C(6)$ & $102,8(14)$ \\
\hline $\mathrm{Mo}(3)$ & $S(4)$ & $\operatorname{Mo}(1)$ & $73,82(9)$ & $\mathrm{C}(3)$ & $\mathrm{P}(1)$ & $\mathrm{Mo}(1)$ & $121,6(12)$ \\
\hline$C(1)$ & $\mathrm{N}(1)$ & $\mathrm{Mo}(1)$ & $114,9(10)$ & $C(6)$ & $\mathrm{P}(1)$ & $C(3)$ & $108,4(16)$ \\
\hline $\mathrm{Mo}(2)$ & $\mathrm{S}(3)$ & $\mathrm{Mo}(3)$ & $75,19(9)$ & $C(6)$ & $\mathrm{P}(1)$ & $\mathrm{Mo}(1)$ & $118,9(10)$ \\
\hline $\operatorname{Mo}(1)$ & $\mathrm{S}(1)$ & $\mathrm{Mo}(2)$ & $72,82(8)$ & $C(9)$ & $\mathrm{N}(2)$ & $\operatorname{Mo}(2)$ & $118,2(8)$ \\
\hline $\operatorname{Mo}(1)$ & $S(1)$ & $\operatorname{Mo}(3)$ & $71,68(8)$ & $C(10)$ & $\mathrm{C}(9)$ & $\mathrm{N}(2)$ & $111,11(11)$ \\
\hline $\mathrm{Mo}(3)$ & $S(1)$ & $\mathrm{Mo}(2)$ & $72,80(8)$ & $C(18)$ & $C(17)$ & $\mathrm{N}(3)$ & $109,6(10)$ \\
\hline$C(22)$ & $\mathrm{P}(3)$ & $\operatorname{Mo}(3)$ & $122,6(5)$ & $C(2)$ & $\mathrm{C}(1)$ & $\mathrm{N}(1)$ & $115(2)$ \\
\hline
\end{tabular}




\subsubsection{ESTRUCTURA DE $\left[\mathrm{W}_{3} \mathrm{~S}_{4} \mathrm{Br}_{3}(\text { ediprp })_{3}\right] \mathrm{BPh}_{4}\left(6\left(\mathrm{BPh}_{4}\right)\right)$}

La cristalización del compuesto $\mathbf{6}\left(\mathrm{BPh}_{4}\right)$ se ha llevado a cabo mediante difusión lenta de pentano sobre una disolución concentrada del complejo en diclorometano. Se observa la presencia de dos clústeres trinucleares cristalográficamente independientes, así como de dos contraiones. El refino se ha realizado en el grupo espacial monoclínico $\mathrm{P} 21 / \mathrm{c}$ de forma exitosa. Todos los átomos de la estructura se han refinado anisotrópicamente, excepto los de hidrógeno que se han generado en posiciones geométricas. En el último mapa de diferencia de Fourier se han localizado una serie de picos cuyas intensidades oscilan entre 3,05 y 1,60 que se encuentran demasiado cerca de los átomos de wolframio para ser incluidos en el modelo cristalográfico.

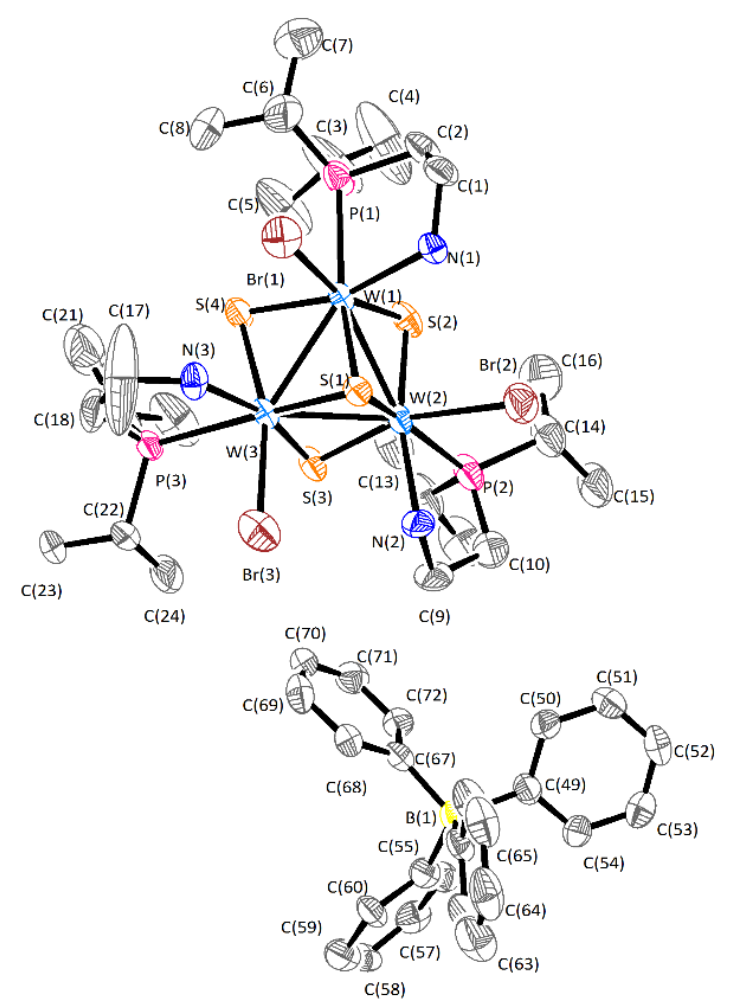

Figura 7.5. Representación ORTEP de uno de los clústeres cristalográficamente independientes $\left[\mathrm{W}_{3} \mathrm{~S}_{4} \mathrm{Br}_{3}(\right.$ ediprp) 3$] \mathrm{BPh}_{4}$ (elipsoides al $50 \%$ de probabilidad). 
Tabla 7.13. Parámetros cristalográficos, condiciones de registro y refino.

Datos cristalográficos

Especie $\quad 2\left(\left[\mathrm{~W}_{3} \mathrm{~S}_{4} \mathrm{Br}_{3}(\text { ediprp })_{3}\right] \mathrm{BPh}_{4}\right)$

Fórmula

$\mathrm{C}_{96} \mathrm{H}_{159} \mathrm{~B}_{2} \mathrm{Br}_{6} \mathrm{~N}_{6} \mathrm{P}_{6} \mathrm{~S}_{8} \mathrm{~W}_{6}$

Grupo espacial

$\mathbf{a}(\AA)$

b $(\AA)$

c $(\AA)$

Z

Color

$\mathbf{d}_{\text {calc }}\left(\mathrm{mg} / \mathrm{mm}^{3}\right)$

Coeficiente de

absorción $\left(\mathrm{mm}^{-1}\right)$
$\mathrm{P} 21 / \mathrm{c}$

13,4292(3)

46,2919(10)

$21,4997(5)$

4

Azul

1,773

7,431
Sistema cristalino

Peso molecular 3443,76

$\alpha\left({ }^{\circ}\right)$

$\boldsymbol{\beta}\left(^{\circ}\right)$

$\gamma\left({ }^{\circ}\right)$

Vol $\left(\AA^{3}\right)$

Tamaño del cristal (mm)

$\lambda(\AA)$

F (000)
Monoclínico

90,00

105,155 (2)

90,00

12900,7(5)

$0,542 \times 0,314 \times$

0,072

0,71073

6636

\section{Parámetros experimentales}

Temperatura $(\mathrm{K}) \quad 200,00(14)$

Tiempo por marco 40

(s)

$\begin{array}{ll}\mathbf{R}(\text { int }) & 0,1174 \\ \mathbf{R}(\boldsymbol{\sigma}) & 0,0843\end{array}$

Límites h, k, $1 \quad-18 \leq \mathrm{h} \leq 18$ (mín/máx) $\quad-55-\leq \mathrm{k} \leq 62$

$-24-\leq 1 \leq 29$

$\mathbf{N}^{\mathbf{o}}$ de reflexiones 22657

independientes

Resolución y refino

\begin{tabular}{|c|c|c|c|}
\hline $\mathbf{N}^{\circ}$ de variables & 1190 & GooF en $F^{2}$ & 1,068 \\
\hline $\mathbf{N}^{o}$ de restricciones & 0 & & \\
\hline R1 final ([I>2sigma(I)]) & 0,0725 & R1 (todos los datos) & 0,0843 \\
\hline wR2 final ([I>2sigma(I)]) & 0,1946 & wR2 (todos los datos) & 0,2053 \\
\hline $\begin{array}{l}\text { Densidad electrónica } \\
\text { residual }\left(e \cdot \AA^{-3}\right)\end{array}$ & $3,05 /-3,06$ & Max. desplazam./ $\sigma$ & 0,002 \\
\hline
\end{tabular}

$$
R 1=\sum|| F_{0}|-| F_{1}|| / \sum F_{0} \quad w R 2=\left[\sum\left[w\left(F_{0}^{2}-F_{c}^{2}\right)^{2}\right] / \sum\left[w\left(F_{0}^{2}\right)^{2}\right]\right]^{1 / 2}
$$


Tabla 7.14. Distancias de enlace representativas $(\AA)$.

\begin{tabular}{cccccc}
\hline Átomo Átomo & Distancia & \multicolumn{2}{c}{ Átomo Átomo } & Distancia \\
\hline $\mathrm{W}(1)$ & $\mathrm{W}(2)$ & $2,7664(7)$ & $\mathrm{W}(4)$ & $\mathrm{W}(5)$ & $2,7516(8)$ \\
$\mathrm{W}(1)$ & $\mathrm{W}(3)$ & $2,7444(7)$ & $\mathrm{W}(4)$ & $\mathrm{W}(6)$ & $2,7590(7)$ \\
$\mathrm{W}(1)$ & $\mathrm{S}(1)$ & $2,364(3)$ & $\mathrm{W}(4)$ & $\mathrm{S}(5)$ & $2,350(3)$ \\
$\mathrm{W}(1)$ & $\mathrm{S}(2)$ & $2,311(3)$ & $\mathrm{W}(4)$ & $\mathrm{S}(6)$ & $2,302(3)$ \\
$\mathrm{W}(1)$ & $\mathrm{S}(4)$ & $2,302(3)$ & $\mathrm{W}(4)$ & $\mathrm{S}(8)$ & $2,310(3)$ \\
$\mathrm{W}(1)$ & $\mathrm{P}(1)$ & $2,557(4)$ & $\mathrm{W}(4)$ & $\mathrm{P}(4)$ & $2,558(4)$ \\
$\mathrm{W}(1)$ & $\mathrm{N}(1)$ & $2,253(10)$ & $\mathrm{W}(4)$ & $\mathrm{N}(4)$ & $2,289(10)$ \\
$\mathrm{W}(1)$ & $\mathrm{Br}(1)$ & $2,616(2)$ & $\mathrm{W}(4)$ & $\mathrm{Br}(4)$ & $2,579(3)$ \\
$\mathrm{W}(2)$ & $\mathrm{S}(1)$ & $2,363(3)$ & $\mathrm{W}(5)$ & $\mathrm{S}(5)$ & $2,350(3)$ \\
$\mathrm{W}(2)$ & $\mathrm{S}(2)$ & $2,312(4)$ & $\mathrm{W}(5)$ & $\mathrm{S}(6)$ & $2,305(3)$ \\
$\mathrm{W}(2)$ & $\mathrm{S}(3)$ & $2,294(3)$ & $\mathrm{W}(5)$ & $\mathrm{S}(7)$ & $2,297(3)$ \\
$\mathrm{W}(2)$ & $\mathrm{P}(2)$ & $2,576(4)$ & $\mathrm{W}(5)$ & $\mathrm{P}(5)$ & $2,563(4)$ \\
$\mathrm{W}(2)$ & $\mathrm{N}(2)$ & $2,268(11)$ & $\mathrm{W}(5)$ & $\mathrm{N}(5)$ & $2,258(12)$ \\
$\mathrm{W}(2)$ & $\mathrm{Br}(2)$ & $2,6394(7)$ & $\mathrm{W}(5)$ & $\mathrm{B}(5)$ & $2,6115(18)$ \\
$\mathrm{W}(3)$ & $\mathrm{S}(1)$ & $2,363(3)$ & $\mathrm{W}(6)$ & $\mathrm{S}(5)$ & $2,350(3)$ \\
$\mathrm{W}(3)$ & $\mathrm{S}(3)$ & $2,306(3)$ & $\mathrm{W}(6)$ & $\mathrm{S}(7)$ & $2,298(3)$ \\
$\mathrm{W}(3)$ & $\mathrm{S}(4)$ & $2,295(3)$ & $\mathrm{W}(6)$ & $\mathrm{S}(8)$ & $2,301(3)$ \\
$\mathrm{W}(3)$ & $\mathrm{P}(3)$ & $2,561(3)$ & $\mathrm{W}(6)$ & $\mathrm{P}(6)$ & $2,570(3)$ \\
$\mathrm{W}(3)$ & $\mathrm{N}(3)$ & $2,252(11)$ & $\mathrm{W}(6)$ & $\mathrm{N}(6)$ & $2,256(11)$ \\
$\mathrm{W}(3)$ & $\mathrm{Br}(3)$ & $2,627(2)$ & $\mathrm{W}(6)$ & $\mathrm{Br}(6)$ & $2,603(2)$ \\
$\mathrm{W}(3)$ & $\mathrm{W}(2)$ & $2,7570(7)$ & $\mathrm{W}(6)$ & $\mathrm{W}(5)$ & $2,7534(7)$ \\
\hline
\end{tabular}


Tabla 7.15. Ángulos de enlace representativos $\left(^{\circ}\right)$.

\begin{tabular}{|c|c|c|c|c|c|c|c|}
\hline \multicolumn{3}{|c|}{ Átomo Átomo Átomo } & \multirow{2}{*}{$\begin{array}{c}\text { Ángulo } \\
60,037(18)\end{array}$} & \multicolumn{3}{|c|}{ Átomo Átomo Átomo } & \multirow{2}{*}{$\begin{array}{r}\text { Ángulo } \\
89,51(12)\end{array}$} \\
\hline $\mathrm{W}(3)$ & $\mathrm{W}(1)$ & $\mathrm{W}(2)$ & & $\mathrm{S}(3)$ & $\mathrm{W}(3)$ & $\mathrm{P}(3)$ & \\
\hline $\operatorname{Br}(1)$ & $\mathrm{W}(1)$ & $\mathrm{W}(3)$ & $96,47(4)$ & $\mathrm{S}(1)$ & $\mathrm{W}(3)$ & $\mathrm{W}(1)$ & $54,53(8)$ \\
\hline $\operatorname{Br}(1)$ & $\mathrm{W}(1)$ & $\mathrm{W}(2)$ & $138,36(5)$ & $S(1)$ & $\mathrm{W}(3)$ & $\mathrm{W}(2)$ & $54,30(8)$ \\
\hline$S(1)$ & $\mathrm{W}(1)$ & $\mathrm{W}(3)$ & $54,49(7)$ & $S(1)$ & $\mathrm{W}(3)$ & $\operatorname{Br}(3)$ & $83,25(9)$ \\
\hline$S(1)$ & $\mathrm{W}(1)$ & $\mathrm{W}(2)$ & $54,15(8)$ & $S(1)$ & $\mathrm{W}(3)$ & $\mathrm{P}(3)$ & $160,99(12)$ \\
\hline $\mathrm{S}(1)$ & $\mathrm{W}(1)$ & $\operatorname{Br}(1)$ & $84,24(9)$ & $\mathrm{P}(3)$ & $\mathrm{W}(3)$ & $\mathrm{W}(1)$ & $135,42(8)$ \\
\hline $\mathrm{S}(1)$ & $\mathrm{W}(1)$ & $\mathrm{P}(1)$ & $161,90(12)$ & $\mathrm{P}(3)$ & $\mathrm{W}(3)$ & W(2) & $142,41(9)$ \\
\hline$S(4)$ & $\mathbb{W}(1)$ & $\mathrm{W}(3)$ & $53,22(8)$ & $\mathrm{P}(3)$ & $\mathrm{W}(3)$ & $\operatorname{Br}(3)$ & $84,85(9)$ \\
\hline$S(4)$ & $\mathrm{W}(1)$ & $\mathrm{W}(2)$ & $98,66(9)$ & $S(4)$ & $\mathrm{W}(3)$ & $\mathrm{W}(1)$ & $53,47(8)$ \\
\hline $\mathrm{S}(4)$ & $\mathrm{W}(1)$ & $\operatorname{Br}(1)$ & $90,85(10)$ & $\mathrm{S}(4)$ & $\mathrm{W}(3)$ & $\mathrm{W}(2)$ & $99,11(9)$ \\
\hline $\mathrm{S}(4)$ & $\mathrm{W}(1)$ & $\mathrm{S}(1)$ & $106,27(11)$ & $\mathrm{S}(4)$ & $\mathrm{W}(3)$ & $\operatorname{Br}(3)$ & $163,68(10)$ \\
\hline$S(4)$ & $\mathrm{W}(1)$ & $\mathrm{P}(1)$ & $87,14(11)$ & $C(42)$ & $\mathrm{P}(6)$ & $C(43)$ & $106,1(6)$ \\
\hline $\mathrm{S}(4)$ & $\mathrm{W}(1)$ & $\mathrm{S}(2)$ & $97,95(13)$ & $\mathrm{W}(6)$ & $\mathrm{S}(8)$ & $\mathrm{W}(4)$ & $73,51(9)$ \\
\hline $\mathrm{P}(1)$ & $\mathrm{W}(1)$ & $\mathrm{W}(3)$ & $140,35(8)$ & $\mathrm{W}(2)$ & $\mathrm{S}(3)$ & $\mathrm{W}(3)$ & $73,64(10)$ \\
\hline $\mathrm{P}(1)$ & $\mathrm{W}(1)$ & $\mathrm{W}(2)$ & $137,14(10)$ & $\mathrm{W}(5)$ & $S(7)$ & $\mathrm{W}(6)$ & $72,62(10)$ \\
\hline $\mathrm{P}(1)$ & $\mathrm{W}(1)$ & $\operatorname{Br}(1)$ & $83,45(11)$ & $\mathrm{W}(4)$ & $S(6)$ & W(5) & $73,35(9)$ \\
\hline$S(2)$ & $\mathrm{W}(1)$ & $\mathrm{W}(3)$ & $99,34(8)$ & $\mathrm{C}(38)$ & $\mathrm{P}(5)$ & $\mathrm{W}(5)$ & $119,8(6)$ \\
\hline$S(2)$ & $\mathrm{W}(1)$ & $\mathrm{W}(2)$ & $53,27(9)$ & $\mathrm{C}(38)$ & $\mathrm{P}(5)$ & $C(34)$ & $103,8(8)$ \\
\hline $\mathrm{S}(2)$ & $\mathrm{W}(1)$ & $\operatorname{Br}(1)$ & $164,17(9)$ & $C(38)$ & $\mathrm{P}(5)$ & $C(35)$ & $106,6(9)$ \\
\hline $\mathrm{S}(2)$ & $\mathrm{W}(1)$ & $\mathrm{S}(1)$ & $105,65(12)$ & C(34) & $\mathrm{P}(5)$ & W(5) & $99,7(5)$ \\
\hline $\mathrm{S}(2)$ & $\mathrm{W}(1)$ & $\mathrm{P}(1)$ & $83,88(14)$ & $C(34)$ & $\mathrm{P}(5)$ & $C(35)$ & $106,7(9)$ \\
\hline $\mathrm{N}(1)$ & $\mathrm{W}(1)$ & $\mathrm{W}(3)$ & $140,9(2)$ & $C(35)$ & $\mathrm{P}(5)$ & $\mathrm{W}(5)$ & $118,1(6)$ \\
\hline $\mathrm{N}(1)$ & $\mathrm{W}(1)$ & $\mathrm{W}(2)$ & $97,9(3)$ & $\mathrm{W}(3)$ & $\mathrm{S}(1)$ & $\mathrm{W}(1)$ & $70,97(9)$ \\
\hline $\mathrm{N}(1)$ & $\mathrm{W}(1)$ & $\operatorname{Br}(1)$ & $79,1(3)$ & $W(2)$ & $\mathrm{S}(1)$ & $\mathrm{W}(1)$ & $71,64(9)$ \\
\hline $\mathrm{N}(1)$ & $\mathrm{W}(1)$ & $S(1)$ & $86,4(2)$ & $\mathrm{W}(2)$ & $S(1)$ & $\mathrm{W}(3)$ & $71,38(9)$ \\
\hline $\mathrm{N}(1)$ & $\mathrm{W}(1)$ & $\mathrm{S}(4)$ & $163,0(3)$ & $C(22)$ & $\mathrm{P}(3)$ & $\mathrm{W}(3)$ & $117,7(5)$ \\
\hline $\mathrm{N}(1)$ & $\mathrm{W}(1)$ & $\mathrm{P}(1)$ & $78,2(3)$ & $C(18)$ & $\mathrm{P}(3)$ & $\mathrm{W}(3)$ & $99,7(6)$ \\
\hline $\mathrm{N}(1)$ & $\mathrm{W}(1)$ & $\mathrm{S}(2)$ & $89,1(3)$ & $C(18)$ & $\mathrm{P}(3)$ & $C(22)$ & $100,6(8)$ \\
\hline $\mathrm{W}(1)$ & $\mathrm{W}(3)$ & $\mathrm{W}(2)$ & $60,378(18)$ & $C(19)$ & $\mathrm{P}(3)$ & $\mathrm{W}(3)$ & $122,9(6)$ \\
\hline $\operatorname{Br}(3)$ & $\mathrm{W}(3)$ & $\mathrm{W}(1)$ & $137,79(5)$ & $C(19)$ & $\mathrm{P}(3)$ & $C(22)$ & $105,4(9)$ \\
\hline $\operatorname{Br}(3)$ & $\mathrm{W}(3)$ & $\mathrm{W}(2)$ & $97,21(5)$ & $C(19)$ & $\mathrm{P}(3)$ & $C(18)$ & $107,4(12)$ \\
\hline $\mathrm{S}(3)$ & $\mathrm{W}(3)$ & $\mathrm{W}(1)$ & $99,36(8)$ & W(5) & $\mathrm{S}(5)$ & $\mathrm{W}(6)$ & $71,71(9)$ \\
\hline $\mathrm{S}(3)$ & $\mathrm{W}(3)$ & $\mathrm{W}(2)$ & $52,98(8)$ & $\mathrm{W}(5)$ & $\mathrm{S}(5)$ & $\mathrm{W}(4)$ & $71,67(9)$ \\
\hline$S(3)$ & $\mathrm{W}(3)$ & $\operatorname{Br}(3)$ & $91,61(10)$ & $\mathrm{W}(4)$ & $\mathrm{S}(5)$ & $\mathrm{W}(6)$ & $71,88(9)$ \\
\hline $\mathrm{S}(3)$ & $\mathrm{W}(3)$ & $\mathrm{S}(1)$ & $105,57(12)$ & $\mathrm{W}(3)$ & $\mathrm{S}(4)$ & $\mathrm{W}(1)$ & $73,31(10)$ \\
\hline
\end{tabular}


Tabla 7.15. Ángulos de enlace representativos $\left(^{\circ}\right)$ (continuación).

\begin{tabular}{|c|c|c|c|c|c|c|c|}
\hline \multicolumn{3}{|c|}{ ÁtomoÁtomoÁtomo } & \multirow{2}{*}{$\begin{array}{c}\text { Ángulo } \\
98,6(5)\end{array}$} & \multicolumn{3}{|c|}{ ÁtomoÁtomo Átomo } & \multirow{2}{*}{$\begin{array}{l}\text { Ángulo } \\
53,17(8)\end{array}$} \\
\hline$C(2)$ & $\mathrm{P}(1)$ & $\mathrm{W}(1)$ & & $S(7)$ & $\mathrm{W}(6)$ & $\mathrm{W}(5)$ & \\
\hline$C(2)$ & $\mathrm{P}(1)$ & $C(6)$ & $102,0(9)$ & $S(7)$ & $\mathrm{W}(6)$ & $\mathrm{W}(4)$ & $98,75(8)$ \\
\hline$C(6)$ & $\mathrm{P}(1)$ & $\mathrm{W}(1)$ & $117,2(6)$ & $S(7)$ & $\mathrm{W}(6)$ & $\operatorname{Br}(6)$ & $92,86(10)$ \\
\hline$C(3)$ & $\mathrm{P}(1)$ & $\mathrm{W}(1)$ & $123,0(9)$ & $S(7)$ & $\mathrm{W}(6)$ & $\mathrm{P}(6)$ & $89,32(11)$ \\
\hline$C(3)$ & $\mathrm{P}(1)$ & $C(2)$ & $107,4(9)$ & $\mathrm{S}(7)$ & $\mathrm{W}(6)$ & $\mathrm{S}(8)$ & $97,32(12)$ \\
\hline$C(3)$ & $\mathrm{P}(1)$ & $\mathrm{C}(6)$ & $105,8(13)$ & $S(7)$ & $\mathrm{W}(6)$ & $S(5)$ & $105,78(12)$ \\
\hline $\mathrm{W}(1)$ & $\mathrm{S}(2)$ & $\mathrm{W}(2)$ & $73,52(10)$ & $S(5)$ & $\mathrm{W}(6)$ & $\mathrm{W}(5)$ & $54,14(9)$ \\
\hline$C(41)$ & $\mathrm{N}(6)$ & $\mathrm{W}(6)$ & $118,1(8)$ & $S(5)$ & $\mathrm{W}(6)$ & $\mathrm{W}(4)$ & $54,05(9)$ \\
\hline $\mathrm{C}(48)$ & $\mathrm{C}(46)$ & $\mathrm{P}(6)$ & $109,8(9)$ & $S(5)$ & $\mathrm{W}(6)$ & $\operatorname{Br}(6)$ & $84,34(10)$ \\
\hline $\mathrm{C}(47)$ & $\mathrm{C}(46)$ & $\mathrm{P}(6)$ & $114,4(10)$ & $S(5)$ & $\mathrm{W}(6)$ & $\mathrm{P}(6)$ & $158,81(11)$ \\
\hline C(45) & $C(43)$ & $\mathrm{P}(6)$ & $113,0(10)$ & $\mathrm{N}(6)$ & $\mathrm{W}(6)$ & $\mathrm{W}(5)$ & $139,8(3)$ \\
\hline$C(44)$ & $C(43)$ & $\mathrm{P}(6)$ & $113,9(11)$ & $\mathrm{N}(6)$ & $\mathrm{W}(6)$ & $\mathrm{W}(4)$ & $94,9(3)$ \\
\hline$C(23)$ & $\mathrm{C}(22)$ & $\mathrm{P}(3)$ & $114,0(10)$ & $\mathrm{N}(6)$ & $\mathrm{W}(6)$ & $\operatorname{Br}(6)$ & $79,8(3)$ \\
\hline$C(24)$ & $C(22)$ & $\mathrm{P}(3)$ & $111,4(11)$ & $\mathrm{N}(6)$ & $\mathrm{W}(6)$ & $\mathrm{P}(6)$ & $77,3(3)$ \\
\hline$S(4)$ & $\mathrm{W}(3)$ & $S(3)$ & $98,04(13)$ & $\mathrm{N}(6)$ & $\mathrm{W}(6)$ & $S(8)$ & $87,1(3)$ \\
\hline$S(4)$ & $\mathrm{W}(3)$ & $\mathrm{S}(1)$ & $106,55(11)$ & $\mathrm{N}(6)$ & $\mathrm{W}(6)$ & $S(7)$ & 165,6(3) \\
\hline$S(4)$ & $\mathrm{W}(3)$ & $\mathrm{P}(3)$ & $82,12(11)$ & $\mathrm{N}(6)$ & $\mathrm{W}(6)$ & $S(5)$ & $86,0(3)$ \\
\hline $\mathrm{N}(3)$ & $\mathrm{W}(3)$ & $\mathrm{W}(1)$ & $94,9(3)$ & $\mathrm{W}(4)$ & $\mathrm{W}(5)$ & $\mathrm{W}(6)$ & $60,155(19)$ \\
\hline $\mathrm{N}(3)$ & $\mathrm{W}(3)$ & $\mathrm{W}(2)$ & $140,7(3)$ & $\operatorname{Br}(5)$ & $\mathrm{W}(5)$ & $\mathrm{W}(6)$ & $137,86(5)$ \\
\hline $\mathrm{N}(3)$ & $\mathrm{W}(3)$ & $\operatorname{Br}(3)$ & $80,9(3)$ & $\operatorname{Br}(5)$ & W(5) & W(4) & $97,87(5)$ \\
\hline $\mathrm{N}(3)$ & $\mathrm{W}(3)$ & $\mathrm{S}(3)$ & $164,9(3)$ & $S(7)$ & $\mathrm{W}(5)$ & $\mathrm{W}(6)$ & $53,21(8)$ \\
\hline $\mathrm{N}(3)$ & $\mathrm{W}(3)$ & $\mathrm{S}(1)$ & $86,7(3)$ & $S(7)$ & $\mathrm{W}(5)$ & $\mathrm{W}(4)$ & $98,98(9)$ \\
\hline $\mathrm{N}(3)$ & $\mathrm{W}(3)$ & $\mathrm{P}(3)$ & $76,9(3)$ & $S(7)$ & W(5) & $\operatorname{Br}(5)$ & $163,13(10)$ \\
\hline $\mathrm{N}(3)$ & $\mathrm{W}(3)$ & $\mathrm{S}(4)$ & $86,6(3)$ & $S(7)$ & W(5) & $S(6)$ & $98,00(11)$ \\
\hline $\mathrm{W}(5)$ & $\mathrm{W}(6)$ & $\mathrm{W}(4)$ & $59,891(19)$ & $S(7)$ & W(5) & $\mathrm{P}(5)$ & $85,16(12)$ \\
\hline $\operatorname{Br}(6)$ & $\mathrm{W}(6)$ & $\mathrm{W}(5)$ & $98,47(5)$ & $\mathrm{S}(7)$ & $\mathrm{W}(5)$ & $S(5)$ & $105,83(11)$ \\
\hline $\operatorname{Br}(6)$ & $\mathrm{W}(6)$ & $\mathrm{W}(4)$ & $138,39(5)$ & $S(6)$ & $\mathrm{W}(5)$ & $\mathrm{W}(6)$ & $99,21(8)$ \\
\hline $\mathrm{P}(6)$ & $\mathrm{W}(6)$ & $\mathrm{W}(5)$ & $142,47(8)$ & $C(40)$ & $\mathrm{C}(38)$ & $\mathrm{P}(5)$ & $114,3(11)$ \\
\hline $\mathrm{P}(6)$ & $\mathrm{W}(6)$ & $\mathrm{W}(4)$ & $139,43(8)$ & $C(39)$ & $C(38)$ & $\mathrm{P}(5)$ & $117,3(14)$ \\
\hline $\mathrm{P}(6)$ & $\mathrm{W}(6)$ & $\operatorname{Br}(6)$ & $80,06(9)$ & $C(10)$ & $\mathrm{P}(2)$ & $\mathrm{W}(2)$ & $100,4(5)$ \\
\hline $\mathrm{S}(8)$ & $\mathrm{W}(6)$ & $\mathrm{W}(5)$ & $98,70(8)$ & $C(10)$ & $\mathrm{P}(2)$ & $\mathrm{C}(11)$ & $103,2(9)$ \\
\hline$S(8)$ & $\mathrm{W}(6)$ & $\mathrm{W}(4)$ & $53,39(8)$ & $C(10)$ & $\mathrm{P}(2)$ & $C(14)$ & $104,3(9)$ \\
\hline$S(8)$ & $\mathrm{W}(6)$ & $\operatorname{Br}(6)$ & $162,83(9)$ & $C(11)$ & $\mathrm{P}(2)$ & $\mathrm{W}(2)$ & $117,3(6)$ \\
\hline$S(8)$ & $\mathrm{W}(6)$ & $\mathrm{P}(6)$ & $86,24(11)$ & $C(11)$ & $\mathrm{P}(2)$ & $C(14)$ & $109,2(8)$ \\
\hline $\mathrm{S}(8)$ & $\mathrm{W}(6)$ & $\mathrm{S}(5)$ & $105,97(12)$ & $C(14)$ & $\mathrm{P}(2)$ & W(2) & $119,7(5)$ \\
\hline
\end{tabular}


Tabla 7.15. Ángulos de enlace representativos $\left(^{\circ}\right)$ (continuación).

\begin{tabular}{|c|c|c|c|c|c|c|c|}
\hline \multicolumn{3}{|c|}{ Átomo Átomo Átomo } & \multirow{2}{*}{$\begin{array}{l}\text { Ángulo } \\
101,3(6)\end{array}$} & \multicolumn{3}{|c|}{ Átomo Átomo Átomo } & \multirow{2}{*}{$\begin{array}{l}\text { Ángulo } \\
98,53(8)\end{array}$} \\
\hline$C(26)$ & $\mathrm{P}(4)$ & $\mathrm{W}(4)$ & & $\mathrm{S}(8)$ & $\mathrm{W}(4)$ & $\mathrm{W}(5)$ & \\
\hline$C(26)$ & $\mathrm{P}(4)$ & $C(27)$ & $97,6(9)$ & $\mathrm{S}(8)$ & $\mathrm{W}(4)$ & $\operatorname{Br}(4)$ & $92,51(10)$ \\
\hline $\mathrm{C}(30)$ & $\mathrm{P}(4)$ & $\mathrm{W}(4)$ & $119,9(9)$ & $\mathrm{S}(8)$ & $\mathrm{W}(4)$ & $S(5)$ & $105,69(11)$ \\
\hline $\mathrm{C}(30)$ & $\mathrm{P}(4)$ & $C(26)$ & $106,4(10)$ & $\mathrm{S}(8)$ & $\mathrm{W}(4)$ & $\mathrm{P}(4)$ & $89,70(12)$ \\
\hline $\mathrm{C}(30)$ & $\mathrm{P}(4)$ & $\mathrm{C}(27)$ & $113,0(13)$ & $S(6)$ & $\mathrm{W}(4)$ & $\mathrm{W}(6)$ & $99,12(8)$ \\
\hline C(24) & $\mathrm{P}(4)$ & $\mathrm{W}(4)$ & $114,7(6)$ & $S(6)$ & $\mathrm{W}(4)$ & W(5) & $53,37(8)$ \\
\hline$C(1)$ & $\mathrm{N}(1)$ & $\mathrm{W}(1)$ & $118,2(8)$ & $S(6)$ & $\mathrm{W}(4)$ & $\operatorname{Br}(4)$ & $164,14(11)$ \\
\hline$C(9)$ & $\mathrm{N}(2)$ & $\mathrm{W}(2)$ & $116,9(9)$ & $\mathrm{S}(6)$ & $\mathrm{W}(4)$ & $S(8)$ & $97,61(11)$ \\
\hline$C(25)$ & $\mathrm{N}(4)$ & $\mathrm{W}(4)$ & $115,3(9)$ & $S(6)$ & $\mathrm{W}(4)$ & $S(5)$ & $105,95(12)$ \\
\hline$C(26)$ & $C(25)$ & $\mathrm{N}(4)$ & $115,9(14)$ & $\mathrm{S}(6)$ & $\mathrm{W}(4)$ & $\mathrm{P}(4)$ & $86,76(13)$ \\
\hline$C(2)$ & $\mathrm{C}(1)$ & $\mathrm{N}(1)$ & $110,8(11)$ & $\mathrm{S}(5)$ & $\mathrm{W}(4)$ & $\mathrm{W}(6)$ & $54,07(8)$ \\
\hline$C(17)$ & $\mathrm{N}(3)$ & $\mathrm{W}(3)$ & $120,2(11)$ & $\mathrm{S}(5)$ & $\mathrm{W}(4)$ & $\mathrm{W}(5)$ & $54,16(9)$ \\
\hline$C(33)$ & $\mathrm{N}(5)$ & $\mathrm{W}(5)$ & $116,7(8)$ & $\mathrm{S}(5)$ & $\mathrm{W}(4)$ & $\operatorname{Br}(4)$ & $82,82(12)$ \\
\hline$S(6)$ & $\mathrm{W}(5)$ & $\mathrm{W}(4)$ & $53,28(8)$ & $\mathrm{S}(5)$ & $\mathrm{W}(4)$ & $\mathrm{P}(4)$ & $158,17(13)$ \\
\hline$S(6)$ & $\mathrm{W}(5)$ & $\operatorname{Br}(5)$ & $92,51(9)$ & $\mathrm{P}(4)$ & $\mathrm{W}(4)$ & $\mathrm{W}(6)$ & $142,73(9)$ \\
\hline$S(6)$ & $\mathrm{W}(5)$ & $\mathrm{P}(5)$ & $88,76(12)$ & $\mathrm{P}(4)$ & $\mathrm{W}(4)$ & W(5) & $139,93(10)$ \\
\hline $\mathrm{S}(6)$ & $\mathrm{W}(5)$ & $S(5)$ & $105,87(13)$ & $\mathrm{P}(4)$ & $\mathrm{W}(4)$ & $\operatorname{Br}(4)$ & $81,09(13)$ \\
\hline $\mathrm{P}(5)$ & $\mathrm{W}(5)$ & $\mathrm{W}(6)$ & $138,23(9)$ & $\mathrm{N}(4)$ & $\mathrm{W}(4)$ & $\mathrm{W}(6)$ & $139,2(3)$ \\
\hline $\mathrm{P}(5)$ & $\mathrm{W}(5)$ & $\mathrm{W}(4)$ & $142,04(9)$ & $\mathrm{N}(4)$ & $\mathrm{W}(4)$ & $\mathrm{W}(5)$ & $94,9(4)$ \\
\hline $\mathrm{P}(5)$ & $\mathrm{W}(5)$ & $\operatorname{Br}(5)$ & $81,90(10)$ & $\mathrm{N}(4)$ & $\mathrm{W}(4)$ & $\operatorname{Br}(4)$ & $80,2(3)$ \\
\hline $\mathrm{S}(5)$ & $\mathrm{W}(5)$ & $\mathrm{W}(6)$ & $54,15(8)$ & $\mathrm{N}(4)$ & $\mathrm{W}(4)$ & $\mathrm{S}(8)$ & $166,0(4)$ \\
\hline $\mathrm{S}(5)$ & $\mathrm{W}(5)$ & W(4) & $54,17(9)$ & $\mathrm{N}(4)$ & $\mathrm{W}(4)$ & $S(6)$ & $87,3(3)$ \\
\hline$S(5)$ & $\mathrm{W}(5)$ & $\operatorname{Br}(5)$ & $83,71(9)$ & $\mathrm{N}(4)$ & $\mathrm{W}(4)$ & $S(5)$ & $85,4(3)$ \\
\hline$S(5)$ & $\mathrm{W}(5)$ & $\mathrm{P}(5)$ & $159,90(12)$ & $\mathrm{N}(4)$ & $\mathrm{W}(4)$ & $\mathrm{P}(4)$ & $77,4(4)$ \\
\hline $\mathrm{N}(5)$ & $\mathrm{W}(5)$ & $\mathrm{W}(6)$ & $95,0(3)$ & $\mathrm{W}(3)$ & $\mathrm{W}(2)$ & $\mathrm{W}(1)$ & $59,585(18)$ \\
\hline $\mathrm{N}(5)$ & $\mathrm{W}(5)$ & $\mathrm{W}(4)$ & $139,6(3)$ & $\operatorname{Br}(2)$ & $\mathrm{W}(2)$ & $\mathrm{W}(1)$ & $99,52(4)$ \\
\hline $\mathrm{N}(5)$ & $\mathrm{W}(5)$ & $\operatorname{Br}(5)$ & $79,4(3)$ & $C(34)$ & $C(33)$ & $\mathrm{N}(5)$ & $107,4(13)$ \\
\hline $\mathrm{N}(5)$ & $\mathrm{W}(5)$ & $S(7)$ & $87,4(3)$ & $\mathrm{N}(2)$ & $\mathrm{C}(9)$ & $C(10)$ & $108,4(11)$ \\
\hline $\mathrm{N}(5)$ & $\mathrm{W}(5)$ & $\mathrm{S}(6)$ & $165,3(3)$ & $C(9)$ & $C(10)$ & $\mathrm{P}(2)$ & $111,0(11)$ \\
\hline $\mathrm{N}(5)$ & $\mathrm{W}(5)$ & $\mathrm{P}(5)$ & $78,0(3)$ & $C(13)$ & $C(11)$ & $\mathrm{P}(2)$ & $112,3(13)$ \\
\hline $\mathrm{N}(5)$ & $\mathrm{W}(5)$ & $S(5)$ & $85,6(3)$ & $C(12)$ & $\mathrm{C}(11)$ & $\mathrm{P}(2)$ & $117,6(15)$ \\
\hline $\mathrm{W}(5)$ & $\mathrm{W}(4)$ & $\mathrm{W}(6)$ & $59,954(19)$ & $C(33)$ & $C(34)$ & $\mathrm{P}(5)$ & $111,6(11)$ \\
\hline $\operatorname{Br}(4)$ & $\mathrm{W}(4)$ & $\mathrm{W}(6)$ & $96,73(7)$ & $\mathrm{N}(6)$ & $\mathrm{C}(41)$ & C(42) & 108,6(11) \\
\hline $\operatorname{Br}(4)$ & $\mathrm{W}(4)$ & $\mathrm{W}(5)$ & $136,99(9)$ & $C(41)$ & $C(42)$ & $\mathrm{P}(6)$ & $109,7(9)$ \\
\hline$S(8)$ & $\mathrm{W}(4)$ & $\mathrm{W}(6)$ & $53,10(8)$ & $\mathrm{C}(1)$ & $C(2)$ & $\mathrm{P}(1)$ & $111,8(11)$ \\
\hline
\end{tabular}


Tabla 7.15. Ángulos de enlace representativos ( $\left(^{\circ}\right)$ (continuación).

\begin{tabular}{cccccccc}
\hline \multicolumn{1}{c}{ ÁtomoÁtomoÁtomo } & \multicolumn{2}{c}{ Ángulo } & \multicolumn{3}{c}{ ÁtomoÁtomoÁtomo } & Ángulo \\
\hline $\mathrm{C}(7)$ & $\mathrm{C}(6)$ & $\mathrm{P}(1)$ & $116,4(15)$ & $\mathrm{N}(2)$ & $\mathrm{W}(2)$ & $\mathrm{Br}(2)$ & $78,7(3)$ \\
$\mathrm{C}(8)$ & $\mathrm{C}(6)$ & $\mathrm{P}(1)$ & $108,0(16)$ & $\mathrm{N}(2)$ & $\mathrm{W}(2)$ & $\mathrm{S}(3)$ & $86,9(3)$ \\
$\mathrm{C}(15)$ & $\mathrm{C}(14)$ & $\mathrm{P}(2)$ & $113,1(13)$ & $\mathrm{N}(2)$ & $\mathrm{W}(2)$ & $\mathrm{S}(1)$ & $85,5(3)$ \\
$\mathrm{Br}(2)$ & $\mathrm{W}(2)$ & $\mathrm{W}(3)$ & $139,02(5)$ & $\mathrm{N}(2)$ & $\mathrm{W}(2)$ & $\mathrm{S}(2)$ & $165,5(3)$ \\
$\mathrm{S}(3)$ & $\mathrm{W}(2)$ & $\mathrm{W}(1)$ & $99,03(9)$ & $\mathrm{N}(2)$ & $\mathrm{W}(2)$ & $\mathrm{P}(2)$ & $78,1(3)$ \\
$\mathrm{S}(3)$ & $\mathrm{W}(2)$ & $\mathrm{W}(3)$ & $53,38(9)$ & $\mathrm{C}(46)$ & $\mathrm{P}(6)$ & $\mathrm{W}(6)$ & $116,0(4)$ \\
$\mathrm{S}(3)$ & $\mathrm{W}(2)$ & $\mathrm{Br}(2)$ & $161,44(10)$ & $\mathrm{C}(43)$ & $\mathrm{P}(6)$ & $\mathrm{W}(6)$ & $124,2(5)$ \\
$\mathrm{S}(3)$ & $\mathrm{W}(2)$ & $\mathrm{S}(1)$ & $105,97(12)$ & $\mathrm{C}(43)$ & $\mathrm{P}(6)$ & $\mathrm{C}(46)$ & $103,4(6)$ \\
$\mathrm{S}(3)$ & $\mathrm{W}(2)$ & $\mathrm{S}(2)$ & $98,69(13)$ & $\mathrm{C}(42)$ & $\mathrm{P}(6)$ & $\mathrm{W}(6)$ & $100,5(4)$ \\
$\mathrm{S}(3)$ & $\mathrm{W}(2)$ & $\mathrm{P}(2)$ & $83,95(12)$ & $\mathrm{C}(42)$ & $\mathrm{P}(6)$ & $\mathrm{C}(46)$ & $104,7(7)$ \\
$\mathrm{S}(1)$ & $\mathrm{W}(2)$ & $\mathrm{W}(1)$ & $54,21(8)$ & $\mathrm{C}(16)$ & $\mathrm{C}(14)$ & $\mathrm{P}(2)$ & $113,7(14)$ \\
$\mathrm{S}(1)$ & $\mathrm{W}(2)$ & $\mathrm{W}(3)$ & $54,32(8)$ & $\mathrm{C}(25)$ & $\mathrm{C}(26)$ & $\mathrm{P}(4)$ & $112,0(12)$ \\
$\mathrm{S}(1)$ & $\mathrm{W}(2)$ & $\mathrm{Br}(2)$ & $84,72(9)$ & $\mathrm{C}(5)$ & $\mathrm{C}(3)$ & $\mathrm{P}(1)$ & $115,9(13)$ \\
$\mathrm{S}(1)$ & $\mathrm{W}(2)$ & $\mathrm{P}(2)$ & $160,36(14)$ & $\mathrm{C}(4)$ & $\mathrm{C}(3)$ & $\mathrm{P}(1)$ & $120,4(18)$ \\
$\mathrm{S}(2)$ & $\mathrm{W}(2)$ & $\mathrm{W}(1)$ & $53,21(9)$ & $\mathrm{C}(17)$ & $\mathrm{C}(18)$ & $\mathrm{P}(3)$ & $118,4(14)$ \\
$\mathrm{S}(2)$ & $\mathrm{W}(2)$ & $\mathrm{W}(3)$ & $98,94(9)$ & $\mathrm{C}(37)$ & $\mathrm{C}(35)$ & $\mathrm{P}(5)$ & $114,3(13)$ \\
$\mathrm{S}(2)$ & $\mathrm{W}(2)$ & $\mathrm{Br}(2)$ & $92,75(10)$ & $\mathrm{C}(36)$ & $\mathrm{C}(35)$ & $\mathrm{P}(5)$ & $115(2)$ \\
$\mathrm{S}(2)$ & $\mathrm{W}(2)$ & $\mathrm{S}(1)$ & $105,65(12)$ & $\mathrm{C}(20)$ & $\mathrm{C}(19)$ & $\mathrm{P}(3)$ & $110,3(15)$ \\
$\mathrm{S}(2)$ & $\mathrm{W}(2)$ & $\mathrm{P}(2)$ & $89,12(14)$ & $\mathrm{C}(21)$ & $\mathrm{C}(19)$ & $\mathrm{P}(3)$ & $119(2)$ \\
$\mathrm{P}(2)$ & $\mathrm{W}(2)$ & $\mathrm{W}(1)$ & $142,31(11)$ & $\mathrm{C}(18)$ & $\mathrm{C}(17)$ & $\mathrm{N}(3)$ & $123(2)$ \\
$\mathrm{P}(2)$ & $\mathrm{W}(2)$ & $\mathrm{W}(3)$ & $137,24(9)$ & $\mathrm{C}(32)$ & $\mathrm{C}(30)$ & $\mathrm{P}(4)$ & $114,0(17)$ \\
$\mathrm{P}(2)$ & $\mathrm{W}(2)$ & $\mathrm{Br}(2)$ & $81,65(10)$ & $\mathrm{C}(31)$ & $\mathrm{C}(30)$ & $\mathrm{P}(4)$ & $121,2(1)$ \\
$\mathrm{N}(2)$ & $\mathrm{W}(2)$ & $\mathrm{W}(1)$ & $139,4(3)$ & $\mathrm{C}(28)$ & $\mathrm{C}(27)$ & $\mathrm{P}(4)$ & $109,8(14)$ \\
$\mathrm{N}(2)$ & $\mathrm{W}(2)$ & $\mathrm{W}(3)$ & $95,2(3)$ & $\mathrm{C}(29)$ & $\mathrm{C}(27)$ & $\mathrm{P}(4)$ & $115,4(16)$ \\
\hline
\end{tabular}




\subsubsection{ESTRUCTURA DE $(P)-\left[\mathrm{Mo}_{3} \mathrm{~S}_{4} \mathrm{Cl}_{3}\left((1 S, 2 R)-\mathrm{PPro}_{3}\right] \mathrm{BF}_{4}\left((P)-7\left(\mathrm{BF}_{4}\right)\right)\right.$}

La obtención de cristales adecuados para la determinación estructural del compuesto $(P)-7\left(\mathrm{BF}_{4}\right)$ se ha llevado a cabo mediante difusión lenta de tolueno sobre una disolución concentrada del clúster en diclorometano. El cambio de contraión de $\mathrm{Cl}^{-}$ a $\mathrm{BF}_{4}{ }^{-}$se ha realizado por elución con una disolución saturada de $\mathrm{KBF}_{4}$ en acetona, tras la absorción de una disolución del clúster en diclorometano en una columna cromatográfica de gel de sílice. El refino se ha realizado con éxito en el grupo espacial no centrosimétrico P2 13 , que presenta un eje ternario a través de la unidad $\mathrm{Mo}_{3} \mathrm{~S}_{4}$, obteniéndose de forma inequívoca el enantiómero con configuración absoluta $P$. Todos los átomos del clúster se han refinado anisotrópicamente, excepto los átomos de hidrógeno que se han generado en posiciones geométricas.

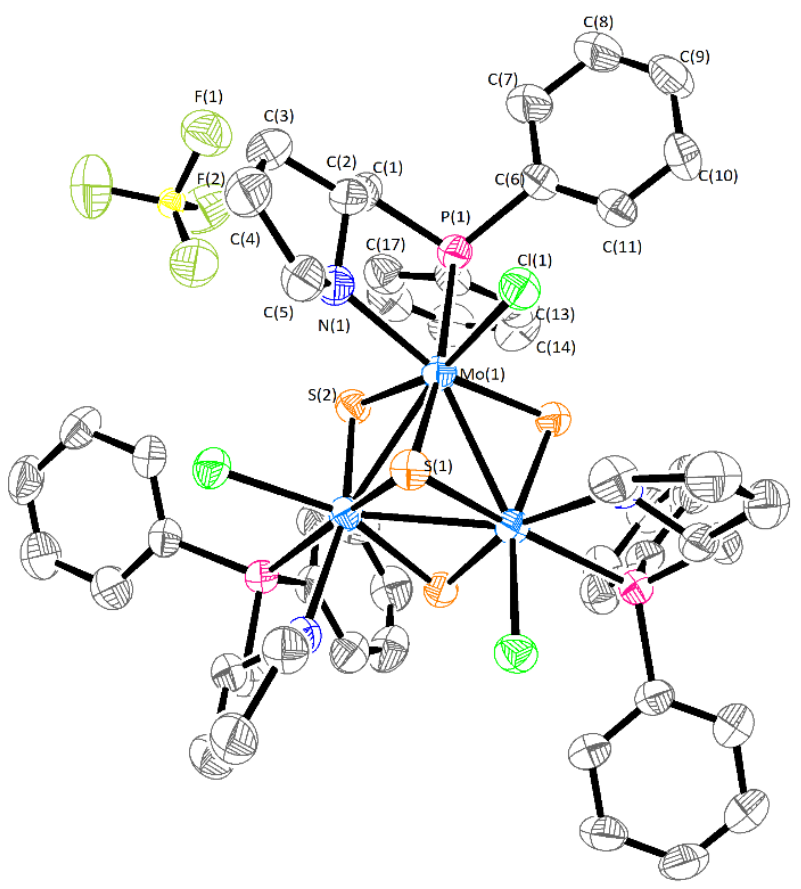

Figura 7.6. Representación ORTEP del clúster $(P)-\left[\mathrm{Mo}_{3} \mathrm{~S}_{4} \mathrm{Cl}_{3}((1 S, 2 \mathrm{R})-\mathrm{PPro})_{3}\right] \mathrm{BF}_{4}$ (elipsoides al 50\% de probabilidad). 
Tabla 7.16. Parámetros cristalográficos, condiciones de registro y refino.

Datos cristalográficos

Especie

Fórmula

Grupo espacial
$\mathbf{a}(\AA)$
b $(\AA)$
c $(\AA)$
$\mathbf{Z}$

Color

$\mathbf{d}_{\text {calc }}\left(\mathrm{mg} / \mathrm{mm}^{3}\right)$

Coeficiente de

absorción $\left(\mathrm{mm}^{-1}\right)$
$\left[\mathrm{Mo}_{3} \mathrm{~S}_{4} \mathrm{Cl}_{3}((1 \mathrm{~S}, 2 \mathrm{R})-\mathrm{PPro})_{3}\right] \mathrm{BF}_{4}$

$\mathrm{C}_{51} \mathrm{H}_{60} \mathrm{BCl}_{3} \mathrm{~F}_{4} \mathrm{Mo}_{3} \mathrm{~N}_{3} \mathrm{P}_{3} \mathrm{~S}_{4}$ Sistema cristalino Peso 1417,15 molecular $\boldsymbol{\alpha}\left(^{\circ}\right) \quad 90,00$ $\boldsymbol{\beta}\left({ }^{\circ}\right) \quad 90,00$ $\boldsymbol{\gamma}\left(^{\circ}\right) \quad 90,00$ Vol $\left(\AA^{3}\right) \quad$ 6938,1(2) Tamaño del $\quad 0,1421 \times 0,097 \times$ cristal (mm) 0,0933 $\lambda(\AA) \quad 0,71073$ F (000) 2856

Parámetros experimentales

Temperatura (K) 200,00(10)

Tiempo por marco 116 (s)

Límites $2 \boldsymbol{\theta}\left(^{\circ}\right) \quad 6,42$ y 49,97

$\mathbf{N}^{\mathrm{o}}$ de reflexiones 14383 registradas

$\begin{array}{ll}\mathbf{R}(\text { int) } & 0,0309 \\ \mathbf{R}(\boldsymbol{\sigma}) & 0,0380\end{array}$

Límites h, k, $1 \quad-22 \leq \mathrm{h} \leq 11$ (mín/máx) $\quad-22-\leq \mathrm{k} \leq 16$ $-25-\leq 1 \leq 23$ $N^{o}$ de reflexiones 3964 independientes

Resolución y refino

$\begin{array}{llll}\mathbf{N}^{\mathbf{o}} \text { de variables } & 217 & \text { GooF en } \mathbf{F}^{2} & 1,158 \\ \mathbf{N}^{\mathbf{0}} \text { de restricciones } & 0 & & \\ \text { R1 final }([\mathbf{I}>2 \text { sigma(I) }]) & 0,0425 & \text { R1 (todos los datos) } & 0,0484 \\ \text { wR2 final }([\mathbf{I}>\text { 2sigma(I)]) } & 0,1469 & \text { wR2 (todos los datos) } & 0,1535 \\ \begin{array}{l}\text { Densidad electrónica } \\ \text { residual }\left(\mathrm{e} \cdot \AA^{-3}\right)\end{array} & 0,91 /-0,45 & \text { Max. desplazam./ } \boldsymbol{\sigma} & 0,000 \\ \text { Parámetro de Flack } & -0,07(2) & & \end{array}$

$$
R 1=\sum|| F_{0}|-| F_{1}|| / \sum F_{0} \quad w R 2=\left[\sum\left[w\left(F_{0}^{2}-F_{c}^{2}\right)^{2}\right] / \sum\left[w\left(F_{0}^{2}\right)^{2}\right]\right]^{1 / 2}
$$


Tabla 7.17. Distancias de enlace representativas $(\AA)$.

\begin{tabular}{cccccc}
\hline Átomo Átomo & Distancia & \multicolumn{2}{c}{ Átomo Átomo } & Distancia \\
\hline $\operatorname{Mo}(1)$ & $\operatorname{Mo}(1)^{\mathrm{A}}$ & $2,7522(13)$ & $\mathrm{Mo}(1)$ & $\mathrm{S}(2)^{\mathrm{A}}$ & $2,277(2)$ \\
$\operatorname{Mo}(1)$ & $\operatorname{Mo}(1)^{\mathrm{B}}$ & $2,7522(13)$ & $\mathrm{Mo}(1)$ & $\mathrm{S}(2)^{\mathrm{B}}$ & $2,277(2)$ \\
$\operatorname{Mo}(1)$ & $\mathrm{S}(1)$ & $2,363(3)$ & $\mathrm{Mo}(1)$ & $\mathrm{P}(1)$ & $2,547(2)$ \\
$\operatorname{Mo}(1)^{\mathrm{A}}$ & $\mathrm{S}(1)$ & $2,363(3)$ & $\mathrm{Mo}(1)$ & $\mathrm{N}(1)$ & $2,302(8)$ \\
$\operatorname{Mo}(1)^{\mathrm{B}}$ & $\mathrm{S}(1)$ & $2,363(3)$ & $\mathrm{Mo}(1)$ & $\mathrm{Cl}(1)$ & $2,461(2)$ \\
$\operatorname{Mo}(1)$ & $\mathrm{S}(2)$ & $2,297(2)$ & & & \\
\hline
\end{tabular}

Transformaciones de simetría utilizadas para generar átomos cristalográficamente equivalentes:

$A=1 / 2-X,-Y, 1 / 2+Z ; B=-1 / 2+X, 1 / 2-Y,-Z$ 
Tabla 7.18. Ángulos de enlace representativos $\left({ }^{\circ}\right)$.

\begin{tabular}{|c|c|c|c|c|c|c|}
\hline \multicolumn{2}{|c|}{ ÁtomoÁtomoÁtomo } & \multirow{2}{*}{$\begin{array}{c}\text { Ángulo } \\
60,0\end{array}$} & \multicolumn{3}{|c|}{ ÁtomoÁtomoÁtomo } & \multirow{2}{*}{$\begin{array}{c}\text { Ángulo } \\
77,9(2)\end{array}$} \\
\hline $\operatorname{Mo}(1)^{A}$ & $\operatorname{Mo}(1) \operatorname{Mo}(1)^{B}$ & & $\mathrm{~N}(1)$ & $\mathrm{Mo}(1)$ & $\mathrm{P}(1)$ & \\
\hline $\mathrm{P}(1)$ & $\operatorname{Mo}(1) \operatorname{Mo}(1)^{B}$ & $137,51(6)$ & $\mathrm{N}(1)$ & $\operatorname{Mo}(1)$ & $S(1)$ & $90,5(2)$ \\
\hline $\mathrm{P}(1)$ & $\operatorname{Mo}(1) \operatorname{Mo}(1)^{A}$ & $131,58(6)$ & $\mathrm{N}(1)$ & $\operatorname{Mo}(1)$ & $\mathrm{Cl}(1)$ & $79,7(2)$ \\
\hline$S(1)$ & $\operatorname{Mo}(1) \operatorname{Mo}(1)^{B}$ & $54,39(5)$ & $C(6)$ & $\mathrm{P}(1)$ & $\operatorname{Mo}(1)$ & $122,2(3)$ \\
\hline$S(1)$ & $\operatorname{Mo}(1) \operatorname{Mo}(1)^{A}$ & $54,39(5)$ & $C(6)$ & $\mathrm{P}(1)$ & $\mathrm{C}(1)$ & $106,4(5)$ \\
\hline$S(1)$ & $\operatorname{Mo}(1) \quad \mathrm{P}(1)$ & $167,32(9)$ & $C(12)$ & $\mathrm{P}(1)$ & $\operatorname{Mo}(1)$ & $115,3(3)$ \\
\hline$S(1)$ & $\mathrm{Mo}(1) \quad \mathrm{Cl}(1)$ & $86,98(8)$ & $\mathrm{C}(12)$ & $\mathrm{P}(1)$ & $C(6)$ & $102,3(4)$ \\
\hline $\mathrm{S}(2)^{\mathrm{B}}$ & $\operatorname{Mo}(1) \operatorname{Mo}(1)^{A}$ & $98,25(6)$ & $C(12)$ & $\mathrm{P}(1)$ & $C(1)$ & $108,6(5)$ \\
\hline $\mathrm{S}(2)$ & $\operatorname{Mo}(1) \operatorname{Mo}(1)^{A}$ & $52,68(6)$ & $\mathrm{C}(1)$ & $\mathrm{P}(1)$ & $\operatorname{Mo}(1)$ & $101,2(4)$ \\
\hline $\mathrm{S}(2)$ & $\operatorname{Mo}(1) \operatorname{Mo}(1)^{\mathrm{B}}$ & $97,77(6)$ & $C(7)$ & $\mathrm{C}(6)$ & $\mathrm{P}(1)$ & $119,4(8)$ \\
\hline $\mathrm{S}(2)^{\mathrm{B}}$ & $\operatorname{Mo}(1) \operatorname{Mo}(1)^{\mathrm{B}}$ & $53,33(6)$ & $\mathrm{C}(11)$ & $C(6)$ & $\mathrm{P}(1)$ & $120,4(7)$ \\
\hline $\mathrm{S}(2)^{\mathrm{B}}$ & $\mathrm{Mo}(1) \quad \mathrm{P}(1)$ & $84,59(8)$ & $C(13)$ & $C(12)$ & $\mathrm{P}(1)$ & $119,3(8)$ \\
\hline $\mathrm{S}(2)$ & $\operatorname{Mo}(1) \quad \mathrm{P}(1)$ & $78,91(8)$ & $\mathrm{C}(17)$ & $\mathrm{C}(12)$ & $\mathrm{P}(1)$ & $121,8(8)$ \\
\hline $\mathrm{S}(2)^{\mathrm{B}}$ & $\mathrm{Mo}(1) \quad \mathrm{S}(1)$ & $106,37(7)$ & $\operatorname{Mo}(1)^{\mathrm{A}}$ & $\mathrm{S}(1)$ & $\operatorname{Mo}(1)^{\mathrm{B}}$ & $71,22(11)$ \\
\hline $\mathrm{S}(2)$ & $\operatorname{Mo}(1) \quad \mathrm{S}(1)$ & $105,73(8)$ & $\operatorname{Mo}(1)^{\mathrm{A}}$ & $\mathrm{S}(1)$ & $\operatorname{Mo}(1)$ & $71,22(11)$ \\
\hline $\mathrm{S}(2)^{\mathrm{B}}$ & $\mathrm{Mo}(1) \quad \mathrm{S}(2)$ & $95,65(12)$ & $\operatorname{Mo}(1)^{\mathrm{B}}$ & $S(1)$ & $\operatorname{Mo}(1)$ & $71,22(11)$ \\
\hline $\mathrm{S}(2)$ & $\operatorname{Mo}(1) \quad \mathrm{Cl}(1)$ & $161,39(9)$ & $\operatorname{Mo}(1)^{\mathrm{A}}$ & $S(2)$ & $\operatorname{Mo}(1)$ & $73,99(8)$ \\
\hline $\mathrm{S}(2)^{\mathrm{B}}$ & $\mathrm{Mo}(1) \quad \mathrm{Cl}(1)$ & $93,63(9)$ & $C(5)$ & $\mathrm{N}(1)$ & $\operatorname{Mo}(1)$ & $124,2(7)$ \\
\hline $\mathrm{S}(2)$ & $\operatorname{Mo}(1) \quad \mathrm{N}(1)$ & $86,5(2)$ & $C(5)$ & $\mathrm{N}(1)$ & $C(2)$ & $104,5(7)$ \\
\hline $\mathrm{S}(2)^{\mathrm{B}}$ & $\operatorname{Mo}(1) \quad \mathrm{N}(1)$ & $161,6(2)$ & $C(2)$ & $\mathrm{N}(1)$ & $\operatorname{Mo}(1)$ & $113,4(6)$ \\
\hline $\mathrm{Cl}(1)$ & $\operatorname{Mo}(1) \operatorname{Mo}(1)^{B}$ & $100,71(7)$ & $\mathrm{N}(1)$ & $C(5)$ & $\mathrm{C}(4)$ & $104,4(9)$ \\
\hline $\mathrm{Cl}(1)$ & $\operatorname{Mo}(1) \operatorname{Mo}(1)^{A}$ & $141,34(6)$ & $C(2)$ & $\mathrm{C}(1)$ & $\mathrm{P}(1)$ & $106,3(7)$ \\
\hline $\mathrm{Cl}(1)$ & $\mathrm{Mo}(1) \quad \mathrm{P}(1)$ & $85,97(8)$ & $\mathrm{N}(1)$ & $C(2)$ & $C(1)$ & $112,0(8)$ \\
\hline $\mathrm{N}(1)$ & $\operatorname{Mo}(1) \operatorname{Mo}(1)^{A}$ & $97,5(2)$ & $\mathrm{N}(1)$ & $C(2)$ & $C(3)$ & $103,3(8)$ \\
\hline $\mathrm{N}(1)$ & $\operatorname{Mo}(1) \operatorname{Mo}(1)^{\mathrm{B}}$ & $144,5(2)$ & & & & \\
\hline
\end{tabular}

Transformaciones de simetría utilizadas para generar átomos cristalográficamente equivalentes:

$A=-1 / 2+X, 1 / 2-Y,-Z ; B=1 / 2-X,-Y, 1 / 2+Z$ 


\subsubsection{ESTRUCTURA DE (P)-[$\left[\mathrm{Mo}_{3} \mathrm{~S}_{4} \mathrm{Cl}_{3}\left((1 S, 2 S)-\mathrm{PPro}_{3}\right] \mathrm{BF}_{4} \cdot 3 \mathrm{C}_{7} \mathrm{H}_{8}((P)-\right.$ $\left.8\left(\mathrm{BF}_{4}\right) \cdot 3 \mathrm{C}_{7} \mathrm{H}_{8}\right)$}

De forma análoga al clúster anterior, los cristales del complejo $(P)-8\left(\mathrm{BF}_{4}\right)$ se han obtenido por difusión lenta de tolueno sobre una disolución del clúster en diclorometano. El cambio de contraión de $\mathrm{Cl}^{-}$a $\mathrm{BF}_{4}^{-}$se ha realizado asimismo por elución de una disolución saturada de $\mathrm{KBF}_{4}$ en acetona, tras la absorción de una disolución del clúster en diclorometano en una columna cromatográfica de gel de sílice. El refino se ha realizado en el grupo espacial no centrosimétrico P2 13 , que presenta un eje ternario a través de la unidad $\mathrm{Mo}_{3} \mathrm{~S}_{4}$, obteniéndose de forma inequívoca el enantiómero con configuración absoluta $P$. Todos los átomos del clúster se han refinado de forma anisotrópica, excepto los átomos de hidrógeno que se han generado en posiciones geométricas. En el último mapa de Fourier se han localizado tres moléculas de tolueno que se han refinado de forma isotrópica debido a los elevados parámetros térmicos que presentan los átomos de carbono.

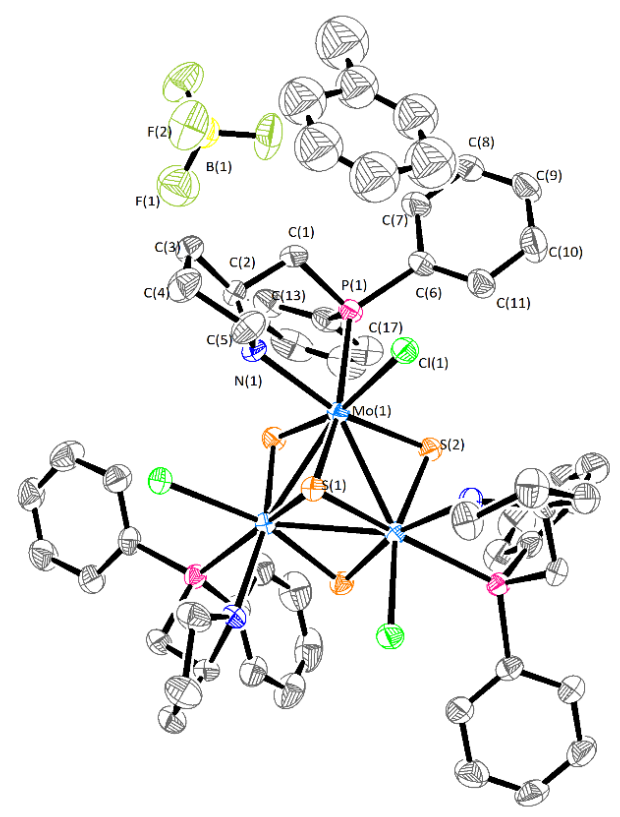

Figura 7.7. Representación ORTEP del clúster $(P)-\left[\mathrm{Mo}_{3} \mathrm{~S}_{4} \mathrm{Cl}_{3}((1 S, 2 S)-\mathrm{PPro})_{3}\right] \mathrm{BF}_{4}$ (elipsoides al $50 \%$ de probabilidad). Las moléculas de tolueno se han omitido por claridad. 
Tabla 7.19. Parámetros cristalográficos, condiciones de registro y refino.

Datos cristalográficos

Especie

Fórmula

Grupo espacial P2,3

$\mathbf{a}(\AA)$

b $(\AA)$

c $(\AA)$

Z

Color

$\mathbf{d}_{\text {calc }}\left(\mathrm{mg} / \mathrm{mm}^{3}\right)$

Coeficiente de

absorción $\left(\mathrm{mm}^{-1}\right)$
$\left[\mathrm{Mo}_{3} \mathrm{~S}_{4} \mathrm{Cl}_{3}((1 S, 2 S)-\mathrm{PPro})_{3}\right] \mathrm{BF}_{4} \cdot 3 \mathrm{C}_{7} \mathrm{H}_{8}$

$\mathrm{C}_{72} \mathrm{H}_{84} \mathrm{BCl}_{3} \mathrm{~F}_{4} \mathrm{Mo}_{3} \mathrm{~N}_{3} \mathrm{P}_{3} \mathrm{~S}_{4}$ Sistema

Cúbico

cristalino

Peso 1693,55

molecular

$\boldsymbol{\alpha}\left(^{\circ}\right) \quad 90,00$

$\boldsymbol{\beta}\left(^{\circ}\right) \quad 90,00$

$\boldsymbol{\gamma}\left(^{\circ}\right) \quad 90,00$

Vol $\left(\AA^{3}\right) \quad 7472,7(3)$

Tamaño del $\quad 0,2206 \times 0,1854$

cristal (mm) x 0,1613

$\lambda(\AA) \quad 0,71073$

F (000) 3456

Parámetros experimentales

Temperatura (K) 200,00(14)

Tiempo por marco 160

(s)

Límites $\mathbf{2 \theta}\left(^{\circ}\right) \quad 5,89$ y 58,65

$\mathbf{N}^{\mathbf{o}}$ de reflexiones 28596

registradas
$\mathbf{R}$ (int)

$\mathbf{R}(\sigma)$

0,0471

0,0416

Límites h, k, $1 \quad-22 \leq \mathrm{h} \leq 17$

(mín/máx) $\quad-26-\leq \mathrm{k} \leq 26$

$-26-\leq 1 \leq 23$

$N^{o}$ de reflexiones 6384

independientes

Resolución y refino

$\mathrm{N}^{\mathrm{o}}$ de variables

$\mathrm{N}^{\mathbf{o}}$ de restricciones

R1 final ([I>2sigma(I)]) $\quad 0,0448$

wR2 final ([I>2sigma(I)]) 0,0992

Densidad electrónica residual $\left(e \cdot \AA^{-3}\right)$

Parámetro de Flack

$246 \quad$ GooF en $F^{2} \quad 1,164$

0

0,80/-0,53 Max. desplazam./ $\boldsymbol{\sigma} \quad 0,001$

R1 (todos los datos) $\quad 0,0694$ wR2 (todos los datos) 0,1183

$-0,03(2)$

$$
R 1=\sum|| F_{0}|-| F_{1}|| / \sum F_{0} \quad w R 2=\left[\sum\left[w\left(F_{0}^{2}-F_{c}^{2}\right)^{2}\right] / \sum\left[w\left(F_{0}^{2}\right)^{2}\right]\right]^{1 / 2}
$$


Tabla 7.20. Distancias de enlace representativas $(\AA)$.

\begin{tabular}{cccccc}
\hline Átomo Átomo & Distancia & \multicolumn{2}{c}{ Átomo Átomo } & Distancia \\
\hline $\operatorname{Mo}(1)$ & $\operatorname{Mo}(1)^{\mathrm{A}}$ & $2,7569(9)$ & $\mathrm{Mo}(1)$ & $\mathrm{S}(2)^{\mathrm{B}}$ & $2,2931(17)$ \\
$\operatorname{Mo}(1)$ & $\operatorname{Mo}(1)^{\mathrm{B}}$ & $2,7569(9)$ & $\operatorname{Mo}(1)^{\mathrm{A}}$ & $\mathrm{S}(2)$ & $2,2930(17)$ \\
$\operatorname{Mo}(1)$ & $\mathrm{S}(1)$ & $2,354(2)$ & $\mathrm{Mo}(1)$ & $\mathrm{P}(1)$ & $2,5509(18)$ \\
$\operatorname{Mo}(1)^{\mathrm{A}}$ & $\mathrm{S}(1)$ & $2,354(2)$ & $\mathrm{Mo}(1)$ & $\mathrm{N}(1)$ & $2,292(5)$ \\
$\operatorname{Mo}(1)^{\mathrm{B}}$ & $\mathrm{S}(1)$ & $2,354(2)$ & $\mathrm{Mo}(1)$ & $\mathrm{Cl}(1)$ & $2,4817(17)$ \\
$\operatorname{Mo}(1)$ & $\mathrm{S}(2)$ & $2,2941(17)$ & & & \\
\hline
\end{tabular}

Transformaciones de simetría utilizadas para generar átomos cristalográficamente equivalentes:

$\mathrm{A}=+\mathrm{X}, 1+\mathrm{Y},-1+\mathrm{Z} ; \mathrm{B}=1+\mathrm{X},+\mathrm{Y},-1+\mathrm{Z}$ 
Tabla 7.21. Ángulos de enlace representativos $\left({ }^{\circ}\right)$.

\begin{tabular}{|c|c|c|c|c|c|c|c|}
\hline \multicolumn{3}{|c|}{ ÁtomoÁtomoÁtomo } & \multirow{2}{*}{$\begin{array}{c}\text { Ángulo } \\
60,0\end{array}$} & \multicolumn{3}{|c|}{ ÁtomoÁtomo Átomo } & \multirow{2}{*}{$\begin{array}{c}\text { Ángulo } \\
78,30(15)\end{array}$} \\
\hline $\operatorname{Mo}(1)^{A}$ & $\mathrm{Mo}(1)$ & $\mathrm{Mo}(1)^{\mathrm{B}}$ & & $\mathrm{N}(1)$ & $\operatorname{Mo}(1)$ & $\mathrm{P}(1)$ & \\
\hline $\mathrm{P}(1)$ & $\mathrm{Mo}(1)$ & $\operatorname{Mo}(1)^{B}$ & $134,90(5)$ & $\mathrm{N}(1)$ & $\operatorname{Mo}(1)$ & $\mathrm{S}(1)$ & $86,35(15)$ \\
\hline $\mathrm{P}(1)$ & $\mathrm{Mo}(1)$ & $\operatorname{Mo}(1)^{\mathrm{A}}$ & $141,41(5)$ & $\mathrm{N}(1)$ & $\mathrm{Mo}(1)$ & $\mathrm{Cl}(1)$ & $83,15(15)$ \\
\hline$S(1)$ & $\operatorname{Mo}(1)$ & $\operatorname{Mo}(1)^{\mathrm{B}}$ & $54,15(4)$ & $C(6)$ & $\mathrm{P}(1)$ & $\operatorname{Mo}(1)$ & $126,1(2)$ \\
\hline$S(1)$ & $\operatorname{Mo}(1)$ & $\operatorname{Mo}(1)^{\mathrm{A}}$ & $54,15(4)$ & $\mathrm{C}(6)$ & $\mathrm{P}(1)$ & $\mathrm{C}(1)$ & $103,8(3)$ \\
\hline$S(1)$ & $\operatorname{Mo}(1)$ & $\mathrm{P}(1)$ & $162,55(6)$ & $C(12)$ & $\mathrm{P}(1)$ & $\operatorname{Mo}(1)$ & $114,4(2)$ \\
\hline$S(1)$ & $\operatorname{Mo}(1)$ & $\mathrm{Cl}(1)$ & $87,23(6)$ & $C(12)$ & $\mathrm{P}(1)$ & $C(6)$ & $101,8(3)$ \\
\hline$S(2)$ & $\mathrm{Mo}(1)$ & $\operatorname{Mo}(1)^{\mathrm{B}}$ & $98,03(4)$ & $C(12)$ & $\mathrm{P}(1)$ & $C(1)$ & $108,3(4)$ \\
\hline $\mathrm{S}(2)^{\mathrm{B}}$ & $\operatorname{Mo}(1)$ & $\operatorname{Mo}(1)^{\mathrm{B}}$ & $53,08(4)$ & $\mathrm{C}(1)$ & $\mathrm{P}(1)$ & $\operatorname{Mo}(1)$ & $101,0(2)$ \\
\hline $\mathrm{S}(2)^{\mathrm{B}}$ & $\operatorname{Mo}(1)$ & $\operatorname{Mo}(1)^{\mathrm{A}}$ & $98,05(4)$ & $C(7)$ & $\mathrm{C}(6)$ & $\mathrm{P}(1)$ & $118,0(6)$ \\
\hline $\mathrm{S}(2)$ & $\operatorname{Mo}(1)$ & $\operatorname{Mo}(1)^{\mathrm{A}}$ & $53,04(5)$ & $C(11)$ & $C(6)$ & $\mathrm{P}(1)$ & $122,9(5)$ \\
\hline $\mathrm{S}(2)^{\mathrm{B}}$ & $\operatorname{Mo}(1)$ & $\mathrm{P}(1)$ & $81,94(6)$ & $C(13)$ & $\mathrm{C}(12)$ & $\mathrm{P}(1)$ & $122,1(6)$ \\
\hline$S(2)$ & $\operatorname{Mo}(1)$ & $\mathrm{P}(1)$ & $88,44(6)$ & $C(17)$ & $C(12)$ & $\mathrm{P}(1)$ & $117,4(6)$ \\
\hline$S(2)$ & $\operatorname{Mo}(1)$ & $\mathrm{S}(1)$ & $105,90(6)$ & $\operatorname{Mo}(1)^{B}$ & $\mathrm{~S}(1)$ & $\operatorname{Mo}(1)^{A}$ & $71,70(7)$ \\
\hline $\mathrm{S}(2)^{\mathrm{B}}$ & $\operatorname{Mo}(1)$ & $\mathrm{S}(1)$ & $105,93(5)$ & $\operatorname{Mo}(1)$ & $\mathrm{S}(1)$ & $\operatorname{Mo}(1)^{\mathrm{B}}$ & $71,70(7)$ \\
\hline $\mathrm{S}(2)^{\mathrm{B}}$ & $\mathrm{Mo}(1)$ & $S(2)$ & $95,61(9)$ & $\mathrm{Mo}(1)$ & $S(1)$ & $\operatorname{Mo}(1)^{A}$ & $71,70(7)$ \\
\hline $\mathrm{S}(2)^{\mathrm{B}}$ & $\mathrm{Mo}(1)$ & $\mathrm{Cl}(1)$ & $163,55(6)$ & $\operatorname{Mo}(1)^{A}$ & $S(2)$ & $\operatorname{Mo}(1)$ & $73,89(5)$ \\
\hline $\mathrm{S}(2)$ & $\mathrm{Mo}(1)$ & $\mathrm{Cl}(1)$ & $90,04(6)$ & $C(5)$ & $\mathrm{N}(1)$ & $\operatorname{Mo}(1)$ & $122,8(4)$ \\
\hline $\mathrm{S}(2)^{\mathrm{B}}$ & $\operatorname{Mo}(1)$ & $\mathrm{N}(1)$ & $87,79(15)$ & $C(5)$ & $\mathrm{N}(1)$ & $C(2)$ & $106,1(5)$ \\
\hline$S(2)$ & $\operatorname{Mo}(1)$ & $\mathrm{N}(1)$ & $165,73(15)$ & $C(2)$ & $\mathrm{N}(1)$ & $\operatorname{Mo}(1)$ & $117,1(4)$ \\
\hline $\mathrm{Cl}(1)$ & $\mathrm{Mo}(1)$ & $\operatorname{Mo}(1)^{\mathrm{A}}$ & $97,69(5)$ & $\mathrm{N}(1)$ & $\mathrm{C}(5)$ & $C(4)$ & $102,9(6)$ \\
\hline $\mathrm{Cl}(1)$ & $\mathrm{Mo}(1)$ & $\operatorname{Mo}(1)^{\mathrm{B}}$ & $141,32(4)$ & $C(2)$ & $\mathrm{C}(1)$ & $\mathrm{P}(1)$ & $110,9(5)$ \\
\hline $\mathrm{Cl}(1)$ & $\operatorname{Mo}(1)$ & $\mathrm{P}(1)$ & $82,78(6)$ & $\mathrm{N}(1)$ & $\mathrm{C}(2)$ & $C(1)$ & $110,6(5)$ \\
\hline $\mathrm{N}(1)$ & $\operatorname{Mo}(1)$ & $\operatorname{Mo}(1)^{\mathrm{B}}$ & $95,10(15)$ & $\mathrm{N}(1)$ & $\mathrm{C}(2)$ & $C(3)$ & $104,5(6)$ \\
\hline $\mathrm{N}(1)$ & $\mathrm{Mo}(1)$ & $\operatorname{Mo}(1)^{A}$ & $140,25(15)$ & & & & \\
\hline
\end{tabular}

Transformaciones de simetría utilizadas para generar átomos cristalográficamente equivalentes:

$\mathrm{A}=+\mathrm{X}, 1+\mathrm{Y},-1+\mathrm{Z} ; \mathrm{B}=1+\mathrm{X},+\mathrm{Y},-1+\mathrm{Z}$ 


\subsubsection{CLÚSTERES FUNCIONALIZADOS CON AMINODIFOSFINAS \\ 7.2.4.1. ESTRUCTURA DE $\left[\mathrm{Mo}_{3} \mathrm{~S}_{4} \mathrm{Cl}_{3}(\mathrm{PNP})_{3}\right] \mathrm{Cl}(9(\mathrm{Cl}))$}

El complejo $\mathbf{9}(\mathrm{Cl})$ se ha cristalizado mediante difusión lenta de hexano sobre una disolución concentrada del clúster en diclorometano. Los cristales, de calidad adecuada, tienen una morfología cúbica y presentan un color verde. El refino se ha realizado en el grupo espacial $\mathrm{Pa}-3$, que contiene un eje ternario que atraviesa la unidad $\mathrm{Mo}_{3} \mathrm{~S}_{4}$. Todos los átomos del clúster se han refinado anisotrópicamente, excepto los átomos de hidrógeno que se han generado en posiciones geométricas. El último mapa de diferencia de Fourier muestra dos picos en posición general (24d) de intensidades similares $\left(c a .2,7 \mathrm{e}^{-} / \AA^{3}\right)$ que han sido asignados a dos átomos de $\mathrm{Cl}$ y refinados anisotrópicamente, fijando el factor de ocupación de cada uno de ellos a $1 / 6$ con el fin de balancear la carga del clúster.

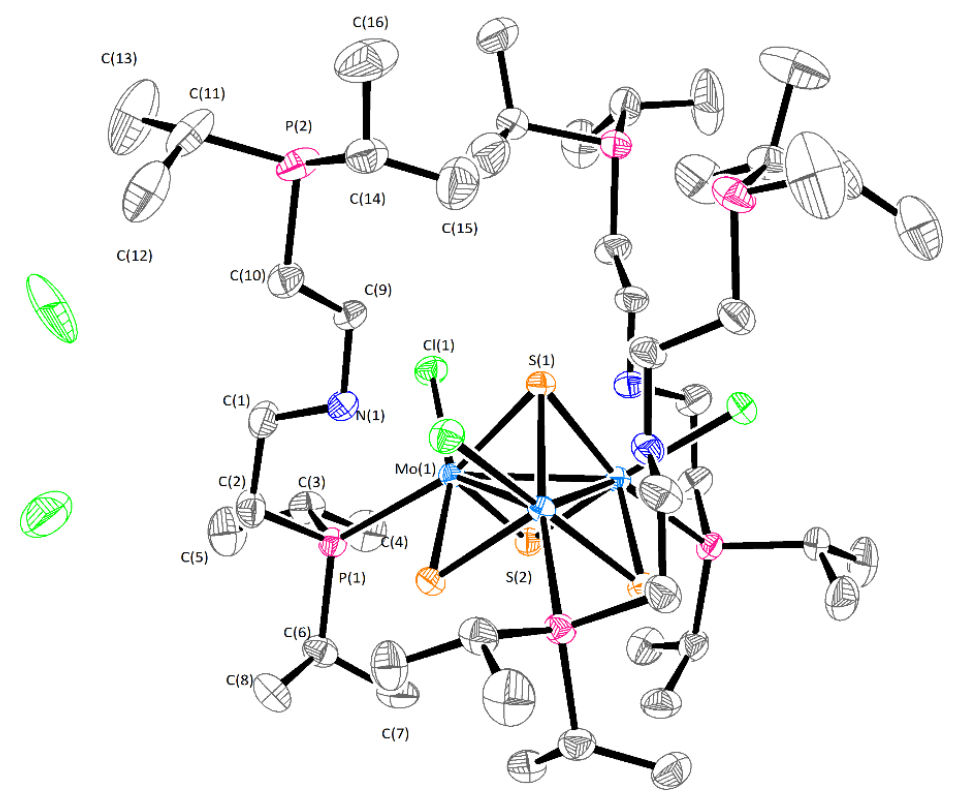

Figura 7.8. Representación ORTEP del clúster $\left[\mathrm{Mo}_{3} \mathrm{~S}_{4} \mathrm{Cl}_{3}(\mathrm{PNP})_{3}\right] \mathrm{Cl}$ (elipsoides al $50 \%$ de probabilidad). 
Tabla 7.22. Parámetros cristalográficos, condiciones de registro y refino.

Datos cristalográficos

Especie

$\left[\mathrm{Mo}_{3} \mathrm{~S}_{4} \mathrm{Cl}_{3}(\mathrm{PNP})_{3}\right] \mathrm{Cl}$

Fórmula

$\mathrm{C}_{48} \mathrm{H}_{111} \mathrm{Cl}_{4} \mathrm{Mo}_{3} \mathrm{~N}_{3} \mathrm{P}_{6} \mathrm{~S}_{4}$

Sistema

Cúbico

Grupo espacial $\mathrm{Pa}-3$

a $(\AA)$

24,21458(13)

cristalino

b $(\AA)$

24,21458(13)

Peso molecular 1474,07

c $(\AA)$

24,21458(13)

Z

8

Color

Verde

$\alpha\left(^{\circ}\right)$

90,00

$\boldsymbol{\beta}\left(^{\circ}\right)$

90,00

$\gamma\left({ }^{\circ}\right)$

90,00

Vol $\left(\AA^{3}\right)$

14198,1(2)

Tamaño del $0,189 \times 0,147 \times$

cristal $(\mathrm{mm}) \quad 0,125$

$\mathbf{d}_{\text {calc }}\left(\mathrm{mg} / \mathrm{mm}^{3}\right)$

1,379

$\lambda(\AA)$

0,957

F (000)

0,71073

Coeficiente de

absorción $\left(\mathrm{mm}^{-1}\right)$

\section{Parámetros experimentales}

\begin{tabular}{|c|c|c|c|}
\hline Temperatura (K) & $199,95(10)$ & $\mathbf{R}$ (int) & 0,0332 \\
\hline $\begin{array}{l}\text { Tiempo por marco } \\
\text { (s) }\end{array}$ & 80 & $\mathbf{R}(\sigma)$ & 0,0219 \\
\hline Límites $2 \theta\left(^{\circ}\right)$ & 6,30 y 58,426 & $\begin{array}{l}\text { Límites h, k, } 1 \\
\text { (mín/máx) }\end{array}$ & $\begin{array}{l}-29 \leq \mathrm{h} \leq 30 \\
-30-\leq \mathrm{k} \leq 22 \\
-31-\leq 1 \leq 32\end{array}$ \\
\hline $\begin{array}{l}\mathrm{N}^{\circ} \text { de reflexiones } \\
\text { registradas }\end{array}$ & 51257 & $\begin{array}{l}\mathrm{N}^{0} \text { de reflexiones } \\
\text { independientes }\end{array}$ & 6122 \\
\hline
\end{tabular}

Resolución y refino

$\begin{array}{llll}\mathbf{N}^{\mathbf{o}} \text { de variables } & 228 & \text { GooF en } \mathbf{F}^{2} & 1,158 \\ \mathbf{N}^{\mathbf{o}} \text { de restricciones } & 0 & & \\ \mathbf{R} 1 \text { final }([\mathrm{I}>\text { 2sigma(I) }]) & 0,0376 & \mathbf{R} 1 \text { (todos los datos) } & 0,0553 \\ \text { wR2 final }([\mathbf{I}>\text { 2sigma(I)]) } & 0,1003 & \text { wR2 (todos los datos) } & 0,1157 \\ \begin{array}{l}\text { Densidad electrónica } \\ \text { residual }\left(\mathrm{e} \cdot \AA^{-3}\right)\end{array} & 1,21 /-0,42 & \text { Max. desplazam. } \boldsymbol{\sigma} & 0,003\end{array}$

$$
R 1=\sum|| F_{0}|-| F_{1}|| / \sum F_{0} \quad w R 2=\left[\sum\left[w\left(F_{0}^{2}-F_{c}^{2}\right)^{2}\right] / \sum\left[w\left(F_{0}^{2}\right)^{2}\right]\right]^{1 / 2}
$$


Tabla 7.23. Distancias de enlace representativas $(\AA)$.

\begin{tabular}{cccccc}
\hline Átomo Átomo & Distancia & Átomo Átomo & Distancia \\
\hline $\operatorname{Mo}(1)$ & $\operatorname{Mo}(1)^{\mathrm{A}}$ & $2,7772(4)$ & $\mathrm{Mo}(1)$ & $\mathrm{S}(2)^{\mathrm{A}}$ & $2,2936(8)$ \\
$\operatorname{Mo}(1)$ & $\operatorname{Mo}(1)^{\mathrm{B}}$ & $2,7772(4)$ & $\mathrm{Mo}(1)^{\mathrm{B}}$ & $\mathrm{S}(2)$ & $2,2935(8)$ \\
$\operatorname{Mo}(1)$ & $\mathrm{S}(1)$ & $2,3539(10)$ & $\mathrm{Mo}(1)$ & $\mathrm{P}(1)$ & $2,5746(8)$ \\
$\operatorname{Mo}(1)^{\mathrm{A}}$ & $\mathrm{S}(1)$ & $2,3539(10)$ & $\mathrm{Mo}(1)$ & $\mathrm{N}(1)$ & $2,346(3)$ \\
$\operatorname{Mo}(1)^{\mathrm{B}}$ & $\mathrm{S}(1)$ & $2,3539(10)$ & $\mathrm{Mo}(1)$ & $\mathrm{Cl}(1)$ & $2,4910(8)$ \\
$\operatorname{Mo}(1)$ & $\mathrm{S}(2)$ & $2,2923(8)$ & & & \\
\hline
\end{tabular}

Transformaciones de simetría utilizadas para generar átomos cristalográficamente equivalentes:

$A=3 / 2-X, 1-Y, 1 / 2+Z ; B=-1 / 2+X, 3 / 2-Y, 1-Z$ 
Tabla 7.24. Ángulos de enlace representativos $\left({ }^{\circ}\right)$.

\begin{tabular}{|c|c|c|c|c|c|c|c|}
\hline \multicolumn{3}{|c|}{ ÁtomoÁtomoÁtomo } & \multirow{2}{*}{$\begin{array}{c}\text { Ángulo } \\
60,0\end{array}$} & \multicolumn{3}{|c|}{ ÁtomoÁtomo Átomo } & \multirow{2}{*}{\begin{tabular}{|l|} 
Ángulo \\
$74,54(3)$
\end{tabular}} \\
\hline $\operatorname{Mo}(1)^{A}$ & $\mathrm{Mo}(1)$ & $\operatorname{Mo}(1)^{\mathrm{B}}$ & & $\operatorname{Mo}(1)$ & $\mathrm{S}(2)$ & $\operatorname{Mo}(1)^{\mathrm{A}}$ & \\
\hline $\mathrm{S}(2)^{\mathrm{B}}$ & $\operatorname{Mo}(1)$ & $\operatorname{Mo}(1)^{A}$ & $98,38(2)$ & $\operatorname{Mo}(1)^{\mathrm{B}}$ & $S(1)$ & $\operatorname{Mo}(1)$ & $72,30(3)$ \\
\hline $\mathrm{S}(2)$ & $\operatorname{Mo}(1)$ & $\operatorname{Mo}(1)^{A}$ & $52,75(2)$ & $\operatorname{Mo}(1)^{\mathrm{B}}$ & $S(1)$ & $\operatorname{Mo}(1)^{A}$ & $72,30(3)$ \\
\hline $\mathrm{S}(2)^{\mathrm{B}}$ & $\mathrm{Mo}(1)$ & $\operatorname{Mo}(1)^{\mathrm{B}}$ & $52,71(2)$ & $\operatorname{Mo}(1)^{\mathrm{A}}$ & $S(1)$ & $\operatorname{Mo}(1)$ & $72,30(3)$ \\
\hline $\mathrm{S}(2)$ & $\operatorname{Mo}(1)$ & $\operatorname{Mo}(1)^{\mathrm{B}}$ & $98,41(2)$ & $\mathrm{C}(2)$ & $\mathrm{P}(1)$ & $\operatorname{Mo}(1)$ & $99,46(12)$ \\
\hline $\mathrm{S}(2)$ & $\mathrm{Mo}(1)$ & $\mathrm{S}(2)^{\mathrm{B}}$ & $97,44(4)$ & $C(2)$ & $\mathrm{P}(1)$ & $C(6)$ & $105,71(17)$ \\
\hline $\mathrm{S}(2)$ & $\operatorname{Mo}(1)$ & $\mathrm{S}(1)$ & $105,18(2)$ & $C(2)$ & $\mathrm{P}(1)$ & $C(3)$ & $104,77(18)$ \\
\hline $\mathrm{S}(2)^{\mathrm{B}}$ & $\mathrm{Mo}(1)$ & $\mathrm{S}(1)$ & $105,14(3)$ & $C(6)$ & $\mathrm{P}(1)$ & $\operatorname{Mo}(1)$ & $123,32(12)$ \\
\hline$S(2)^{B}$ & $\mathrm{Mo}(1)$ & $\mathrm{P}(1)$ & $79,78(3)$ & $C(3)$ & $\mathrm{P}(1)$ & $\operatorname{Mo}(1)$ & $117,71(13)$ \\
\hline $\mathrm{S}(2)$ & $\operatorname{Mo}(1)$ & $\mathrm{P}(1)$ & $87,61(3)$ & $C(3)$ & $\mathrm{P}(1)$ & $\mathrm{C}(6)$ & $103,70(16)$ \\
\hline$S(2)$ & $\operatorname{Mo}(1)$ & $\mathrm{Cl}(1)$ & $90,25(3)$ & $C(10)$ & $\mathrm{P}(2)$ & $C(11)$ & $100,9(2)$ \\
\hline $\mathrm{S}(2)^{\mathrm{B}}$ & $\operatorname{Mo}(1)$ & $\mathrm{Cl}(1)$ & $162,95(3)$ & $\mathrm{C}(14)$ & $\mathrm{P}(2)$ & $C(10)$ & $102,04(18)$ \\
\hline$S(2)^{B}$ & $\mathrm{Mo}(1)$ & $\mathrm{N}(1)$ & $88,32(7)$ & $C(14)$ & $\mathrm{P}(2)$ & $\mathrm{C}(11)$ & $101,7(2)$ \\
\hline $\mathrm{S}(2)$ & $\mathrm{Mo}(1)$ & $\mathrm{N}(1)$ & $163,64(7)$ & $C(1)$ & $\mathrm{N}(1)$ & $\operatorname{Mo}(1)$ & $114,2(2)$ \\
\hline $\mathrm{S}(1)$ & $\mathrm{Mo}(1)$ & $\operatorname{Mo}(1)^{\mathrm{B}}$ & $53,850(17)$ & $\mathrm{C}(9)$ & $\mathrm{N}(1)$ & $\operatorname{Mo}(1)$ & $119,4(2)$ \\
\hline $\mathrm{S}(1)$ & $\mathrm{Mo}(1)$ & $\operatorname{Mo}(1)^{A}$ & $53,851(17)$ & $C(9)$ & $\mathrm{N}(1)$ & $C(1)$ & $108,1(3)$ \\
\hline $\mathrm{S}(1)$ & $\operatorname{Mo}(1)$ & $\mathrm{P}(1)$ & $165,31(3)$ & $\mathrm{C}(1)$ & $\mathrm{C}(2)$ & $\mathrm{P}(1)$ & $109,5(3)$ \\
\hline $\mathrm{S}(1)$ & $\mathrm{Mo}(1)$ & $\mathrm{Cl}(1)$ & $87,32(3)$ & $\mathrm{N}(1)$ & $\mathrm{C}(1)$ & $C(2)$ & $111,7(3)$ \\
\hline $\mathrm{P}(1)$ & $\operatorname{Mo}(1)$ & $\operatorname{Mo}(1)^{\mathrm{B}}$ & $132,48(2)$ & $C(8)$ & $C(6)$ & $\mathrm{P}(1)$ & $114,8(3)$ \\
\hline $\mathrm{P}(1)$ & $\mathrm{Mo}(1)$ & $\operatorname{Mo}(1)^{A}$ & $140,01(2)$ & $C(7)$ & $\mathrm{C}(6)$ & $\mathrm{P}(1)$ & $112,9(3)$ \\
\hline $\mathrm{Cl}(1)$ & $\operatorname{Mo}(1)$ & $\operatorname{Mo}(1)^{\mathrm{B}}$ & $141,16(2)$ & $C(4)$ & $\mathrm{C}(3)$ & $\mathrm{P}(1)$ & $111,1(3)$ \\
\hline $\mathrm{Cl}(1)$ & $\mathrm{Mo}(1)$ & $\operatorname{Mo}(1)^{A}$ & $98,39(2)$ & $C(5)$ & $\mathrm{C}(3)$ & $\mathrm{P}(1)$ & $113,8(3)$ \\
\hline $\mathrm{Cl}(1)$ & $\mathrm{Mo}(1)$ & $\mathrm{P}(1)$ & $85,39(3)$ & $\mathrm{C}(9)$ & $\mathrm{C}(10)$ & $\mathrm{P}(2)$ & $112,1(3)$ \\
\hline $\mathrm{N}(1)$ & $\operatorname{Mo}(1)$ & $\operatorname{Mo}(1)^{A}$ & $141,67(7)$ & $\mathrm{N}(1)$ & $\mathrm{C}(9)$ & $C(10)$ & $113,8(3)$ \\
\hline $\mathrm{N}(1)$ & $\mathrm{Mo}(1)$ & $\operatorname{Mo}(1)^{B}$ & $97,26(7)$ & $C(15)$ & $C(14)$ & $\mathrm{P}(2)$ & $110,6(3)$ \\
\hline $\mathrm{N}(1)$ & $\operatorname{Mo}(1)$ & $\mathrm{S}(1)$ & $87,91(7)$ & $C(16)$ & $C(14)$ & $\mathrm{P}(2)$ & $110,8(3)$ \\
\hline $\mathrm{N}(1)$ & $\mathrm{Mo}(1)$ & $\mathrm{P}(1)$ & $78,31(7)$ & $C(12)$ & $C(11)$ & $\mathrm{P}(2)$ & $117,6(3)$ \\
\hline $\mathrm{N}(1)$ & $\mathrm{Mo}(1)$ & $\mathrm{Cl}(1)$ & $80,43(7)$ & $C(13)$ & $\mathrm{C}(11)$ & $\mathrm{P}(2)$ & $109,2(4)$ \\
\hline
\end{tabular}

Transformaciones de simetría utilizadas para generar átomos cristalográficamente equivalentes:

$A=-1 / 2+X, 3 / 2-Y, 1-Z ; B=3 / 2-X, 1-Y, 1 / 2+Z$ 


\subsection{CÁlCULOS TEÓRICOS}

Todas las geometrías optimizadas y cálculos NBO descritos en el capítulo 5 de esta tesis doctoral se han llevado a cabo con el paquete de software Gaussian09.[16] La Teoría del Funcional de la Densidad (DFT) se ha aplicado con el funcional híbrido de Becke.[17-19] Se ha utilizado el conjunto de funciones de base doble- $\xi$ con pseudo orbitales LanL2DZ, que incluyen el potencial interno relativista LanL2 de Los Álamos. Las optimizaciones de geometría se han realizado en fase gas sin ninguna restricción de simetría, seguidas de cálculos de frecuencia analítica para confirmar que se ha alcanzado un mínimo. El análisis ELF se ha llevado a cabo con el paquete TopMod,[20] considerando un mallado cúbico de distancia de paso menor de 0,05 bohr sobre la función de onda obtenida al nivel teórico B3LYP/3-21G//B3LYPLanL2DZ.

\subsubsection{GEOMETRÍAS OPTIMIZADAS}

Los detalles estructurales de las geometrías optimizadas descritas en el capítulo 5 de esta tesis se muestran a continuación. En el caso de los isómeros $P$, los parámetros estructurales iniciales para la construcción del modelo teórico se han extraído de los datos cristalográficos detallados en los apartados 7.2.3.4 y 7.2.3.5. Los contraiones y las moléculas de disolvente se han eliminado para evitar la ralentización de los cálculos. Los isómeros $M$ se han construido intercambiando las posiciones de los ligandos aminofosfina y cloro de las estructuras anteriores, preservando las configuraciones de los centros estereogénicos. A continuación se muestra una lista detallada de distancias y ángulos de enlace de las geometrías optimizadas, con el objetivo de que sean comparadas con los datos obtenidos de forma experimental para los isómeros $P$. Tal y como se ha especificado anteriormente, estas optimizaciones se han llevado a cabo sin ninguna restricción de simetría, por lo que las distancias y ángulos que aquí se muestran son el resultado de una media aritmética entre átomos equivalentes. La desviación estándar se presenta entre corchetes. 


\subsubsection{1. (P)-[$\left[\mathrm{Mo}_{3} \mathrm{~S}_{4} \mathrm{Cl}_{3}((1 S, 2 R)-\mathrm{PPro})_{3}\right]^{+}\left((P)-7^{+}\right)$}

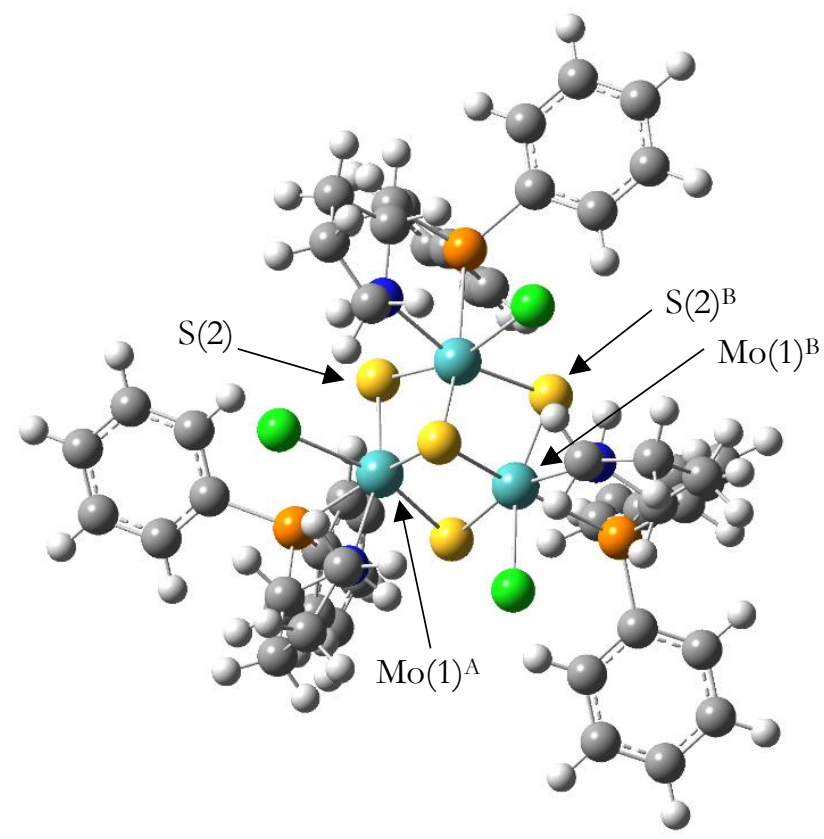

Figura 7.9. Geometría optimizada del complejo $(P)-\left[\mathrm{Mo}_{3} \mathrm{~S}_{4} \mathrm{Cl}_{3}((1 S, 2 \mathrm{R})-\mathrm{PPro})_{3}\right]^{+}$.

Tabla 7.25. Distancias de enlace representativas $(\AA)$.

\begin{tabular}{|c|c|c|c|c|c|}
\hline Átomo & Átomo & Distancia & Átomo & Átomo & Distancia \\
\hline Mo & Mo & $2,8074[8]$ & Mo & $\mathrm{P}$ & $2,6605[3]$ \\
\hline Mo & $\mu_{3}-S$ & $2,4377[5]$ & Mo & $\mathrm{N}$ & $2,2885[14]$ \\
\hline Mo & $\mu-S^{(a)}$ & $2,3788[1]$ & Mo & $\mathrm{Cl}$ & $2,5500[9]$ \\
\hline Mo & $\mu-S^{(b)}$ & $2,3800[1]$ & & & \\
\hline
\end{tabular}

(a) Distancia trans al enlace Mo-Cl.

(b) Distancia trans al enlace Mo-N.

[] Desviación estándar del promedio. 
Tabla 7.26. Ángulos de enlace representativos $\left(^{\circ}\right)$.

\begin{tabular}{|c|c|c|c|c|c|c|c|}
\hline \multicolumn{3}{|c|}{ Átomo Átomo Átomo } & \multirow{2}{*}{$\begin{array}{l}\text { Ángulo } \\
60,00[3]\end{array}$} & \multicolumn{3}{|c|}{ Átomo Átomo Átomo } & \multirow{2}{*}{$\begin{array}{c}\text { Ángulo } \\
78,31[2]\end{array}$} \\
\hline $\operatorname{Mo}(1)^{A}$ & $\operatorname{Mo}(1)$ & $\operatorname{Mo}(1)^{B}$ & & $\mathrm{~N}(1)$ & $\operatorname{Mo}(1)$ & $\mathrm{P}(1)$ & \\
\hline $\mathrm{P}(1)$ & $\operatorname{Mo}(1)$ & $\operatorname{Mo}(1)^{B}$ & $140,47[2]$ & $\mathrm{N}(1)$ & $\operatorname{Mo}(1)$ & $\mathrm{S}(1)$ & $86,34[4]$ \\
\hline $\mathrm{P}(1)$ & $\operatorname{Mo}(1)$ & $\operatorname{Mo}(1)^{A}$ & $133,40[11]$ & $\mathrm{N}(1)$ & $\operatorname{Mo}(1)$ & $\mathrm{Cl}(1)$ & $80,06[5]$ \\
\hline $\mathrm{S}(1)$ & $\operatorname{Mo}(1)$ & $\operatorname{Mo}(1)^{B}$ & $54,84[1]$ & $C(6)$ & $\mathrm{P}(1)$ & $\operatorname{Mo}(1)$ & $123,95[23]$ \\
\hline $\mathrm{S}(1)$ & $\operatorname{Mo}(1)$ & $\operatorname{Mo}(1)^{A}$ & $54,84[3]$ & $C(6)$ & $\mathrm{P}(1)$ & $\mathrm{C}(1)$ & $105,03[8]$ \\
\hline $\mathrm{S}(1)$ & $\operatorname{Mo}(1)$ & $\mathrm{P}(1)$ & $163,30[7]$ & $C(12)$ & $\mathrm{P}(1)$ & $\operatorname{Mo}(1)$ & $119,65[12]$ \\
\hline $\mathrm{S}(1)$ & $\operatorname{Mo}(1)$ & $\mathrm{Cl}(1)$ & $85,43[1]$ & $\mathrm{C}(12)$ & $\mathrm{P}(1)$ & $C(6)$ & $102,07[14]$ \\
\hline $\mathrm{S}(2)$ & $\operatorname{Mo}(1)$ & $\operatorname{Mo}(1)^{B}$ & $99,36[6]$ & $\mathrm{C}(12)$ & $\mathrm{P}(1)$ & $\mathrm{C}(1)$ & $105,38[14]$ \\
\hline $\mathrm{S}(2)^{\mathrm{B}}$ & $\operatorname{Mo}(1)$ & $\operatorname{Mo}(1)^{B}$ & $53,83[1]$ & $\mathrm{C}(1)$ & $\mathrm{P}(1)$ & $\operatorname{Mo}(1)$ & $98,41[3]$ \\
\hline $\mathrm{S}(2)^{\mathrm{B}}$ & $\operatorname{Mo}(1)$ & $\operatorname{Mo}(1)^{A}$ & $99,33[2]$ & $C(7)$ & $C(6)$ & $\mathrm{P}(1)$ & $119,50[14]$ \\
\hline $\mathrm{S}(2)$ & $\operatorname{Mo}(1)$ & $\operatorname{Mo}(1)^{A}$ & $53,87[2]$ & $\mathrm{C}(11)$ & $C(6)$ & $\mathrm{P}(1)$ & $120,78[13]$ \\
\hline $\mathrm{S}(2)^{\mathrm{B}}$ & $\mathrm{Mo}(1)$ & $\mathrm{P}(1)$ & $86,910[3]$ & $C(13)$ & $C(12)$ & $\mathrm{P}(1)$ & $118,60[14]$ \\
\hline $\mathrm{S}(2)$ & $\operatorname{Mo}(1)$ & $\mathrm{P}(1)$ & $79,55[10]$ & $C(17)$ & $C(12)$ & $\mathrm{P}(1)$ & $121,87[15]$ \\
\hline $\mathrm{S}(2)$ & $\operatorname{Mo}(1)$ & $\mathrm{S}(1)$ & $107,00[5]$ & $\operatorname{Mo}(1)^{A}$ & $\mathrm{~S}(1)$ & $\operatorname{Mo}(1)^{\mathrm{B}}$ & $70,31[3]$ \\
\hline $\mathrm{S}(2)^{\mathrm{B}}$ & $\operatorname{Mo}(1)$ & $\mathrm{S}(1)$ & $106,97[2]$ & $\operatorname{Mo}(1)^{A}$ & $\mathrm{~S}(1)$ & $\operatorname{Mo}(1)$ & $70,31[3]$ \\
\hline $\mathrm{S}(2)^{\mathrm{B}}$ & $\operatorname{Mo}(1)$ & $\mathrm{S}(2)$ & $97,57[5]$ & $\operatorname{Mo}(1)^{B}$ & $\mathrm{~S}(1)$ & $\operatorname{Mo}(1)$ & $70,31[3]$ \\
\hline $\mathrm{S}(2)^{\mathrm{B}}$ & $\operatorname{Mo}(1)$ & $\mathrm{Cl}(1)$ & $89,42[5]$ & $\operatorname{Mo}(1)^{A}$ & $\mathrm{~S}(2)$ & $\operatorname{Mo}(1)$ & $72,31[3]$ \\
\hline $\mathrm{S}(2)$ & $\operatorname{Mo}(1)$ & $\mathrm{Cl}(1)$ & $163,07[5]$ & $C(5)$ & $\mathrm{N}(1)$ & $\operatorname{Mo}(1)$ & $122,71[1]$ \\
\hline $\mathrm{S}(2)^{\mathrm{B}}$ & $\operatorname{Mo}(1)$ & $\mathrm{N}(1)$ & $162,42[4]$ & $C(5)$ & $\mathrm{N}(1)$ & $\mathrm{C}(2)$ & $102,62[3]$ \\
\hline $\mathrm{S}(2)$ & $\operatorname{Mo}(1)$ & $\mathrm{N}(1)$ & $89,13[4]$ & $C(2)$ & $\mathrm{N}(1)$ & $\operatorname{Mo}(1)$ & $115,59[3]$ \\
\hline $\mathrm{Cl}(1)$ & $\operatorname{Mo}(1)$ & $\operatorname{Mo}(1)^{A}$ & $140,21[3]$ & $\mathrm{N}(1)$ & $C(5)$ & $C(4)$ & $105,44[4]$ \\
\hline $\mathrm{Cl}(1)$ & $\operatorname{Mo}(1)$ & $\operatorname{Mo}(1)^{B}$ & $97,22[4]$ & $\mathrm{C}(2)$ & $\mathrm{C}(1)$ & $\mathrm{P}(1)$ & $107,82[2]$ \\
\hline $\mathrm{Cl}(1)$ & $\operatorname{Mo}(1)$ & $\mathrm{P}(1)$ & $85,48[14]$ & $\mathrm{N}(1)$ & $\mathrm{C}(2)$ & $\mathrm{C}(1)$ & $111,58[3]$ \\
\hline $\mathrm{N}(1)$ & $\operatorname{Mo}(1)$ & $\operatorname{Mo}(1)^{B}$ & $141,12[3]$ & $\mathrm{N}(1)$ & $C(2)$ & $C(3)$ & $103,55[1]$ \\
\hline $\mathrm{N}(1)$ & $\operatorname{Mo}(1)$ & $\operatorname{Mo}(1)^{A}$ & $97,75[3]$ & & & & \\
\hline
\end{tabular}




\subsubsection{2. (P)-[ $\left.\mathrm{Mo}_{3} \mathrm{~S}_{4} \mathrm{Cl}_{3}((1 S, 2 S)-P P r o)_{3}\right]^{+}\left((P)-8^{+}\right)$}

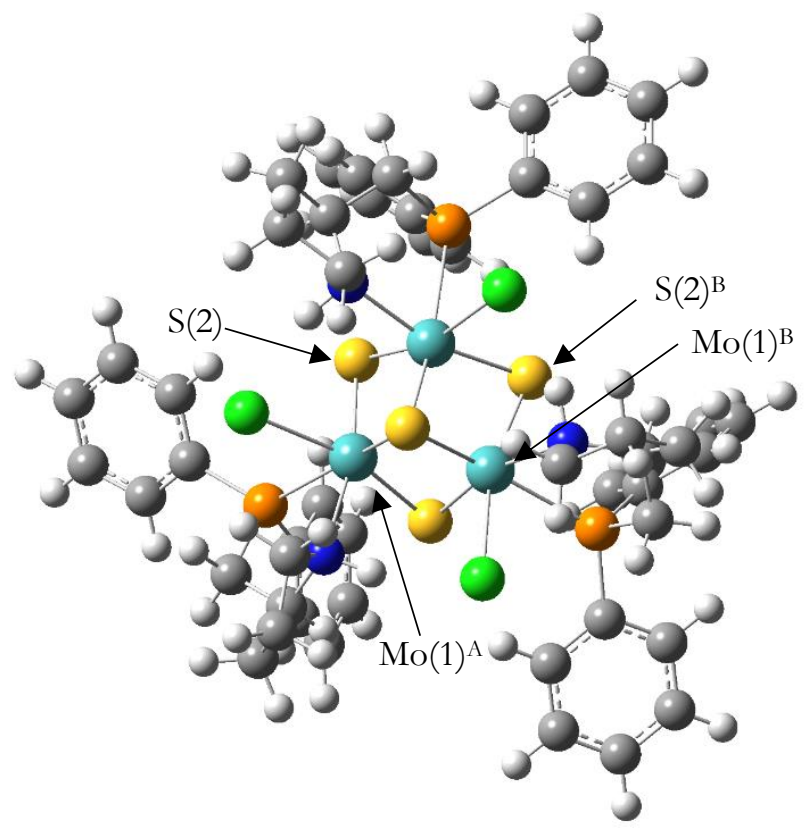

Figura 7.10. Geometría optimizada del complejo $(P)-\left[\mathrm{Mo}_{3} \mathrm{~S}_{4} \mathrm{Cl}_{3}((1 S, 2 S)-\mathrm{PPro})_{3}\right]^{+}$.

Tabla 7.27. Distancias de enlace representativas $(\AA)$.

\begin{tabular}{cccccc}
\hline \multicolumn{2}{c}{ Átomo } & Átomo & Distancia & \multicolumn{3}{c}{ Átomo } & Átomo & Distancia \\
\hline Mo & Mo & $2,8054[3]$ & Mo & P & $2,6309[14]$ \\
Mo & $\mu_{3}-S$ & $2,4431[9]$ & Mo & N & $2,2912[16]$ \\
Mo & $\mu-S^{(a)}$ & $2,3673[6]$ & Mo & $\mathrm{Cl}$ & $2,5699[9]$ \\
Mo & $\mu-S^{(b)}$ & $2,3869[2]$ & & & \\
\hline
\end{tabular}

(a) Distancia trans al enlace $\mathrm{Mo}-\mathrm{Cl}$.

(b) Distancia trans al enlace Mo-N.

[] Desviación estándar del promedio. 
Tabla 7.28. Ángulos de enlace representativos $\left(^{\circ}\right)$.

\begin{tabular}{|c|c|c|c|c|c|c|}
\hline \multicolumn{2}{|c|}{ Átomo Átomo Átomo } & \multirow{2}{*}{$\begin{array}{c}\text { Ángulo } \\
60,00[1]\end{array}$} & \multicolumn{3}{|c|}{ Átomo Átomo Átomo } & \multirow{2}{*}{$\begin{array}{l}\text { Ángulo } \\
79,17[2]\end{array}$} \\
\hline $\operatorname{Mo}(1)^{A}$ & $\operatorname{Mo}(1) \operatorname{Mo}(1)^{B}$ & & $\mathrm{~N}(1)$ & $\operatorname{Mo}(1)$ & $\mathrm{P}(1)$ & \\
\hline $\mathrm{P}(1)$ & $\operatorname{Mo}(1) \operatorname{Mo}(1)^{B}$ & $140,21[6]$ & $\mathrm{N}(1)$ & $\operatorname{Mo}(1)$ & $\mathrm{S}(1)$ & $85,73[5]$ \\
\hline $\mathrm{P}(1)$ & $\operatorname{Mo}(1) \operatorname{Mo}(1)^{A}$ & $137,75[10]$ & $\mathrm{N}(1)$ & $\operatorname{Mo}(1)$ & $\mathrm{Cl}(1)$ & $83,42[8]$ \\
\hline $\mathrm{S}(1)$ & $\operatorname{Mo}(1) \operatorname{Mo}(1)^{B}$ & $54,96[3]$ & $\mathrm{C}(6)$ & $\mathrm{P}(1)$ & $\operatorname{Mo}(1)$ & $126,23[5]$ \\
\hline $\mathrm{S}(1)$ & $\operatorname{Mo}(1) \operatorname{Mo}(1)^{A}$ & $54,96[3]$ & $C(6)$ & $\mathrm{P}(1)$ & $\mathrm{C}(1)$ & $104,65[4]$ \\
\hline $\mathrm{S}(1)$ & $\operatorname{Mo}(1) \quad \mathrm{P}(1)$ & $160,84[3]$ & $C(12)$ & $\mathrm{P}(1)$ & $\operatorname{Mo}(1)$ & $117,79[4]$ \\
\hline $\mathrm{S}(1)$ & $\mathrm{Mo}(1) \quad \mathrm{Cl}(1)$ & $84,95[5]$ & $\mathrm{C}(12)$ & $\mathrm{P}(1)$ & $\mathrm{C}(6)$ & $101,69[5]$ \\
\hline $\mathrm{S}(2)$ & $\operatorname{Mo}(1) \operatorname{Mo}(1)^{B}$ & $99,18[4]$ & $\mathrm{C}(12)$ & $\mathrm{P}(1)$ & $\mathrm{C}(1)$ & $106,18[3]$ \\
\hline $\mathrm{S}(2)^{\mathrm{B}}$ & $\operatorname{Mo}(1) \operatorname{Mo}(1)^{B}$ & $53,51[1]$ & $\mathrm{C}(1)$ & $\mathrm{P}(1)$ & $\operatorname{Mo}(1)$ & $98,03[2]$ \\
\hline $\mathrm{S}(2)^{\mathrm{B}}$ & $\operatorname{Mo}(1) \operatorname{Mo}(1)^{A}$ & $98,70[2]$ & $\mathrm{C}(7)$ & $C(6)$ & $\mathrm{P}(1)$ & $118,38[4]$ \\
\hline $\mathrm{S}(2)$ & $\operatorname{Mo}(1) \operatorname{Mo}(1)^{A}$ & $54,16[1]$ & $\mathrm{C}(11)$ & $\mathrm{C}(6)$ & $\mathrm{P}(1)$ & $121,95[4]$ \\
\hline $\mathrm{S}(2)^{\mathrm{B}}$ & $\operatorname{Mo}(1) \quad \mathrm{P}(1)$ & $86,70[6]$ & $\mathrm{C}(13)$ & $\mathrm{C}(12)$ & $\mathrm{P}(1)$ & $122,92[2]$ \\
\hline $\mathrm{S}(2)$ & $\mathrm{Mo}(1) \quad \mathrm{P}(1)$ & $83,64[9]$ & $C(17)$ & $\mathrm{C}(12)$ & $\mathrm{P}(1)$ & $117,75[3]$ \\
\hline $\mathrm{S}(2)$ & $\operatorname{Mo}(1) \quad S(1)$ & $107,52[3]$ & $\operatorname{Mo}(1)^{A}$ & $\mathrm{~S}(1)$ & $\operatorname{Mo}(1)^{B}$ & $70,08[2]$ \\
\hline $\mathrm{S}(2)^{\mathrm{B}}$ & $\operatorname{Mo}(1) \quad S(1)$ & $106,90[4]$ & $\operatorname{Mo}(1)^{A}$ & $\mathrm{~S}(1)$ & $\operatorname{Mo}(1)$ & $70,08[2]$ \\
\hline $\mathrm{S}(2)^{\mathrm{B}}$ & $\operatorname{Mo}(1) \quad S(2)$ & $96,41[4]$ & $\operatorname{Mo}(1)^{B}$ & $\mathrm{~S}(1)$ & $\operatorname{Mo}(1)$ & $70,08[2]$ \\
\hline $\mathrm{S}(2)^{\mathrm{B}}$ & $\mathrm{Mo}(1) \quad \mathrm{Cl}(1)$ & $89,45[8]$ & $\operatorname{Mo}(1)^{A}$ & $\mathrm{~S}(2)$ & $\operatorname{Mo}(1)$ & $72,33[1]$ \\
\hline $\mathrm{S}(2)$ & $\mathrm{Mo}(1) \quad \mathrm{Cl}(1)$ & $163,86[1]$ & $\mathrm{C}(5)$ & $\mathrm{N}(1)$ & $\operatorname{Mo}(1)$ & $122,41[4]$ \\
\hline $\mathrm{S}(2)^{\mathrm{B}}$ & $\operatorname{Mo}(1) \quad \mathrm{N}(1)$ & $162,94[7]$ & $C(5)$ & $\mathrm{N}(1)$ & $\mathrm{C}(2)$ & $104,90[1]$ \\
\hline $\mathrm{S}(2)$ & $\operatorname{Mo}(1) \quad \mathrm{N}(1)$ & $87,25[7]$ & $\mathrm{C}(2)$ & $\mathrm{N}(1)$ & $\operatorname{Mo}(1)$ & $117,70[7]$ \\
\hline $\mathrm{Cl}(1)$ & $\operatorname{Mo}(1) \operatorname{Mo}(1)^{A}$ & $139,79[5]$ & $\mathrm{N}(1)$ & $C(5)$ & $C(4)$ & $103,57[4]$ \\
\hline $\mathrm{Cl}(1)$ & $\operatorname{Mo}(1) \operatorname{Mo}(1)^{B}$ & $96,40[5]$ & $\mathrm{C}(2)$ & $\mathrm{C}(1)$ & $\mathrm{P}(1)$ & $111,05[2]$ \\
\hline $\mathrm{Cl}(1)$ & $\operatorname{Mo}(1) \quad \mathrm{P}(1)$ & $81,54[11]$ & $\mathrm{N}(1)$ & $\mathrm{C}(2)$ & $C(1)$ & $110,86[4]$ \\
\hline $\mathrm{N}(1)$ & $\operatorname{Mo}(1) \operatorname{Mo}(1)^{B}$ & $140,39[4]$ & $\mathrm{N}(1)$ & $\mathrm{C}(2)$ & $C(3)$ & $105,75[2]$ \\
\hline $\mathrm{N}(1)$ & $\operatorname{Mo}(1) \operatorname{Mo}(1)^{A}$ & $95,28[9]$ & & & & \\
\hline
\end{tabular}




\subsubsection{3. $(M)-\left[\mathrm{Mo}_{3} \mathrm{~S}_{4} \mathrm{Cl}_{3}((1 S, 2 R)-\mathrm{PPro})_{3}\right]^{+}\left((M)-7^{+}\right)$}

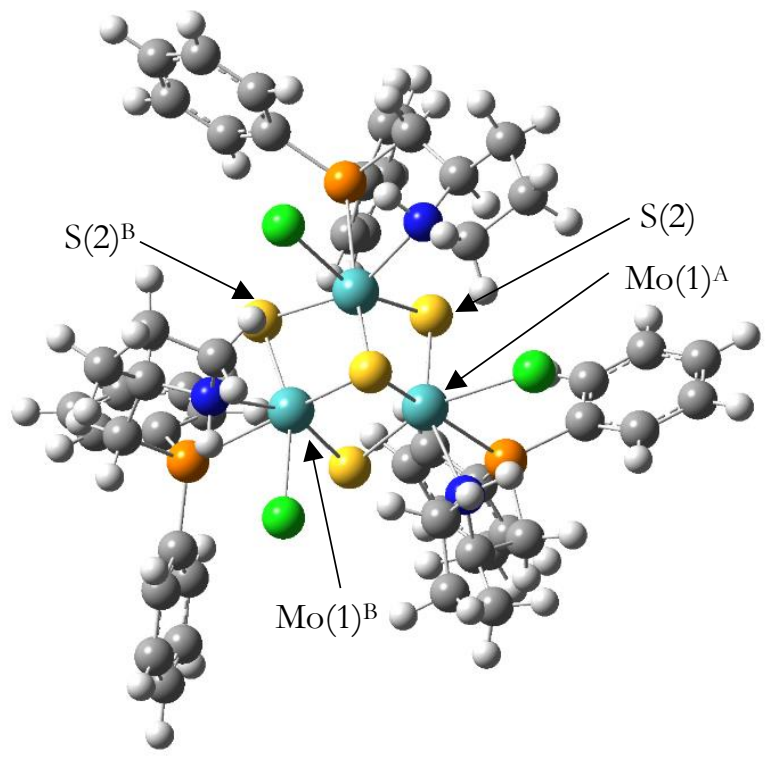

Figura 7.11 Geometría optimizada del complejo $(M)-\left[\mathrm{Mo}_{3} \mathrm{~S}_{4} \mathrm{Cl}_{3}((1 S, 2 \mathrm{R})-\mathrm{PPro})_{3}\right]^{+}$.

Tabla 7.29. Distancias de enlace representativas $(\AA)$.

\begin{tabular}{cccccc}
\hline \multicolumn{2}{c}{ Átomo } & Átomo & Distancia & \multicolumn{3}{c}{ Átomo } & Átomo & Distancia \\
\hline Mo & Mo & $2,8329[12]$ & Mo & P & $2,6720[41]$ \\
Mo & $\mu_{3}-S$ & $2,4604[19]$ & Mo & N & $2,2957[5]$ \\
Mo & $\mu-S^{(a)}$ & $2,3558[5]$ & Mo & $\mathrm{Cl}$ & $2,6009[17]$ \\
Mo & $\mu-S^{(b)}$ & $2,3763[7]$ & & & \\
\hline
\end{tabular}

(a) Distancia trans al enlace Mo-Cl.

(b) Distancia trans al enlace Mo-N.

[] Desviación estándar del promedio. 
Tabla 7.30. Ángulos de enlace representativos $\left(^{\circ}\right)$.

\begin{tabular}{|c|c|c|c|c|c|c|c|}
\hline \multicolumn{3}{|c|}{ Átomo Átomo Átomo } & \multirow{2}{*}{$\begin{array}{c}\text { Ángulo } \\
60,00[4]\end{array}$} & \multicolumn{3}{|c|}{ Átomo Átomo Átomo } & \multirow{2}{*}{$\begin{array}{c}\text { Ángulo } \\
75,68[2]\end{array}$} \\
\hline $\operatorname{Mo}(1)^{A}$ & $\operatorname{Mo}(1)$ & $\operatorname{Mo}(1)^{B}$ & & $\mathrm{~N}(1)$ & $\operatorname{Mo}(1)$ & $\mathrm{P}(1)$ & \\
\hline $\mathrm{P}(1)$ & $\operatorname{Mo}(1)$ & $\operatorname{Mo}(1)^{B}$ & $137,68[25]$ & $\mathrm{N}(1)$ & $\operatorname{Mo}(1)$ & $\mathrm{S}(1)$ & $90,66[16]$ \\
\hline $\mathrm{P}(1)$ & $\operatorname{Mo}(1)$ & $\operatorname{Mo}(1)^{A}$ & $135,69[24]$ & $\mathrm{N}(1)$ & $\operatorname{Mo}(1)$ & $\mathrm{Cl}(1)$ & $74,21[5]$ \\
\hline $\mathrm{S}(1)$ & $\operatorname{Mo}(1)$ & $\operatorname{Mo}(1)^{B}$ & $54,84[4]$ & $C(6)$ & $\mathrm{P}(1)$ & $\operatorname{Mo}(1)$ & $121,66[48]$ \\
\hline $\mathrm{S}(1)$ & $\operatorname{Mo}(1)$ & $\operatorname{Mo}(1)^{A}$ & $54,84[5]$ & $C(6)$ & $\mathrm{P}(1)$ & $\mathrm{C}(1)$ & $105,94[36]$ \\
\hline $\mathrm{S}(1)$ & $\operatorname{Mo}(1)$ & $\mathrm{P}(1)$ & $164,45[10]$ & $C(12)$ & $\mathrm{P}(1)$ & $\operatorname{Mo}(1)$ & $120,17[37]$ \\
\hline $\mathrm{S}(1)$ & $\operatorname{Mo}(1)$ & $\mathrm{Cl}(1)$ & $89,66[21]$ & $\mathrm{C}(12)$ & $\mathrm{P}(1)$ & $C(6)$ & $101,27[16]$ \\
\hline $\mathrm{S}(2)$ & $\operatorname{Mo}(1)$ & $\operatorname{Mo}(1)^{B}$ & $99,60[5]$ & $\mathrm{C}(12)$ & $\mathrm{P}(1)$ & $\mathrm{C}(1)$ & $105,32[10]$ \\
\hline $\mathrm{S}(2)^{\mathrm{B}}$ & $\operatorname{Mo}(1)$ & $\operatorname{Mo}(1)^{B}$ & $52,90[2]$ & $\mathrm{C}(1)$ & $\mathrm{P}(1)$ & $\operatorname{Mo}(1)$ & $100,76[7]$ \\
\hline $\mathrm{S}(2)^{\mathrm{B}}$ & $\operatorname{Mo}(1)$ & $\operatorname{Mo}(1)^{A}$ & $99,12[4]$ & $C(7)$ & $C(6)$ & $\mathrm{P}(1)$ & $118,67[36]$ \\
\hline $\mathrm{S}(2)$ & $\operatorname{Mo}(1)$ & $\operatorname{Mo}(1)^{A}$ & $53,53[2]$ & $\mathrm{C}(11)$ & $C(6)$ & $\mathrm{P}(1)$ & $122,04[35]$ \\
\hline $\mathrm{S}(2)^{\mathrm{B}}$ & $\mathrm{Mo}(1)$ & $\mathrm{P}(1)$ & $84,92[26]$ & $C(13)$ & $C(12)$ & $\mathrm{P}(1)$ & $120,42[15]$ \\
\hline $\mathrm{S}(2)$ & $\operatorname{Mo}(1)$ & $\mathrm{P}(1)$ & $82,19[27]$ & $C(17)$ & $C(12)$ & $\mathrm{P}(1)$ & $120,24[13]$ \\
\hline $\mathrm{S}(2)$ & $\operatorname{Mo}(1)$ & $\mathrm{S}(1)$ & $106,49[10]$ & $\operatorname{Mo}(1)^{A}$ & $\mathrm{~S}(1)$ & $\operatorname{Mo}(1)^{\mathrm{B}}$ & $70,31[5]$ \\
\hline $\mathrm{S}(2)^{\mathrm{B}}$ & $\operatorname{Mo}(1)$ & $\mathrm{S}(1)$ & $105,88[7]$ & $\operatorname{Mo}(1)^{A}$ & $\mathrm{~S}(1)$ & $\operatorname{Mo}(1)$ & $70,31[5]$ \\
\hline $\mathrm{S}(2)^{\mathrm{B}}$ & $\operatorname{Mo}(1)$ & $\mathrm{S}(2)$ & $99,20[7]$ & $\operatorname{Mo}(1)^{B}$ & $\mathrm{~S}(1)$ & $\operatorname{Mo}(1)$ & $70,31[5]$ \\
\hline $\mathrm{S}(2)^{\mathrm{B}}$ & $\operatorname{Mo}(1)$ & $\mathrm{Cl}(1)$ & $86,97[14]$ & $\operatorname{Mo}(1)^{A}$ & $\mathrm{~S}(2)$ & $\operatorname{Mo}(1)$ & $73,57[3]$ \\
\hline $\mathrm{S}(2)$ & $\operatorname{Mo}(1)$ & $\mathrm{Cl}(1)$ & $160,17[24]$ & $C(5)$ & $\mathrm{N}(1)$ & $\operatorname{Mo}(1)$ & $124,61[13]$ \\
\hline $\mathrm{S}(2)^{\mathrm{B}}$ & $\operatorname{Mo}(1)$ & $\mathrm{N}(1)$ & $154,95[10]$ & $C(5)$ & $\mathrm{N}(1)$ & $\mathrm{C}(2)$ & $106,95[2]$ \\
\hline $\mathrm{S}(2)$ & $\operatorname{Mo}(1)$ & $\mathrm{N}(1)$ & $93,77[19]$ & $C(2)$ & $\mathrm{N}(1)$ & $\operatorname{Mo}(1)$ & $114,68[8]$ \\
\hline $\mathrm{Cl}(1)$ & $\operatorname{Mo}(1)$ & $\operatorname{Mo}(1)^{A}$ & $144,38[26]$ & $\mathrm{N}(1)$ & $C(5)$ & $C(4)$ & $106,09[1]$ \\
\hline $\mathrm{Cl}(1)$ & $\operatorname{Mo}(1)$ & $\operatorname{Mo}(1)^{B}$ & $99,12[25]$ & $\mathrm{C}(2)$ & $\mathrm{C}(1)$ & $\mathrm{P}(1)$ & $110,83[14]$ \\
\hline $\mathrm{Cl}(1)$ & $\operatorname{Mo}(1)$ & $\mathrm{P}(1)$ & 79,59[29] & $\mathrm{N}(1)$ & $\mathrm{C}(2)$ & $\mathrm{C}(1)$ & $109,91[3]$ \\
\hline $\mathrm{N}(1)$ & $\operatorname{Mo}(1)$ & $\operatorname{Mo}(1)^{B}$ & $145,26[21]$ & $\mathrm{N}(1)$ & $C(2)$ & $C(3)$ & $103,76[2]$ \\
\hline $\mathrm{N}(1)$ & $\operatorname{Mo}(1)$ & $\operatorname{Mo}(1)^{A}$ & $105,81[11]$ & & & & \\
\hline
\end{tabular}


7.3.1.4. $(M)-\left[\mathrm{Mo}_{3} \mathrm{~S}_{4} \mathrm{Cl}_{3}((1 S, 2 S)-\mathrm{PPro})_{3}\right]^{+}\left((M)-8^{+}\right)$

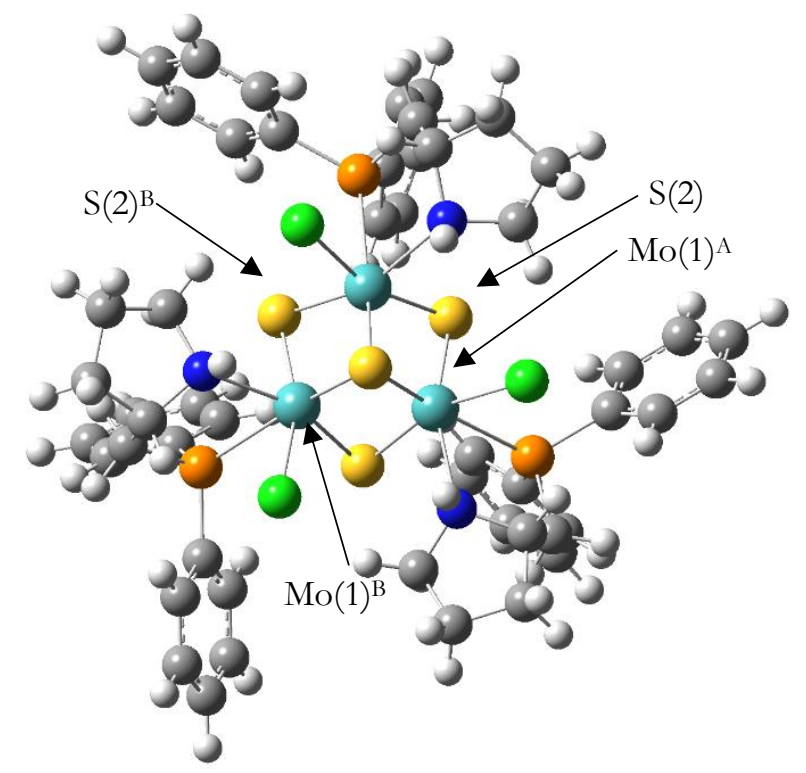

Figura 7.12. Geometría optimizada del complejo $(M)-\left[\mathrm{Mo}_{3} \mathrm{~S}_{4} \mathrm{Cl}_{3}((1 S, 2 S)-\mathrm{PPro})_{3}\right]^{+}$.

Tabla 7.31. Distancias de enlace representativas $(\AA)$.

\begin{tabular}{|c|c|c|c|c|c|}
\hline Átomo & Átomo & Distancia & Átomo & Átomo & Distancia \\
\hline Mo & Mo & $2,8446[18]$ & Mo & $\mathrm{P}$ & $2,6334[19]$ \\
\hline Mo & $\mu_{3}-S$ & $2,4491[11]$ & Mo & $\mathrm{N}$ & $2,3615[9]$ \\
\hline Mo & $\mu-S^{(a)}$ & $2,3712[7]$ & Mo & $\mathrm{Cl}$ & $2,5438[25]$ \\
\hline Mo & $\mu-S^{(b)}$ & 2,3761[8] & & & \\
\hline
\end{tabular}

(a) Distancia trans al enlace Mo-Cl.

(b) Distancia trans al enlace Mo-N.

[] Desviación estándar del promedio. 
Tabla 7.32. Ángulos de enlace representativos $\left(^{\circ}\right)$.

\begin{tabular}{|c|c|c|c|c|c|c|}
\hline \multicolumn{2}{|c|}{ Átomo Átomo Átomo } & \multirow{2}{*}{$\begin{array}{c}\text { Ángulo } \\
60,00[6]\end{array}$} & \multicolumn{3}{|c|}{ Átomo Átomo Átomo } & \multirow{2}{*}{$\begin{array}{c}\text { Ángulo } \\
75,49[10]\end{array}$} \\
\hline $\operatorname{Mo}(1)^{A}$ & $\operatorname{Mo}(1) \operatorname{Mo}(1)^{B}$ & & $\mathrm{~N}(1)$ & $\operatorname{Mo}(1)$ & $\mathrm{P}(1)$ & \\
\hline $\mathrm{P}(1)$ & $\operatorname{Mo}(1) \operatorname{Mo}(1)^{B}$ & $136,15[25]$ & $\mathrm{N}(1)$ & $\operatorname{Mo}(1)$ & $\mathrm{S}(1)$ & $93,48[12]$ \\
\hline $\mathrm{P}(1)$ & $\operatorname{Mo}(1) \operatorname{Mo}(1)^{A}$ & $141,97[22]$ & $\mathrm{N}(1)$ & $\operatorname{Mo}(1)$ & $\mathrm{Cl}(1)$ & $77,20[28]$ \\
\hline $\mathrm{S}(1)$ & $\operatorname{Mo}(1) \operatorname{Mo}(1)^{B}$ & $54,50[1]$ & $C(6)$ & $\mathrm{P}(1)$ & $\operatorname{Mo}(1)$ & $118,88[9]$ \\
\hline $\mathrm{S}(1)$ & $\operatorname{Mo}(1) \operatorname{Mo}(1)^{A}$ & $54,50[5]$ & $C(6)$ & $\mathrm{P}(1)$ & $\mathrm{C}(1)$ & $105,67[10]$ \\
\hline $\mathrm{S}(1)$ & $\operatorname{Mo}(1) \quad \mathrm{P}(1)$ & $160,92[40]$ & $C(12)$ & $\mathrm{P}(1)$ & $\operatorname{Mo}(1)$ & $121,46[5]$ \\
\hline $\mathrm{S}(1)$ & $\mathrm{Mo}(1) \quad \mathrm{Cl}(1)$ & $81,14[10]$ & $\mathrm{C}(12)$ & $\mathrm{P}(1)$ & $C(6)$ & $101,98[10]$ \\
\hline $\mathrm{S}(2)$ & $\operatorname{Mo}(1) \operatorname{Mo}(1)^{B}$ & $98,42[9]$ & $\mathrm{C}(12)$ & $\mathrm{P}(1)$ & $\mathrm{C}(1)$ & $103,93[5]$ \\
\hline $\mathrm{S}(2)^{\mathrm{B}}$ & $\operatorname{Mo}(1) \operatorname{Mo}(1)^{B}$ & $53,11[1]$ & $\mathrm{C}(1)$ & $\mathrm{P}(1)$ & $\operatorname{Mo}(1)$ & $103,09[3]$ \\
\hline $\mathrm{S}(2)^{\mathrm{B}}$ & $\operatorname{Mo}(1) \operatorname{Mo}(1)^{A}$ & $98,31[11]$ & $\mathrm{C}(7)$ & $C(6)$ & $\mathrm{P}(1)$ & $119,23[6]$ \\
\hline $\mathrm{S}(2)$ & $\operatorname{Mo}(1) \operatorname{Mo}(1)^{A}$ & $53,27[5]$ & $\mathrm{C}(11)$ & $\mathrm{C}(6)$ & $\mathrm{P}(1)$ & $121,34[6]$ \\
\hline $\mathrm{S}(2)^{\mathrm{B}}$ & $\operatorname{Mo}(1) \quad \mathrm{P}(1)$ & $83,19[27]$ & $\mathrm{C}(13)$ & $\mathrm{C}(12)$ & $\mathrm{P}(1)$ & $120,77[8]$ \\
\hline $\mathrm{S}(2)$ & $\mathrm{Mo}(1) \quad \mathrm{P}(1)$ & $88,73[17]$ & $C(17)$ & $\mathrm{C}(12)$ & $\mathrm{P}(1)$ & $1219,78[6]$ \\
\hline $\mathrm{S}(2)$ & $\operatorname{Mo}(1) \quad S(1)$ & $106,32[13]$ & $\operatorname{Mo}(1)^{A}$ & $\mathrm{~S}(1)$ & $\operatorname{Mo}(1)^{B}$ & $71,01[4]$ \\
\hline $\mathrm{S}(2)^{\mathrm{B}}$ & $\operatorname{Mo}(1) \quad S(1)$ & $106,17[5]$ & $\operatorname{Mo}(1)^{A}$ & $\mathrm{~S}(1)$ & $\operatorname{Mo}(1)$ & $71,01[4]$ \\
\hline $\mathrm{S}(2)^{\mathrm{B}}$ & $\operatorname{Mo}(1) \quad S(2)$ & $96,27[18]$ & $\operatorname{Mo}(1)^{B}$ & $\mathrm{~S}(1)$ & $\operatorname{Mo}(1)$ & $71,01[4]$ \\
\hline $\mathrm{S}(2)^{\mathrm{B}}$ & $\mathrm{Mo}(1) \quad \mathrm{Cl}(1)$ & $96,85[2]$ & $\operatorname{Mo}(1)^{A}$ & $\mathrm{~S}(2)$ & $\operatorname{Mo}(1)$ & $73,63[5]$ \\
\hline $\mathrm{S}(2)$ & $\mathrm{Mo}(1) \quad \mathrm{Cl}(1)$ & $162,40[4]$ & $C(5)$ & $\mathrm{N}(1)$ & $\operatorname{Mo}(1)$ & $122,02[19]$ \\
\hline $\mathrm{S}(2)^{\mathrm{B}}$ & $\operatorname{Mo}(1) \quad \mathrm{N}(1)$ & $158,47[22]$ & $C(5)$ & $\mathrm{N}(1)$ & $\mathrm{C}(2)$ & $102,68[7]$ \\
\hline $\mathrm{S}(2)$ & $\operatorname{Mo}(1) \quad \mathrm{N}(1)$ & $86,33[18]$ & $\mathrm{C}(2)$ & $\mathrm{N}(1)$ & $\operatorname{Mo}(1)$ & $117,05[4]$ \\
\hline $\mathrm{Cl}(1)$ & $\operatorname{Mo}(1) \operatorname{Mo}(1)^{A}$ & $135,52[13]$ & $\mathrm{N}(1)$ & $C(5)$ & $C(4)$ & $104,92[2]$ \\
\hline $\mathrm{Cl}(1)$ & $\operatorname{Mo}(1) \operatorname{Mo}(1)^{B}$ & $98,82[11]$ & $\mathrm{C}(2)$ & $\mathrm{C}(1)$ & $\mathrm{P}(1)$ & $112,17[18]$ \\
\hline $\mathrm{Cl}(1)$ & $\operatorname{Mo}(1) \quad \mathrm{P}(1)$ & $81,25[39]$ & $\mathrm{N}(1)$ & $\mathrm{C}(2)$ & $C(1)$ & $110,04[4]$ \\
\hline $\mathrm{N}(1)$ & $\operatorname{Mo}(1) \operatorname{Mo}(1)^{B}$ & $147,76[14]$ & $\mathrm{N}(1)$ & $\mathrm{C}(2)$ & $C(3)$ & $104,77[8]$ \\
\hline $\mathrm{N}(1)$ & $\operatorname{Mo}(1) \operatorname{Mo}(1)^{A}$ & $100,30[38]$ & & & & \\
\hline
\end{tabular}




\subsection{BIBLIOGRAFÍA}

[1] MassLynx 4.1 ed, Waters Ltd., Milford, MA, 2006.

[2] Cotton, F. A.; Kibala, P. A.; Matusz, M.; McCaleb, C. S.; Sandor, R. B. W. Inorg. Chem. 1989, 28, 2623.

[3] Fedin, V. P.; Sokolov, M. N.; Gerasko, O. A.; Kolesov, B. A.; Fedorov, V. Y.; Mironov, A. V.; Yufit, D. S.; Slovohotov, Y. L.; Struchkov, Y. T. Inorg. Chim. Acta 1990, 175, 217.

[4] Ginsberg, A. P. Inorganic Syntheses; Vol. 27: Hoboken, NJ, USA, 1990.

[5] Fedin, V. P.; Sokolov, M. N.; Mironov, Y. V.; Kolesov, B. A.; Tkachev, S. V.; Fedorov, V. Y. Inorg. Chim. Acta 1990, 167, 39.

[6] Sasaki, M.; Sakane, G.; Ouchi, T.; Shibahara, T. J. Clust. Sci. 1998, 9, 25.

[7] Estevan, F.; Feliz, M.; Llusar, R.; Mata, J. A.; Uriel, S. Polyhedron 2001, 20, 527.

[8] Jager-Fiedler, U.; Klahn, M.; Arndt, P.; Baumann, W.; Spannenberg, A.; Burlakov, V. V.; Rosenthal, U. J. Mol. Catal. A-Chem. 2007, 261, 184.

[9] CrysAlis ${ }^{\text {Pro }}$, Agilent Technologies: Santa Clara, CA, USA, 2012.

[10] Blessing, R. H. Acta Crystallogr. Sect. A 1995, 51, 33.

[11] Sheldrick, G. M. Acta Crystallogr. Sect. A 2008, 64, 112.

[12] Sheldrick, G. M. Acta Crystallogr. Sect. C-Struct. Chem. 2015, 71, 3.

[13] Palatinus, L.; Chapuis, G. J. Appl. Crystallogr. 2007, 40, 786.

[14] Dolomanov, O. V.; Bourhis, L. J.; Gildea, R. J.; Howard, J. A. K.; Puschmann, H. J. Appl. Crystallogr. 2009, 42, 339.

[15] Farrugia, L. J. Appl. Crystallogr. 1997, 30, 565.

[16] Gaussian 09, M. J. Frisch; H. B. Schlegel; G. E. Scuseria; J. R. C. M. A. Robb, G. S., V. Barone, B. Mennucci, , H. N. G. A. Petersson, M. Caricato, X. Li, H. P. Hratchian, , J. B. A. F. Izmaylov, G. Zheng, J. L. Sonnenberg, M. Hada, , K. T. M. Ehara, R. Fukuda, J. Hasegawa, M. Ishida, T. Nakajima, , O. K. Y. Honda, H. Nakai, T. Vreven, J. A. Montgomery, Jr., , F. O. J. E. Peralta, M. Bearpark, J. J. Heyd, E. Brothers, V. N. Staroverov, K. N. Kudin, T. Keith, 
R. Kobayashi, J. Normand, A. Rendell, K. Raghavachari, J. C. Burant, S. S. Iyengar, J. Tomasi, , N. R. M. Cossi, J. M. Millam, M. Klene, J. E. Knox, J. B. Cross, C. Adamo, V. Bakken, J. Jaramillo, R. Gomperts, R. E. Stratmann, A. J. Austin, O. Yazyev, R. Cammi, C. Pomelli, J. W. Ochterski, , K. Morokuma, R. L. Martin, V. G. Zakrzewski, G. A. Voth, , J. J. Dannenberg P. Salvador, S. Dapprich, A. D. Daniels, J. B. Foresman, O. Farkas, J. V. Ortiz, J. Cioslowski, and G. and D. J. Fox; Gaussian, Inc., Wallingford CT, 2010.

[17] Becke, A. D. Phys. Rev. A 1988, 3098.

[18] Becke, A. D. J. Chem. Phys. 1993, 5648.

[19] C. T. Lee; Yang, W. T.; Parr, R. G. Phys. Rev. B 1988, 785.

[20] S. Noury, X. K., F. Fuster and B. Silvi Comput. Chem 1999, 597. 
8. CONCLUSIONES 


\section{CONCLUSIONES}

"Muchos fracasos de la vida han sido de hombres que no supieron darse cuenta de lo cerca que estaban del éxito cuando se rindieron." 
De los estudios experimentales y teóricos llevados a cabo en esta tesis doctoral se pueden extraer las siguientes conclusiones:

(i) La sustitución de cloruros por hidruros en el clúster precursor halogenado $\left[\mathrm{Mo}_{3} \mathrm{~S}_{4} \mathrm{Cl}_{3}(\text { dppe })_{3}\right]^{+}$mediante tratamiento con borohidruro conduce al complejo $\left[\mathrm{Mo}_{3} \mathrm{~S}_{4} \mathrm{H}_{3}(\mathrm{dppe})_{3}\right]^{+}$en rendimientos moderados. Este nuevo hidruro cuboidal preserva la simetría $C_{3}$ de su precursor, así como el estado de oxidación de los metales.

(ii) Los hidruros de fórmula $\left[\mathrm{M}_{3} \mathrm{~S}_{4} \mathrm{H}_{3}(\mathrm{dppe})_{3}\right]^{+}(\mathrm{M}=\mathrm{Mo}, \mathrm{W})$ catalizan de forma regioselectiva la hidrodefluoración de la pentafluoropiridina en posición para en presencia de silanos como fuente de hidrógeno. Una disminución de la basicidad de la difosfina, dppe frente a dmpe, resulta en clústeres más activos. La reacción catalizada por el clúster $\left[\mathrm{W}_{3} \mathrm{~S}_{4} \mathrm{H}_{3}(\text { dppe })_{3}\right]^{+}$transcurre bajo activación térmica, mientras que para el resto de los complejos se requiere radiación microondas.

(iii) La reacción entre los precursores halogenados de fórmula $\left[\mathrm{M}_{3} \mathrm{~S}_{4} \mathrm{X}_{3}(\mathrm{dppe})_{3}\right]^{+}(\mathrm{M}=\mathrm{Mo}, \mathrm{W} ; \mathrm{X}=\mathrm{Cl}, \mathrm{Br})$ y una sal fluorada como el $\mathrm{CsF}$ resulta en un intercambio de ligandos halogenuro y constituye una vía de síntesis de los fluoruros $\left[\mathrm{M}_{3} \mathrm{~S}_{4} \mathrm{~F}_{3}(\mathrm{dppe})_{3}\right]^{+}$en rendimientos moderados. En presencia de silanos, estos compuestos son precatalizadores en el proceso de hidrodefluoración de la pentafluoropiridina, con rendimientos menores que en el caso de los hidruros análogos.

(iv) El precursor molecular de fórmula $\left[\mathrm{Mo}_{3} \mathrm{~S}_{4} \mathrm{Cl}_{4}\left(\mathrm{PPh}_{3}\right)_{3}\left(\mathrm{H}_{2} \mathrm{O}\right)_{2}\right]$ reacciona con los ligandos aminofosfina 2-(aminoetil)di-i-propilfosfina (ediprp), (R)-2-[(difenilfosfino)metil]pirrolidina ((R)-PPro) y (S)-2-[(difenilfosfino)metil]pirrolidina ((S)-PPro) para dar clústeres de fórmula $\left.\left[\mathrm{Mo}_{3} \mathrm{~S}_{4} \mathrm{Cl}_{3} \text { (aminofosfina) }\right]_{3}\right]^{+}$en altos rendimientos. La aminofosfina se coordina de una forma específica, con el átomo de fósforo en posición 
trans al azufre apuntado y el átomo de nitrógeno en posición trans a los azufres puente. En el caso de las aminofosfinas quirales (R)-PPro y $(S)$ PPro, los clústeres obtenidos de fórmula $(P)-\left[\mathrm{Mo}_{3} \mathrm{~S}_{4} \mathrm{Cl}_{3}((1 S, 2 \mathrm{R})-\mathrm{PPro})_{3}\right]^{+}$ y $(P)-\left[\mathrm{Mo}_{3} \mathrm{~S}_{4} \mathrm{Cl}_{3}((1 S, 2 S)-P P r o)_{3}\right]^{+}$son ópticamente puros. La resolución de su estructura cristalina nos permite asignar la configuración absoluta como $P$ en ambos casos y evidencia la presencia de tres centros estereogénicos en cada complejo.

(v) Las estructuras de $\quad(P)-\left[\mathrm{Mo}_{3} \mathrm{~S}_{4} \mathrm{Cl}_{3}((1 S, 2 \mathrm{R})-\mathrm{PPro})_{3}\right]^{+}$y $\quad(P)-$ $\left[\mathrm{Mo}_{3} \mathrm{~S}_{4} \mathrm{Cl}_{3}((1 S, 2 S)-\mathrm{PPro})_{3}\right]^{+}$así como las de los posibles isómeros $(M)-$ $\left[\mathrm{Mo}_{3} \mathrm{~S}_{4} \mathrm{Cl}_{3}((1 S, 2 \mathrm{R})-\mathrm{PPro})_{3}\right]^{+}$y $(M)-\left[\mathrm{Mo}_{3} \mathrm{~S}_{4} \mathrm{Cl}_{3}((1 S, 2 S)-\mathrm{PPro})_{3}\right]^{+}$se han optimizado mediante cálculos DFT. Los parámetros estructurales optimizados para los isómeros $P$ concuerdan con los obtenidos experimentalmente. Tras la realización de análisis de distribución electrónica mediante cálculos de NBO y ELF, se ha confirmado que la mayor estabilidad de los isómeros $P$ frente a los $M$ se debe a interacciones vecinales entre los ligandos cloruro y los hidrógenos de los grupos amino coordinados al metal adyacente.

(vi) La escisión de la fase sólida polimérica $\left\{\mathrm{W}_{3} \mathrm{~S}_{7} \mathrm{Br}_{4}\right\}_{\mathrm{n}}$ en presencia del ligando ediprp permite aislar el clúster de fórmula $\left[\mathrm{W}_{3} \mathrm{~S}_{4} \mathrm{Br}_{3}(\text { ediprp) })_{3}\right]\left(\mathrm{BPh}_{4}\right)$ en rendimientos moderados. La coordinación específica del ligando aminofosfina, con el átomo de fósforo en posición trans al azufre apuntado y el de nitrógeno en posición trans a los azufres puente, es equivalente a la observada en los clústeres análogos de molibdeno. Este clúster bromado reacciona con borohidruro para dar el complejo $\left[\mathrm{W}_{3} \mathrm{~S}_{4} \mathrm{H}_{3}(\text { ediprp) })_{3}\right]\left(\mathrm{BPh}_{4}\right)$, que se obtiene tras la sustitución de los ligandos bromuro por hidruros.

(vii) La coordinación del ligando aminodifosfina bis[(2-di-i-propilfosfino)etil]amina (PNP) a la unidad trinuclear $\mathrm{Mo}_{3} \mathrm{~S}_{4}$ tiene lugar a través 
de un átomo de fósforo, que se sitúa trans al azufre apuntado, y uno de nitrógeno, en posición trans a los azufres puente, de manera que un grupo fosfino permanece sin coordinar. El clúster $\left[\mathrm{Mo}_{3} \mathrm{~S}_{4} \mathrm{Cl}_{3}(\mathrm{PNP})_{3}\right]^{+}$reacciona con $\mathrm{AgBF}_{4}$, que abstrae los átomos de cloro y facilita la coordinación de este grupo fosfino para dar el complejo $\left[\mathrm{Mo}_{3} \mathrm{~S}_{4}(\mathrm{PNP})_{3}\right]^{4+}$.

(viii) Los clústeres de fórmula $\left[\mathrm{Mo}_{3} \mathrm{~S}_{4} \mathrm{Cl}_{3} \text { (aminofosfina) }\right]^{+}$catalizan la reducción de cetonas a alcoholes en presencia de agentes reductores tales como una mezcla de ácido fórmico y trietilamina o diferentes silanos. El catalizador más activo es el complejo $\left.\left[\mathrm{Mo}_{3} \mathrm{~S}_{4} \mathrm{Cl}_{3} \text { (ediprp) }\right]_{3}\right]^{+}$que, en combinación con $\mathrm{PhSiH}_{3}$ a $70{ }^{\circ} \mathrm{C}$ en THF, permite la obtención de $\alpha$ (trifluorometil)bencil alcohol en conversiones y rendimientos elevados. La presencia de un grupo amino primario y de cadenas alquílicas voluminosas enlazadas al fósforo favorece el proceso catalítico.

(ix) Los grupos fosfino no coordinados del clúster $\left[\mathrm{Mo}_{3} \mathrm{~S}_{4} \mathrm{Cl}_{3}(\mathrm{PNP})_{3}\right]^{+}$ reaccionan con $\left[\left\{\mathrm{RuCl}_{2}(\mathrm{Ar})\right\}_{2}\right](\mathrm{Ar}=p$-cimeno, benceno), lo que resulta en la coordinación de cada uno de estos grupos a un átomo de rutenio para generar los clústeres heterodimetálicos de fórmula $\left[\mathrm{Mo}_{3} \mathrm{~S}_{4} \mathrm{Cl}_{3}\left(\kappa^{2}(\mathrm{P}, \mathrm{N})-\left(\mathrm{RuCl}_{2}(\mathrm{Ar})\left(\kappa^{1}(\mathrm{P})-\mathrm{PNP}\right)\right)_{3}\right]^{+}\right.$, que contienen seis átomos metálicos. El clúster de fórmula $\left[\mathrm{Mo}_{3} \mathrm{~S}_{4} \mathrm{Cl}_{3}\left(\kappa^{2}(\mathrm{P}, \mathrm{N})-\left(\mathrm{RuCl}_{2}(\not p-\right.\right.\right.$ cimeno $\left.\left.\left(\kappa^{1}(\mathrm{P})-\mathrm{PNP}\right)\right)_{3}\right]^{+}$es activo en la hidrogenación de $\mathrm{CO}_{2}$ para formar $\mathrm{HCOOH}$. Cuando se utiliza $\mathrm{KHCO}_{3}$ como aditivo, la actividad del catalizador aumenta. 


\section{(ENGLISH VERSION)}

From the experimental and theoretical studies carried out in this thesis, the following conclusions can be extracted:

(i) The substitution of chlorine by hydrogen in $\left[\mathrm{Mo}_{3} \mathrm{~S}_{4} \mathrm{Cl}_{3}(\mathrm{dppe})_{3}\right]^{+}$occurs in the presence of borohydride salts and leads to the obtention of $\left[\mathrm{Mo}_{3} \mathrm{~S}_{4} \mathrm{H}_{3}(\mathrm{dppe})_{3}\right]^{+}$in moderate yields. This hydrido cluster maintains the $\mathrm{C}_{3}$ symmetry observed in the halogenated precursor. The oxidation state of molybdenum is also preserved.

(ii) The family of hydrido clusters $\left[\mathrm{M}_{3} \mathrm{~S}_{4} \mathrm{H}_{3}(\mathrm{dppe})_{3}\right]^{+}(\mathrm{M}=\mathrm{Mo}, \mathrm{W})$ catalyze the hydrodefluorination of pentafluoropyridine, that proceeds selectively at the para position in the presence of silanes. A diminution of the diphosphine basicity when replacing dmpe by dppe increases the catalytic activity of these clusters. When $\left[\mathrm{W}_{3} \mathrm{~S}_{4} \mathrm{H}_{3}(\mathrm{dppe})_{3}\right]^{+}$is used as a catalyst, the reaction takes place through thermal activation. However, microwave radiation is demanded for the rest of $\mathrm{M}_{3} \mathrm{~S}_{4}$ complexes.

(iii) The reaction of $\left[\mathrm{M}_{3} \mathrm{~S}_{4} \mathrm{X}_{3}(\mathrm{dppe})_{3}\right]^{+}(\mathrm{M}=\mathrm{Mo}, \mathrm{W} ; \mathrm{X}=\mathrm{Cl}, \mathrm{Br})$ and a fluorinated salt such as $\mathrm{CsF}$ results in a ligand exchange process where $\left[\mathrm{M}_{3} \mathrm{~S}_{4} \mathrm{~F}_{3}(\mathrm{dppe})_{3}\right]^{+}$clusters are synthesized in moderate yields. In the presence of silanes, these clusters show activity as precatalysts in the hydrodefluorination of pentafluoropyridine. Yields are lower than those obtained with the hydride analogous clusters.

(iv) The molecular precursor $\left[\mathrm{Mo}_{3} \mathrm{~S}_{4} \mathrm{Cl}_{4}\left(\mathrm{PPh}_{3}\right)_{3}\left(\mathrm{H}_{2} \mathrm{O}\right)_{2}\right]$ reacts with several aminophosphine ligands such as 2-(aminoethyl)di-i-propylphosphine (ediprp), (R)-2-[(diphenylphosphino)-methyl]pyrrolidine ((R)-PPro) and (S)-2-[(diphenylphosphino)methyl]pyrrolidine ((S)-PPro) to yield clusters of formula $\left.\left[\mathrm{Mo}_{3} \mathrm{~S}_{4} \mathrm{Cl}_{3} \text { (aminophosphine }\right)_{3}\right]^{+}$in high yields. The aminophosphines coordinate in a specific mode, with the phosphorus atom trans to the capping sulphur and the nitrogen atom trans to the 
bridging sulphur. When chiral aminophosphines (R)-PPro and ( $S$ )-PPro are used, optically pure clusters of formulae $(P)-\left[\mathrm{Mo}_{3} \mathrm{~S}_{4} \mathrm{Cl}_{3}((1 S, 2 \mathrm{R})\right.$ PPro $\left.)_{3}\right]^{+}$and $(P)-\left[\mathrm{Mo}_{3} \mathrm{~S}_{4} \mathrm{Cl}_{3}((1 S, 2 S)-\mathrm{PPro})_{3}\right]^{+}$are obtained. Their absolute configuration can be assigned as $P$ due to the study of their crystal structures. This study also indicates the presence of three stereogenic centers in every cluster complex.

(v) The structures of $(P)-\left[\mathrm{Mo}_{3} \mathrm{~S}_{4} \mathrm{Cl}_{3}((1 S, 2 \mathrm{R})-\mathrm{PPro})_{3}\right]^{+},(P)-\left[\mathrm{Mo}_{3} \mathrm{~S}_{4} \mathrm{Cl}_{3}((1 S, 2 S)-\right.$ PPro $\left.)_{3}\right]^{+},(M)-\left[\mathrm{Mo}_{3} \mathrm{~S}_{4} \mathrm{Cl}_{3}((1 S, 2 \mathrm{R})-\mathrm{PPro})_{3}\right]^{+}$and $(M)-\left[\mathrm{Mo}_{3} \mathrm{~S}_{4} \mathrm{Cl}_{3}((1 S, 2 S)-\right.$ PPro $\left.)_{3}\right]^{+}$have been optimized by means of DFT calculations. The optimized parameters in $P$ isomers agree with the experimental ones. $\mathrm{NBO}$ and ELF analyses prove that the larger stability of $P$ isomers is due to the vicinal interactions that take place between chlorine ligands and the hydrogens bonded to the amino groups, which are coordinated to an adjacent metal center.

(vi) The excision reaction of the polymeric phase $\left\{\mathrm{W}_{3} \mathrm{~S}_{7} \mathrm{Br}_{4}\right\}_{\mathrm{n}}$ with ediprp in acetonitrile represents a general synthetic route to prepare the trinuclear cluster of formula $\left[\mathrm{W}_{3} \mathrm{~S}_{4} \mathrm{Br}_{3}(\text { ediprp })_{3}\right]^{+}$in moderate yields. The coordination of the aminophosphine ligand, with the phosphorus atom placed trans to the capping sulphur and the nitrogen atom trans to the bridging sulphur, is equivalent to that observed in molybdenum analogous clusters. This bromide cluster reacts with borohydride to yield $\left[\mathrm{W}_{3} \mathrm{~S}_{4} \mathrm{H}_{3} \text { (ediprp) }\right]^{+}$through a ligand exchange reaction.

(vii) The coordination of the aminodiphosphine bis[(2-di-i-propylfosfino)ethyl]amine (PNP) to the trinuclear $\mathrm{Mo}_{3} \mathrm{~S}_{4}$ unit occurs through a phosphorus atom, placed trans to the capping sulphur, and the nitrogen atom, trans to the bridging sulphur, resulting in a dangling phosphino group. The cluster of formula $\left[\mathrm{Mo}_{3} \mathrm{~S}_{4} \mathrm{Cl}_{3}(\mathrm{PNP})_{3}\right]^{+}$reacts with $\mathrm{AgBF}_{4}$ to yield $\left[\mathrm{Mo}_{3} \mathrm{~S}_{4}(\mathrm{PNP})_{3}\right]^{4+}$, due to the reaction of the silver salt with chloride 
ligands that allows the coordination of the dangling phosphino group to the trimetallic unit.

(viii) Clusters of formula $\left[\mathrm{Mo}_{3} \mathrm{~S}_{4} \mathrm{Cl}_{3} \text { (aminophosphine) }{ }_{3}\right]^{+}$show activity in the catalytic reduction of ketones to alcohols in the presence of reducing agents such as silanes or a mixture of formic acid and triethylamine. $\left[\mathrm{Mo}_{3} \mathrm{~S}_{4} \mathrm{Cl}_{3}(\text { ediprp) })_{3}\right]^{+}$is the most active catalyst when $\mathrm{PhSiH}_{3}$ is used as a reducing agent in THF at $70{ }^{\circ} \mathrm{C}$, in order to obtain $\alpha$ (trifluoromethyl)benzyl alcohol with good conversions and yields. The use of aminophosphines which present primary amines and bulky alkyl groups bonded to the phosphorus atom, enhance the results of this catalytic process.

(ix) Uncoordinated phosphino groups in $\left[\mathrm{Mo}_{3} \mathrm{~S}_{4} \mathrm{Cl}_{3}(\mathrm{PNP})_{3}\right]^{+}$react with $\left[\left\{\mathrm{RuCl}_{2}(\mathrm{Ar})\right\}_{2}\right](\mathrm{Ar}=p$-cymene, benzene) resulting in the coordination of every phosphine to a ruthenium atom to yield the heterodimetallic clusters $\left[\mathrm{Mo}_{3} \mathrm{~S}_{4} \mathrm{Cl}_{3}\left(\kappa^{2}(\mathrm{P}, \mathrm{N})-\left(\mathrm{RuCl}_{2}(\mathrm{Ar})\left(\kappa^{1}(\mathrm{P})-\mathrm{PNP}\right)\right)_{3}\right]^{+}\right.$, which contain six metal atoms. The heterodimetallic cluster of formula $\left[\mathrm{Mo}_{3} \mathrm{~S}_{4} \mathrm{Cl}_{3}\left(\kappa^{2}(\mathrm{P}, \mathrm{N})-\left(\mathrm{RuCl}_{2}(p \text {-cymene })\left(\kappa^{1}(\mathrm{P})-\mathrm{PNP}\right)\right)_{3}\right]^{+}\right.$shows activity in the hydrogenation of $\mathrm{CO}_{2}$ to form $\mathrm{HCOOH}$. When $\mathrm{KHCO}_{3}$ is used as an additive, the catalytic activity of this heterodimetallic cluster increases. 
9. PUBLICACIONES 
9. PUBLICACIONES

"La ciencia más útil es aquella cuyo fruto es el más comunicable." Leonardo Da Vinci 
Los resultados presentados en esta tesis doctoral se han incluido en las siguientes publicaciones:

1) "Influence of the Diphosphine Coordinated to Molybdenum and Tungsten Triangular $\mathrm{M}_{3} \mathrm{~S}_{4}$ Cluster Hydrides in the Catalytic Hydrodefluorination of Pentafluoropyridine." Carmina Alfonso, Tomás F. Beltrán, Marta Feliz y Rosa Llusar, Journal of Cluster Science, 2015, 26, 199-209

2) " $\mathrm{C}_{3}$-symmetry $\mathrm{Mo}_{3} \mathrm{~S}_{4}$ Aminophosphino Clusters Combining Three Sources of Stereogenicity: Stereocontrol Directed by Hydrogen Bond Interactions and Ligand Configuration.” Carmina Alfonso, Marta Feliz, Vicent S. Safont y Rosa Llusar, Dalton Transactions, 2016, 45, 7829-7835

3) "Triangular $\mathrm{Mo}_{3} \mathrm{~S}_{4}$ Clusters Bearing PNP Ligands with Potential Applications in Catalysis." Carmina Alfonso, Marta Feliz y Rosa Llusar. Manuscrito en preparación.

4) "Synthesis of $\mathrm{M}_{3} \mathrm{~S}_{4}$ Aminophosphino Clusters and Their Catalytic Activity in the Reduction of Ketones". Carmina Alfonso, Marta Feliz y Rosa Llusar. Manuscrito en preparación. 
